FINAL REPORT

FHWA/IN/JTRP-2004/27

\title{
USING IMAGING TECHNOLOGY TO EVALUATE HIGHWAY SAFETY
}

\author{
by \\ Praprut Songchitruksa \\ Graduate Research Assistant \\ and \\ Andrew P. Tarko \\ Associate Professor of Civil Engineering \\ School of Civil Engineering \\ Purdue University \\ Joint Transportation Research Program \\ Project No: C-36-59JJ \\ File No: $8-5-36$ \\ SPR-2663 \\ Conducted in Cooperation with the \\ Indiana Department of Transportation \\ and the \\ U.S. Department of Transportation \\ Federal Highway Administration
}

The contents of this report reflect the views of the authors, who are responsible for the facts and the accuracy of the data presented herein. The contents do not necessarily reflect the official views or policies of the Indiana Department of Transportation or the Federal Highway Administration at the time of publication. The report does not constitute a standard, specification, or regulation.

Purdue University

West Lafayette, IN 47907

July 2004 


\section{TECHNICAL Summary}

INDOT Research

Technology Transfer and Project Implementation Information

TRB Subject Code: 54-9 Traffic Performance Measures

July 2004

Publication No.: FHWA/IN/JTRP-2004/27, SPR-2663

Final Report

\section{USING IMAGING TECHNOLOGY TO EVALUATE HIGHWAY SAFETY}

\section{Introduction}

Crash-based safety analysis is set back by several shortcomings such as randomness and rarity of crash occurrences, lack of timeliness, and inconsistency in crash reporting. Safety analysis based on observable traffic characteristics more frequent than crashes is one promising alternative. Traditional approach to alternative safety analysis relies on the assumption of constant risk across locations. In addition, the current practice of collecting surrogate data often suffers from inherent subjectivity of humans involved in the task. In this research, we proposed a novel statistical approach to safety estimation based on observable traffic characteristics. We evaluated the proposed method by applying to right-angle collisions at signalized intersections. 8-hour traffic movements at selected intersections were recorded using a Purdue University mobile traffic lab. A traffic characteristic so-called postencroachment time (PET) was observed as a surrogate safety measure to evaluate the risk of collisions. In addition, traditional approach to safety estimation using regression analyses was examined as well. The feasibility of facilitating the measurement of PET with digital video and image processing technology is also examined. Measurement alternatives considered in this study are image detection system 1 (commercial video detection system), image detection system 2 (proprietary developed software), and manual measurement.

\section{Findings}

Based upon the results of the measurement evaluation, both image detection systems were not sufficiently accurate for the purpose of our research. However, system 2 was found to perform better than system 1 if all of the following conditions are satisfied: (a) no camera vibration, (b) no obstacles in the field of view, and (c) no more than one through lane per approach. Post-detection of digitized video clips using the manual frame-by-frame analysis was therefore chosen for a collection of the evaluation data.

Poisson and negative binomial regression analyses indicate a significant relationship between PET counts and observed crash counts. A novel safety estimation approach alternative to regression analyses applies the extreme value theory to describe the behavior of PETs. The proposed method allows (a) estimation of risk and crash frequency, (b) model calibration using data from individual location, and (c) model calibration without historical crash data. The evaluation of the proposed method indicates a promising relationship between safety estimates and observed crash counts. The current problem of the proposed approach is a large variance of estimates due to insufficient observation period. A simulation experiment was conducted to examine this issue. It was found that the proposed method requires a few weeks of PET observation to obtain crash frequency estimates with confidence intervals comparable to those being obtained from 3 -year observed crash counts. Once a reliable automated measurement method is available in the future, the proposed safety estimation method will immediately offer a new possibility for unprecedented rapid highway safety evaluation. The proposed method can be applied to other types of collisions and locations as well. 


\section{Implementation}

Based upon the results in this study, a simple method to evaluate the risk of right-angle collisions at signalized intersections was provided. Using a count of short PETs at a studied intersection or individual conflict zone within an intersection, the corresponding annual frequency of right-angle collisions can be estimated. Also given is a guideline to help evaluate whether there is excessive risk of right-angle collisions.

\section{Contacts}

For more information:

Prof. Andrew Tarko

Principal Investigator

School of Civil Engineering

Purdue University

West Lafayette IN 47907

Phone: (765) 494-5027

Fax: (765) 496-7996

E-mail: tarko@ecn.purdue.edu

\section{Indiana Department of Transportation}

Division of Research

1205 Montgomery Street

P.O. Box 2279

West Lafayette, IN 47906

Phone: (765) 463-1521

Fax: (765) 497-1665

\section{Purdue University}

Joint Transportation Research Program

School of Civil Engineering

West Lafayette, IN 47907-1284

Phone: (765) 494-9310

Fax: (765) 496-7996

E-mail: jtrp@ecn.purdue.edu

http://www.purdue.edu/jtrp 


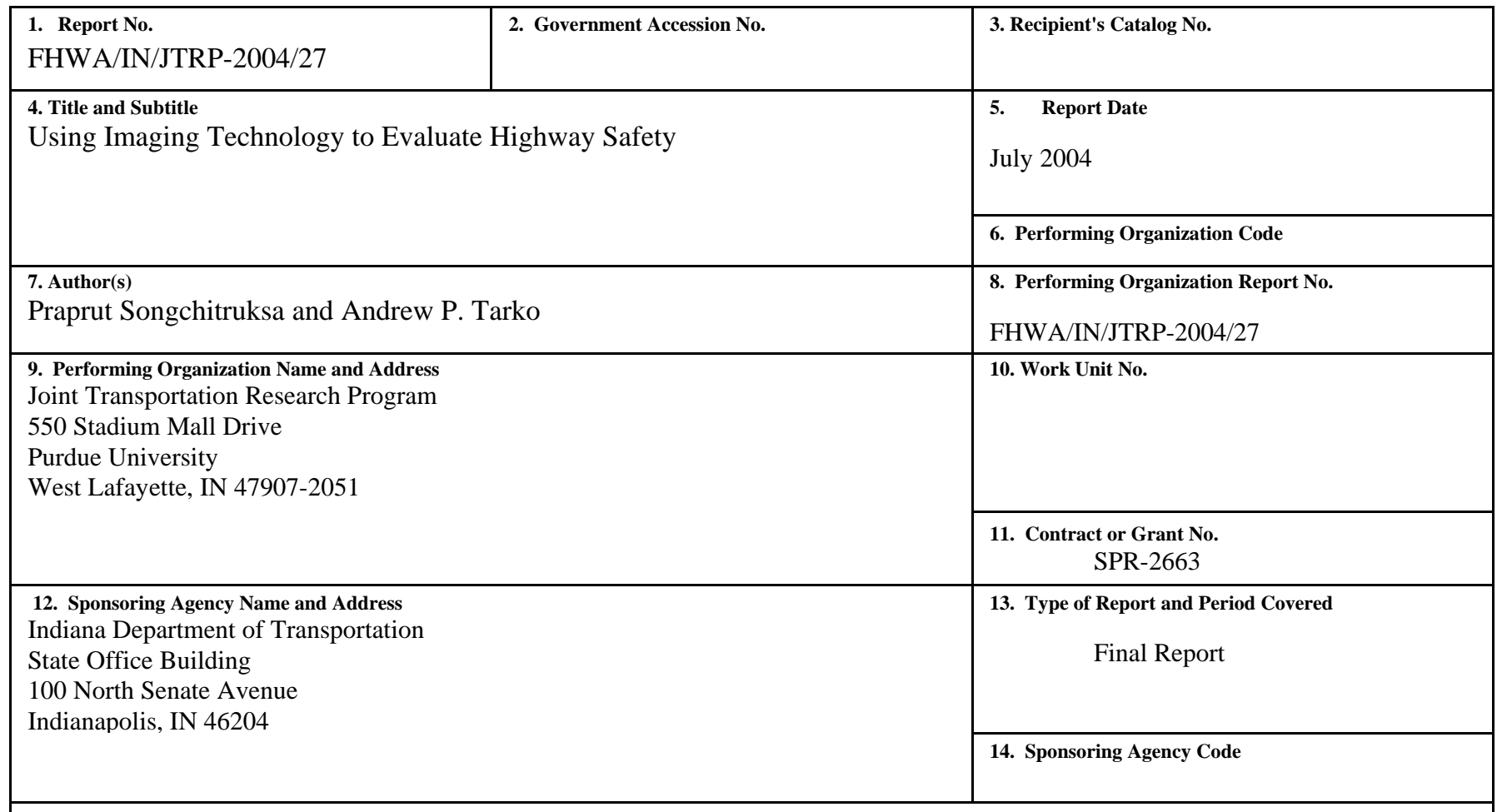

\section{Supplementary Notes}

Prepared in cooperation with the Indiana Department of Transportation and Federal Highway Administration.

\section{Abstract}

Crash-based safety analysis is set back by several shortcomings such as randomness and rarity of crash occurrences, lack of timeliness, and inconsistency in crash reporting. Non-crash-based safety analysis has been around for more than three decades but its potential was limited due to a difficulty in the data collection and the evaluation. Recent advancement in digital videos and image detection technology renewed our interest in facilitating the data collection and improving the evaluation method. Two image detection systems for the measurement of traffic characteristics were evaluated: (a) a commercial video detection system and (b) proprietary image processing software. The measurement evaluation revealed that both systems were still not sufficiently accurate for the safety evaluation purpose and thus a manual measurement from digitized video clips was preferred for a collection of evaluation data. We proposed a novel application of extreme value theory to safety evaluation based on observable traffic characteristics. The proposed method was evaluated by applying to right-angle collisions at signalized intersections. A traffic characteristic so-called post-encroachment time (PET) was collected at selected intersections as a surrogate safety measure. Based on PET characteristics, risk and frequency of rightangle crashes at the studied intersection or individual conflict zone can be estimated using only the data collected at the location. For comparison, a traditional approach to safety analysis using Poisson and negative binomial regression analyses was also examined. Both evaluation methods - extreme value approach and regression - indicate a significant relationship between PETs and historical crash data. Simulation experiments were carried out to examine the effect of observation period on a variance of estimates obtained the proposed method. Advantages and problems with the proposed method are described in this study. A simple method for an evaluation of the risk of right-angle collisions at signalized intersections is also provided in the appendix.

\section{Key Words}

safety evaluation, extreme value theory, risk estimation, surrogate safety measure, right-angle collision, video image processing, post-encroachment time.

\section{Distribution Statement}

No restrictions. This document is available to the public through the National Technical Information Service, Springfield, VA 22161

\begin{tabular}{|c|c|c|c|}
\hline $\begin{array}{c}\text { 19. Security Classif. (of this report) } \\
\text { Unclassified }\end{array}$ & 20. Security Classif. (of this page) & 21. No. of Pages & 22. Price \\
Unclassified & 428 & \\
\hline
\end{tabular}




\section{IMPLEMENTATION REPORT}

Based upon the results in this study, a simple method for an evaluation of risk of rightangle collisions at signalized intersections was provided in the Appendix B. This method estimates annual frequency of right-angle crashes based on a count of short PETs at individual conflict zone or the entire intersection depending on the study objective. Two methods for PET count were described - PET count from a video clip and on-site observation. The first method requires only one observer provided that desirable equipment specification is met. Short PETs can be counted accurately using the first method. The second method requires no special equipment other than stop watches and can be carried out on-site. However, more human observers are required and the method is prone to error if it is applied to an intersection that has (a) more than one through lane per approach and (b) heavy traffic volume.

If short PETs are counted for individual conflict zone, annual frequency of right-angle collisions can be estimated for that conflict zone using the given equation. This allows safety engineers to evaluate a location of safety concern within the intersection. In addition, the method can also estimate the right-angle crash frequency for the entire intersection using PET counts from all conflict zones. Based on 61 conflict zones observed in this study, we established a cumulative distribution of estimated right-angle crash frequency which can be used as a guideline to determine the risk level of the estimated right-angle crash frequency. 


\section{TABLE OF CONTENTS}

LIST OF TABLES vii

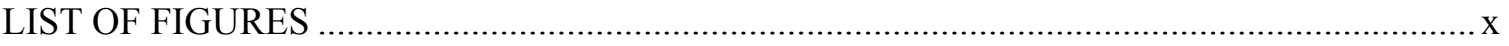

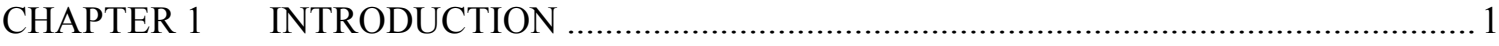

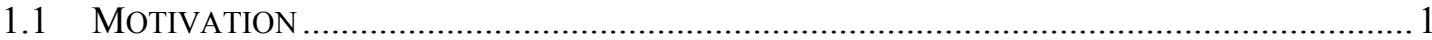

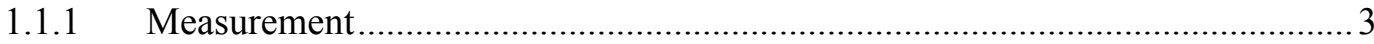

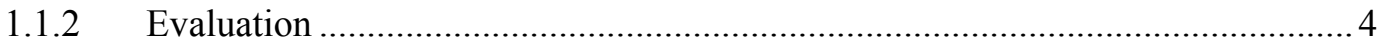

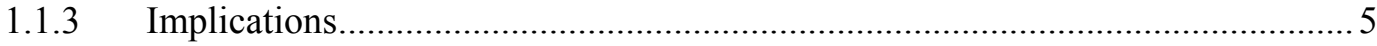

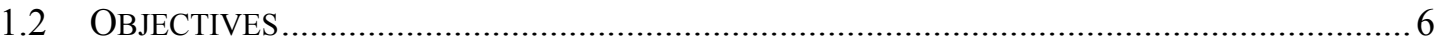

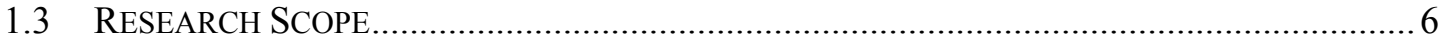

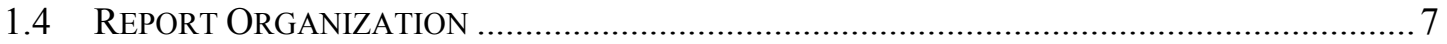

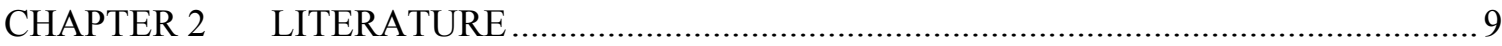

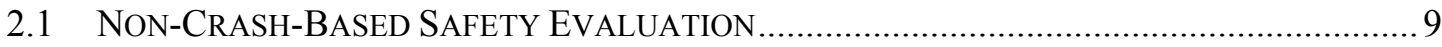

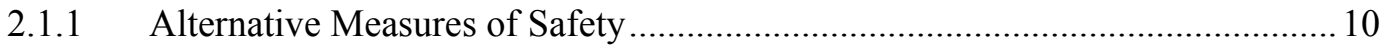

2.1.2 Past Practices of Traffic Conflict Technique .................................................... 15

2.1.3 Reliability and Validity of Non-Crash-Based Safety Measures......................... 19

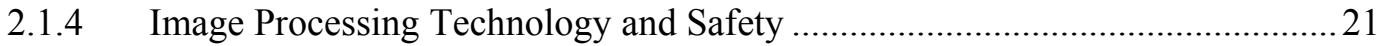

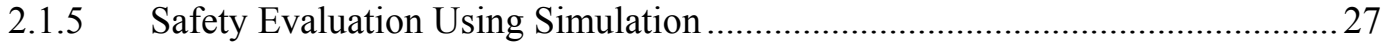

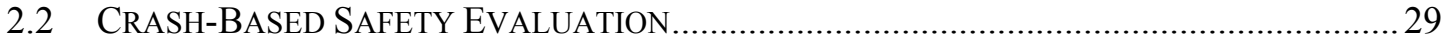

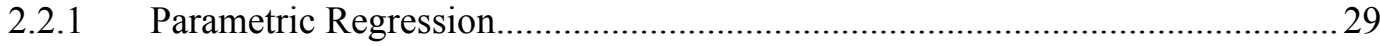

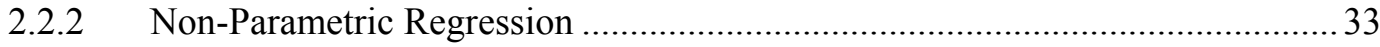

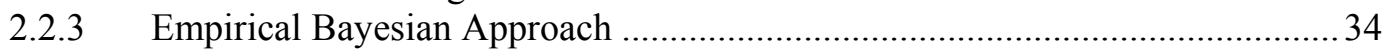

2.3 Signal Timings, Driver Attitudes, AND Right-Angle CRASHES............................ 37

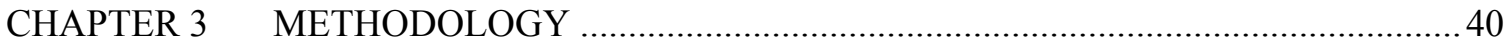

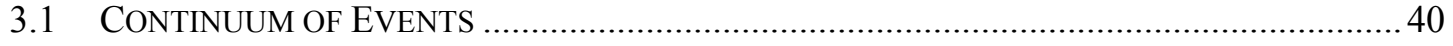

3.2 CURRENT APPROACH TO NON-CRASH-BASED SAFETY ANALYSIS .............................. 41

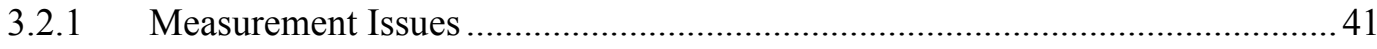

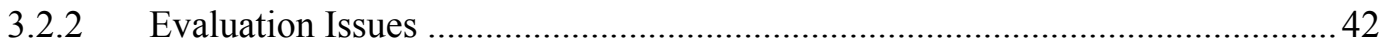




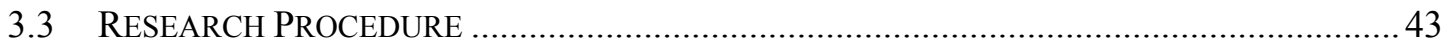

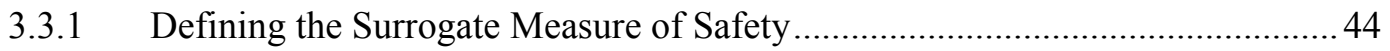

3.3.2 Data Collection and Measurement Evaluation................................................ 45

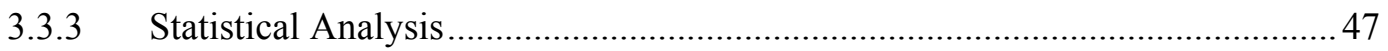

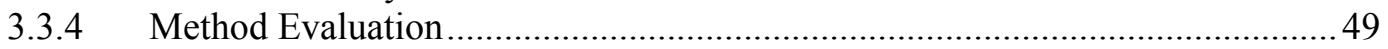

CHAPTER 4 DATA COLLECTION AND MEASUREMENT EVALUATION....................51

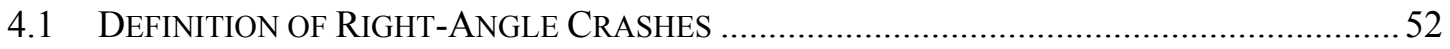

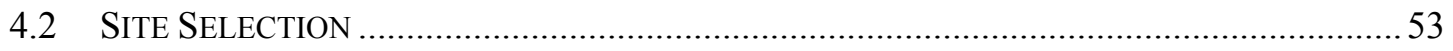

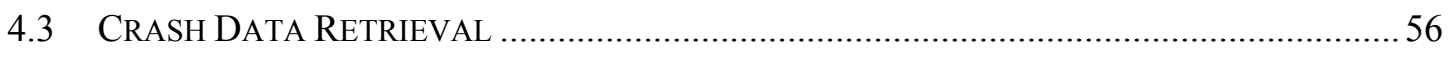

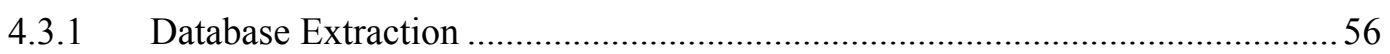

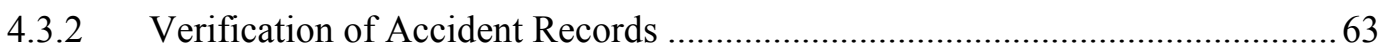

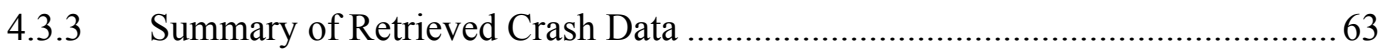

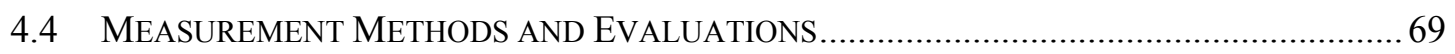

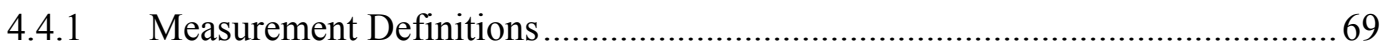

4.4.2 Overview of Measurement Methods and Limitations......................................... 71

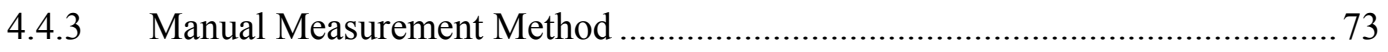

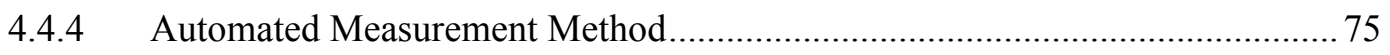

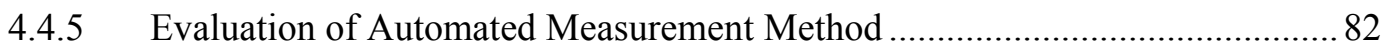

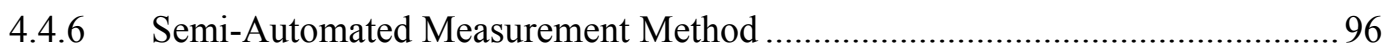

4.5 Data Collection Procedure ANd Collected Data .............................................. 101

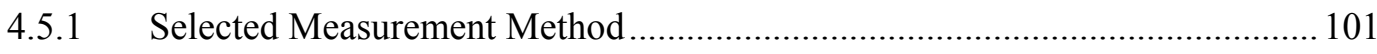

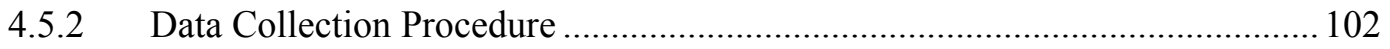

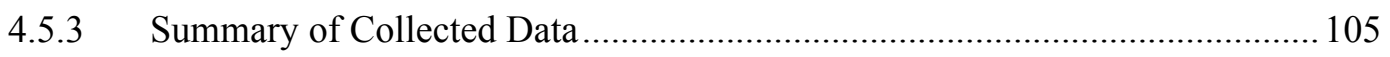

CHAPTER 5 REGRESSION ANALYSES OF SAFETY RELATIONSHIPS ...................... 113

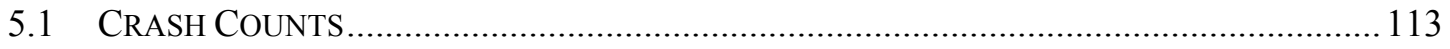

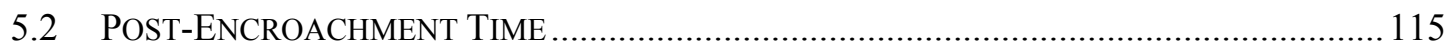

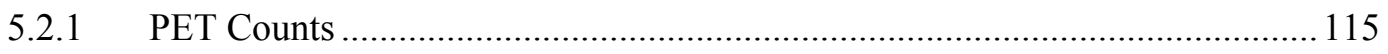

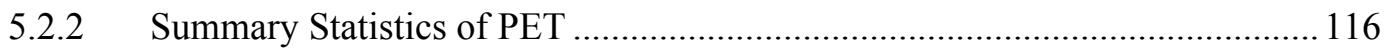

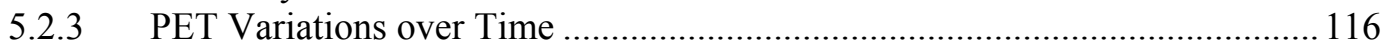

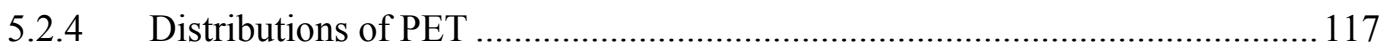

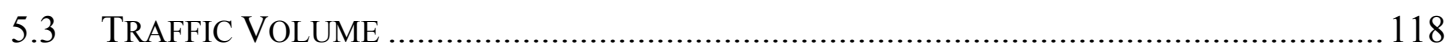

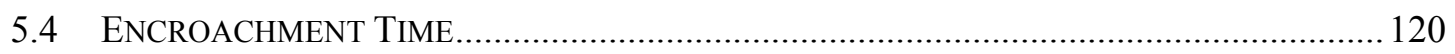

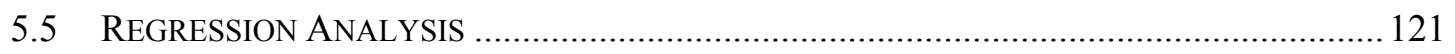

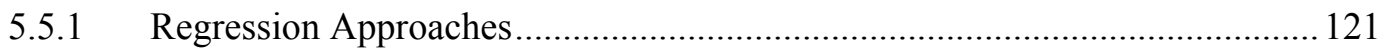

5.5.2 PET Counts versus Right-Angle Crash Counts .............................................. 126

5.5.3 Traffic Volume versus Right-Angle Crashes .................................................. 131

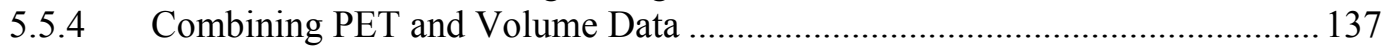




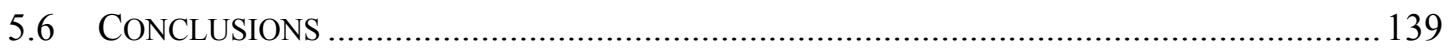

CHAPTER 6 EXTREME VALUE APPROACH FOR SAFETY ESTIMATIONS.............. 141

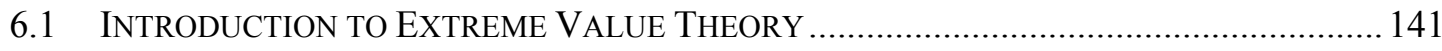

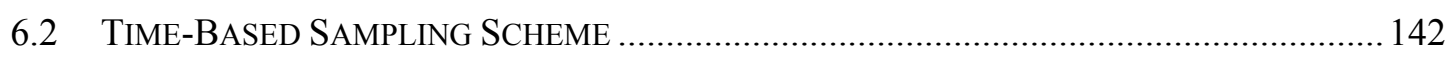

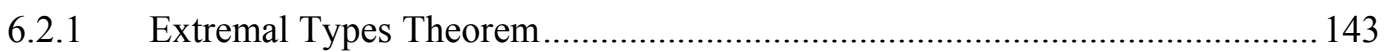

6.2.2 Generalized Extreme Value Model ................................................................. 143

6.2.3 Model Generalization: the r Largest Order Statistic Model............................. 148

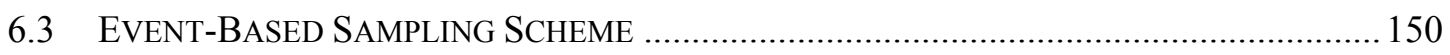

6.3.1 The Generalized Pareto Distribution............................................................ 150

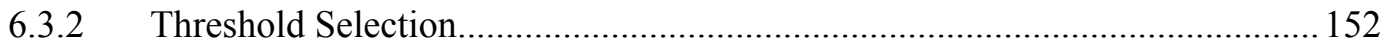

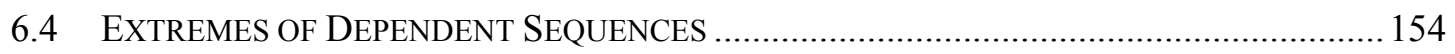

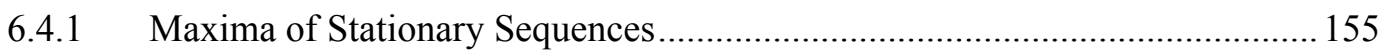

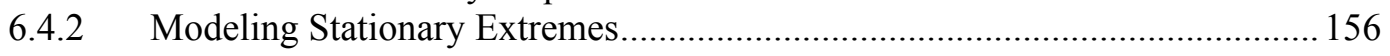

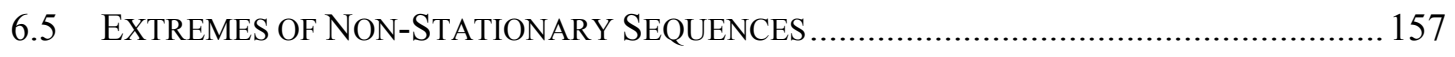

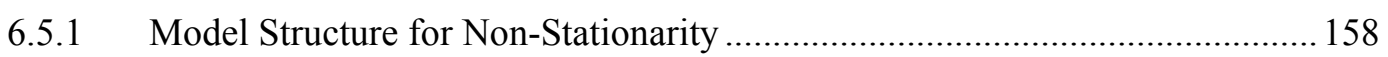

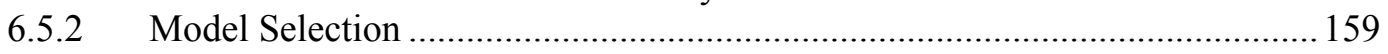

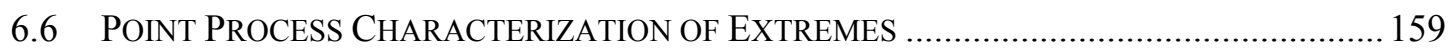

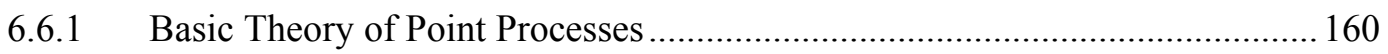

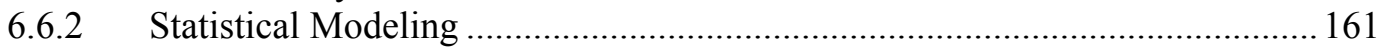

6.6.3 Connections with Threshold Excess Model Likelihood .................................. 162

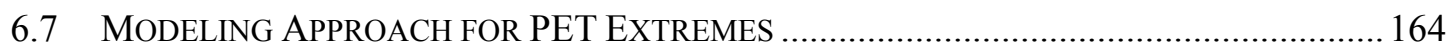

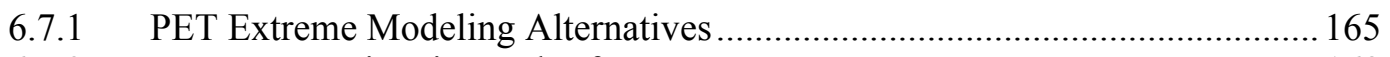

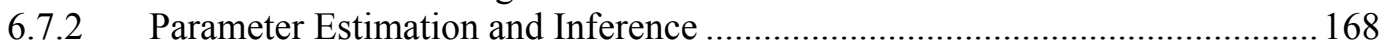

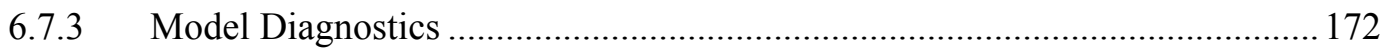

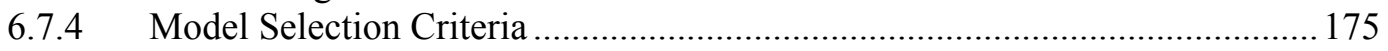

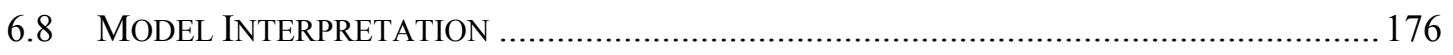

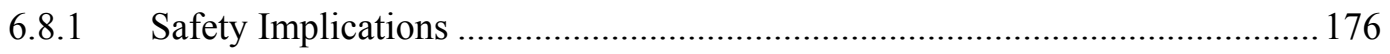

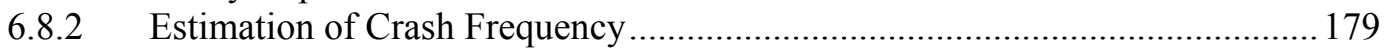

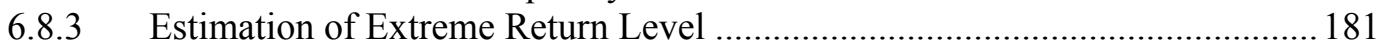

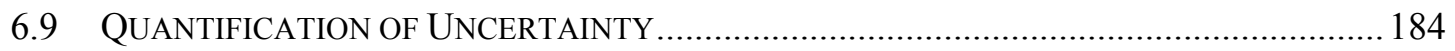

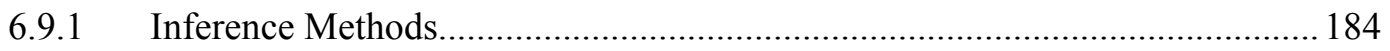

6.9.2 Guidelines for Method Selection ........................................................... 187

CHAPTER 7 MODEL DEVELOPMENT AND ESTIMATION RESULTS......................... 188 


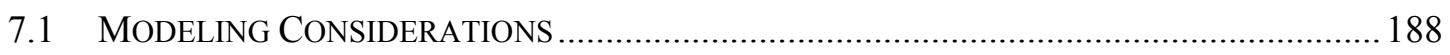

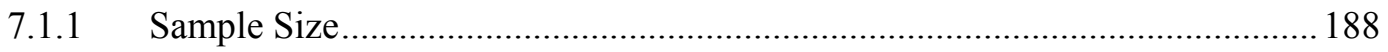

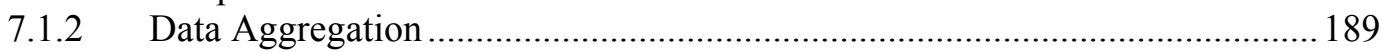

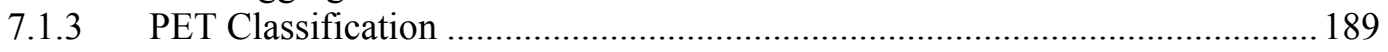

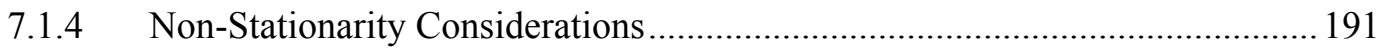

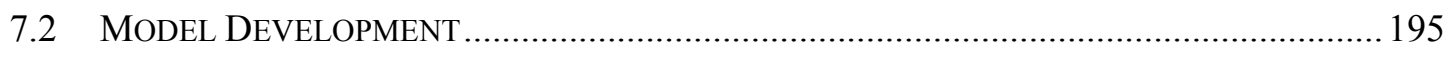

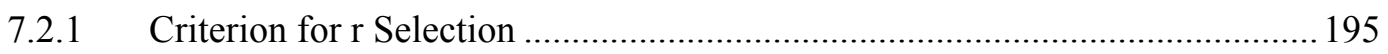

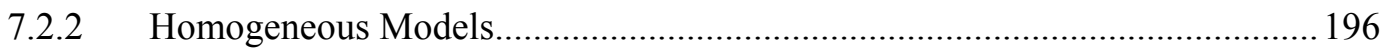

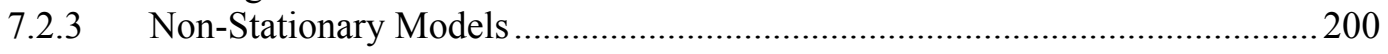

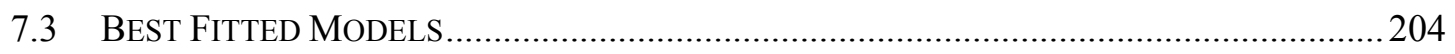

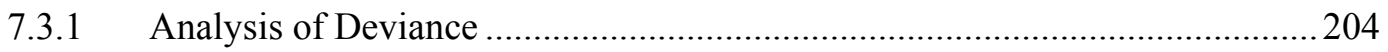

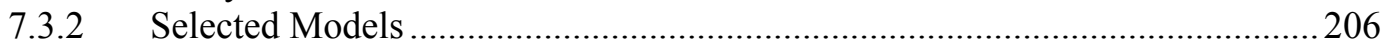

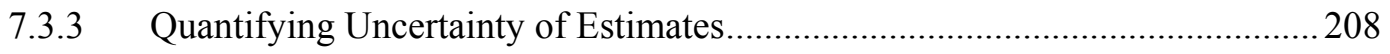

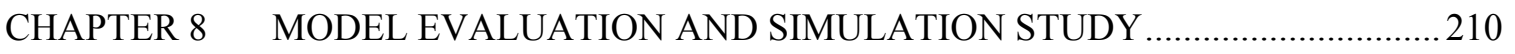

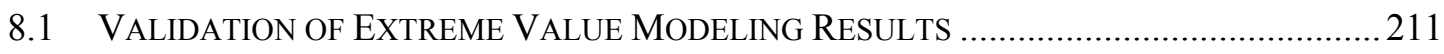

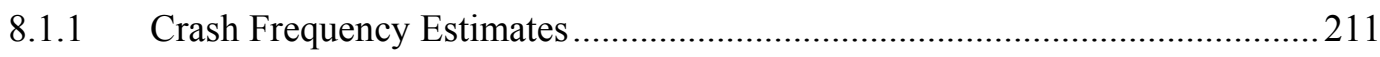

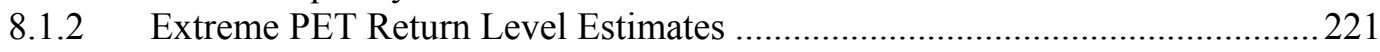

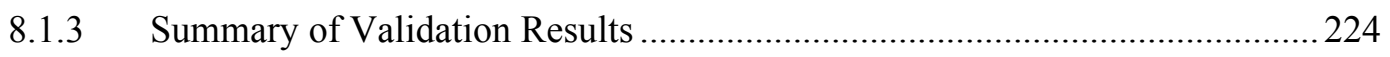

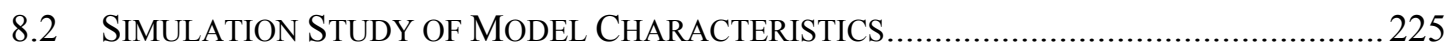

8.2.1 Constructing Underlying Parametric Distribution .......................................226

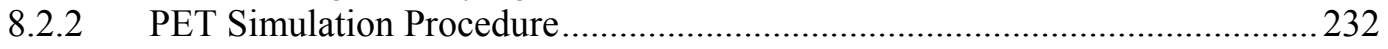

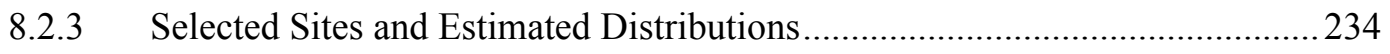

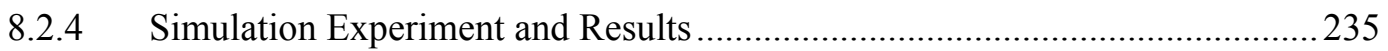

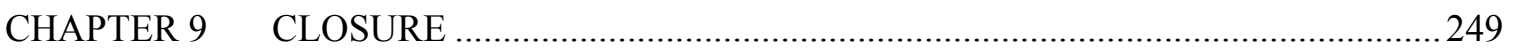

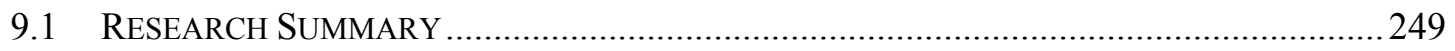

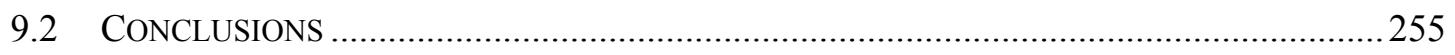

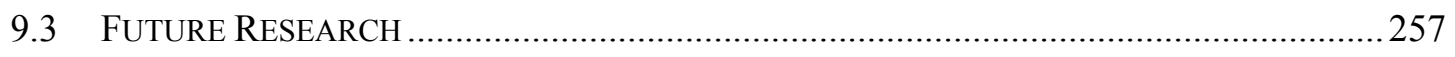

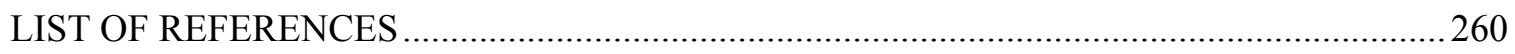

\section{APPENDICES}

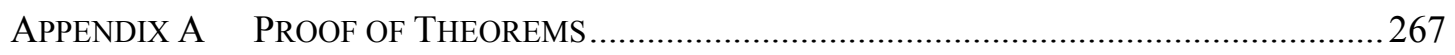

APPENDiX B EVALUATION OF Risk OF Right-ANGLE COLLISIONS...................................269

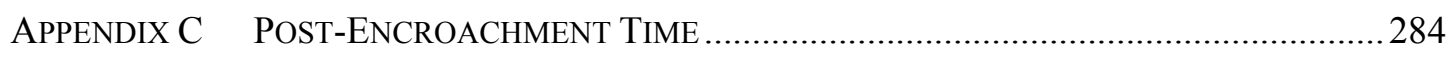

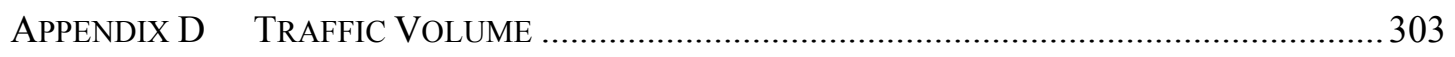

APPEndix E Estimation Results And Model Diagnostics ...................................... 312 


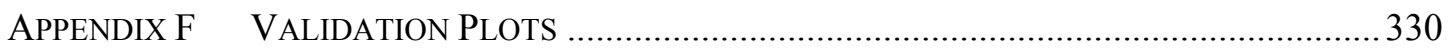

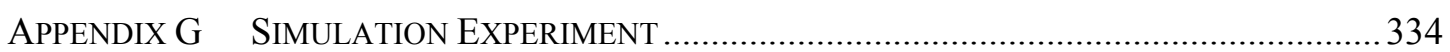

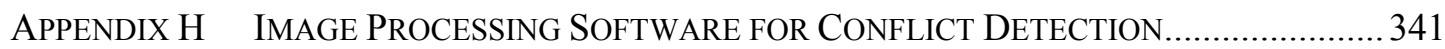




\section{LIST OF TABLES}

Table

Page

Table 2-1: Suggested Improvements for Signalized, 4-leg, 4-lane Intersections..... 16

Table 2-2: Suggested Improvements for Unsignalized, 4-leg, 2-lane Intersections 17

Table 2-3: Suggested Improvements for Unsignalized, 3-leg, 2-lane Intersections ...................... 18

Table 4-1: Preliminary List of Potential Studied Sites ................................................................ 55

Table 4-2: List of Possible Names of Intersecting Roads at Selected Intersections ......................57

Table 4-3: Results of Manual Verification with Crash Reports..................................................... 66

Table 4-4: Monthly Daytime Period for Crash Data Extraction .................................................. 67

Table 4-5: Summary of Right-Angle Crash Counts by Intersections (1997-2000) .......................67

Table 4-6: Summary of Right-Angle Crash Counts by Collision Zones (1997-2000) ..................68

Table 4-7: Example of Autoscope Event Data File ................................................................... 77

Table 4-8: Example of Output File with Gap Time ..................................................................... 79

Table 4-9: List of Selected Conflict Spots for Validation......................................................... 85

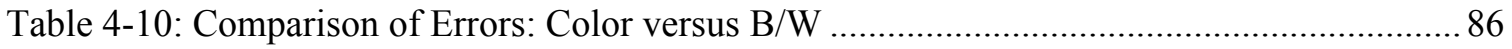

Table 4-11: Two-Sample t-tests of the Differences in Mean Errors: Color versus B/W ............... 86

Table 4-12: Conflict Spots and Configured Detector Numbers ................................................... 90

Table 4-13: Conditions of Observed Conflict Spots .................................................................. 91

Table 4-14: MOEs of Automated Method Classified by Conditions of Conflict Spots ................ 92

Table 4-15: Automated Method MOEs Grouped by Specific Conditions ..................................... 93

Table 4-16: Fitted Regression Model for Error Prediction ..................................................... 95

Table 4-17: Covariance Matrix of Parameter Estimates of the Fitted Regression Model .............98

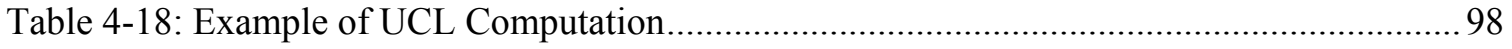

Table 4-19: MOEs of Semi-Automated Method Classified by Conditions of Conflict Spots..... 100

Table 4-20: MOEs of Semi-Automated Method (Excluding Far Conflict Spots) ....................... 100

Table 4-21: Summary of Periods of Extracted Surrogate Data................................................... 108

Table 4-22: Example of Recorded PET, GT, and ET Values ................................................. 110 


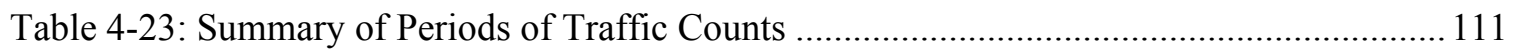

Table 5-1: Summary of Right-Angle Crash Counts in 1997 - 2000......................................... 114

Table 5-2: Count Summary of Observed PETs ........................................................................ 115

Table 5-3: Summary Statistics of Post-Encroachment Time and Signal Settings ....................... 116

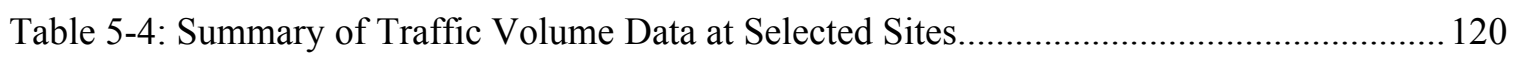

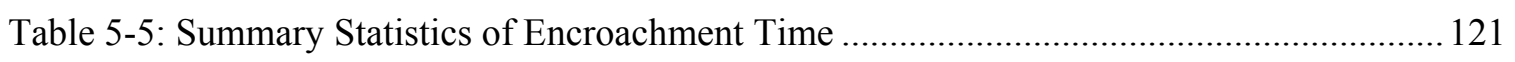

Table 5-6: Model-Selection Decision Guidelines at 95\% Confidence Level using Vuong Statistic

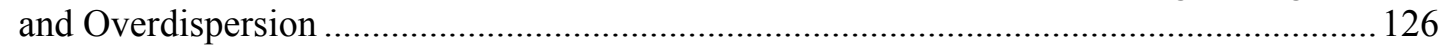

Table 5-7: Negative Binomial Model of PET Counts by Sites ................................................... 128

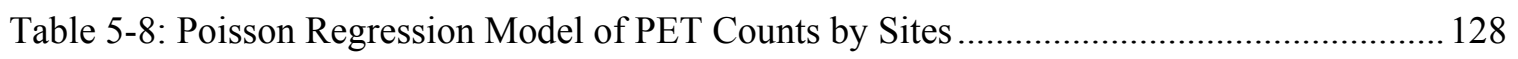

Table 5-9: Negative Binomial Model of PET Counts by Conflict Zones..................................... 130

Table 5-10: Zero-Inflated Negative Binomial Model of PET Counts by Conflict Zones............ 131

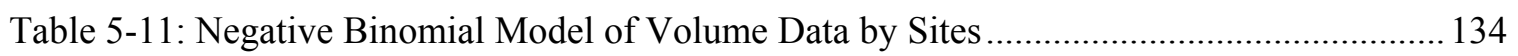

Table 5-12: Poisson Regression Model of Volume Data by Sites ............................................... 135

Table 5-13: Negative Binomial Model of Volume Data by Conflict Zones................................ 136

Table 5-14: Negative Binomial Model using PET and Volume Data by Sites............................. 137

Table 5-15: Poisson Regression Model using PET and Volume Data by Sites............................ 138

Table 5-16: Negative Binomial Model using PET and Volume Data by Conflict Zones............. 138

Table 5-17: Summary of Log-Likelihoods at Convergence of Selected Models.......................... 139

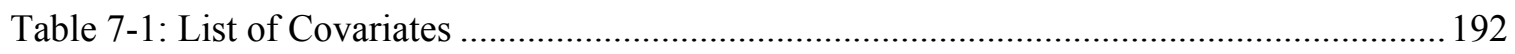

Table 7-2: Non-Stationary Models with Different Link Structures - Site 87933 ........................ 193

Table 7-3: Non-Stationary Models with Different Link Structures - Site 97901 ......................... 194

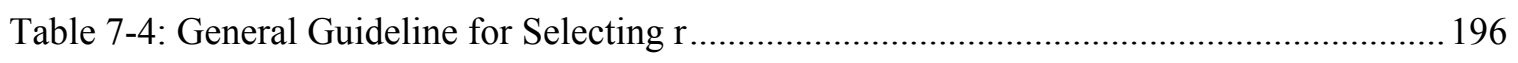

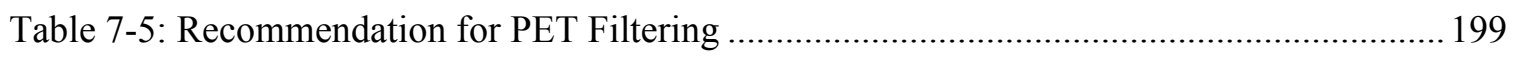

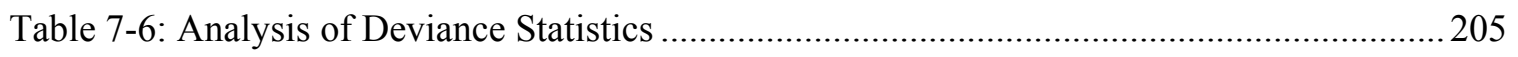

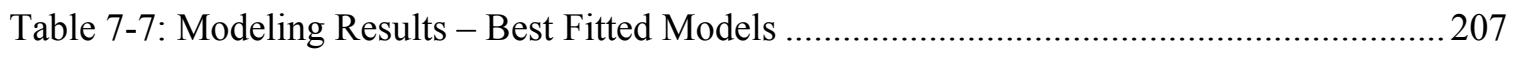

Table 8-1: Model-based Estimates of Crash Frequency and Inferences .....................................212

Table 8-2: Model-based Estimates of Return Level of Negated PETs and Inferences.................222

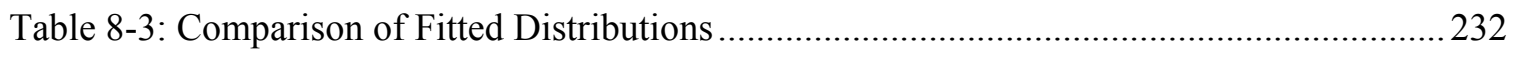

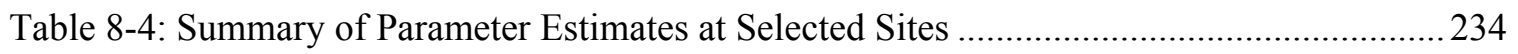

Table B-1: Example of Estimation of Right-Angle Crash Frequency at Site 87907 ...................278

Table B-2: Example of Estimation of Right-Angle Crash Frequency at Site 87909 ....................2 278 
Table B-3: Example of Estimation of Right-Angle Crash Frequency at Site 97901 ..................279

Table B-4: Annual Right-Angle Crash Frequency Estimates at $85^{\text {th }}$ and $90^{\text {th }}$ Percentiles ........... 282

Table E-1: Estimation Results of All PET Data Fitted to Homogeneous Models ........................ 313

Table E-2: Results - Homogeneous Models Using All PET Data............................................... 318

Table E-3: Results - Homogeneous Models Using Filtered PETs .............................................. 319

Table E-4: Results - Non-Stationary Models Using " $\mathrm{V}_{\mathrm{xth}}$ " ...................................................... 323

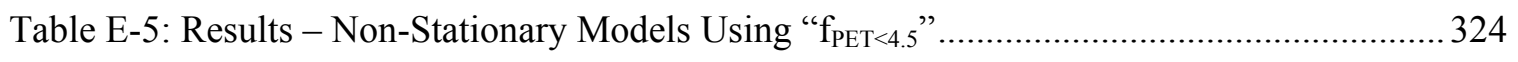

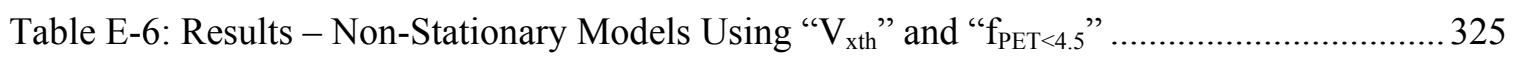

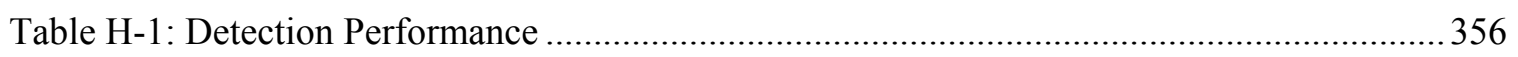

Table H-2: Detection Performance after Manual Check of False Detections.............................. 356

Table H-3: Processing Times of Video Clips (in multiples of real-time duration) ........................358

Table H-4: Coordinates of Objects Selected for the Camera Stabilization (for 720x480) ..........381

Table H-5: Results Obtained after Applying Vibration Removal Algorithm ............................... 383

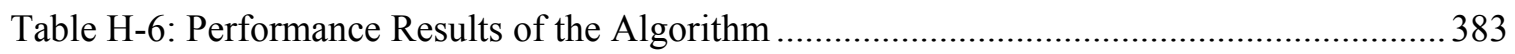

Table H-7: Relative Video Clips Processing Times in the Batch Mode between Previous and New

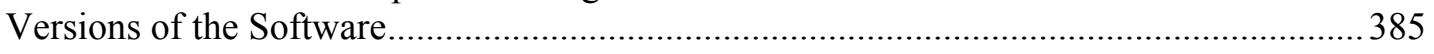

Table H-8: Results Obtained Using 3 Different Tracked Objects (Clip 97901_10) .................... 386

Table H-9: Results as in Table H-6 Using 3 Different Tracked Objects (Clip 97901_10).......... 386 


\section{LIST OF FIGURES}

Figure

Page

Figure 2-1: Frequency Distribution of Traffic Events (Chin and Quek, 1997)........................... 12

Figure 2-2: Proximity to Collision for Car-Following Situation (Allen et al., 1978) .................... 12

Figure 2-3: Concept of Time-Exposed TTC and Time-Integrated TTC ...................................... 14

Figure 2-4: Example of Regression Tree Using HTBR (Karlaftis and Golias, 2002) ................... 33

Figure 2-5: Logic of Estimation Using Empirical Bayesian Approach ........................................ 35

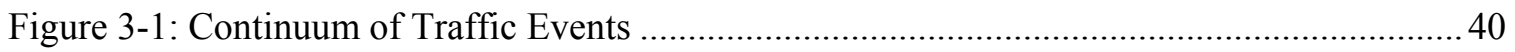

Figure 4-1: Straight versus Left-turn Right-Angle Collisions .....................................................52

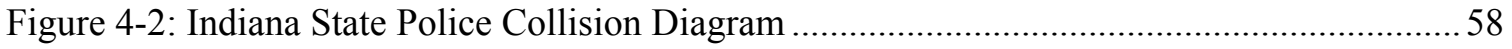

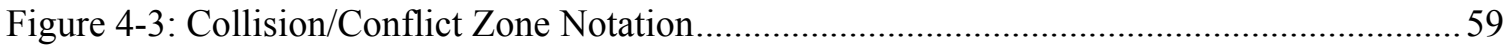

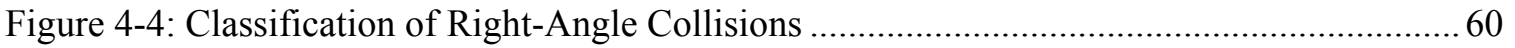

Figure 4-5: Classification of Opposing Left-Turn Collisions and Other Types ........................... 61

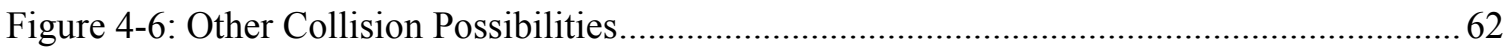

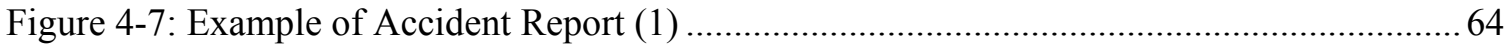

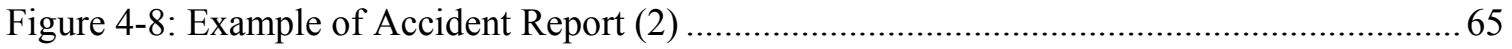

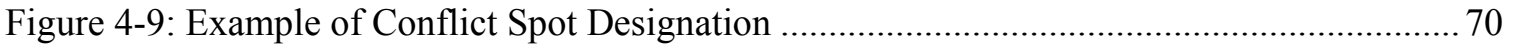

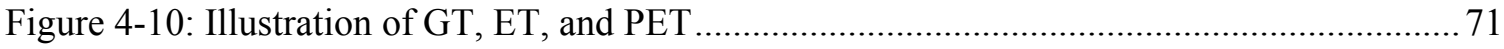

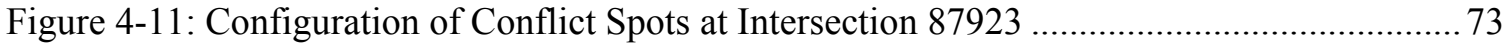

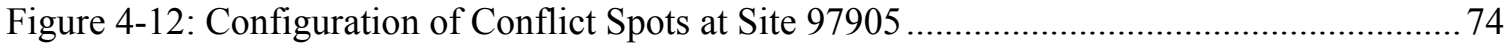

Figure 4-13: Example of Autoscope Detector Configuration for One Conflict Spot ................... 76

Figure 4-14: Detecting False Calls in the Autoscope Event File ................................................ 80

Figure 4-15: Interface for Processing of Autoscope Event File .................................................. 82

Figure 4-16: SR 26 @ Farabee Dr in Monochrome (B/W) mode.................................................. 83

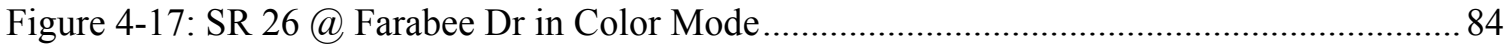

Figure 4-18: Detector Configuration with Corresponding ID................................................... 85

Figure 4-19: Field of View of SR-26 @ Earl Ave (No Shadow)................................................... 88 
Figure 4-20: Field of View of SR-26 @ Earl Ave (With Shadow)............................................... 88

Figure 4-21: Detector Configuration for TH-TH Conflicts ......................................................... 89

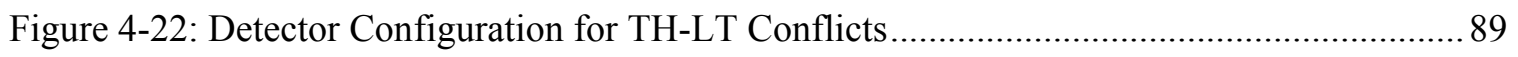

Figure 4-23: Histogram of Measurement Errors Using All Observations ..................................... 94

Figure 4-24: Purdue University Mobile Traffic Laboratory ....................................................... 103

Figure 4-25: Configuration of Conflict Spots at Intersection 97901 ........................................ 103

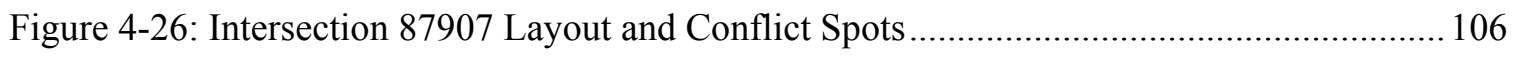

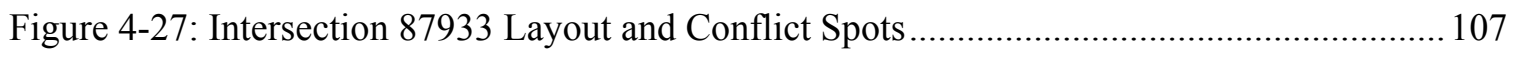

Figure 4-28: Excel Worksheet for Manual PET Measurement.................................................. 109

Figure 4-29: Autoscope Detector Configuration at Intersection 87907 ................................... 110

Figure 4-30: Autoscope Detector Configuration at Intersection 87933 …................................. 111

Figure 5-1: 15-Minute Traffic Volume Variations over Time at Site 87915 ................................ 118

Figure 5-2: Scatter Plots of 4-Year Crash Counts Versus 8-Hour PET Counts.............................. 127

Figure 5-3: Conflict Zone Counts of Right-Angle Collisions versus PET Counts ....................... 129

Figure 5-4: Scatter Plots of Volume Information versus Actual Counts of Crashes .................... 132

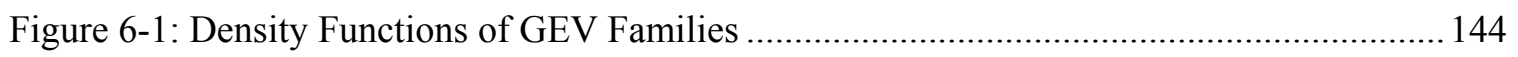

Figure 6-2: Behavior of Fréchet Density Function for Varying Shape Parameters ..................... 145

Figure 6-3: Behavior of Weibull Density Function for Varying Shape Parameters .................... 145

Figure 8-1: Model-based Crash Estimates versus Actual Crash Counts......................................213

Figure 8-2: Model-based Crash Estimates versus Actual Crash Counts (without 97903)...........214

Figure 8-3: Model Estimates versus Crash-based 95\% Poisson Confidence Intervals ................ 219

Figure 8-4: Model Estimates versus Crash-based 99\% Poisson Confidence Intervals ................ 219

Figure 8-5: Actual Counts of Collisions versus Extreme Return Level Estimates ...................... 222

Figure 8-6: Estimates of Crash Frequency versus Estimates of Return Level.............................223

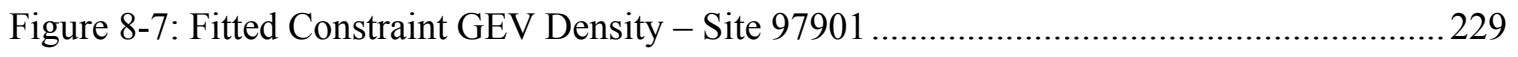

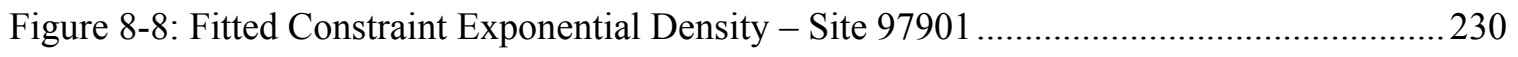

Figure 8-9: Fitted Constraint Generalized Pareto Distribution - Site 97901 ..............................231

Figure 8-10: Fitted Constraint Generalized Pareto Distribution - Site 87906 ............................. 234

Figure 8-11: Effect of Block Length on Crash Frequency Estimates - Site 87906 ..................... 237

Figure 8-12: Effect of Block Length on Crash Frequency Estimates - Site 97901 .....................238

Figure 8-13: Estimated Crash Frequency at Site 87906 Using $r=1$ and 30-minute Blocks ....... 238 
Figure 8-14: Diagnostic Plots of Model Fitted to 10 Days of Simulated PET at Site 87906 (block length $=15$ minutes and $r=1$ ).

Figure 8-15: Diagnostic Plots of Model Fitted to 10 Days of Simulated PET at Site 87906 (block length $=1$ hour and $r=1$ )

Figure 8-16: Effect of Choice of $r$ on Crash Frequency Estimates - Site 87906 (Observation = 1 to 10 days).

Figure 8-17: Effect of Choice of $r$ on Crash Frequency Estimates - Site 87906 (Observation $=10$ to 100 days).

Figure 8-18: Effect of Choice of $r$ on Crash Frequency Estimates - Site 97901 (Observation = 1 to 10 Days).

Figure 8-19: Effect of Choice of $r$ on Crash Frequency Estimates - Site 97901 (Observation $=10$ to 100 days).....

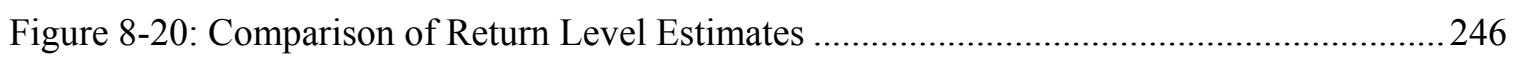

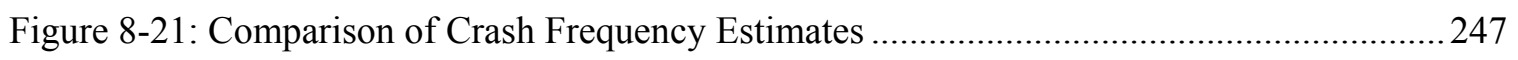

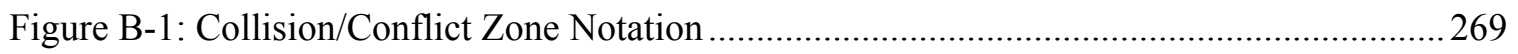

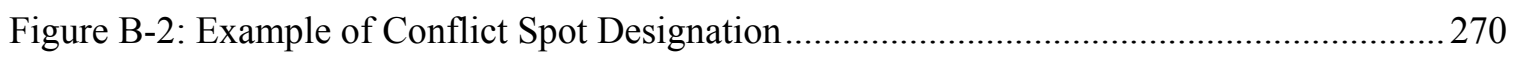

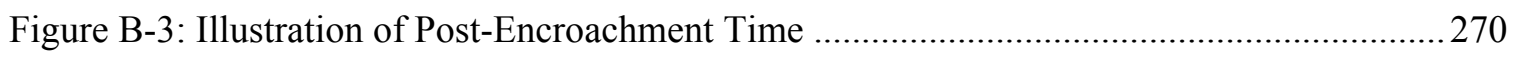

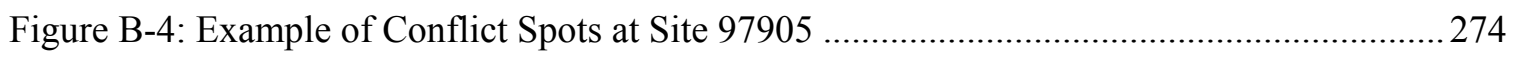

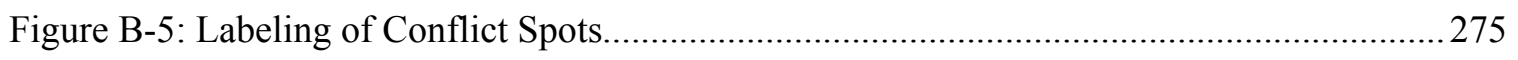

Figure B-6: Illustration of a Left-Turn Right-Angle Collision ................................................280

Figure B-7: Empirical Cumulative Distribution of Annual Daytime Right-Angle Crash Frequency

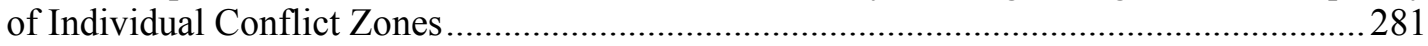

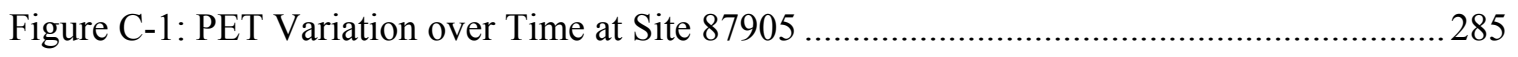

Figure C-2: PET Variation over Time by Conflict Zones at Site 87905 ....................................285

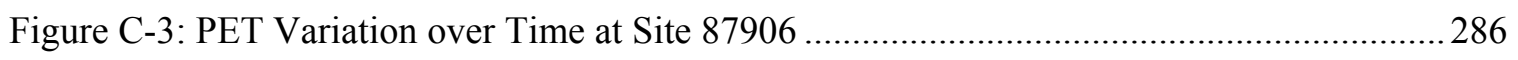

Figure C-4: PET Variation over Time by Conflict Zones at Site 87906 ....................................286

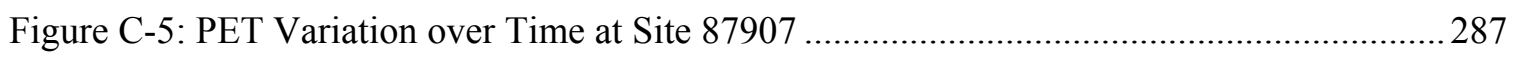

Figure C-6: PET Variation over Time by Conflict Zones at Site 87907 .....................................287

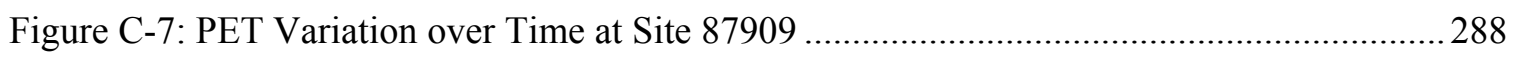

Figure C-8: PET Variation over Time by Conflict Zones at Site 87909 .......................................28

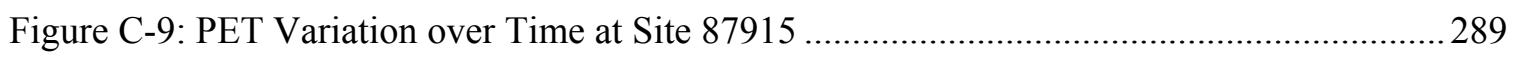

Figure C-10: PET Variation over Time by Conflict Zones at Site 87915 ....................................289

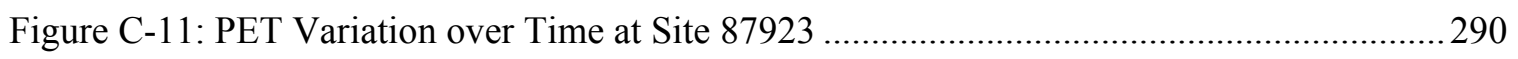

Figure C-12: PET Variation over Time by Conflict Zones at Site 87923 .................................290 


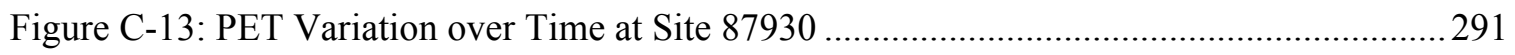

Figure C-14: PET Variation over Time by Conflict Zones at Site 87930 ..................................291

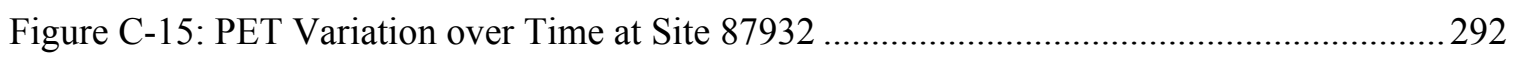

Figure C-16: PET Variation over Time by Conflict Zones at Site 87932 ................................... 292

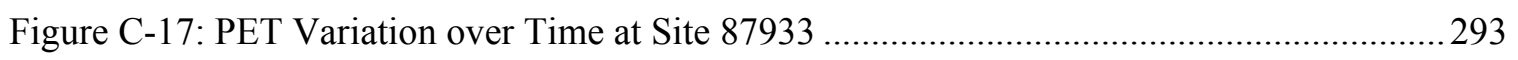

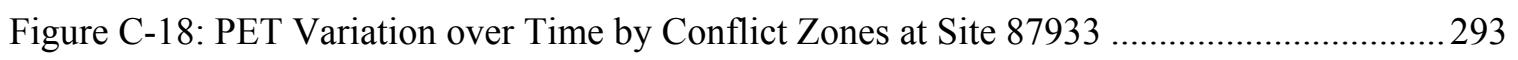

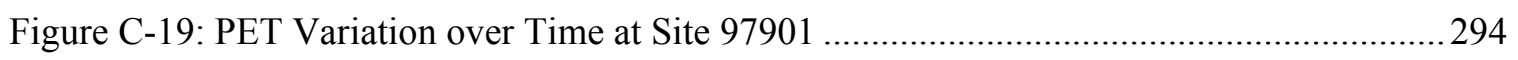

Figure C-20: PET Variation over Time by Conflict Zones at Site 97901 .....................................294

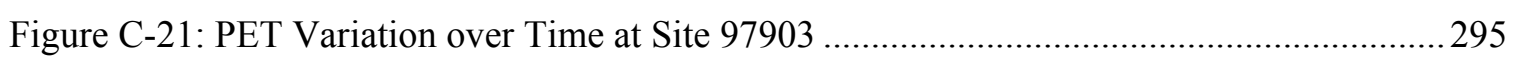

Figure C-22: PET Variation over Time by Conflict Zones at Site 97903 ..................................295

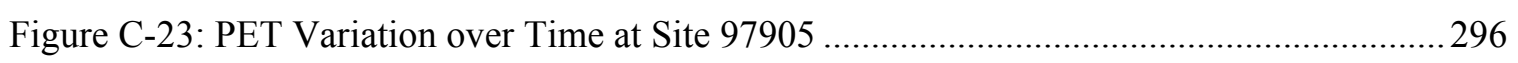

Figure C-24: PET Variation over Time by Conflict Zones at Site 97905 ...................................296

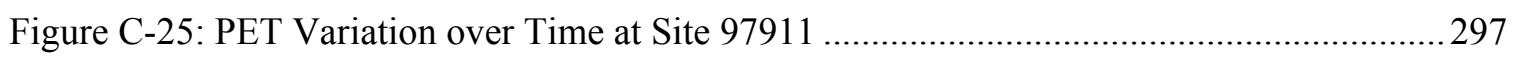

Figure C-26: PET Variation over Time by Conflict Zones at Site 97911 ....................................297

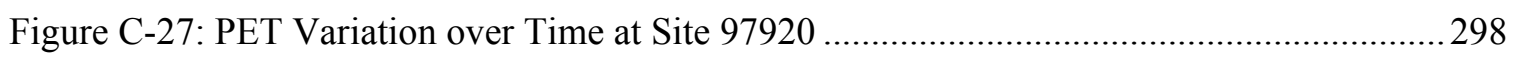

Figure C-28: PET Variation over Time by Conflict Zones at Site 97920 ....................................298

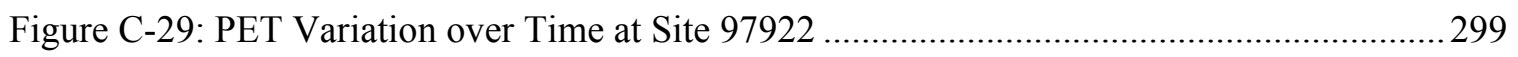

Figure C-30: PET Variation over Time by Conflict Zones at Site 97922 ...................................299

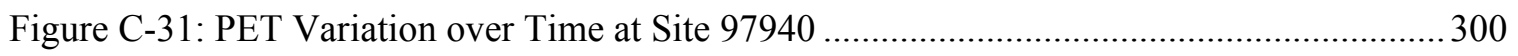

Figure C-32: PET Variation over Time by Conflict Zones at Site 97940 ................................... 300

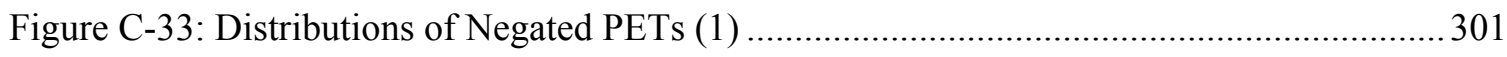

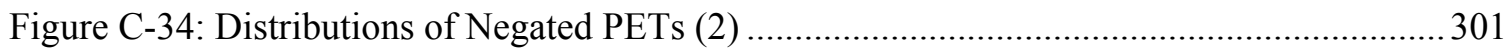

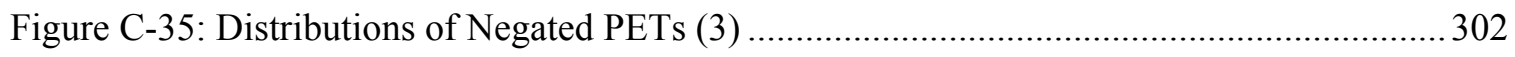

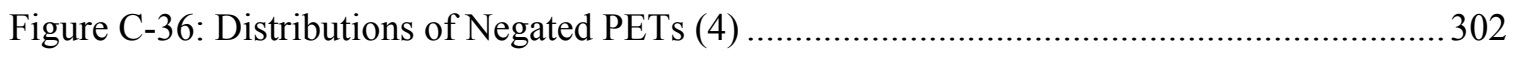

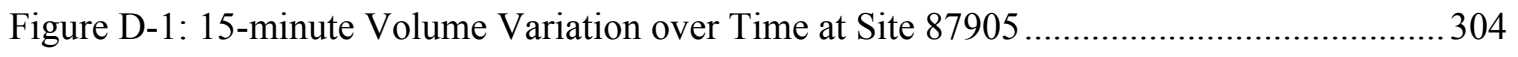

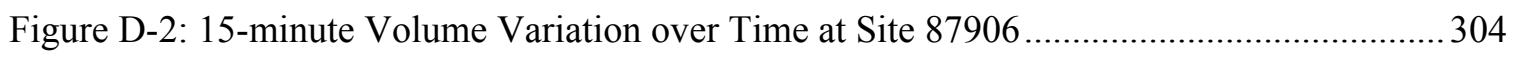

Figure D-3: 15-minute Volume Variation over Time at Site 87907 .......................................... 305

Figure D-4: 15-minute Volume Variation over Time at Site 87909 …...................................... 305

Figure D-5: 15-minute Volume Variation over Time at Site 87915 …........................................ 306

Figure D-6: 15-minute Volume Variation over Time at Site 87923 ........................................... 306

Figure D-7: 15-minute Volume Variation over Time at Site 87930 …........................................ 307

Figure D-8: 15-minute Volume Variation over Time at Site 87932 ........................................... 307 
Figure D-9: 15-minute Volume Variation over Time at Site 87933 .......................................... 308

Figure D-10: 15-minute Volume Variation over Time at Site 97901 .......................................... 308

Figure D-11: 15-minute Volume Variation over Time at Site 97903 ........................................... 309

Figure D-12: 15-minute Volume Variation over Time at Site 97905 ........................................... 309

Figure D-13: 15-minute Volume Variation over Time at Site 97911 ........................................... 310

Figure D-14: 15-minute Volume Variation over Time at Site 97920 ........................................ 310

Figure D-15: 15-minute Volume Variation over Time at Site 97922 ........................................ 311

Figure D-16: 15-minute Volume Variation over Time at Site 97940 ........................................ 311

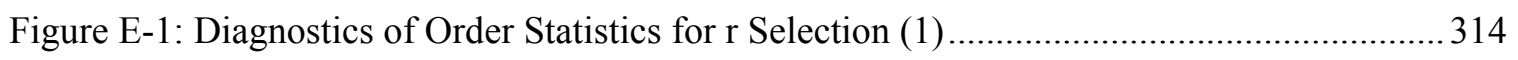

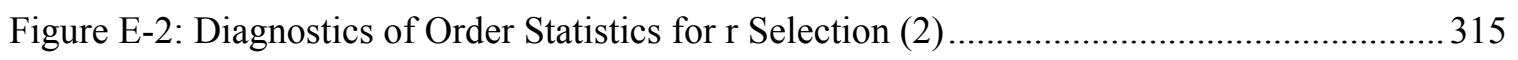

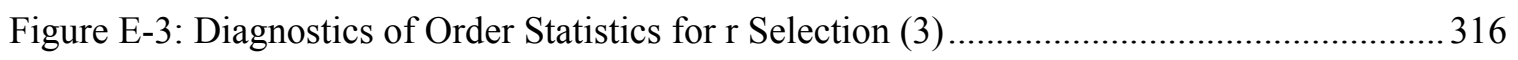

Figure E-4: Diagnostics of Order Statistics for r Selection (4) ............................................... 317

Figure E-5: Selected Diagnostics for Homogeneous Models Using All PET Data (1) ................ 320

Figure E-6: Selected Diagnostics for Homogeneous Models Using All PET Data (2) ............... 321

Figure E-7: Diagnostics for Sites with PETs Filtered - Homogeneous Models ........................... 322

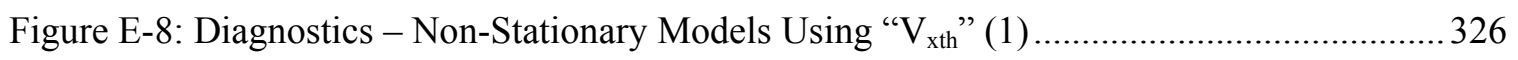

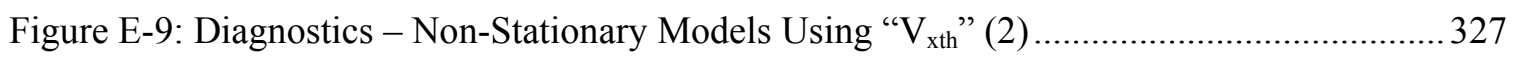

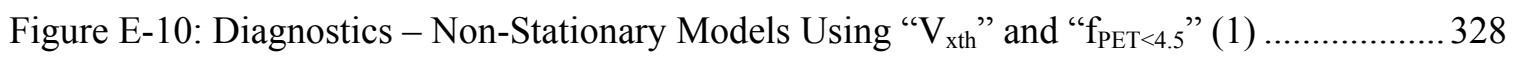

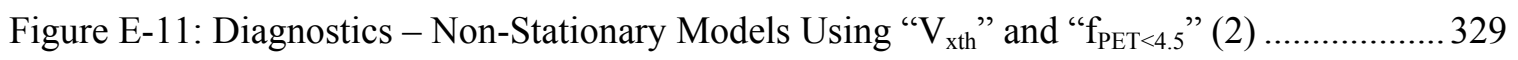

Figure F-1: Validation Plots - Homogeneous Models Using All PETs ...................................... 331

Figure F-2: Validation Plots - Homogeneous Models Using Filtered PETs ............................... 331

Figure F-3: Validation Plots - Non-Stationary Models Using " $\mathrm{V}_{\mathrm{xth}}$ " ........................................... 332

Figure F-4: Validation Plots of 12 Sites - Non-Stationary Models Using " $\mathrm{V}_{\mathrm{xth}}$ "........................ 332

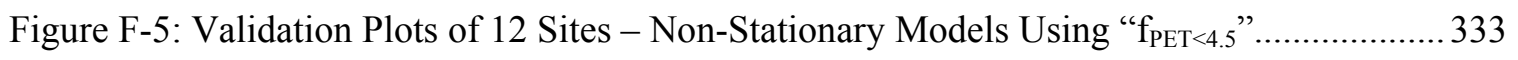

Figure F-6: Validation Plots of 12 Sites - Non-Stationary Models Using Both Covariates........ 333

Figure G-1: Estimated Crash Frequency at Site 87906 using $r=1$ and 15-minute Blocks ......... 335

Figure G-2: Estimated Return Level at Site 87906 using $r=1$ and 15-minute Blocks ............... 335

Figure G-3: Estimated Crash Frequency at Site 87906 using $r=1$ and 1-hour Blocks ............... 336

Figure G-4: Estimated Return Level at Site 87906 using $r=1$ and 1-hour Blocks ..................... 336

Figure G-5: Estimated Crash Frequency at Site 87906 using r $=2$ and 15-minute Blocks ......... 337

Figure G-6: Estimated Return Level at Site 87906 using r = 2 and 15-minute Blocks ............... 337

Figure G-7: Estimated Crash Frequency at Site 97901 using r $=1$ and 15-minute Blocks .......... 338 
Figure $\quad$ Page

Figure G-8: Estimated Return Level at Site 97901 using $r=1$ and 15-minute Blocks ............... 338

Figure G-9: Estimated Crash Frequency at Site 97901 using $r=1$ and 1-hour Blocks ............... 339

Figure G-10: Estimated Return Level at Site 97901 using $r=1$ and 1-hour Blocks ................... 339

Figure G-11: Estimated Crash Frequency at Site 97901 using $r=3$ and 15-minute Blocks ....... 340

Figure G-12: Estimated Return Level at Site 97901 using $r=3$ and 15-minute Blocks ............. 340

Figure H-1: Conflict spots, STOP lines and Region of Interest on the Screen Capture of the

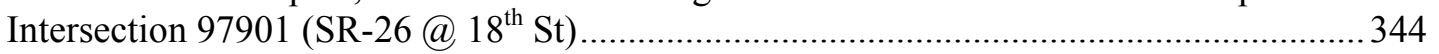

Figure H-2: Possible Relations between the Main and Auxiliary List........................................ 350

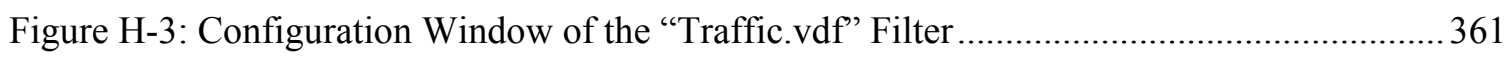

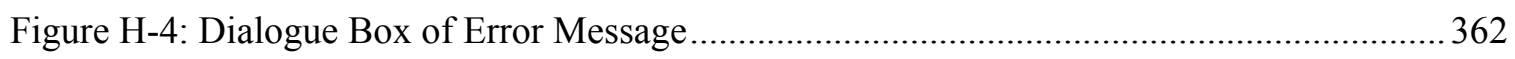

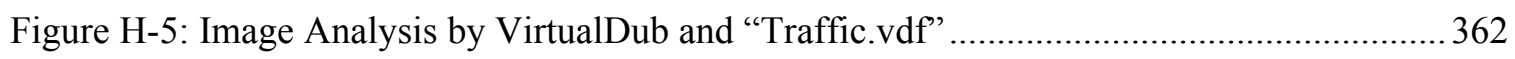

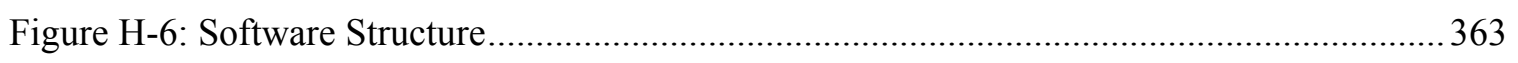

Figure H-7: Writing the Script to be Executed in Batch Mode.................................................. 365

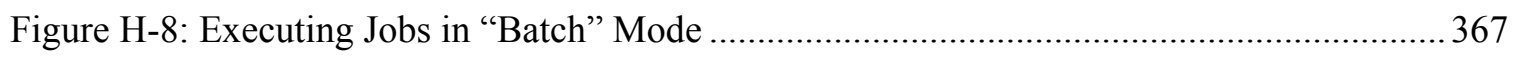

Figure H-9: Graphic Configuration File for Clip 87923 - "routes” Section in Box H.3 ............. 372

Figure H-10: Overlaid Configuration Elements at Clip 97901 (Left) and Configuration File

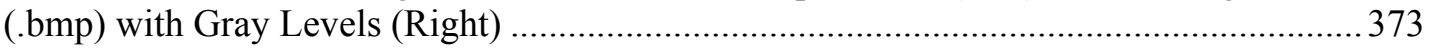

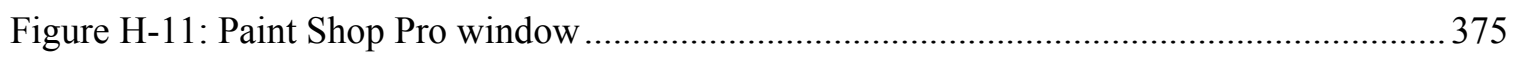

Figure H-12: Consecutive Stages of Generation of the Graphic File with the Occluded Areas

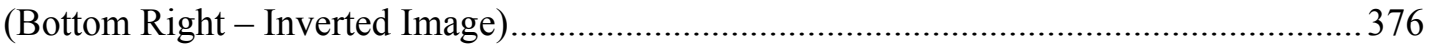

Figure H-13: Locations of the Selected Reference Points for the Vibration Removal Algorithm

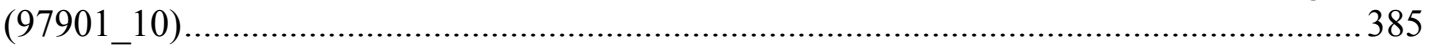

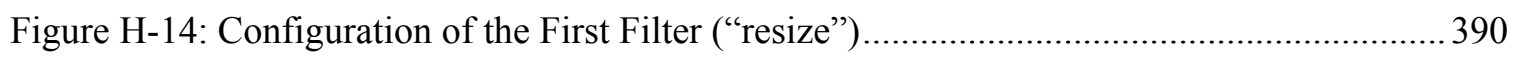

Figure H-15: The "unshaken" Filter - Specifying the Coordinates of the Object Selected.......... 390

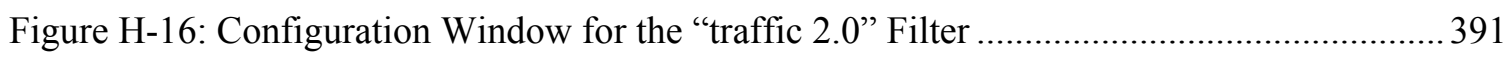

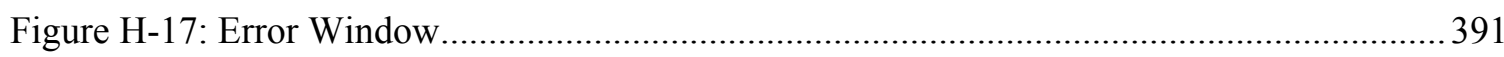

Figure H-18: Image Analysis by VirtualDub and User-Defined Filters .................................... 392

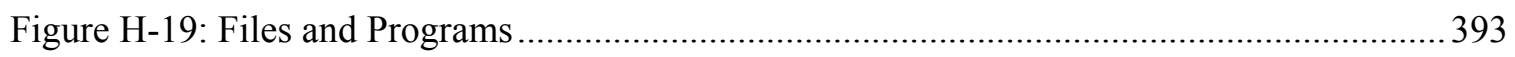

Figure H-20: Writing the Script to be Executed in Batch Mode.................................................. 395

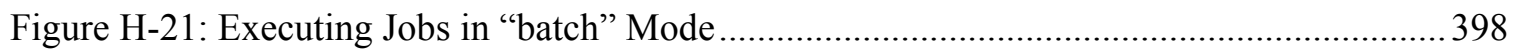




\section{CHAPTER 1 INTRODUCTION}

\subsection{Motivation}

The occurrence of traffic crashes is among the top-priority transportation problems in the United States. The National Highway Traffic Safety Administration reported that the economic cost of motor vehicle crashes that occurred in 2000 totaled \$230.6 billion (NHTSA, 2002). The importance of transportation safety has long been emphasized in U.S. transportation policies; in fact, enhancing safety is first among the five strategic goals of the U.S. Department of Transportation (US DOT, 1998).

This leads to one of the most important questions among traffic safety researchers; "How do we measure safety or unsafety to be precise in the traffic system?" We can gain better insight into this issue from the viewpoint of traffic interactions. The traffic system is the interaction among three major entities - road users, vehicles, and the environment. There is an interaction between road users and vehicles, road users and the environment, vehicles and the environment, and also among road users themselves. A flawless traffic system would require perfect interactions among these three entities. Such a flawless system can never be realized when there is involvement with human behavior, which is known to be sometimes erratic and oftentimes unpredictable. As a quality of these interactions deteriorates, the likelihood of a breakdown in the traffic system escalates. There is no question that traffic crashes indicate total breakdowns in these interactions and thus must be considered as being unsafe, but, the near total breakdown situations are relatively more frequent to observe as a result of these imperfect interactions. These nearbreakdown situations have crash potential and therefore must be considered to be unsafe as well. These near-breakdown situations are sometimes referred to as "surrogate measure of safety." It is apparent that the level of unsafety can be measured differently from this notion. The next question emerges; "Is there any linkage between these two?"

The traditional approach of measuring unsafety has mainly been focused on the occurrence of traffic crashes and their consequences. This approach implies that the analysis of traffic crashes is 
a valid method for safety estimation. This implication is quite natural as we equate reported accidents with traffic unsafety. However, there are several well-known issues of using reported accident data for traffic safety analysis, which include:

- The rarity and randomness of accident occurrences. The accidents are rare events and are therefore associated with the random variation inherent in a small number. It is not sufficient to gather the crash data for weeks or months. The typical period to be considered sufficient is as long as three years (Nicholson, 1985).

- A traffic safety analysis using traffic accidents is not a proactive approach. This basically forces us to wait for accidents to take place before we can apply any corrective measures. This is particularly true for a new traffic infrastructure where historical crash data is unavailable.

- Not all accidents are reported and the level of reporting is unevenly distributed with regard to crash participants, locations, severity of injuries, etc.

- The accident data does not provide an insight into the pre-crash process. Pre-accident driver behaviors or situational aspects are often vaguely reported in the police crash reports, which make it difficult to understand the connection between the crashes and the causes of the safety problem.

The problems stemming from the accident data analysis have prompted the need for an alternative method of safety analysis. The safety measures alternative to accident data should satisfy the following requirements (Svensson, 2000):

- The events must be more frequent than accident data.

- The events must be observable in the traffic system.

- The events must have a causal relationship with accidents.

- These events must be characterized as crashes at the extremes.

An impressive amount of work has been done in the past to search and analyze the traffic characteristics that may complement the crash data. The most acknowledged ones include traffic conflicts (Chin et al., 1992; Chin and Quek, 1997; Glauz and Migletz, 1980; Parker and Zegeer, 1989a), critical events, e.g., aggressive lane merging, speeding, and running on red (Kloeden et al., 1997; Porter et al., 1999); acceleration noise (Shoarian-Sattari and Powell, 1987); postencroachment time (Allen et al., 1978); and time-integrated time-to-collision (Minderhoud and 
Bovy, 2001). Other proposed measures are volume, speed, delay, accepted gaps, headways, shock-waves, and deceleration-to-safety-time (FHWA, 1981). Although some of the latter measures are safety factors rather than surrogate measures, they are listed to adequately reflect the past work. The attempts to confirm the statistical linkage with accident data yielded mixed findings at best. In addition, not all the proposed indicators in the past research satisfy the desirable properties of surrogate measures. For example, a measure such as time-integrated timeto-collision is so data-intensive that it is attainable only in the simulation environment and is therefore not observable in the field (Minderhoud and Bovy, 2001). The traditional approach to the analysis of a surrogate measure of safety shares several commom traits, which we can discuss from two aspects, measurement and evaluation.

\subsubsection{Measurement}

Two major issues frequently encountered in the measurement of surrogate data are accuracy and efficiency. The sources of an accuracy problem stem from the subjectivity inherent in human observers and the technical limitations of a measurement procedure. Let us consider a traffic conflict measurement for example. A traditional traffic conflict count requires the observation of evasive maneuvers, which can be very subjective. The revised measurement method, using timeto-collision, still cannot do away with human intervention. Post-processing of a video recording of traffic movement was one possibility to help reduce inter-person, as well as intra-person, variations in the measurement, see Chin et al. (1992) for example. Efficiency is always an issue in the measurement of surrogate data. A video recording helps minimize the accuracy problem only at the expense of the data extraction effort. This was particularly true in the past where the digital video was not widely available and the cost of digital data storage plus a processing platform was prohibitive. The post-processing of VHS media with an analog video cassette recorder (VCR) is very cumbersome and impractical for a large-scale study.

This technological barrier is no longer an issue nowadays. The advent of commercial video image processing (VIP) units, an affordable digital computing platform, and a cheaper unit cost for digital storage has renewed our interest in improving accuracy and efficiency in the measurement of surrogate data. Their potential on the measurement of surrogate data is yet to be explored. This feasibility study may also entail a refinement in a working definition of a surrogate measure of interest to overcome certain limitations of the technology being considered. 


\subsubsection{Evaluation}

The evaluation of surrogate measures of safety has been done in several respects in the past. Traffic conflict attracted the most research attention, among others. One prominent issue, and probably the most critical, is the linkage between the surrogate data and the accident data. Inconsistent findings and a failure to establish a safety relationship can be attributed to three hypotheses: risk assumption, measurement error, and randomness of surrogate data.

Safety researchers have typically evaluated the validity of surrogate data using a regressional relationship between these surrogates and crashes. The implicit assumption underlying this approach is a constant risk across locations being considered. By doing so, surrogate data are treated as traffic exposure while the risk is fixed. The increase in the observed exposure implies greater expected accident frequency. The important issue here is whether the risk should be the same across locations. There rarely exist two identical traffic systems and therefore why should it be for the risk. Researchers worked around this issue by restricting the analysis to a group of similar locations. In addition, similarity is a term subject to interpretation, i.e., the extent to which we must have similar entities in a traffic system and similar interactions among these entities before we can adequately justify this term is unknown. Therefore, there is a definite need for a new analytical framework that can relax this assumption.

Subjectivity and reliability in the measurement of surrogate data have been a subject of controversy in both the practicality and validity of the approach. The impact of measurement errors on the evaluation results has never been fully understood because the precise measurement is not attainable in the field. The lack of standard definitions for either traffic conflicts or crashes in the past has been another factor in this inextricable relationship with safety (Williams, 1981). There is potential for improvement in the measurement aspects with the recent advancement in digital video and image processing technology. There is potential to gain more insight into the safety relationship between surrogate data and crashes when these measurement errors are adequately addressed.

The repeatability of surrogate data depends on the randomness in its occurrences and this problem has been underserved by researchers' interest. Repeatability refers to an acceptable level of variations in repeated observations under nominally identical conditions. The variance-to-mean ratio of crash counts is about 1 if Poisson assumption is appropriate and greater than 1 if the count process (particularly for a specific type of crashes) is more irregular and thus the negative 
binomial assumption is more appropriate (Nicholson, 1985). For surrogate data, Glauz and Migletz (1980) reported the variance-to-mean ratio and the coefficient of variations (CV) of 15minute traffic conflict counts for various types of conflicts. The variance-to-mean ratio was found to range from 1.5 to 3.5 , depending on the conflict types and the coefficient of variation ranged from $73 \%$ to $685 \%$. All cross-traffic conflict types and opposing left-turn conflicts had CV's of more than $200 \%$. How long the observation period should be and how acceptable repeatability should be defined have not been well researched. This can be answered if there is a wellestablished framework to link surrogate data with crashes. A new approach that allows conversion of surrogate data into crashes using site-specific data would be useful in determining the observation period needed to attain acceptable repeatability of the surrogate data. Better understanding of the impact of random variation of surrogate data may also offer a clue to inconsistent findings in the safety relationship between surrogate data and crashes.

In addition to the issues described above, the traditional approach of surrogate data analysis is not a crash-free calibration process. The regression approach requires observed crash counts for model calibration and this somewhat contradicts the concept of proactive safety analysis, which aims to identify unsafe locations and appropriate preventive countermeasures before crashes will occur.

\subsubsection{Implications}

Difficulties in measuring and evaluating the unsafety of a traffic system bring about several consequential implications. The safety evaluation results are less convincing or even unreliable when the safety measurement can be questioned. The identification of hazardous locations cannot pinpoint the locations that actually have safety problems. These will in turn result in inefficient allocation of resources for safety improvements; thereby hampering the overall effectiveness of the safety management system.

The frequency of crashes and their severity are the measures of road safety preferred by transportation agencies and by safety specialists for their unquestionable connection with safety. Unfortunately, due to the random and infrequent occurrence of crashes, collecting crash data at intersections and road segments can require several years of monitoring; and even then, the estimation precision of the crash frequency is far from ideal. This intricacy hampers safety modeling, evaluation, and enhancement. Identification of hazards and evaluation of safety 
impacts do not necessarily yield timely and even correct answers, which may result in an inefficient allocation of resources.

Modern technologies are increasingly being used in transportation systems. Technological innovations applied to transportation have a relatively short life compared to the traditional infrastructure; however, their effects on safety cannot be easily evaluated using crash-based methods. Fortunately, technological progress has also opened unprecedented opportunities for collecting traffic data that may carry safety information.

These issues renew our interest in facilitating a measurement method and call for a better approach to safety estimation.

\subsection{OBJECTIVES}

The objectives of this research can be summarized as follows:

- Develop a non-crash-based safety estimation method that allows (a) invidualization of a risk using observable traffic characteristics from specific time, location, and condition; (b) direct linkage between observed traffic characteristics and crashes; and (c) model calibration without historical crash data.

- Evaluate the feasibility of digital video and image processing technology in facilitating the measurement of traffic characteristics.

The successful development of the method will provide more insight into the inextricable linkage between surrogate measures of safety and crashes. A better understanding of this relationship may offer a new possibility for unprecedented rapid safety evaluation which may be a breakthrough in the area of highway safety science. Knowledge of the capability and limitations of the new technology will help identify important requirements and lay important ground work for development towards automation of the measurement of surrogate data in the future.

\subsection{RESEARCH SCOPE}

We will develop a framework to demonstrate the use of microscopic traffic data for a safety estimation task. A surrogate measure for detecting an excessive degree of hazard of collisions 
will be proposed. Then, its usefulness for safety assessment will be evaluated at selected intersections. The proposed surrogate safety measure must be observable in the field and measurable objectively. In addition, it should be amenable for image-based detection.

To illustrate the approach in this study, we will preliminarily focus on one particular type of traffic crashes - straight right-angle collisions - at signalized intersections. A straight right-angle collision is caused by a crossing conflict of two straight traffic flows. An area where a straight right-angle collision may be expected is relatively well-defined, static, and detached from the approach. Moreover, driver behaviors are more or less predictable at signalized intersections. These properties suit the need for the research starting point and increase the chance for successful development of detection and analysis of a surrogate safety measure.

Primary methods to detect potential safety-related events will be based on digital video and image processing technology. A manual measurement method using digital video is expected to give attainable accuracy of $1 / 30$ seconds and is likely to save post-processing time compared to a VCR because of the capability of instantaneous access to specific frames in the video.

A commercial video image processing (VIP) unit, Autoscope, will be evaluated as to whether it can help detect and measure the surrogate data efficiently and reliably. The Autoscope is widely used across the country and has been found to be a mature and reliable tripwire system (Grenard et al., 2001).

In addition, a safety relationship between proposed surrogates and crashes will be examined using a regression approach as traditionally implemented in the evaluation of surrogate data. A potential linkage between these measures and crashes would increase the chance for successful development of a new safety estimation method.

\subsection{REPORT ORGANIZATION}

In this research, the main part of the report is organized into nine chapters. Other relevant materials and the results of this study are documented in the appendices.

Chapter 1 provides the research overview, the motivation for this study, the research objectives and the scope of study. 
Chapter 2 summarizes the literature related to the current state of practices in crash-based and non-crash-based safety estimations. This chapter also discusses image processing technology, applications, and potential for safety analysis.

Chapter 3 discusses the methodology pursued in this research. The limitations of the current approach to safety analysis, the requirements for the proposed surrogate measure of safety, and the desirable properties of a new method are examined in details. The research procedure is described in this chapter as well.

Chapter 4 discusses the proposed surrogate measure of safety, the selection of studied sites, the evaluated measurement methods, and the evaluation results. The collected data and the data collection procedure conclude the chapter.

Chapter 5 examines the collected traffic characteristics and observed crash counts. Safety relationships between these characteristics and crashes are examined using regression analyses.

Chapter 6 proposes a new approach to analyze the surrogate measure of safety. This chapter provides an overview of the extreme value theory and important considerations for extreme value modeling of the surrogate data. Then, the selected extreme value modeling approach and the proposed safety estimation method are discussed.

Chapter 7 discusses the general considerations in the model development process, using the selected extreme value models. The final estimated models at selected sites, along with the safety estimates and their inferences, conclude the chapter.

Chapter 8 describes the evaluation of the safety estimates derived from the proposed method. A simulation study was conducted to examine the effects of different model settings on the confidence of safety estimates. Using the proposed method, the repeatability of the surrogate measure is also examined in terms of the observation period required to obtain safety estimates with acceptable confidence intervals.

Chapter 9 summarizes the research approach and important findings. The directions for further research are also discussed. 


\section{CHAPTER 2 LITERATURE}

In general, measures of highway safety can be categorized into two groups: crash-based and noncrash-based safety measures. Examples of crash-based safety measures are observed changes in the number, rate, and severity of traffic accidents resulting from the implementation of safety countermeasures. Non-crash-based safety measures are indirect ways to measure safety. Noncrash-based measures are often needed when crash data are (a) not available, (b) insufficient for crash-based evaluation, or (c) when rapid evaluation of safety is desirable. Examples of noncrash-based safety measures are traffic volume, speed, travel time and delay, intersection delay, traffic conflicts and erratic maneuver, gap acceptance, headway distribution (smaller headways are relatively unsafe), time-to-collision distribution (a change in distribution signifies a change in safety characteristics), the number and severity of shock waves, time-to-accident, postencroachment-time (PET), and deceleration-to-safety-time (FHWA, 1981; Minderhoud and Bovy, 2001). Nevertheless, non-crash-based measures are not intended to be a substitute for the ultimate safety measure (crash and severity reduction) since definitive quantitative relationships between crash experiences and many non-crash-based measures have not been well understood (FHWA, 1981).

This chapter aims to provide an overview of the past research on non-crash-based safety measures. In addition, several methods proposed for crash-based safety evaluation are summarized for comparison. Research findings on the effects of traffic control and driver attitudes on the risk of right-angle crashes are also reviewed.

\subsection{NON-CRASH-BASED SAFETY EVALUATION}

Crash data is the most widely used and direct measure of safety at an intersection. However, crash data alone is often not suitable for diagnosing safety problems at intersections or for evaluating the effectiveness of safety improvements. The use of crash data to evaluate safety exposes itself to several underlying problems, such as unreported crashes, randomness in crash 
occurrences, and a considerably long waiting time for an adequate amount of crash data. In addition, crash data analysis is a reactive approach in that collisions have to occur before an improvement program can be applied. Therefore, at times, an indirect and proactive safety measurement to allow for a rapid and reliable safety evaluation is highly desirable. Non-crashbased safety measures generally focus on the traffic operational behaviors at the site level. This section reviews past research attempts in order to define the current state of this research area. The technology aspects and simulation approaches for applications in safety evaluation are also discussed.

\subsubsection{Alternative Measures of Safety}

Traffic conflict has been one widely-used indirect safety measurement tool for more than three decades. One of its appealing aspects is that conflict data can be gathered within a much shorter period (Chin and Quek, 1997). Traffic conflict and its measurement technique have been modified and implemented by several researchers and practitioners over the years.

The history of traffic conflict technique began as early as the 1960s. Perkin and Harris (1967) defined conflicts based on evasive actions taken by drivers. However, there are problems with evasive actions since (a) it is rather difficult to differentiate a precautionary action from a truly evasive one, particularly when a quick assessment is demanded on spot; and (b) sometimes evasive actions may be absent in critical situations.

A number of research efforts have been undertaken in order to develop conflict measurements in such a manner that the results are objective and repeatable based on the fact that traditional observation of traffic conflict relies on trained human observers that are more or less subjective in nature. More objective definitions and measurements of traffic conflicts are of interest among traffic safety researchers.

Hayward (1972) suggested the use of time-measured-to-collision, the time to collide with the leading vehicle if both vehicles continue in the same path without changing their speeds.

The first agreeable and improved definition for conflicts is proposed by Amundsen and Hyden (1977). A conflict is "an observable situation in which two or more road users approach each 
other in space and time to such an extent that there is a risk of collision if their movements remain unchanged."

Allen et al. (1978) measured several candidates for revised definitions of traffic conflicts which include gap time (GT), post-encroachment time (PET), encroachment time (ET), initial-attempted post-encroachment time (IAPE), proportion of stopping distance (PSD), and deceleration rate (DR).

Gap time (GT) is the time difference between the arrival times of the involved vehicles at the point of crossing if no evasive actions are taken by either vehicle. A zero-value GT can be recorded when (a) there is a crash, and (b) precautionary actions are taken (Glauz and Migletz, 1980).

Post-encroachment time (PET) is the clearance interval between two vehicles with crossing paths; any value less than some threshold is defined to be a conflict. One problem about PET is that although it can be objectively measured, it is uncertain how to distinguish between the true conflict severity and the willingness of the drivers in accepting the risk (Chin and Quek, 1997).

Proportion of stopping distance (PSD) is the ratio of the distance available for a driver to maneuver to the distance remaining to the projected collision point. A PSD value of at least 1 is required to stop safely. The ratio is given as

$$
P S D=R D / M S D,
$$

where RD is the remaining distance to the potential collision point and MSD is the acceptable minimum stopping distance.

Deceleration rate (DR) is used as an indicator of the degree of hazard perceived by the second driver in response to the erratic maneuver of the first vehicle. Encroachment time (ET) is defined as time during which the first vehicle encroaches into the right-of-way of the second vehicle. The ET depends on the conflict type being considered; for example, a right-angle traffic conflict should have a mean ET less than the opposing left-turn traffic conflict. IAPE is the postencroachment time that is calculated based on the initial deceleration rate as a forecast of its value. 
Based on the evaluation of proposed indicators, Allen et al. (1978) proposed a revised definition of cross-traffic conflict as whenever the two following conditions are satisfied: (a) the gap time is less than a threshold value while the two vehicles are not necessarily on a collision path; and (b) the post-encroachment time should be less than some threshold value to eliminate the confusion on the evasive maneuver and a maneuver due to an intersection control or turning movements.

Time-to-intersection $\left(\mathrm{TTI}_{\mathrm{br}}\right)$ is the time expected for a vehicle to enter the intersection at a constant instantaneous speed just at the onset of braking (van der Horst, 1990). This has been used for a single-vehicle interaction at unsignalized intersection.

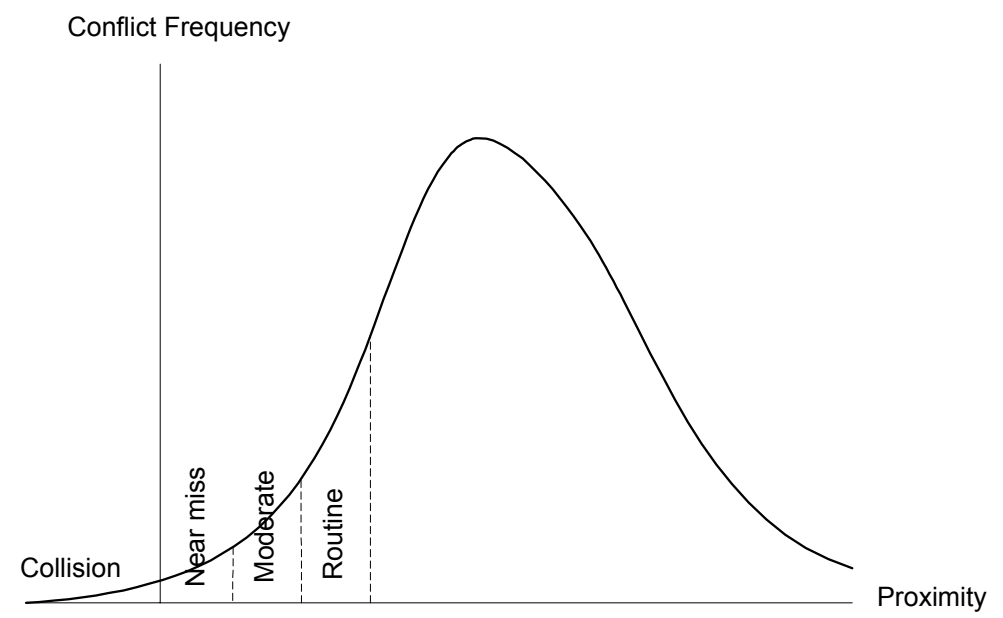

Figure 2-1: Frequency Distribution of Traffic Events (Chin and Quek, 1997)

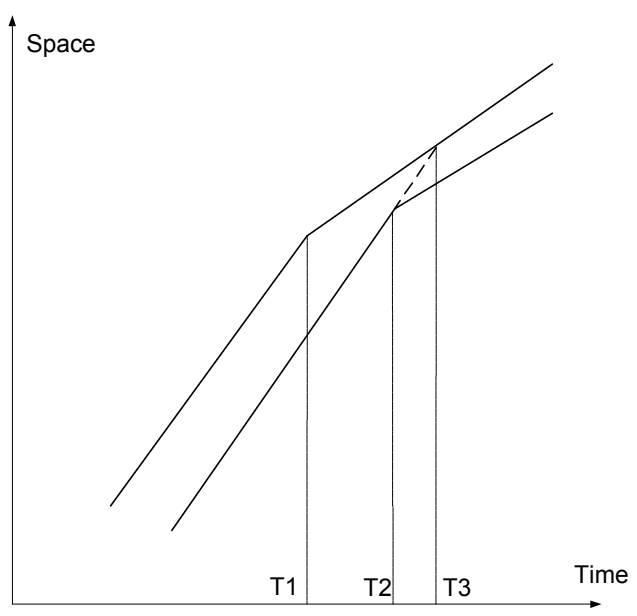

Figure 2-2: Proximity to Collision for Car-Following Situation (Allen et al., 1978) 
Time proximity to collision, time to collision, and measure of nearness to collision were used by several researchers (Sayed et al., 1994; Glauz and Migletz, 1980). Frequency distribution (see Figure 2-1) and cumulative frequency distribution of proximity to collision can be used to help define traffic conflict severity (Chin and Quek, 1997). This curve could be generated for a specific location as an intersection characteristic. Proximity to collision for car-following situation is illustrated in Figure 2-2 where the proximity to collision is T3-T2.

The traffic conflict technique (TCT) is a procedure of systematically observing traffic conflicts and employing some quantitative measures to deduce the accident potential of a location (Chin et al., 1991). TCT has been widely applied to safety evaluation at an intersection, but only to a limited extent in expressway merging (Chin et al., 1991). Conceptually, a conflict can be presented in the form of an ordinal severity scale, ranging from slight to very serious. The two extremes are non-conflict and crashes (Chin and Quek, 1997).

Chin et al. (1992) suggested the use of a reciprocal of TTC instead of TTC itself. Variations of the reciprocal of TTC get larger as the conflict severity increases. A significant drop in severity was observed for short serious traffic conflicts. In addition, it was reported that the Weibull distribution gave the best fit to the empirical data.

Chin et al. (1992) defined the magnitude of the average deceleration that the conflicted vehicle is required to take just to avoid a collision as deceleration-to-avoid-collision. However, this measurement requires an intensive post-processing task from the video taping data.

Shoarian-Sattari and Powell (1987) considered acceleration noise and the mean velocity gradient as safety indicators. Acceleration noise is defined as the root mean square deviation of the acceleration of the vehicle and can be written as

$$
\sigma^{2}=\frac{1}{X} \int_{0}^{X}[a(x)-\bar{a}(x)]^{2} d x
$$

where $\mathrm{X}$ is the total distance covered, $a(x)$ is the acceleration at distance $\mathrm{x}$, and $\bar{a}(x)$ is the average acceleration over distance X. Computationally, the distance was divided into small increments of distance, $\Delta \mathrm{x}$. The measurements of each small increment can be added up to determine the value of an entire link. 
It was found that the acceleration noise decreases with speed. The mean velocity gradient (MVG) was proposed to account for this effect, which is expressed as

$$
M V G=\frac{\sigma}{\bar{V}(x)}
$$

There are two variations of the indicators, depending on whether the origin (zero) or the mean is used in the calculation of acceleration noise.

Minderhoud and Bovy (2001) proposed two indicators to consider the occurrences of the small TTC values of all traffic participants, at any moment, for a specified road length $\mathrm{L}$ and duration H. Time-exposed time-to-collision (TET) is defined as the duration of exposure to safety-critical TTC values over a specified duration $\mathrm{H}$. In other words, it is a summation of all instants (over the analyzed duration) that a driver approaches a front vehicle with a TTC value below the threshold value. Since the TET indicator does not account for the difference from the threshold values, time-integrated TTC (TIT) indicator uses the integral of the time-to-collision profile of drivers to express the level of safety. Figure 2-3 shows the conceptual illustration of these two indicators. However, the methods are limited only to a microscopic simulation model.

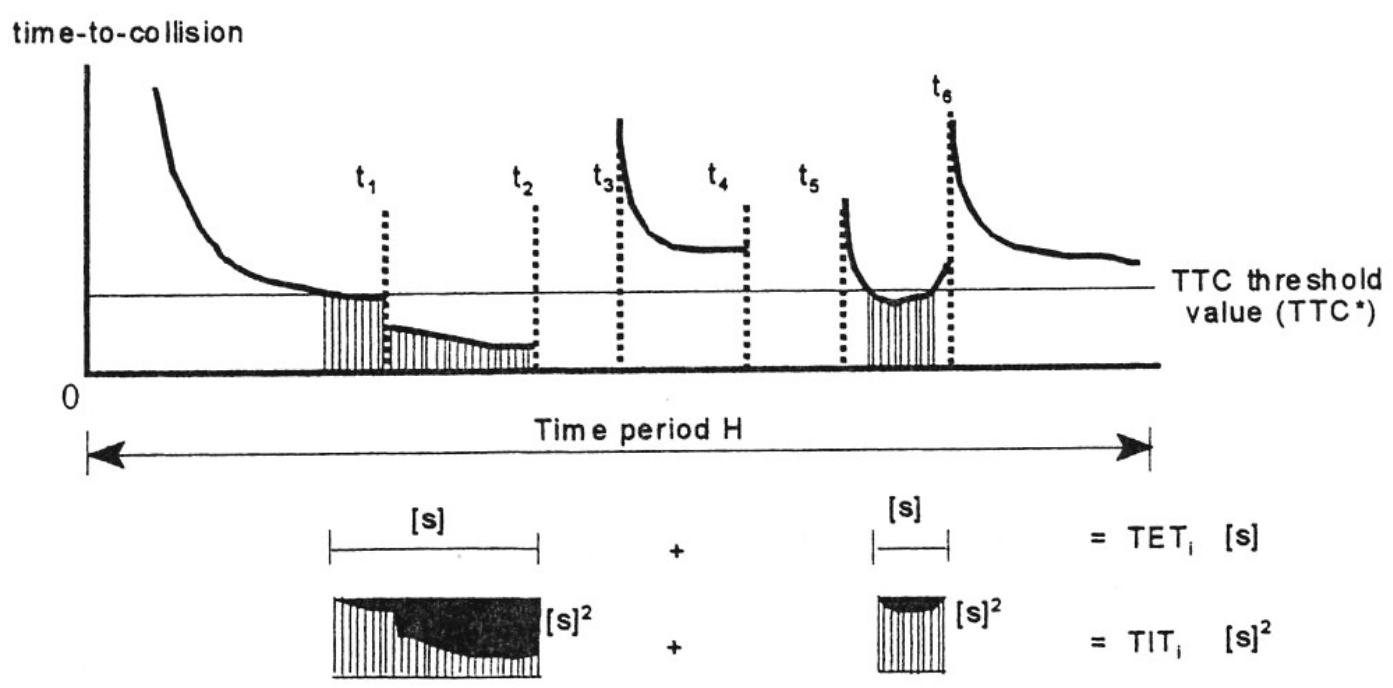

Figure 2-3: Concept of Time-Exposed TTC and Time-Integrated TTC

It is clear that several alternative measures of safety have been proposed to date. One major problem for traffic conflicts is that there exists an inconsistency among the traffic conflict 
definitions as well as their measurement. Currently, there is still no perfect definition or flawless measurement methods for traffic conflict techniques. The use of an evasive maneuver in the definition requires an exhaustive list of possible evasive actions. Another inherent problem in the definitions is the theoretical argument as to whether crash counts should be excluded from the conflicts if the conflicts are defined as events prior to crashes. In addition, research on how to measure the severity of traffic conflict are still ongoing. The threshold values to justify a conflict situation appear to be arbitrary.

Other than the problem of inconsistencies, there are two additional major problems regarding the application of alternative measures of safety, reliability and validity of the techniques. These issues are discussed in the subsequent section.

\subsubsection{Past Practices of Traffic Conflict Technique}

The TCT has been primarily used as a tool for diagnosing the safety/operational problems of intersections that have previously been identified for attention. Typical sources of such attention are dominant crash frequencies, citizen complaints, serious or fatal crashes, or short-term rash of crashes at a particular intersection. In addition, TCT is often used in before/after evaluations of safety countermeasures. One caveat for this evaluation is that the collected conflict types must correlate to the type of implemented countermeasures.

Although TCT was used by some researchers to identify hazardous locations (Katamine and Hamarneh, 1998), it should be noted that TCT is not cost-effective to be used for this purpose, particularly for a large-scale study (Glauz and Migletz, 1980).

Traffic conflicts can be analyzed and interpreted in several different ways. One common use is the counts normalized by traffic volumes, i.e., conflict rates. The absolute counts itself as a safety indicator at present cannot be stated unequivocally. Based on the FHWA procedure detailed in Parker and Zegeer (1989a and 1989b), Salman and Al-Maita (1995) illustrated the determination of average and abnormal limits to be used to evaluate the level of safety among 18 three-leg, unsignalized intersections. The conflict frequency distribution also can be obtained to help identify the appropriate type of safety improvement. A risk index was developed based on various conflict types and related crashes to priority rank the intersections for adopting countermeasures. The linear regression analysis technique was used to model the relationships between traffic 
conflicts and volumes, crashes and traffic volumes, and crashes and traffic conflicts. The hourly conflicts were compared with the sums and square root of the product of the concerned flows generating these conflicts for various intersection categories. Intersection categories included a two-lane intersection roadway, a four-lane intersection roadway, and all intersections. The relationship between crashes and total conflicts was found to be linear and statistically significant.

Traffic conflict counts can be used in the before/after evaluation of safety countermeasures, provided the traffic volume remains more or less constant. The counts may be compared using the standard statistical tests, such as t-tests. High traffic conflict rates of specific types may suggest the need for intersection improvements. Suggested improvements for three types of intersections are proposed as shown in Table 2-1 through Table 2-3 (Glauz and Migletz, 1980).

\section{Table 2-1: Suggested Improvements for Signalized, 4-leg, 4-lane Intersections}

\begin{tabular}{|c|c|c|c|c|c|c|c|c|c|c|c|}
\hline Improvement & $\begin{array}{r}\begin{array}{r}\text { Sam } \\
\text { Direct } \\
\text { (Rear-1 }\end{array} \\
\end{array}$ & 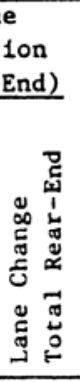 & 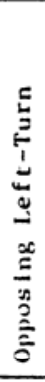 & $\begin{array}{l}\text { Ri } \\
\text { Tu } \\
\text { Cr } \\
\text { Tra } \\
\end{array}$ & 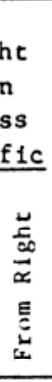 & $\begin{array}{l}\text { Le } \\
\text { Tu } \\
\text { Cr } \\
\text { Tra } \\
\end{array}$ & 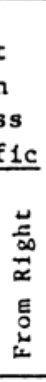 & $\begin{array}{l}\text { Th } \\
\text { Cro } \\
\text { Tra } \\
\end{array}$ & 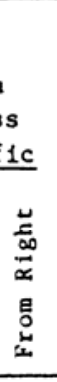 & 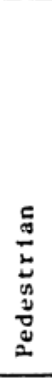 & 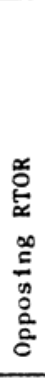 \\
\hline Left-Turn Bay & $\mathrm{x}$ & $\mathrm{x}$ & $\mathrm{x}$ & & & & & & & & \\
\hline Left-Turn Phase & $\mathrm{x}$ & $\mathrm{x}$ & $\mathrm{x}$ & & & & & & & & \\
\hline $\begin{array}{l}\text { Left-Turn Restriction } \\
\text { Right-Turn Bay }\end{array}$ & $x_{x}$ & $\begin{array}{l}\mathrm{x} \\
\mathrm{x}\end{array}$ & $\mathrm{x}$ & & & & & & & & \\
\hline $\begin{array}{l}\text { Right-Turn Radius or } \\
\text { Roadway }\end{array}$ & $\begin{array}{l}\mathrm{x} \\
\mathrm{x}\end{array}$ & $\begin{array}{l}x \\
x\end{array}$ & & $\mathrm{x}$ & $\mathrm{x}$ & & & & & & \\
\hline $\begin{array}{l}\text { Signal Cycle or Phase } \\
\text { Length }\end{array}$ & $\mathrm{x} \quad \mathrm{x}$ & $\mathrm{x} x$ & $\mathrm{x}$ & & & & & & & $\mathrm{x}$ & \\
\hline Actuated Signals & & & & & $\mathrm{x}$ & $\mathrm{x}$ & $\mathrm{x}$ & $\mathrm{x}$ & $\mathrm{x}$ & & \\
\hline $\begin{array}{l}\text { Longer Amber or all Red } \\
\text { Clearance }\end{array}$ & & $x$ & & & $\mathrm{x}$ & $\mathrm{x}$ & $\mathrm{x}$ & $\mathrm{x}$ & $\mathrm{x}$ & & \\
\hline RTOR Restrictions & & & & $\mathrm{x}$ & $\mathrm{x}$ & & & & & & $\mathrm{x}$ \\
\hline Pedestrian Barriers & & & & & & & & & & $\mathrm{x}$ & \\
\hline Pedestrian Phase & & & & & & & & & & $\mathrm{x}$ & \\
\hline Add Lanes & $\mathrm{x} \times \mathrm{x}$ & $\mathrm{x}$ & & $\mathrm{x}$ & & & & & & & \\
\hline Parking Restrictions & $\mathrm{x} x$ & & & & & & & & & & \\
\hline Install Median & & & & $\mathrm{x}$ & & & & & & & \\
\hline $\begin{array}{l}\text { Improve Corner Sight } \\
\text { Distance }\end{array}$ & & & & & $\mathrm{x}$ & & & & & $\mathrm{x}$ & \\
\hline Speed Zone & $\mathrm{x} \times \mathrm{x}$ & $\mathrm{x}$ & & & $\mathrm{x}$ & & & & & & \\
\hline $\begin{array}{l}\text { Advance Warning or } \\
\text { Sight Distance Control }\end{array}$ & $\mathrm{x} \times \mathrm{x}$ & $\mathrm{x} \times$ & & & $\mathrm{x}$ & & & & & & \\
\hline $\begin{array}{l}\text { Advance Street Name Sign } \\
\text { Enforcement }\end{array}$ & $\mathrm{x} \times \mathrm{x}$ & $\mathrm{x} x$ & & & $\mathrm{x}$ & $x$ & $\mathrm{x}$ & $\mathrm{x}$ & $\mathrm{x}$ & & \\
\hline
\end{tabular}


Table 2-2: Suggested Improvements for Unsignalized, 4-leg, 2-lane Intersections

\begin{tabular}{|c|c|c|c|c|c|c|c|c|c|c|}
\hline \multirow[b]{2}{*}{ Improvement } & \multicolumn{2}{|c|}{$\begin{array}{c}\text { Same } \\
\text { Direction } \\
\text { (Rear-End) } \\
\end{array}$} & \multirow{2}{*}{ 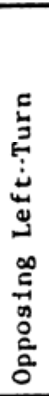 } & \multicolumn{2}{|c|}{$\begin{array}{l}\text { Right } \\
\text { Turn } \\
\text { Cross } \\
\text { Traffic }\end{array}$} & \multicolumn{2}{|c|}{$\begin{array}{l}\text { Left } \\
\text { Turn } \\
\text { Cross } \\
\text { Traff1c } \\
\end{array}$} & \multicolumn{2}{|c|}{$\begin{array}{l}\text { Thru } \\
\text { Cross } \\
\text { Traff1c } \\
\end{array}$} & \multirow[b]{2}{*}{ 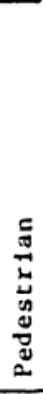 } \\
\hline & 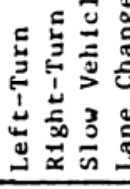 & 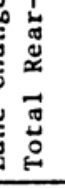 & & 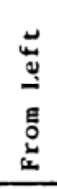 & 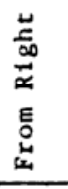 & 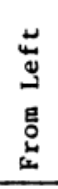 & 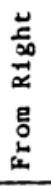 & 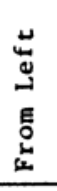 & 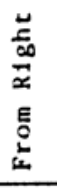 & \\
\hline Add S1gnal & $\mathrm{x} x$ & $\mathrm{x}$ & & & & $\mathrm{x}$ & $\mathrm{x}$ & $x$ & $x$ & $\mathrm{x}$ \\
\hline Left-Turn Bay & $\mathrm{x}$ & & $\mathrm{x}$ & & & & & & & \\
\hline Right-Turn Bay & $\mathrm{x}$ & & & & & & & & & \\
\hline $\begin{array}{l}\text { Right-Turn Radius or } \\
\text { Roadway }\end{array}$ & $\mathrm{x}$ & & & $x$ & $\mathrm{x}$ & & & & & \\
\hline Pedestrian Barriers & & & & & & & & & & $\mathrm{x}$ \\
\hline Add Lanes & $\mathrm{x} \times \mathrm{x}$ & $\mathrm{x}$ & & $\mathrm{x}$ & & & & & & \\
\hline Parking Restrictions & $\mathrm{x} \times$ & & & & & & & & & $\mathrm{x}$ \\
\hline $\begin{array}{l}\text { Improve Corner Sight } \\
\text { Distance }\end{array}$ & & & & & $\mathrm{x}$ & $x$ & $\mathrm{x}$ & $\mathrm{x}$ & $\mathrm{x}$ & $\mathrm{x}$ \\
\hline Speed Zone & $\mathrm{x} \times \mathrm{x}$ & $\mathrm{x}$ & & & $\mathrm{x}$ & $\mathrm{x}$ & $\mathrm{x}$ & $\mathrm{x}$ & $\mathrm{x}$ & \\
\hline $\begin{array}{l}\text { Advance Warning or } \\
\text { Sight Distance to } \\
\text { Traffic Control }\end{array}$ & $\mathrm{x} \times \mathrm{x}$ & $\mathrm{x}$ & & & $\mathrm{x}$ & & & & & \\
\hline Advance Street Name Sign & $\mathrm{x} \times \mathrm{x}$ & $\mathrm{x}$ & & & $\mathrm{x}$ & $x$ & $x$ & $x$ & $x$ & \\
\hline
\end{tabular}

Detailed analysis, such as the distribution of TTC, distribution of the reciprocal TTC, and variation of TTC can also be determined if the objective measurements of traffic conflicts are employed. Chin et al. (1991) and Chin et al. (1992) used video recordings to analyze the safety performance of expressway merging situations. In addition, the conflict probability can also be obtained. The risk of collision in an expressway merging process was determined using the traffic conflict technique. The conflict severity is quantified by the inverse of the time to collision. Data was collected from the Singapore Expressway over eight periods (approximately 45 minutes each), and the arrival times of vehicles at constantly-spaced intervals were subsequently extracted during video playback at slower speed. The systematic errors in the data set were adjusted. The computed measure from about 200 merging events in each observation period was best fitted by the Weibull distribution. Using the tail end of the distribution, the probability of a near-accident per merge in each period can then be established. 
Table 2-3: Suggested Improvements for Unsignalized, 3-leg, 2-lane Intersections

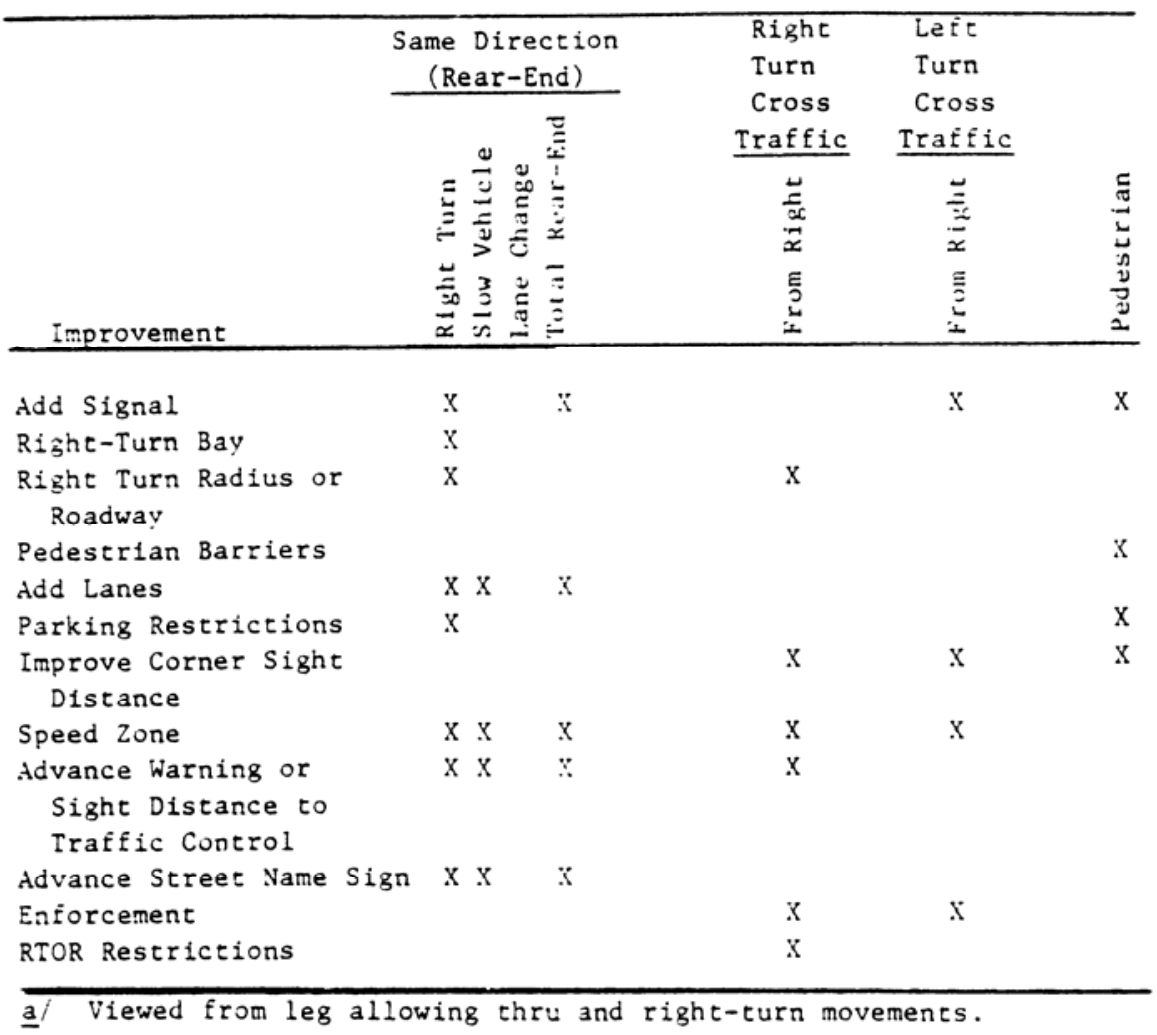

Traffic conflicts are also often used as indicators of safety performance for simulation models where objective measurements can be derived. Sayed et al. (1994) used time-to-collision with a threshold value of 1.5 seconds to define the conflict situation in the model. The conflicts detected in the model were also calibrated against the real data observation. The effects of the following factors versus traffic conflicts were studied in the simulation: volume, speed, intersection control, driver characteristics, and speed (versus conflict severity). Based on the TTC concepts, the timeexposed TTC and time-integrated TTC were proposed and used by Minderhoud and Bovy (2001) to assess the potential safety impacts of the simulated vehicles with driver support systems.

Sayed and Zein (1998) used the data collected from 94 conflict surveys to establish traffic conflict frequency and severity standards for signalized and unsignalized intersections. Using these standards, the relative comparison of the conflict risk at various intersections was achieved. An Intersection Conflict Index (ICI) was developed to summarize the conflict risk and provide an indicator regarding the relative risk of being involved in a conflict at an intersection. Sayed's and Zein's work is one of the recent attempts to standardize traffic conflict technique through a 
relatively well-defined concept of time-to-collision and risk-of-collision scores. However, the technique still relies on human observation and judgment.

\subsubsection{Reliability and Validity of Non-Crash-Based Safety Measures}

\subsubsection{Validity Issues}

Non-crash-based safety measures are usually considered valid if they can accurately represent the true safety level of a transportation system. Although several researchers proposed alternative safety measures, relatively few have examined their validity. In addition, most of the validity checks were carried out only for traffic conflicts.

Validity of safety measures relies heavily on the question of what the absolute measure of safety is. The most acceptable answer would still be the frequency and severity of crashes. Suppose that we have one intersection with a significant number of conflicts but no crash at all. Traffic conflict counts at such intersection would yield no more than a meaningless number since it by no means relates to crashes. Therefore, the validity of TCT is often judged by how good the TCT is in estimating the expected number of crashes (Hauer and Garder, 1986) or the adequacy of the correlation between the observed conflict counts and crash records (Chin and Quek, 1997).

Although several researchers reported the successful use of TCT (Zegeer and Deen, 1978; Glauz et al., 1985; Salman and Al-Maita, 1995), some researchers questioned the TCT seriously. The evaluation of TCT by Williams (1981) failed to establish a relationship between crashes and conflicts. Traffic conflicts cannot well reflect a hazard in the road system. Major reasons for the inconsistency in research findings are a lack of standard operational definition for either traffic conflicts or crashes and an unsound conflict definition that appears to bear little relationship with crash occurrences (Williams, 1981). Glennon et al. (1977) also called for reassessment of the entire concept of TCT.

Hauer and Garder (1986) addressed the issue of validity more fundamentally rather than merely seeking a good statistical correlation between conflicts and crashes. They argued that validation of the techniques based on their crash-predictive ability is unnecessary since the TCT should be used mainly as a diagnostic and evaluative tool rather than as a predictive one. The validity of the 
techniques should be best judged by comparing the variance of the estimates. The most valid method is the one that produces an unbiased estimate with the smallest variance.

Shoarian-Sattari and Powell (1987) demonstrated the relationship between behavioral-based traffic indicators and accident risks. The acceleration noise and mean velocity gradient were considered. The data collection and results were based on a 4.4-km route in East London which had experienced a total of 414 reported personal injury accidents for the three-year period 19791981. The studied route represented a mixture of segments and junctions. The equipment used to collect field data was an onboard servo-accelerometer mounted horizontally and aligned in the longitudinal direction of the motion. The objective safety measures used were accident counts and accident risks. The accident risks were computed by dividing the three-year crash counts by the appropriate traffic volumes. The correlation coefficients were computed to investigate the statistical relationship between accidents and the proposed indicators.

It was shown that both the acceleration noise and the mean velocity gradient offered useful correlations with the accident risk. The mean velocity gradient yielded the strongest correlation coefficient $\left(\mathrm{R}^{2}=0.91\right)$. The traffic volume was tested as a comparative candidate for the predictor of accident counts. The traffic volume exhibited a good correlation with crash history as well $\left(\mathrm{R}^{2}\right.$ $=0.77$ ), but it still was not as good as the proposed mean velocity gradient. However, the high $\mathrm{R}^{2}$ obtained should be viewed with caution. There were only eight data points used in the correlation analysis and therefore the confidence level of the correlation can be questionable.

\subsubsection{Reliability of Measurement}

The aspects of unreliability in the measurement can be classified into two categories (Chin and Quek, 1997; Glauz and Migletz, 1980):

- Intra-rater variation or consistency problem: inconsistency in a recording made by an individual observer

- Inter-rater variation or repeatability problem: variability in the interpretations and recordings of a given situation between different observers

Measurements for traffic conflicts, as well as other potential non-crash-based measures, are inherently subjective whenever it comes down to involvement with human observers. 
Traffic conflicts can be measured by the direct on-site observers or post-processing observations from videotape. Other measures, such as PET, ET, and TTC, are more difficult to measure on-site precisely. On-site observations suffer from the disadvantage that they are difficult to verify. Video recordings, though capable of facilitating repeated viewings, are limited as a twodimensional, sequential view of video images. Human observers are more likely to make better judgment using simultaneous views of traffic events (Chin and Quek, 1997). Also, the postprocessing time required to extract the data from the video clips can be time-consuming. There is the potential, however, for automating some measurements due to the recent advancements in image processing technology, which would reduce the processing time. The required capability and current limitations of the technology and past applications for safety analysis are discussed next.

\subsubsection{Image Processing Technology and Safety}

Intelligent Transportation Systems (ITS) can use image processing for many application nowadays. Researchers are incrementally embedding machine vision into the existing system for various applications, which poses a challenge. There are three fundamental components for image processing (Masaki, 1998):

- Image-acquisition hardware. Conventional television cameras allow an intensity contrast of 500:1 in the same image frame. Most ITS application requires a 10,000:1 dynamic range for each frame and 100,000:1 for a short string of frames.

- Real-time image processors. The challenge for this component is to carry out real-time image processing with compact and inexpensive processors.

- Algorithms. A key issue for ITS applications is how to obtain 3D information from a machine vision.

The majority of practical applications of image processing for transportation systems can be classified into one of following three categories (Masaki, 1998; Ozawa, 1999):

- Driving or cruise assistance. Machine vision is being used to detect lane markings, preceding vehicles, and obstacles. There has been significant research progress in highway driving assistance, however, the application has been found to be more difficult 
for urban driving assistance as the driving conditions are complicated by stop-and-go situations and pedestrians.

- Traffic and road monitoring. Vision-based traffic-monitoring systems involve three steps: (a) segmentation, (b) reasoning about traffic parameters such as vehicle counts, average speeds, and queue lengths, and (c) reasoning about area-wide traffic parameters such as link travel times and origin-destination counts. Vehicle identification from reading license plate numbers at the entrances and exits of roads is also becoming a popular method for travel time estimation.

- Driver monitoring. Machine vision is being used to detect driver behaviors that could lead to unsafe driving such as fatigue and drowsiness.

\subsubsection{Vehicle Tracking}

The recent rapid advances in video imaging technology make it a potential means to detect certain operational traffic characteristics with minimal, or even without, human involvement. Video Image Processing Systems (VIPS) that employ machine vision technology can be used to interpret video material collected with video cameras. VIPS generally fall into three categories: (a) tripwire systems, (b) tracking systems, and (c) spatial analysis. Tripwire systems use a camera to emulate conventional detectors by using small-localized regions of the image as detector sites. Tracking systems follow individual vehicles through the camera field of view and provide a microscopic description of their movements. Spatial analysis is used in the Image Processing for Automatic Computer Traffic Surveillance (IMPACTS) system and concentrates on analyzing the two-dimensional information that video images provide. Some commercial VIPS are tripwire systems that do not track vehicles (e.g. Autoscope) while some other systems do tracking (PEEK, 2001 and Nestor, 2001). Vehicle tracking poses the biggest challenge to researchers while it aims to provide the most complete information for the road monitoring task. Vehicle tracking has the biggest potential for safety measurement applications as information about individual vehicles in time and space is often needed. At present, vehicle-tracking strategies can be classified into four categories (Coifman et al., 1998):

1. 3D model-based tracking. This strategy focuses on recovering trajectories and models with high accuracy for a small number of vehicles. However, the weakness of this 
approach is its reliance on detailed geometric object models, which makes the method unfeasible for highway traffic with a variety of vehicle models and classes.

2. Region-based tracking. The algorithm identifies a connected region in the image, a "blob" that is associated with each vehicle, and tracks it over time using a crosscorrelation measure. The process is typically initialized by the background subtraction technique. A Kalman-filter-based adaptive background model allows the background estimate to evolve as the weather and time of day affect lighting conditions. This approach works fairly well in free-flow condition, but it fails under congested conditions where vehicles partially occlude one another.

3. Active contour-based tracking. Active contour models maintain a representation of the bounding contour of the object and dynamically update it. This method can address partial occlusion if the vehicles initially do not occlude each other; however, this condition is difficult to meet for congested traffic.

4. Feature-based tracking. Instead of tracking an entire object, this approach tracks subfeatures such as distinguishable points or lines on the object. The approach was found to apply well, even in the presence of partial occlusion. The features are grouped together using a common motion constraint. It also works well in different lighting conditions, i.e., day, night, and twilight.

Vehicle tracking has been employed in several ITS applications. The feature-based tracking approach used by Coifman et al. (1998) measures traffic parameters, i.e., generalized flow, occupancy, and velocity. This approach is considered to be an area-wide detector. By averaging trajectories over space and time, the traditional traffic parameters are more stable than corresponding measurements from point detectors, which can only average over time.

Taktak et al. (1996) proposed a two-stage detection methodology to detect vehicles on highways, namely, an initialization phase and a detection phase. Malik et al. (1994) developed a prototype of automatic traffic scene analysis for IVHS (Intelligent Vehicle Highway Systems) applications that combined a vision-based traffic surveillance system with a dynamic belief network and symbolic reasoning. An explicit occlusion reasoning algorithm was developed by Koller et al. (1994) to compensate for shifts in vehicle trajectories caused by vehicles overlapping. Pflugfelder and Bischof (2000) proposed a Kalman filter tracking approach to track cars traveling in a tunnel. The algorithm can detect only a pair of lights, which excludes bicycles and motorcycles and vehicles with broken lights. Kamoji et al. (2000) used a Spatio-Temporal Markov Random Field 
(MRF) model to segment and track traffic images at an intersection. The Spatio-Temporal MRF was found to be robust against random occlusions.

In summary, the primary challenges for a vehicle tracking strategy at present are: (a) real-time processing, (b) robust detection and tracking of various vehicles, (c) robustness against different lighting conditions and (d) partial occlusion (Pflugfelder and Bischof, 2000).

\subsubsection{VIPS for Safety Applications}

Limited research for safety application using image processing technology has been one mainly because the current capability of the technology still does not meet the exacting requirements for the safety applications. For example, there is still no vehicle tracking algorithm that can address all the issues described in the previous section effectively and efficiently. Certain research attempts in the past employed image processing units designed primarily for traffic control purposes to help detect certain traffic events. To the best of our knowledge, there is still no commercialized software and/or hardware developed specifically for safety analysis and/or the evaluation task to date.

Tarko and Lakshmikanth (2001) checked the feasibility of using Autoscope-based video detection to monitor red light running. The concept of monitoring red light running with video detection systems and the evaluated prototype method were described. The evaluation methodology is given and the results are discussed. Out of 107 violations observed during five days, 55 were detected. Thirty-four false detections were also reported. No significant difference in performance was observed between daytime and nighttime periods. Monitoring of through lanes was considerably more effective than of left-turn lanes. The performance of a fully-automated approach was not satisfactory enough and therefore the semi-automated method was proposed. Human reviewers were required to check the Autoscope-based outputs manually.

Mizun et al. (2002) investigated the image processing technique for the driver aid system. They recommended location of a CCD camera for the driver monitoring system inside a vehicle based on the calculated distribution result of driver's eyellipse and data collected through an actual driving test. Furthermore, a method to evaluate the driver's awareness level is explained. This experiment measured how long the drivers closed their eyes while driving by using an image processing technology to assess their awareness level. 
Abbas and Bonneson (2003) provided some guidelines for VIP unit detection placement and configuration. The developed guidelines refer to four vendors of commercial VIP units: Vantage, Traficon, Peek, and Autoscope. Although the guidelines are meant primarily for traffic control applications, several rules are applicable for vehicle detections in general. These guidelines should be taken into account when VIP units are considered for vehicle detections in safety applications. The authors discussed and evaluated proposed guidelines for urban/suburban settings with design speeds of less than $60 \mathrm{mph}$. The proposed detection design was found to reduce both the max-out frequency and the vehicle waiting time based on six selected intersections in Texas. Some considerations and guidelines pertinent to the performance of the detection zone are briefly summarized as follows:

\section{Effect of Camera Perspective}

A major functional difference between VIP detectors and loop detectors is the effect of the camera perspective on the vehicle effective length. The low camera height, small pitch angle, and a long perpendicular distance from the camera to the vehicles can increase the effective vehicle length.

\section{Monitored Road Length and Camera Height}

When placing the detectors on the far side of the screen, the "1:10 rule" was suggested. This means one foot of camera height is required for each 10 feet of monitored road length. Therefore, for a height of 45 feet, the monitored road length should be within 450 feet for optimal detection where the speed and count are important. Otherwise, the "1:17 rule" was reported to be acceptable.

\section{Optimizing the Field of View}

The calibration of the field of view should include: (a) adjusting the camera zoom and focus such that the detected area is shown in the screen image, (b) adjusting the pitch angle of the camera such that the view excludes the horizon, and (c) the detection zones should not be placed over 
high contrast objects such as stop line, lane markers, crosswalks, span wires, street signs, commercial signs, etc.

\section{Detection Functions}

The VIP detectors can be joined to each other using logical functions. The general use of the AND function is to reduce false detection caused by vehicles (and their shadows) in the adjacent lanes. The OR function is commonly used to increase the sensitivity of the detection area by linking several detectors together.

\section{Detection Size}

The Autoscope manual recommends that the detection zones should be about three-quarters to one vehicle long and one and one-half times the width of the vehicle headlight. Smaller detection zones are recommended for smaller objects further upstream. However, the Autoscope manual also cautions against too small detection zones as they will limit the ability to compensate for visual distortions caused by shadows, video noise, reflections, and camera motion.

The authors also provided a procedure to calculate the effective vehicle length and design stop bar and advance detections. The data was collected from the field for the before-and-after study. The measures of effectiveness are the frequency of max-out and the vehicle waiting time.

The proposed guidelines were found to improve the operational performance of VIP based on the considered MOEs. The study revealed no significant difference in the improvement for two different VIP units considered, Vantage and Autoscope. Midday operation was found to improve more than the other periods. A different set of detector configurations was suggested for nighttime operation as the detectors are triggered by the headlight, not the object. The detectors tend to recognize the calls earlier than usual and drop the calls before the trailing edge of a vehicle left the detection zone. 


\subsubsection{Safety Evaluation Using Simulation}

As certain non-crash-based measures of safety are difficult to obtain in the field, some researchers employed simulation techniques in the hope that the parameters extracted from simulation would be able to replicate those of the field conditions.

Sayed et al. (1994) extracted time-to-collision with a threshold value of 1.5 seconds to define the conflict situation in the simulation model. The conflicts detected in the model were also calibrated against the real data observation. The effects of the following factors versus traffic conflicts were studied in the simulation: volume, intersection control, driver characteristics, and speed (versus conflict severity).

Minderhoud and Bovy (2001) extracted the time-exposed time-to-collision (TET) and timeintegrated time-to-collision (TIT) from a microscopic simulation model in order to assess the safety impacts of driver support systems, i.e., Autonomous Intelligent Cruise Control or AICC. These measurements were found to be sensitive to the specified threshold values. The benefits and interpretation of TET in relation to safety performance were not well understood.

One recent project conducted by Gettman and Head (2003) was to evaluate the assessment of surrogate measures of safety for intersection traffic from existing, commercially-available traffic simulation models. The authors reviewed the capabilities of traffic simulation models for obtaining surrogate safety measures, the functional requirements for a surrogate safety assessment software tool, and a set of surrogate measures to collect from traffic simulation models to assess the safety of various intersection designs and timing strategies. The surrogate measures proposed in the report were:

- Minimum time to collision during the conflict event

- Minimum post-encroachment time during the conflict event

- Initial deceleration rate of the reacting vehicle

- Maximum speed of the two vehicles

- Maximum speed difference of the two vehicles during the conflict event

- Location of the starting and ending points of the conflict event.

The first three measures were based on the work of Allen et al. (1978). The maximum speed and maximum speed difference were used to measure the severity of a conflict event. The possible 
situations where the traffic conflict events can occur were presented. These situations were classified into three categories:

- Conflict points: interacting vehicles cause a crossing conflict

- Conflict lines: interacting vehicles cause a merging conflict

- Rear-end conflict lines: one vehicle follows another (leading vehicle).

Computational algorithms to calculate these measures were presented in the report. The algorithms for conflict lines and rear-end conflicts require successive computations to determine the worst cases.

At the implementation level, the new software was written as an external module that would allow users to import the event file from the simulation software to perform surrogate safety analysis. The entire process of computing, extracting, and analyzing the surrogate measures from the traffic simulation models was dubbed "Surrogate Safety Assessment Methodology (SSAM)."

The validation procedures to assess the connection between the surrogate measures and intersection safety were discussed in terms of hypothesis testing. The first hypothesis was to verify that different intersection designs can be discriminated by the distributions of surrogate measures from the simulation model. The second one was to assess the correlation between the traffic conflicts from the simulation and the field study. This by no means guarantees the relationship between simulated conflicts and actual crashes. The last hypothesis was that the surrogate measures produced by the simulation model would predict (be correlated with) the difference in traffic conflicts, as experienced in real-world condition, between "before" and "after" conditions of an intersection subject to safety improvements.

Huang and Pant (1994) developed a simulation-neural network model to help evaluate the dilemma zone problems at low-volume, isolated, high-speed signalized intersections. The alternative evaluation approaches were briefly discussed together with their limitations. The neural network was used to predict driver behavior in terms of speeds at different zones. Key ANN model inputs consisted of current speed, vehicle type, traffic control devices (types and locations), signal status, vehicle movement, and intersection geometry. The output of the ANN model was the speed of the next zone. The ANN model was integrated with the simulation model which produced vehicle arrivals, controlled the traffic signals, and kept updating system activities and collecting relevant statistics. Several outputs were collected from the simulation. The safety- 
related measures of effectiveness (MOE) for evaluating dilemma zone problems were: (a) probability of being caught in dilemma zone (PBCDZ) and (b) vehicle conflict rates. The conflicts in this research were defined as: (a) abrupt stop, (b) acceleration on yellow, and (c) red light running. The conflict rate was the number of conflicts per 1,000 vehicles. The data was colleted from four signalized intersections for model calibration. The PBCDZ was obtained only from the model, however, by tracing individual vehicles and checking whether it was caught in the dilemma zone at the onset of yellow indication. PBCDZ was not observed from the field. The model was tested with one other intersection to evaluate its performance. The conflict rates were found to vary from $22 \%$ to $47 \%$.

The developed ANN model can be used as a non-crash-based safety evaluation procedure for high-speed signalized intersections. However, it needs more data collection to cover a wide range of existing conditions before its validity can be proved. PBCDZ estimation is still limited to only simulation. Field data is still needed to verify this information.

It is obvious that the benefits of the simulation approach are: (a) it requires the least human involvement, which is the main source of subjective error; and (b) it can extract certain parameters that are difficult to obtain in the field. Nevertheless, the simulation approach is hampered by its reliance on the assumption that the simulation-based outputs would be representative of the field conditions. Often, this is proven to be unrealistic, which therefore lessens the utility of the simulation approach.

\subsection{CRASH-BASED SAFETY EVALUATION}

To supplement the non-crash-based safety evaluation, this section reviews past studies on crashbased safety evaluation, particularly at signalized intersections. The techniques frequently used to model crash frequency can be classified into three major categories: (a) parametric regression, (b) non-parametric regression, and (c) the Bayesian approach.

\subsubsection{Parametric Regression}

Parametric regression using generalized linear models (GLM) is the most widely-used method in safety modeling. GLM allows flexible assumptions on the distributions of dependent variables. 
When the response variable is normally distributed with an identity link to the mean parameter, the GLM approach is commonly known as a normal linear regression. A GLM with a Poissondistributed response variable is frequently used to model crash counts; this method is called Poisson regression. When we further allow the Poisson mean parameter to be a gammadistributed random variable, the model becomes a negative binomial regression.

Turner and Nicholson (1998) reviewed models used in practice to relate accidents to traffic flows, with particular emphasis on the appropriateness of the model form and the statistical analysis technique employed for parameter estimation. The relationships between crashes and traffic flows can be developed either directly or indirectly. The direct approach involves the development of a relationship between crashes and traffic flows. The indirect method seeks to identify the relationship between a crash and its exposure, which in turn relates to traffic flow. The indirect method takes into account the mechanism of crash occurrence while the direct approach, which rests mainly on statistical correlations appears to have a logical fault (Hauer et al., 1989).

The typical relationship used for the normal linear regression is of the form

$$
Y=\beta_{0}+\beta_{1} X_{1}+\beta_{2} X_{2}+\ldots
$$

with a normal-distributed error structure having zero mean and constant variance. However, there are some problems with this type of model: (a) crash frequencies are discrete but normal distribution is continuous, (b) the variance of crash counts tends to increase with the flow and expected crash counts, and (c) crash counts are nonnegative.

The Poisson or Negative Binomial error structure is found to be more appropriate (Maher and Summersgill, 1996). The choice of which depends on the variability of crash counts for a particular flow situation. If the variance is about the mean, the Poisson distribution is appropriate. If the variance is significantly greater than the mean, the negative binomial distribution should be used. For GLM, the Poisson and Negative Binomial error structures can be easily incorporated into a multiplicative model

$$
Y=\beta_{0} X_{1}^{\beta_{1}} X_{2}^{\beta_{2}} \cdots
$$

Turner and Nicholson (1998) tested for the appropriate functions of volumes in the crash models. They concluded that the model with conflicting crashes related to the product of the conflicting 
traffic volumes appeared to be the most appropriate logically. However, it was found that this form did not outperform the other types in general. The nonlinear model forms were preferred over linear and quadratic types since it is consistent with the evidence that the rate of increase of crashes tends to decline as the flow rate increases. The drivers are likely to exercise more care while driving as the flows increase, thereby resulting in a lower chance for a crash. Crash rates tend to level off as the flow increases. The analysis on the role of the non-collision flows showed that some crashes involving particular movements are affected by the flows associated with other movements. The addition of non-collision flows into the model based on the mechanism of crash occurrences requires considerable care to avoid omitting important non-collision flows. The alternative approach is to include all flows initially and eliminate the statistically insignificant ones later. The collision flows, as logic dictates, cannot be removed even if they are not statistically significant.

Affum and Ap Taylor (1996) developed regression models to help predict crash frequencies by type based on different types of traffic flow movement, signal parameters, intersection geometry, and location factors. The data of 115 selected signalized intersections in metropolitan Adelaide were assembled for the analysis. For each intersection, the following information was gathered: (a) signal phasing diagram, (b) coordinated or not, (c) fixed time or actuated, (d) cycle length and timing plans, (e) intersection geometry, (f) number of intersection legs, and (g) number and width of lanes. The types of accidents considered in the model development were rear end, side swipe, right angle, and right turn.

The exposure measure used was based on the total traffic flow contributing to each accident type. The sum of the flows was used for the traffic traveling in the same direction. The product of conflicting flows was used for right-angle and right-turn accidents. Right-angle accidents were found to be poorly explained by exposure. This implied that some factors other than exposure may be present at a right-angle crash occurrence, such as red light violations, during certain periods of time. Drivers may be more likely to run on red during periods of low flows, thereby independent of traffic exposure. It was suggested that the actual traffic flow during the period of crash occurrence would be beneficial for the improvement of model efficiency. Some other traffic control factors, such as right turn prohibition during certain times of day, were also found to be statistically significant for right-angle crash prediction. 
Poch and Mannering (1995) used negative binomial regression to estimate the frequency of accidents at intersection approaches. Seven-year accident histories from 63 intersections in Bellevue, Washington were used in the model estimation. One model with all types of accidents and three other models with specific types (rear-end, angle, and approach-turn) were estimated using geometric and traffic-related elements as explanatory factors. Abdel-Aty and Radwan (2000) used negative binomial regression to model the frequency of accident occurrence and involvement on a principal arterial in Central Florida. The model illustrated the significance of the AADT; the degree of horizontal curve; lane, shoulder, and median widths; whether urban or rural; and the segment length. Akaike's information criterion (AIC) was used to decide which subset of independent variables should be included in the model. AIC identifies the best approximating model among a class of competing models with a different number of parameters. To measure the overall goodness-of-fit statistics, the deviance value, which is $\chi^{2}$-distributed, was used. The elasticity of each variable was computed to determine the relative significance of the variable on the dependent variable, which is crash frequency. Three categories of the model were developed: (a) overall model, (b) models by gender and (c) models by age group. The paper attempted to add human factors into the models by considering gender and age group. Female drivers were found to experience more crashes than male drivers in heavy traffic volume, reduced mean width, narrow lane width, and larger number of lanes. Male drivers had a greater tendency to be involved in traffic accidents while speeding. The models also indicated that young and older drivers experienced more accidents than middle-aged drivers in heavy traffic volume and when there were reduced shoulder and median widths. Younger drivers had a greater tendency to be involved in accidents on roadway curves and while speeding.

Chin and Quddus (2003) used the random effect negative binomial (RENB) model to analyze traffic accident occurrence at signalized intersection in Singapore. The RENB model allows the data to be treated as a time-series cross-section panel, therefore addressing the spatial and temporal effects in the data. Although negative binomial regression relaxes the variance-to-mean unity assumption and allows between-site variations, it still suffers from the limitation that the temporal variations are not well considered. Consequently, the standard error in the model coefficients may be underestimated and the t-ratios may be inflated. Hence, the RENB model was used to address the possible serial correlation of crashes at each specific location. The model explanatory variables included traffic volumes, geometric elements, and traffic control data. The goodness-of-fit statistics of the model showed that the RENB model was appropriate. 


\subsubsection{Non-Parametric Regression}

In contrast to the parametric-based approach, there exists an alternative approach to model accident frequencies without the need to rely on the distributional assumptions of the models. Karlaftis and Golias (2002) used a non-parametric statistical methodology known as hierarchical tree-based regression (HTBR) to quantitatively assess the effects of geometric elements, pavement conditions and traffic volumes on the accident rates of rural roads. The HTBR was used separately for the data from two-lane and multilane roads in Indiana from 1991 to 1995. The key advantages of HTBR over the widely-used negative binomial regression are: (a) it allows for quantitative assessment of the effects of each factor on accident rates and quick prediction of accident rates given a set of factors; (b) it is non-parametric and does not require specification of a functional form; (c) it does not require a pre-selected set of variables since it uses a stepwise method to determine optimal splitting rules; (d) it can handle data sets with a complex (nonhomogeneous) structure; (e) it is robust to the effects of outliers; and (f) it is not affected by multicollinearity between the independent variables. Example of a regression tree obtained from HTBR for rural two-lane roads is shown in Figure 2-4.

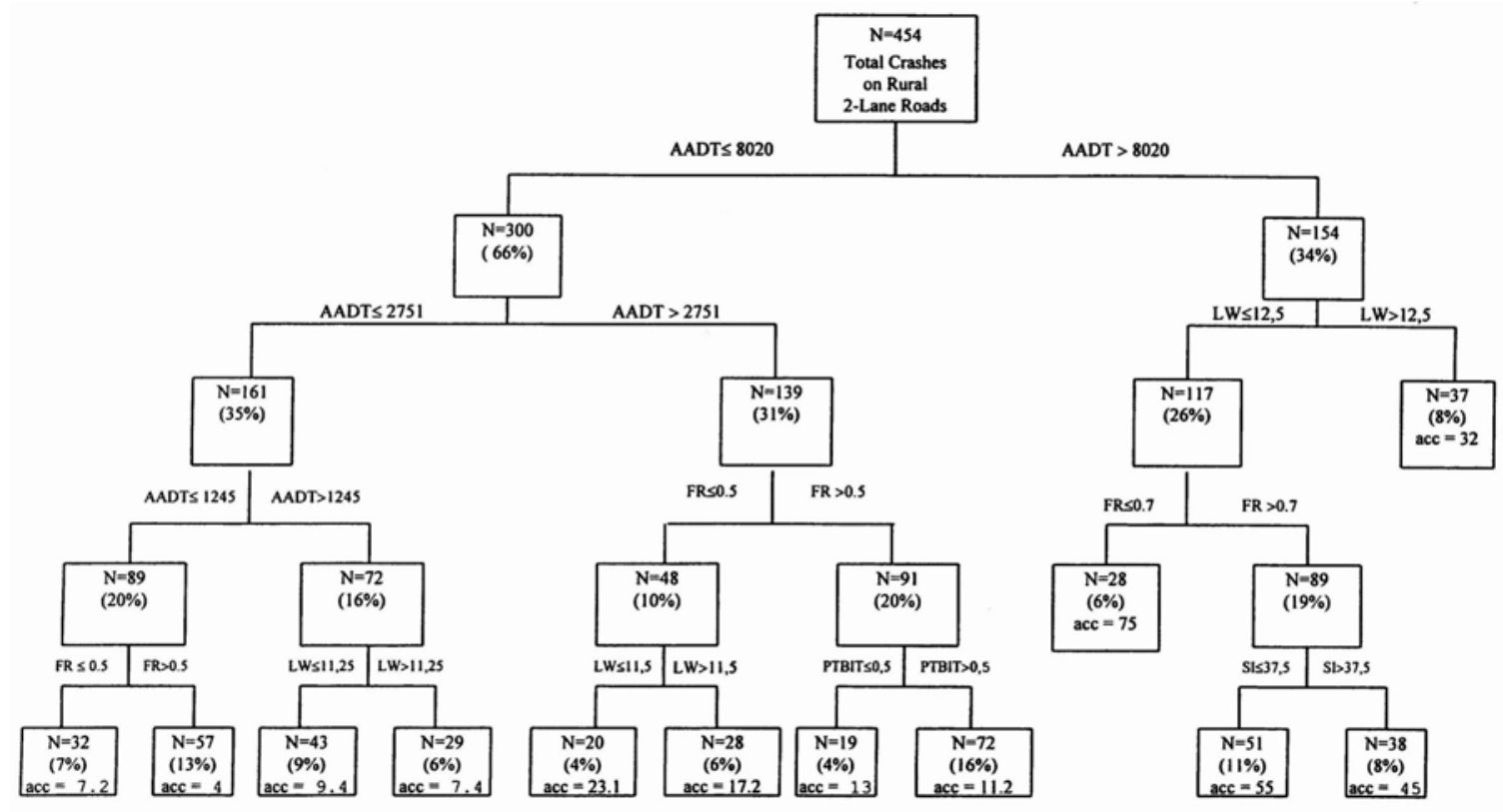

Figure 2-4: Example of Regression Tree Using HTBR (Karlaftis and Golias, 2002)

In Poisson or negative binomial regression, the relative significance of each variable can be measured by the elasticity. For HTBR, the importance scores can be calculated to determine the effect of each element on accident rates. Since HTBR uses an if-then structure in the regression 
tree, the method has the potential for some specific application in SMS, such as a rule-based expert system.

\subsubsection{Empirical Bayesian Approach}

In an observational study, there is likely to be a link between the decision to treat a selected site and its crash history. This link causes a so-called "selection bias" or "regression-to-mean" bias. It makes $\mathrm{K}$ a biased estimate of $\kappa$ where $\mathrm{K}$ and $\kappa$ denote the actual and expected crash counts of the before period respectively. The "before" period ended when the treatment was applied. However, there is no fixed time when the period had begun. The empirical Bayesian (EB) method aims to address the regression-to-mean bias and improve the statistical precision of parametricbased estimations.

\subsubsection{1 $\quad \underline{\text { Regression-to-Mean Bias }}$}

The regression-to-mean bias was often discussed in literature (Abbess et al., 1981; Hauer and Persaud, 1983; Hauer and Persaud, 1987). The phenomenon occurs when the entity is treated because its "before" crash counts $(\mathrm{K})$ was abnormally high or unusually low such that $\mathrm{K}$ cannot be a good estimate of $\kappa$. The $\mathrm{K}$ cannot be a good estimate of what is normal and usual by using abnormal crash counts. If the entity has been selected because of a high number of crashes, $\mathrm{K}$ would tend to overestimate $\kappa$.

\subsubsection{Methodology}

The empirical Bayesian approach accounts for two types of information to estimate the safety of the entity: (a) safety traits such as the geometry of intersections, traffic, driver behavior, and the environment and (b) crash history. The use of both estimates results in mean safety towards which the individual estimates appear to regress (Hauer, 1997).

The safety estimates for the first type of information can be derived using the reference population. The reference population is defined as the group of entities that share the same set of traits as the entity in the safety of which we are focusing. Safety traits are characteristics that can 
influence safety. Traffic control type, traffic volume, and geometry can play a pivotal role in the safety estimates of the reference population.

The estimation of safety using the empirical Bayesian approach has been investigated by several researchers (Abbess et al., 1981; Hauer and Persaud, 1983; Hauer, 1997; and Garder et al., 2001). The logic of the estimation is depicted in Figure 2-5.

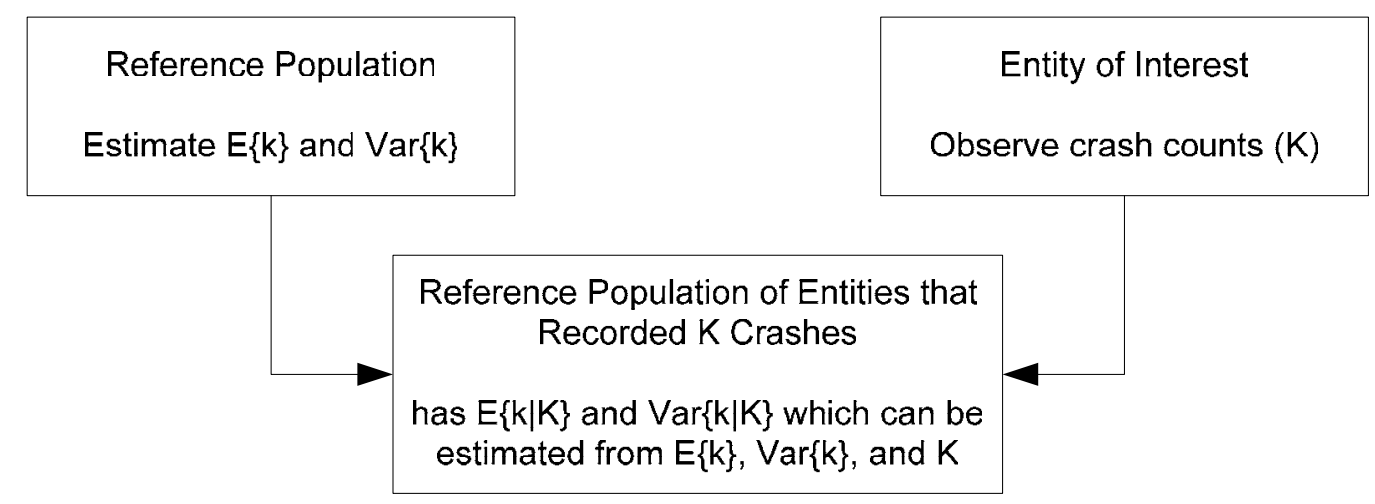

Figure 2-5: Logic of Estimation Using Empirical Bayesian Approach

The estimate of $\kappa$ and its variance for the specific entity can be obtained by the following equations:

$$
\begin{gathered}
E\{\kappa \mid K\}=\alpha E\{\kappa\}+(1-\alpha) K, \text { where } 0<\alpha<1, \\
\alpha=\frac{1}{1+\frac{\operatorname{Var}\{\kappa\}}{E\{\kappa\}}}, \text { and } \\
\operatorname{var}\{\kappa \mid K\}=(1-\alpha) E\{\kappa \mid K\} .
\end{gathered}
$$

It should be noted that if we evaluated the variance of $\kappa$ using only $K(\alpha=0)$ and assuming $K$ is Poisson distributed, the variance of the estimate would be $\mathrm{E}\{\kappa \mid \mathrm{K}\}$. When both clues of safety are used, the variance becomes smaller.

Sometimes it is useful to model the entire probability distribution function of $\kappa \mid \mathrm{K}$. It is often assumed that the expected "before" crash counts for a reference population ( $\kappa)$ follow Gamma distribution (Al-Masaeid and Sinha, 1994 and Hauer, 1997). If the crash count for an entity (K) is Poisson-distributed and the expected counts of reference population $\kappa$ are gamma-distributed, the 
distribution of crash counts in the population of entities obeys the negative binomial probability distribution.

\subsubsection{3 $\quad \underline{\text { Applications }}$}

Belanger (1994) applied the empirical Bayesian method to estimate the safety of four-legged unsignalized intersections. First, multivariate models were developed to estimate the number of accidents from various flow functions at these intersections. The best model was obtained from the product of major and minor flows, raised to a power. Attempts were made to develop models for specific patterns of collisions and to incorporate variables other than traffic flow functions to these models. The modeling results were then combined with the accident count of a four-legged unsignalized intersection to estimate its safety. Results were used to identify blackspot locations and to evaluate the effects of treatments more accurately.

Persaud and Nguyen (1998) developed disaggregate safety performance models for three-legged and four-legged signalized intersections on Ontario provincial roads. Models were disaggregated by time period, accident severity, and environmental class. Two levels of models were calibrated for different levels of data availability and model requirements. For level 1, the crash frequency was estimated as a function of the sum of all entering flows. Separate estimates were obtained for three different types of crashes: rear-end, right angle, and turning movement. In level 2, specific patterns were defined by pre-accident vehicle movements and crashes were estimated as a function of flows relevant to each pattern.

The models were calibrated based on the empirical Bayesian framework. The estimate obtained by combining the accident count $(\mathrm{x})$ of a specific intersection in the most recent $\mathrm{n}$ years with an estimate of the expected annual number of accidents $(\mathrm{P})$, based on the accident history of similar intersections. This estimate of $\mathrm{m}$ is: $\mathrm{m}=\mathrm{w}_{1}(\mathrm{x})+\mathrm{w}_{2}(\mathrm{P}) . \mathrm{w}_{1}$ and $\mathrm{w}_{2}$ are estimated from the mean and variance of the regression estimate. The selected model form of a safety performance function is

$$
P=\alpha S_{1}^{\beta_{1}} S_{2}^{\beta_{2}}
$$

where $S_{1}$ and $S_{2}$ are relevant flows; $\alpha, \beta_{1}$, and $\beta_{2}$ are parameters to be calibrated. In the level 2 model, $S_{2}$ and $\beta_{2}$ are dropped out and $S_{1}$ becomes the sum of all flows. Obviously, the model is of 
a nonlinear functional form and the dependent variable is nonnormal. The calibration of this equation was done using a generalized linear model approach. The models in both levels can be used to provide estimates for identifying intersections for treatment or evaluating the safety effectiveness of countermeasures.

\subsection{SIgNAL TIMINGS, DRIVER ATTITUDES, AND RIGHT-ANGLE CRASHES}

Rear-end and right-angle collisions are the two most common types of accidents at signalized intersections. Rear-end collisions occur when a leading driver decides to stop and the follower does not anticipate the stop and fails to perform an evasive maneuver. Right-angle collisions, on the other hand, happen as vehicles from two conflicting approaches decide to enter the intersection at the same time. It is well known that one significant factor in right-angle crashes at signalized intersections is signal timing. This section provides overview of the effects of the signal timing and driver attitudes on the risk of right-angle crashes.

Studies in the past revealed that the risk of right-angle collisions can be significantly reduced by up to $40 \%$ when all-red intervals are used (Benioff et al., 1980; Butler, 1983; Hagenauer et al., 1982). An experiment in Virginia conducted by Retting et al. (2002) showed that a slight modification of signal timing (yellow and all-red intervals) can reduce a crash risk for overall crashes and pedestrian/bicyclist related crashes. However, the reductions were not statistically significant for specific types of crashes, i.e., right-angle and rear-end collisions.

Hulscher (1984) concluded that the timing of three-second yellow was too short for the majority of intersections. The yellow interval was suggested to be varied according to the approach speed and road conditions. The approach grades were not taken into account in the timing design. The increase in yellow time was found to reduce the rate of red runners only at the beginning. The violation rate was not sustained and, in contrast, deteriorated again within two years. From the operational efficiency point of view, the slight increase in the effective lost time due to the increase in change interval had only a marginal effect upon intersection capacity or delay, provided that the intersection was not operating under saturated conditions.

From the deterrence aspect, the risk of accident involvement or punishment under the law has no substantial effect on run-on-red behaviors. The only effective deterrent is the presence of police officers at intersections. Hulscher (1984) suggested that the most promising countermeasure 
appears to lie in a change in societal attitude to traffic rule violations in general. One possible approach to change this is a sustained promotional activity combined with suitable restraints on advertising and entertainment.

Mahalel et al. (1985) studied the effectiveness of a flashing green program prior to the yellow, leading to a red interval. The deleterious effects of flashing green program were dominant at lowspeed approaches and at urban intersections. A laboratory experiment was set up to investigate the rate of inappropriate decisions under various driving conditions. Inappropriate decisions were defined as a "go" decision after the red light and a "stop" decision when the drivers can actually proceed. The probability of stopping as a function of the potential time to reach the intersection was plotted. The potential time to reach the intersection is the time lapsed from the onset of yellow to the stop bar if drivers maintain the prevailing speed at that instant. The logit function was fitted to the data. It was found that the likelihood of stopping was very high even if the potential time was very short. This implies more rear-end conflicting drivers during a flashing green program. There also was no evidence that right-angle conflicts were reduced.

Van der Horst and Wilmink (1986) discussed factors which may influence the driver's decisionmaking process during the yellow time, which are:

- The driver's attitude

- The amount of predictability of the situation

- An estimate of the consequences of not stopping, e.g., the risk of right-angle collisions, running on red and, getting a ticket

- An estimate of the consequences of stopping, e.g., discomfort, delay, the risk of rear-end collisions

- Driver's estimates of the required decelerations based on the speed and the distance to the stop bar

- The yellow and all-red periods

- The influence from other road users, such as the leader's stopping.

In addition, van der Horst and Wilmink's study also summarizes several findings of other researchers on the effects of the duration of yellow and all-red intervals on run-on-red disciplines. It was found that overlong all-red times decreased the red-light discipline. When the yellow period was extended from three to four seconds, the number of red phases with at least one 
violation decreased from $7 \%$ to $2 \%$. In addition to that, yellow duration of less than three seconds was also found to increase the number of red light violations significantly.

It was also found that the extension of the yellow time in the urban areas from three to four seconds appears to reduce the number of red light violations by half (van der Horst and Wilmink, 1986). Nevertheless, the adaptation of driver's behavior was found to be minimal based on several plots of the stopping probability functions versus the time to the stop bar, the distance to the stop bar, and the required deceleration rate. No significant change was found between sixmonth and twelve-month "after" periods.

In another study by van der Horst (1988), he comparatively studied the driver decision-making process at signalized intersections (fixed time versus actuated controllers), signals for drawbridges, and signals for railroad grade crossing.

At signalized intersections, he confirmed, based on the literature study, that a one-second increase of the yellow interval can diminish the number of run-on-red offenses by half due in large to reduced exposure. However, only a slight behavior adaptation of drivers was observed. There exists a one-second shift between the probability of stopping for fixed-time controllers and that of actuated ones. At actuated intersections, the drivers were found to decide to proceed in the early stage of the approach process, compared to fixed-timed intersections. The drivers expect to see green extension at actuated controllers and thereby counter their anticipations when the green time is maxed out. This also implies that drivers are more disciplined at fixed-time intersections.

Comparative studies of driver decisions at drawbridges and railway grade crossings yielded some interesting findings. Yellow signals were absent for both types of locations. At drawbridges, it was found that driver willingness to stop seemed to be poor. One possible explanation is that the motorists are familiar with the system and know that the bridgekeeper can interrupt the procedure. This, however, can also be partially attributed to the operational deficiencies of the signal system itself. In contrast, the drivers were found to be more willing to stop at railway grade crossings than those at signalized intersections. The drivers appear to recognize the risk of running on red at this type of locations. Some emergency braking (more than $4 \mathrm{~m} / \mathrm{s}^{2}$ ) was also observed. 


\section{CHAPTER 3 METHODOLOGY}

The research procedure utilized in this study will be described in this chapter. We will begin with a very important concept called the continuum of events. This concept is the crux of our argument about surrogate measures of safety. Then, we will highlight issues typically encountered in the measurement and validation of surrogate safety measures. The methodology used in this study aims to address these issues using a right-angle collision as a research starting point.

\subsection{CONTINUUM OF EVENTS}

It is interesting to note that the concept of safety may be linked to some events during crash-free operations. Let us refer to these events as crash-generating events. What events can be considered as crash-generating events? If we depict this notion as a pyramid representing the continuum of events (see Figure 3-1), safety alternatively might be recognized by some traffic events; some turn into accidents and others near misses.

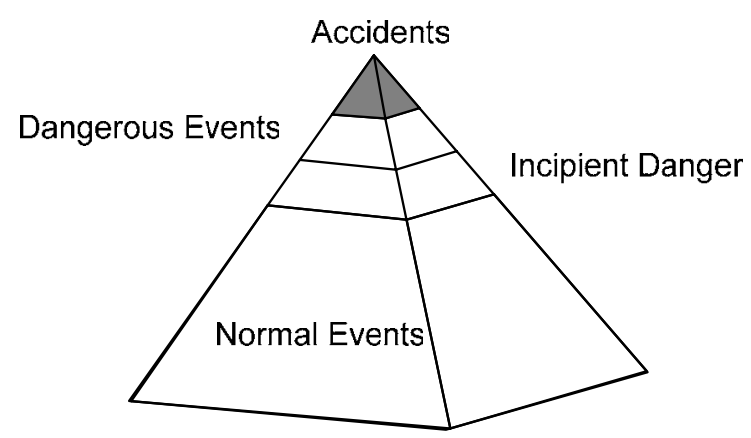

Figure 3-1: Continuum of Traffic Events

The volume of each layer represents the frequency of the events. An accident is a very rare event; hence, there is a great challenge to define safety in terms of the frequency of dangerous traffic events instead of crashes. There is one important assumption underlying this pyramid in order to confirm a linkage between dangerous events and accidents. The crash-contributing factors present 
during the time that we observed these dangerous events must be present at the time of crash occurrences as well. Past research attempts on non-crash-based safety measures were to search for the potentially dangerous events and then confirm their linkage with historical crash data. Without the underlying hypothesis of the continuum of traffic events, these research attempts in the past would have been invalid.

\subsection{CURRENT APPROACH TO NON-CRASH-BASED SAFETY ANALYSIS}

Currently, difficulties encountered in the analysis of non-crash-based safety measures can be classified into two major categories, measurement and evaluation. This section describes the limitations of current practice from these two aspects. Then, in the next section, we will propose the research methodology and describe how these issues can be addressed with the proposed analytical framework.

\subsubsection{Measurement Issues}

Two major measurement problems are accuracy and efficiency. The accuracy of non-crash-based safety measures is often compromised by the involvement of human observers. Traditional traffic conflict technique, for example, relies on the subjective judgment of humans as to whether the situation can be defined as a conflict. The introduction of the time-to-collision (TTC) concept can reduce the subjective nature only to a certain extent. The complexity of certain measures, such as time-integrated TTC, rendered themselves more difficult to be measured in the field. Some researchers therefore resolved this complication using a simulation approach. The obvious shortcoming of the measurements extracted from simulation is that it by no means assures that the actual field conditions are being replicated. The efficiency of the measurement methods is characterized by the amount of effort needed for the data collection task. In general, there exists an accuracy-efficiency tradeoff in the measurement process. A sophisticated safety measure may require an excessive amount of time and thus makes it impractical for a large-scale study. Let us consider TTC as an example. A precise measurement of TTC would require a computation of the time remaining to the collision point of the approaching vehicles for every instant over time. This would apply to all vehicles entering the intersection. This is practically impossible to implement in real-time as of today (a robust algorithm is also a challenge other than the computational complexity). 


\subsubsection{Evaluation Issues}

Safety researchers have attempted to examine the relationship between non-crash-based safety measures and actual crashes for more than four decades. Scores of research records yielded mixed findings at best. The validation failure may be attributed to several sources: (a) measurement errors, (b) arbitrary threshold specifications for certain measures, and (c) the assumption of constant risk across locations.

The extent of the impact that measurement errors could have on evaluation results has not been fully understood to date. Frequently, researchers worked around this issue by either treating them as negligible or minimizing them with the help of technology such as digital video recording.

In general, not all observed events are of interest. A wide spectrum of collision and non-collision events can be viewed as a pyramid shown in Figure 3-1. "Safer" and more frequent events occupy the pyramid's lower part while more "dangerous" events are located above. The threshold must be specified as to which event should be treated as dangerous; for example, a TTC lower than 1.5 seconds or a PET less than three seconds. Referring to past practices, a threshold is invariably chosen as a constant and usually arbitrarily. The consequence of an incorrect threshold specification can be severe because it can influence all components of the study, ranging from data collection to evaluation tasks. In addition, there is no basis to support the practice of a constant threshold other than simplicity.

Past research attempted to demonstrate that the frequency of collisions (the top of the pyramid) and the frequency of other events are related in the form of the following expression

$$
C=k \cdot E,
$$

where $C=$ crash frequency (crashes/year), $k=$ coefficient (typically estimated with regression) and $E=$ surrogate measure; here frequency of non-crash events (vehicle passages, traffic conflicts, and etc). $E$ is sometimes called exposure, particularly when $E$ is the number of vehicle passages.

Hauer (1982) discussed the fundamental flaws in attempts to use conflict and exposure interchangeably. If the conflicts are being used to estimate safety, the risk of two systems being compared must be the same. However, if the conflicts are to be treated as exposure for the risk estimation, this would imply varying risks from system to system, thereby rendering the conflict 
method invalid. This issue can be resolved by careful definitions of the risk and exposure for a system being studied. In this research, we will further extend this concept by illustrating how the risk and exposure can be defined and estimated simultaneously using the proposed method.

The relationship (3-1) postulates that the frequency of crashes is proportional to the frequency of a specific class of events, e.g., dangerous events. The postulated relationship meets the intuitively acceptable boundary condition of zero crash frequency at a location with a zero frequency of dangerous events. This proportion is assumed to be unchanged across all similar locations. There is no basis for this claim. In reality, there rarely exist two locations that share identical traffic event behavior even if they are of the same type. Consequently, the relationship in equation (3-1) calibrated for some locations is not transferable to other locations without adding additional variability to the crash frequency estimates. This fact is already addressed in safety performance functions where the $E$ variable is traffic volume and other covariates are typically present in equation (3-1) to reduce the variability of the estimate of $C$.

In addition, the major limitation of current practice of non-crash-based safety evaluation is that it requires a crash-dependent calibration process. A safety evaluation of a new infrastructure system requires crash records of similar locations and the constant risk assumption. The dependence on crash data prevents the non-crash-based safety estimation from reaping its full benefits.

\subsection{RESEARCH PROCEDURE}

The research procedure proposed in this study aims to address the present limitations of the noncrash-based safety evaluation. We studied one particular type of crashes, i.e., right-angle crashes, to evaluate the feasibility of the entire procedure. For the initial attempt, the right-angle crash was selected due to its relatively well-defined vehicle traveling paths prior to the collision and the collision spots are well detached from intersection approaches. The research procedure described herein, however, is not exclusively limited to right-angle crashes. With some modifications, the procedure can be generalized for other types of collisions, as well as other surrogate measures of safety. 


\subsubsection{Defining the Surrogate Measure of Safety}

A traffic crossing event is a crossing maneuver which may result in right-angle crashes. Each crossing event is associated with different degrees of hazard of a right-angle collision. To measure the degree of hazard, two potential candidates for the non-crash-based safety measure are considered: (a) time-to-collision (TTC) and (b) post-encroachment time (PET). The selected measure should consider the following:

- The selected measure should correspond to the type of crashes being studied. For example, the rear-end traffic conflict is well-suited for rear-end collisions while the rightangle traffic conflict is appropriate for right-angle collisions.

- The objective measurement of the selected measure must be feasible and require minimal subjectivity.

- The selected measure should be amenable for future automation of the measurement procedure.

- The selected measure must possess the continuous characteristic, which can represent traffic events during normal traffic operations, as well as crash occurrences on the same scale.

- The selected measure should have a crisp boundary between crash and non-crash events.

Considering these requirements, the PET between straight vehicles from two adjacent approaches is chosen as a measure for the degree of hazard of right-angle collisions at intersections. PET is the time measured from the moment the first vehicle leaves the right-of-way of the second vehicle to the moment the second vehicle enters into the right-of-way of the first one. PET was first introduced by Allen et al. (1978) and the preliminary evaluation of PET revealed that it was a preferred alternative to TTC in several respects.

PET is relatively simple to measure compared to TTC. It does not require the estimation of time remaining to the collision spot, which is rather complicated to obtain precisely. To measure PET, we need to know only two points in time: (a) when the first vehicle leaves the right-of-way infringement zone; and (b) when the second vehicle enters the right-of-way infringement zone. Due to its simplicity, PET is also relatively more amenable to automated measurement methods using techniques such as video image processing. TTC is estimated by the instantaneous speed at the moment when a driver initiates an evasive maneuver. To obtain precise TTCs, the exact positions and instantaneous speeds of all vehicles approaching the intersection are required to 
determine the worst case. An algorithm to differentiate between straight and turning vehicles must be developed. A real-time automated measurement for TTC would be difficult to achieve given the current state of image processing technology.

Another important property of PET is that it is continuous from crash-free operations to crash occurrences with a distinct boundary at zero. The smaller value of PET implies a greater risk of right-angle collisions. A collision is defined when PET becomes zero or less. On the contrary, a single and low value of TTC may represent either a crash or a non-crash event, depending on the outcome of evasive maneuvers. Some evasive maneuvers initiated 0.5 second prior to the collision may be successful while some others may not. In some circumstances, the TTC may not exist if none of the involved drivers attempt to avoid the collision. A distinct boundary between crash and non-crash events does not exist for TTC.

\subsubsection{Data Collection and Measurement Evaluation}

Once we defined the surrogate measure of safety, the next task was to collect the data needed for the study. First, we had to select the sites to be studied. Occasionally, we may refer to "intersections" as "sites" throughout this report. The site selection process should be carried out without looking at the crash history to avoid the potential bias from the regression-to-the-mean effect (see Section 2.2.3.1). This phenomenon occurs when a site is selected because of abnormally high or unusually low crash counts.

Two categories of data were acquired in this study: field data and crash data. For each intersection, field data consist of video recordings of traffic movements, traffic volumes, intersection control, and geometry. Traffic movements and volume data are used in the operational analysis while geometry data is used in the field of view calibration. Crash data are the objective measure of safety that is typically used in the evaluation of a surrogate safety measure. In the traditional regression analysis, the crash data is the response variable in which we attempt to find a group of covariates that can best describe its variability. Our scope of study focused on right-angle crashes. The exposure and condition associated with each crash occurrence will also be extracted and verified.

For the field data collection, a Purdue University mobile traffic laboratory will be used to monitor and digitally archive traffic movements at selected signalized intersections around Lafayette, 
Indiana. Detailed data, such as PETs and traffic volumes at certain periods of time, will be postprocessed later in the lab.

Crash data will be first retrieved from the Indiana State Police (ISP) numerical database. Then, each crash will be verified manually with the police accident report archived in a microfiche format to ensure that the type of crashes and the conditions during the crash occurrence are accurately recorded and to determine the collision spot within the intersection.

Traffic movements recorded at intersections will be post-processed to extract for PET data. Three alternatives are identified for the task: (a) manual measurement, (b) automated measurement, and (c) semi-automated measurement.

The manual measurement method requires full human intervention in the procedure. The video clips will be digitized at 30 frames per second (fps) and reviewed frame-by-frame to measure the exact values of the PETs. The measurement results extracted from the manual method are considered as ground truth data.

Autoscope is a tripwire image processing system primarily used for a traffic control purpose. Autoscope virtual detectors aim to emulate the functions of inductive loop detectors embedded in the pavement of intersection approaches at an actuated signal control. There is a possibility to automate the measurement procedure if the Autoscope detectors are configured properly and the Autoscope event files are carefully interpreted. From the practical viewpoint, the key benefit of a video image processing tool such as Autoscope is that we can configure and change the detector configurations at our own convenience without disrupting the traffic flows. However, Autoscope still suffers from technological limitations typically encountered in image processing systems commercially available in the market today. It is therefore important to evaluate whether a system such as Autoscope is applicable for this measurement task, which is different from its design purposes.

We will consider proprietary image processing software specifically developed for the measurement task as another possibility for the automated measurement method. The video input required by the software will be in the same format as required by other alternatives. Reported outputs should be easily interpretable and compatible with other methods. The performance of the software will be evaluated in a similar manner with the other methods. 
The semi-automated method will be considered if the performance of the automated measurement method is unsatisfactory. It is semi-automated in the sense that human intervention is required in order to obtain the final results. A trained observer may be required to select a portion of the video clips to review them manually, depending on the results from the automated method. The performance of this method is contingent upon the selection criteria and the reliability of results obtained from the automated method.

The performance evaluation of measurement alternatives will depend on two important criteria: accuracy and efficiency. The measurement results from other alternatives can be compared against those from the manual method. The most appropriate method will be selected based upon evaluation results. The selected method will be used to extract the data from video materials collected from all the selected sites.

\subsubsection{Statistical Analysis}

Using regression analyses, we will first check the relationships between the proposed surrogate measure of safety and the crash counts as typically implemented in the traditional analysis of surrogate measures of safety. Any promising correlation between these measures and crashes would confirm the potential for successful development of a new method that can address our concerns in the traditional analysis.

The regression analyses treat a surrogate measure of safety as exposure and assume a constant risk across a group of similar locations. In a new method, instead of assuming a constant ratio for frequencies in equation (3-1) or incorporating additional covariates to better explain the variability of the $C$ estimates, we propose to estimate this ratio direct from data collected at a particular location. Equation (3-1) is therefore slightly modified to better incorporate the known statistical properties of crashes as

$$
C=R \cdot E,
$$

where $C=$ crash frequency (crashes/year), $R=$ likelihood of crash associated with unit exposure, and $E=$ exposure (exposure units/year).

In the proposed framework, estimation of crash frequency includes estimation of the exposure and estimation of the likelihood of crash associated with the unit exposure. For the sake of 
brevity, the likelihood of a crash will be referred to as "risk." The risk is defined and estimated in relation to the exposure unit. The method of estimating the risk may require a specific definition of exposure unit. It can be a traffic event (event-based) or a time interval (time-based). In the latter case, the risk is the likelihood of a crash in a unit of time interval. If the time interval is sufficiently short, then the likelihood of two crashes in one interval is negligible. Risk $R$ preserves its meaning as the proportion of the time intervals in a period of interest (one year for example) that experiences crashes. By this notion, the risk $R$ in equation (3-2) becomes a timebased risk and the estimation of a corresponding time-based exposure is straightforward.

Based on the premise a PET satisfies the continuum-of-event assumption, we propose the approach to estimate the risk of right-angle collisions in the context of the extreme value theory (EVT), which enables the extrapolation of typically observed PET levels to a rarely observed crash occurrence level on a sound mathematical basis. The risk estimation in EVT is a crashindependent estimation procedure. This implies that crash data are not required in the risk estimation. We can define the risk based on the mechanism of the PET occurrence. The risk of a right-angle collision is defined by the likelihood of the PET exceeding the boundary between non-crash and crash events. PET data can be treated as extremes by blocking a series of observations over time or specifying a threshold. In contrast to the conventional approach, we do not have to arbitrarily specify the threshold for extreme events. Different definitions of extremes can lead to different extreme value distributions. Temporal variation of extremes may be modeled by linking model parameters with time-varying covariates such as traffic volume. We can convert the estimated risk from the model to the estimated crash frequency by adjusting the estimated risk with appropriate exposure.

In summary, this step comprises the following tasks:

1. Analyze the safety relationships of the surrogate measure of safety, traffic data, and historical crash counts using regression analyses.

2. Identify and evaluate extreme value modeling alternatives. The model considerations include sampling schemes, extreme value distributions, dependence of observations, and non-stationarity of the process. Select the modeling approach that is well-suited to the proposed surrogate measure of safety. 
3. Develop the safety estimation method. The analytical, as well as numerical, solutions will be established for the selected approach. Note that there are several possibilities to interpret safety from the extreme value models.

4. Quantify the uncertainty of the model estimates. Several alternatives are available, including the delta method, profile (concentrated) likelihood method, simulation, and bootstrapping. Certain methods may not approximate the confidence of the estimates as good as others due to some underlying assumptions. The approach suitable for the task will be examined.

5. Select the best fitted models. The incorporation of non-stationary covariates, if necessary, made feasible a large catalogue of modeling possibilities. The model adequacy and goodness-of-fit signify how well the data can be described by the extreme value model. The additional decision factors may include the confidence of estimates and the computational complexity. The most appropriate approach can be determined based on these metrics.

\subsubsection{Method Evaluation}

The true level of safety of the intersection is unknown theoretically. However, the historical crash counts for a long period of time are widely accepted as the best objective measure of safety and there is no other credible alternative to date. The safety estimates derived from the extreme value models will be validated against historical crash counts. By doing so, we are considering the expected crash counts as an objective measure of safety that can best represent the level of the safety of the intersection.

The validity of the proposed estimation method can be checked by examining the relationship between the safety estimates from the proposed models and crash counts. A strong correlation would confirm the validity of the method while a weak correlation would not necessarily indicate the method's failure. In fact, there is a wide range of issues that may influence the estimation results and consequently the validity of the method. Certain issues cannot be studied from the field data alone due to the limited time and resources available. A simulation experiment is a viable alternative to address these issues, which include the effect of model settings and the optimal observation period. 
A simulation routine to analyze the characteristics of extreme value models fitted to the surrogate measure of safety will be proposed. We will conduct a preliminary investigation on these issues in a simulated environment and provide directions for future research in accordance with our findings. 


\section{CHAPTER 4 DATA COLLECTION AND MEASUREMENT EVALUATION}

In this chapter, we will first describe the site selection procedure and crash data retrieval. Occasionally, we will refer to "intersections" as "sites" in this report. The measurement methods and evaluation results will be presented. Based on the measurement evaluation results, the most suitable measurement method will be selected to extract the surrogate data from the selected studied sites.

This chapter documents our efforts to obtain data that are as accurate as possible. The amount of data we collected for measurement evaluation and subsequent analyses was limited by the time and resource available. All the collected data required human intervention at varying degrees to ensure the best quality. The detailed procedure to obtain each type of data and its limitations will be described.

The three main sources of data required in this study are:

- Historical crash data

- Surrogate data

- Traffic volume data

As mentioned in the scope of this study, we will be focusing on right-angle collisions in particular. The required crash data are therefore the counts of right-angle crashes. We had the crash data available from 1997 to 2000. The surrogate data are the measured values of the proposed surrogate measure of safety. The traffic volume will be collected during the same period as the surrogate data are collected. In addition, general information about studied sites will also be collected which includes intersection geometry, traffic control, and weather conditions during the period of observation. 


\subsection{DEFINITION OF RIGHT-ANGLE CRASHES}

First, let us begin with the specific definition of the right-angle crashes considered in this study. We classified the right-angle crashes into two types in this study, which are (a) straight rightangle crashes and (b) left-turn right-angle crashes. These two types of collisions are illustrated in Figure 4-1. Only the first type is considered in the scope of this study due to the time and resource constraints in the data collection and its relative ease in the measurement of the corresponding surrogate data. We will describe the measurement of surrogate data in detail later in this chapter.
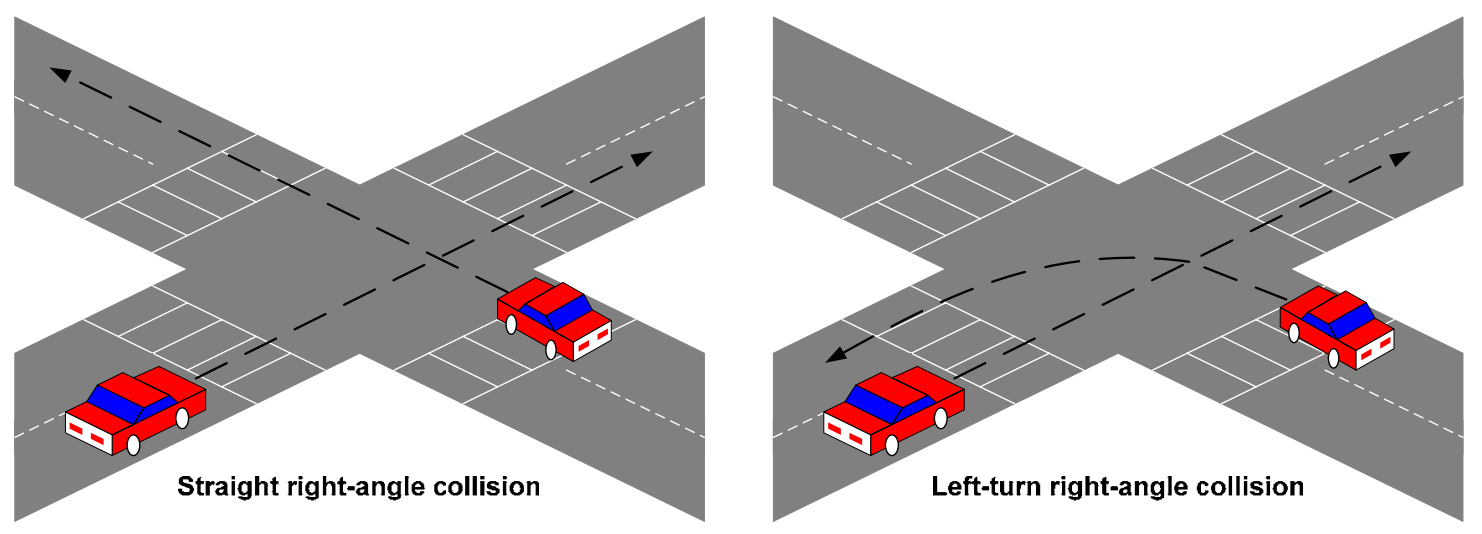

Figure 4-1: Straight versus Left-turn Right-Angle Collisions

The straight right-angle crash refers to a right-angle crash caused by two vehicles traveling straight in a pre-accident direction. The left-turn right-angle crash is a collision caused by a vehicle traveling straight versus a vehicle turning left into the approach of the oncoming straight vehicle.

These two types of right-angle crashes are not clearly distinguished in the numerical accident database. These two types also require separate measurement of PETs if both are to be studied. For the left-turn right-angle collision, it is interesting to observe that the first portion of the leftturn traveling path is somewhat similar to a vehicle that is going straight. However, the speed of the vehicle could differ and the left-turn right-angle crashes are more likely to occur at intersections that have significant left-turn volumes.

We will describe later in this chapter how we ensured that only straight right-angle collisions were counted in the retrieval of crash data for selected intersections. For simplicity, we will occasionally abbreviate "straight right-angle collisions" as "right-angle collisions" unless specifically noted otherwise. 


\subsection{SITE SELECTION}

Our first estimate of the number of intersections for which we were able to collect the field data and analyze within a reasonable amount of time was about 20 locations. Two major concerns that we recognized in the process of the selection of studied sites are:

- Selected sites should represent a good balance between a number of safe and unsafe intersections. A lack of variability in the safety levels of the selected sites can lead to a problem in the regression analysis and the comparative examination of results from the proposed models.

- The selection procedure should not identify unsafe locations on the basis of crash counts as it can lead to the problem of selectivity bias. Selecting studied sites based on crash counts may trigger certain safe locations to be identified as unsafe due to the randomness of crash occurrences.

Sampling studied intersections by examining crash counts is prone to a problem of regression-tomean bias, which is attributed to the randomness of crash occurrences, resulting in the overrepresentation or underrepresentation of the number of crashes observed at certain locations with respect to the true mean value. One effective way to mitigate this problem is random sampling, which requires a number of sites to be selected in a random manner. However, this does not guarantee a satisfactory balance between the safe and unsafe intersections for a relatively small sample size in this study. Hence, the following selection procedure was conducted to determine a list of studied sites.

Previous research by Tarko and DeSalle (2002) showed that motorist feedback can provide a good account of the locations of the safety concerns. In that study a web-based tool was used to collect the motorist feedback over a five-month trial period from road users in Tippecanoe County, Indiana in 2001 and the results were evaluated against the actual crash data. It was found that motorist feedback can be a very effective supplement to crash data and can guide highway agencies in the examination and identification of hazardous highway locations. This implies that we can still select unsafe intersections, without examining the crash counts, based on the volume of feedback from motorists.

Inspection of the reported locations documented in Tarko and DeSalle (2002) gives a preliminary list of potentially unsafe locations based on motorist feedback. This procedure allows us to 
include potentially unsafe locations in our small sample while avoiding selectivity bias from the examination of crash data. A preliminary list contains a total of 97 locations with 29 locations having at least two responses from the survey.

For each reported location in a preliminary list, the following criteria were used to filter out the locations that can complicate the study or do not meet the scope of this research:

- The location must be a four-legged intersection.

- For the intersection layout, the N-S approaches should be approximately perpendicular to the E-W approaches. This criterion aims to avoid confusion regarding the vehicle traveling directions recorded in the crash database.

- Non-unique intersections must be removed. Non-unique intersections refer to the case where two roads intersect more than once. Because intersections are identified in the crash database using local names of crossroads, these non-unique locations will be registered in the accident database as the same location despite the fact that they are not. Crash counts at these locations are overrepresented and unusable.

- The intersection must be signalized and must have at least one available parking space at the corner for field data collection using a mobile traffic laboratory.

All the 97 locations in a preliminary list were checked against the first three criteria using U.S. maps available from several sources (http://mappoint.msn.com, http://map.yahoo.com, and http://www.mapquest.com). There were only 20 locations left usable after the verification. Hence, we added 19 intersections that meet the first three criteria by random selection from the Tippecanoe county map. At this step, we had a total of 39 intersections ready for the final criterion check.

The last criterion was confirmed by site visits. The final set of selected sites that met all the criteria was reduced from 39 to 22 intersections. Table 4-1 shows the list of 39 sites visited and 22 intersections usable in this study. The final list was reduced to 18 intersections because of the problems encountered during data collection. Four sites that were removed from the list are $87904,87934,97913$, and 97914. We could not obtain parking permission at some of these sites and some available parking spots are too close to a high-voltage power line. For safety purposes, a 10-foot clearance must be maintained between the van's mast and the power line all the time. 
Table 4-1: Preliminary List of Potential Studied Sites

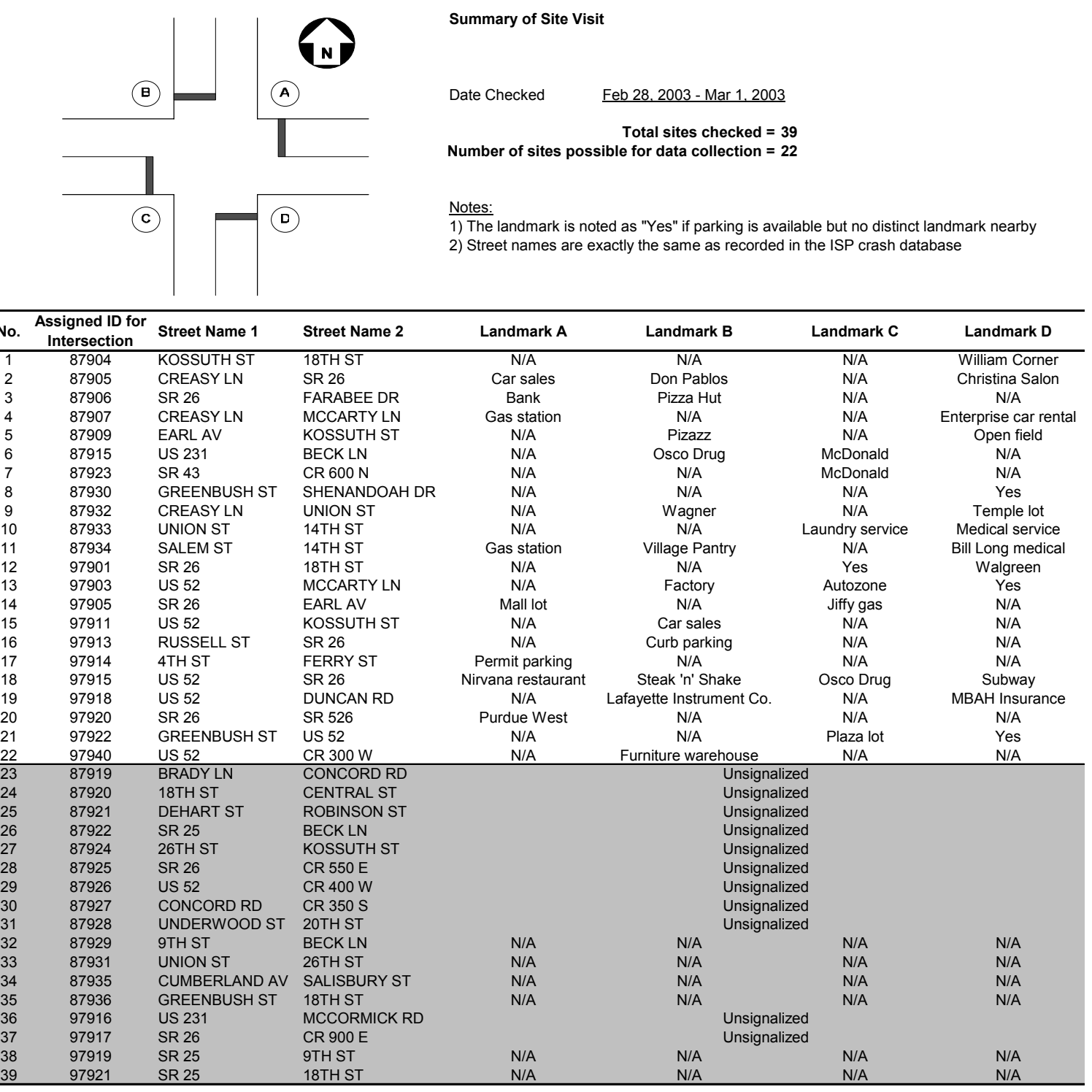

The list above shows the landmark nearest to the corner of each intersection. The shaded rows indicate the intersections that are excluded from further consideration either because they are unsignalized or have no available parking spaces. The ID assigned to the intersection is a fivedigit unique number which is of the format XYYZZ. The $X=8$ is assigned to intersections that have only one unique pair of crossing road names. The $X=9$ is assigned to intersections having crossing roads with more than one local name. The YY $=79$ is the ISP code for the Tippecanoe county. The $\mathrm{ZZ}$ is the intersection number running from 01 to 99. 


\subsection{CRASH DATA RETRIEVAL}

\subsubsection{Database Extraction}

The Indiana State Police (ISP) crash database was available for 1997 to 2000. The crash data were imported into Microsoft Access, and the extraction codes were written in the Visual Basic environment using the data access and management capabilities of SQL and ActiveX Data Object (ADO). The straight right-angle crashes are obtainable from the database with better confidence than the left-turn right-angle crashes because when a left-turn vehicle is involved in a crash, the direction of travel recorded in the database is ambiguous as to whether it is a pre-crash, duringcrash, or post-crash direction. It is up to the police officer's judgment at the scene. Each rightangle collision has to be classified by the location of its occurrence within the intersection. Therefore, a procedure to retrieve right-angle crashes has to be supported by the manual verification of each crash occurrence using police accident reports.

The accident master record consists of five categories: (a) environment record, (b) vehicle record, (c) driver record, (d) pedestrian record, and (e) injured record. In order to classify the type of collisions and the location of the occurrence within the intersection, the attributes of the environment and the vehicle records are the key identifiers.

The environment record describes the circumstances, location, and surrounding conditions of the accident. Each accident has one unique environment record. The vehicle record describes the vehicle and the circumstances of the accident unique to each vehicle. Each vehicle involved in an accident will appear as one record. It was found during the crash extraction process that vehicle records could be missing for some accidents, although those accidents were recorded in the environment database.

\subsubsection{List of Local Names}

The intersections were identified in the database using a pair of pseudocodes. A pseudocode is unique to a road name. Therefore, if a road has more than one local name (e.g., SR-26, State Street, and South Street are the same street), the same intersection may appear in crash records as different intersections when different road names were recorded in the crash reports. To address this problem, all possible local names of the intersecting roads at selected sites were checked 
manually from the maps. All the possible combinations of a pair of local names were put together in a MS Access table as shown in Table 4-2.

Table 4-2: List of Possible Names of Intersecting Roads at Selected Intersections

\begin{tabular}{|c|c|c|c|c|}
\hline IntxID & Pseudocode 1 & N-S Road Name & Pseudocode 2 & E-W Road Name \\
\hline 87904 & 9104300 & 18TH ST & 9048800 & KOSSUTH ST \\
\hline 87905 & 9022300 & CREASY LN & 9026001 & SR 26 \\
\hline 87906 & 9030300 & FARABEE DR & 9026001 & SR 26 \\
\hline 87907 & 9022300 & CREASY LN & 9164300 & MCCARTY LN \\
\hline 87909 & 9026100 & EARL AV & 9048800 & KOSSUTH ST \\
\hline 87915 & 9231002 & US 231 & 9004800 & BECK LN \\
\hline 87923 & 9043001 & SR 43 & 9115200 & CR $600 \mathrm{~N}$ \\
\hline 87930 & 9033700 & GREENBUSH ST & 9078100 & SHENANDOAH DR \\
\hline 87932 & 9094100 & UNION ST & 9022300 & CREASY LN \\
\hline 87933 & 9094100 & UNION ST & 9103800 & 14TH ST \\
\hline 87934 & 9075100 & SALEM ST & 9103800 & 14TH ST \\
\hline 97901 & 9104300 & 18TH ST & 9026001 & SR 26 \\
\hline 97901 & 9104300 & 18TH ST & 9079900 & SOUTH ST \\
\hline 97903 & 9025001 & SR 25 & 9164300 & MCCARTY LN \\
\hline 97903 & 9052002 & US 52 & 9164300 & MCCARTY LN \\
\hline 97903 & 9131500 & SAGAMORE PKWY & 9164300 & MCCARTY LN \\
\hline 97905 & 9026100 & EARL AV & 9026001 & SR 26 \\
\hline 97905 & 9026100 & EARL AV & 9079900 & SOUTH ST \\
\hline 97911 & 9052002 & US 52 & 9048800 & KOSSUTH ST \\
\hline 97911 & 9131 & SAGAMORE PKWY & 9048800 & KOSSUTH ST \\
\hline 97913 & 9074700 & RUSSELL ST & 9026001 & SR 26 \\
\hline 97913 & 9074700 & RUSSELL ST & 9087900 & STATE ST \\
\hline 97914 & 9102700 & 4TH ST & 9030500 & FERRY ST \\
\hline 97914 & 9231002 & US 231 & 9030500 & FERRY ST \\
\hline 97915 & 9052002 & US 52 & 9026001 & SR 26 \\
\hline 97915 & 9131500 & SAGAMORE PKWY & 9026001 & SR 26 \\
\hline 97918 & 9156800 & DUNCAN RD & 9052002 & US 52 \\
\hline 97918 & 9156800 & DUNCAN RD & 9131500 & SAGAMORE PKWY \\
\hline 97920 & 9055500 & MCCORMICK RD & 9026001 & SR 26 \\
\hline 97920 & 9055500 & MCCORMICK RD & 9087900 & STATE ST \\
\hline 97920 & 9526001 & SR 526 & 9026001 & SR 26 \\
\hline 97920 & 9526001 & SR 526 & 9087900 & STATE ST \\
\hline 97922 & 9052002 & US 52 & 9033700 & GREENBUSH ST \\
\hline 97922 & 9131500 & SAGAMORE PKWY & 9033700 & GREENBUSH ST \\
\hline 97940 & 9111500 & CR $300 \mathrm{~W}$ & 9052002 & US 52 \\
\hline 97940 & 9111500 & CR $300 \mathrm{~W}$ & 9231002 & US 231 \\
\hline
\end{tabular}

\subsubsection{2 $\quad$ Order of Pseudocodes}

Based on the list in Table 4-2, all the crashes at the listed intersections were queried from the database as to where the recorded location of the crash occurrence was at the intersection. The swapping of the order in which the pseudocodes were keyed into the crash database can cause the same intersection to be misidentified as a different one if it was not treated accordingly. Hence, this issue was addressed in the construction of queries to retrieve the crash data. 


\subsubsection{Classification of Types of Collision}

The collision diagram used by the Indiana State Police is shown in Figure 4-2. The straight and left-turn right-angle crashes considered in this study are Nos. 6 and 9 respectively. Note that the accident record does not include the specific collision spot within the intersection. It is possible in certain cases to identify a collision spot from vehicle records that include pre-accident maneuvers and vehicle traveling directions. However, the aforementioned traveling directions recorded in the accident database were subject to the judgment calls of police officers as to whether they are precrash or post-crash directions. As such, the specific collision spots cannot always be confirmed using the numerical database alone. The verification of a collision spot from the collision diagram and accident description in the police crash report is necessary.

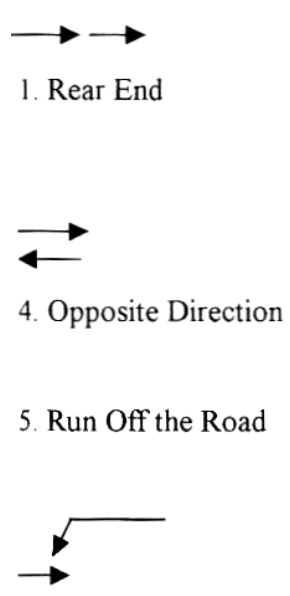

8. Left Turn

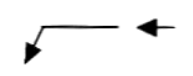

11. Left Turn

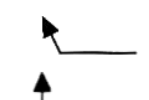

14. Right Turn

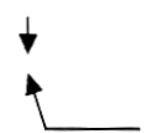

17. Right Turn

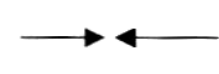

2. Head On

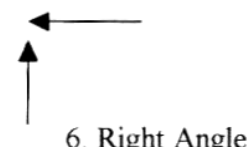

6. Right Angle

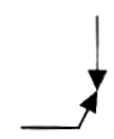

9. Left Turn

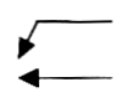

12. Left Turn

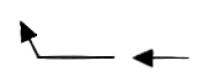

15. Right Turn

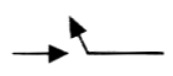

18. Right Turn
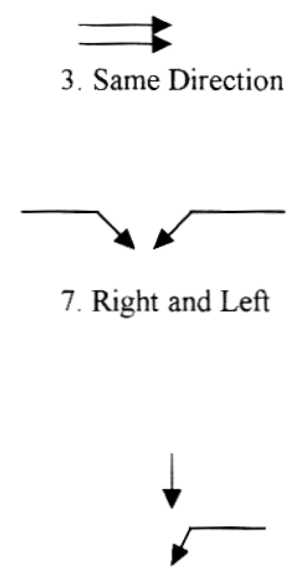

10. Left Turn

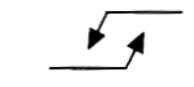

13. Left Turn

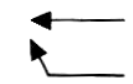

16. Right Turn

Figure 4-2: Indiana State Police Collision Diagram 
In order to help classify the locations of crash occurrences, the region inside the intersection was split into four quadrants as shown in Figure 4-3. Each quadrant represents the area where collisions may take place from two conflicting traffic streams. The "cz" stands for "collision zone" and "conflict zone" in the context of accident data and surrogate data respectively. For example, the collision zone of a right-angle collision between a northbound vehicle versus an eastbound vehicle was denoted as "czNBEB".

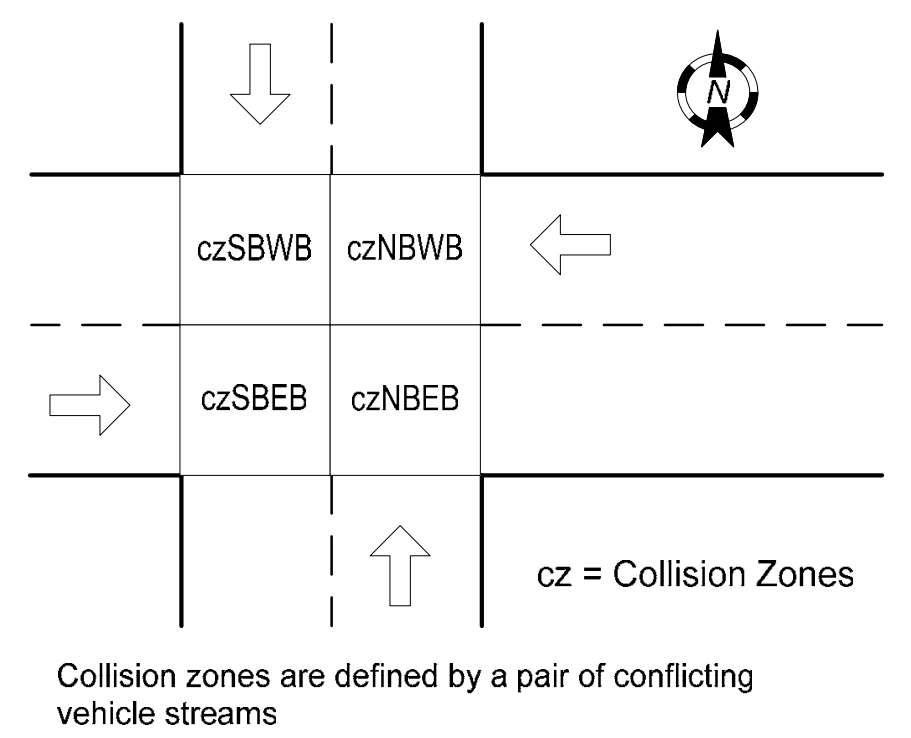

Figure 4-3: Collision/Conflict Zone Notation

To facilitate the classification procedure, the possible conflicting directions of each type of collision were coded as shown from Figure 4-4 to Figure 4-6. The code numbers 901 to 904 represent the straight right-angle crashes. The code numbers 905 to 908 represent the left-turn right-angle crashes. The opposing left-turn collisions are coded as 909 to 912. Although the scope of this study does not include the opposing left-turn collision, it was included in the classification procedure as it accounts for a significant proportion of all collisions recorded as right-angle at signalized intersections. The code number 931 refers to the rear-end collision. Any unverifiable crashes were coded as 998 and all other types of collisions were coded as 999 . Only the primary accident was considered in the classification, i.e., only the first two participants involved in a crash were taken into account. The only way to confidently confirm the types of collision and conflicting directions is to manually examine accident reports. 


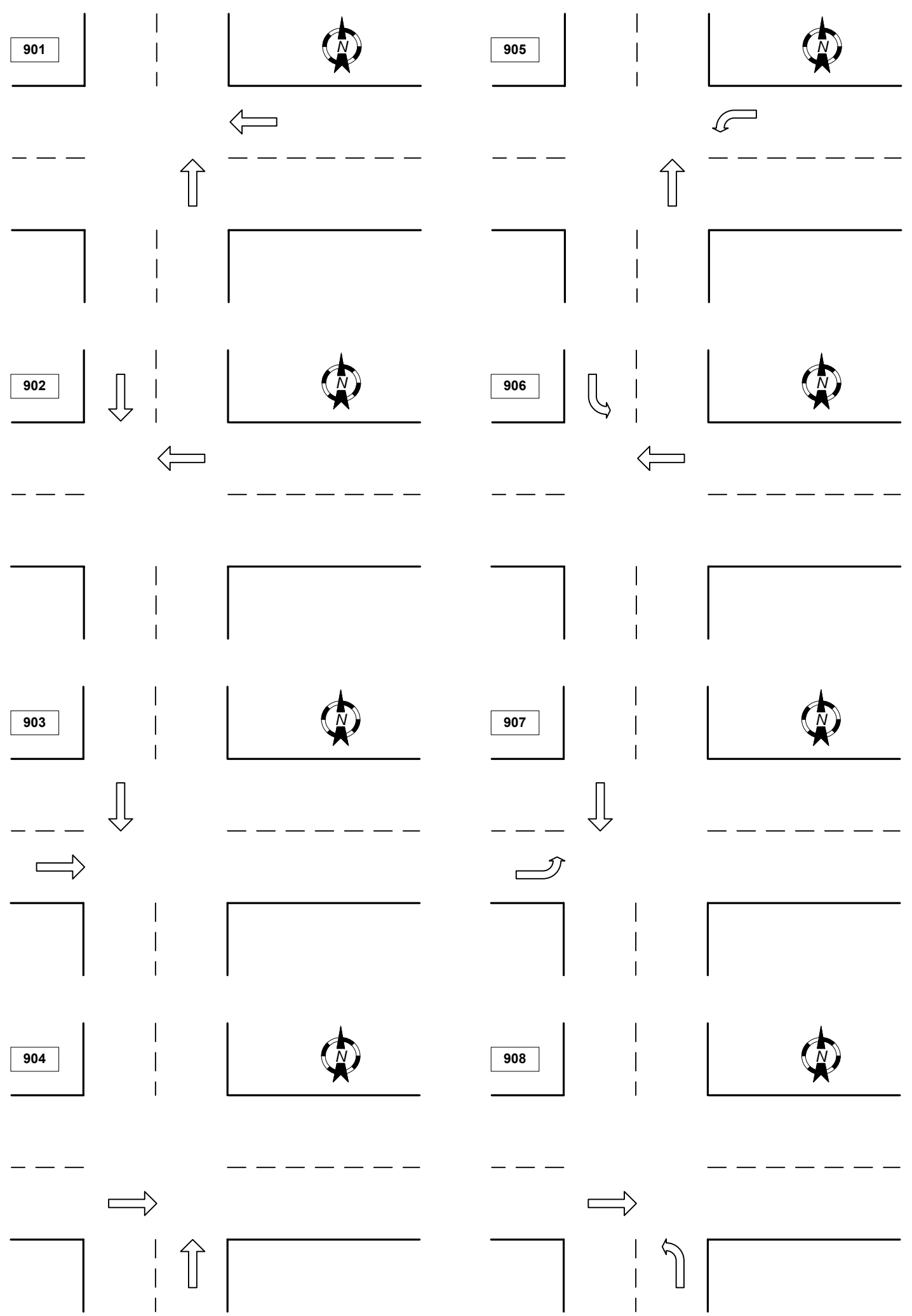

Figure 4-4: Classification of Right-Angle Collisions 

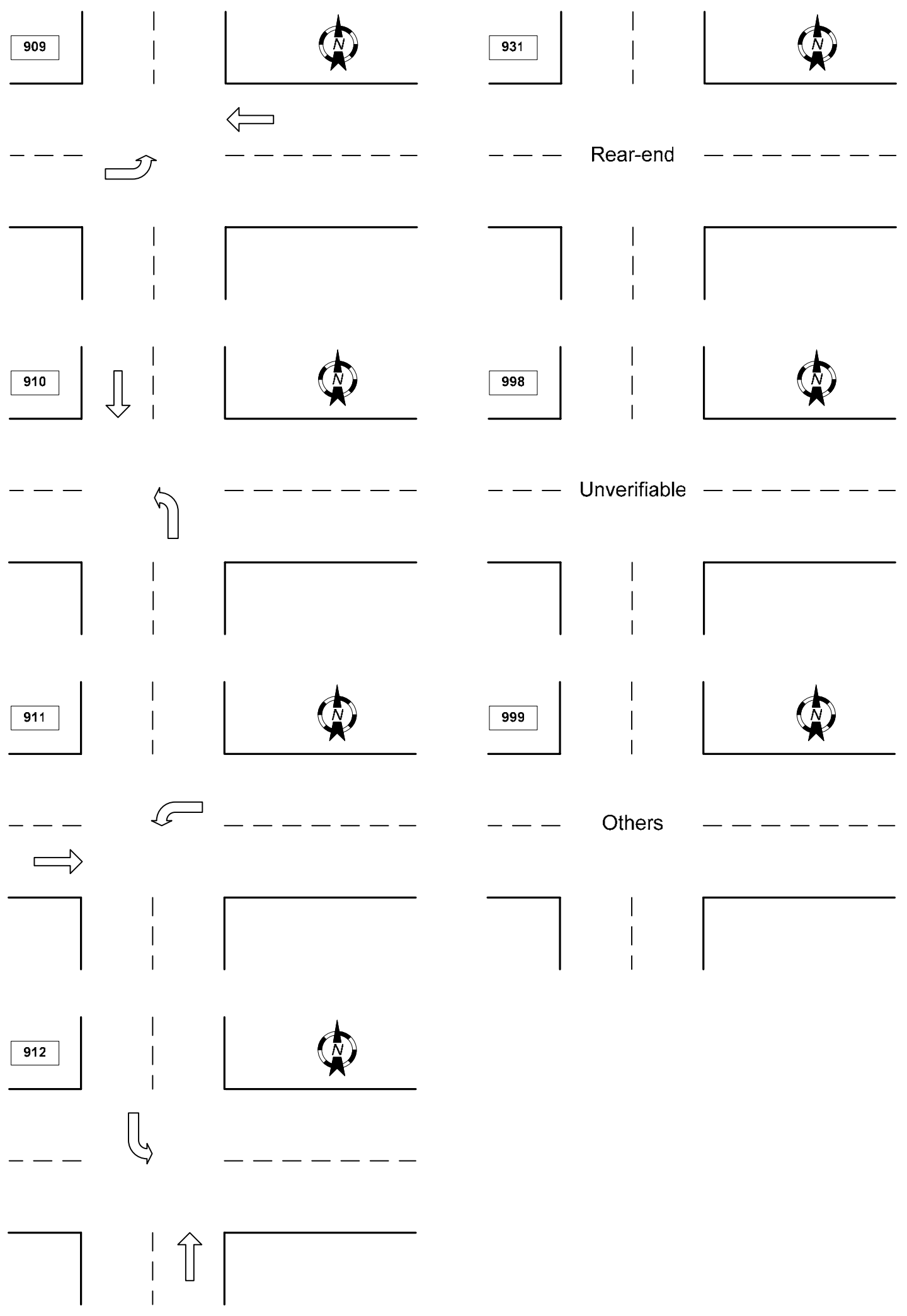

Figure 4-5: Classification of Opposing Left-Turn Collisions and Other Types 


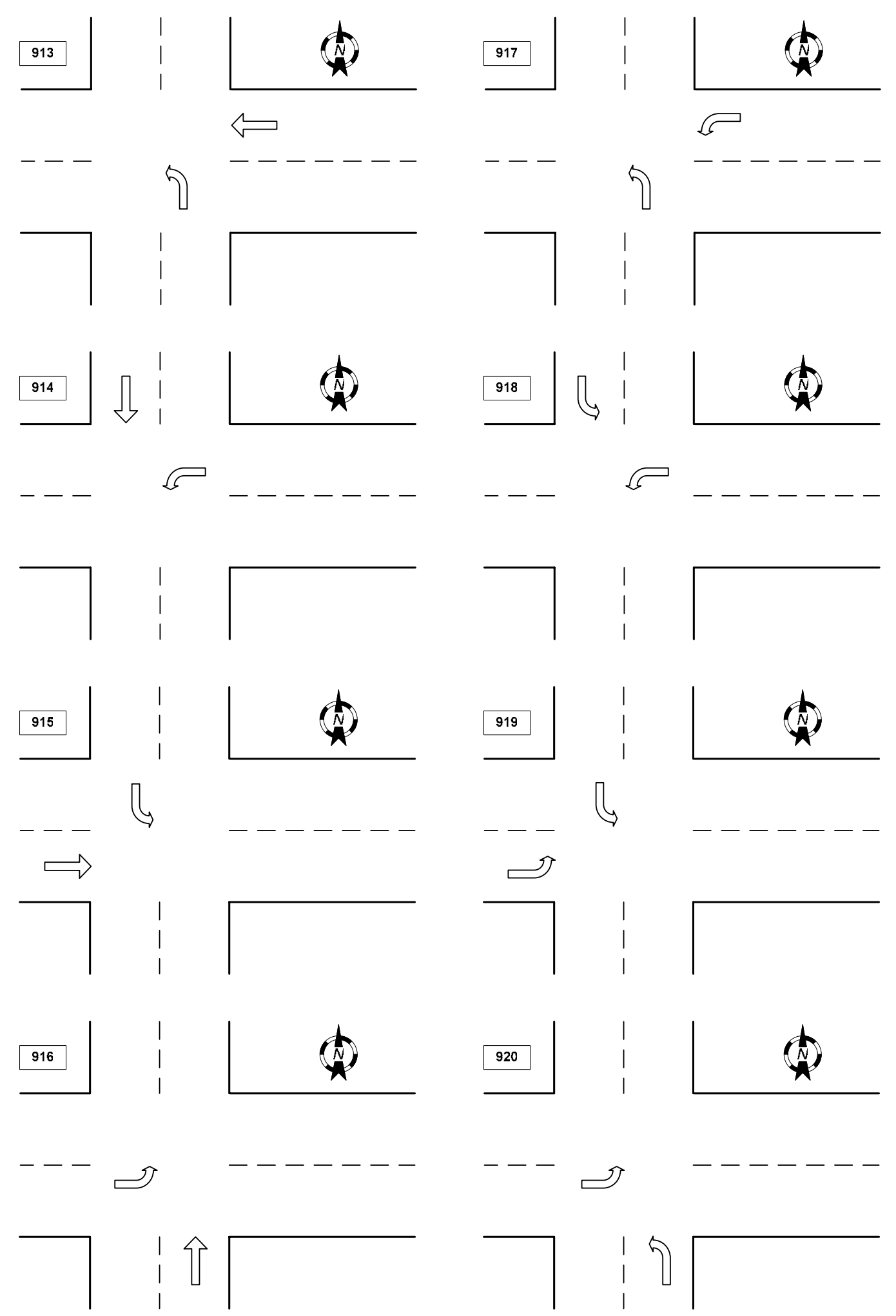

Figure 4-6: Other Collision Possibilities 


\subsubsection{Verification of Accident Records}

Based on the classification diagrams described in the previous section, each collision at selected intersections was verified with microfilm maintained at the Indiana Department of Transportation (INDOT). From the numerical database, there were a total of 654 crashes at 18 selected signalized intersections from 1997 to 2000 (this was reduced from the original list of 22 intersection because of problems during the data collection).

The recorded collision diagram in the database was used to screen out certain records that do not require manual verification. These records were either usually recorded correctly, e.g., rear-end, head-on, and side-swipe, or were unlikely to be confusing with the considered types of collisions, e.g., right-turn collisions. Hence, only crash records with an ISP collision diagram recorded for Nos. 6 to 13 were further verified with actual accident reports (see ISP collision diagrams in Figure 4-2). This reduced the number of collisions to be verified to 384 .

The numerical crash database contains a microfilm index uniquely assigned to the crash record. These microfilm indices are used to look up the accident reports of interest. The collision diagrams drawn by police officers and the narrative descriptions of the accident event in these reports are the main clues for identification of actual vehicle movements and collision zones. The examples of accident reports are shown in Figure 4-7 and Figure 4-8.

\subsubsection{Summary of Retrieved Crash Data}

The verification results are summarized in Table 4-3. There were a total of 654 crashes recorded at 18 selected signalized intersections. The number of verified crashes represents those crashes about which we are uncertain as to its type and the conflicting directions. Those crashes that are outside the scope of this study were excluded from the verification.

From Table 4-3, there were 337 crashes to be verified. Three types of crashes were checked specifically: (a) straight right-angle crashes, (b) left-turn right-angle crashes and (c) opposing leftturn crashes. About $6 \%$ of the verified crashes were unverifiable because of either missing microfilms or poor collision diagram/descriptions. About $20 \%$ of all crashes at these intersections

were straight right-angle crashes. Opposing left-turn crashes account for a significant proportion of crash counts $(18 \%)$ at selected intersections as well. 


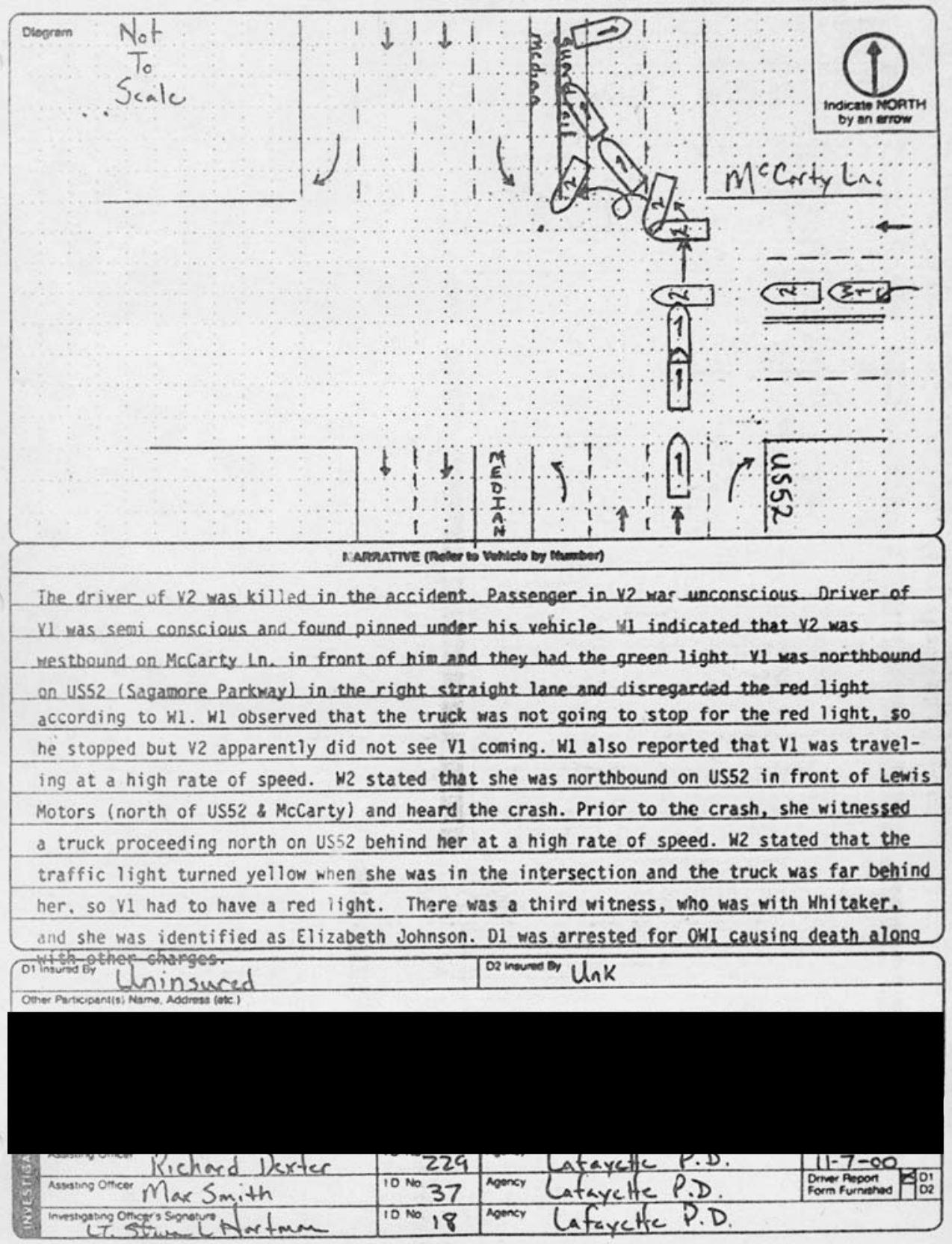

Figure 4-7: Example of Accident Report (1) 


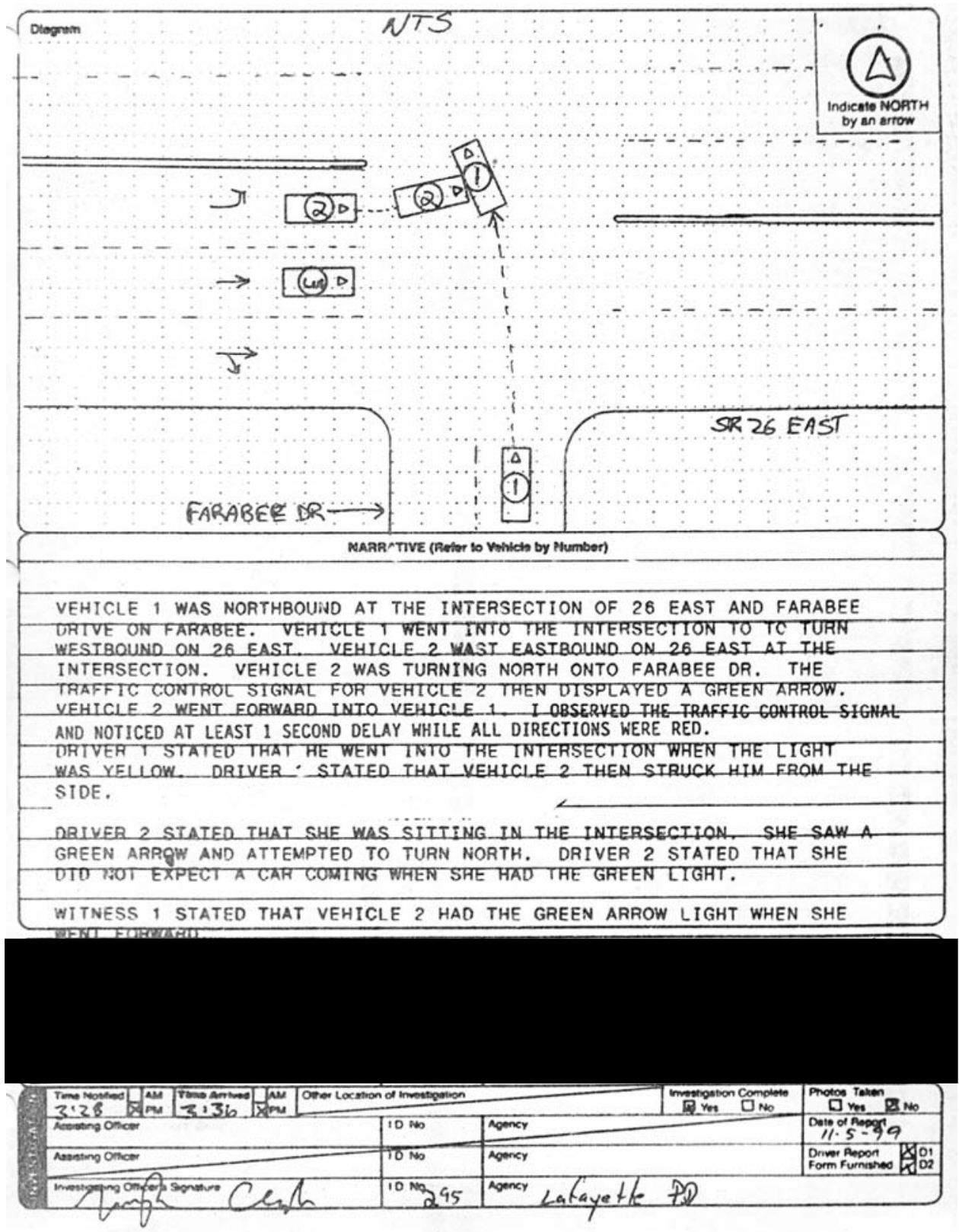

Figure 4-8: Example of Accident Report (2) 
Table 4-3: Results of Manual Verification with Crash Reports

\begin{tabular}{|c|c|c|c|c|c|c|c|}
\hline \multirow[b]{2}{*}{ Site } & \multirow{2}{*}{$\begin{array}{c}\text { Total Crashes of } \\
\text { All Types }\end{array}$} & \multirow{2}{*}{$\begin{array}{c}\text { Number of } \\
\text { Crashes Verified } \\
\text { Manually }\end{array}$} & \multicolumn{5}{|c|}{ Results of Manual Verification with Crash Reports (based on number of crashes verified) } \\
\hline & & & $\begin{array}{l}\text { Straight Right- } \\
\text { Angle Crashes }\end{array}$ & $\begin{array}{l}\text { Left-Turn Right- } \\
\text { Angle Crashes }\end{array}$ & $\begin{array}{l}\text { Opposing Left- } \\
\text { Turn Crashes }\end{array}$ & Others & Unverifiable \\
\hline 87905 & 74 & 26 & 2 & 0 & 14 & 9 & 1 \\
\hline 87906 & 52 & 30 & 10 & 1 & 16 & 2 & 1 \\
\hline 87907 & 49 & 27 & 5 & 1 & 11 & 8 & 2 \\
\hline 87909 & 27 & 18 & 13 & 0 & 4 & 0 & 1 \\
\hline 87915 & 28 & 13 & 5 & 0 & 4 & 1 & 3 \\
\hline 87923 & 15 & 9 & 5 & 0 & 1 & 3 & 0 \\
\hline 87930 & 4 & 1 & 0 & 0 & 1 & 0 & 0 \\
\hline 87932 & 20 & 9 & 2 & 2 & 4 & 1 & 0 \\
\hline 87933 & 25 & 15 & 1 & 0 & 1 & 11 & 2 \\
\hline 97901 & 58 & 46 & 32 & 1 & 11 & 1 & 1 \\
\hline 97903 & 67 & 37 & 14 & 0 & 16 & 2 & 5 \\
\hline 97905 & 49 & 27 & 16 & 1 & 8 & 2 & 0 \\
\hline 97911 & 37 & 20 & 6 & 0 & 11 & 3 & 0 \\
\hline 97915 & 68 & 19 & 5 & 0 & 3 & 10 & 1 \\
\hline 97918 & 21 & 2 & 1 & 0 & 0 & 1 & 0 \\
\hline 97920 & 5 & 3 & 1 & 0 & 1 & 1 & 0 \\
\hline 97922 & 40 & 23 & 5 & 0 & 9 & 7 & 2 \\
\hline 97940 & 15 & 12 & 8 & 0 & 1 & 3 & 0 \\
\hline Total & 654 & 337 & 131 & 6 & 116 & 65 & 19 \\
\hline
\end{tabular}

The retrieved crash data represent all the crashes that took place during the four-year period regardless of the time of day, weather, season, etc. However, the data collection at the selected intersections may not cover a variety of conditions within a relatively short period. It was decided to restrict the field conditions to non-winter, weekday, and daytime only. This is to avoid the complexity that may arise from the crash contributing factors that are present only under certain circumstances. Hence, the retrieved crash counts are filtered by the following set of criteria to reflect the expected field conditions:

- Daytime period: the hours of daylight vary from month to month (based on http://www.weather.com). See Table 4-4 for details. The average daytime duration for the entire year is about 12.09 hours per day.

- Non-winter months: April to November

- Weekday: Monday to Friday

- Normal weather: Difficult weather conditions are usually noted in the accident database

The straight right-angle crash counts classified by intersections and by collision zones are summarized in Table 4-5 and Table 4-6 respectively. 
Table 4-4: Monthly Daytime Period for Crash Data Extraction

\begin{tabular}{ccc}
\hline Month & Daytime Period & Duration (hours) \\
\hline January & 0830 to 1730 & 9.0 \\
February & 0745 to 1815 & 10.5 \\
March & 0700 to 1900 & 12.0 \\
April & 0600 to 1930 & 13.5 \\
May & 0530 to 2000 & 14.5 \\
June & 0530 to 2000 & 14.5 \\
July & 0530 to 2000 & 14.5 \\
August & 0600 to 1930 & 13.5 \\
September & 0630 to 1900 & 12.5 \\
October & 0700 to 1800 & 11.0 \\
November & 0730 to 1730 & 10.0 \\
December & 0800 to 1730 & 9.5 \\
\hline
\end{tabular}

Table 4-5: Summary of Right-Angle Crash Counts by Intersections (1997-2000)

\begin{tabular}{|c|c|c|c|c|c|c|}
\hline \multirow[b]{2}{*}{ ID } & \multirow[b]{2}{*}{ Intersection } & \multicolumn{5}{|c|}{ Conditions } \\
\hline & & $\begin{array}{c}\text { All } \\
\text { Conditions }\end{array}$ & Daytime & $\begin{array}{l}\text { Daytime and } \\
\text { Non-winter }\end{array}$ & $\begin{array}{l}\text { Daytime, non- } \\
\text { winter, and } \\
\text { weekday }\end{array}$ & $\begin{array}{l}\text { Daytime, non- } \\
\text { winter, weekday, } \\
\text { and normal } \\
\text { weather }\end{array}$ \\
\hline 87905 & Creasy Ln @ SR-26 & 2 & 1 & 1 & 1 & 1 \\
\hline 87906 & SR-26@ Farabee Dr & 10 & 6 & 6 & 5 & 5 \\
\hline 87907 & Creasy Ln @ McCarty Ln & 5 & 3 & 2 & 2 & 1 \\
\hline 87909 & Earl Av @ Kossuth St & 13 & 7 & 4 & 3 & 3 \\
\hline 87915 & US-231 @ Beck Ln & 5 & 3 & 2 & 2 & 2 \\
\hline 87923 & SR-43 @ CR-600 N & 5 & 2 & 2 & 1 & 0 \\
\hline 87930 & Greenbush St @ Shenandoah Dr & 0 & 0 & 0 & 0 & 0 \\
\hline 87932 & Creasy Ln @ Union St & 2 & 2 & 2 & 2 & 2 \\
\hline 87933 & Union St @ 14th St & 1 & 1 & 1 & 0 & 0 \\
\hline 97901 & SR-26 @ 18th St & 32 & 18 & 12 & 10 & 5 \\
\hline 97903 & US-52@ McCarty Ln & 14 & 7 & 6 & 4 & 4 \\
\hline 97905 & SR-26 @ Earl Av & 16 & 7 & 4 & 3 & 2 \\
\hline 97911 & US-52 @ Kossuth St & 6 & 4 & 0 & 0 & 0 \\
\hline 97915 & US-52 @ SR-26 & 5 & 1 & 1 & 0 & 0 \\
\hline 97918 & US-52 @ Duncan Rd & 1 & 0 & 0 & 0 & 0 \\
\hline 97920 & SR-26 @ SR-526 & 1 & 1 & 0 & 0 & 0 \\
\hline 97922 & Greenbush St @ US-52 & 5 & 3 & 2 & 1 & 1 \\
\hline 97940 & US-52 @ CR-300 W & 8 & 7 & 6 & 4 & 4 \\
\hline & Total & 131 & 73 & 51 & 38 & 30 \\
\hline
\end{tabular}


Table 4-6: Summary of Right-Angle Crash Counts by Collision Zones (1997-2000)

\begin{tabular}{|c|c|c|c|c|c|c|}
\hline \multirow[b]{2}{*}{ Site ID } & \multirow[b]{2}{*}{$\begin{array}{l}\text { Collision } \\
\text { Zone }\end{array}$} & \multicolumn{5}{|c|}{ Conditions } \\
\hline & & $\begin{array}{c}\text { All } \\
\text { Conditions }\end{array}$ & Daytime & $\begin{array}{l}\text { Daytime and Non- } \\
\text { winter }\end{array}$ & $\begin{array}{l}\text { Daytime, Non-winter, } \\
\text { and Weekday }\end{array}$ & $\begin{array}{l}\text { Daytime, Non-winter, } \\
\text { Weekday, and } \\
\text { Normal Weather }\end{array}$ \\
\hline 87905 & czNBWB & 0 & 0 & 0 & 0 & 0 \\
\hline 87905 & czSBWB & 0 & 0 & 0 & 0 & 0 \\
\hline 87905 & czSBEB & 1 & 0 & 0 & 0 & 0 \\
\hline 87905 & czNBEB & 1 & 1 & 1 & 1 & 1 \\
\hline 87906 & czNBWB & 3 & 0 & 0 & 0 & 0 \\
\hline 87906 & czSBWB & 2 & 2 & 2 & 2 & 2 \\
\hline 87906 & czSBEB & 4 & 3 & 3 & 2 & 2 \\
\hline 87906 & czNBEB & 1 & 1 & 1 & 1 & 1 \\
\hline 87907 & czNBWB & 2 & 1 & 1 & 1 & 0 \\
\hline 87907 & czSBWB & 2 & 1 & 0 & 0 & 0 \\
\hline 87907 & czSBEB & 0 & 0 & 0 & 0 & 0 \\
\hline 87907 & czNBEB & 1 & 1 & 1 & 1 & 1 \\
\hline 87909 & czNBWB & 4 & 1 & 0 & 0 & 0 \\
\hline 87909 & czSBWB & 2 & 0 & 0 & 0 & 0 \\
\hline 87909 & czSBEB & 1 & 0 & 0 & 0 & 0 \\
\hline 87909 & czNBEB & 6 & 6 & 4 & 3 & 3 \\
\hline 87915 & czNBWB & 0 & 0 & 0 & 0 & 0 \\
\hline 87915 & czSBWB & 0 & 0 & 0 & 0 & 0 \\
\hline 87915 & czSBEB & 4 & 3 & 2 & 2 & 2 \\
\hline 87915 & czNBEB & 1 & 0 & 0 & 0 & 0 \\
\hline 87923 & czNBWB & 1 & 0 & 0 & 0 & 0 \\
\hline 87923 & czSBWB & 1 & 0 & 0 & 0 & 0 \\
\hline 87923 & czSBEB & 3 & 2 & 2 & 1 & 0 \\
\hline 87923 & cZNBEB & 0 & 0 & 0 & 0 & 0 \\
\hline 87930 & czNBWB & 0 & 0 & 0 & 0 & 0 \\
\hline 87930 & czSBWB & 0 & 0 & 0 & 0 & 0 \\
\hline 87930 & czSBEB & 0 & 0 & 0 & 0 & 0 \\
\hline 87930 & czNBEB & 0 & 0 & 0 & 0 & 0 \\
\hline 87932 & czNBWB & 1 & 1 & 1 & 1 & 1 \\
\hline 87932 & czSBWB & 1 & 1 & 1 & 1 & 1 \\
\hline 87932 & czSBEB & 0 & 0 & 0 & 0 & 0 \\
\hline 87932 & czNBEB & 0 & 0 & 0 & 0 & 0 \\
\hline $87933^{*}$ & czSBEB & 1 & 1 & 1 & 0 & 0 \\
\hline $87933^{*}$ & czNBEB & 0 & 0 & 0 & 0 & 0 \\
\hline 97901 & czNBWB & 9 & 7 & 4 & 3 & 2 \\
\hline 97901 & czSBWB & 12 & 7 & 5 & 4 & 1 \\
\hline 97901 & czSBEB & 7 & 1 & 1 & 1 & 1 \\
\hline 97901 & czNBEB & 4 & 3 & 2 & 2 & 1 \\
\hline 97903 & czNBWB & 2 & 1 & 1 & 0 & 0 \\
\hline 97903 & czSBWB & 4 & 1 & 1 & 1 & 1 \\
\hline 97903 & czSBEB & 4 & 4 & 3 & 2 & 2 \\
\hline 97903 & czNBEB & 4 & 1 & 1 & 1 & 1 \\
\hline 97905 & czNBWB & 2 & 0 & 0 & 0 & 0 \\
\hline 97905 & czSBWB & 4 & 1 & 0 & 0 & 0 \\
\hline 97905 & czSBEB & 7 & 5 & 4 & 3 & 2 \\
\hline 97905 & czNBEB & 3 & 1 & 0 & 0 & 0 \\
\hline 97911 & czNBWB & 2 & 1 & 0 & 0 & 0 \\
\hline 97911 & czSBWB & 0 & 0 & 0 & 0 & 0 \\
\hline 97911 & czSBEB & 2 & 1 & 0 & 0 & 0 \\
\hline 97911 & czNBEB & 2 & 2 & 0 & 0 & 0 \\
\hline 97915 & czNBWB & 3 & 1 & 1 & 0 & 0 \\
\hline 97915 & czSBWB $^{\star \star}$ & 1 & 0 & 0 & 0 & 0 \\
\hline 97915 & czSBEB $^{\star \star}$ & 0 & 0 & 0 & 0 & 0 \\
\hline 97915 & czNBEB & 1 & 0 & 0 & 0 & 0 \\
\hline 97918 & czNBWB & 0 & 0 & 0 & 0 & 0 \\
\hline 97918 & czSBWB & 0 & 0 & 0 & 0 & 0 \\
\hline 97918 & czSBEB & 0 & 0 & 0 & 0 & 0 \\
\hline 97918 & czNBEB & 1 & 0 & 0 & 0 & 0 \\
\hline 97920 & czNBWB & 1 & 1 & 0 & 0 & 0 \\
\hline 97920 & czSBWB & 0 & 0 & 0 & 0 & 0 \\
\hline 97920 & czSBEB & 0 & 0 & 0 & 0 & 0 \\
\hline 97920 & czNBEB & 0 & 0 & 0 & 0 & 0 \\
\hline 97922 & czNBWB & 0 & 0 & 0 & 0 & 0 \\
\hline 97922 & czSBWB $^{* *}$ & 2 & 2 & 2 & 1 & 1 \\
\hline 97922 & czSBEB & 1 & 1 & 0 & 0 & 0 \\
\hline 97922 & czNBEB & 2 & 0 & 0 & 0 & 0 \\
\hline 97940 & czNBWB & 1 & 1 & 1 & 1 & 1 \\
\hline 97940 & czSBWB & 6 & 5 & 4 & 3 & 3 \\
\hline 97940 & czSBEB & 0 & 0 & 0 & 0 & 0 \\
\hline \multirow[t]{2}{*}{97940} & czNBEB & 1 & 1 & 1 & 0 & 0 \\
\hline & Total & 131 & 73 & 51 & 38 & 30 \\
\hline
\end{tabular}

Notes: * Site 87933 is a one-way street intersecting with a two-way street

** Unobservable in the field of view during the data collection 


\subsection{MEASUREMENT METHODS AND EVALUATIONS}

\subsubsection{Measurement Definitions}

A straight right-angle crash is caused by two vehicles traveling straight in perpendicular conflicting directions. Let us define a crash-generating event as a traffic event leading to a collision. In this case, a crossing passage of two straight vehicles is a crash-generating event because it must take place before a collision. We will simply refer to a crossing passage of two straight vehicles as a crossing event. The smaller separation between these two crossing vehicles implies a greater risk of a collision. A traditional time-to-collision measurement based on an evasive maneuver has three key disadvantages: (a) it fails to include all the scenarios that can lead to a collision if the conflict observation is based on an evasive maneuver; (b) it is difficult to measure accurately; and (c) it requires the subjective judgment of human observers. A better approach to measure the separation in the crossing event should focus on the characteristics of a crossing event that can be measured accurately and requires minimal subjectivity.

A conflict zone is an area defined as the intersection of two crossing traffic flows. One intersection may have up to four conflict zones. For example, a conflict zone between northbound versus westbound traffic flows is abbreviated as "czNBWB." A graphical explanation was given in Figure 4-3.

A conflict spot is an intersection area of two crossing vehicle paths in a conflict zone. For instance, a crossing of vehicles between a northbound approach with two through lanes and a westbound approach with one through lane will generate one conflict zone and two conflict spots. The lane number is assigned as an integer starting from 1 in increasing order from east to west for northbound and southbound approaches and from north to south for eastbound and westbound approaches. The lane number is appended to the traffic flow direction to designate a conflict spot (see the example in Figure 4-9). For an intersection where all approaches have only one through lane, a conflict zone is equivalent to a conflict spot. Additional conflict spots may be added to a conflict zone if a single vehicle path cannot properly describe actual traffic movements. This situation can occur, but infrequently, at an intersection approach with unusually wide lanes. This varying vehicle path is more common for a left-turn movement (excluded from the scope of this study). 


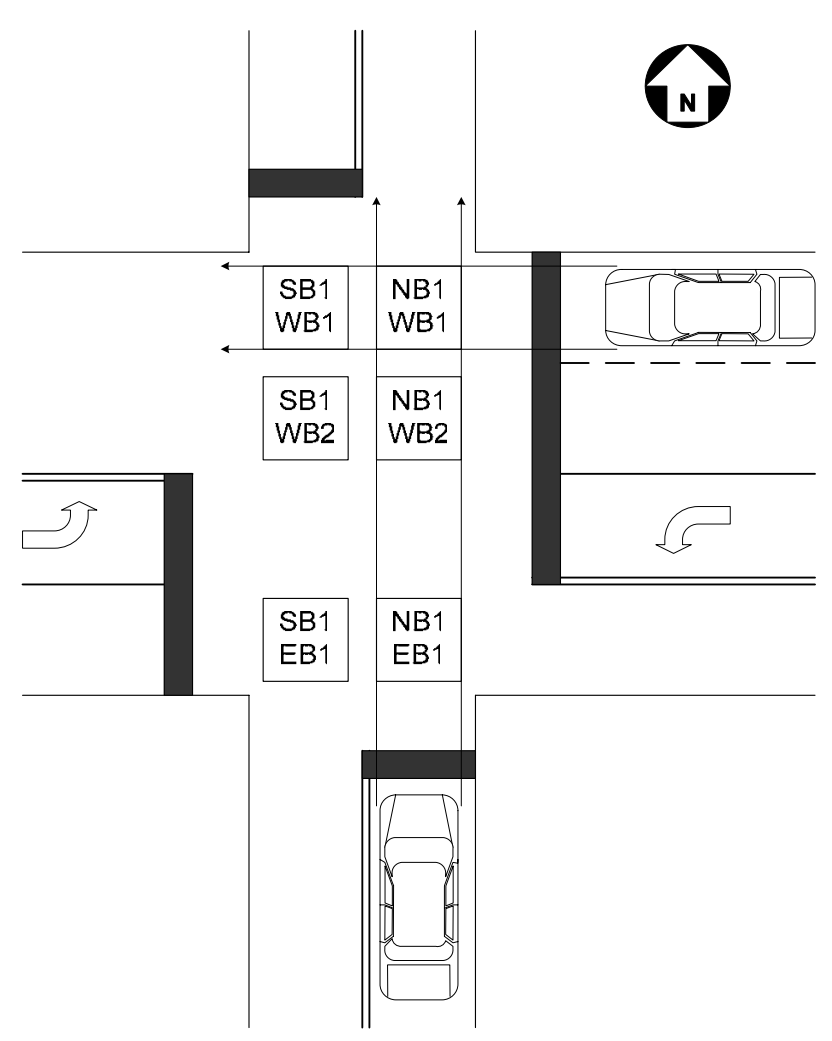

Figure 4-9: Example of Conflict Spot Designation

Gap time (GT) is defined as the time between the entry into the conflict spot of two vehicles, measured from the front bumper to the front bumper. The gap time can be degenerated into two components according to the terms coined by Allen et al. (1978): encroachment time (ET) and post-encroachment time (PET). ET is the time that the first vehicle entering the conflict spot infringes upon the right-of-way of the second vehicle, measured from the rear bumper to the front bumper. PET is the time it takes from the end of the right-of-way infringement of the first vehicle for the second vehicle to reach the conflict spot, measured from the rear bumper to the front bumper. These three components can be explained graphically as shown in Figure 4-10.

As discussed by Allen et al. (1978), ET would be an accurate indicator of conflict severity only if the second vehicle enters the conflict spot at a uniform speed. Although the speed uniformity assumption is unlikely to hold true in reality, ET may be helpful in explaining its relationship with PET and GT. The ET is usually small for the last vehicle of the through lanes from the green-terminating approach. The ET is typically larger for the left-turn vehicle yielding to the through traffic. PET is a more obvious measurement of how nearly a crash has been avoided. A PET value of zero or less would indicate a crash occurrence. Allen et al. (1978) also concluded 
that PET was the most promising indicator among others for its relative ease of measurement and safety implication. However, neither the standardized measurement method nor the relationship with crash likelihood was mentioned in the study of Allen et al. (1978).
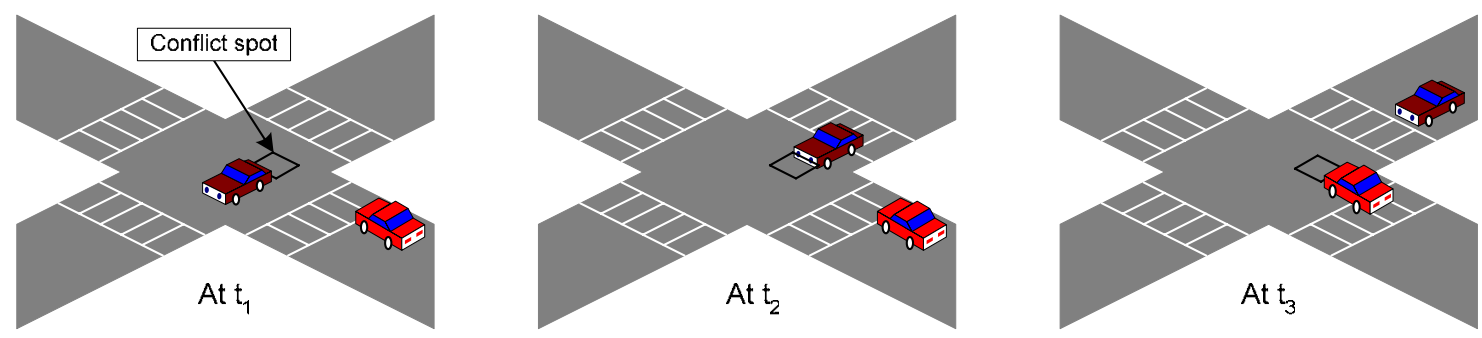

Encroachment Time, ET $=t_{2}-t_{1} ;$ Post-encroachment Time, PET $=t_{3}-t_{2} ;$ Gap time, $G T=t_{3}-t_{1}=E T+$ PET

Figure 4-10: Illustration of GT, ET, and PET

In brief, there are three measurable characteristics for a crossing event - ET, PET and GT. A right-angle crash occurrence is defined when a value of PET becomes zero or when the GT value becomes less than the ET (since GT is a summation of ET and PET).

\subsubsection{Overview of Measurement Methods and Limitations}

As mentioned in the previous section, there are three measurable event characteristics. Small values of GT and PET are potential indicators of safety concerns for right-angle collisions at signalized intersections. Three measurement methods are considered in this study: (a) manual method, (b) automated method, and (c) semi-automated method.

For the manual method, a digitized video clip of traffic movement at the intersection was reviewed manually on a frame-by-frame basis. The measurement obtained from this method is considered ground truth data. The resolution of the measurement depends on the frame rate of the digitized video clips. Theoretically, the event characteristics can be measured up to the resolution of $1 / 30$ second since the frame rate of a video signal is about 30 frames per second (fps). The virtual detectors drawn on the screen (by any VIP units) can be used to assist the reviewer in determining the precise positions of the conflict spots. This method is, however, time-consuming and labor intensive. 
The automated method measures the event characteristics using the commercial VIP units. At Purdue University we have two VIP units, Autoscope and VideoTrak. The earlier study by Grenard et al. (2000) concluded that Autoscope is a more reliable system at present. The feasibility of automated measurement using Autoscope was therefore investigated in this study. This method is efficient if the detection reliability is excellent, however, is unlikely given the many challenges in the field condition. Therefore, the evaluation of this method is needed to determine if Autoscope can be used for the measurement of the surrogate data.

Based on our preliminary evaluation, the PET measurement using Autoscope was hampered by the following problems:

- The measurement of PET using Autoscope requires a presence detector to determine the departure from the conflict spot. The accuracy of the presence detector is far from satisfactory for this task.

- The alternative method is to place additional count detectors with logical operands. In general, there are four conflict zones with at least four conflict spots. This would significantly increase the processing load of the Autoscope thus leading to an excessive number of missing detections.

- An attempt to decrease the processing load by running the Autoscope zone by zone may be a viable idea, but the measurement procedure would no longer be efficient and still far less reliable than the manual method.

To solve this issue, we decided to evaluate whether the GT can be measured automatically using the Autoscope. GT detection requires only the proper configuration of count detectors with speed traps. The processing load is not a major issue as in the case of PET. The PET can be obtained later by a manual review of video clips only at the reported occurrence times with sufficiently short GTs. If the Autoscope-based method is successful, we can still substantially reduce the amount of effort originally required in the manual measurement method. Therefore, the evaluation objective of the Autoscope-based method is to check whether the GT can be reliably measured.

As a part of Joint Transportation Research Program project SPR-2663, proprietary image processing software was developed for the PET measurement task. This software development 
aims to provide the automated measurement method alternative to the Autoscope-based approach. The algorithm and the performance of the software are documented in Appendix H.

The semi-automated method is the combination of a manual method and an automated one. The VIP unit is used to process the video clip to extract possible events of interest. The human reviewer manually checks certain reported events and measures the characteristics of interest. This method is expected to cut down the extraction time of the manual method while still retaining a better accuracy than the fully-automated one. This method still inherits, to a certain degree, the detection problem of the VIP unit.

\subsubsection{Manual Measurement Method}

For the manual measurement method, the recorded video clips at the intersections were digitized at a resolution of $30 \mathrm{fps}$. Then, two computers were set up for the task. Computer 1 controlled the playback of the digital videos on a frame-by-frame basis and on computer 2 were configured the observed conflict spots that were overlaid on the video screen. Computer 2 was also used to record the data manually into the Excel worksheet. Examples of the configuration of the virtual conflict spots are shown in Figure 4-11 and Figure 4-12.

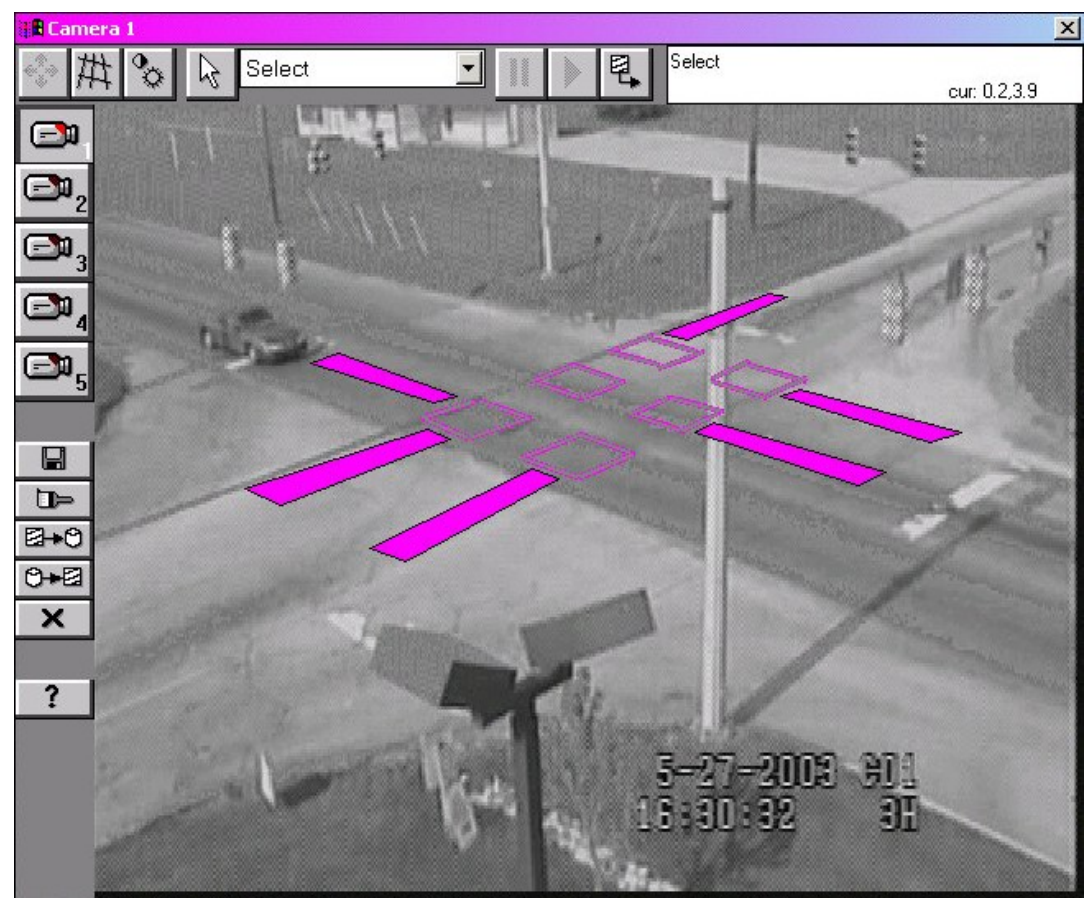

Figure 4-11: Configuration of Conflict Spots at Intersection 87923 


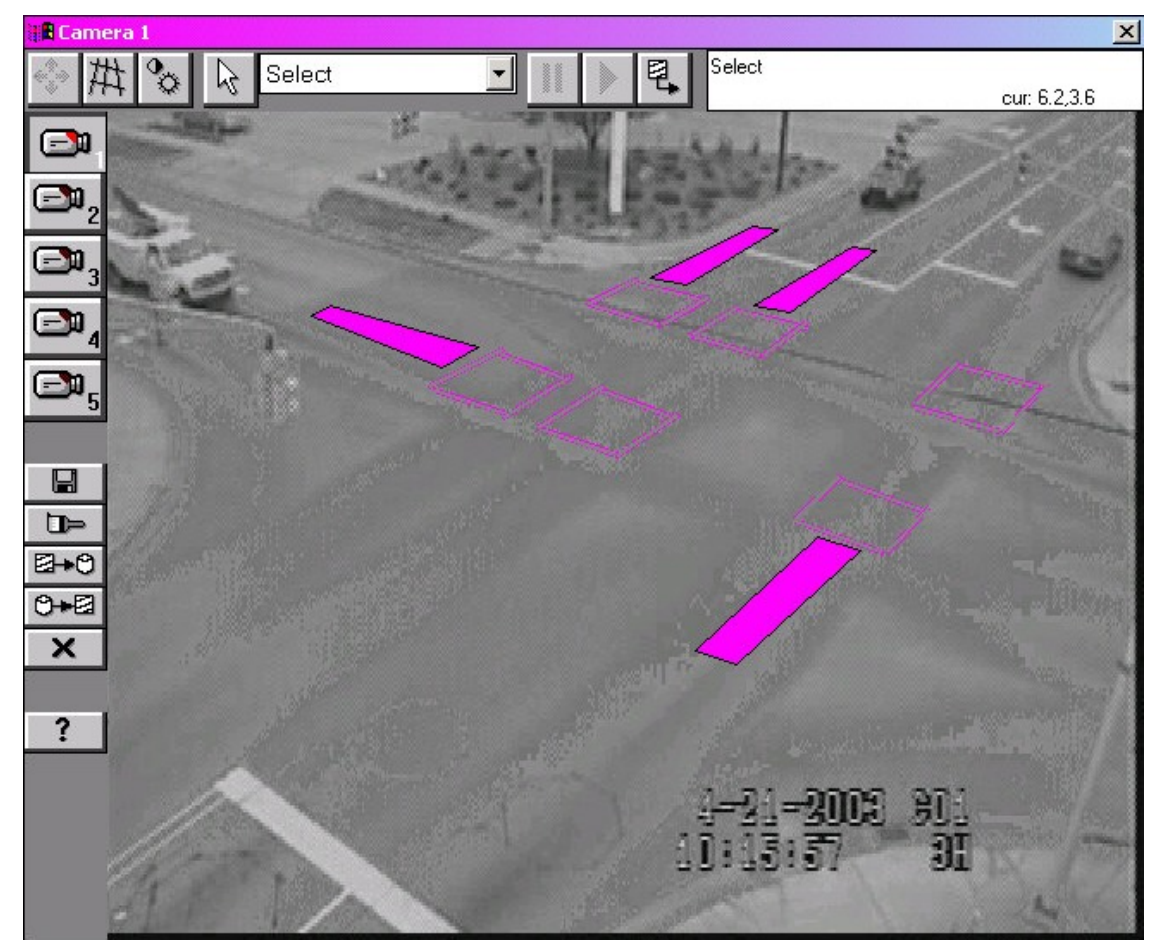

Figure 4-12: Configuration of Conflict Spots at Site 97905

The requirements for the video viewing software and the graphic card on computer 1 were: (a) the capability to access the video clip at a particular frame; (b) the capability to playback the video frame-by-frame; and (c) the capability to convert the video output into analog signal. The last requirement is necessary because Autoscope was used to help configure the virtual conflict spots on computer 2 and Autoscope takes only analog signal input.

For computer 2 we used the Autoscope virtual detectors to help configure conflict spots. The virtual conflict spots drawn on the screen were used to assist a human reviewer in determining the precise positions of the vehicles' departures and entries for PET measurement. We conducted a two-hour test on the measurement accuracy by having two trained observers extract the PET data from the same video clip. Each person configured their own conflict spots using a given guideline for conflict spot configurations. It was found that the standard error from the inter-person variations was about 0.3 second. When two persons used the same pre-configured conflict spots, the variance of the measurement errors was reduced by approximately $40 \%$. 
For each crossing event, we can measure the PET, ET and GT in seconds. In the manual measurement method, it is impractical to measure all the values for a long period of time. In addition, large values of GT and PET indicate a low-risk situation. Therefore, an upper observation threshold can be specified to save the amount of time and labor needed in the manual method. This upper threshold is applied to the GT for the evaluation of automated and semiautomated methods using Autoscope.

We will describe the procedure and evaluation results of the automated and semi-automated in subsequent sections. As we will explain, the manual measurement method was still found to be the most desirable among all the evaluated methods. Therefore, the manual method was selected for the full-scale measurement task at all the selected sites. The eight-second upper observation threshold was applied in the manual method.

\subsubsection{Automated Measurement Method}

The Autoscope unit is a relatively mature tripwire image-processing unit designed primarily for traffic control. The Autoscope detectors aim to emulate the functions of inductive loop detectors usually embedded at intersection approaches. Since we can draw the Autoscope virtual detectors anywhere on the screen, this enables us to configure the detectors around the conflict spots of interest to obtain the time that a vehicle places a call on the detector. All the events detected by these virtual detectors will be recorded into a text file, called the Autoscope event data file. The post-processing of this event data file allows us to extract the gap time and its approximate time of occurrence. This method is referred to as the Autoscope-based or Autoscope-aided measurement method. The measurement results were subsequently evaluated against those obtained from the manual measurement.

\subsubsection{Detector Configuration}

For each conflict spot, a pair of Autoscope speed detectors is configured as shown in Figure 4-13. The event time recorded in the event data file is the time when a vehicle places a call on the count detector at the end of the speed detector (the Autoscope speed detector must always be coupled with a count detector). The Autoscope speed detectors are preferred to other detector types because the speed data obtained in addition to the event time can be used to filter out false calls. 


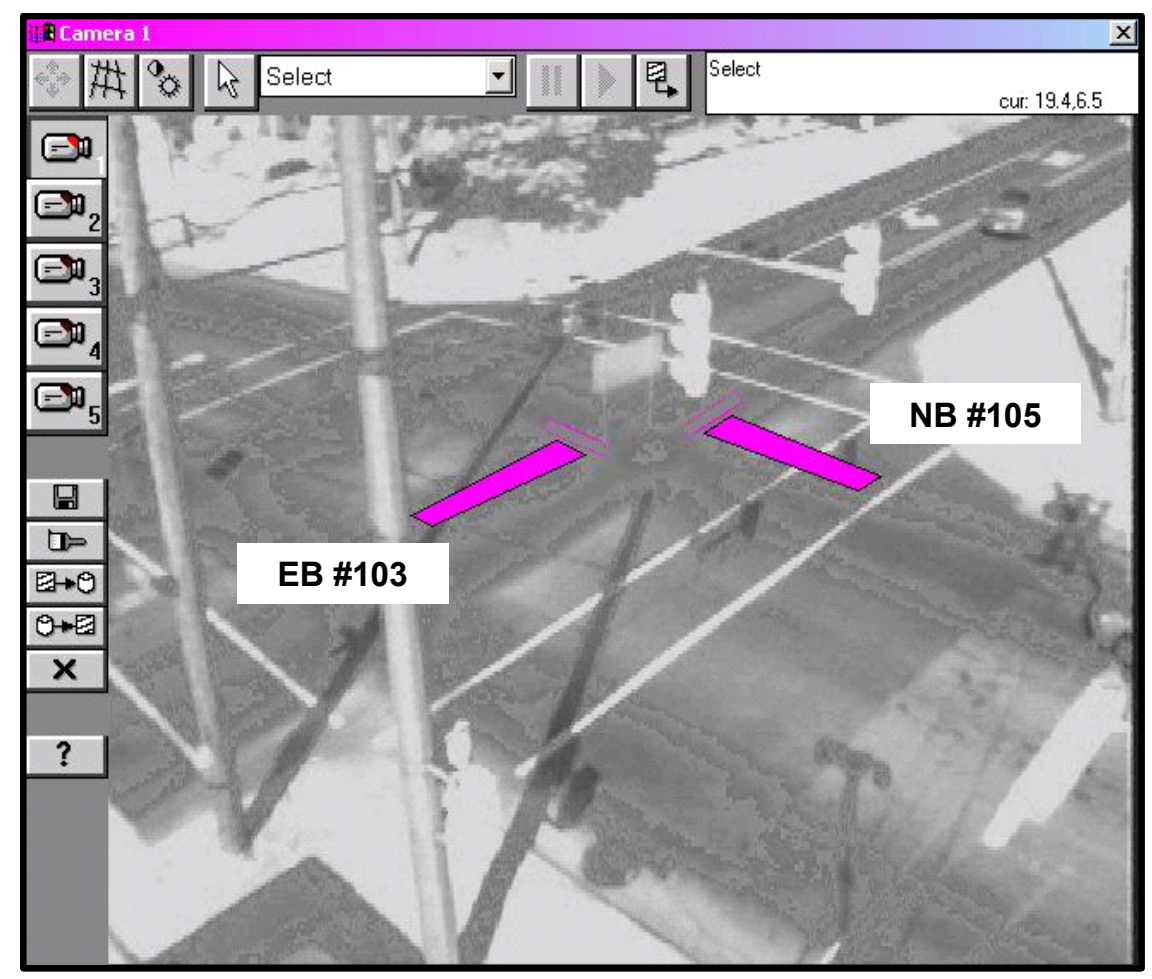

Figure 4-13: Example of Autoscope Detector Configuration for One Conflict Spot

The conflict spot is defined on the screen by two speed detectors. Therefore, the consistency in the configuration of these virtual detectors for various observation periods and locations is central to the accuracy of the measured data. The measurements can be very inaccurate if these virtual detectors are misplaced. To minimize this problem, the following procedure is a basic guideline to help configure the detectors consistently:

1. Feed the video and calibrate the field of view.

2. Trace the wheels of the vehicles that are involved in a conflict spot of interest.

3. Draw a line using a presence detector for each traffic stream in a direction that follows the observed trails (where the wheels contact the pavement).

4. Observe the traffic and check whether the majority of the vehicles follow the lines. Adjust the lines if necessary. This process will help uncover any unaccounted variations of vehicle paths, which may justify for additional conflict spots.

5. Draw a pair of Autoscope speed traps (one detector for each vehicle direction). Adjust the count detector of the speed trap to keep it in line with the outlines drawn in the previous step. Make sure that the size of the speed trap is reasonable. Detectors that are too small may be insensitive to the traffic movement, thus creating excessive missed calls. 
Oversized detectors may also trigger a number of false calls from many sources, such as adjacent-lane vehicles, opposing vehicles, truck shadows, etc.

6. Once all the speed traps are configured, recheck the vehicle path and adjust the position and size if necessary.

7. Assign and note the detector numbers of all the configured speed traps.

8. Delete all the lines drawn using a presence detector in the earlier step. Then, feed the video into the Autoscope unit and start collecting the event data.

\subsubsection{Autoscope Event Data File}

All the events occurring at the speed detectors are collected and recorded into the Autoscope event data file. An example record is shown in Table 4-7.

Table 4-7: Example of Autoscope Event Data File

\begin{tabular}{cccccc}
\hline Time & Detector & On Time & Speed & Length & Vehicle Class \\
\hline 15:37:36.148 & 107 & 434 & -1 & 0 & -1 \\
$15: 37: 35.981$ & 109 & 701 & 21 & 18 & 0 \\
15:37:36.716 & 114 & 733 & 21 & 17 & 0 \\
$15: 37: 47.193$ & 145 & 767 & -1 & 0 & -1 \\
$15: 37: 47.626$ & 138 & 1068 & -1 & 0 & -1 \\
$15: 37: 47.593$ & 144 & 1101 & -1 & 0 & -1 \\
$15: 37: 49.295$ & 107 & 233 & -1 & 0 & -1 \\
$15: 37: 49.094$ & 109 & 468 & 31 & 17 & 0 \\
$15: 37: 49.628$ & 114 & 534 & 27 & 16 & 0 \\
$15: 37: 50.796$ & 127 & 467 & -1 & 0 & -1 \\
$15: 37: 50.763$ & 132 & 634 & 30 & 19 & 0 \\
$15: 37: 51.397$ & 138 & 400 & 32 & 16 & 0 \\
$15: 37: 51.363$ & 145 & 467 & -1 & 0 & -1 \\
\hline
\end{tabular}

The file has six fields for each row of record. Each row represents the event occurring at a specific detector. Three important fields used further for extracting gap time are:

- Time: the Autoscope time when the vehicle triggers the count detector located at the end of the speed trap.

- Detector: the specific number assigned to the count detector (Autoscope records the count detector number instead of the speed detector number).

- Speed: the speed of a vehicle is reported in mph. The reported speed is -1 if the measured speed is invalid. 


\subsubsection{Data Extraction}

The codes were written in the Visual Basic environment with a user-friendly interface to extract the data from the Autoscope event data file. The key steps of the procedure to extract the gap time from the Autoscope event file can be briefly summarized as follows:

\section{Data Preparation}

A MS Access database, "Detectors.mdb", is set up under the subfolder "Files" of the directory in which the program was installed. The event data file must be imported into this database as a table. The table can be given any name as long as it does not violate the MS Access convention of table names.

\section{Compute Event Time}

The program modifies the Autoscope reported event time into two formats: (a) second index (SecIndex) and (b) reference time (refTime). The "secIndex" is basically an event time in units of seconds with reference to the time of the first event. The "refTime" is a second index converted into a conventional format, "hh:mm:ss." The former format is computationally convenient while the latter is compatible with the time in the video viewing software (which is Adobe Premiere). Users must specify a table name in this step.

\section{Compute Gap Time}

Users must provide the two detector numbers that were configured for each conflict spot. The program then queries all the related events sorted by the event time. Ideally, the data should appear as an alternate series of event from two detectors where a switching point from one series to another indicates a gap time. In reality, false calls from any detector can interrupt a perfect series, thus creating a false gap time if not removed. Therefore, an algorithm to eliminate false calls is incorporated into this step (see details in Section 4.4.4.4). The gap time can be computed accordingly once the false calls are removed. The measured gap time will be recorded as a new table in the same database. The name of the output table is modified from the original data table 
name by appending the original name with a user-given extension. The example of an output file for a particular conflict spot is shown in Table 4-8.

The gap time (gap) is reported in seconds as shown in Table 4-8. The gap type is determined from a sequence of vehicles entering into a conflict spot. The gap type is 1 if the main-street vehicle enters into the conflict spot first, and the gap type is 2 if the side-street vehicle enters first. The "refTime" reported in the same row is the time at the end of the crossing event.

Table 4-8: Example of Output File with Gap Time

\begin{tabular}{cccccccccc}
\hline Time & Detector & On Time & Speed & Length & Vehicle Class & Seclndex & refTime & Gap & GapType \\
\hline $15: 12: 14.913$ & 109 & 567 & 21 & 15 & 0 & 374.958 & $0: 6: 43.958$ & & \\
$15: 12: 17.316$ & 109 & 567 & 21 & 15 & 0 & 377.361 & $0: 6: 46.361$ & & \\
$15: 12: 19.319$ & 109 & 501 & 26 & 16 & 0 & 379.364 & $0: 6: 48.364$ & & 1 \\
$15: 12: 43.318$ & 107 & 668 & 20 & 17 & 0 & 403.363 & $0: 7: 12.363$ & 23.999 & 1 \\
$15: 12: 45.454$ & 107 & 668 & 19 & 18 & 0 & 405.499 & $0: 7: 14.499$ & & \\
$15: 12: 47.390$ & 107 & 635 & 19 & 17 & 0 & 407.435 & $0: 7: 16.435$ & & \\
$15: 12: 49.527$ & 107 & 467 & 23 & 15 & 0 & 409.572 & $0: 7: 18.572$ & & \\
$15: 12: 56.603$ & 107 & 802 & 16 & 17 & 0 & 416.648 & $0: 7: 25.648$ & & \\
$15: 12: 58.439$ & 107 & 968 & 17 & 19 & 0 & 418.484 & $0: 7: 27.484$ & & \\
$15: 13: 00.509$ & 107 & 968 & 18 & 21 & 0 & 420.554 & $0: 7: 29.554$ & & \\
$15: 13: 02.211$ & 107 & 768 & 17 & 18 & 0 & 422.256 & $0: 7: 31.256$ & & \\
$15: 13: 12.792$ & 109 & 735 & 14 & 14 & 0 & 432.837 & $0: 7: 41.837$ & 10.581 & \\
$15: 13: 14.361$ & 109 & 668 & 20 & 16 & 0 & 434.406 & $0: 7: 43.406$ & & \\
$15: 13: 16.498$ & 109 & 701 & 19 & 16 & 0 & 436.543 & $0: 7: 45.543$ & & \\
$15: 13: 18.667$ & 109 & 634 & 21 & 16 & 0 & 438.712 & $0: 7: 47.712$ & & \\
$15: 13: 21.237$ & 109 & 601 & 21 & 16 & 0 & 441.282 & $0: 7: 50.282$ & & \\
\hline
\end{tabular}

\subsubsection{Algorithm to Detect and Remove False Calls}

Let us define the switching time as the time between two calls from the same detector, say detector 1 . The false calls are identified and removed by successive computation of the switching time and comparison with the threshold value. If the calls from the detector on the other approach (say detector 2) are between these two calls from detector 1 and the switching time is less than the threshold, then these calls are marked as false calls. One possible threshold is the interchange period plus the minimum green time. A lower threshold value may also be preferred in order to avoid losing the short gap time from red-light runners.

The flowchart of the algorithm is shown in Figure 4-14. Once the data are queried for two specified detectors, the algorithm begins from the first row by reading the current detector number (curDet) and current event time (curTime). Then, the pointer moves forward to search for the next series of valid calls from the same detector utilizing the switching time criterion. 


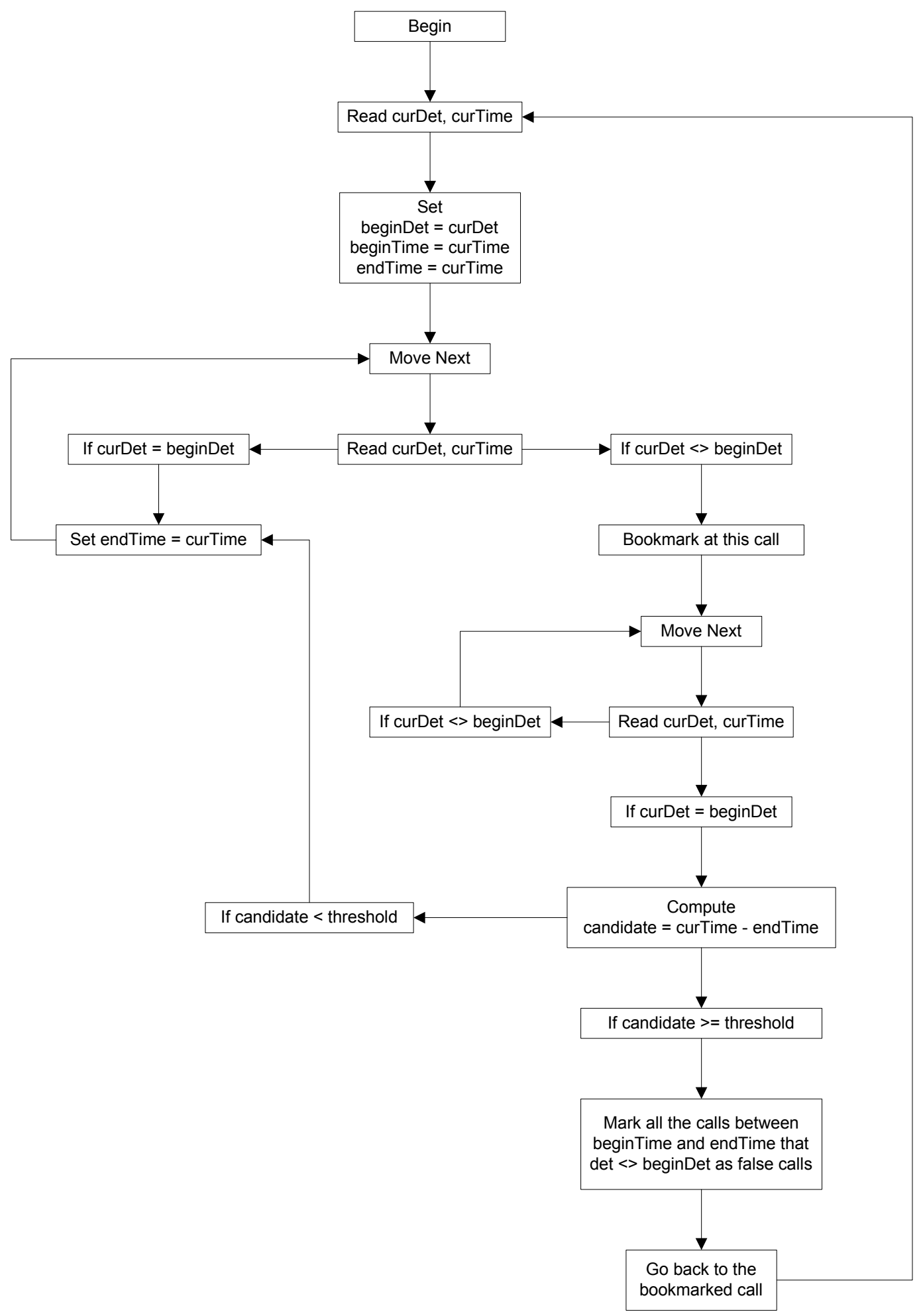

Figure 4-14: Detecting False Calls in the Autoscope Event File 
Once the next series of valid calls are identified, all the false calls from another prior detector are then marked and removed. There are two important considerations in the details of this algorithm: (a) false call at the end of the phase and (b) the effect of the threshold specification.

\section{False Call at the End of the Phase}

The first valid call of the next phase will be marked as false if the false call from the previous phase takes place soon enough to pass the threshold. We can decrease the threshold, however, so that the false call is more likely to be addressed in the next phase consideration, instead of being treated as false.

\section{Effect of Threshold Specification}

A small threshold is a conservative specification because the smaller the threshold, the more gap times that will be reported because the chance to mistreat the false call as the starting vehicle of the next phase is increased when the vehicle headway is large (e.g., for the low-volume or offpeak condition). This problem is minimal during the peak hour. However, the valid gaps are more likely to be detected.

From a conservative viewpoint, a small threshold decreases the efficiency of the method but no valid calls will be removed except for the case discussed above. If the semi-automated method is to be considered, this will generate a number of candidate gap times for manual verification.

\subsubsection{Software Implementation}

The algorithm is coded using SQL and ActiveX Data Object (ADO) in the Visual Basic Development environment. The software interface is shown in Figure 4-15.

Users can specify the name of the data table to be processed. The button "Get Time Index" is used to compute the event time. In the gap time calculation, users need to specify two specific detector numbers for a conflict spot and the extension to create the output table. The "Compute Gap" button is used to compute the gap time. 


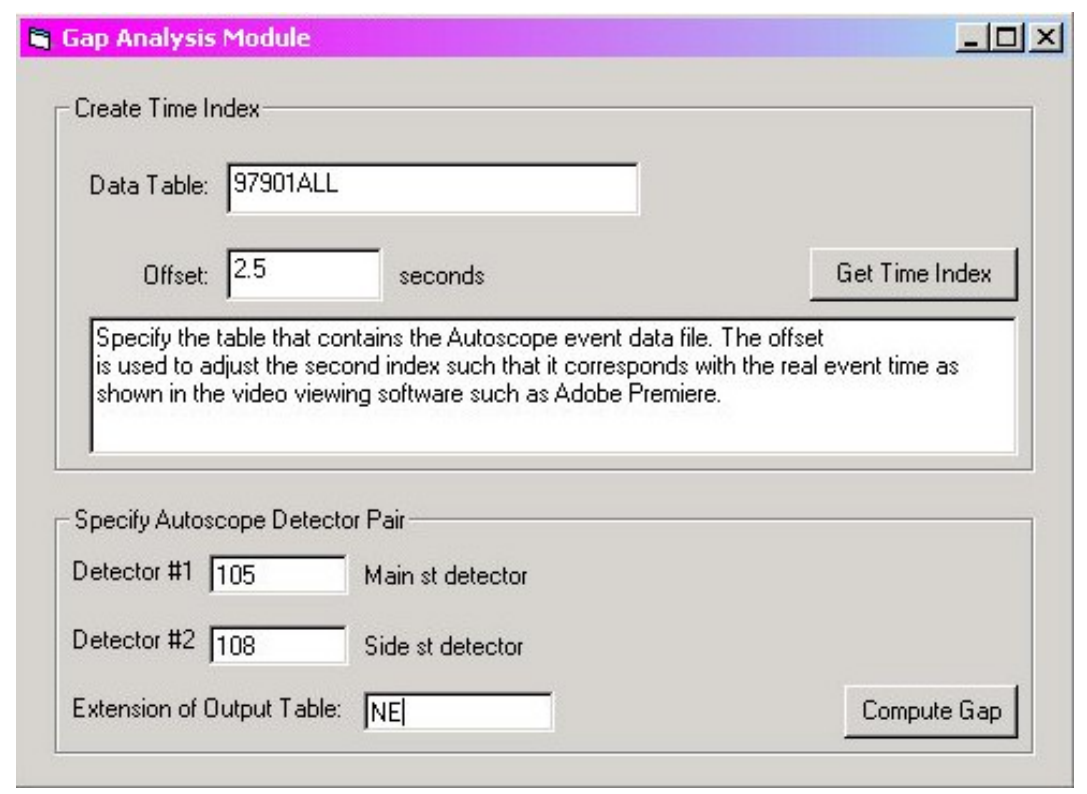

Figure 4-15: Interface for Processing of Autoscope Event File

\subsubsection{Alternative Software for Automated Measurement}

The software specifically designed to help automate the measurement of the GTs and PETs from digitized video clips was developed (see Appendix H). The method was found to perform better than the Autoscope-based method. However, the measurement accuracy is still insufficient for the evaluation purpose in this study. Evaluation results and usage instructions are also documented.

\subsubsection{Evaluation of Automated Measurement Method}

\subsubsection{Autoscope Performance Evaluation: Monochrome versus Color Video}

As Autoscope is not the sole tool possible in our measurement, it was desirable to have intersection traffic recorded in a format suitable for other alternatives as well. Autoscope SoloPro and research-level image processing software are examples of the alternatives that require color video for the best performance. For this reason we are interested in evaluating the performance of the Autoscope for our application if color video is recorded instead of monochrome or black-andwhite $(\mathrm{B} / \mathrm{W})$ video. When the color video is fed into the Autoscope, only the $\mathrm{B} / \mathrm{W}$ portion of the video signal will be processed. 
We selected one intersection (SR $26 @$ Farabee Dr) to record the traffic movements for about 1.5 hours. Using a mobile traffic laboratory, we set up two cameras on the top of the mast to focus on the intersection area. Both cameras were adjusted to obtain approximately the same field of view (FOV). Since both cameras (Panasonic WV-CS854) are capable of switching between the B/W and color mode, one camera was set to the $\mathrm{B} / \mathrm{W}$ mode and another was set to the color mode. The $\mathrm{B} / \mathrm{W}$ video was recorded directly into a computer. The color video was recorded into a digital VCR (Panasonic AG-DTL1). The FOVs from both cameras are shown in Figure 4-16 and Figure 4-17.

On the main street (SR 26), the intersection has two through lanes, one right-turn lane, and one left-turn bay for both approaches. On the side street (Farabee Dr), the NB approach has only one lane with flare for right turns while the SB approach has one through lane and one left-turn lane. The intersection is fully actuated with protected-permitted leading left turns. The traffic was recorded on Tuesday, November 26, 2002 from 10:40 AM to 12:10 PM. The mobile traffic laboratory was parked at the corner between the SB and EB approaches. Note that the time stamps shown in the video captures are inaccurate because they were not properly adjusted.

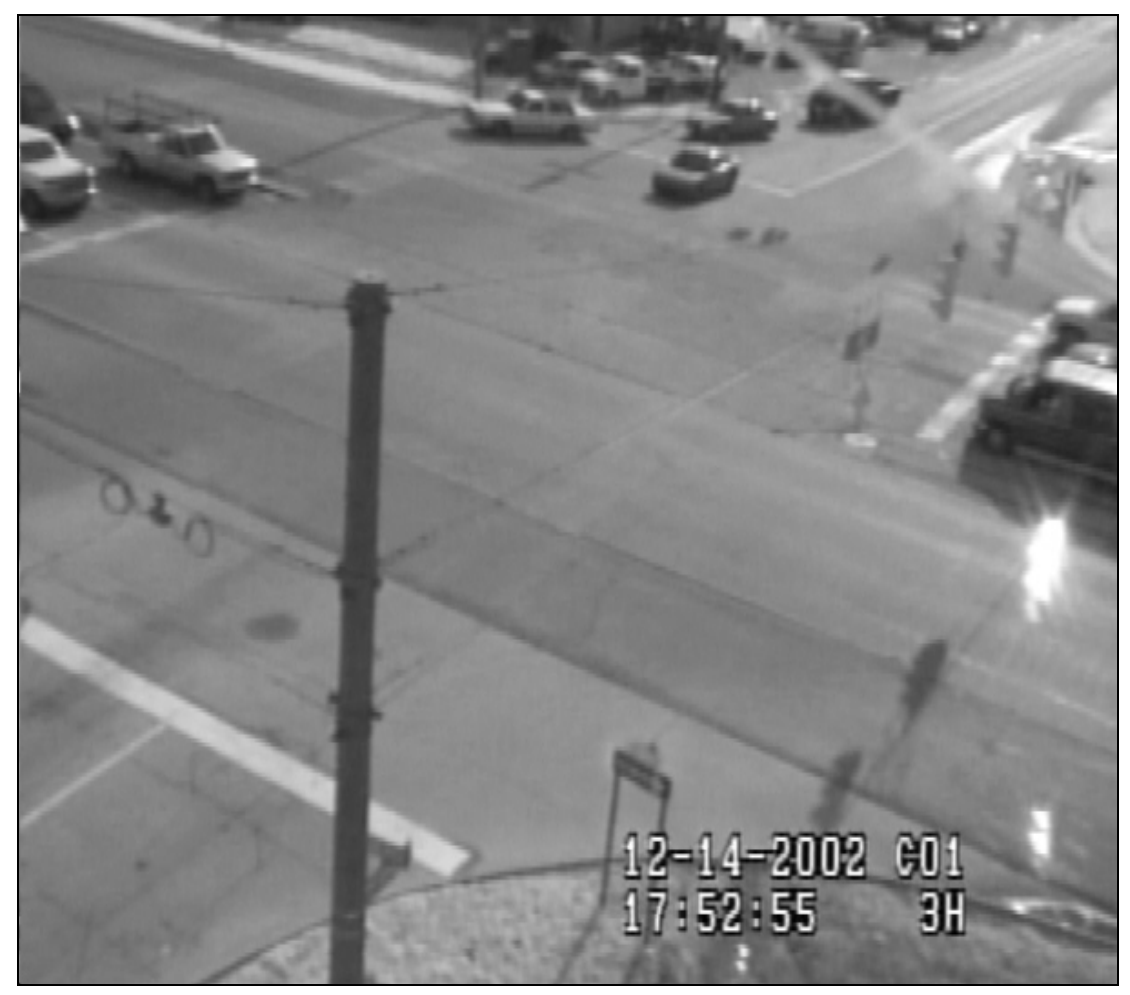

Figure 4-16: SR 26 @ Farabee Dr in Monochrome (B/W) mode 


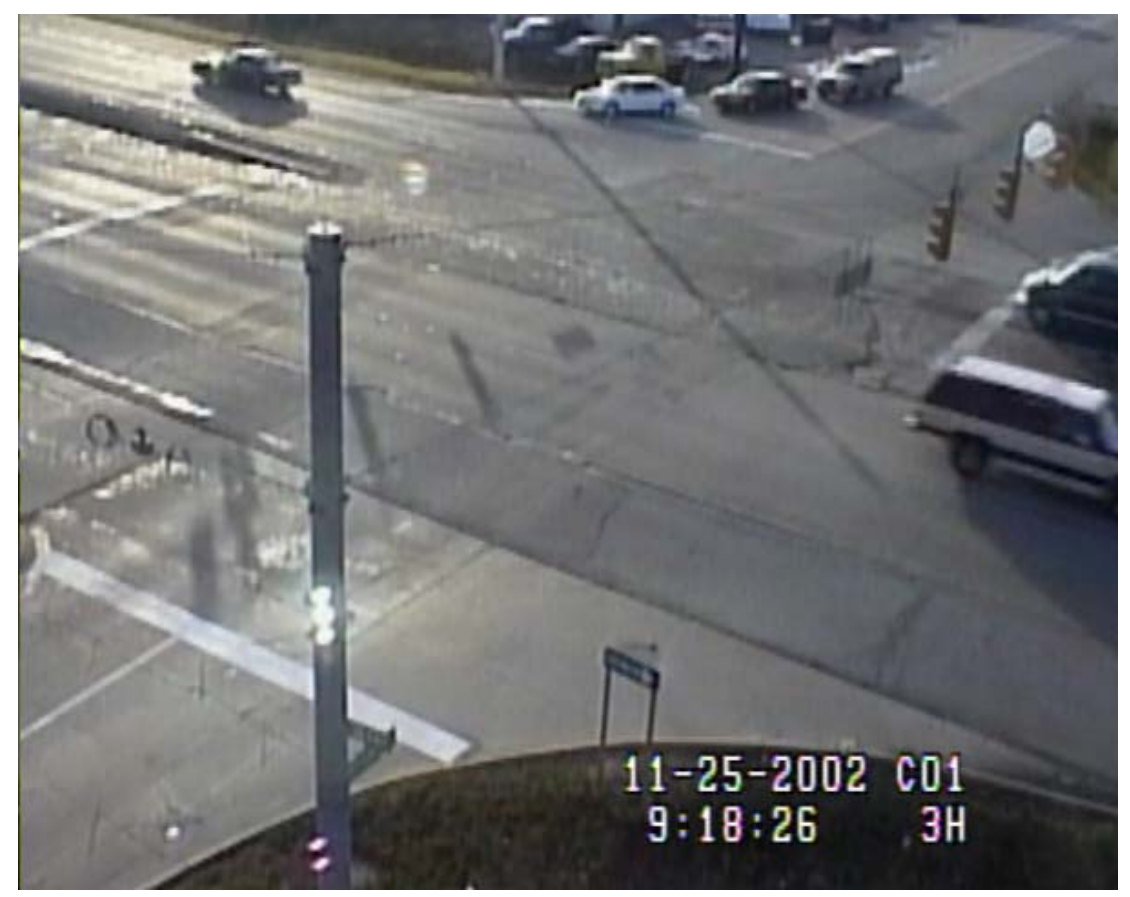

Figure 4-17: SR 26 @ Farabee Dr in Color Mode

Both recorded videos were processed using Autoscope to collect the event data files. Twelve speed traps were used (or six conflict spots equivalently) in the detector configuration. The coded software was used to extract the gap time and the occurrence time from the event data files. Four conflict zones were selected for manual validation.

For this evaluation, all the GTs of 10 seconds or less at the four selected conflict spots were measured by watching the video clip and playback frame-by-frame for gap measurement. Some gaps of slightly over 10 seconds were also included randomly to have an adequate sample size for the evaluation. The total observation period was nine spot-hours.

\section{Detector Layout}

The detectors with their corresponding IDs were configured as shown in Figure 4-18. Twelve speed traps were used to define six conflict zones. Four conflict zones were selected for manual validation as listed in Table 4-9. 


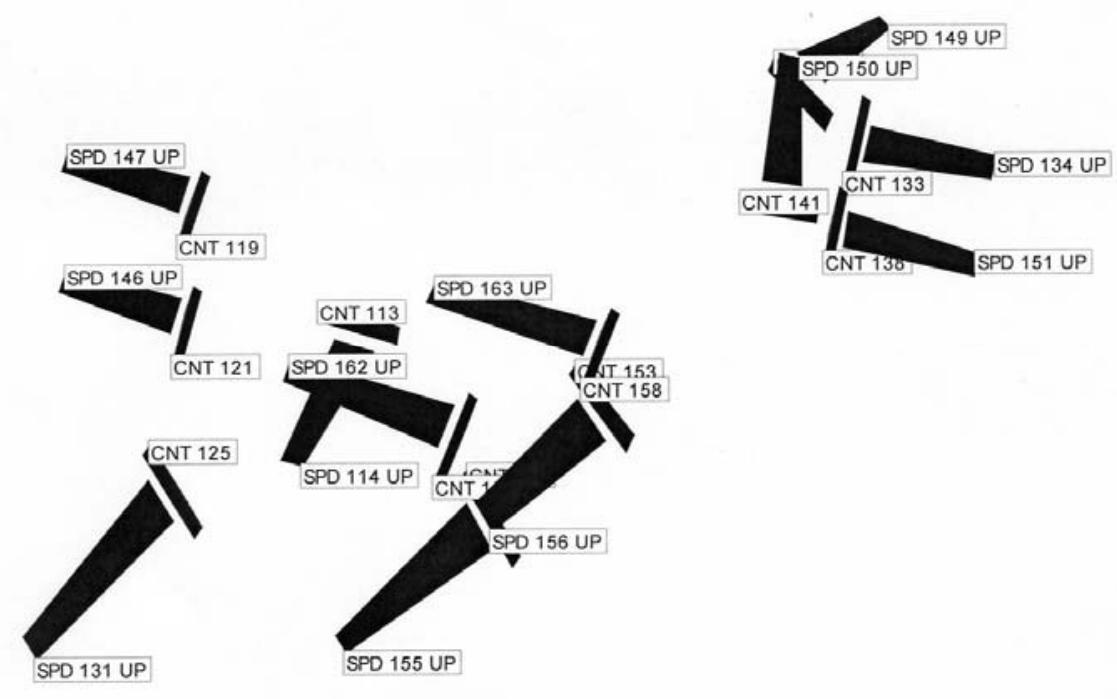

Figure 4-18: Detector Configuration with Corresponding ID

Table 4-9: List of Selected Conflict Spots for Validation

\begin{tabular}{|c|c|c|c|}
\hline Conflict Spot & Main St. Detector & Side St. Detector & Remarks \\
\hline 1 & 121 & 125 & Through vs. Left-turn \\
\hline 2 & 119 & 113 & Through vs. Left-turn \\
\hline 3 & 153 & 158 & Through vs. Through \\
\hline 4 & 133 & 117 & $\begin{array}{c}\text { Through vs. Through, Far side of } \\
\text { the intersection }\end{array}$ \\
\hline
\end{tabular}

\section{Evaluation Results}

The evaluation results consisted of three conditions of measured gap times: (a) Autoscopedetected gap times using color video, (b) Autoscope-detected gap times using B/W video, and (c) measured gap times using a manual frame-by-frame review. Conflict zone No. 4 was excluded from the comparison because it was the far spot in the FOV and thus did not give a consistent performance with the other three selected zones. There were a total of 31 gaps manually measured in the validation process at conflict zones 1,2 and 3 for the observed period. Most of the measured gaps are less than 10 seconds. The gap time differences between the color and $\mathrm{B} / \mathrm{W}$ videos were computed only when the gap times were detected by both videos. The errors of both videos compared to actual values were also computed. 
The number of missed detections by both videos differed by less than 10 percent. The mean errors are somewhat different but this appears to be accounted for by a large standard error. The standard deviation (SD) of errors for the $\mathrm{B} / \mathrm{W}$ video appears to be larger than that of the color video when all the data points were used. This is attributed to one large error $(16.836 \mathrm{sec})$ in the $\mathrm{B} / \mathrm{W}$ mode due to a missed call. The SD of the errors for both the $\mathrm{B} / \mathrm{W}$ and color videos was very close when we removed this outlier. Table 4-10 summarizes the error comparison when the outlier is removed.

Table 4-10: Comparison of Errors: Color versus B/W

\begin{tabular}{|c|c|c|c|}
\hline \multicolumn{4}{|c|}{${ }^{* *}$ Remove one outlier in BW case } \\
\hline Total counted gaps & 31 & & \\
\hline \multirow[t]{2}{*}{ Exposure } & 3.5 & zone-hours & $\begin{array}{l}\text { * Exposure is computed by a sum of products between number of } \\
\text { observed conflict zones and observation period at each zone }\end{array}$ \\
\hline & Color & BW & \\
\hline Missed Gaps & 10 & 13 & ${ }^{*}$ missed gaps $=$ Autoscope reported no gaps comparing to actual gaps \\
\hline Mean Error & 0.469 & -1.452 & \\
\hline SD Error & 2.999 & 3.035 & \\
\hline $\mathbf{N}$ & 21 & 18 & $\begin{array}{l}{ }^{*} \mathrm{~N}=\text { sample size, number of matched gaps between the actual } \\
\text { and Autoscope-detected ones }\end{array}$ \\
\hline
\end{tabular}

Assuming the errors in both cases have equal variances, it is hypothesized that there is no difference between the mean errors of the $\mathrm{B} / \mathrm{W}$ versus the color video (null hypothesis). The twosample t-test is used to test against the null hypothesis. The test results are summarized in Table 4-11 for two scenarios: with and without removing an outlier.

Table 4-11: Two-Sample t-tests of the Differences in Mean Errors: Color versus B/W

\begin{tabular}{lcrrr}
\hline & \multicolumn{1}{c}{ Color } & $B / W$ & \multicolumn{1}{c}{ Color } & \multicolumn{1}{c}{$B / W$} \\
\hline & ${ }^{*}$ No outlier is removed & ${ }^{*}$ An outlier is removed \\
\cline { 2 - 5 } Mean & 0.469 & -0.489 & 0.469 & -1.452 \\
Variance & 8.994 & 26.300 & 8.994 & 9.210 \\
Observations & 21 & 19 & 21 & 18 \\
Pooled Variance & 17.192 & & 9.093 & \\
Hypothesized Mean Difference & 0 & & 0 & \\
df & 38 & & 37 & \\
t Stat & 0.729 & & 0.055 & \\
P(T<=t) two-tail & 0.470 & & 2.026 & \\
t Critical two-tail & 2.024 & &
\end{tabular}

From Table 4-11, in the case where the outlier is not removed, the two-tailed p-value is 0.470 , which is far greater than 0.05 . When the outlier is removed, the p-value is reduced to 0.055 but still greater than 0.05 . Therefore, we have no strong statistical evidence to reject the null hypothesis. This leads to the conclusion that the color and $\mathrm{B} / \mathrm{W}$ videos yield a comparable 
Autoscope performance in our application. This finding allows us to continue all the recording of traffic movement at selected intersections in the color mode.

\subsubsection{Evaluation of the Autoscope-based Automated Measurement}

In the previous section, we showed that the detection performance using color and $\mathrm{B} / \mathrm{W}$ videos is comparable. In this section, we will evaluate the detection performance of the Autoscope-based measurement with respect to specific field conditions such as the lighting condition and the distance from the camera to the conflict spot. The procedure to configure the Autoscope detectors and extract the data was described earlier in Section 4.4.4.

\section{Evaluation Procedure}

The selected test intersection is SR 26 @ Earl Ave (Intersection 97905) in Lafayette, Indiana. The traffic movement was recorded for the entire intersection for about four hours on December 2, 2002 from 9:21 AM to 12:21 PM and 12:34 PM to 13.35 PM. The intersection geometry was collected for the calibration of the field of view in Autoscope. The observed period includes the long-shadow condition for almost two hours. Figure 4-19 and Figure 4-20 show the field of view of the studied intersection from the camera without and with a long shadow respectively.

The video clips were digitized and reviewed manually to collect all the gap times of 10 seconds or less. The 10-second observation threshold was chosen arbitrarily in this case in order to have an adequate sample size for comparison between the actual and the Autoscope-reported results.

Two sets of detector configurations were used for (a) all through versus through conflicts and (b) all through versus left-turn conflicts (see Figure 4-21 and Figure 4-22). Left-turn movements were included in this evaluation to determine how the automated method would fare when dealing with non-through traffic. Two sets of detector configuration were run separately to avoid the excessive processing load on the Autoscope unit, which could have a negative impact on detection reliability. The Autoscope load depends on the number and type of detectors used. The detectors and conflict spots are summarized in Table 4-12. The northbound through versus westbound left-turn traffic requires two conflict spots because the left-turn traveling path varies significantly. 


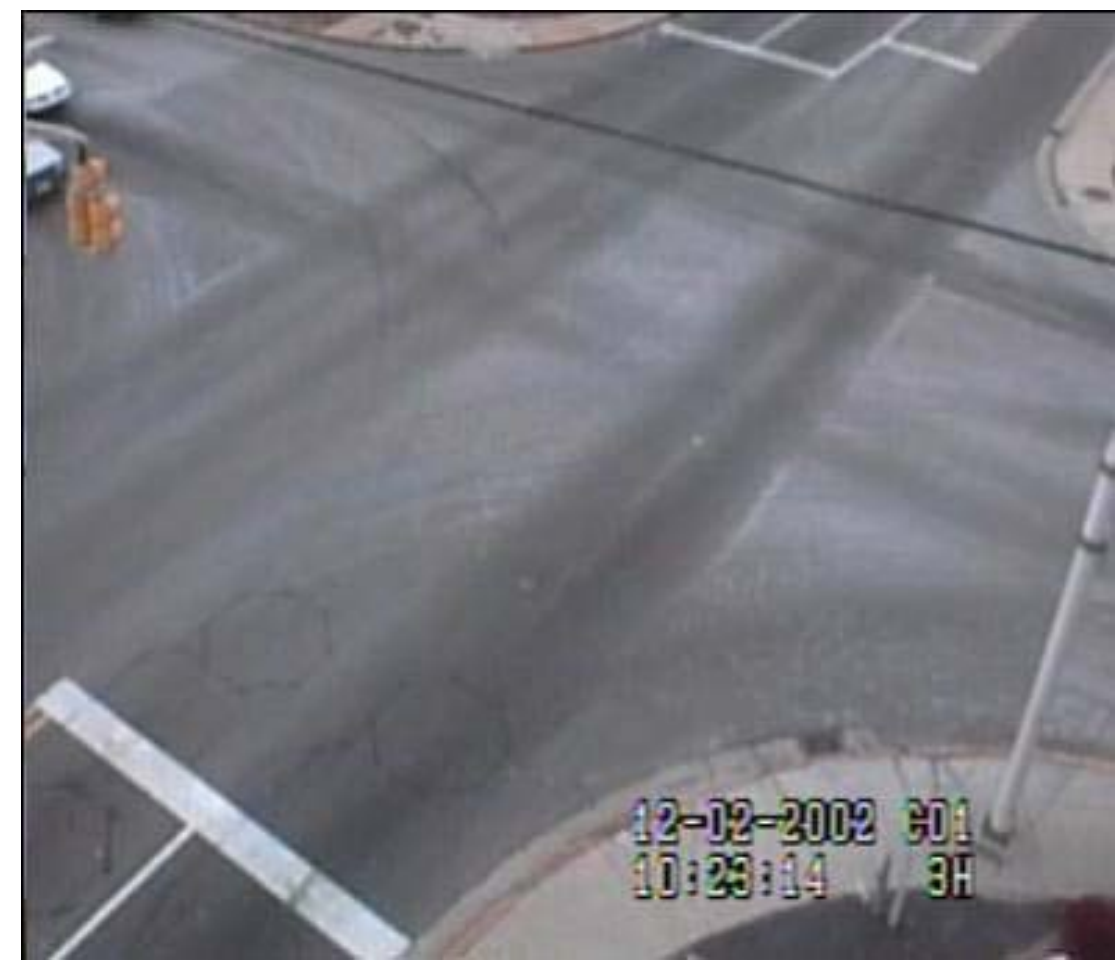

Figure 4-19: Field of View of SR-26 @ Earl Ave (No Shadow)

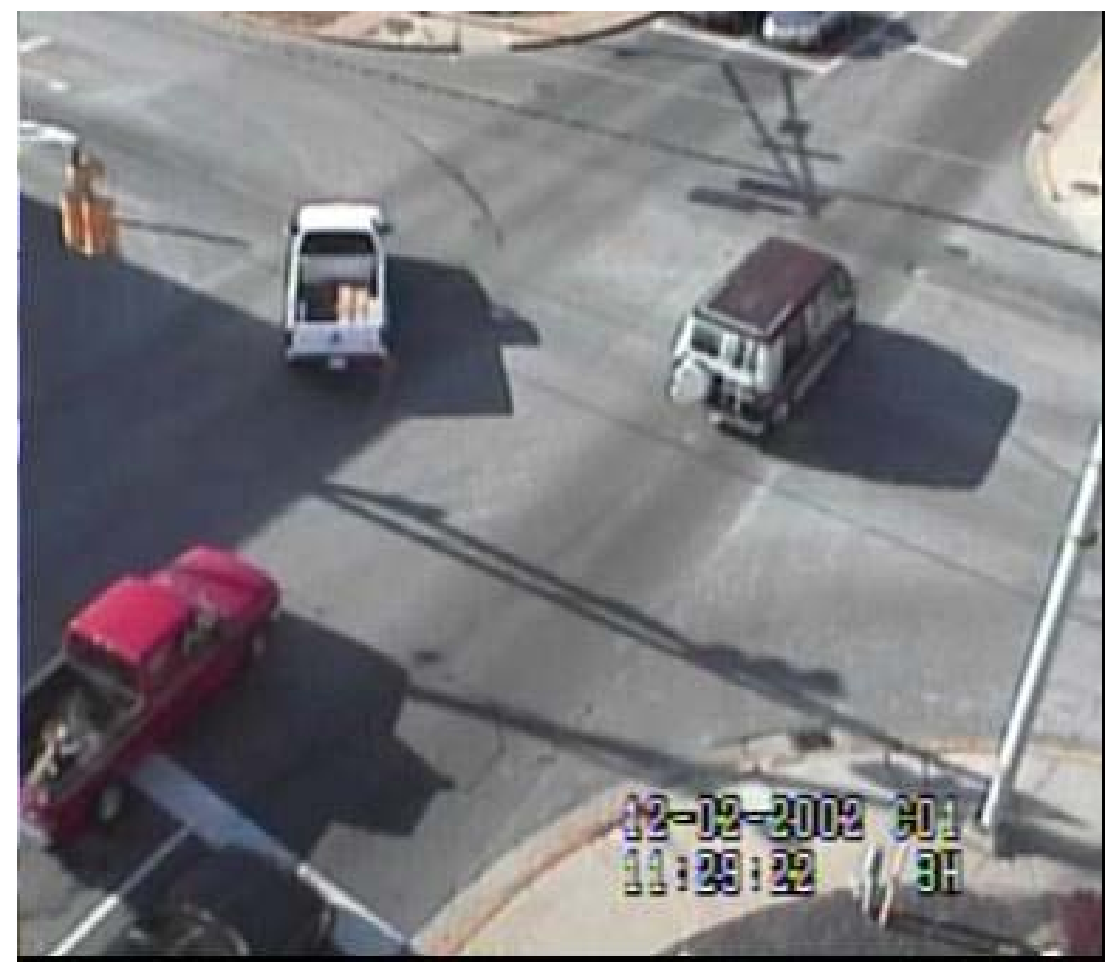

Figure 4-20: Field of View of SR-26 @ Earl Ave (With Shadow) 


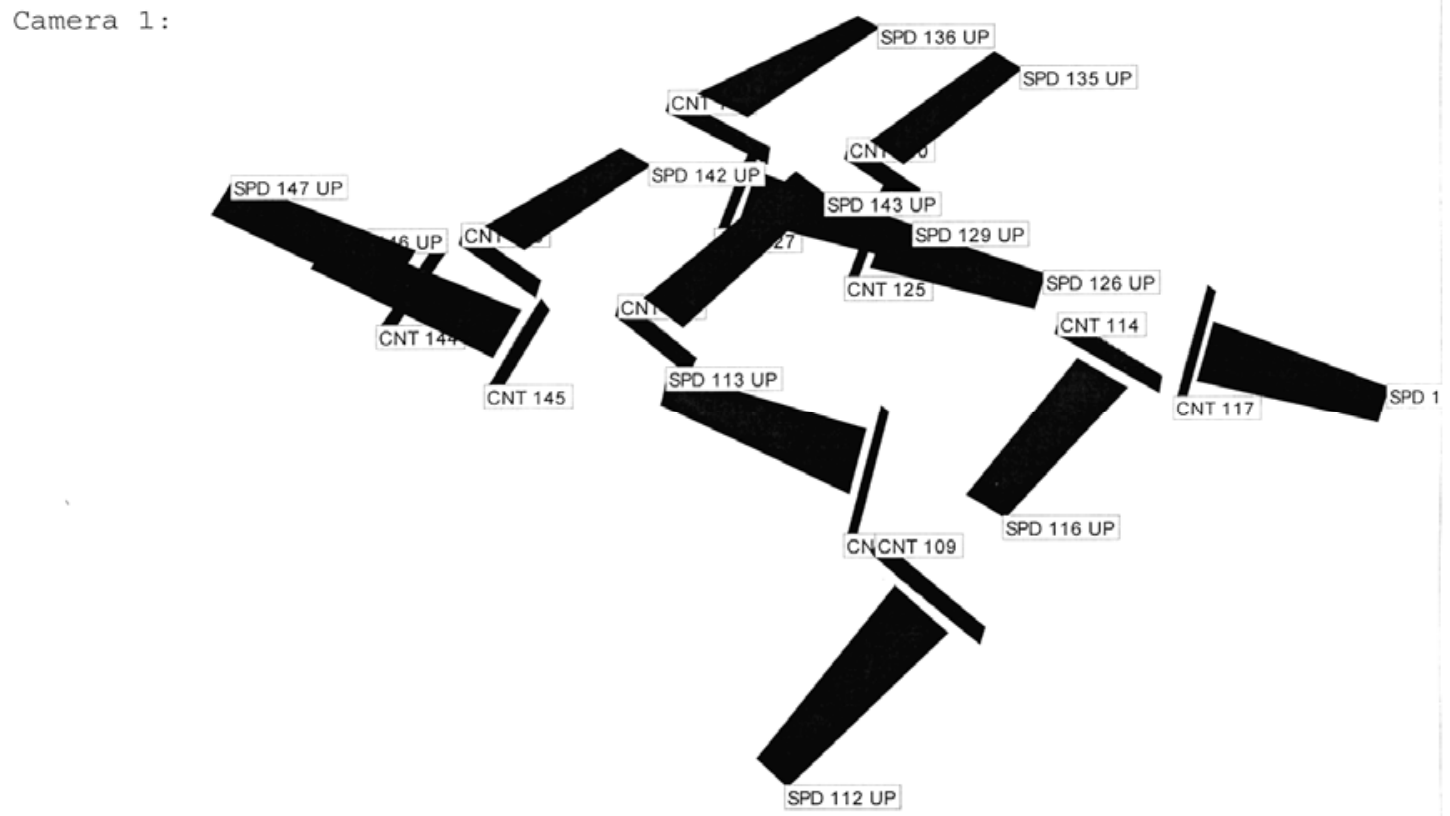

Figure 4-21: Detector Configuration for TH-TH Conflicts

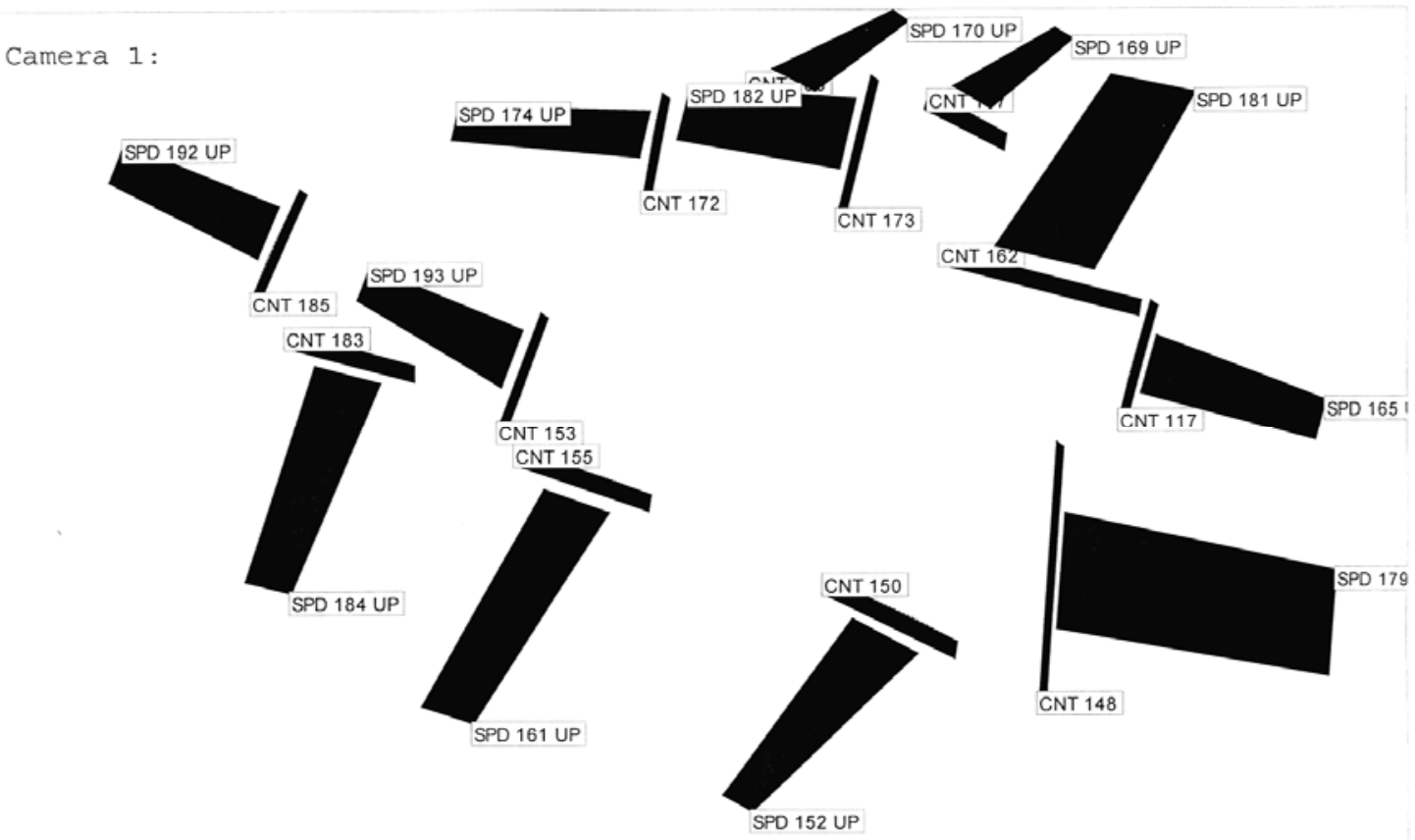

Figure 4-22: Detector Configuration for TH-LT Conflicts

To measure the gap time manually for comparison, the entry times of two vehicles at a conflict spot were recorded in an Excel spreadsheet. For each record, the entry time of the second vehicle were matched with the Autoscope-reported time (the end of the event) if both differed by no more than three seconds. 
Table 4-12: Conflict Spots and Configured Detector Numbers

\begin{tabular}{lcc} 
Detector File & 97905TH.TF & \\
\hline Conflict Spot & Main st. Detector & Side st. Detector \\
\hline NB1WB1 & 109 & 107 \\
SB1WB1 & 114 & 117 \\
SB1EB2 & 132 & 127 \\
SB1EB1 & 130 & 125 \\
NB1EB2 & 138 & 144 \\
NB1EB1 & 139 & 145 \\
\hline
\end{tabular}

\begin{tabular}{lcc} 
Detector File & 97905LT.TF & \\
\hline Conflict Spot & Main st. Detector & Side st. Detector \\
\hline SBL1WB1 & 150 & 148 \\
SB1EBL1 & 162 & 117 \\
NBL1EB2 & 166 & 172 \\
NBL1EB1 & 167 & 173 \\
NB1WBL101* & 183 & 185 \\
NB1WBL102* & 155 & 153 \\
\hline${ }^{*}$ two conflict spots are used for the NB versus WBL
\end{tabular}

\section{Measures of Effectiveness (MOEs)}

In the evaluation procedure, we manually measured all the gap times of 10 seconds or less and then compare them with the reported ones. The total number of measured gap times was 287 . The following MOEs were used to evaluate the method:

- Number of reported gap times: the number of reported gap times from the postprocessing of the Autoscope event data file. In this evaluation procedure, all the reported values of 10 seconds or less were counted.

- Number of detected gap times: the number of actual gap times that can be detected, defined by the matching of the occurrence times between the actual and reported ones within $+/-3$ seconds.

- Number of false detections: the number of reported gap times that cannot be matched with the actual gap times.

- Detection rate: a percentage of the gap times detectable by the Autoscope-based method.

- False detection rate: a percentage of the gap times falsely reported by the Autoscopebased method.

- Mean error of measurement: the mean of the differences between the actual and detected gap times.

- Standard error: the standard deviation of the differences between the actual and detected gap times.

\section{Factors Affecting the Performance of the Method}

The performance of the Autoscope-based method can be quantified as follows: 
- Detection performance: relevant MOEs are the detection rate and the false detection rate.

- Measurement accuracy: relevant MOEs are the mean error and the standard error.

Several factors can influence the performance of the method. From our evaluation test, we can identify four factors that are likely to have impacts on the performance of the method:

- Distance from the camera to the conflict spot. The distance factor is classified into three categories: near (D1), moderate (D2), and far (D3). The increasing occlusion, limited camera perspective, and smaller object size complicate detection as the distance from the camera to the conflict spot increases.

- Crossing Movement. This factor considers the effect of the movements between through versus through and through versus left turn. Left-turn vehicles have relatively more varying travel paths compared to through vehicles.

- Lighting. This factor indicates whether long shadows are present. Long shadows are expected to be the main source of false calls.

- Number of lanes. This factor indicates the number of crossing lanes (one direction) at a conflict zone.

The conditions of each observed conflict spot are summarized in Table 4-13. Note that the lighting condition is not listed because its presence is the same for all spots.

Table 4-13: Conditions of Observed Conflict Spots

\begin{tabular}{cccc}
\hline Conflict Spot & $\begin{array}{c}\text { Crossing Movement } \\
\text { (main st vs site st) }\end{array}$ & Distance & $\begin{array}{c}\text { Number of Lanes } \\
\text { (main st x side st) }\end{array}$ \\
\hline NB1WB1 & TH-TH & Near & $1 \times 1$ \\
SB1WB1 & TH-TH & Moderate & $1 \times 1$ \\
SB1EB2 & TH-TH & Far & $2 \times 1$ \\
SB1EB1 & TH-TH & Far & $2 \times 1$ \\
NB1EB2 & TH-TH & Moderate & $2 \times 1$ \\
NB1EB1 & TH-TH & Moderate & $2 \times 1$ \\
SBL1WB1 & TH-LT & Near & $1 \times 1$ \\
SB1EBL1 & LT-TH & Moderate & $1 \times 1$ \\
NBL1EB2 & TH-LT & Far & $2 \times 1$ \\
NBL1EB1 & TH-LT & Far & $2 \times 1$ \\
NB1WBL101 & LT-TH & Moderate & $1 \times 1$ \\
NB1WBL102 & LT-TH & Moderate & $1 \times 1$ \\
\hline
\end{tabular}




\section{Evaluation Results}

The MOEs for the automated method classified by different sets of conditions are tabulated in Table 4-14. Regarding a sample size, only the first two cases have the number of actual gaps of less than 10 . When all the observations are included, the detection rate and the false rate are $86 \%$ and $69 \%$ respectively. Excluding the cases that involve far spots, the detection rates range from $80 \%$ to $100 \%$ except for one case. The false detection rates are very high in all cases, thus dictating human intervention in the measurement procedure.

Table 4-14: MOEs of Automated Method Classified by Conditions of Conflict Spots

\begin{tabular}{|c|c|c|c|c|c|c|c|c|c|c|c|c|c|c|c|c|}
\hline \multirow{4}{*}{ Item } & Distance & Near & Near & Near & Near & Mod & Mod & Mod & Mod & Mod & Mod & Far & Far & Far & Far & \multirow{4}{*}{$\begin{array}{c}\text { All } \\
\text { Conditions }\end{array}$} \\
\hline & $\begin{array}{c}\text { Crossing Movement } \\
\text { (main st-side st) }\end{array}$ & TH-TH & TH-TH & TH-LT & TH-LT & TH-TH & TH-TH & TH-TH & TH-TH & LT-TH & LT-TH & TH-TH & TH-TH & TH-LT & TH-LT & \\
\hline & $\begin{array}{c}\text { Lighting (Yes: } \\
\text { presence of shadow) }\end{array}$ & No & Yes & No & Yes & No & No & Yes & Yes & No & Yes & No & Yes & No & Yes & \\
\hline & $\begin{array}{l}\text { Number of Lanes } \\
\text { (main st } x \text { side st) }\end{array}$ & $1 \times 1$ & $1 \times 1$ & $1 \times 1$ & $1 \times 1$ & $1 \times 1$ & $2 \times 1$ & $1 \times 1$ & $2 \times 1$ & $1 \times 1$ & $1 \times 1$ & $2 \times 1$ & $2 \times 1$ & $2 \times 1$ & $2 \times 1$ & \\
\hline 1 & Exposure (spot-hours) & 2.22 & 1.08 & 2.22 & 1.08 & 2.22 & 4.44 & 1.8 & 3.6 & 6.66 & 5.4 & 4.44 & 3.6 & 4.44 & 3.6 & 46.80 \\
\hline 2 & No. of actual GTs $\leq 10 \mathrm{~s}$ & 9 & 5 & 51 & 40 & 25 & 17 & 17 & 10 & 17 & 23 & 36 & 14 & 12 & 11 & 287 \\
\hline 3 & $\begin{array}{l}\text { No. of reported GTs } \leq 10 \\
\mathrm{~s}\end{array}$ & 27 & 39 & 55 & 39 & 33 & 45 & 68 & 38 & 32 & 64 & 163 & 63 & 17 & 15 & 698 \\
\hline 4 & $\begin{array}{l}\text { No. of detected GTs } \leq \\
10 \mathrm{~s}\end{array}$ & 6 & 4 & 47 & 33 & 25 & 16 & 14 & 10 & 9 & 15 & 28 & 7 & 3 & 1 & 218 \\
\hline 5 & $\begin{array}{l}\text { No. of detected GTs > } \\
10 \mathrm{~s} \\
\text { Total no. of detected }\end{array}$ & 3 & 0 & 4 & 6 & 0 & 1 & 1 & 0 & 2 & 4 & 1 & 2 & 1 & 3 & 28 \\
\hline 6 & $\begin{array}{l}\text { GTs comparing to actual } \\
\text { GTs } \leq 10 \mathrm{~s}\end{array}$ & 9 & 4 & 51 & 39 & 25 & 17 & 15 & 10 & 11 & 19 & 29 & 9 & 4 & 4 & 246 \\
\hline 7 & $\begin{array}{l}\text { No. of false detections } \\
\text { out of reported GTs } \leq 10 \\
\text { s }\end{array}$ & 21 & 35 & 8 & 6 & 8 & 29 & 54 & 28 & 23 & 49 & 135 & 56 & 14 & 14 & 480 \\
\hline 8 & $\begin{array}{l}\text { Detection rate at } 10 \mathrm{~s} \\
\text { max threshold (item } \\
6 / \text { item } 2 \text { ) } \\
\text { False detection rate at }\end{array}$ & $100.0 \%$ & $80.0 \%$ & $100.0 \%$ & $97.5 \%$ & $100.0 \%$ & $100.0 \%$ & $88.2 \%$ & $100.0 \%$ & $64.7 \%$ & $82.6 \%$ & $80.6 \%$ & $64.3 \%$ & $33.3 \%$ & $36.4 \%$ & $85.7 \%$ \\
\hline 9 & $\begin{array}{l}10 \text { s max threshold (item } \\
7 / \text { item 3) }\end{array}$ & $77.8 \%$ & $89.7 \%$ & $14.5 \%$ & $15.4 \%$ & $24.2 \%$ & $64.4 \%$ & $79.4 \%$ & $73.7 \%$ & $71.9 \%$ & $76.6 \%$ & $82.8 \%$ & $88.9 \%$ & $82.4 \%$ & $93.3 \%$ & $68.8 \%$ \\
\hline 10 & $\begin{array}{l}\text { Mean error of } \\
\text { measurement }\end{array}$ & 0.155 & -0.193 & 0.769 & 3.296 & 0.104 & 0.133 & 0.374 & -1.580 & 2.655 & 3.128 & -0.276 & 1.791 & 0.431 & 18.231 & 1.359 \\
\hline 11 & Standard error & 1.117 & 0.715 & 3.441 & 9.177 & 0.792 & 0.771 & 2.996 & 3.372 & 7.865 & 8.894 & 1.386 & 7.729 & 0.247 & 23.993 & 6.392 \\
\hline
\end{tabular}

The MOEs of the automated method when the observations are grouped by individual factors are presented in Table 4-15. From the aspect of detection performance, the detection rate decreases and the false detection rate increases as the distance to the observed conflict spot increases. A similar decreasing trend for the detection rate was found when the number of crossing lanes at the conflict zone increase from 1 by 1 . The measurement accuracy deteriorates significantly as indicated by the increase in standard error when (a) there was the presence of a shadow and (b) a conflict spot involves LT movements.

In summary, the findings based on the MOEs presented in Table 4-15 are: 
- The mean errors of the measurement are positive in all cases. This bias indicates that the reported values were frequently longer than the actual ones. The manual review of the video clips revealed that the Autoscope frequently missed detection of the first vehicle from the approach.

- The distance to the observed spot and the number of lanes are likely to impact detection performance.

- The presence of a shadow and LT movements at a conflict spot tend to affect the measurement accuracy.

Table 4-15: Automated Method MOEs Grouped by Specific Conditions

\begin{tabular}{|c|c|c|c|c|c|c|c|c|c|c|}
\hline \multirow[t]{2}{*}{ Item } & \multirow[t]{2}{*}{ Descriptions } & \multicolumn{3}{|c|}{ Distance } & \multicolumn{2}{|c|}{$\begin{array}{c}\text { Lighting (Yes: } \\
\text { presence of shadow) }\end{array}$} & \multicolumn{2}{|c|}{$\begin{array}{c}\text { Crossing } \\
\text { Movements }\end{array}$} & \multicolumn{2}{|c|}{$\begin{array}{l}\text { Number of } \\
\text { Lanes }\end{array}$} \\
\hline & & Near & Mod & Far & No & Yes & TH-TH & TH-LT & $1 \times 1$ & $1 \times 2$ \\
\hline 1 & Exposure (spot-hours) & 6.6 & 24.12 & 16.08 & 26.64 & 20.16 & 23.4 & 23.4 & 22.68 & 24.12 \\
\hline 2 & No. of actual GTs $\leq 10$ s & 105 & 109 & 73 & 167 & 120 & 133 & 154 & 187 & 100 \\
\hline 3 & No. of reported GTs $\leq 10 \mathrm{~s}$ & 160 & 280 & 258 & 372 & 326 & 476 & 222 & 357 & 341 \\
\hline 4 & No. of detected GTs $\leq 10 \mathrm{~s}$ & 92 & 89 & 39 & 134 & 86 & 112 & 108 & 155 & 65 \\
\hline 5 & No. of detected GTs > $10 \mathrm{~s}$ & 11 & 8 & 7 & 12 & 14 & 6 & 20 & 18 & 8 \\
\hline 6 & $\begin{array}{l}\text { Total no. of detected GTs } \\
\text { comparing to actual GTs } \leq 10 \mathrm{~s}\end{array}$ & 103 & 97 & 46 & 146 & 100 & 118 & 128 & 173 & 73 \\
\hline 7 & $\begin{array}{l}\text { No. of false detections out of } \\
\text { reported gaps } \leq 10 \mathrm{~s}\end{array}$ & 68 & 191 & 219 & 238 & 240 & 364 & 114 & 202 & 276 \\
\hline 8 & $\begin{array}{l}\text { Detection rate at } 10 \mathrm{~s} \text { max } \\
\text { threshold (item 6/item 2) }\end{array}$ & $98.1 \%$ & $89.0 \%$ & $63.0 \%$ & $87.4 \%$ & $83.3 \%$ & $88.7 \%$ & $83.1 \%$ & $92.5 \%$ & $73.0 \%$ \\
\hline 9 & $\begin{array}{l}\text { False detection rate at } 10 \mathrm{~s} \text { max } \\
\text { threshold (item } 7 / \text { item } 3 \text { ) }\end{array}$ & $42.5 \%$ & $68.2 \%$ & $84.9 \%$ & $64.0 \%$ & $73.6 \%$ & $76.5 \%$ & $51.4 \%$ & $56.6 \%$ & $80.9 \%$ \\
\hline 10 & Mean error of measurement & 1.635 & 0.859 & 1.799 & 0.468 & 2.660 & 0.029 & 2.586 & 1.533 & 0.948 \\
\hline 11 & Standard error & 6.248 & 5.118 & 8.783 & 3.083 & 9.181 & 2.703 & 8.302 & 6.052 & 7.161 \\
\hline
\end{tabular}

\subsubsection{Quantifying the Factor Impacts}

It is useful to know how factors or some combination of factors influence measurement accuracy. A multiple regression modeling approach was used to quantify the effects of factors discussed in the previous section. The explanatory variables considered are defined as follows:

- D1: 1 - close distance to the conflict spot, 0 - otherwise

- D2: 1 - moderate distance to the conflict spot, 0 - otherwise

- D3: 1 - far distance to the conflict spot, 0 - otherwise

- $\mathrm{M}: 1-$ conflict involves with left-turn vehicles, 0 - otherwise

- L: 1 - presence of shadow, 0 - otherwise

- $\quad \mathrm{N}: 1-1 \times 2$ lanes, 0 - otherwise 


\section{Variability of Measurement Errors}

First, the histogram of errors was plotted to check the range and variability of the observations (see Figure 4-23). The errors range from -10 to 55 seconds based on a total of 246 observations. The errors are limited on the negative side because the observed actual gap values were cut off at 10 seconds. There is no boundary on positive errors.

From the histogram of errors, it can be observed that the range is discontinuous at 12 seconds or greater. These large errors are likely to be attributable to Autoscope glitches that happen every once in a while. For example, the detector may lock in the presence status even when the vehicle has left and thereby miss detection of the following vehicles. This could result in large reported gap values. It was decided to remove errors greater than 15 seconds to reduce the amount of unexplained variability in the measurement errors. These outliers account for 10 observations or about $4 \%$ of the total sample size. The final sample size used in the regression modeling was 236 observations. In addition, the absolute measurement errors were used as a dependent variable in the regression model.

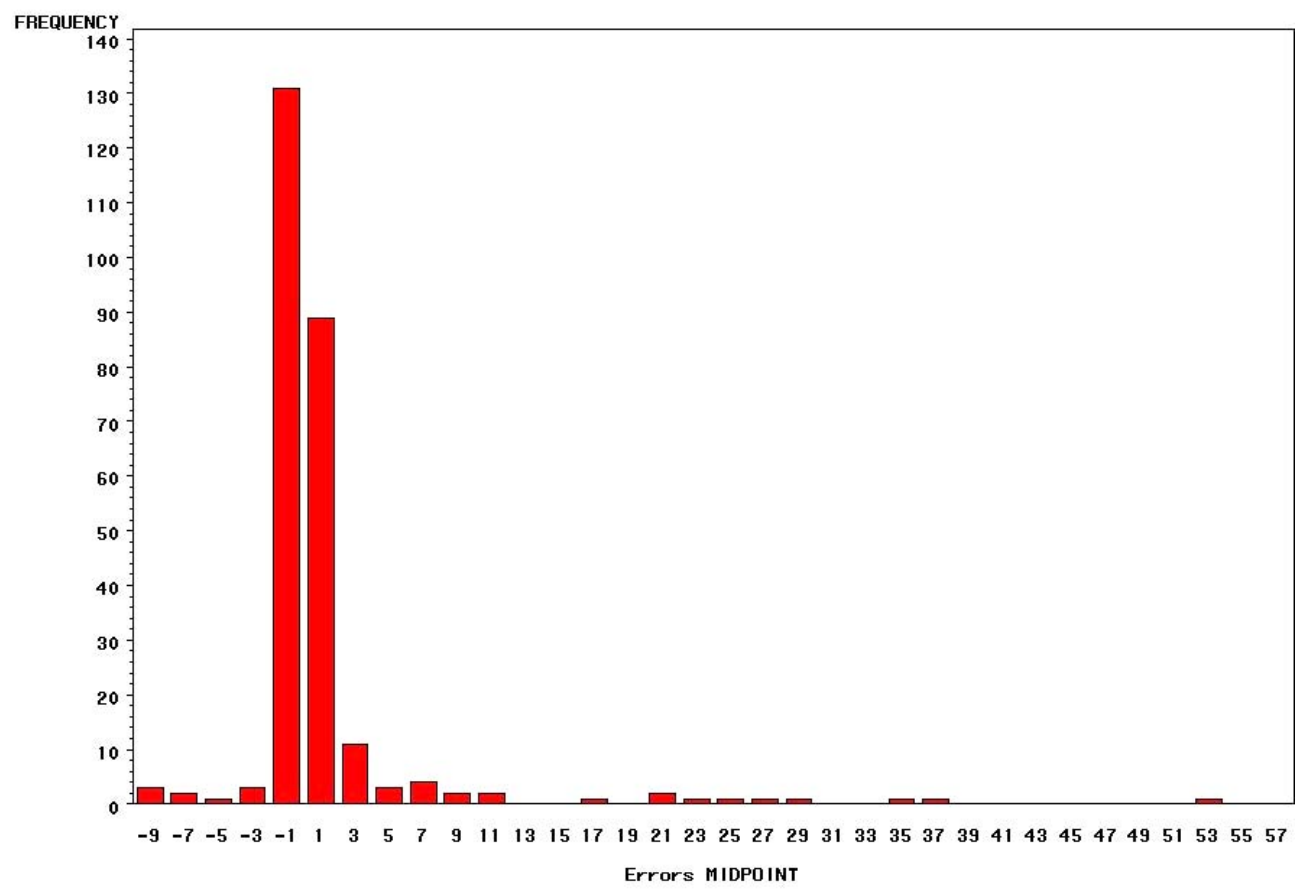

Figure 4-23: Histogram of Measurement Errors Using All Observations 


\section{Model Selection Procedure}

Several variable selection procedures were considered in this problem, including forward selection, backward selection and stepwise regression. Given that the number of candidate variables is not large for today's computer capability, an all-possible-regression procedure was adopted. The all-possible-regression procedure calls for considering all possible subsets of the pool of potential explanatory variables and identifying a few good subsets for detailed examination (Neter et al., 1996). The adjusted $\mathrm{R}^{2}$ was used to identify good subsets. The final selection of the model considers whether it offers a valid interpretation while using a minimal number of variables.

In the selection procedure, it was found that the adjusted $\mathrm{R}^{2}$ improved when using the absolute errors as a dependent variable, instead of the actual errors. The intercept was found to be consistently statistically insignificant ( $p$-value $>0.4$ ); therefore, the intercept term was dropped out of the model in the subsequent fittings.

\section{Best Fitted Model}

The parameter estimates for both models, with and without interactions, are presented in Table 4-16. The Durbin-Watson statistics for both models are close to 2.0 (1.95-1.96) and therefore indicate no presence of serial correlation problem. The fitted regression model can be used to predict the error for the specification of verification threshold in the semi-automated method.

Table 4-16: Fitted Regression Model for Error Prediction

\begin{tabular}{|c|c|c|c|c|}
\hline Variable & $\begin{array}{c}\text { Parameter } \\
\text { Estimate }\end{array}$ & $\begin{array}{l}\text { Standard } \\
\text { Error }\end{array}$ & t-ratio & $\operatorname{Pr}>|t|$ \\
\hline $\begin{array}{l}\text { D2 (1 if moderate distance to the } \\
\text { conflict spot, } 0 \text { if otherwise) }\end{array}$ & 0.5839 & 0.2149 & 2.72 & 0.0071 \\
\hline $\begin{array}{l}\text { D3 (1 if far distance to the } \\
\text { conflict spot, } 0 \text { if otherwise) }\end{array}$ & 1.0571 & 0.2844 & 3.72 & 0.0003 \\
\hline $\begin{array}{l}M(1 \text { if left-turn related, } 0 \text { if } \\
\text { otherwise) }\end{array}$ & 0.3960 & 0.1995 & 1.98 & 0.0483 \\
\hline $\begin{array}{l}L(1 \text { if shadow is present, } 0 \text { if } \\
\text { otherwise) }\end{array}$ & 0.8750 & 0.2494 & 3.51 & 0.0005 \\
\hline
\end{tabular}


The number of lanes and a near conflict spot were found to have no statistically significant impact on the error magnitude. The error magnitude was affected most by the far distance, followed by the presence of a shadow, moderate distance, and a left-turn movement.

\subsubsection{Semi-Automated Measurement Method}

The semi-automated method aims to address the problem of a high false detection rate in the automated approach. The false detection rate in the semi-automated method is reduced when the reported gap times are manually verified. The detection rate depends on the number of gap times that are verified, which can be controlled by specifying the verification threshold on reported values. The maximum detection rate of this method is equal to the detection rate of the automated method (by assuming that all the reported gap times are manually verified).

\subsubsection{Procedure}

The semi-automated method takes the reported outputs from the Autoscope-based method to verify manually using a frame-by-frame video playback. In each record of the output from the automated method, the reported time stamp at the end of the gap time event can be used to locate the portion of the video clip to be verified. A digitized video format allows us to access the video data at a particular frame. However, the number of reported gap times is excessively large, a majority of them being false. This brings about an efficiency problem to verify all of the reported values, which for this method is no different from the manual measurement. Hence, the upper control limit (UCL) and lower control limit (LCL) are specified for a set of reported values to save the time required in the manual verification.

The UCL is specified from the knowledge of the prediction intervals computed from the fitted regression model (see Section 4.4.5.3). The LCL is optional and shoud be set at zero to ensure the best detection rate. In this evaluation, we set the LCL at 0.5 second only to favor efficient verification, which is still not really efficient as the results will explain later. 


\subsubsection{Specification of Verification Threshold}

The UCLs are derived from the fitted regression model in Section 4.4.5.3. The $1-\alpha$ prediction interval of the error magnitude provides a range in which the true error magnitude will fall at significance level $\alpha$. To compute the prediction interval of the magnitude of error for given values denoted by $X_{h 1}, \ldots, X_{h, p-1}$, we define the vector $X_{h}$ as

$$
X_{h}=\left[\begin{array}{c}
1 \\
X_{h 1} \\
\mathrm{M} \\
X_{h, p-1}
\end{array}\right] .
$$

Note that the element " 1 " in the vector $X_{h}$ is dropped out in the case of regression through origin. The estimated variance of a predicted error, defined as $s^{2}\left\{\hat{Y}_{h}\right\}$, is given by

$$
s^{2}\left\{\hat{Y}_{h}\right\}=X_{h}^{\prime} s^{2}\{b\} X_{h}
$$

$s^{2}\{b\}$ is a covariance matrix of parameter estimates from the fitted regression model (see Table 4-17).

Hence, the $1-\alpha$ prediction limits for a predicted $Y_{h(\text { new })}$ corresponding to $X_{h}$, the specified values of the $\mathrm{X}$ variables, are

$$
\hat{Y}_{h} \pm t\left(1-\frac{\alpha}{2}, n-p\right) . s\{\text { pred }\}
$$

where

$$
s^{2}\{\text { pred }\}=M S E+s^{2}\left\{\hat{Y}_{h}\right\} .
$$


Table 4-17: Covariance Matrix of Parameter Estimates of the Fitted Regression Model

\begin{tabular}{|c|c|c|c|c|}
\hline Variable & $\mathrm{D} 2$ & D3 & $\mathrm{M}$ & $\mathrm{L}$ \\
\hline D2 & 0.04616 & 0.00544 & -0.00104 & -0.02110 \\
\hline D3 & 0.00544 & 0.08089 & -0.00052 & -0.01185 \\
\hline$M$ & -0.00104 & -0.00052 & 0.03980 & -0.02325 \\
\hline L & -0.02110 & -0.01185 & -0.02325 & 0.06218 \\
\hline
\end{tabular}

However, the UCL can be computed using one-tailed t-value because the lower limit of error magnitude must be zero. The formula for the UCL at level $\alpha$ is

$$
U C L=G T_{o b s}+\hat{Y}_{h}+t(1-\alpha, n-p) . s\{\text { pred }\}
$$

where $G T_{o b s}$ is the observation threshold for the gap times and $\hat{Y}_{h}$ is the predicted error magnitude evaluated at a given set of variables.

Table 4-18: Example of UCL Computation

\begin{tabular}{|c|c|c|c|c|}
\hline Variable & $X_{h}$ & $\begin{array}{l}\text { Parameter } \\
\text { Estimates }\end{array}$ & $\begin{aligned} N & = \\
\text { Predicted } Y & =\end{aligned}$ & $\begin{array}{r}236 \\
1.4589\end{array}$ \\
\hline D2 & 1 & 0.5839 & MSE $=$ & 3.4250 \\
\hline D3 & 0 & 1.0571 & $\mathrm{~s}\{$ pred $\}=$ & 1.8685 \\
\hline $\mathrm{M}$ & 0 & 0.3960 & alpha = & 0.15 \\
\hline $\mathrm{L}$ & 1 & 0.8750 & GT threshold = & 6.0000 \\
\hline $\begin{array}{l}{ }^{*} \mathrm{UCL} \text { is C } \\
\text { value and } \\
\text { assumed }\end{array}$ & $\mathrm{s}$ & $\begin{array}{l}\text { one-tailed t- } \\
\text { threshold is }\end{array}$ & $\mathrm{UCL}=$ & 9.3998 \\
\hline
\end{tabular}

The example of UCL computation for a moderate-distance conflict spot with the presence of a shadow is shown in Table 4-18. The $\alpha$-value of 0.15 appears to be a good compromise between detection and efficiency rates. The computed UCL value should be considered as a guideline and therefore is flexible for adjustment if necessary.

\subsubsection{Measures of Effectiveness}

The following MOEs are used to evaluate the performance of the semi-automated method:

- Number of verified gaps: the number of Autoscope-reported gap times within the specified limits, which are to be verified manually. 
- Number of detected gaps: the number of actual gap times that can be detected by manually verifying the Autoscope-reported ones.

- Number of missed detections or undetected gaps: the missed detections are classified into three types: (a) overreporting, (b) non-reporting and (c) underreporting. The first type can be reduced at the expense of the efficiency rate by increasing the UCL, while the third type can be eliminated if the LCL is set to zero.

- Detection rate: the ratio of the number of detected gap times to the number of actual gap times.

- Missed detection rate: the ratio of the number of undetected gap times to the actual gap times, classified into three types: (a) missed detection rate by overreporting, (b) missed detection rate by non-reporting, and (c) missed detection rate by underreporting.

- Efficiency rate: the ratio of the number of detected gap times to the number of verified gap times.

\subsubsection{Evaluation Results}

We set the observation threshold of gap times at six seconds for this evaluation to reflect the probable working threshold if this method is to be used. Table 4-19 presents a summary of the MOEs of the semi-automated method classified by a set of conditions. The LCL was specified at 0.5 second only to improve the efficiency rate. The UCLs were computed for each case using the procedure described earlier. The final selected UCLs were done by grouping the sets of conditions that have similar ranges of UCL and then assigning one common UCL for each group.

The detection rate is almost $80 \%$, which is comparable to the automated method. However, the efficiency rate is very low ( $9 \%$ ), which is very undesirable. This basically implies that out of every 100 reported gap times verified, only about nine of them are correctly reported. The

efficiency rate is extremely poor for the condition of far conflict spots. Thus, this case may be avoided, which leads to the performance summary in Table 4-20 in which the case of far conflict spots is excluded from the consideration. 
Table 4-19: MOEs of Semi-Automated Method Classified by Conditions of Conflict Spots

\begin{tabular}{|c|c|c|c|c|c|c|c|c|c|c|c|c|c|c|c|c|}
\hline \multirow{4}{*}{ Item } & $\begin{array}{c}\text { Distance } \\
\end{array}$ & Near & Near & Near & Near & Mod & Mod & Mod & Mod & Mod & Mod & Far & Far & Far & Far & \multirow{4}{*}{$\begin{array}{c}\text { All } \\
\text { Conditions }\end{array}$} \\
\hline & $\begin{array}{l}\text { Crossing Movement (main st- } \\
\text { side st) }\end{array}$ & TH-TH & TH-TH & TH-LT & TH-LT & TH-TH & TH-TH & TH-TH & TH-TH & LT-TH & LT-TH & TH-TH & TH-TH & TH-LT & TH-LT & \\
\hline & $\begin{array}{l}\text { Lighting (Yes: presence of } \\
\text { shadow) }\end{array}$ & No & Yes & No & Yes & No & No & Yes & Yes & No & Yes & No & Yes & No & Yes & \\
\hline & $\begin{array}{c}\text { Number of Lanes (main st } X \\
\text { side st) }\end{array}$ & $1 \times 1$ & $1 \times 1$ & $1 \times 1$ & $1 \times 1$ & $1 \times 1$ & $2 \times 1$ & $1 \times 1$ & $2 \times 1$ & $1 \times 1$ & $1 \times 1$ & $2 \times 1$ & $2 \times 1$ & $2 \times 1$ & $2 \times 1$ & \\
\hline 1 & Selected UCL & 7.922 & 8.454 & 8.454 & 8.454 & 8.454 & 8.454 & 8.454 & 9.400 & 9.400 & 9.400 & 9.400 & 9.400 & 10.282 & 10.282 & \\
\hline 2 & Exposure (spot-hours) & 2.22 & 1.08 & 2.22 & 1.08 & 2.22 & 4.44 & 1.8 & 3.6 & 6.66 & 5.4 & 4.44 & 3.6 & 4.44 & 3.6 & 46.8 \\
\hline 3 & No. of actual gap times $\leq 6 \mathrm{~s}$ & 1 & 12 & 3 & 11 & 11 & 0 & 2 & 4 & 2 & 7 & 8 & 1 & 2 & 2 & 66 \\
\hline 4 & $\begin{array}{l}\text { No. of verified gap times: range of } \\
\text { reported values }=(0.5, \mathrm{UCL})\end{array}$ & 18 & 46 & 27 & 34 & 28 & 34 & 28 & 46 & 27 & 60 & 148 & 15 & 61 & 14 & 586 \\
\hline 5 & No. of detected gap times & 1 & 11 & 2 & 9 & 11 & 0 & 1 & 2 & 2 & 6 & 6 & 0 & 1 & 0 & 52 \\
\hline 6 & Number of overreported values & 0 & 1 & 0 & 1 & 0 & 0 & 0 & 1 & 0 & 1 & 0 & 0 & 0 & 1 & 5 \\
\hline 7 & Number of non-reported values & 0 & 0 & 1 & 1 & 0 & 0 & 1 & 0 & 0 & 0 & 2 & 0 & 1 & 1 & 7 \\
\hline 8 & Number of underreported values & 0 & 0 & 0 & 0 & 0 & 0 & 0 & 1 & 0 & 0 & 0 & 1 & 0 & 0 & 2 \\
\hline 9 & Total no. of undetected gaps & 0 & 1 & 1 & 2 & 0 & 0 & 1 & 2 & 0 & 1 & 2 & 1 & 1 & 2 & 14 \\
\hline 10 & Detection rate (item $5 /$ item 3 ) & $100.0 \%$ & $91.7 \%$ & $66.7 \%$ & $81.8 \%$ & $100.0 \%$ & NA & $50.0 \%$ & $50.0 \%$ & $100.0 \%$ & $85.7 \%$ & $75.0 \%$ & $0.0 \%$ & $50.0 \%$ & $0.0 \%$ & $78.8 \%$ \\
\hline 11 & Efficiency rate (item $5 /$ item 4 ) & $5.6 \%$ & $23.9 \%$ & $7.4 \%$ & $26.5 \%$ & $39.3 \%$ & $0.0 \%$ & $3.6 \%$ & $4.3 \%$ & $7.4 \%$ & $10.0 \%$ & $4.1 \%$ & $0.0 \%$ & $1.6 \%$ & $0.0 \%$ & $8.9 \%$ \\
\hline 12 & $\begin{array}{l}\text { Missed detection rate by } \\
\text { overreporting (item 6/item 3) }\end{array}$ & $0.0 \%$ & $8.3 \%$ & $0.0 \%$ & $9.1 \%$ & $0.0 \%$ & NA & $0.0 \%$ & $25.0 \%$ & $0.0 \%$ & $14.3 \%$ & $0.0 \%$ & $0.0 \%$ & $0.0 \%$ & $50.0 \%$ & $7.6 \%$ \\
\hline 13 & $\begin{array}{l}\text { Missed detection rate by } \\
\text { nonreporting (item } 7 / \text { item } 3 \text { ) }\end{array}$ & $0.0 \%$ & $0.0 \%$ & $33.3 \%$ & $9.1 \%$ & $0.0 \%$ & NA & $50.0 \%$ & $0.0 \%$ & $0.0 \%$ & $0.0 \%$ & $25.0 \%$ & $0.0 \%$ & $50.0 \%$ & $50.0 \%$ & $10.6 \%$ \\
\hline 14 & $\begin{array}{l}\text { Missed detection rate by } \\
\text { underreporting (item } 8 / \text { item } 3 \text { ) }\end{array}$ & $0.0 \%$ & $0.0 \%$ & $0.0 \%$ & $0.0 \%$ & $0.0 \%$ & NA & $0.0 \%$ & $25.0 \%$ & $0.0 \%$ & $0.0 \%$ & $0.0 \%$ & $100.0 \%$ & $0.0 \%$ & $0.0 \%$ & $3.0 \%$ \\
\hline
\end{tabular}

Table 4-20: MOEs of Semi-Automated Method (Excluding Far Conflict Spots)

\begin{tabular}{|c|c|c|c|c|c|c|c|c|}
\hline \multirow[t]{2}{*}{ Item } & \multirow[t]{2}{*}{ Descriptions } & \multirow{2}{*}{$\begin{array}{c}\text { Overall } \\
\text { Performance } \\
\text { without Far } \\
\text { Conflict Spots }\end{array}$} & \multicolumn{2}{|c|}{$\begin{array}{l}\text { Lighting (Yes: } \\
\text { presence of } \\
\text { shadow) }\end{array}$} & \multicolumn{2}{|c|}{$\begin{array}{l}\text { Distance from } \\
\text { Camera to } \\
\text { Conflict Spot }\end{array}$} & \multicolumn{2}{|c|}{$\begin{array}{l}\text { Crossing } \\
\text { Movements }\end{array}$} \\
\hline & & & No & Yes & Near & Mod & TH-TH & TH-LT \\
\hline 2 & Exposure (spot-hours) & 30.72 & 11.76 & 18.96 & 6.6 & 24.12 & 21.36 & 9.36 \\
\hline 3 & No. of actual gap times $\leq 6 \mathrm{~s}$ & 53 & 26 & 27 & 27 & 26 & 21 & 32 \\
\hline 4 & $\begin{array}{l}\text { No. of verified gap times: range of } \\
\text { reported values }=(0.5, \mathrm{UCL})\end{array}$ & 348 & 154 & 194 & 125 & 223 & 180 & 168 \\
\hline 5 & No. of detected gap times & 45 & 24 & 21 & 23 & 22 & 18 & 27 \\
\hline 6 & Number of overreported values & 4 & 1 & 3 & 2 & 2 & 1 & 3 \\
\hline 7 & Number of non-reported values & 3 & 1 & 2 & 2 & 1 & 1 & 2 \\
\hline 8 & Number of underreported values & 1 & 0 & 1 & 0 & 1 & 1 & 0 \\
\hline 9 & Total no. of undetected gaps & 8 & 2 & 6 & 4 & 4 & 3 & 5 \\
\hline 10 & Detection rate (item 5/item 3) & $84.9 \%$ & $92.3 \%$ & $77.8 \%$ & $85.2 \%$ & $84.6 \%$ & $85.7 \%$ & $84.4 \%$ \\
\hline 11 & Efficiency rate (item 5/item 4) & $12.9 \%$ & $15.6 \%$ & $10.8 \%$ & $18.4 \%$ & $9.9 \%$ & $10.0 \%$ & $16.1 \%$ \\
\hline 12 & $\begin{array}{l}\text { Missed detection rate by overreporting } \\
\text { (item 6/item 3) }\end{array}$ & $7.5 \%$ & $3.8 \%$ & $11.1 \%$ & $7.4 \%$ & $7.7 \%$ & $4.8 \%$ & $9.4 \%$ \\
\hline 13 & $\begin{array}{l}\text { Missed detection rate by nonreporting } \\
\text { (item } 7 \text { /item 3) }\end{array}$ & $5.7 \%$ & $3.8 \%$ & $7.4 \%$ & $7.4 \%$ & $3.8 \%$ & $4.8 \%$ & $6.3 \%$ \\
\hline 14 & $\begin{array}{l}\text { Missed detection rate by underreporting } \\
\text { (item 8/item 3) }\end{array}$ & $1.9 \%$ & $0.0 \%$ & $3.7 \%$ & $0.0 \%$ & $3.8 \%$ & $4.8 \%$ & $0.0 \%$ \\
\hline
\end{tabular}

The overall performance of the semi-automated method slightly improves when the far conflict spots are excluded. From Table 4-20, the presence of a shadow appears to most affect the method performance both in terms of the detection rates and the efficiency rates. The detection rates are slightly affected by the distance and movement while the efficiency rates drop as the distances increase. The higher efficiency rate for the TH-LT movement may be attributable to the fact that the left-turn volumes are much less than the through volumes, thereby producing a lower number of false calls. 
The efficiency rate of the semi-automated method is still far from desirable while the detection rate is comparable to the automated method. The automated method suffers from the high false detection rates. There appears to be no easy solution in order to continue the data collection using either the automated or semi-automated method. Therefore, the manual method is selected for the full-scale data collection needed in this study.

\subsection{DATA Collection Procedure AND Collected DATA}

\subsubsection{Selected Measurement Method}

The Autoscope-based automated method produced an excessive false detection rate and large measurement errors and thus was not a suitable approach. The semi-automated method addresses the problems of false detection and measurement accuracy in the automated method at the expense of the data collection time; which appears to have no advantage over the manual method. Furthermore, the detection performance in the Autoscope-based method also suffers from the following issues:

- Poor detection performance at far conflict spots.

- A high rate of false calls when a long shadow is present.

- Occlusions of vehicles from adjacent lanes.

- Limited processing load on the Autoscope unit. An unusually high processing load can seriously impair the detection capability.

In addition, the PET cannot be measured reliably using the Autoscope as discussed earlier. The manual method, on the other hand, can accurately give the GT, PET, and ET for each crossing event without the restrictions encountered in the Autoscope-based method. The time savings gained from the semi-automated method compared to the manual method is marginal if at all. The detection rates of both automated and semi-automated methods still fall short of our expectations in this study. A critical event is rare and therefore we cannot afford to lose as much as $10 \%$ of the detections in the Autoscope-based method. The manual method appears to be the most reasonable method in this study thus far. Hence, the manual measurement method is chosen for extracting all the surrogate data collected from the digitized video clips. 


\subsubsection{Data Collection Procedure}

Let us recall the important definitions frequently used in the data collection:

- Conflict zone is an area defined by the intersection of two crossing traffic flows. One intersection may have up to four conflict zones (see Figure 4-3).

- Conflict spot is an intersection area of two crossing vehicle paths in a conflict zone (see example in Figure 4-9).

- Gap time (GT) is the time between the entries into the conflict spot of two vehicles, measured from the front bumper to the front bumper. The GT can be broken down into two components based on the terms coined by Allen et al. (1978): encroachment time (ET) and post-encroachment time (PET). GT is a summation of ET and PET.

- Encroachment time (ET) is the time that the first vehicle entering the conflict spot infringes upon the right-of-way of the second vehicle, measured from the rear bumper to the front bumper.

- Post-encroachment time (PET) is the time it takes from the end of the right-of-way infringement of the first vehicle for the second vehicle to reach the conflict spot, measured from the rear bumper to the front bumper.

All of above were described graphically in Figure 4-10. GT would be a good measure of crash proximity only if the first vehicles occupy the conflict for the same amount of time. This assumption does not hold true in reality because vehicles vary in length and move at different speeds. PET is a better choice; its value of zero or less represents a crash occurrence. Allen et al. (1978) also concluded that PET was the most promising indicator for its relative ease of measurement and safety implication. PET has a specific value for any crossing event (consecutive two passages from conflicting directions). Unlike TTC, the PET measurement does not involve human judgment and is relatively easier for automation, which is an important factor of a method's practicality. It was observed that a long ET is usually caused by the following situations: (a) a slow-speed vehicle, (b) a truck or semi-truck, and (c) a vehicle idling due to queue overflows. Allen et al. (1978) also noted that the ET is usually long for a left-turn related movement. 


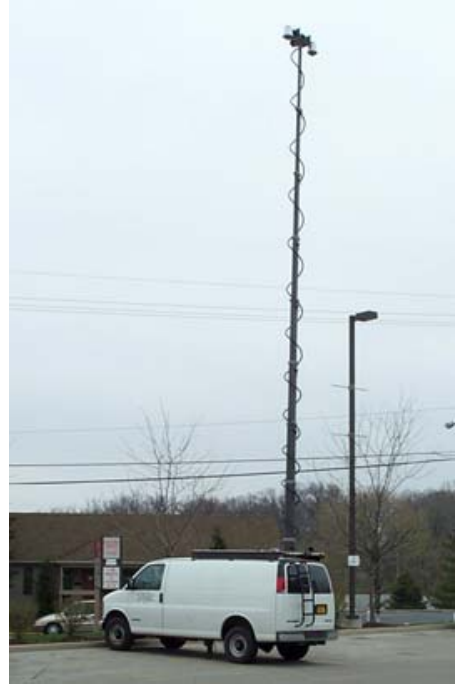

(a) During the data collection

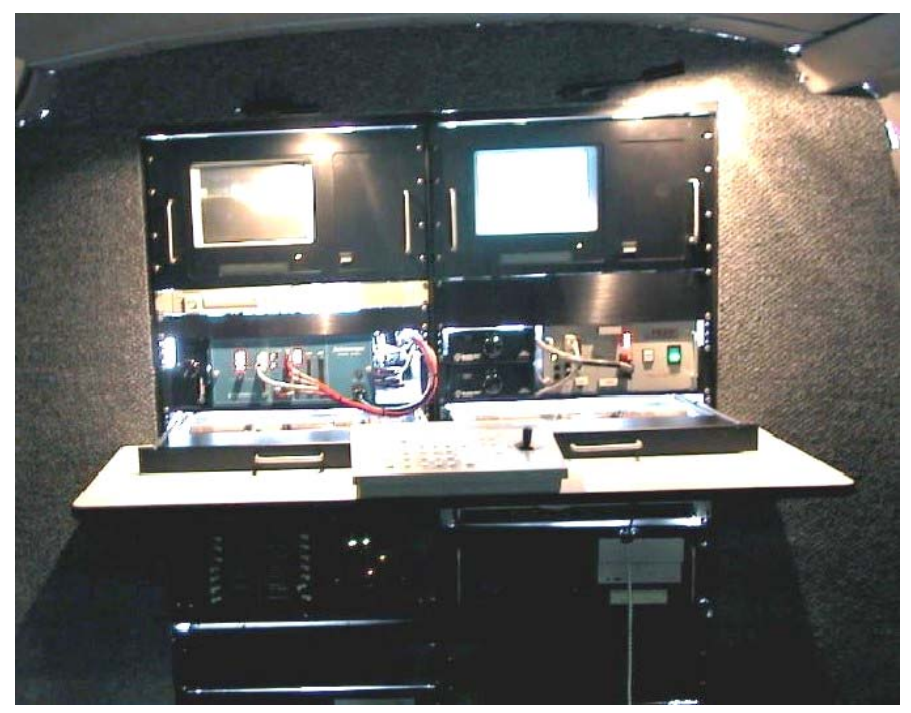

(b) Inside the van

Figure 4-24: Purdue University Mobile Traffic Laboratory

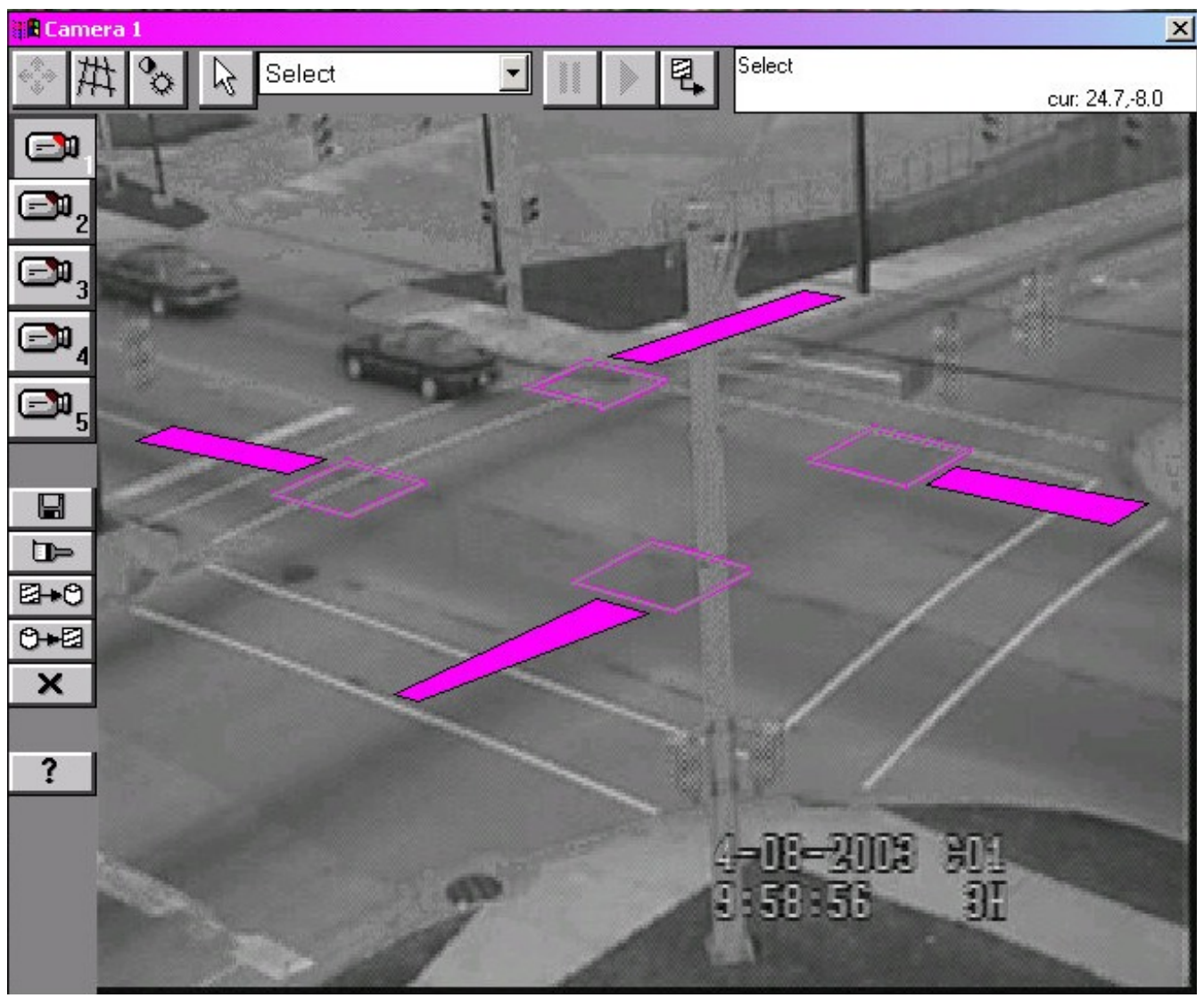

Figure 4-25: Configuration of Conflict Spots at Intersection 97901 
To demonstrate the data collection procedure, we selected the suburban signalized intersection of SR-26@18th St (intersection 97901) from a list of selected studied sites. This is a four-leg intersection with one through lane and one left-turn lane on each approach. All left-turns are permitted except the eastbound left-turn, which is protected-permitted. Traffic movement data were collected by videotaping the traffic at this intersection using the Purdue University mobile traffic laboratory (see Figure 4-24). The mobile traffic laboratory is equipped with a 42-foot pneumatic mast with two surveillance cameras. Eight hours of traffic (9:00 AM - 4:00 PM and 4:30 PM - 5:30 PM) were recorded in a digital format on April 8, 2003. All four conflict zones were observable in the camera field of view.

The PET values were measured by watching the video material frame by frame. The manual method was used to reduce the measurement error to a minimum. For the manual measurement method, the recorded video clips at the intersections were digitized at the resolution of $30 \mathrm{fps}$, which gives the attainable average precision of $1 / 30 \mathrm{~s}$. Conflict spots were marked on the video monitor with Autoscope virtual detectors (Figure 4-25). The virtual conflict spots assisted a human reviewer in determining specific frames where vehicles entered and exited the conflict spots.

In the process of data extraction, we recorded $t_{1}, t_{2}$, and $t_{3}$ as shown in Figure 4-10 for each crossing event and then computed the GT, ET and PET. The PET values that were larger than eight seconds were not recorded since they were not useful for safety estimation purposes. The corresponding PET values were too long to believe that they are in close proximity with crashes.

To summarize, the following data were collected at each site:

- Historical crash data

- Sketch of intersection layout

- Archived detector layout

- Eight hours of PET, ET, and GT data

- Signal cycle data

- Eight-hour 15-minute directional counts of traffic volume 


\subsubsection{Summary of Collected Data}

\subsubsection{Historical Crash Data}

The right-angle crashes from 1997 to 2000 for all of the selected 18 sites were verified with the actual police accident report archived in the microfiche format. The collision zone was also recorded for each collision. The environment condition at the time of crash occurrence was also retrieved from the database, which includes the date, time, and weather condition if available.

\subsubsection{2 $\quad \underline{\text { Sketch of Intersection Layout }}$}

This is important for the calibration of Autoscope in the automated measurement method. Although the manual method does not require this information, it still provides a good reference for the intersection studied. Examples of the sketches of sites 89707 and 87933 are displayed in Figure 4-26 and Figure 4-27 respectively. There are a total of 18 intersection sketches in this study. The following information is included in the sketch:

- Names of crossroads

- Nearby landmarks

- Date and weather conditions

- Type of traffic control signal

- Yellow and all-red intervals

- Traffic signal phasing diagram

- Lane assignment

- Lane widths

- Lane markings

- Crosswalks

- Stop bar

- Van parking location

- Conflict spot notation 
Intersection Inventory Worksheet

Site Code: 87907

Main st: Creasy Ln

Side st:

Sketch:

Items: Lane width, lane markings, crosswalks, stop

bar, van position, landmark, loop detector locations
Date: May 22,2003

Weather: Mostly Sunr

Temp: $50^{\circ} \mathrm{F}-68^{\circ} \mathrm{F}$

Wind: Light

Traffic Control: Fully actuated

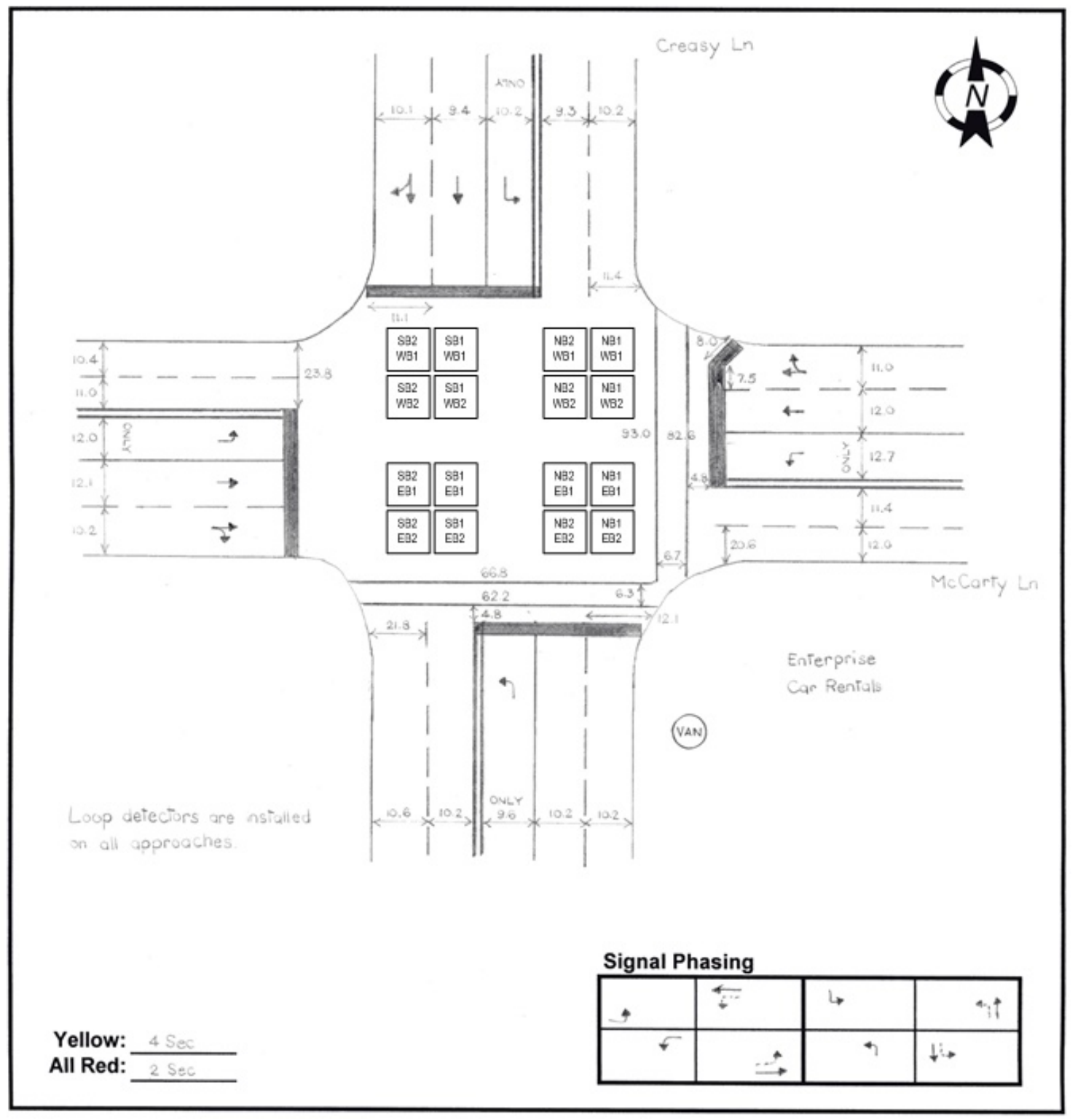

Figure 4-26: Intersection 87907 Layout and Conflict Spots 
Intersection Inventory Worksheet

$\begin{aligned} \text { Site Code: } & 87933 \\ \text { Main st: } & \text { Union St (EB one-way) } \\ \text { Side st: } & 14^{\text {th }} \text { St }\end{aligned}$

\section{Sketch:}

Items: Lane width, lane markings, crosswalks, stop bar, van position, landmark

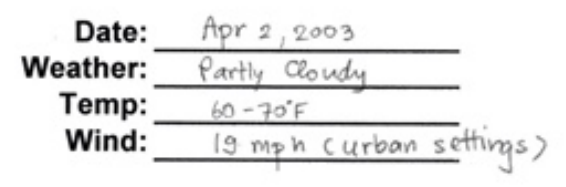

Pretimed:

Actuated:

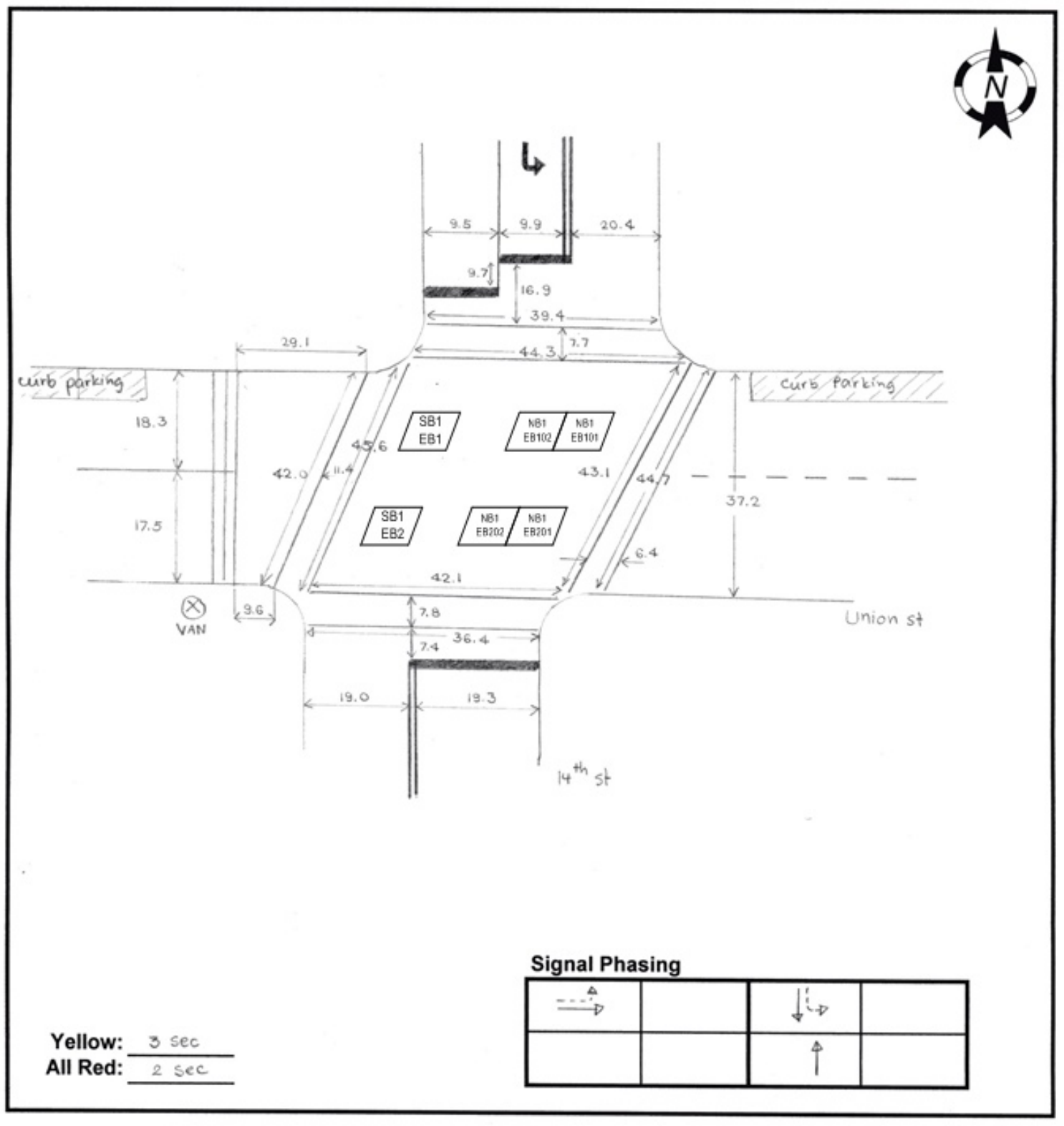

Figure 4-27: Intersection 87933 Layout and Conflict Spots 


\subsubsection{Surrogate Data}

The PET, GT, and ET data were collected at each site for eight hours. The list of collection periods and observation dates for all sites is given in Table 4-21. We aimed to cover the morning, mid-day, and afternoon peak periods in the eight-hour duration. The observed duration at site 97915 was less than eight hours due to the intermittent loss of the video signal. Sites 97915 and 97918 have very few PETs shorter than eight seconds observed during the entire observation period.

Table 4-21: Summary of Periods of Extracted Surrogate Data

\begin{tabular}{|c|c|c|c|}
\hline Site & Date & Periods of Extracted Gaps & Total Duration \\
\hline 87905 & Friday, June 13, 2003 & $0745-0845,1000-1600,1630-1730$ & $08 \mathrm{hrs}$ \\
\hline 87906 & Monday, June 16, 2003 & $0730-0830,1000-1600,1630-1730$ & $08 \mathrm{hrs}$ \\
\hline 87907 & Thursday, May 22, 2003 & $0900-1000,1000-1600,1630-1730$ & $08 \mathrm{hrs}$ \\
\hline 87909 & Wednesday, June 25, 2003 & $0800-0900,1000-1600,1630-1730$ & $08 \mathrm{hrs}$ \\
\hline 87915 & Friday, April 11, 2003 & $0800-0900,1000-1600,1630-1730$ & $08 \mathrm{hrs}$ \\
\hline 87923 & Tuesday, May 27, 2003 & $0800-0900,1000-1600,1630-1730$ & $08 \mathrm{hrs}$ \\
\hline 87930 & Wednesday, July 02, 2003 & $0730-0830,1000-1600,1630-1730$ & $08 \mathrm{hrs}$ \\
\hline 87932 & Monday, July 14, 2003 & 0730-0830, 1000-1600, 1630-1730 & $08 \mathrm{hrs}$ \\
\hline 87933 & Wednesday, April 02, 2003 & $0900-1000,1000-1600,1630-1730$ & $08 \mathrm{hrs}$ \\
\hline 97901 & Tuesday, April 08, 2003 & $0900-1000,1000-1600,1630-1730$ & $08 \mathrm{hrs}$ \\
\hline 97903 & Tuesday, April 29, 2003 & 0815-0915, 1000-1600, 1630-1730 & $08 \mathrm{hrs}$ \\
\hline 97905 & Monday, April 21, 2003 & $0745-0845,1000-1600,1630-1730$ & $08 \mathrm{hrs}$ \\
\hline 97911 & Wednesday, May 21, 2003 & $0830-0930,1000-1600,1630-1730$ & $08 \mathrm{hrs}$ \\
\hline $97915^{(1,2)}$ & Wednesday, April 09, 2003 & $0745-0945,1000-1100,1230-1500,1615-1815$ & $07 \mathrm{hrs} 30 \mathrm{mins}$ \\
\hline $97918^{(1)}$ & Wednesday, July 09, 2003 & $0730-0830,1000-1600,1600-1700$ & $08 \mathrm{hrs}$ \\
\hline 97920 & Tuesday, April 01, 2003 & $0745-0845,1000-1600,1630-1730$ & $08 \mathrm{hrs}$ \\
\hline 97922 & Wednesday, April 16, 2003 & 0800-0900, 1000-1600, 1630-1730 & $08 \mathrm{hrs}$ \\
\hline 97940 & Friday, April 18, 2003 & $0745-0845,1000-1600,1615-1715$ & $08 \mathrm{hrs}$ \\
\hline
\end{tabular}

To facilitate the manual measurement of the PET data, an Excel worksheet as shown in Figure 4-28 was designed for data recording. To use the worksheet, the time events $t_{1}$ (the front bumper of the first vehicle entering the conflict spot), $\mathrm{t}_{2}$ (the rear bumper of the first vehicle leaving the conflict spot), and $t_{3}$ (the front bumper of the second vehicle entering the conflict spot) are recorded in the worksheet in which the corresponding PET and GT will be automatically calculated. The $t_{1}, t_{2}$ and $t_{3}$ are respectively recorded as "mainEnt," "mainExit," and "sideEnt" if the first vehicle was coming from the main street. On the other hand, the $t_{1}, t_{2}$, and $t_{3}$ was recorded in sequence as "sideEnt," "sideExit," and "mainEnt" if the first vehicle was coming from the side street. 


\begin{tabular}{|c|c|c|c|c|c|c|c|c|c|c|c|c|c|c|c|c|c|c|c|c|c|c|}
\hline \multirow{7}{*}{$\begin{array}{l}\text { Site Code } \\
\text { Main St } \\
\text { Side St } \\
\text { Date } \\
\text { Time } \\
\text { Detector File } \\
\text { Observed Collision } \\
\text { Zones } \\
\text { Number of Conflict } \\
\text { Spots } \\
\text { GT Observation } \\
\text { Threshold (sec) } \\
\text { Notes }\end{array}$} & \multicolumn{22}{|l|}{87907} \\
\hline & \multicolumn{22}{|c|}{ May 22, 2003} \\
\hline & \multicolumn{22}{|c|}{$\begin{array}{l}\text { 0900-1000, } 1000-1100,1100-1300,1300-1400,1400-1500,1500-1600,1630-1730 \\
\text { 87907A.TF }\end{array}$} \\
\hline & & \multicolumn{16}{|c|}{ czNBEB, czNBWB, czSBEB, czSBWB } \\
\hline & \multicolumn{22}{|c|}{16} \\
\hline & \multicolumn{22}{|l|}{8} \\
\hline & \multicolumn{22}{|c|}{ By Robert, Melanie, Heintze } \\
\hline \multirow{2}{*}{ VdoTime (hhmmss) } & \multirow{2}{*}{ Czone } & \multirow{2}{*}{ Cspot } & \multicolumn{4}{|c|}{ MainEnt } & \multicolumn{4}{|c|}{ MainExit } & \multicolumn{4}{|c|}{ SideEnt } & \multicolumn{4}{|c|}{ SideExit } & \multirow{2}{*}{ GT } & \multirow{2}{*}{ PET } & \multirow{2}{*}{ Type } & \multirow{2}{*}{ Remarks } \\
\hline & & & $\overline{\mathrm{hr}}$ & $\mathrm{mm}$ & ss & $\mathrm{fr}$ & $\overline{\mathrm{hr}}$ & $\mathrm{mm}$ & ss & $\mathrm{fr}$ & $\overline{\mathrm{hr}}$ & $\mathrm{mm}$ & ss & $\mathrm{fr}$ & $\mathrm{hr}$ & $\mathrm{mm}$ & ss & $\mathrm{fr}$ & & & & \\
\hline 90011 & czSBWB & SB2WB2 & 0 & 1 & 6 & 3 & 0 & 1 & 6 & 14 & 0 & 1 & 13 & 1 & & & & & 6.933 & 6.567 & 1 & \\
\hline 91511 & CZSBEB & SB1EB1 & 0 & 16 & 5 & 0 & 0 & 16 & 5 & 20 & 0 & 16 & 10 & 27 & & & & & 5.900 & 5.233 & 1 & \\
\hline 102813 & czNBEB & NB1EB1 & 0 & 29 & 10 & 24 & & & & & 0 & 29 & 5 & 24 & 0 & 29 & 6 & 65 & 5.000 & 4.600 & 2 & \\
\hline 102813 & czNBEB & NB2EB1 & 0 & 29 & 10 & 29 & & & & & 0 & 29 & 6 & 0 & 0 & 29 & 6 & 13 & 4.967 & 4.533 & 2 & \\
\hline 103144 & CZSBEB & SB2EB1 & 0 & 32 & 36 & 3 & 0 & 32 & 36 & 16 & 0 & 32 & 43 & 8 & & & & & 7.167 & 6.733 & 1 & \\
\hline 104525 & cZNBWB & NB2WB1 & 0 & 46 & 16 & 14 & 0 & 46 & 17 & 2 & 0 & 46 & 23 & 28 & & & & & 7.467 & 6.867 & 1 & van hidden by bus \\
\hline 104629 & CZSBEB & SB2EB1 & 0 & 47 & 20 & 4 & 0 & 47 & 20 & 23 & 0 & 47 & 26 & 7 & & & & & 6.100 & 5.467 & 1 & \\
\hline 105347 & CZSBEB & SB2EB1 & 0 & 54 & 38 & 7 & 0 & 54 & 38 & 22 & 0 & 54 & 45 & 19 & & & & & 7.400 & 6.900 & 1 & \\
\hline 105348 & cZSBEB & SB1EB1 & 0 & 54 & 38 & 25 & 0 & 54 & 39 & 15 & 0 & 54 & 44 & 28 & & & & & 6.100 & 5.433 & 1 & \\
\hline 110538 & cZNBEB & NB2EB1 & 0 & 6 & 22 & 25 & 0 & 6 & 23 & 8 & 0 & 6 & 29 & 22 & & & & & 6.900 & 6.467 & 1 & \\
\hline : & : & : & : & : & : & : & : & : & : & : & : & : & : & : & : & : & : & : & : & : & : & : \\
\hline : & : & : & : & : & : & : & : & : & : & : & $:$ & : & : & : & : & : & : & : & : & $:$ & : & : \\
\hline
\end{tabular}

Figure 4-28: Excel Worksheet for Manual PET Measurement

The PET data were extracted from the video clips digitized at 30 frames per second during May 2003 to January 2004. Several undergraduate students at Purdue University were trained for about four to eight hours before participating in the data extraction. A trained observer needed approximately one to three hours to extract data from one hour of video material, depending on the complexity of the traffic operations. The final data set consists of a series of events as tabulated in Table 4-22. Each recorded event contains the following information:

- $\quad$ Site ID (Site)

- Video time stamp at the beginning of the recorded event (VdoTime)

- Conflict zone of the event being observed (Czone)

- Conflict spot of the event being observed (Cspot)

- Gap time (GT)

- Post-encroachment time (PET)

- Encroachment time (ET), which is obtained by subtracting PET from GT

- Sequence of the vehicle entering the conflict spot (GapType) - 1 if the main-street vehicle enters first and 0 if otherwise. 
Table 4-22: Example of Recorded PET, GT, and ET Values

\begin{tabular}{cccccccc}
\hline Site & VdoTime & Czone & Cspot & GT & PET & ET & GapType \\
\hline 87907 & 90011 & czSBWB & SB2WB2 & 6.933 & 6.567 & 0.367 & 1 \\
87907 & 91511 & czSBEB & SB1EB1 & 5.900 & 5.233 & 0.667 & 1 \\
87907 & 92935 & czSBEB & SB1EB1 & 4.567 & 4.100 & 0.467 & 1 \\
87907 & 94208 & czSBEB & SB1EB1 & 3.767 & 3.367 & 0.400 & 1 \\
87907 & 100415 & czSBEB & SB1EB1 & 6.333 & 5.567 & 0.767 & 1 \\
87907 & 101229 & czSBEB & SB1EB2 & 7.933 & 7.300 & 0.633 & 2 \\
87907 & 101720 & czSBEB & SB2EB1 & 6.267 & 5.200 & 1.067 & 1 \\
87907 & 102813 & czNBEB & NB1EB1 & 5.000 & 4.600 & 0.400 & 2 \\
87907 & 102813 & czNBEB & NB2EB1 & 4.967 & 4.533 & 0.433 & 2 \\
87907 & 103144 & czSBEB & SB2EB1 & 7.167 & 6.733 & 0.433 & 1 \\
87907 & 104525 & czNBWB & NB2WB1 & 7.467 & 6.867 & 0.600 & 1 \\
87907 & 104629 & czSBEB & SB2EB1 & 6.100 & 5.467 & 0.633 & 1 \\
87907 & 105347 & czSBEB & SB2EB1 & 7.400 & 6.900 & 0.500 & 1 \\
87907 & 105348 & czSBEB & SB1EB1 & 6.100 & 5.433 & 0.667 & 1 \\
$:$ & $:$ & $:$ & $:$ & $:$ & $:$ & $:$ & $:$ \\
$:$ & $:$ & $:$ & $:$ & $:$ & $:$ & $:$ & $:$ \\
\hline
\end{tabular}

Examples of archived detector configurations at sites 87907 and 87933 for PET measurement are shown in Figure 4-29 and Figure 4-30 respectively.

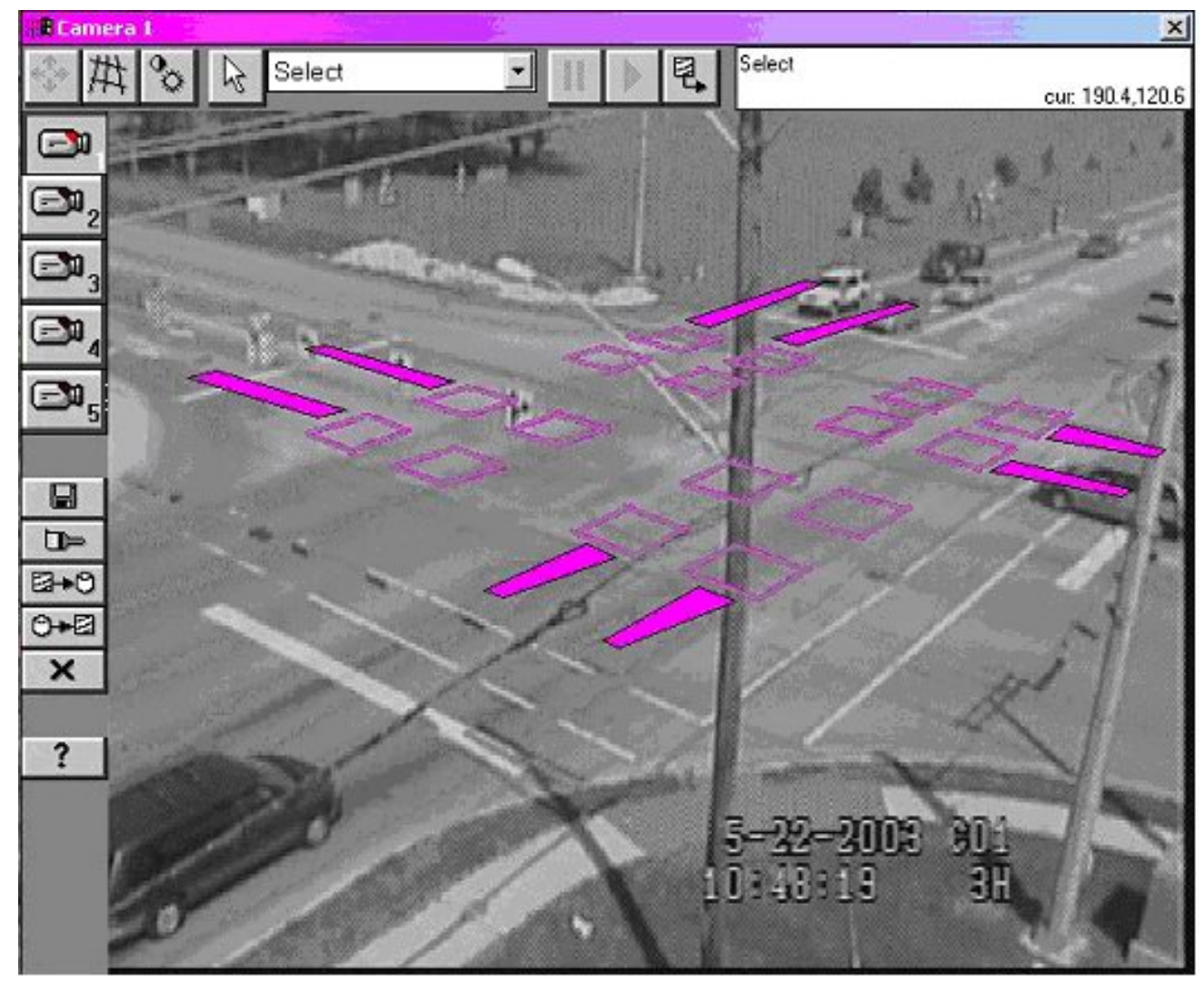

Figure 4-29: Autoscope Detector Configuration at Intersection 87907 


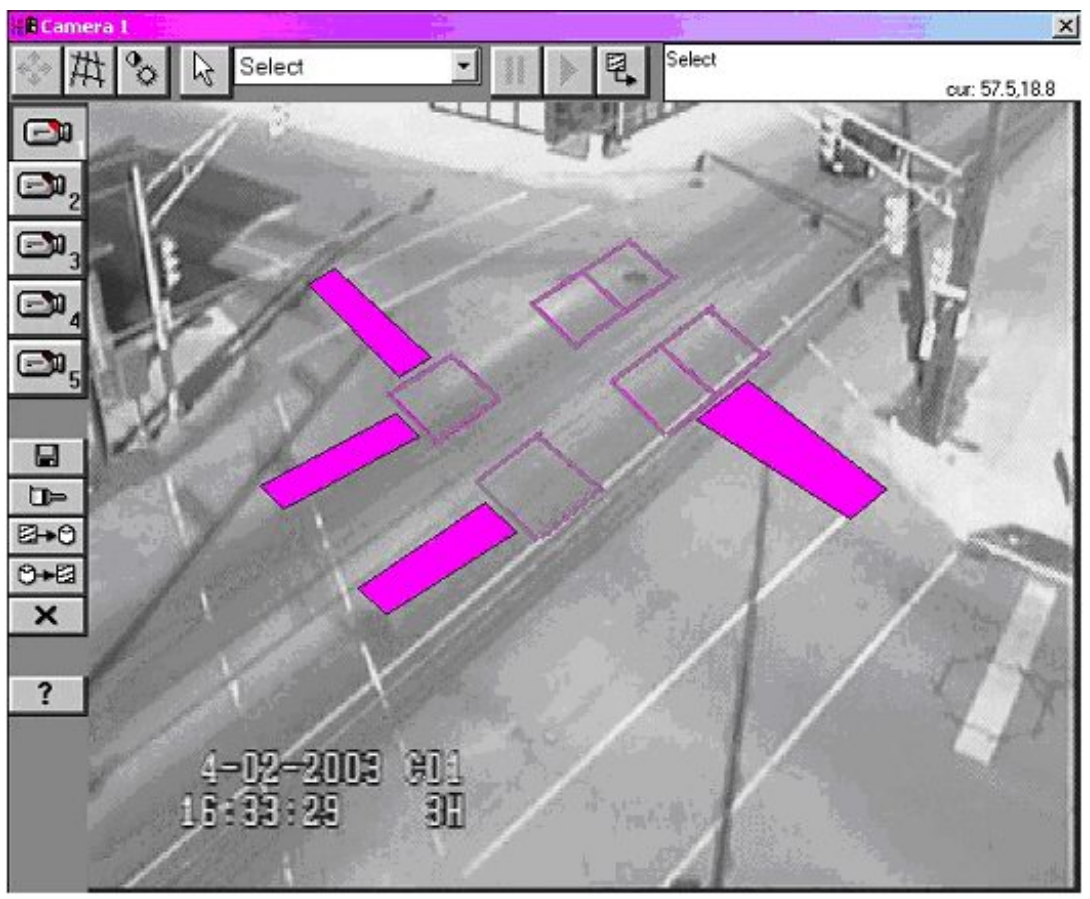

Figure 4-30: Autoscope Detector Configuration at Intersection 87933

Table 4-23: Summary of Periods of Traffic Counts

\begin{tabular}{llll}
\hline Site & Date & Periods of Counted Traffic Volume & Total Duration \\
\hline 87905 & Friday, June 13, 2003 & $0745-0845,1000-1730$ & $08 \mathrm{hrs} 30 \mathrm{mins}$ \\
87906 & Monday, June 16, 2003 & $0730-1730$ & $10 \mathrm{hrs}$ \\
87907 & Thursday, May 22, 2003 & $0845-1730$ & $08 \mathrm{hrs} 45 \mathrm{mins}$ \\
87909 & Wednesday, June 25, 2003 & $0730-1730$ & $10 \mathrm{hrs}$ \\
87915 & Friday, April 11, 2003 & $0800-1730$ & $09 \mathrm{hrs} 30 \mathrm{mins}$ \\
87923 & Tuesday, May 27, 2003 & $0800-0900,1000-1730$ & $08 \mathrm{hrs} 30 \mathrm{mins}$ \\
87930 & Wednesday, July 02, 2003 & $0730-0900,1000-1345,1400-1730$ & $08 \mathrm{hrs} 45 \mathrm{mins}$ \\
87932 & Monday, July 14, 2003 & $0730-0900,1000-1730$ & $09 \mathrm{hrs}$ \\
87933 & Wednesday, April 02, 2003 & $0815-1730$ & $09 \mathrm{hrs} 15 \mathrm{mins}$ \\
97901 & Tuesday, April 08, 2003 & $0815-1730$ & $09 \mathrm{hrs} 15 \mathrm{mins}$ \\
97903 & Tuesday, April 29, 2003 & $0815-0915,1000-1730$ & $08 \mathrm{hrs} 30 \mathrm{mins}$ \\
97905 & Monday, April 21, 2003 & $0745-0900,1000-1730$ & $08 \mathrm{hrs} 45 \mathrm{mins}$ \\
97911 & Wednesday, May 21, 2003 & $0815-1730$ & $09 \mathrm{hrs} 15 \mathrm{mins}$ \\
$97915^{(1)}$ & Wednesday, April 09, 2003 & $0745-1100,1215-1500,1600-1815$ & $08 \mathrm{hrs} 15 \mathrm{mins}$ \\
$97918^{(2)}$ & Wednesday, July 09, 2003 & $0730-0900,1000-1200,1400-1700$ & $06 \mathrm{hrs} 30 \mathrm{mins}$ \\
97920 & Tuesday, April 01, 2003 & $0745-0900,1000-1730$ & $08 \mathrm{hrs} 45 \mathrm{mins}$ \\
97922 & Wednesday, April 16, 2003 & $0800-0900,1000-1730$ & $08 \mathrm{hrs} 30 \mathrm{mins}$ \\
97940 & Friday, April 18, 2003 & $0730-0900,1000-1730$ & $09 \mathrm{hrs}$ \\
\hline
\end{tabular}

Notes:

(1) Intermittent loss of video signal during the observation period.

(2) The traffic volume data are incomplete at this location because of poor visibility of traffic movements caused by the rain interruption and limited field of view. 


\subsubsection{15-minute Directional Traffic Counts}

The 15-minute directional traffic counts were collected manually using the Jamar ${ }^{\circledR}$ traffic counter. About $50 \%$ of the counts were collected in the field and the remainder was completed later in the laboratory from the recorded video materials. The list of periods with traffic counted at each site is provided in Table 4-23. The periods of counted traffic volume coincide with those of the measured PETs.

The characteristics of the collected data and the safety relationships will be examined in the next chapter. 


\section{CHAPTER 5 REGRESSION ANALYSES OF SAFETY RELATIONSHIPS}

The crash count and traffic characteristics collected are examined in this chapter prior to discussion of the development of the extreme value modeling approach in Chapter 6 . The three traffic characteristics analyzed are post-encroachment times, encroachment times, and traffic volumes. We will check the relationships between these data and the crash counts as typically implemented in traditional analysis of surrogate measures of safety. Any correlation between these measure and crashes would confirm a chance for successful model building. Then, we will proceed from a standard regression for a group of locations to extreme value modeling of safety at individual sites.

\subsection{CRASH COUNTS}

Table 5-1 presents a summary of right-angle crash counts at the selected sites. The crash counts in this table reflect adjustment of the counts at the locations where the camera's field of view could cover only a part of the intersection. Those crashes that occurred in the area where traffic movements were not observable from the videos were not counted (see Table 4-5 and Table 4-6 for complete crash data). The right-angle crash counts in this table are also classified by a set of conditions during the crash occurrences.

Sites 97915 and 97918 were not considered in the subsequent analysis because of incomplete data at both sites. The video material at site 97915 was incomplete because of the intermittent loss of video signal. Site 97918 had incomplete volume data due to a rain interruption and a restricted field of view from the recorded videos. It is interesting, however, to note that these two sites had very low counts of PETs during the entire observation period. Both of these sites have protected left-turn phases with heavy left-turn traffic volume. Short PETs between straight traffic flows are prevented from occurring by being separated in time with the leading left-turn phase in each cycle. 
A good amount of dispersion of crash counts across the locations is important to ensure that the sample locations have varying levels of safety. This property is desirable for the validation process where we determine if the method is capable of discriminating various safety levels at the studied locations. The amount of dispersion can be estimated by the overdispersion parameter, which is

$$
\hat{\alpha}=\frac{\operatorname{var} c-a}{a^{2}}
$$

where $\hat{\alpha}$ is estimated overdispersion parameter, var $c$ is a sample variance of crash counts from all sites, and $a$ is a sample mean of crash counts from all sites.

Table 5-1: Summary of Right-Angle Crash Counts in 1997 - 2000

\begin{tabular}{|c|c|c|c|c|c|c|}
\hline \multirow[b]{2}{*}{ Site ID } & \multirow[b]{2}{*}{ Intersection } & \multicolumn{5}{|c|}{ Conditions } \\
\hline & & $\begin{array}{c}\text { All } \\
\text { Conditions }\end{array}$ & Daytime & $\begin{array}{c}\text { Daytime and } \\
\text { Non-winter }\end{array}$ & $\begin{array}{c}\text { Daytime, non- } \\
\text { winter, and } \\
\text { weekday }\end{array}$ & $\begin{array}{l}\text { Daytime, non- } \\
\text { winter, weekday, } \\
\text { and normal } \\
\text { weather }^{(1)}\end{array}$ \\
\hline 87905 & Creasy Ln@ @ SR-26 & 2 & 1 & 1 & 1 & 1 \\
\hline 87906 & SR-26@Farabee Dr & 10 & 6 & 6 & 5 & 5 \\
\hline 87915 & US-231@Beck Ln & 5 & 3 & 2 & 2 & 2 \\
\hline 87923 & SR-43@CR-600 N & 5 & 2 & 2 & 1 & 0 \\
\hline 87930 & Greenbush St @ Shenandoah Dr & 0 & 0 & 0 & 0 & 0 \\
\hline 87932 & Creasy Ln @ Union St & 2 & 2 & 2 & 2 & 2 \\
\hline 87933 & Union St @ 14th St & 1 & 1 & 1 & 0 & 0 \\
\hline 97911 & US-52@ Kossuth St & 6 & 4 & 0 & 0 & 0 \\
\hline $97915^{(2)}$ & US-52 @ SR-26 & 4 & 1 & 1 & 0 & 0 \\
\hline 97918 & US-52@ Duncan Rd & 1 & 0 & 0 & 0 & 0 \\
\hline 97920 & SR-26 @ SR-526 & 1 & 1 & 0 & 0 & 0 \\
\hline $97922^{(3)}$ & Greenbush St @ US-52 & 3 & 1 & 0 & 0 & 0 \\
\hline \multirow[t]{5}{*}{97940} & US-52 @ CR-300 W & 8 & 7 & 6 & 4 & 4 \\
\hline & Total & 128 & 71 & 49 & 37 & 29 \\
\hline & Mean & 7.111 & 3.944 & 2.722 & 2.056 & 1.611 \\
\hline & Variance & 61.516 & 18.997 & 9.977 & 6.644 & 3.428 \\
\hline & Overdispersion ( $\alpha)$ & 1.076 & 0.967 & 0.979 & 1.086 & 0.700 \\
\hline
\end{tabular}

\section{Notes:}

(1) Normal weather is defined as no precipitation and good visibility for driving conditions.

(2) czSBEB (conflict zone between southbound and eastbound traffic) and czSBWB (conflict zone between southbound and westbound traffic) are not observable in the camera field of view during the data collection.

(3) czSBWB (conflict zone between southbound and westbound traffic) is not observable in the camera field of view during the data collection.

The overdispersion parameter is non-negative and the zero value is equivalent to the undispersed data. The further the overdispersion parameter is from zero indicates an increasing amount of 
dispersion. The overdispersion parameter shown in the table is close to 1 in all cases except for the last column, which is about 0.700 . This implies that crash counts from the sample sites are overdispersed and therefore comprise varying levels of safety, which is desirable for our study.

\subsection{POST-ENCROACHMENT TIME}

The basic summary statistics of PET data are presented for all sites. PET data are the key surrogate measure of safety, which we later will use to build the models.

\subsubsection{PET Counts}

Counts of PETs shorter than the threshold values varied from one second to eight seconds for all 16 sites, except the two with incomplete data are tabulated in Table 5-2.

Table 5-2: Count Summary of Observed PETs

\begin{tabular}{|c|c|c|c|c|c|c|c|c|c|c|c|c|c|c|c|}
\hline \multirow{2}{*}{ Site } & \multicolumn{15}{|c|}{ 8-hour counts of PETs $\leq$} \\
\hline & $8.0 \mathrm{~s}$ & $7.5 \mathrm{~s}$ & $7.0 \mathrm{~s}$ & $6.5 \mathrm{~s}$ & $6.0 \mathrm{~s}$ & $5.5 \mathrm{~s}$ & $5.0 \mathrm{~s}$ & $4.5 \mathrm{~s}$ & $4.0 \mathrm{~s}$ & $3.5 \mathrm{~s}$ & $3.0 \mathrm{~s}$ & $2.5 \mathrm{~s}$ & $2.0 \mathrm{~s}$ & $1.5 \mathrm{~s}$ & $1.0 \mathrm{~s}$ \\
\hline 87905 & 68 & 68 & 59 & 47 & 31 & 25 & 16 & 13 & 8 & 5 & 2 & 0 & 0 & 0 & 0 \\
\hline 87906 & 190 & 186 & 162 & 129 & 98 & 66 & 42 & 26 & 15 & 7 & 3 & 2 & 1 & 0 & 0 \\
\hline 87907 & 95 & 94 & 81 & 68 & 54 & 35 & 19 & 10 & 3 & 1 & 0 & 0 & 0 & 0 & 0 \\
\hline 87909 & 538 & 537 & 457 & 357 & 268 & 184 & 121 & 72 & 38 & 14 & 7 & 2 & 2 & 0 & 0 \\
\hline 87915 & 250 & 244 & 216 & 164 & 116 & 81 & 47 & 23 & 15 & 3 & 1 & 1 & 1 & 1 & 0 \\
\hline 87923 & 70 & 69 & 56 & 40 & 24 & 15 & 11 & 4 & 2 & 1 & 0 & 0 & 0 & 0 & 0 \\
\hline 87930 & 90 & 90 & 70 & 54 & 38 & 30 & 19 & 11 & 7 & 2 & 1 & 0 & 0 & 0 & 0 \\
\hline 87932 & 60 & 59 & 45 & 39 & 27 & 20 & 8 & 4 & 1 & 0 & 0 & 0 & 0 & 0 & 0 \\
\hline 87933 & 180 & 180 & 159 & 132 & 104 & 81 & 48 & 30 & 17 & 8 & 3 & 2 & 1 & 1 & 1 \\
\hline 97901 & 573 & 571 & 508 & 413 & 311 & 219 & 149 & 79 & 35 & 14 & 9 & 4 & 0 & 0 & 0 \\
\hline 97903 & 550 & 544 & 493 & 398 & 327 & 262 & 196 & 146 & 115 & 81 & 59 & 43 & 34 & 27 & 17 \\
\hline 97905 & 182 & 180 & 153 & 135 & 106 & 78 & 47 & 30 & 16 & 7 & 4 & 0 & 0 & 0 & 0 \\
\hline 97911 & 113 & 112 & 105 & 87 & 69 & 55 & 42 & 24 & 12 & 5 & 1 & 0 & 0 & 0 & 0 \\
\hline 97920 & 164 & 162 & 134 & 113 & 79 & 56 & 42 & 24 & 10 & 3 & 0 & 0 & 0 & 0 & 0 \\
\hline 97922 & 60 & 59 & 48 & 37 & 26 & 14 & 10 & 5 & 1 & 0 & 0 & 0 & 0 & 0 & 0 \\
\hline 97940 & 28 & 27 & 23 & 19 & 15 & 10 & 5 & 1 & 1 & 1 & 0 & 0 & 0 & 0 & 0 \\
\hline
\end{tabular}

At the eight-second threshold, the eight-hour PET counts range from 28 to 538, which indicates a significant amount of variability in the PET data necessary for modeling as well as for validation purposes. Also, it should be noted that a PET of three seconds or less is very infrequent at most sites. Site 97903 is overwhelmed by a number of PETs shorter than three seconds and a review of the video clips at this intersection revealed that a number of extremely short PETs were caused by queue spillbacks during congested periods. 


\subsubsection{Summary Statistics of PET}

The summary statistics of the collected PETs between straight traffic flows are shown in Table 5-3. The yellow, all-red, and intergreen periods measured from the field at each intersection are also presented. PETs of six seconds or shorter are used to calculate the summary statistics. The six-second threshold was selected as it gives a sample mean close to a sample median at most sites. In addition, decreasing trends of PETs are more consistent from six seconds to shorter values. The slight variations of the sample means and the sample medians from site to site do not constitute any distinct pattern. The intergreen periods range from five to six seconds for selected sites. The minimum observed PETs range from 0.1 second to 3.9 seconds for eight hours of observation. The value of 0.1 second at site 97903 was caused by queue spillbacks while the value of 0.867 at site 87933 was caused by a single event of red light violation.

Table 5-3: Summary Statistics of Post-Encroachment Time and Signal Settings

\begin{tabular}{|c|c|c|c|c|c|c|c|c|c|c|}
\hline \multirow[b]{2}{*}{$\begin{array}{l}\text { Site } \\
\text { ID }\end{array}$} & \multicolumn{7}{|c|}{ Summary Statistics based on PET $\leq 6$ seconds } & \multicolumn{3}{|c|}{ Signal Settings (sec) } \\
\hline & $\begin{array}{c}\text { 8-hr } \\
\text { Counts }\end{array}$ & Min & $\begin{array}{c}\text { 1st } \\
\text { Quartile }\end{array}$ & Median & Mean & $\begin{array}{c}\text { 3rd } \\
\text { Quartile }\end{array}$ & Max & $\begin{array}{c}\text { Yellow } \\
\text { (Y) }\end{array}$ & $\begin{array}{c}\text { All-Red } \\
\text { (AR) }\end{array}$ & $\begin{array}{c}\text { Intergreen } \\
(\mathrm{Y}+\mathrm{AR})\end{array}$ \\
\hline 87905 & 31 & 2.633 & 4.050 & 4.900 & 4.646 & 5.300 & 5.967 & 4.0 & NA & NA \\
\hline 87906 & 98 & 1.633 & 4.442 & 5.167 & 4.951 & 5.667 & 6.000 & NA & NA & NA \\
\hline 87907 & 54 & 3.367 & 4.600 & 5.350 & 5.136 & 5.592 & 6.000 & 4.0 & 2.0 & 6.0 \\
\hline 87909 & 268 & 1.700 & 4.467 & 5.167 & 4.971 & 5.642 & 6.000 & 4.0 & 1.0 & 5.0 \\
\hline 87915 & 116 & 1.400 & 4.592 & 5.300 & 5.060 & 5.633 & 6.000 & 3.0 & 2.0 & 5.0 \\
\hline 87923 & 24 & 3.067 & 4.592 & 5.167 & 5.040 & 5.675 & 5.833 & 4.0 & 2.0 & 6.0 \\
\hline 87930 & 38 & 2.700 & 4.375 & 4.983 & 4.846 & 5.433 & 5.967 & 3.5 & 1.5 & 5.0 \\
\hline 87932 & 27 & 3.600 & 4.967 & 5.200 & 5.156 & 5.450 & 6.000 & 4.0 & 1.0 & 5.0 \\
\hline 87933 & 104 & 0.867 & 4.392 & 5.100 & 4.846 & 5.467 & 6.000 & 3.0 & 2.0 & 5.0 \\
\hline 97901 & 311 & 2.133 & 4.500 & 5.067 & 4.963 & 5.633 & 6.000 & 3.0 & 2.0 & 5.0 \\
\hline 97903 & 327 & 0.100 & 3.617 & 4.700 & 4.277 & 5.400 & 6.000 & 3.0 & 2.0 & 5.0 \\
\hline 97905 & 106 & 2.933 & 4.392 & 5.133 & 4.925 & 5.533 & 6.000 & NA & NA & NA \\
\hline 97911 & 69 & 2.800 & 4.100 & 4.833 & 4.746 & 5.433 & 6.000 & 3.0 & 2.0 & 5.0 \\
\hline 97920 & 79 & 3.267 & 4.317 & 5.000 & 4.908 & 5.600 & 6.000 & NA & NA & NA \\
\hline 97922 & 26 & 3.933 & 4.800 & 5.467 & 5.174 & 5.700 & 5.867 & 3.0 & 2.0 & 5.0 \\
\hline 97940 & 15 & 3.300 & 4.750 & 5.300 & 5.116 & 5.633 & 5.967 & 3.0 & 2.0 & 5.0 \\
\hline
\end{tabular}

Note: NA = Data is not available

\subsubsection{PET Variations over Time}

The PETs were plotted over time for the entire site as well as for each conflict zone (see Figure C-1 to Figure C-32). Preliminary examination of these plots reveals that the PET values were more likely to be shorter in the afternoon period, particularly for the sites with frequent PET occurrences. This may be the result of increasing volumes during the afternoon period. The declining trends of PETs are less distinct for the sites with low PET counts. A few short PETs 
were observed randomly at many sites. These short PETs may significantly influence the modeling results for those intersections with infrequent occurrences of PETs.

In the plots the sequence of the two vehicles entering a conflict spot for each PET is distinguished as $0 / 1$ indicator where 1 signifies the PET that the main-street vehicle reaches the conflict spot first and 0 signifies the PET that the side-street vehicle reaches the conflict spot first. The 0/1 vehicle entering sequences were somewhat evenly distributed for most sites observed. The PET variation plots by conflict zones, however, reveal that PETs are more likely to cluster at certain conflict zones for sites such as 87906, 87907 and 97903. Apart from the mentioned sites, distributions of PET counts by conflict zones were more or less random and therefore the aggregation of PET data from all conflict zones at the same site was expected to have minimal impact on the safety modeling from a theoretical viewpoint.

\subsubsection{Distributions of PET}

The distributions of PETs for all sample sites are plotted in Figure C-33 to Figure C-36. The PETs of eight seconds or less from all conflict zones are presented. Negated PETs were used instead of regular PETs for two reasons: (a) it is relatively simpler to compare the distributions between sites as the abscissa are left-truncated at the same point and the heavier right tail implies a greater degree of hazard; and (b) it is consistent with the extreme value modeling of PET presented in the next chapter where PETs are negated and grouped into blocks.

The following points are observed from the distribution plots:

- In general, the distributions of negated PETs tend to decrease rapidly at the beginning and then level off as negated PETs approach zero.

- The empirical distributions are disconnected at the right tail for sites 87909,87915 , 87933, and 97940. For sites with low PET frequency, these few values at the right tail can have influential impacts on extreme value modeling.

- The patterns of PETs greater than six seconds are somewhat random. PETs in this range are less governed by the effect of signal settings and driver behavior from two crossing flows. Instead, the values of PETs strongly depend on the arrival times of vehicles at intersections. The crossing event with PETs in this range are usually far from hazardous situations. 


\subsection{TRAFFIC VOLUME}

Traffic volume is perhaps the most popular exposure variable in both crash-based and non-crashbased safety estimations. The importance of traffic volume is often explained in simple words there is no crash if there is no traffic. The traffic volumes in 15-minute intervals corresponding to the eight-hour periods of PET observation were collected using Jamar® traffic counters.

Two types of volume trends, total entering volumes and conflicting volumes, were examined at each intersection. Figure 5-1 is an example of the 15-minute traffic volume variations over time at intersection 87915 (see Figure D-1 to Figure D-16 for all intersections).
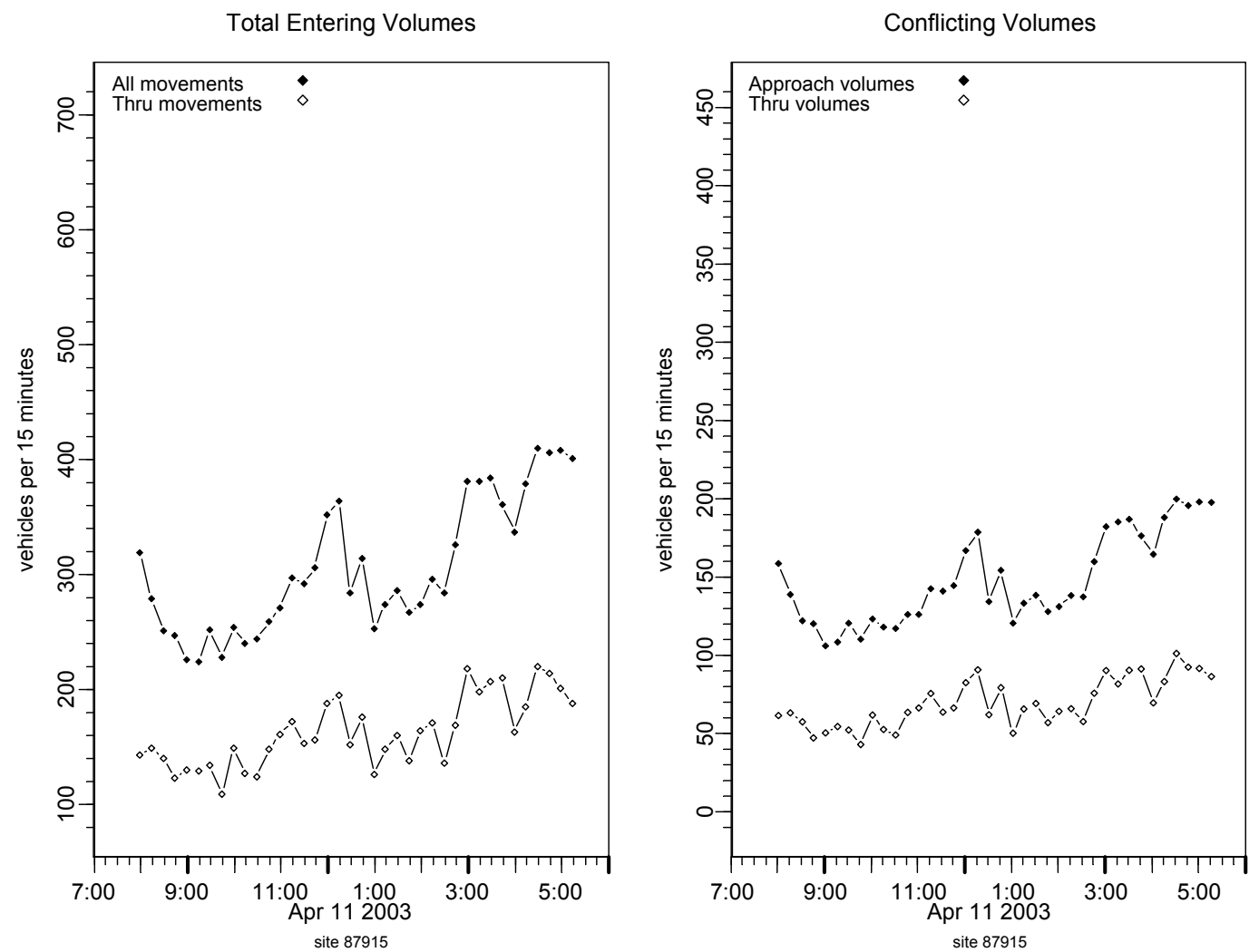

Figure 5-1: 15-Minute Traffic Volume Variations over Time at Site 87915

The upper line in the figure on the left is the total traffic volume entering the intersection during 15-minute intervals $\left(V_{\text {sum }}\right)$. The lower line in the same figure is the total straight-ahead traffic 
volume in 15-minute intervals $\left(V_{\text {sumth }}\right)$. The intersection volume $\left(V_{\text {sum }}\right)$ and intersection through volume $\left(V_{\text {sumth }}\right)$ can be written as

$$
V_{\text {sum }}=V_{N B}+V_{S B}+V_{E B}+V_{W B}
$$

where $V_{N B}, V_{S B}, V_{E B}$ and $V_{W B}$ are total approach volumes from northbound, southbound, eastbound and westbound approaches respectively, and

$$
V_{\text {sumth }}=V_{\text {NBTH }}+V_{S B T H}+V_{E B T H}+V_{W B T H},
$$

where $V_{N B T H}, V_{S B T H}, V_{E B T H}$ and $V_{W B T H}$ are through traffic volumes from northbound, southbound, eastbound and westbound approaches respectively.

The figure on the right is a summation of a product of crossing volumes from all conflict zones during each 15-minute interval. In order to maintain the same unit as in the left figure, we take a square root of this sum, which is referred to as a "conflicting volume." Conflicting volumes can be considered as the amount of conflicting exposure which may lead to right-angle collisions.

The upper line in the right figure is a conflicting volume computed using approach volumes, which can be expressed as

$$
V_{x}=\sqrt{\left(V_{N B} V_{E B}+V_{S B} V_{E B}+V_{N B} V_{W B}+V_{S B} V_{W B}\right)} .
$$

The lower line in the right figure represents a conflicting through volume computed using only through volumes, which can be written as

$$
V_{x t h}=\sqrt{\left(V_{\text {NBTH }} V_{E B T H}+V_{S B T H} V_{E B T H}+V_{\text {NBTH }} V_{\text {WBTH }}+V_{\text {SBTH }} V_{W B T H}\right)} .
$$

Table 5-4 shows a summary of the traffic volume data at the studied sites. From this table, we can see that the traffic volume varies considerably, thus a wide range of traffic is sufficiently represented in the sample. 
Table 5-4: Summary of Traffic Volume Data at Selected Sites

\begin{tabular}{|c|c|c|c|c|c|}
\hline $\begin{array}{l}\text { Site } \\
\text { ID }\end{array}$ & $\begin{array}{l}\text { Hourly Average } \\
\text { Intersection } \\
\text { Volume }\left(\mathbf{V}_{\text {sum }}\right)\end{array}$ & $\begin{array}{l}\text { Hourly Average } \\
\text { Intersection Thru } \\
\text { Volume }\left(\mathbf{V}_{\text {sumth }}\right)\end{array}$ & $\begin{array}{l}\text { Hourly Average } \\
\text { Conflicting Volume } \\
\qquad\left(\mathbf{V}_{\mathbf{x}}\right)\end{array}$ & $\begin{array}{c}\text { Hourly Average } \\
\text { Conflicting Thru } \\
\text { Volume }\left(\mathbf{V}_{\mathrm{xth}}\right)\end{array}$ & $\begin{array}{l}\text { Average Ratio of } \\
\text { Left-turn to Total } \\
\text { Volume (LTratio) }\end{array}$ \\
\hline 87905 & 4463 & 2484 & 2224 & 1195 & 0.223 \\
\hline 87906 & 2674 & 1940 & 1014 & 377 & 0.127 \\
\hline 87907 & 2256 & 1341 & 1015 & 379 & 0.210 \\
\hline 87909 & 1450 & 1019 & 734 & 516 & 0.152 \\
\hline 87915 & 1246 & 666 & 612 & 290 & 0.248 \\
\hline 87923 & 1198 & 888 & 472 & 231 & 0.131 \\
\hline 87930 & 938 & 698 & 348 & 144 & 0.122 \\
\hline 87932 & 1584 & 889 & 734 & 272 & 0.213 \\
\hline 87933 & 965 & 739 & 362 & 183 & 0.225 \\
\hline 97901 & 1781 & 1523 & 896 & 766 & 0.090 \\
\hline 97903 & 2603 & 1852 & 1135 & 737 & 0.145 \\
\hline 97905 & 2303 & 1636 & 1142 & 791 & 0.169 \\
\hline 97911 & 2684 & 1899 & 1209 & 738 & 0.144 \\
\hline 97920 & 1092 & 521 & 536 & 228 & 0.262 \\
\hline 97922 & 2766 & 1878 & 1316 & 829 & 0.149 \\
\hline 97940 & 1682 & 1048 & 715 & 240 & 0.187 \\
\hline
\end{tabular}

\subsection{ENCROACHMENT TIME}

Encroachment time (ET) has an inverse relationship with the speed of a vehicle traversing the conflict spot. The summary statistics of ET are shown in Table 5-5. CV is a coefficient of variation obtained by dividing the standard deviation with the mean. Percent-norm (\%-norm) is the ET value at a specific percentile under the normal distribution assumption. Percentile (\%-tile) represents the empirical quantile of ET at a predefined probability value. For examples, $90 \%$ quantile of ET is a value of ET that is greater than $90 \%$ of the sample and $90 \%$-norm can be calculated as

$$
\begin{aligned}
90 \% \text {-norm } & =\text { sample mean }+\left(\mathrm{z}_{0.90}\right)(\text { sample } \mathrm{SD}) \\
& =\text { sample mean }+(1.282)(\text { sample } \mathrm{SD})
\end{aligned}
$$

where $\mathrm{z}_{0.90}$ is a $90 \%$-quantile of a standard normal distribution.

ET is useful in detecting cases of queue spillback at intersections. An excessively long ET indicates a slow vehicle blocked by the queue ahead. In this table, queue spillback appears to be a problem at site 97903. The maximum value of ET and the 95\% quantile of ET at site 97903 are excessively longer than those of other sites. 
Table 5-5: Summary Statistics of Encroachment Time

\begin{tabular}{ccccccccccccc}
\hline Site & Freq & Min & Max & Mean & SD & CV & 85\%-norm & 90\%-norm & 95\%-norm & 85\%-tile & 90\%-tile & 95\%-tile \\
\hline 87905 & 68 & 0.367 & 1.200 & 0.694 & 0.179 & 0.257 & 0.879 & 0.923 & 0.988 & 0.867 & 0.877 & 1.044 \\
87906 & 190 & 0.300 & 3.567 & 0.614 & 0.327 & 0.532 & 0.953 & 1.033 & 1.152 & 0.767 & 0.867 & 0.933 \\
87907 & 95 & 0.200 & 1.067 & 0.468 & 0.124 & 0.265 & 0.597 & 0.627 & 0.673 & 0.600 & 0.600 & 0.667 \\
87909 & 538 & 0.267 & 1.767 & 0.589 & 0.162 & 0.275 & 0.756 & 0.796 & 0.855 & 0.700 & 0.733 & 0.833 \\
87915 & 250 & 0.233 & 2.167 & 0.601 & 0.243 & 0.404 & 0.853 & 0.912 & 1.001 & 0.833 & 0.900 & 1.018 \\
87923 & 70 & 0.167 & 2.933 & 0.597 & 0.329 & 0.551 & 0.937 & 1.018 & 1.137 & 0.688 & 0.740 & 0.888 \\
87930 & 90 & 0.267 & 1.233 & 0.502 & 0.149 & 0.297 & 0.656 & 0.693 & 0.747 & 0.588 & 0.603 & 0.818 \\
87932 & 60 & 0.300 & 1.500 & 0.552 & 0.179 & 0.324 & 0.737 & 0.781 & 0.846 & 0.700 & 0.700 & 0.735 \\
87933 & 180 & 0.400 & 4.300 & 0.704 & 0.345 & 0.489 & 1.061 & 1.146 & 1.271 & 0.833 & 0.900 & 1.067 \\
97901 & 573 & 0.167 & 3.133 & 0.638 & 0.295 & 0.462 & 0.943 & 1.016 & 1.123 & 0.767 & 0.867 & 1.000 \\
97903 & 550 & 0.300 & 7.400 & 0.934 & 0.878 & 0.941 & 1.844 & 2.059 & 2.378 & 1.033 & 1.487 & 2.655 \\
97905 & 182 & 0.400 & 3.200 & 0.703 & 0.321 & 0.457 & 1.036 & 1.115 & 1.232 & 0.800 & 0.867 & 1.000 \\
97911 & 113 & 0.333 & 2.933 & 0.668 & 0.294 & 0.440 & 0.972 & 1.044 & 1.151 & 0.767 & 0.833 & 0.947 \\
97920 & 164 & 0.333 & 1.933 & 0.545 & 0.236 & 0.434 & 0.790 & 0.847 & 0.933 & 0.623 & 0.813 & 1.026 \\
97922 & 60 & 0.233 & 1.067 & 0.500 & 0.219 & 0.438 & 0.727 & 0.780 & 0.860 & 0.777 & 0.870 & 1.002 \\
97940 & 28 & 0.233 & 2.400 & 0.676 & 0.468 & 0.693 & 1.162 & 1.277 & 1.447 & 1.059 & 1.120 & 1.448 \\
\hline
\end{tabular}

\subsection{REGRESSION ANALYSIS}

To better understand the safety relationships between crashes and surrogate data, we conducted the regression analysis on the crash count data using the traffic and PET data as model covariates. Crash counts are non-negative integer values and therefore the standard least-square regression models are inappropriate. Linear regression models yield predicted values that are non-integers and can also predict values that are negative. There are a number of methods that can be used to properly model crash counts. The most commonly used ones are Poisson and negative binomial regression models. The limitation of Poisson regression is that it restricts the mean of the count process to be equal to its variance. In reality, the variance of actual data can be significantly larger than the mean; in other words, the data is said to be overdispersed. Typically, overdispersed count data is modeled using a negative binomial model. Washington et al. (2003) discussed these count data models in detail and provided several examples of modeling results.

\subsubsection{Regression Approaches}

Two widely used count data models are considered in this analysis, Poisson and negative binomial regressions. In addition, zero-inflated models are also examined for the case where the number of zero counts present in the data is excessive and may be better explained by a two-state count process. 


\subsubsection{Poisson Regression}

In a Poisson regression, the probability of location $i$ having $y_{i}$ counts of traffic crashes is given by

$$
P\left(y_{i}\right)=\frac{e^{-\lambda_{i}} \lambda_{i}^{y_{i}}}{y_{i} !}
$$

where $P\left(y_{i}\right)$ is the probability of location $i$ having $y_{i}$ counts of traffic crashes and $\lambda_{i}$ is the Poisson parameter for location $i$, which is equal to expected number of crash counts at location $i$. Poisson regression models are estimated by specifying the Poisson parameter $\lambda_{i}$ as a function of explanatory variables. The most common relationship is the log-linear link, which is expressed as

$$
\begin{aligned}
\lambda_{i} & =\exp \left(\beta X_{i}\right), \text { or } \\
\log \left(\lambda_{i}\right) & =\beta X_{i},
\end{aligned}
$$

where $X_{i}$ is a vector of explanatory variables and $\beta$ is a vector of estimable coefficients. The likelihood function is

$$
L(\beta)=\prod_{i} \frac{\exp \left[-\exp \left(\beta X_{i}\right)\right]\left[\exp \left(\beta X_{i}\right)\right]^{y_{i}}}{y_{i} !}
$$

Maximum likelihood estimation is commonly used for parameter estimations since the estimates are consistent, asymptotically normal, and asymptotically efficient. The log-likelihood function is easier to manage and more appropriate for the maximum likelihood estimation procedure, and is given as

$$
1(\beta)=\sum_{i=1}^{n}\left[-\exp \left(\beta X_{i}\right)+y_{i} \beta X_{i}-\log \left(y_{i} !\right)\right]
$$

The likelihood ratio test is a common test procedure used to assess the goodness-of-fit between nested models. The likelihood ratio test statistic is 


$$
-2\left[1\left(\beta_{R}\right)-1\left(\beta_{U}\right)\right]
$$

where $1\left(\beta_{R}\right)$ is the log-likelihood at convergence of the restricted model (only constant term is added) and $1\left(\beta_{U}\right)$ is the log-likelihood at convergence of the unrestricted model. This statistic follows $\chi^{2}$ distribution with the degrees of freedom equal to the difference in the number of parameters of two competing models.

Another commonly used measure of overall model fit is the $\rho^{2}$ statistic, which is given by

$$
\rho^{2}=1-\frac{1\left(\beta_{U}\right)}{1\left(\beta_{R}\right)}
$$

The value of $\rho^{2}$ is between 0 and 1 where the value closer to 1 implies a better model fit; in other words, the more variance the estimated model is explaining.

\subsubsection{Negative Binomial Regression}

The common error in the Poisson regression analysis is when the data violate the equality assumption $E\left(y_{i}\right)=\operatorname{var}\left(y_{i}\right)$. The data are said to be underdispersed when $E\left(y_{i}\right)>\operatorname{var}\left(y_{i}\right)$ or overdispersed when $E\left(y_{i}\right)<\operatorname{var}\left(y_{i}\right)$. As a result, the estimated coefficients will be biased if corrective measures are not taken. The negative binomial model aims to address this issue. The model is derived by rewriting equation (5-7) such that, for each observation $i$,

$$
\lambda_{i}=\exp \left(\beta X_{i}+\varepsilon_{i}\right)
$$

where $\exp \left(\varepsilon_{i}\right)$ is a gamma-distributed error term with mean 1 and variance $\alpha^{2}$. The addition of this term allows the variance to differ from the mean as

$$
\operatorname{var}\left(y_{i}\right)=E\left(y_{i}\right)\left[1+\alpha E\left(y_{i}\right)\right]=E\left(y_{i}\right)+\alpha E\left(y_{i}\right)^{2} .
$$


The Poisson regression model is a limiting model of the negative binomial model as $\alpha \rightarrow 0$. This implies that the selection between these two is dependent upon the estimated value of $\alpha$ (the overdispersion parameter). The negative binomial distribution is of the following form

$$
P\left(y_{i}\right)=\frac{\Gamma\left((1 / \alpha)+y_{i}\right)}{\Gamma(1 / \alpha) y_{i} !}\left(\frac{1 / \alpha}{(1 / \alpha)+\lambda_{i}}\right)^{1 / \alpha}\left(\frac{\lambda_{i}}{(1 / \alpha)+\lambda_{i}}\right)^{y_{i}}
$$

where $\Gamma(\cdot)$ is a gamma function. The corresponding likelihood function is

$$
L\left(\lambda_{i}\right)=\prod_{i} \frac{\Gamma\left((1 / \alpha)+y_{i}\right)}{\Gamma(1 / \alpha) y_{i} !}\left(\frac{1 / \alpha}{(1 / \alpha)+\lambda_{i}}\right)^{1 / \alpha}\left(\frac{\lambda_{i}}{(1 / \alpha)+\lambda_{i}}\right)^{y_{i}}
$$

When the data are overdispersed, the estimated variance term is larger than under a true Poisson process. Consequently, all of the standard errors of coefficient estimates become inflated.

\subsubsection{Zero-Inflated Poisson and Negative Binomial Regression Models}

An observation of zero events during the observation period can arise from two qualitatively different conditions. A normal count-process state can at times lead to a zero count due to the random nature of the count process. A zero-count state, on the other hand, is the inability to experience an event.

There is a fine line between these two states in reality. Let us consider the right-angle crashes for a particular area within the intersection. For intersections with a heavy left-turn volume, light through traffic, appropriate signal setting, and good visibility, the likelihood of a straight rightangle collision is minimal but yet still present, since human erratic behavior can lead to an accident. However, when we have taken the count data for four years for a particular region and still we have not yet observed any right-angle crashes. These areas can be considered in a zerocount state because the likelihood of a collision is extremely small. Thus, the zero-count state in this case may refer to a situation where the likelihood of a crash is extremely rare compared to the normal-count state where the occurrence of events is more likely and follows some known count process. 
Data obtained from two-state regimes often suffer from overdispersion if treated as a normal count process only because the number of zero events is inflated by the zero-count state. Usually, the preponderance of zeros in the data often hints that the two-state count process may be a more appropriate approach. Models that account for this dual-state system are referred to as zeroinflated models.

The zero-inflated Poisson (ZIP) model assumes that the events $Y=\left(y_{1}, y_{2}, \ldots, y_{n}\right)$ are independent and the model is

$$
\begin{aligned}
& \operatorname{Pr}\left\{y_{i}=0\right\}=p_{i}+\left(1-p_{i}\right) e^{-\lambda_{i}} \\
& \operatorname{Pr}\left\{y_{i}=y\right\}=\frac{\left(1-p_{i}\right) e^{-\lambda_{i}} \lambda_{i}^{y}}{y !} .
\end{aligned}
$$

Similarly, the zero-inflated negative binomial regression model (ZINB) for independent $Y=\left(y_{1}, y_{2}, \ldots, y_{n}\right)$ is

$$
\begin{aligned}
& \operatorname{Pr}\left\{y_{i}=0\right\}=p_{i}+\left(1-p_{i}\right)\left[\frac{1 / \alpha}{(1 / \alpha)+\lambda_{i}}\right]^{1 / \alpha} \\
& \operatorname{Pr}\left\{y_{i}=y\right\}=\left(1-p_{i}\right)\left[\frac{\Gamma((1 / \alpha)+y) u_{i}^{1 / \alpha}\left(1-u_{i}\right)^{y}}{\Gamma(1 / \alpha) y !}\right] .
\end{aligned}
$$

Maximum likelihood estimations can be used to estimate both zero-inflated models. The splitting process can be assumed to follow a logit (logistic) or probit (normal) probability process. To test the appropriateness of using a zero-inflated model, Vuong (1989) proposed a test statistic for nonnested models which is well suited for situations where the distributions are specified. The statistic for each observation $i$ is calculated as

$$
m_{i}=\log \left(\frac{f_{1}\left(y_{i} \mid X_{i}\right)}{f_{2}\left(y_{i} \mid X_{i}\right)}\right)
$$

where $f_{1}\left(y_{i} \mid X_{i}\right)$ and $f_{2}\left(y_{i} \mid X_{i}\right)$ are the probability density functions of models 1 and 2 respectively. Vuong's statistic for testing the non-nested hypothesis of model 1 versus model 2 is given as (Shankar et al., 1997), 


$$
V=\frac{\sqrt{n}\left[\left(\frac{1}{n}\right) \sum_{i=1}^{n} m_{i}\right]}{\sqrt{\left(\frac{1}{n}\right) \sum_{i=1}^{n}\left(m_{i}-\bar{m}\right)^{2}}}=\frac{\sqrt{n}(\bar{m})}{S_{m}}
$$

where $\bar{m}$ is the mean, $S_{m}$ is the standard deviation, and $\mathrm{n}$ is the sample size. Vuong's statistic follows the standard normal distribution asymptotically; and if $|V|$ is less than $V_{\text {critical }}\left(\mathrm{z}_{0.95}=\right.$ 1.96 for a $95 \%$ confidence level), the test is inconclusive. If $V>V_{\text {critical }}$, then the test favors model 1 . Vice versa, model 2 is preferred to model 1 if $V<-V_{\text {critical }}$.

Using Vuong's test and t-statistic of overdispersion parameter $(\alpha)$, Shankar et al. (1997) proposed a guideline to help select the appropriate model among Poisson, negative binomial, zero-inflated Poisson, and zero-inflated negative binomial models. The guidelines for a 95\% confidence level are given in Table 5-6.

Table 5-6: Model-Selection Decision Guidelines at 95\% Confidence Level using Vuong Statistic and Overdispersion

\begin{tabular}{cccc}
\hline & & $\begin{array}{c}\text { t-statistic of the negative binomial } \\
\text { overdispersion parameter }(\alpha)\end{array}$ \\
\cline { 2 - 4 } & $<-1.96$ & ZIP or Poisson & $>|1.96|$ \\
\hline Vuong statistic for comparison & $<|1.96|$ & NB \\
between ZINB $\left(f_{1}\right)$ and NB $\left(f_{2}\right)$ & $>1.96$ & ZIP & ZINB \\
\hline
\end{tabular}

\subsubsection{PET Counts versus Right-Angle Crash Counts}

\subsubsection{Analysis by Sites}

First, we produced scatter plots to visually examine the relationship between the PET counts and actual crash counts. PET counts are specific to the predetermined threshold. The scatter plots of eight-hour PET counts versus four-year crash counts in Figure 5-2 are for PET $\leq 6.5$ seconds. Different thresholds were tested and were found to produce similar trends. Different conditions considered in counting crashes are shown in each quadrant of the plot. The trends suggest no 
significant difference whether all crashes or specific conditions such as daytime only are used. Hence, daytime crash counts were considered for our subsequent analysis. This choice represents a reasonable compromise between the adequacy of field conditions during data collection and the attempt to use as many crash counts as possible to increase the confidence of results.
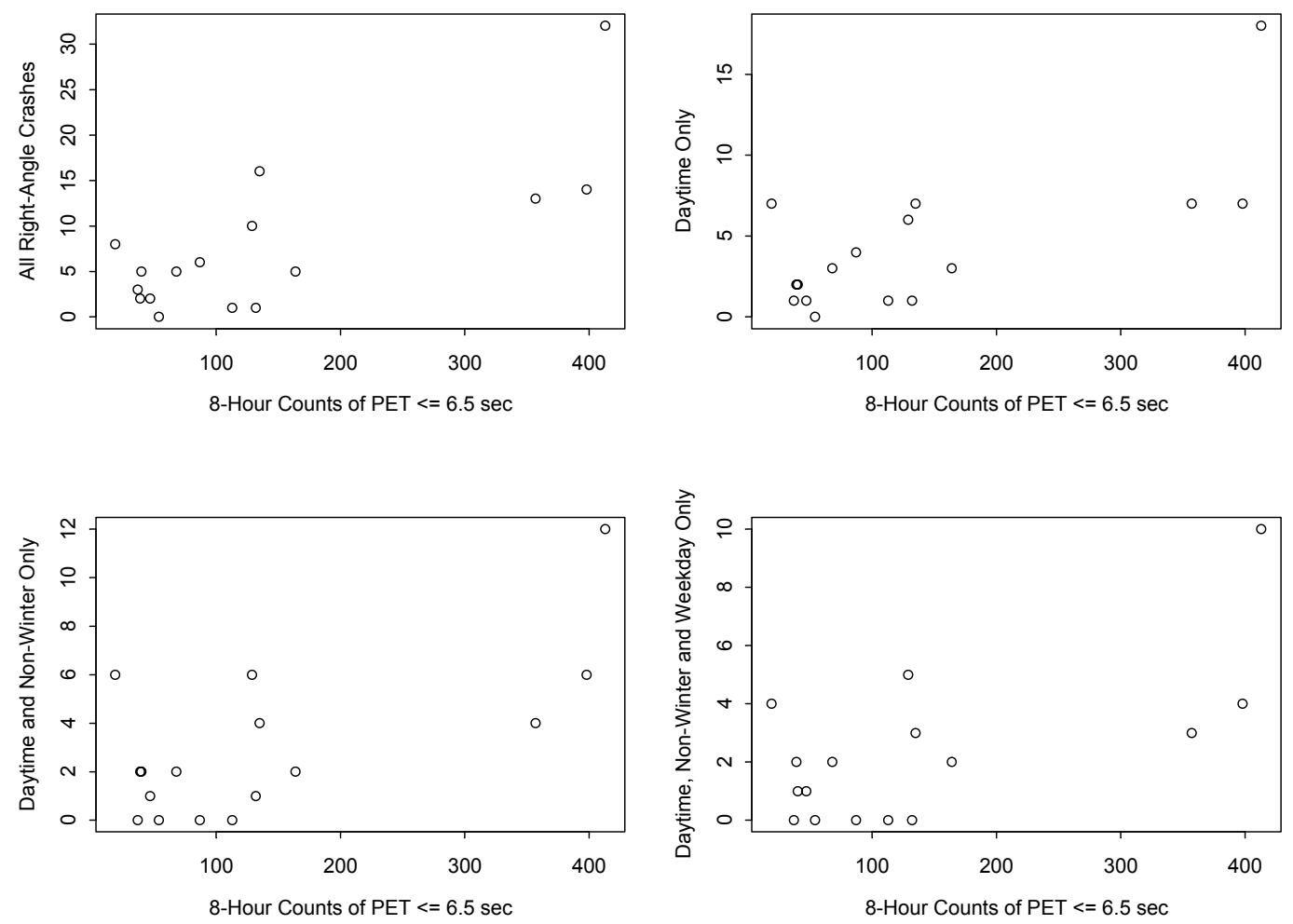

Figure 5-2: Scatter Plots of 4-Year Crash Counts Versus 8-Hour PET Counts

An important observation can be made here that a short period of PET observation may be sufficiently representative for the entire period of crash counting. The crash counting period includes periods with conditions different from those in the PET observation period. It could be supported by the fact that the overdispersion parameter of crash counts does not change significantly between different conditions despite changing means (see Table 5-1).

Using daytime crash counts as a response variable, a series of negative binomial regression models was fitted to PET counts at thresholds varying from eight seconds to one second at 0.5 second spacings. The model goodness-of-fit was evaluated in terms of the t-ratio of the estimated 
coefficients, the likelihood ratio test statistic, and the $\rho^{2}$-statistic for overall model fit. The 6.5second threshold was found to give the best fitted models when the count data were grouped by sites. The fitted negative binomial model is shown in Table 5-7. The log-linear link structure is given as

$$
\lambda_{i}=\exp \left(\beta_{0}+\beta_{1} f_{P E T, i}+\varepsilon_{i}\right)
$$

where $f_{P E T, i}$ is the 8-hour counts of PET less than the specified threshold at location $i$ and $\varepsilon_{i}$ is a gamma-distributed error term.

Table 5-7: Negative Binomial Model of PET Counts by Sites

Negative Binomial Regression

$Y=4$-Year Daytime Counts of Right-Angle Collisions

\begin{tabular}{lcc}
\hline Independent Variable & $\begin{array}{c}\text { Estimated } \\
\text { Coefficient }\end{array}$ & t-ratio \\
\hline Constant & 0.7098 & 3.070 \\
8-Hour Counts of PET $\leq 6.5 \mathrm{sec}$ & 0.0042 & 3.563 \\
Overdispersion, $(\alpha=1 / \theta)$ & 0.1699 & 0.741 \\
& & \\
\hline Number of Observations & 16 & \\
Restricted Log-likelihood (Poisson assumption) & -51.910 & \\
Restricted Log-likelihood (NB assumption) & -40.801 & \\
Log-likelihood at convergence & -36.025 & \\
$\chi^{2}$-statistic and associated p-value & 9.551 & 0.002 \\
$\rho^{2}$-statistic & 0.117 & \\
\hline
\end{tabular}

Table 5-8: Poisson Regression Model of PET Counts by Sites

\begin{tabular}{lcc}
$\begin{array}{l}\text { Poisson Regression } \\
\text { Y }=4 \text {-Year Daytime Counts of Right-Angle Collisions }\end{array}$ & \\
\hline Independent Variable & $\begin{array}{c}\text { Estimated } \\
\text { Coefficient }\end{array}$ & t-ratio \\
\hline Constant & 0.6894 & 3.216 \\
8-Hour Counts of PET $\leq 6.5 \mathrm{sec}$ & 0.0043 & 5.590 \\
& & \\
\hline Number of Observations & 16 & \\
Restricted Log-likelihood & -51.910 & \\
Log-likelihood at convergence & -37.329 & \\
$X^{2}$-statistic and associated p-value & 29.161 & $<0.0000001$ \\
$\rho^{2}$-statistic & 0.281 & \\
\hline
\end{tabular}

The low t-ratio of the estimated overdispersion parameter in Table 5-7 suggests no evidence of overdispersion in the data set and that the Poisson regression model may be allowed. Hence, the 
same set of variables in Table 5-7 is fitted to the Poisson regression model as shown in Table 5-8. The comparison of log-likelihoods at the convergence between both models indicates only a marginal improvement from the Poisson model. The corresponding deviance statistic is 2($36.025+37.329)=2.608 \sim \chi_{d f=1}^{2}$. The overdispersion in the negative binomial model is therefore not statistically significant at a 95\% confidence level. In addition, the goodness-of-fit statistics shown in the Table 5-8 indicates that the Poisson regression model may be allowed in this case.

The positive coefficient estimate of the PET counts implies that an intersection with higher PET counts is likely to experience more right-angle collisions. The positive estimate of the constant implies a tendency to experience right-angle collisions at intersections despite zero PET counts. This constant captures the variability of crash counts unexplained by the PET counts alone.

\subsubsection{Analysis by Conflict Zones}

The count data are split into four different conflict zones to build the models at a disaggregate level. PET counts that belong to a conflict zone of interest are treated as an explanatory variable. The relationship between the PET counts and four-year daytime right-angle crash counts by conflict zones is plotted in Figure 5-3.

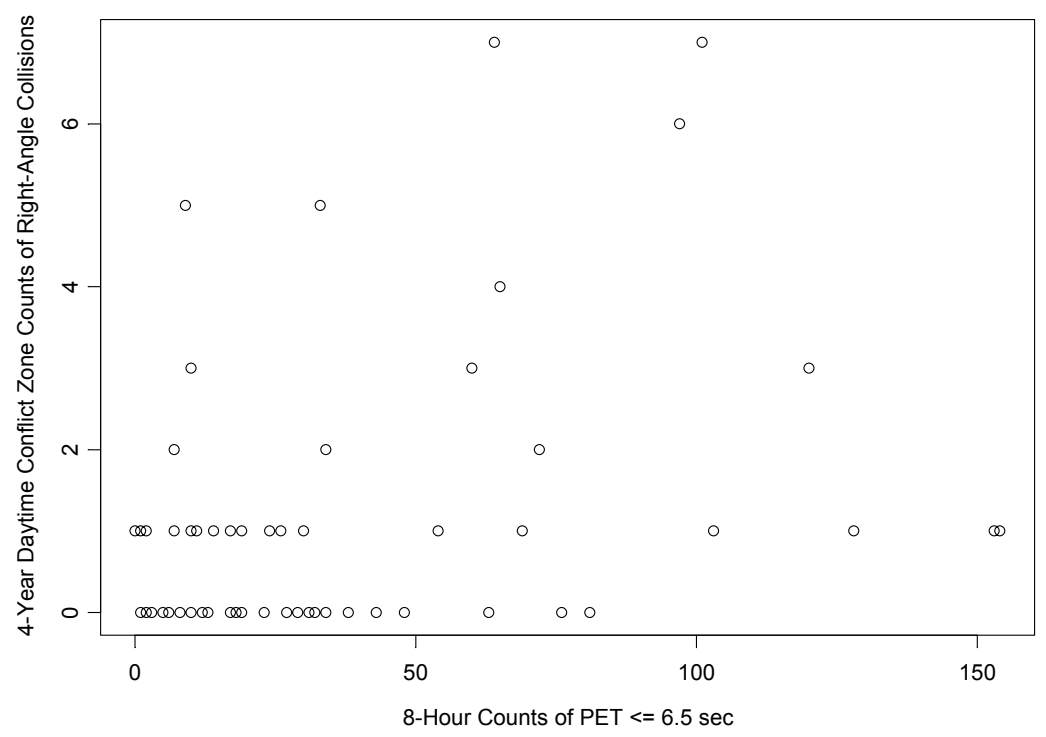

Figure 5-3: Conflict Zone Counts of Right-Angle Collisions versus PET Counts 
It is interesting to note that there are a number of zero counts in the data set when the counts are broken down into different conflict zones. The count process of right-angle collisions is more irregular at a disaggregate level and the risk of collisions at certain conflict zones may be extremely low, such that it may be treated as a zero-risk situation. This leads to the possibility of modeling the crash counts by conflict zones using a two-state count process: normal-count and zero-count states. Therefore, zero-inflated models (Poisson or negative binomial) may be taken into consideration in addition to standard Poisson and negative binomial regression models.

The negative binomial model is estimated using the same log-linear link structure as in equation (5-20). Note that the eight-hour count of PETs is for individual conflict zones in this case, not the entire intersection. Table 5-9 summarizes the estimated negative binomial model. The estimated overdispersion of 0.934 with the corresponding t-ratio of 2.014 indicates that the data are overdispersed and the negative binomial model is an appropriate choice in this case.

Table 5-9: Negative Binomial Model of PET Counts by Conflict Zones

Negative Binomial Regression

$Y=4-Y e a r$ Daytime Conflict Zone Counts of Right-Angle Collisions

\begin{tabular}{lcc}
\hline Independent Variable & $\begin{array}{c}\text { Estimated } \\
\text { Coefficient }\end{array}$ & t-ratio \\
\hline Constant & -0.3908 & -1.406 \\
8-Hour Counts of PET $\leq 6.5 \mathrm{sec}$ & 0.0117 & 2.464 \\
Overdispersion, $(\alpha=1 / \theta)$ & 0.9343 & 2.014 \\
& & \\
\hline Number of Observations & 61 \\
Restricted Log-likelihood (Poisson assumption) & -104.204 & \\
Restricted Log-likelihood (NB assumption) & -90.361 & \\
Log-likelihood at convergence & -87.054 & \\
$\chi^{2}$-statistic and associated p-value & 6.615 & 0.010 \\
$\rho^{2}$-statistic & 0.037 & \\
\hline
\end{tabular}

A zero-inflated negative binomial model is also estimated to check whether the preponderance of zero counts in the data set can be justified by a two-state count process. The logic behind the zero-inflated models was described earlier in Section 5.5.1.3. The estimated zero-inflated model using a logistic distribution splitting rule is shown in Table 5-10.

The t-ratios of the estimated model coefficients of the zero-inflated model are not statistically significant. The Vuong statistic is also used to compare between the negative binomial and zeroinflated models. The computed Vuong statistic is -0.707 , which indicates no evidence to favor the zero-inflated model. The crash occurrences at each conflict zone are therefore adequately 
explained by a normal-count process. This finding also allows the aggregation of counts from all conflict zones at the same intersection. In other words, there is no evidence of a zero-count state at a conflict-zone level, thus, the aggregation of counts from all conflict zones in the site model (e.g., Section 5.5.2.1) is sufficiently explained by a normal-count process. The standard Poisson and negative binomial regression models are sufficient for the modeling of crash counts using this data set.

Table 5-10: Zero-Inflated Negative Binomial Model of PET Counts by Conflict Zones Zero-Inflated Negative Binomial Regression Y = 4-Year Daytime Conflict Zone Counts of Right-Angle Collisions

\begin{tabular}{lcc}
\hline Independent Variable & $\begin{array}{c}\text { Estimated } \\
\text { Coefficient }\end{array}$ & t-ratio \\
\hline Negative Binomial Count State & & \\
Constant & 0.1376 & 0.439 \\
8-Hour Counts of PET $\leq 6.5 \mathrm{sec}$ & 0.0057 & 1.800 \\
Overdispersion, ( $\alpha=1 / \theta)$ & 0.6709 & 0.787 \\
Zero-Count State & & \\
Constant & -5.1422 & -0.421 \\
& & \\
\hline Number of Observations & 61 & \\
Log-Likelihood at Convergence (Poisson) & -97.348 & \\
Log-Likelihood at Convergence (Negative Binomial) & -87.054 & \\
Log-Likelihood at Convergence (ZINB) & -88.555 & \\
Poisson Zeros (Actual/Predicted) & $28 / 21.2$ & \\
Negative Binomial Zeros (Actual/Predicted) & $28 / 29.5$ & \\
ZINB Zeros (Actual/Predicted) & $28 / 28.2$ & \\
Vuong Statistic & -0.707 & \\
\hline
\end{tabular}

\subsubsection{Traffic Volume versus Right-Angle Crashes}

Traffic volume is the exposure commonly used in safety modeling and has been shown to be statistically significant in numerous regression studies of crash frequency. However, the significance of traffic volume may diminish for a specific type of counts, such as right-angle crashes. Affum and Ap Taylor (1996) found that right-angle accidents are poorly explained by exposure data such as traffic volume. Contributing factors for right-angle crashes may be present only for a specific time of day; for example, run-on-red violations of through vehicles without the separating effect of left-turn phases may occur during periods of low turning volumes at actuated signals. Traffic volume is unable to capture these conditions. 
We will examine whether the traffic volume alone can explain a variability of right-angle crash counts. Two types of models are considered as in the PET-based models: site models and conflict zone models.

\subsubsection{Analysis by Sites}

First, we visually examine the relationship between the volume and crash counts from the plots in Figure 5-4. The square root of products of crossing flows, $\mathrm{V}_{\mathrm{xflows}}$, is defined as

$$
\mathrm{V}_{\text {xflows }}=\sqrt{\mathrm{V}_{\text {major }} \mathrm{V}_{\text {minor }}}
$$

where $\mathrm{V}_{\text {major }}$ is the hourly average through traffic on the major street and $\mathrm{V}_{\text {minor }}$ is the hourly average through traffic on the minor street.
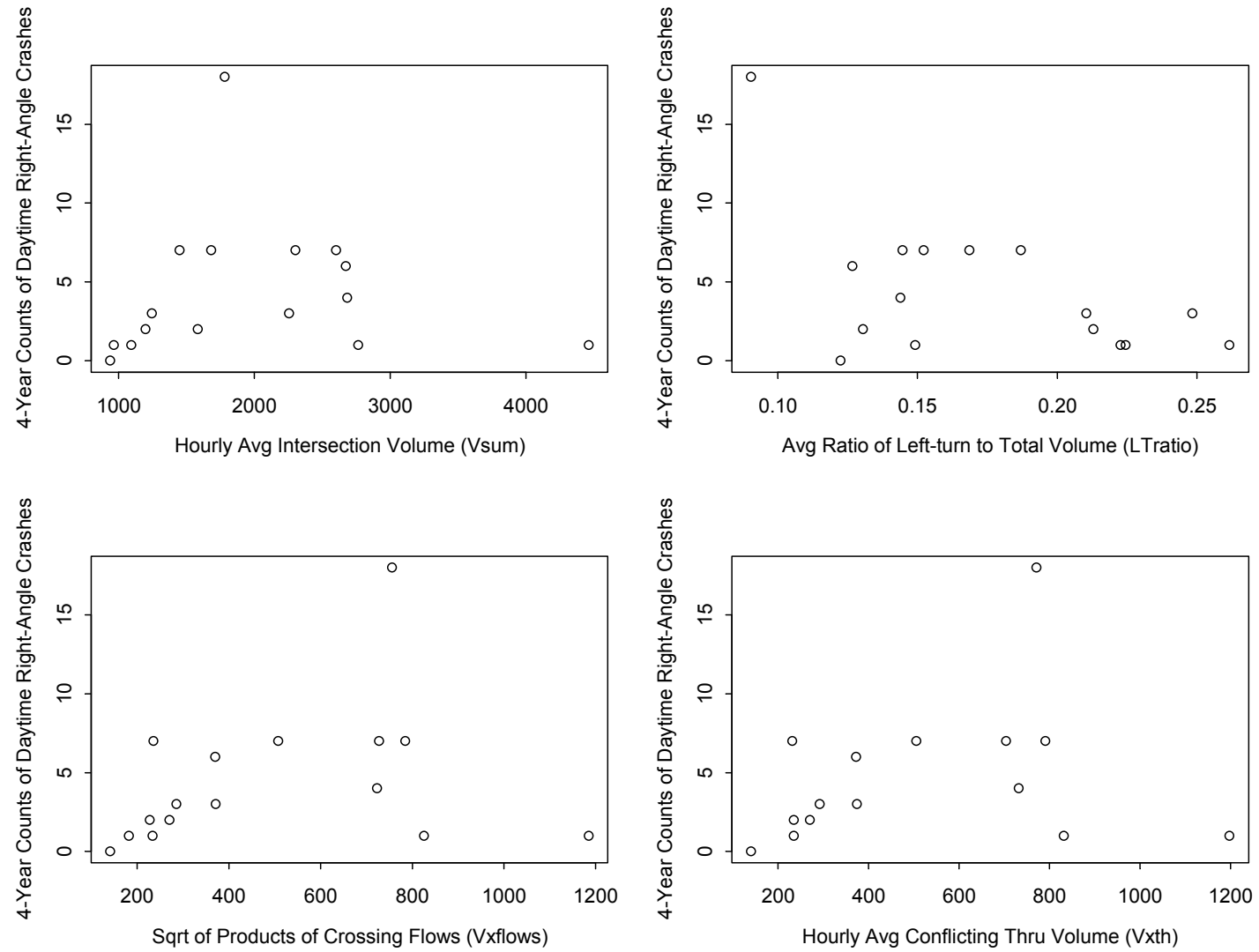

Figure 5-4: Scatter Plots of Volume Information versus Actual Counts of Crashes 
The trend between the hourly average intersection volume $\left(\mathrm{V}_{\text {sum }}\right)$ and the crash counts is very weak. Slightly increasing trends are observed for the hourly average conflicting through volumes $\left(\mathrm{V}_{\mathrm{xth}}\right)$ and the square root of products of crossing flows $\left(\mathrm{V}_{\mathrm{xflows}}\right) . \mathrm{V}_{\mathrm{xflows}}$ is a simplified indicator of the degree of crossing interactions alternative to $V_{x t h} . V_{\text {sum }}$ and $V_{x t h}$ were defined earlier in equations (5-2) and (5-5) respectively. A slightly decreasing trend is noticeable for the average ratio of left-turn to total volume (LTratio).

Four different model structures using volume were investigated, of which are summarized below. Model structure 1 is modified from a typical highway safety performance function where the average hourly traffic volumes on the major street $\left(\mathrm{V}_{\text {major }}\right)$ and the minor street $\left(\mathrm{V}_{\text {minor }}\right)$ are used in place of the major-street and minor-street AADTs respectively. This product form implies a meaningful and intuitive physical interpretation since the expected mean of right-angle collisions is equal to zero if there is no traffic on either one of the streets. The model structure 2 is similar to structure 1 but it assigns equivalent weights to both the $\mathrm{V}_{\text {major }}$ and $\mathrm{V}_{\text {minor }}$. The model structure 3 takes a sum of products of crossing flows for all four conflict zones. This sum of crossing flow products aims to represent the amount of crossing interactions an intersection is experiencing. The last model structure is similar to structure 2 but all the explanatory variables are in the exponent term.

Structure 1:

$$
\begin{aligned}
\lambda_{i} & =\beta_{0} V_{\text {major }}^{\alpha_{1}} V_{\text {minor }}^{\alpha_{2}} \exp \left(\beta_{1}(\text { LTratio })\right), \text { or } \\
\log \lambda_{i} & =\log \beta_{0}+\alpha_{1} \log V_{\text {major }}+\alpha_{2} \log V_{\text {minor }}+\beta_{1}(\text { LTratio })
\end{aligned}
$$

Structure 2:

$$
\begin{aligned}
\lambda_{i} & =\beta_{0}\left(\sqrt{V_{\text {major }} V_{\text {minor }}}\right)^{\alpha_{1}} \exp \left(\beta_{1}(\text { LTratio })\right), \text { or } \\
\log \lambda_{i} & =\log \beta_{0}+\alpha_{1} \log \sqrt{V_{\text {major }} V_{\text {minor }}}+\beta_{1}(\text { LTratio })
\end{aligned}
$$

Structure 3:

$$
\begin{aligned}
\lambda_{i} & =\beta_{0}\left(V_{x t h}\right)^{\alpha_{1}} \exp \left(\beta_{1}(\text { LTratio })\right), \text { or } \\
\log \lambda_{i} & =\log \beta_{0}+\alpha_{1} \log V_{x t h}+\beta_{1} \text { (LTratio) }
\end{aligned}
$$


Structure 4:

$$
\begin{aligned}
\lambda_{i} & =\exp \left(\beta_{0}+\beta_{1} \sqrt{V_{\text {major }} V_{\text {minor }}}+\beta_{2}(\text { LTratio })\right), \text { or } \\
\log \lambda_{i} & =\beta_{0}+\beta_{1} \sqrt{V_{\text {major }} V_{\text {minor }}}+\beta_{2}(\text { LTratio })
\end{aligned}
$$

The ratio of the left-turn volume to the total intersection volume (LTratio) is considered in all structures as it consistently improves the model goodness-of-fit. Through-traffic volumes were found to give a better model fit than approach-traffic volumes (all movements) for all the considered structures. Model structure 2 outperforms the other structures in terms of the t-ratios of estimated coefficients and the overall goodness-of-fit of the models. The constant term is neglected in the fitted models as its t-ratio is not statistically significant. The fitted negative binomial regression model using model structure 2 is shown in Table 5-11.

Table 5-11: Negative Binomial Model of Volume Data by Sites

Negative Binomial Regression

\begin{tabular}{|c|c|c|}
\hline Independent Variable & $\begin{array}{l}\text { Estimated } \\
\text { Coefficient }\end{array}$ & t-ratio \\
\hline $\begin{array}{l}\text { Log of (Average Hourly Thru Traffic on Major Road } \times \\
\text { Average Hourly Thru Traffic on Minor Road) }\end{array}$ & 0.473 & 4.302 \\
\hline Ratio of left-turn volume to total intersection volume & -8.797 & -2.222 \\
\hline Overdispersion, $(\alpha=1 / \theta)$ & 0.244 & 1.111 \\
\hline Number of Observations & 16 & \\
\hline Restricted Log-likelihood (Poisson assumption) & -51.910 & \\
\hline Restricted Log-likelihood (NB assumption) & -40.801 & \\
\hline Log-likelihood at convergence & -36.850 & \\
\hline$x^{2}$-statistic and associated $p$-value & 7.902 & 0.019 \\
\hline$\rho^{2}$-statistic & 0.097 & \\
\hline
\end{tabular}
$\mathrm{Y}=4$-Year Daytime Counts of Right-Angle Collisions

The estimated overdispersion parameter is slightly greater than zero, which indicates that the data is only slightly overdispersed. The t-ratio of the overdispersion parameter is 1.111 ; this implies that the Poisson regression model may be allowed as in the case of the PET-based model. Therefore, the data set is re-estimated using Poisson regression and the results are given in Table 5-12. It should be noted that the Poisson regression model generally provided a higher confidence of model estimates as well as better overall fit of the model because the overdispersion is not allowed in the model $(\alpha=0)$. A similar trend was also observed in the estimated Poisson regression model for the PET-based model. 
Table 5-12: Poisson Regression Model of Volume Data by Sites

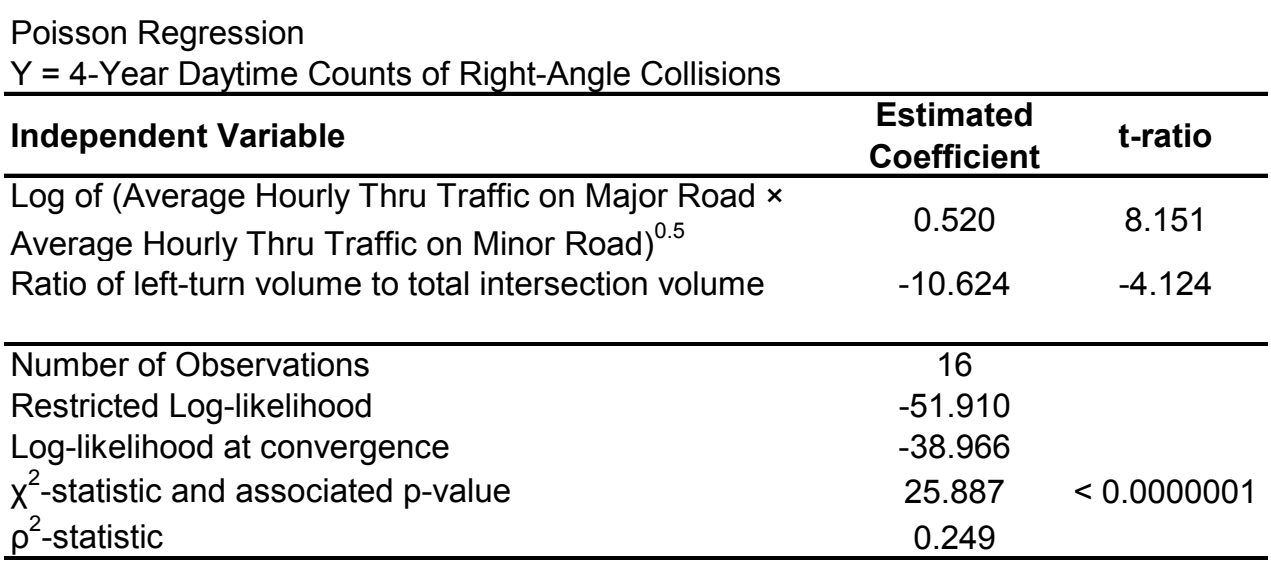

\subsubsection{Analysis by Conflict Zones}

The intersection data were broken down into different conflict zones for each intersection. As shown below, two potential model structures were considered in this case. Both model structures incorporate the product of crossing flows into the models. Model structure 1 assigns different weights on each flow, whereas model structure 2 assumes no difference between these two crossing flows. $V_{i}$ and $V_{j}$ are crossing through-traffic volumes at a conflict zone of interest. For example, northbound through-traffic $\left(\mathrm{V}_{\mathrm{NBTH}}\right)$ and eastbound through-traffic $\left(\mathrm{V}_{\mathrm{EBTH}}\right)$ are considered for the conflicts between the northbound and eastbound traffic flows.

Structure 1:

$$
\begin{aligned}
\lambda_{i} & =\beta_{0} V_{i}^{\alpha_{1}} V_{j}^{\alpha_{2}} \exp \left(\beta_{1}(\text { LTratio })\right), \text { or } \\
\log \lambda_{i} & =\log \beta_{0}+\alpha_{1} \log V_{i}+\alpha_{2} \log V_{j}+\beta_{1}(\text { LTratio }) \\
V_{i} & >V_{j}
\end{aligned}
$$

Structure 2:

$$
\begin{aligned}
\lambda_{i} & =\beta_{0}\left(\sqrt{V_{i} V_{j}}\right)^{\alpha_{1}} \exp \left(\beta_{1}(\text { LTratio })\right), \text { or } \\
\log \lambda_{i} & =\log \beta_{0}+\alpha_{1} \log \sqrt{V_{i} V_{j}}+\beta_{1} \text { (LTratio) }
\end{aligned}
$$


Both model structures were tested and model structure 2 is preferred to the model structure 1 , which was not unexpected since we have no basis on which to claim that the effect of each crossing flow on the crash frequency estimate should differ from one another.

Using model structure 2, Table 5-13 summarizes the estimated negative binomial regression model using a conflict-zone data. The constant term was dropped from the model since the t-ratio is not statistically significant. The overdispersion parameter estimate is far from zero and the tratio is statistically significant. This indicates overdispersion in the data set and the negative binomial model is therefore appropriate in this case. The positive coefficient estimate of the product of the crossing flows implies that more right-angle crashes can be expected with the increase in the amount of crossing interactions at a conflict zone. The negative coefficient estimate of the left-turn ratio suggests that intersections with a higher proportion of left-turn volumes are less prone to right-angle collisions, which could be anticipated because the traffic controls at intersections with heavy left-turn volume are usually assigned with a protected leftturn phase and this could lead to a significant reduction in the amount of crossing maneuvers between through-traffic.

Table 5-13: Negative Binomial Model of Volume Data by Conflict Zones Negative Binomial Regression Y = 4-Year Daytime Conflict Zone Counts of Right-Angle Collisions

\begin{tabular}{|c|c|c|}
\hline Independent Variable & $\begin{array}{l}\text { Estimated } \\
\text { Coefficient }\end{array}$ & t-ratio \\
\hline $\begin{array}{l}\text { Log of (Product of Average Hourly Thru Traffic Volume } \\
\left.\text { from Interested Approaches }{ }^{*}\right)^{0.5}\end{array}$ & 0.315 & 3.137 \\
\hline Ratio of left-turn volume to total intersection volume & -9.758 & -3.106 \\
\hline Overdispersion, $(\alpha=1 / \theta)$ & 0.778 & 1.930 \\
\hline Number of Observations & 61 & \\
\hline Restricted Log-likelihood (Poisson assumption) & -104.204 & \\
\hline Restricted Log-likelihood (NB assumption) & -90.361 & \\
\hline Log-likelihood at convergence & -85.576 & \\
\hline$x^{2}$-statistic and associated $p$-value & 9.570 & 0.008 \\
\hline$\rho^{2}$-statistic & 0.053 & \\
\hline
\end{tabular}

Zero-inflated negative binomial regression was also tested. The corresponding Vuong statistic for comparison between the NB and ZINB models is -0.8973 which indicates that the test is inconclusive. This is also consistent with the earlier findings in Section 5.5.2.2. 


\subsubsection{Combining PET and Volume Data}

In the last scenario, we combined the PET and volume data to estimate the models. Site and conflict zone models were established as in previous cases. We adopted the same volume structure as in the volume-based models for the combined-data model. The variable indicating the PET counts is then added into the exponent term of the models to combine these two types of data.

\subsubsection{Analysis by Sites}

The negative binomial was first estimated in this case. The t-ratio of the overdispersion parameter reveals that overdispersion was not a problem and the model could be reduced to the Poisson regression model. The estimated negative binomial and Poisson regression models are shown in Table 5-14 and Table 5-15 respectively.

The signs of the coefficient estimates are consistent with the findings in the PET-based and volume-based models. The combination of both data still produces coefficient estimates with statistically significant t-statistics; this implies a potential synergy in the explanatory power when both the PET data and the volume are combined. The PET carries additional safety information which is unexplained by traffic volume alone.

Table 5-14: Negative Binomial Model using PET and Volume Data by Sites Negative Binomial Regression Y=4-Year Daytime Counts of Right-Angle Collisions

\begin{tabular}{lcc}
\hline Independent Variable & $\begin{array}{c}\text { Estimated } \\
\text { Coefficient }\end{array}$ & t-ratio \\
\hline Log of (Average Hourly Thru Traffic on Major Road $\times$ & 0.2940 & 1.831 \\
Average Hourly Thru Traffic on Minor Road) & 0.5 & -1.018 \\
Ratio of left-turn volume to total intersection volume & -5.3006 & 2.091 \\
8-hour Counts of PET $\leq 6.5$ seconds & 0.0029 & 0.292 \\
Overdispersion, $(\alpha=1 / \theta)$ & 0.0420 & \\
& & \\
\hline Number of Observations & 16 & \\
Restricted Log-likelihood (Poisson assumption) & -51.910 & \\
Restricted Log-likelihood (NB assumption) & -40.801 & \\
Log-likelihood at convergence & -34.524 & \\
$X^{2}$-statistic and associated p-value & 12.555 & \\
$\rho^{2}$-statistic & 0.006 \\
\hline
\end{tabular}


Table 5-15: Poisson Regression Model using PET and Volume Data by Sites

Poisson Regression

$\mathrm{Y}=4$-Year Daytime Counts of Right-Angle Collisions

\begin{tabular}{|c|c|c|}
\hline Independent Variable & $\begin{array}{l}\text { Estimated } \\
\text { Coefficient }\end{array}$ & t-ratio \\
\hline $\begin{array}{l}\text { Log of (Average Hourly Thru Traffic on Major Road } \times \\
\text { Average Hourly Thru Traffic on Minor Road) }\end{array}$ & 0.3086 & 3.270 \\
\hline Ratio of left-turn volume to total intersection volume & -5.7341 & -2.024 \\
\hline 8-hour Counts of PET $\leq 6.5$ seconds & 0.0028 & 2.917 \\
\hline $\begin{array}{l}\text { Number of Observations } \\
\text { Restricted Log-likelihood } \\
\text { Log-likelihood at convergence } \\
x^{2} \text {-statistic and associated p-value } \\
\rho^{2} \text {-statistic }\end{array}$ & $\begin{array}{c}16 \\
-51.910 \\
-34.606 \\
34.608 \\
0.333 \\
\end{array}$ & $<0.0000001$ \\
\hline
\end{tabular}

\subsubsection{Analysis by Conflict Zones}

Using both the PET and volume data, the right-angle crash counts for each conflict zone were modeled using the negative binomial regression. The modeling results are given in Table 5-16.

Table 5-16: Negative Binomial Model using PET and Volume Data by Conflict Zones Negative Binomial Regression

$\mathrm{Y}=4$-Year Daytime Conflict Zone Counts of Right-Angle Collisions

\begin{tabular}{lcc}
\hline Independent Variable & $\begin{array}{c}\text { Estimated } \\
\text { Coefficient }\end{array}$ & t-ratio \\
\hline Log of (Product of Average Hourly Thru Traffic Volume & 0.2062 & 1.594 \\
from Interested Approaches $\left.{ }^{0}\right)^{0.5}$ & -7.8554 & -2.297 \\
Ratio of left-turn volume to total intersection volume & 0.0064 & 1.262 \\
8-hour Counts of PET $\leq 6.5$ seconds & 0.7241 & 1.767 \\
Overdispersion, ( $\alpha=1 / \theta)$ & & \\
& & \\
\hline Number of Observations & -104.204 & \\
Restricted Log-likelihood (Poisson assumption) & -90.361 & \\
Restricted Log-likelihood (NB assumption) & -84.687 & \multirow{2}{*}{0.010} \\
Log-likelihood at convergence & 11.349 \\
$X^{2}$-statistic and associated p-value & 0.063 & \\
$\rho^{2}$-statistic & \\
${ }^{*}$ For example, use a product of NB thru traffic and EB thru traffic to predict the right- \\
angle crash frequency generated by NB versus EB crossing traffic
\end{tabular}

The signs of the coefficient estimates indicate the same findings as in the PET-based and volumebased models. The t-ratio of the overdispersion parameter indicates overdispersion in the data set and thus the negative binomial model was suitable. 


\subsection{CONCLUSIONS}

The characteristics of crash counts, traffic volume, PET, and ET were examined in this chapter. Then, the usefulness of PETs and traffic volume in explaining the variability of crash counts was checked. A summary of the selected regression models for PET-based, volume-based, and combined models with the values of log-likelihood at convergence is presented in Table 5-17.

Table 5-17: Summary of Log-Likelihoods at Convergence of Selected Models

\begin{tabular}{cccc}
\hline Model Type & $\begin{array}{c}\text { Response Variable: } \\
\text { Frequency of Right- } \\
\text { Angle Collisions }\end{array}$ & Selected Regression Approach & $\begin{array}{c}\text { Log-likelihood at } \\
\text { Convergence }\end{array}$ \\
\hline \multirow{2}{*}{ PET-based model } & $\begin{array}{c}\text { Site } \\
\text { Conflict Zone }\end{array}$ & Pogative Binomial Regression & -87.054 \\
\hline \multirow{2}{*}{ Volume-based model } & Site & Poisson Regression & -38.996 \\
& Conflict Zone & Negative Binomial Regression & -85.576 \\
\hline Combined data model & Site & Poisson Regression & -34.606 \\
& Conflict Zone & Negative Binomial Regression & -84.687 \\
\hline
\end{tabular}

To choose the appropriate model for the task, the model-selection guidelines are as follows:

- Use of a model depends on the data availability. For example, a PET-based model is preferred if the PET data is available. If both PET and traffic volume data are available, the combined data model can be applied.

- If the directional traffic count data or the PET counts for each conflict zone are available, the conflict zone model should be selected as it is capable of predicting the expected right-angle crash frequency at a more disaggregate level.

- For planning purpose or in cases where no detailed traffic data are available, the site model can be used to predict the expected crash frequency. For example, if only the AADTs of the major and minor streets are available, the traffic adjustment factor can be applied to the AADT to obtain the hourly average traffic volume during the daytime period. Then, the directional split factors from some known similar locations can be applied to obtain only the through-traffic volume. A left-turn ratio of 0.15 to 0.25 can be assumed if no other information is available; this approximation accounts for typical suburban signalized intersections.

The log-likelihood at convergence can be used to approximately compare the goodness-of-fit of the estimated models. A greater value of log-likelihood at convergence implies a better model fit. At the site level, the comparison of the log-likelihood at convergence of the Poisson regression 
model reveals that the PET-based model explains the variability in the count data slightly better than the volume-based model. On the other hand, the volume-based model is slightly better than the PET-based model at the conflict zone level. When both types of data are combined, the loglikelihood at convergence indicates that more variability in the data set is being explained in addition to the use of either PET or volume as a sole explanatory variable. In other words, there is an increase in the explanatory power of the model when both types of data are combined.

In the regression analysis, PET counts and traffic volume are viewed as exposure data. The positive coefficient estimates of PET counts, as well as the products of crossing flows correspond to the notion that the increase in exposure is likely to give rise to the expected right-angle crash frequency.

Based on the regression analysis results, we can conclude that the frequency of short PETs is a potential indicator in discriminating varying safety levels within a location (conflict zone models) and across locations (site models). The performance of PET as a surrogate measure of safety is equivalent or better than traffic-related data, but PET carries additional safety information unexplained by the traffic data alone. Hence, PET data can complement the traffic data in highway safety evaluation applications. There is a potential gain in having both types of data available in safety analysis tasks.

In addition, the most important benefit of PET lies in its continuous characteristic that exists during both crash-free operations as well as crash occurrences. Traffic volume data do not possess this characteristic. Based on the premise that a collision is defined as when the PET becomes less than zero, PET gives the possibility to build a safety model at individual sites using data from the site. The traditional regression approach needs to rely on the trend from a group of similar locations and model transferability. In the proposed approach, PET measures enable simultaneous estimation of collision risk and exposure, which can be converted into crash frequency straightforwardly. In the next chapter, we will outline a theory which provides a tool for estimating safety from PET data. 


\section{CHAPTER 6 EXTREME VALUE APPROACH FOR SAFETY ESTIMATIONS}

In order to obtain an individualized risk estimate and consequently a crash frequency estimate, we propose the extreme value theory (EVT) approach to model the variability of post-encroachment times. This chapter begins with the fundamentals of EVT and common applications. Different sampling schemes and resulting distributions considered in this study are discussed and the most suitable approach is selected. Then, we propose a method to estimate the models and predict the safety estimates. Discussions on alternatives to quantify the uncertainty of model estimates and selected techniques conclude the chapter.

\subsection{INTRODUCTION TO EXTREME VALUE THEORY}

Extreme value theory (EVT) has emerged as an important statistical discipline which has found its way to a wide range of applications. Some examples of these include alloy strength prediction, ocean wave modeling, wind engineering, thermodynamics of earthquakes, and assessment of meteorological change (Coles, 2001). The distinguishing feature of an extreme value analysis is the capability to model the stochastic behavior of the process that is unusually large or small in nature. This extreme behavior is typically very rare and unobservable within a reasonable data collection time period. The EVT often involves the challenge to estimate the probability of extreme events over an extended period of time given very short and limited historical data. The extreme value paradigm comprises a principle for model extrapolation based on the implementation of mathematical limits as finite-level approximations. The key implicit assumption of the EVT is that the underlying stochastic behavior of the process being modeled is sufficiently smooth to enable extrapolations to unobserved levels. No other credible alternative has been proposed to date.

EVT has been regularly applied in various fields such as meteorology, the financial market, the insurance business, structural reliability, and telecommunications. Examples of EVT applications include the prediction of annual maximum sea level, wave height modeling, wind speed 
prediction, investment return modeling, returns of exchange rates modeling, internet traffic congestion modeling, and the distribution of sizable claims in insurance business (Coles, 2001; Galambos et al., 1994; Reiss and Thomas, 1997).

We can classify sampling schemes in extreme value modeling into two approaches, time-based and event-based. In the time-based sampling scheme, a series of observations is blocked into fixed intervals over time and the block maxima (or minima) are treated as extremes. For the event-based sampling scheme, the observations are treated as extremes if a predetermined threshold is being exceeded. The resulting distributions from these sampling schemes, if applied properly, are independent of a true underlying distribution, typically unknown in practice.

There are several references available for the extreme value theory. In this report, we will closely follow mathematical formulations and proofs as presented in Coles (2001) and Leadbetter et al. (1983).

\subsection{TIME-BASED SAMPLING SCHEME}

The model focuses on the statistical behavior of

$$
M_{n}=\max \left\{X_{1}, \ldots, X_{n}\right\},
$$

where $X_{1}, \ldots, X_{n}$ is a sequence of independent random variables having a common distribution function $F$. In this sampling scheme, $X_{i}$ usually represents the values of a process measured on a regular time-scale so that $M_{n}$ represents the maximum of the process over $n$ time units of observation.

Theoretically, the exact distribution of $\mathrm{M}_{\mathrm{n}}$ can be derived for all values of $\mathrm{n}$ as

$$
\operatorname{Pr}\left\{M_{n} \leq z\right\}=\operatorname{Pr}\left\{X_{1} \leq z, \ldots, X_{n} \leq z\right\}=\{F(z)\}^{n}
$$

Since the true distribution of $\mathrm{F}$ is usually unknown in practice, the alternative approach is to focus on approximate families of models for $F_{n}$, which can be estimated on the basis of extreme data only. 


\subsubsection{Extremal Types Theorem}

If there exist sequences of constants $\left\{a_{n}>0\right\}$ and $\left\{b_{n}\right\}$ such that

$$
\operatorname{Pr}\left\{\left(M_{n}-b_{n}\right) / a_{n} \leq z\right\} \rightarrow G(z) \text { as } \mathrm{n} \rightarrow \infty,
$$

where $G$ is a non-degenerate distribution function, then $G$ belongs to one of the following families

$$
\begin{aligned}
& \mathrm{I}: G(z)=\exp \left\{-\exp \left[-\left(\frac{z-b}{a}\right)\right]\right\},-\infty<\mathrm{z}<\infty \\
& \mathrm{II}: G(z)= \begin{cases}0 & , \mathrm{z} \leq \mathrm{b} \\
\exp \left\{-\left(\frac{z-b}{a}\right)^{-\alpha}\right\}, & \mathrm{z}>\mathrm{b}\end{cases} \\
& \text { III: } G(z)=\left\{\begin{aligned}
\exp \left\{-\left[-\left(\frac{z-b}{a}\right)^{\alpha}\right]\right\} & , \mathrm{z}>\mathrm{b} \\
1 & , \mathrm{z} \geq \mathrm{b}
\end{aligned}\right.
\end{aligned}
$$

for parameters $\mathrm{a}>0, \mathrm{~b}$ and in case of families II and III, $\alpha>0$. These three classes of distributions are referred to as extreme value distributions, with type I, II, and III widely known as the Gumbel, Fréchet, and Weibull families respectively.

This theorem implies that, when $M_{n}$ can be stabilized with suitable sequences $\left\{\mathrm{a}_{\mathrm{n}}\right\}$ and $\left\{\mathrm{b}_{\mathrm{n}}\right\}$, the corresponding normalized variable $M_{n}^{*}$ has a limiting distribution that must be one of the three types of extreme value distribution.

\subsubsection{Generalized Extreme Value Model}

The three types of limits have distinct forms of behavior, corresponding to the different forms of tail behavior for the distribution function $\mathrm{F}$ of the $\mathrm{X}_{\mathrm{i}}$. For the Weibull distribution $\mathrm{Z}_{+}$is finite, while for both the Fréchet and Gumbel distributions $\mathrm{Z}_{+}=\infty$. The density of $\mathrm{G}$ decays exponentially for the Gumbel distribution and polynomially for the Fréchet distribution. 
All three types of limits can be combined into a single family of models having distribution functions of the form

$$
G(z)=\exp \left\{-\left[1+\xi\left(\frac{z-\mu}{\sigma}\right)\right]^{-1 / \xi}\right\}
$$

defined on the set $\{z: 1+\xi(z-\mu) / \sigma>0\}$, where the parameters satisfy $-\infty<\mu<\infty, \sigma>0$, and $-\infty<\xi<\infty$. Equation (6-1) is the generalized extreme value (GEV) distribution. The model has three parameters: $\mu$ (the location parameter), $\sigma$ (the scale parameter), and $\xi$ (the shape parameter). The type II and type III classes of extreme value distribution correspond respectively to the cases $\xi>0$ and $\xi<0$ in this parameterization. Three types of density function with the same location and scale parameter are plotted in Figure 6-1 for comparison. Note that $\mathrm{z}_{\text {- }}$ is finite for the Fréchet density while $\mathrm{z}_{+}$is finite for the Weibull density.

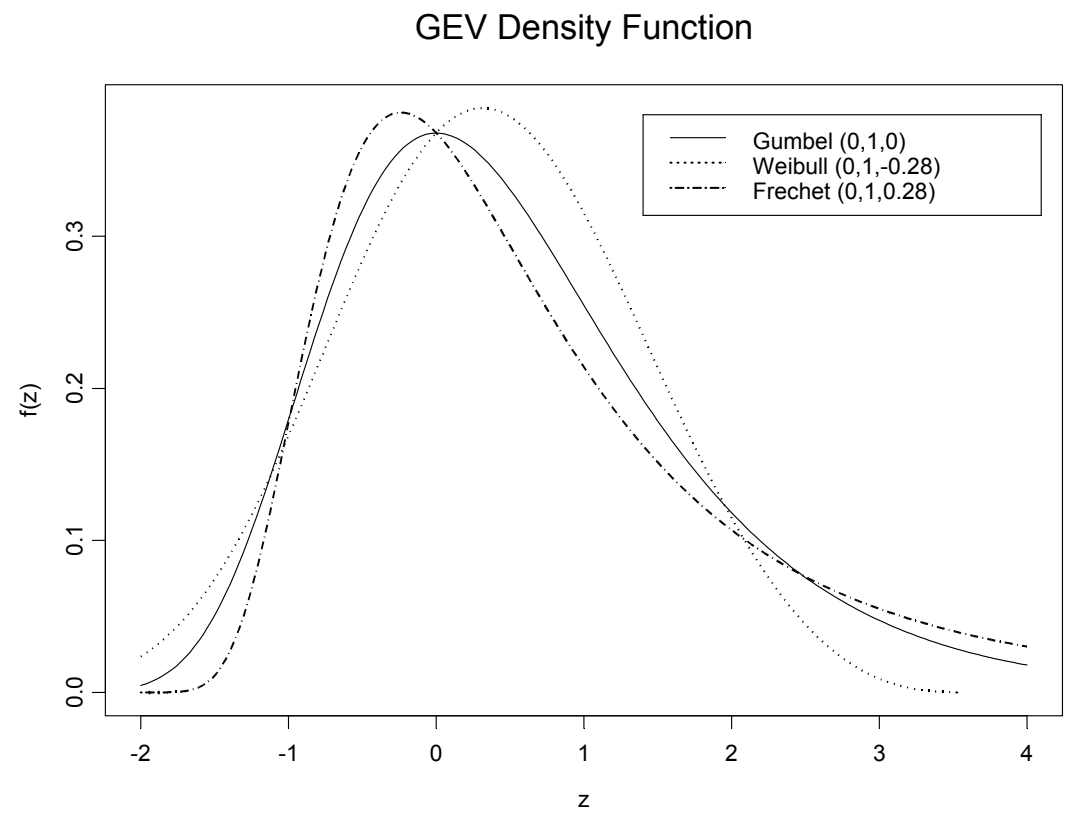

Figure 6-1: Density Functions of GEV Families

The subset of the GEV family with $\xi=0$ is interpreted as the limit of equation (6-1) as $\xi \rightarrow 0$, leading to the Gumbel family with distribution function 


$$
G(z)=\exp \left[-\exp \left\{-\left(\frac{z-\mu}{\sigma}\right)\right\}\right],-\infty<z<\infty .
$$

\section{Frechet Density Function}

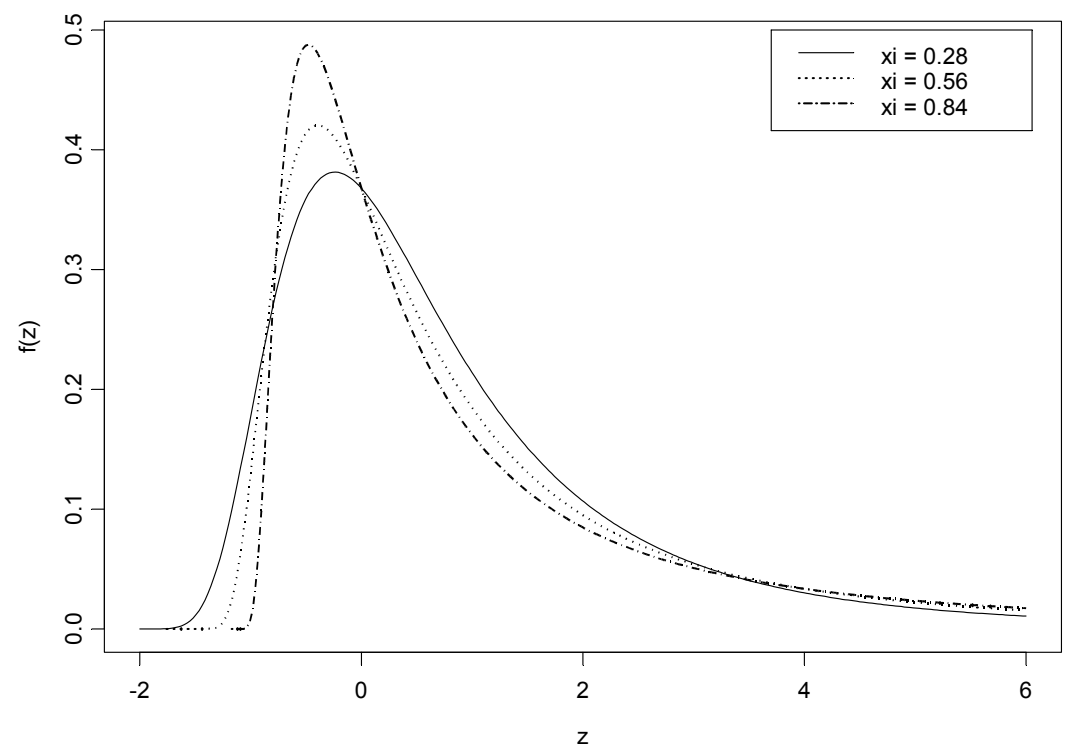

Figure 6-2: Behavior of Fréchet Density Function for Varying Shape Parameters

Weibull Density Function

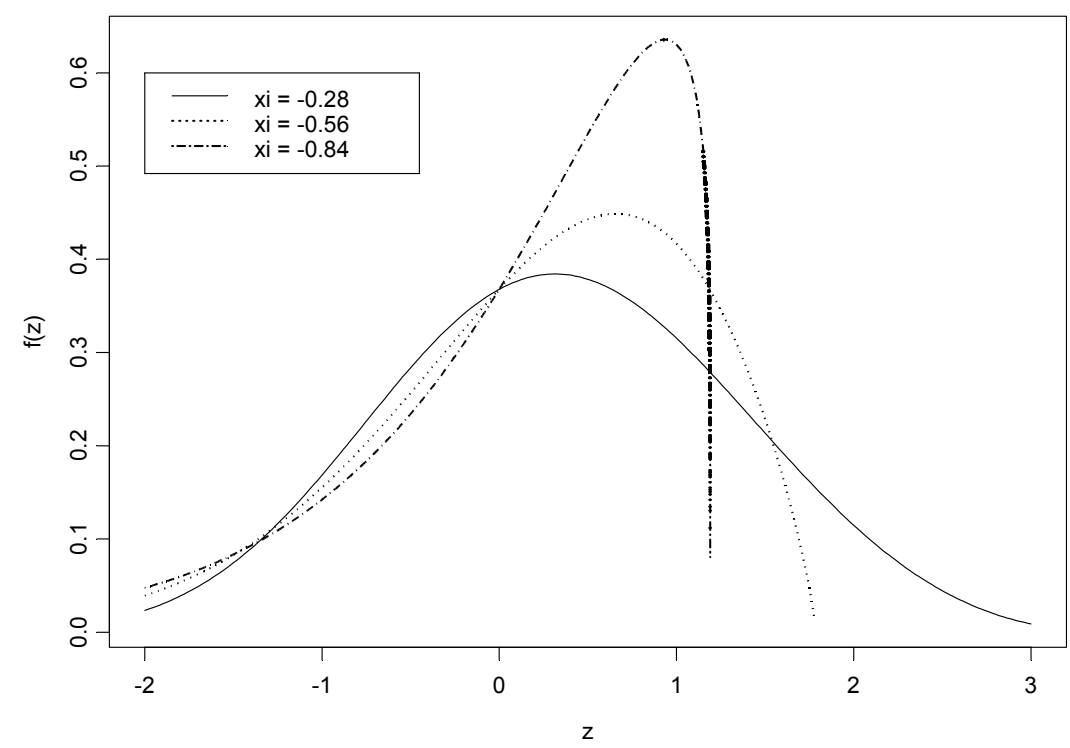

Figure 6-3: Behavior of Weibull Density Function for Varying Shape Parameters 
Figure 6-2 and Figure 6-3 illustrate the effects of different shape parameters on the tail behavior of the Fréchet and Weibull density functions respectively. For the Fréchet distribution $(\xi>0)$, the density function shifts to the left with the tighter lower bound and the heavier tail as $\xi$ increases. In contrast, the Weibull density function shifts to the right with a tighter upper bound as $\xi$ decreases.

The upper or lower limit of $z$, depending on the value of $\xi$, on which $G(z)$ is defined can be expressed as

$$
z_{\text {limit }}=\mu-\frac{\sigma}{\xi}
$$

\subsubsection{1 $\quad$ Return Level and Return Period}

To model the extremes of a series of independent observations $X_{1}, X_{2}, \ldots$, data are blocked into sequences of observations of length $n$, for some large value of $n$, generating a series of block maxima, $M_{n, 1}, \ldots, M_{n, m}$, to which the GEV distribution can be fitted. Estimates of the extreme quantile of the block maximum distribution are then obtained by inverting equations (6-1) and $(6-2)$ :

$$
z_{p}=\left\{\begin{array}{lc}
\mu-\frac{\sigma}{\xi}\left[1-\{-\log (1-p)\}^{-\xi}\right] & \text { for } \xi \neq 0 \\
\mu-\sigma \log \{-\log (1-p)\} & \text { for } \xi=0
\end{array},\right.
$$

where $G\left(z_{p}\right)=1-p$. In common terminology, $\mathrm{z}_{\mathrm{p}}$ is the return level associated with the return period $1 / \mathrm{p}$. In other words, the level $z_{\mathrm{p}}$ is expected to be exceeded, on average, once every $1 / \mathrm{p}$ time blocks. More precisely, the level $z_{p}$ is exceeded by the block maximum in any particular time block with probability $\mathrm{p}$.

To graph a return level plot, defining $y_{p}=-\log (1-p)$ so that 


$$
z_{p}= \begin{cases}\mu-\frac{\sigma}{\xi}\left[1-y_{p}^{-\xi}\right] & \text { for } \xi \neq 0 \\ \mu-\sigma \log y_{p} & \text { for } \xi=0\end{cases}
$$

It follows that, if $z_{p}$ is plotted against $y_{p}$ on a logarithmic scale - or equivalently, if $z_{p}$ is plotted against $\log y_{p}-$ the plot is linear in case $\xi=0$. If $\xi<0$, the plot is convex with the asymptotic limit as $p \rightarrow 0$ at $\mu-\sigma / \xi$. If $\xi>0$, the plot is concave and has no finite bound. The return level plot is useful in highlighting the effect of extrapolation at the tail of the distribution.

\subsubsection{2 $\quad$ Asymptotic Models for Minima}

Let $M_{n}^{0}=\min \left\{X_{1}, \ldots, X_{n}\right\}$, where $\mathrm{X}_{\mathrm{i}}$ denote each individual observation which is assumed to be independent and identically distributed. Letting $Y_{i}=-X_{i}$ for $\mathrm{i}=1, \ldots, \mathrm{n}$, the change of sign means that the small values of $\mathrm{X}_{\mathrm{i}}$ correspond to the large values of $\mathrm{Y}_{\mathrm{i}}$. Therefore, if $M_{n}^{0}=\min \left\{X_{1}, \ldots, X_{n}\right\}$ and $M_{n}^{0}=\max \left\{Y_{1}, \ldots, Y_{n}\right\}$, then $M_{n}^{0}=-M_{n}$. The GEV distribution for minima can be derived in analogous to that for maxima, which results in the following theorem:

If there exist sequences of constants $\left\{a_{n}>0\right\}$ and $\left\{b_{n}\right\}$ such that

$$
\operatorname{Pr}\left\{\left(M_{n}^{0}-b_{n}\right) / a_{n} \leq z\right\} \rightarrow G^{o}(\rho z) \text { as } \mathrm{n} \rightarrow \infty
$$

defined on $\{z: 1-\xi(z-\not</ \sigma>0\}$, where $-\infty<\mu<\infty$, $8 / \infty>0$, and $-\infty<\xi<\infty$.

This implies that the maximum likelihood estimates obtained from fitting the GEV distribution of the maxima to the data $-z_{1}, \ldots,-z_{m}$ correspond exactly to that of the required GEV distribution of the minima except for only the location parameter where the sign correction is needed: $\hat{\mu} / \sigma=-\hat{\mu}$.

The asymptotic model for the block maxima presented earlier can be applied for the block minima as well. The maximum likelihood estimate of the parameters of the GEV distribution for the block minima corresponds exactly to that of the block maxima, except for the sign correction of the location parameter. Therefore, the better way to handle the block minima with the GEV 
model without the need for concern about the sign correction is to negate the block minima before estimating the model.

\subsubsection{Model Generalization: the $\mathbf{r}$ Largest Order Statistic Model}

Extremes are scarce, by the nature of the problem, so that model estimates as well as extreme return levels have a large variance. This issue motivated the search for characterizations of extreme value behavior that enable the modeling of data other than just the block maxima. There are two well-known general characterizations. One is based on the behavior of the $r$ largest order statistics within a block, for small values of $r$. The other is based on exceedances of a high threshold. We will focus on the first characterization in this section. The second approach is discussed in the next section in the context of the event-based sampling scheme.

Suppose that $\mathrm{X}_{1}, \mathrm{X}_{2}, \ldots$ is a sequence of independent and identically-distributed random variables. Let us define,

$$
M_{n}^{(k)}=k^{\text {th }} \text { largest of }\left\{X_{1}, \ldots, X_{n}\right\}
$$

The limiting behavior of this variable, for fixed $\mathrm{k}$, as $n \rightarrow \infty$, can be described by the following generalization.

If there exists sequences of constants $\left\{a_{n}>0\right\}$ and $\left\{b_{n}\right\}$ such that

$$
\operatorname{Pr}\left\{\left(M_{n}-b_{n}\right) / a_{n} \leq z\right\} \rightarrow G(z) \text { as } n \rightarrow \infty
$$

for some non-degenerate distribution function G, so that G is the GEV distribution function given by equation (6-1), then, for fixed $\mathrm{k}$,

$$
\operatorname{Pr}\left\{\left(M_{n}^{(k)}-b_{n}\right) / a_{n} \leq z\right\} \rightarrow G_{k}(z)
$$

on $\{z: 1+\xi(z-\mu) / \sigma>0\}$, where

$$
G_{k}(z)=\exp \{-\tau(z)\} \sum_{s=0}^{k-1} \frac{\tau(z)^{s}}{s !}
$$


with

$$
\tau(z)=\left[1+\xi\left(\frac{z-\mu}{\sigma}\right)\right]^{-1 / \xi}
$$

This generalization implies that, if the $\mathrm{k}^{\text {th }}$ largest order statistic within a block is normalized in exactly the same way as the block maximum, then its limiting distribution is of the form given by equation (6-6), the parameters of which correspond to the parameters of the limiting GEV distribution of the block maximum.

Although the block maxima from different blocks are independent by assumption, the components within a block cannot be independent. For example, $M_{n}^{(2)}$ cannot be greater than $M_{n}^{(1)}$. The outcome of each component influences the distribution of the other. Hence, we require a characterization of the limiting joint distribution of the entire vector $\boldsymbol{M}_{n}^{(r)}$ where

$$
\boldsymbol{M}_{n}^{(r)}=\left\{M_{n}^{(1)}, \ldots, M_{n}^{(r)}\right\}
$$

The following theorem gives the joint density function of the limiting distribution.

If there exist sequences of constants $\left\{a_{n}>0\right\}$ and $\left\{b_{n}\right\}$ such that

$$
\operatorname{Pr}\left\{\left(M_{n}-b_{n}\right) / a_{n} \leq z\right\} \rightarrow G(z) \text { as } n \rightarrow \infty
$$

for some non-degenerate distribution function $\mathrm{G}$, then, for fixed $\mathrm{r}$, the limiting distribution as $n \rightarrow \infty$ of

$$
\boldsymbol{M}_{n}^{0}{ }^{(r)}=\left\{\frac{M_{n}^{(1)}-b_{n}}{a_{n}}, \ldots, \frac{M_{n}^{(r)}-b_{n}}{a_{n}}\right\}
$$

falls within the family having joint probability density function

$$
f\left(z^{(1)}, \ldots, z^{(r)}\right)=\exp \left\{-\left[1+\xi\left(\frac{z^{(r)}-\mu}{\sigma}\right)\right]^{-1 / \xi}\right\} \cdot \prod_{k=1}^{r} \sigma^{-1}\left[1+\xi\left(\frac{z^{(k)}-\mu}{\sigma}\right)\right]^{-\frac{1}{\xi}-1}
$$


where $-\infty<\mu<\infty, \sigma>0,-\infty<\xi<\infty ; z^{(r)} \leq z^{(r-1)} \leq \ldots \leq z^{(1)} ; z^{(k)}: 1+\xi\left(z^{(k)}-\mu\right) / \sigma>0$ for $k=1, \ldots, r$.

In the case $r=1$, equation (6-7) reduces to the GEV family of density functions. The case $\xi=0$ is interpreted as the limiting form as $\xi \rightarrow 0$, leading to the family of density functions

$$
f\left(z^{(1)}, \ldots, z^{(r)}\right)=\exp \left\{-\exp \left[-\left(\frac{z^{(r)}-\mu}{\sigma}\right)\right]\right\} \cdot \prod_{k=1}^{r} \sigma^{-1} \exp \left[-\left(\frac{z^{(k)}-\mu}{\sigma}\right)\right]
$$

The parameters of the $r$ largest order statistic model correspond to those of the GEV distribution of the block maxima while incorporating more of the observed extreme data. Therefore, relative to a standard block maxima analysis, the interpretation of parameters is unaltered, but precision should be improved due to the inclusion of extra information.

\subsection{EVENT-BASED SAMPLING SCHEME}

The time-based sampling scheme which models only the block maxima can be a wasteful approach to extreme value analysis if one block happens to contain more extreme events than another. The second approach for model generalization defines the extreme events where the prespecified threshold is exceeded. The entire time series data may be better used by avoiding the procedure of blocking.

\subsubsection{The Generalized Pareto Distribution}

Let $\mathrm{X}_{1}, \mathrm{X}_{2}, \ldots$ be a sequence of independent and identically distributed random variables, having a marginal distribution function $F$. $X_{i}$ is regarded as extreme events if it exceeds some high threshold $\mathrm{u}$. It follows that a stochastic behavior of extreme events can be described by the conditional probability

$$
\operatorname{Pr}\{X>u+y \mid X>u\}=\frac{1-F(u+y)}{1-F(u)} ; y>0 .
$$


If the parent distribution $\mathrm{F}$ were known, the exact distribution of threshold exceedances would also be known. However, $\mathrm{F}$ is usually unknown in practice. Approximation of distributions in this case parallels the use of the GEV for the distribution of the block maxima when the true parent distribution is unknown. The threshold excess model is described by the following theorem.

Let $\mathrm{X}_{1}, \mathrm{X}_{2}, \ldots$ be a sequence of independent random variables with common distribution $\mathrm{F}$, and let

$$
M_{n}=\max \left\{X_{1}, \ldots, X_{n}\right\}
$$

Suppose that F satisfies the extremal types theorem (see Section 6.2.1), so that for large $n$,

$$
\operatorname{Pr}\left\{M_{n} \leq z\right\} \approx G(z)
$$

where

$$
G(z)=\exp \left\{-\left[1+\xi\left(\frac{z-\mu}{\sigma}\right)\right]^{-1 / \xi}\right\}
$$

for some $\mu, \sigma>0$ and $\xi$. Then, for large enough $\mathrm{u}$, the distribution function of $(X-u)$, conditional upon $X>u$, is approximately

$$
H(y)=1-\left(1+\frac{\xi y}{8 / 0}\right)^{-1 / \xi}
$$

defined on $\{y: y>0$ and $(1+\xi y / \&)>0\}$, where

$$
\stackrel{8}{\sigma}=\sigma+\xi(u-\mu)
$$

The family of distributions defined by equation (6-10) is called the generalized Pareto family. The parameters of the generalized Pareto distribution of threshold excesses are uniquely determined by those of the associated GEV distribution of block maxima. The parameter $\xi$ in equation (6-10) is equal to that of the corresponding GEV distribution. 
The duality between the GEV and generalized Pareto families means that the shape parameter $\xi$ is dominant in determining the qualitative behavior of the generalized Pareto distribution. If $\xi<0$, the distribution of threshold excesses has an upper bound of $u-\delta / \phi \xi$; if $\xi \geq 0$, the distribution has no upper limit. The case of $\xi=0$ requires a separate treatment by taking limit $\xi \rightarrow 0$ in equation (6-10), leading to

$$
H(y)=1-\exp \left(-\frac{y}{8}\right) ; y>0
$$

corresponding to an exponential distribution with parameter $1 / 8 \%$

\subsubsection{Threshold Selection}

In the block maxima approach, there is a bias-variance tradeoff for the choice of block length. The small block length generates more extremes but is more likely to violate the asymptotic basis of the model, thereby producing biased estimates. The large block length is vice versa; a few extremes can lead to estimates with a high variance.

The issue of block length in the block maxima approach is analogous to the threshold choice in the threshold excess model. Too low a threshold is likely to violate the asymptotic assumption of the model, leading to bias; too high a threshold will generate few extremes to be used in the model estimation, leading to high variance. The standard practice is to adopt as low a threshold as possible while the model still provides a reasonable approximation (Coles, 2001). There are two methods which can be used as tools to help determine the appropriate threshold: (a) the mean residual life plot which can be carried out prior to the model estimation and (b) the assessment of the stability of parameter estimates, based on fitting models across the range of varying thresholds.

\subsubsection{1 $\quad$ Mean Residual Life Plot}

If $\mathrm{Y}$ has a generalized Pareto distribution with parameters $\sigma$ and $\xi$, then 


$$
E(Y)=\frac{\sigma}{1-\xi}
$$

provided that $\xi<1$. When $\xi \geq 1$, the mean is infinite. Let $\sigma_{u_{0}}$ denote the scale parameter corresponding to the excesses of the threshold $\mathrm{u}_{0}$. If $\mathrm{u}_{0}$ is a valid threshold, then it should equally be valid for all thresholds $\mathrm{u}>\mathrm{u}_{0}$. Hence, for $\mathrm{u}>\mathrm{u}_{0}$,

$$
E(X-u \mid X>u)=\frac{\sigma_{u}}{1-\xi}=\frac{\sigma_{u_{0}}+\xi\left(u-u_{0}\right)}{1-\xi}
$$

by virtue of equation (6-11). $\sigma_{u}$ is the scale parameter corresponding to the excesses of the threshold $\mathrm{u}$. Consequently, for $\mathrm{u}>\mathrm{u}_{0}, E(X-u \mid X>u)$ is a linear function of $\mathrm{u}$. $E(X-u \mid X>u)$ is simply the mean of the excesses of the threshold $\mathrm{u}$ which can be estimated by the sample mean of excesses of the threshold $\mathrm{u}$. This leads to the mean residual life plot which consists of the locus of points

$$
\left\{\left(u, \frac{1}{n_{u}} \sum_{i=1}^{n_{u}}\left(X_{(i)}-u\right)\right): u<x_{\max }\right\}
$$

where $x_{(1)}, \ldots, x_{\left(n_{u}\right)}$ consist of the $\mathrm{n}_{\mathrm{u}}$ observations that exceed $\mathrm{u}$, and $\mathrm{x}_{\max }$ is the largest of the $\mathrm{X}_{\mathrm{i}}$. Above the threshold $\mathrm{u}_{0}$ at which the generalized Pareto distribution provides a valid approximation of the excess distribution, the mean residual life plot should be approximately linear in $\mathrm{u}$.

\subsubsection{Assessment of Parameter Stability}

A complementary technique to the mean residual life plot is to fit the generalized Pareto distribution at a range of thresholds and then examine stability of parameter estimates. If $\mathrm{u}_{0}$ is a valid threshold for the generalized Pareto distribution, then all thresholds $\mathrm{u}>\mathrm{u}_{0}$ should also be valid. From equation (6-11), we can show that

$$
\sigma_{u}=\sigma_{u_{0}}+\xi\left(u-u_{0}\right)
$$


The scale parameter changes as $\mathrm{u}$ varies unless $\xi=0$. This difficulty can be remedied by reparameterizing the scale parameter of the generalized Pareto distribution as

$$
\sigma^{*}=\sigma_{u}-\xi u
$$

$\sigma^{*}$ is now a constant with respect to u. Consequently, this suggests plotting both $\hat{\sigma}^{*}$ and $\hat{\xi}$ against $\mathrm{u}$, together with confidence intervals for each of these quantities, and selecting $\mathrm{u}_{0}$ as the lowest value of $u$ for which the estimates remain stable. These quantities will not be exactly constant due to a sampling variability, but they should be approximately stable after allowance

for their sampling errors. Confidence intervals of $\hat{\xi}$ can be obtained straightforwardly from the variance-covariance matrix. Confidence intervals for $\hat{\sigma}^{*}$ can be determined using the delta method.

\subsection{EXTREMES OF DEPENDENT SEQUENCES}

Preliminary visual evaluation of PET variations over time (see Figure C-1 to Figure C-32) reveals that PETs tend to exhibit a certain degree of dependency as clusters. The occurrences of short PETs are more likely to be followed by a sequence of short PETs. Two hypotheses can be suggested for this behavior. First, the PET could be time-varying per se or certain underlying covariates may influence the PET occurrences. This issue calls for the examination of the process non-stationarity which is discussed in the next section. The second hypothesis is that the occurrences of PETs are temporally dependent on prior events. The latter issue treats the PET occurrence process as identically distributed but not independent. The aforementioned sampling schemes and resulting distributions address the extremes under the independent and identically distributed (iid) assumption. This section examines how the possible dependency in a series of observations can be handled in the extreme value models.

The most natural generalization of a sequence of independent random variables is to a stationary series. A stationary process is a series whose variables may be mutually dependent but whose stochastic properties are homogeneous through time. Dependence in stationary series can take many different forms. We will follow the treatment of this issue as limited long-range dependence at extreme levels (Coles, 2001). This consideration is often plausible for physical processes and particularly for our application. In other words, extreme events are close to 
independent at times that are reasonably far apart. This leads to a relatively simple quantification of such effects on the standard extreme value limits.

\subsubsection{Maxima of Stationary Sequences}

Coles (2001) shows that, if a series has limited long-range dependence at extreme levels, the serial dependency has no influence on the limit result and the maxima of the stationary series follow the same distributional limit laws as those of an independent series. However, the parameters of the limit distribution are affected by the dependence in the series. This impact is summarized in the following theorem.

Let $X_{1}, X_{2}, \ldots$ be a stationary process and $X_{1}^{*}, X_{2}^{*}, \ldots$ be a sequence of independent variables with the same marginal distribution. Let $M_{n}=\max \left\{X_{1}, \ldots X_{n}\right\}$ and $M_{n}^{*}=\max \left\{X_{1}^{*}, \ldots, X_{n}^{*}\right\}$. Under suitable regularity conditions,

$$
\operatorname{Pr}\left\{\left(M_{n}^{*}-b_{n}\right) / a_{n} \leq z\right\} \rightarrow G_{1}(z)
$$

as $n \rightarrow \infty$ for normalizing sequences $\left\{a_{n}>0\right\}$ and $\left\{b_{n}\right\}$, where $\mathrm{G}_{1}$ is a non-degenerate distribution function, if and only if

$$
\operatorname{Pr}\left\{\left(M_{n}-b_{n}\right) / a_{n} \leq z\right\} \rightarrow G_{2}(z)
$$

where

$$
G_{2}(z)=G_{1}^{\theta}(z)
$$

for a constant $\theta$ such that $0<\theta \leq 1$. This implies that the effect of dependence in the stationary series (which satisfies the limited long-range dependence condition) is simply a replacement of $G_{1}$ as the limit distribution with $G_{1}^{\theta}$. If $G_{1}$ corresponds to the GEV distribution with parameters $(\mu, \sigma, \xi)$ and $\xi \neq 0$, then 


$$
\begin{aligned}
G_{1}^{\theta}(z) & =\exp \left\{-\left[1+\xi\left(\frac{z-\mu}{\sigma}\right)\right]^{-1 / \xi}\right\} \\
& =\exp \left\{-\left[1+\xi\left(\frac{z-\mu^{*}}{\sigma^{*}}\right)\right]^{-1 / \xi}\right\}
\end{aligned}
$$

where $\mu^{*}=\mu-\frac{\sigma}{\xi}\left(1-\theta^{-\xi}\right)$ and $\sigma^{*}=\sigma \theta^{\xi}$.

Similarly, if $G_{1}$ corresponds to the Gumbel distribution with parameters $(\mu, \sigma), G_{2}$ is also a Gumbel distribution, with parameters $\mu^{*}=\mu-\sigma \log \theta$ and $\sigma^{*}=\sigma$.

The quantity $\theta$ defined in equation (6-17) is termed the extremal index. A more precise statement together with proofs is given in Leadbetter et al. (1983). The extremal index $\theta$ of a stationary series can be interpreted in terms of the propensity of the process to cluster at extreme levels. Loosely,

$$
\theta=(\text { limiting mean cluster size })^{-1} \text {. }
$$

For an independent series, the extremal index $\theta=1$. The converse, however, is not true. A stationary series with $\theta=1$ does not necessarily have to be a series of independent observations.

\subsubsection{Modeling Stationary Extremes}

In this section, we examine how the dependent series has an impact on extreme value modeling in practice.

For block maxima data, provided long-range dependence at extreme levels is weak, the distribution of the block maxima is still within the GEV family. From the results in Section 6.4.1, the parameters estimated from the stationary series will be different from what would have been had the series been independent. But, since the parameters are to be estimated anyway, the conclusion is that dependence in the data is already addressed in the model estimation procedure and can be ignored. 
For threshold excess models, the fact that extremes in a stationary series have a tendency to cluster means that some change of practice is needed. The asymptotic equivalents suggest that the distribution of any individual threshold excess may be modeled using the generalized Pareto distribution. However, the clustering induces dependence in the observations, invalidating the typical log-likelihood. One widely-used method to deal with dependent exceedances in threshold excess models is declustering. The declustering technique is a filtering of dependent observations to obtain a set of threshold excesses that are approximately independent. This method works in steps as follows: (a) using an empirical rule to define clusters of exceedances, (b) identifying the maximum excess within each cluster, (c) assuming cluster maxima to be independent with conditional excess distribution given by the generalized Pareto distribution, and (d) fitting the generalized Pareto distribution to the cluster maxima.

In conclusion, the block maxima models have an advantage over the threshold excess models when dependency exists in a series of extremes due to its simplicity. A declustering scheme can be helpful for the threshold excess models but the results can be sensitive to the arbitrary choices made in the cluster determination. In addition, the declustering is a waste of data, which is contrary to one of the key benefits we try to achieve from the threshold excess models.

\subsection{EXTREMES OF NON-STATIONARY SEQUENCES}

In the previous section, we examined the effect of dependent extremes in the context of a stationary series, provided that a series has a weak long-range dependence. It is possible that the PET occurrence process could be time-varying per se or influenced by certain underlying covariates. In such a case, the distribution of extremes is not identical and the standard extreme value modeling may be invalid. Non-stationary processes have characteristics that change systematically over time. The fact that PET variations over time exhibit similar patterns across sites suggests that the identically-distributed assumption may be invalid and some underlying mechanisms may characterize the PET occurrence processes. This section will focus on the nonstationary extremes and how the non-stationarity can be incorporated in the model structure. 


\subsubsection{Model Structure for Non-Stationarity}

A variable that appears to relate to the extreme behavior of a series is referred to as a covariate. To incorporate the covariates into the model structure, the extreme value parameters can be expressed in the following form

$$
\theta(t)=h\left(\boldsymbol{X}^{T} \boldsymbol{\beta}\right)
$$

where $\theta$ denotes either $\mu, \sigma$ or $\xi$, h is a specified function, $\beta$ is a vector of parameters, and $\boldsymbol{X}$ is a model vector. In this context, $\mathrm{h}$ is usually referred to as the inverse-link function. For example, a linear trend over time in the location parameter $\mu$ can be expressed by letting $\mathrm{h}$ be the identity function and

$$
\mu(t)=\left[\begin{array}{ll}
1 & t
\end{array}\right]\left[\begin{array}{l}
\beta_{0} \\
\beta_{1}
\end{array}\right] .
$$

The log-linear model for $\sigma$ has a similar structure to equation $(6-21) ; \mu(t)$ is replaced by $\sigma(t)$ and the inverse-link $h$ is taken as the exponential function. For example, a log-link of $\sigma$ with covariates $X_{1}$ and $X_{2}$ can be expressed as

$$
\log \sigma(t)=\left[\begin{array}{lll}
1 & X_{1} & X_{2}
\end{array}\right]\left[\begin{array}{l}
\beta_{0} \\
\beta_{1} \\
\beta_{2}
\end{array}\right]
$$

There is a similarity between the class of models implied by equation (6-20) and generalized linear models (GLMs), whose theory is well developed and computing algorithms are standard implementations in several statistical analysis software programs (e.g., SAS, LIMDEP, and SPlus). For detailed discussions about GLMs, interested readers can consult a number of textbooks available on this topic, e.g., see Dobson (2002). The standard results or computational tools for GLMs are not directly transferable to extreme value modeling. The major difference is that the GLM family is restricted only to the distributions that fall within the exponential family (Coles, 2001) while the standard extreme value models generally fall outside this scope. 


\subsubsection{Model Selection}

With the possibility of modeling any extreme value parameters as functions of a combination of time and/or any covariates, there is a large catalogue of models from which to select, and choosing an appropriate combination becomes an important issue. Probably the most widely adopted principle is to choose the best model on the ground of parsimony, i.e., selecting the simplest model possible that can explain the variability in the data set as much as possible.

Maximum likelihood estimation of nested models leads to a simple test procedure of one model against the other. With models $\mathrm{M}_{0} \subset \mathrm{M}_{1}$, the deviance statistic is defined as

$$
D=2\left\{1_{1}\left(\mathrm{M}_{1}\right)-1_{0}\left(\mathrm{M}_{0}\right)\right\}
$$

where $1_{0}\left(M_{0}\right)$ and $1_{1}\left(M_{1}\right)$ are the maximized log-likelihoods under models $M_{0}$ and $M_{1}$ respectively. Large values of $D$ indicate that the model $\mathrm{M}_{1}$ substantially explains the variability in the data more than the model $\mathrm{M}_{0}$. The formal statistical test is provided by the asymptotic distribution of the deviance function, which can be summarized as follows.

The model $\mathrm{M}_{0}$ is rejected at the $\alpha$-level significance test if $D>c_{\alpha}$, where $c_{\alpha}$ is the $(1-\alpha)$ quantile of the $\chi_{k}^{2}$ distribution, and $k$ is the difference in the dimensionality of $\mathrm{M}_{0}$ and $\mathrm{M}_{1}$. In other words, the formal criterion specifies how large $\mathrm{D}$ should be before the model $\mathrm{M}_{1}$ is to be preferred.

\subsection{POINT PRocess CHARACTERIZATION OF EXTREMES}

The extreme value behavior of a process can be characterized in many different ways. One elegant formulation is derived from the theory of point process. The benefits of point process characterization of extremes are of twofold: (a) it provides a unified framework to interpret the extreme value behavior for all the models introduced so far; and (b) the model leads directly to a likelihood that enables a more natural formulation of non-stationarity in threshold excesses than the use of the generalized Pareto model. 
The basic theory of point process is discussed briefly in this section. Except for the threshold excess models, the point process characterization of extreme value behavior does not offer any significant advantage over standard formulations. Therefore, only the connection between the point process and the threshold excess models is further discussed. Coles (2001) and Leadbetter et al. (1983) provide a more in-depth discussion on this generalization.

\subsubsection{Basic Theory of Point Processes}

A point process on a set $\mathrm{T}$ is a stochastic rule for the occurrence and position of point events. In a modeling context, with $\mathrm{T}$ representing a period of time, a point process model might be used to describe the occurrence of thunderstorms, earthquakes, and PETs in our particular case.

For each $\tau \subset \mathrm{T}, N(\tau)$ is the number of points in the set $\tau$. The intensity measure of the process is defined as

$$
\Lambda(\tau)=E\{N(\tau)\}
$$

The intensity measure $\Lambda(\tau)$ is the expected number of points in any subset $\tau \subset \mathrm{T}$.

Assuming $\tau=\left[t_{1}, x_{1}\right] \times \ldots \times\left[t_{k}, x_{k}\right] \subset \mathfrak{R}^{k}$, and provided it exists, the derivative function

$$
\lambda(x)=\frac{\partial \Lambda(\tau)}{\partial x_{1} \cdots \partial x_{k}}
$$

is the intensity (density) function of the process.

A point process on $\mathrm{T} \subset \mathfrak{R}^{k}$ is said to be a k-dimensional non-homogeneous Poisson process, with intensity density function $\lambda(\cdot)$, if it satisfies the property of independent counts on nonoverlapping subsets and, for all $\tau \subset \mathrm{T}$,

$$
N(\tau): \text { Poisson }(\Lambda(\tau))
$$

where 


$$
\Lambda(\tau)=\int_{\tau} \lambda(x) d x
$$

We now assume that the intensity function $\lambda(\cdot)$ is within a family of parametric models $\lambda(\cdot ; \theta)$. Therefore, the only issue is the estimation of the unknown parameter vector $\theta$.

Suppose points $x_{1}, \ldots x_{n}$ have been observed in a region $\mathrm{T} \subset \mathfrak{R}$, and that these are the realization of a Poisson process on $\mathrm{T}$, with intensity function $\lambda(\cdot ; \theta)$, for some value of $\theta$. This leads to, by the Poisson property, the following likelihood function

$$
L\left(\theta ; x_{1}, \ldots, x_{n}\right)=\exp \{-\Lambda(\mathrm{T} ; \theta)\} \prod_{i=1}^{n} \lambda\left(x_{i} ; \theta\right)
$$

where $\Lambda(\mathrm{T} ; \theta)=\int_{\mathrm{T}} \lambda(x ; \theta) d x$. Likelihood (6-26) also applies to the more general case of a Poisson process on a k-dimensional set $\mathrm{T}$.

\subsubsection{Statistical Modeling}

In order to enable modeling extremes within the point process framework, we need the result from the following theorem.

Let $X_{1}, \ldots, X_{n}$ be a series of independent and identically distributed random variables, and let

$$
N_{n}=\left\{\frac{i}{n+1}, X_{i}\right\} ; i=1, \ldots, n .
$$

The scaling in the first ordinate ensures that the time axis is always mapped to $(0,1)$. Then, for sufficiently large $\mathrm{u}$, on regions of the form $(0,1) \times[u, \infty), N_{n}$ is approximately a Poisson process, with intensity measure on $\tau=\left[t_{1}, t_{2}\right] \times(z, \infty)$ given by

$$
\Lambda(\tau)=\left(t_{2}-t_{1}\right)\left[1+\xi\left(\frac{z-\mu}{\sigma}\right)\right]^{-1 / \xi}
$$


First, select a high threshold $u$ and set $\tau=(0,1) \times[u, \infty)$. The $N(\tau)$ points observed in the region $\tau$ are re-labeled $\left\{\left(t_{1}, x_{1}\right), \ldots,\left(t_{N(\tau)}, x_{N(\tau)}\right)\right\}$. Assuming the limiting Poisson process is an acceptable approximation to the process of $N_{n}$ on $\tau$, an approximate likelihood can be derived. Maximizing this likelihood leads to estimates of parameters $(\mu, \sigma, \xi)$ of the limiting intensity function. The small adjustment is needed for (6-27). If PET data is observed for eight hours, the parameters obtained will correspond to an eight-hour maximum. By replacing (6-27) with

$$
\Lambda(\tau)=n_{y}\left(t_{2}-t_{1}\right)\left[1+\xi\left(\frac{z-\mu}{\sigma}\right)\right]^{-1 / \xi}
$$

where $n_{y}=$ the number of time blocks of observation. If we discretize the PET data into hourly intervals, by letting $n_{y}=8$, the estimates of parameters $(\mu, \sigma, \xi)$ will correspond to the hourly maximum distribution of the observed process. Similarly, if we let $n_{y}=32$, the estimates will correspond to the 15-minute maximum distribution of the observed process.

Substituting (6-28) into (6-26), with $\left[t_{1}, t_{2}\right]=[0,1]$, leads to the likelihood function in (6-29).

$$
\begin{aligned}
L_{\tau}\left(\mu, \sigma, \xi ; x_{1}, \ldots, x_{n}\right) & =\exp \{-\Lambda(\tau)\} \prod_{i=1}^{N(\tau)} \lambda\left(t_{i}, x_{i}\right) \\
& =\exp \left\{-n_{y}\left[1+\xi\left(\frac{u-\mu}{\sigma}\right)\right]^{-1 / \xi}\right\} \prod_{i=1}^{N(\tau)} \sigma^{-1}\left[1+\xi\left(\frac{x_{i}-\mu}{\sigma}\right)\right]^{-\frac{1}{\xi}-1}(6-2)
\end{aligned}
$$

This function can be treated as a typical likelihood function to obtain maximum likelihood estimates, standard errors, and approximate confidence intervals.

\subsubsection{Connections with Threshold Excess Model Likelihood}

From the threshold excess models, we know that the distribution of the excesses of a high threshold $u$ follows the generalized Pareto distribution. The likelihood function of threshold excess models originally ignores $X_{i}$ that fails to exceed threshold $\mathrm{u}$. We now supplement the 
likelihood to include partial information on these observations in order to demonstrate the connections between the likelihoods of the point process and the threshold excess models.

From the knowledge of the generalized Pareto distribution, we have

$$
\zeta=\operatorname{Pr}\left\{X_{i}>u\right\}=1-F(u) \approx \frac{1}{n}\left[1+\xi\left(\frac{u-\mu}{\sigma}\right)\right]^{-1 / \xi}
$$

The rate of exceedances $\zeta$ is the probability of any individual observation $X_{i}$ exceeding a high threshold $u$. Next, recall from equation (6-11),

$$
\stackrel{q}{\sigma}=\sigma+\xi(u-\mu)
$$

The likelihood contribution of individual observation $X_{i}$ that fails to exceed the threshold $u$ is

$$
\operatorname{Pr}\left\{X_{i}<u\right\}=1-\zeta
$$

On the contrary, the likelihood contribution of each $X_{i}$ that exceeds $u$ is

$$
\operatorname{Pr}\left\{X_{i}=x\right\}=\operatorname{Pr}\left\{X_{i}=x \mid X_{i}>u\right\}=\zeta f(x-u ; \& g \xi)
$$

where $f(\cdot ; \delta g \xi)$ denotes the probability density function of the generalized Pareto distribution. Taking products across independent realizations gives the likelihood

$$
L\left(\zeta, 8 / \rho \xi ; x_{1}, \ldots, x_{n}\right)=(1-\zeta)^{n-n_{u}} \prod_{i=1}^{n_{u}} \zeta 8^{-1}\left[1+\xi\left(\frac{x_{i}-u}{8 \%}\right)\right]^{-\frac{1}{\xi}-1}
$$

where $n_{u}$ is the number of exceedances of $u$. For a high threshold, $n_{u}$ will be relatively smaller than $n$, therefore

$$
(1-\zeta)^{n-n_{u}} \approx(1-\zeta)^{n} \approx \exp \{-n \zeta\}
$$

Additionally, by (6-30) and repeated use of (6-11), 


$$
\zeta \delta^{\circ}\left[1+\xi\left(\frac{x_{i}-u}{\sigma_{0}}\right)\right]^{-\frac{1}{\xi}-1}=(n \sigma)^{-1}\left[1+\xi\left(\frac{x_{i}-u}{\sigma}\right)\right]^{-\frac{1}{\xi}-1}
$$

Substituting (6-34) and (6-35) into (6-33) gives the likelihood in (6-29) with $n_{y}=1$. The point process likelihood gives the threshold excess models reparameterized in terms of the usual GEV parameters.

The equivalence of the threshold excess models and point process characterization confirms that any statistical inference made within the point process framework is equal to those obtained within the threshold excess model framework. However, the immediate advantages of the point process characterization over standard threshold excess models are: (a) the natural model parameterization in terms of usual GEV parameters, (b) the scale parameter $\sigma$ is invariant to the thresholds, and (c) the threshold exceedance rates form a part of the statistical inference. These benefits are particularly useful for modeling non-stationarity in threshold excesses. It is not difficult to incorporate time or other covariates into the location parameter $\mu$, which is usually simple in the block maxima models. In addition, point process likelihood facilitates the estimation of models with time-varying thresholds.

\subsection{MODELING APPROACH FOR PET EXTREMES}

Two major approaches for modeling PET extremes are considered in this study - time-based versus event-based extreme value sampling schemes. For both approaches, the negated PET values are modeled instead of the actual PET values. The lower PET values correspond to situations associated with a greater risk of collisions; therefore, the large negated PETs now correspond to a more hazardous situation. There are two immediate advantages of doing so. First, it is more convenient mathematically to model the right tail of the extreme value distributions using the classes of models presented earlier. Moreover, we demonstrated in Section 6.2.2.2 the connection of asymptotic models for maxima and those for minima. We can avoid the need to correct the sign of parameter estimates by using the negated observed values. Second, the threshold excesses of a high threshold approximately follow the generalized Pareto distribution, but not for a low threshold in our case of actual PET values. Threshold excess models will require 
the negated observations where low values are of interest. To avoid any inconsistency in model considerations, the negated observed PETs are preferred henceforth.

\subsubsection{PET Extreme Modeling Alternatives}

Two categories of extreme value modeling approach are considered for modeling PET extremes time-based and event-based sampling schemes.

\subsubsection{Time-Based PET Extremes}

In the time-based sampling scheme, we discretize the negated PET data into intervals and select the maximum from each block. The block maximum distribution is approximated by the GEV distribution. The more flexible approach is the model generalization as the $r$ largest order statistic in which we can select $r$ largest observations from each block.

A bias-variance tradeoff is considered in choosing the suitable block length. A too large block length will generate few data points leading to estimates with large variance while a too small block length is likely to violate the asymptotic assumption of the GEV distribution. We have chosen 15-minute intervals, which result in 32 blocks for the eight-hour observation period. This is equivalent to 32 block maxima, provided that the block is sufficiently large to have at least one PET. In addition, as a general rule of thumb in statistical sampling, a sample size of 30 or more is deemed to be sufficiently large for many limit theorems to be applicable. A block length larger than 15 minutes is not practical due to the limited observation period in this study. A larger block length would generate too few extreme data points and the parameter estimation algorithm would be difficult to converge. Also, the 15-minute interval is a natural block length for our application since it also corresponds with a standard practice in traffic volume measurement.

However, not all the blocks experience at least one PET in the field for two main reasons: (a) we observed only the PETs that are less than a specified threshold, which is eight seconds in this study; therefore, large PET values usually associated with normal crossing traffic operations are omitted and (b) heavy left-turn volume at intersections with a protected left-turn phase separates conflicting through-phases and prevents sufficiently low PET values from occurring. For these reasons, it should be noted that some sites may have less than 32 block extremes where low PET 
values do not exist in certain blocks. The sample size issue can be addressed at some sites using the $r$ largest order statistic model which enables the sampling of more than just a block maximum.

The utmost important benefit of modeling PET using block maxima distribution is that the dependency across the series of observations is automatically handled in the parameter estimation procedure (see Section 6.4). On the other hand, the shortcoming of this modeling approach is wastage of data, which may result in a large variance of model estimates and subsequent model predictions.

\subsubsection{Event-Based PET Extremes}

This approach defines the negated PETs as extremes where they exceed a specified threshold. The choice of a threshold is analogous to the choice of the block length in the case of a timebased sampling scheme. Threshold excesses can be approximated by the generalized Pareto distribution as described in Section 6.3.1. The more flexible approach is to model threshold excesses using point process characterization. The connection between point process and generalized Pareto likelihoods was discussed in Section 6.6.3. The point process likelihood has the intensity density function characterized in terms of the usual GEV parameters. In addition, under the point process framework, the rate of exceedance forms a part of the likelihood function and the scale parameter is invariant to the threshold.

Selecting an appropriate threshold is a relatively difficult task and there is no standardized practice on this issue. In Section 6.3.2, we discussed two techniques to help select the appropriate threshold, provided that a time-constant threshold is sufficient. These two techniques require a visual evaluation, which is subjective. In cases where a non-constant threshold is more appropriate, one may adopt a time-varying threshold within the point process framework in order to maintain a constant rate of exceedance. The determination of time-varying thresholds is usually carried out using a trial-and-error procedure.

The major advantage of this modeling approach is the more efficient use of data. However, the effect of data dependency across a series of observations is a serious concern. Computationally, the degree of dependency can be approximately determined by the extremal index. The data dependency, if left unhandled, can lead to biased model estimates. Declustering is a commonlyused method for dealing with data dependency in threshold excess as well as point process 
models (see Section 6.4.2). A declustering method, however, still suffers from the lack of a standardized procedure and the degree to which declustering is needed remains subjective.

In brief, we need to apply a declustering method to a series of PET data and then select an appropriate threshold from a declustered series prior to model estimation. Then, PET threshold excesses as extremes can be modeled within the framework of the point process likelihood.

\subsubsection{Selected Modeling Approach}

Due to the dependency embedded in a series of observation, the first method, time-based sampling scheme, is more suitable for modeling PET extremes. Although a sample size issue is of our concern, the problem is partially addressed by the $r$ largest order statistic model. The model estimation results from the second method is likely to be less robust than the first one due in large to the subjectivity associated with a declustering method and a threshold selection required prior to model estimation. The advantage of additional extremes achieved from the event-based sampling scheme is considerably offset by the potentially biased estimates and the arbitrary nature of the method.

The modeling procedure for PET extremes can be summarized as follows:

1. Block a series of negated PET observations into 15-minute time intervals.

2. Select the $r$ largest values from each block. The choice of $r$ depends on the actual sample size. Note that the GEV distribution is a special case of the $r$ largest order statistic model where $r=1$.

3. Prepare the covariate data (e.g., total intersection volume, PET frequency, and left-turn proportion) for each corresponding 15-minute interval.

4. Estimate the models based on the PET and covariate data. The log-likelihood value at convergence can be used to compare the goodness-of-fit between nested models.

The detailed modeling procedure is described in subsequent sections. Then, we will describe the method to predict the extreme return level and the crash frequency based on the parameter estimates. 


\subsubsection{Parameter Estimation and Inference}

Many techniques have been proposed for parameter estimation in extreme value models. These include graphical techniques based on model of probability plots, method of moments, and likelihood-based methods. However, the all-around utility and adaptability to complex modelbuilding make the likelihood-based approach particularly attractive.

One potential difficulty with the use of likelihood-based methods concerns the regularity conditions required for the standard asymptotic properties associated with maximum likelihood estimates. The violation of the regularity conditions implies that the usual asymptotic results are not automatically applicable. Smith (1985) studied this issue and concluded the findings as follows:

- For $\xi>-0.5$, the maximum likelihood estimators are regular and possess the usual asymptotic properties.

- For $-1.0<\xi<-0.5$, the maximum likelihood estimators are estimable but the usual asymptotic properties do not apply.

- For $\xi<-1.0$, the maximum likelihood estimators are unlikely attainable.

\subsubsection{Maximum Likelihood Estimation}

The maximum likelihood estimation is used to estimate the model parameters in this study. Extreme value distributions are not a standard package in statistical analysis software, so we developed a source code for this task in S language, which can be executed in S-Plus or R platforms. Parts of the code are modified from the S-code originally written by Coles (2001).

\section{Generalized Extreme Value Model}

GEV distribution is a special case of the $r$ largest order statistic model where $r=1$. Under the assumption that $Z_{1}, \ldots, Z_{m}$ are GEV-distributed independent variables, the log-likelihood function for $\xi \neq 0$ is 


$$
1(\mu, \sigma, \xi)=-m \log \sigma-\left(1+\frac{1}{\xi}\right) \sum_{i=1}^{m} \log \left[1+\xi\left(\frac{z_{i}-\mu}{\sigma}\right)\right]-\sum_{i=1}^{m}\left[1+\xi\left(\frac{z_{i}-\mu}{\sigma}\right)\right]^{-1 / \xi}
$$

defined on

$$
1+\xi\left(\frac{z_{i}-\mu}{\sigma}\right)>0 ; i=1, \ldots, m
$$

The condition (6-37) ensures that none of the observed data points falls beyond the end-point of the distribution.

The case $\xi=0$ requires separate treatment using the Gumbel limit, which leads to the loglikelihood

$$
1(\mu, \sigma)=-m \log \sigma-\sum_{i=1}^{m}\left(\frac{z_{i}-\mu}{\sigma}\right)-\sum_{i=1}^{m} \exp \left\{-\left(\frac{z_{i}-\mu}{\sigma}\right)\right\}
$$

\section{The r Largest Order Statistic Model}

This generalization of block maximum distribution enables a more efficient use of information from a series of observed PETs. In practice, we would select the $r$ value as high as possible subject to the model adequacy. For each block $i$, the largest $r_{i}$ observations are recorded as

$$
\boldsymbol{M}_{i}^{\left(r_{i}\right)}=\left\{z_{i}^{(1)}, \ldots, z_{i}^{\left(r_{i}\right)}\right\} ; \text { for } i=1, \ldots, m
$$

Usually we set $r_{1}=r_{2}=\ldots=r_{m}=r$ but fewer data points are allowed in certain blocks where the data are unavailable.

Taking products across blocks with the density function defined in (6-7) gives the likelihood function, for $\xi \neq 0$,

$$
L(\mu, \sigma, \xi)=\prod_{i=1}^{m}\left(\exp \left\{-\left[1+\xi\left(\frac{z_{i}^{\left(r_{i}\right)}-\mu}{\sigma}\right)\right]^{-1 / \xi}\right\} \cdot \prod_{k=1}^{r_{i}} \sigma^{-1}\left[1+\xi\left(\frac{z_{i}^{(k)}-\mu}{\sigma}\right)\right]^{-\frac{1}{\xi}-1}\right)
$$


provided that

$$
1+\xi\left(\frac{z^{(k)}-\mu}{\sigma}\right)>0 ; k=1, \ldots, r_{i}, i=1, \ldots, m
$$

For the case $\xi=0$, we have the likelihood derived from (6-8) as

$$
L(\mu, \sigma, \xi)=\prod_{i=1}^{m}\left(\exp \left\{-\exp \left[-\left(\frac{z_{i}^{\left(r_{i}\right)}-\mu}{\sigma}\right)\right]\right\} \cdot \prod_{k=1}^{r_{i}} \sigma^{-1} \exp \left[-\left(\frac{z_{i}^{(k)}-\mu}{\sigma}\right)\right]\right)
$$

\section{Non-stationary Models}

The advantage of likelihood-based methods for model estimation is the ability to incorporate time variables or covariates to describe non-stationary behavior based on the existing model structures.

For a non-stationary model, we describe the distribution of $Z_{t}$ for $t=1, \ldots, m$ having extreme value distribution $F$ as

$$
Z_{t}=F(\mu(t), \sigma(t), \xi(t))
$$

where each parameter is linked with a combination of covariates as described in (6-20). Let us denote $\boldsymbol{\beta}$ as a complete vector of parameters. Then, we can express the likelihood function for the non-stationary versions as

$$
L(\boldsymbol{\beta})=\prod_{t=1}^{m} f\left(z_{t} ; \mu(t), \sigma(t), \xi(t)\right)
$$

where $f$ is the corresponding probability density function.

\subsubsection{Inferences for Maximum Likelihood Estimates}

Standard asymptotic results of maximum likelihood estimates stated that, under suitable regularity conditions, for large $\mathrm{n}$, 


$$
\hat{\theta}_{0}: M V N_{d}\left(\theta_{0}, I_{E}\left(\theta_{0}\right)^{-1}\right)
$$

where $\hat{\theta}_{0}$ is a vector of maximum likelihood estimates of d-dimensional model parameter vector $\theta_{0}$ and the matrix $I_{E}(\theta)$ is the expected information matrix that measures the expected curvature of the log-likelihood surface.

As a result of standard asymptotic results, under suitable regularity conditions, the approximate distribution of $\hat{\theta}$ is a multivariate normal with mean $\theta$ and a variance-covariance matrix equal to the inverse of the observed information matrix, $I_{o}(\theta)$, evaluated at the maximum likelihood estimates. Let $1(\cdot)$ be the log-likelihood function. The matrix $I_{o}(\theta)$, an approximation of $I_{E}(\theta)$, is defined by

$$
I_{o}(\theta)=\left[\hat{e}_{i, j}(\theta)\right]_{\mathrm{d} \times \mathrm{d}} ; \text { evaluated at } \theta=\hat{\theta}
$$

where

$$
\hat{e}_{i, j}(\theta)=\left\{\begin{array}{c}
-\frac{\partial^{2} 1(\theta)}{\partial \theta_{i} \partial \theta_{j}} ; i \neq j \\
-\frac{\partial^{2} 1(\theta)}{\partial \theta_{i}^{2}} ; i=j
\end{array} .\right.
$$

Denoting the diagonal elements of the inverse of the matrix $I_{o}(\theta)$ by $\psi_{i, i}$, it follows that an approximate $(1-\alpha)$ confidence interval for $\theta_{i}$ is

$$
\hat{\theta}_{i} \pm z_{\alpha / 2} \sqrt{\varphi_{i, i}}
$$

where $z_{\alpha / 2}$ is the $(1-\alpha / 2)$ quantile of the standard normal distribution.

In our PET modeling context, $\theta$ can be either a standard parameter vector $(\mu, \sigma, \xi)$ or a vector $\boldsymbol{\beta}$ in a non-stationary case. 


\subsubsection{Model Diagnostics}

The fitted extreme value models have two components: (a) the observed component and (b) the extrapolation component. The validity check of the extrapolation is usually very difficult and it is often impossible to check the validity of extrapolation in practice. We will discuss this concern in subsequent chapters. However, we can assess the quality of model fit with reference to the observed data. This is typically done by graphical evaluations known as probability and quantile plots. Other plots, such as return level plot and density plot, are also used in the diagnostics of fitted extreme value models.

\subsubsection{Diagnostic Plots for GEV Models}

\section{Probability and Quantile Plots}

The probability plot is a comparison of the empirical and fitted distribution functions. With the ordered block maxima (negative PETs) $z_{(1)} \leq z_{(2)} \leq \ldots \leq z_{(m)}$, the empirical distribution function is defined as

$$
G^{\prime}\left(z_{(i)}\right)=\frac{i}{m+1}
$$

The corresponding model estimates $\hat{G}\left(z_{(i)}\right)$ can be obtained by substituting $z_{(i)}$ into (6-1), evaluated at $(\hat{\mu}, \hat{\sigma}, \hat{\xi})$. A probability plot consists of the locus of points

$$
\left\{\hat{G}\left(z_{(i)}\right), G^{\prime}\left(z_{(i)}\right) ; i=1, \ldots, m\right\}
$$

which should lie close to unit diagonal. A weakness of the probability plot for extreme value models is that both $\hat{G}\left(z_{(i)}\right)$ and $G^{\prime}\left(z_{(i)}\right)$ are bound to approach 1 as $z_{(i)}$ increases, while it is usually the tail region of the extreme value distribution that is of the greatest concern. This can be avoided by the quantile plot, which consists of the points 


$$
\left\{\hat{G}^{-1}\left(\frac{i}{m+1}\right), z_{(i)} ; i=1, \ldots, m\right\} .
$$

The quantile plot has an advantage over the probability plot in that the data points are not clustered when $\mathrm{z}_{(\mathrm{i})}$ increases. Any substantial departure from a unit diagonal for both probability and quantile plots may indicate a model inadequacy.

Return Level Plot

A return level plot consists of the points

$$
\left\{\left(\log y_{p}, \hat{z}_{p}\right): 0<p<1\right\}
$$

where $y_{p}=-\log (1-p)$ and $\hat{z}_{p}$ is defined in (6-5). The tail of the distribution is compressed so that return level estimates for long return periods are displayed, while the linearity of the plot in the case $\xi=0$ provides a baseline against which to judge the effect of the estimated shape parameter.

\section{Probability Density Function Plot}

For completeness, the probability density function can be plotted against the histogram of the actual data. However, this is generally less informative than the other plots since the shape of the histogram can vary substantially depending on the choice of grouping intervals.

\subsubsection{Diagnostic Plots for the r Largest Order Statistic Models}

The previous section discussed the plots for examining the quality of fit of the models fitted to only the block maximum in the GEV models. The $r$ largest order statistic model is the generalization of the GEV case; we can examine the quality of fit in a similar manner for any $\mathrm{k}^{\text {th }}$ largest values from the block $(k=1, \ldots, r)$. 


\section{Probability and Quantile Plots}

These are obtained by comparing the distribution of the $\mathrm{k}^{\text {th }}$ order statistic, model (6-6), with parameters replaced by maximum likelihood estimates. For each $\mathrm{k}^{\text {th }}$ order statistic, the probability plots are straightforward, as in the case of the GEV models.

The quantile plot is more complicated since model (6-6) cannot be inverted analytically. For the model estimate of the $(1-p)$ quantile, $z_{p}$ is to be solved numerically from the expression

$$
G_{k}\left(z_{p}\right)=1-p
$$

where $G_{k}(z)$ is the distribution function of the $\mathrm{k}^{\text {th }}$ order statistic defined in (6-6).

As usual, any substantial departure from the unit diagonal for any $\mathrm{k}^{\text {th }}$ order statistic in these plots may signify a fundamental lack-of-fit problem.

\subsubsection{Diagnostic Plots for Non-Stationary Models}

The model checking for the non-stationary case is more complicated due to the lack of homogeneity in the distributional assumptions across the series of observations. The approximate extreme value distributions are not identically distributed in the non-stationary case. We need to apply the diagnostic plots to a standardized version of the data instead, conditional upon the fitted parameter estimates.

For the block maxima distributed as non-stationary GEV, the estimated model is

$$
Z_{t}=G E V(\hat{\mu}(t), \hat{\sigma}(t), \hat{\xi}(t))
$$

The standardized variables $Z_{t}^{0}$ is obtained by

$$
Z_{t} / 0=\frac{1}{\hat{\xi}(t)} \log \left\{1+\hat{\xi}(t)\left(\frac{Z_{t}-\hat{\mu}(t)}{\hat{\sigma}(t)}\right)\right\}
$$


Each $Z_{t}^{0}$ now follows the standard Gumbel distribution $(\mu=0, \sigma=1)$, with probability function

$$
\operatorname{Pr}\left\{Z_{t}^{0} \leq z\right\}=\exp \left(-e^{-z}\right), z \in \mathrm{i}
$$

This means that probability and quantile plots of the observed non-stationary $2 t^{\circ}$ can be made with reference to the distribution (6-50). Let $2_{(9)}, \ldots, 2_{(m)}$ be the ordered values of $2_{t}$, the probability plot consists of the pairs

$$
\left\{\frac{i}{m+1}, \exp (-\exp (-2 /(i))) ; i=1, \ldots, m\right\}
$$

while the quantile plot consists of the locus of points

$$
\left\{\left(2 /(?)-\log \left(-\log \left(\frac{i}{m+1}\right)\right)\right) ; i=1, \ldots, m\right\} .
$$

The probability plot is invariant to the choice of Gumbel as a reference distribution, but this is not the case for the quantile plot. Choices other than Gumbel would lead to a different quantile plot. Despite this arbitrariness, the Gumbel distribution is arguably the most natural choice given its connection with GEV family.

\subsubsection{Model Selection Criteria}

For each site, the model goodness-of-fit can be evaluated by checking the probability and quantile plots. With the possibility to model PETs as a non-stationary process, a number of combinations of covariates can be considered in the modeling procedure. The appropriate link structure is also important. The selected set of covariates should logically relate to the mechanism of PET occurrences. The test for statistical significance between different nested models was described in Section 6.5.2. 
The best model should be selected on the grounds of parsimony. The more complicated models should be preferred only if additional covariates can substantially (in a statistical sense) explain the variability in a series of the observed data in a logical manner.

\subsection{MODEL INTERPRETATION}

We can estimate the risk of right-angle collisions using the information from the tail behavior of the extreme value distributions. Generally, the extreme return level is the value of interest estimated from the fitted models in typical applications of extreme value analysis. Alternative interpretation is to examine the area under the tail region of the extreme value distributions as estimated crash frequency. This section describes the safety interpretation from the distribution tails of estimated models and the computational procedure to determine safety estimates.

\subsubsection{Safety Implications}

The tail behaviors of the fitted models are the results of the selected extreme value distribution and its properties. Extreme value theory enables the extrapolation of the distributions fitted to the observed PETs to the unobserved levels on a sound mathematical argument. The extrapolated component of the distributions fitted to a series of PETs is crucial since it implies the safety levels associated with an individual site. There exist two values of interest in this extrapolated tail region. The first one is the risk defined by the area under the extreme tail region; the second one is the extreme return level defined by the value of the negated PET corresponding to the given area under the tail region where the extreme return level is exceeded.

\subsubsection{Definition of Risk}

Recall the traffic crossing event, which is the underlying mechanism of PET occurrences. As the degree of hazard of right-angle collisions increases as the separation between crossing traffic becomes narrower. The degree of separation is quantified in terms of the PET which in turn directly relates to the degree of hazard of the crossing situations. For a normal traffic operation, the PET is usually very large and therefore does not carry much information about safety. At the other extreme, the failure to perform normal crossing maneuvers will result in a right-angle crash, 
which implies a zero separation. These extreme cases or right-angle crashes, in other words, if observable in the field, will correspond to the measured PET value of zero or less. However, we know that actual crashes are a rare event and require a considerable amount of waiting time. Therefore, it is unlikely to observe actual crashes in a relatively short period of field data collection. But, based on the knowledge of the PET occurrence mechanism, the collision event is characterized by the PET $\leq 0$. In extreme value modeling, the negated PETs are fitted to the distributions instead of actual PETs; hence, the collision event is defined as the event that has the negated PET $\geq 0$.

For the GEV and the $\mathrm{r}$ largest order statistic models, a series of PETs is blocked into 15-minute intervals. The area under the tail region where a negated PET is positive corresponds to the probability of experiencing a collision in a 15-minute interval. We refer to this probability as the risk. The risk alternatively can be recognized as the expected frequency of collisions during a 15minute interval. The latter interpretation simplifies the conversion of the risk to the expected frequency of collisions for any given period of time. The detailed procedure is described in Section 6.8.2.

\subsubsection{Extreme Return Level}

Although the return level is a standard prediction in extreme value analysis, the interpretation in the case of PET modeling is more subtle. By definition, the return level $z_{p}$ corresponds to the value which gives

$$
G\left(z_{p}\right)=\operatorname{Pr}\left\{Z \leq z_{p}\right\}=1-p
$$

where $G(\cdot)$ is the extreme value distribution function and $\mathrm{p}$ is the specified probability under the tail region. The value $1 / p$ is usually referred to as the return period.

Let us consider the extreme value analysis of the annual maximum sea level as a typical example. Assume that we have the maximum sea levels collected on a daily basis. If observed sea levels are blocked annually and the annual block maxima are fitted to the GEV distribution, the 100year return level can be computed by letting $p=1 / 100=0.01$. The return level $z_{p=0.01}$ here will correspond to the 100-year return level of the annual maximum sea level or, equivalently, the 
annual maximum sea level which is expected to be exceeded on average once every 100 years. In other words, the probability of the annual maximum sea level exceeding $z_{p=0.01}$ in any particular year is 0.01 .

The same analogy applies to the PET extremes. The PET data are blocked into 15-minute intervals. To compute the n-year return level of maximum negated PETs, we need to specify $p$ based on the number of 15-minute blocks equivalent to $\mathrm{n}$ years of observation. For example, a one-year return level of maximum negated PET is the negated PET level, which is expected to be exceeded on average once every year. Alternatively, the probability of the maximum negated PET exceeding the one-year return level in any particular year is $1 /$ (number of 15 -minute blocks in a year).

Let $z_{\mathrm{p}}$ be the $\mathrm{n}$-year return level of maximum negated PETs. The condition $z_{p} \geq 0$ implies that positive crash frequency is expected during the n-year period; while the condition $z_{p}<0$ indicates that zero crash frequency is expected during the n-year period. The greater value of $z_{p}$ on a positive axis implies a greater degree of hazard of right-angle collisions and that collisions are more likely. On the other hand, the smaller value of $z_{p}$ on a negative axis indicates a safer situation for the same type of collisions and that collisions are less likely.

Section 6.8.3 explains the computational procedure to obtain the extreme return level for different types of extreme value models.

\subsubsection{Advantages and Disadvantages}

There is a relationship between the risk and the extreme return level. However, these two entities are neither convertible nor interchangeable. The tail behaviors of extreme value distributions represented by two different approaches have their own pros and cons.

The advantages of the first approach are: (a) it is more intuitive and relatively simple to understand; (b) it can be converted to frequency of collisions; and (c) the validation check is straightforward by direct comparison with actual crashes. However, the risk is non-negative and low-risk intersections tend to yield estimates close to zero. Because of small values, a meaningful comparison is not possible in certain cases. 
The extreme return level obtained in the second approach is unrestricted by the constraint encountered in the first approach. For this reason, the second approach may fare better in discriminating varying safety levels across different locations. However, the shortcomings of the return level estimate are: (a) it is not convertible to frequency of crashes, which is a standard measure of safety; (b) the validation check relies on the correlation with actual crashes; (c) the estimation procedure is computationally cumbersome; and (d) the safety interpretation is less intuitive than the first approach.

\subsubsection{Estimation of Crash Frequency}

The risk of right-angle collisions in the $i^{\text {th }} 15$-minute block is defined by

$$
R_{i}=\operatorname{Pr}\left\{Z_{i} \geq 0\right\}=1-G_{i}(0)
$$

where $Z_{i}$ is the maximum negated PET of block i and $G_{i}(\cdot)$ is the extreme value distribution function. The $R_{i}$ is a non-negative quantity, which becomes zero if one of the following conditions is satisfied:

- No low PET is observed in the $\mathrm{i}^{\text {th }}$ block where the observation threshold for PET in the field was set at eight seconds. If there exists no PET equal to or shorter than eight seconds in that interval, this implies a near-zero-risk situation for that particular block $\mathrm{i}$ and zero risk will be assumed.

- The maximum return level of block $i, z_{\max , i}$, is not greater than zero. This implies zero probability that a maximum negated PET in block $\mathrm{i}$ will be greater than zero, the threshold for crash occurrence.

The maximum return level of the $\mathrm{i}^{\text {th }}$ block, $z_{\text {max }, i}$, is obtained by

$$
z_{\max , i}=\lim _{p \rightarrow 0} z_{p, i}=\left\{\begin{array}{r}
\mu_{i}-\frac{\sigma_{i}}{\xi_{i}} ; \\
\infty \quad \xi_{i}<0 \\
\infty \quad \xi_{i} \geq 0
\end{array},\right.
$$


where $z_{p, i}$ is the $1 / \mathrm{p}$ return level of block $\mathrm{i}$ as defined in (6-4) and $\left(\mu_{i}, \sigma_{i}, \xi_{i}\right)$ is a parameter vector associated with block i.

In the case of GEV models, the distribution of block maxima is fitted and $G_{i}(0)$ follows the model (6-1). In the model generalization as the $r$ largest order statistics, the $r$ largest values of block i are modeled, but only the block maxima is of our concern; this corresponds to the model (6-6) where $k=1$. Therefore, the definitions of $G_{i}(0)$ are the same for both the GEV and the $\mathrm{r}$ largest order statistic models. Provided that $R_{i}$ is non-zero, this leads to

$$
G_{i}(0)=\left\{\begin{array}{l}
\exp \left\{-\left[1-\xi_{i}\left(\frac{\mu_{i}}{\sigma_{i}}\right)\right]^{-1 / \xi_{i}}\right\} ; \xi_{i} \neq 0 \\
\exp \left\{-\exp \left(\frac{\mu_{i}}{\sigma_{i}}\right)\right\} \quad ; \xi_{i}=0
\end{array}\right.
$$

The risk $R_{i}$ can be alternatively viewed as the expected number of right-angle collisions during block $i$. The summation of $R_{i}$ over a period of interest $\mathrm{T}$ is therefore equivalent to the expected number of right-angle collisions during time $\mathrm{T}$, which is

$$
C_{T}=\sum_{i=1}^{N_{T}} R_{i}
$$

where $N_{T}$ is the number of 15-minute blocks during period T. If T is one year, then $C_{T}$ is the expected number of collisions during a year, or the annual frequency.

It is impractical to observe the PET data for a long period. To estimate the frequency of collisions during long period $\mathrm{T}$ based on partial observation period $\mathrm{t}$, we need to assume that the period of observation $t$ is representative of the entire period $\mathrm{T}$. In our case, the collected PET data represent only the daytime traffic operations and therefore the estimated frequency of collisions during period $\mathrm{T}$ is restricted to only daytime right-angle collisions. 
Let $N_{t}$ be the number of 15-minute blocks during the period of data collection $\mathrm{t}$ and $N_{T}$ be the number of 15-minute blocks having similar conditions as period t during the entire period of interest $\mathrm{T}$. The estimated frequency of collisions for a period $\mathrm{T}$ is

$$
\hat{C}_{T}=\frac{N_{T}}{N_{t}} \sum_{i=1}^{N_{t}} \hat{R}_{i}
$$

where $\hat{R}_{i}$ is the estimated risk for the $\mathrm{i}^{\text {th }}$ block obtained by replacing parameter estimates into equations (6-51) and (6-53).

To be specific, the period of observation $\mathrm{t}=8$ hours is equal to $N_{t}=32$ blocks and, given period of interest $\mathrm{T}=4$ years, the annual average daytime period in Indiana, which is approximately 12.09 hours per day leads to $N_{T}=12.09 \times 365 \times 4=17,651$ blocks. For homogeneous models, $R_{i}$ is constant for all $i$ with exception to the case of assumed $R_{i}=0$ which corresponds to the blocks that have no PETs. In a non-stationary case, $R_{i}$ varies across a series of blocks depending on covariates associated with each block.

\subsubsection{Estimation of Extreme Return Level}

\subsubsection{1 $\quad \underline{\text { Homogeneous Models }}$}

Under the homogeneity assumption, the block maxima are identically distributed and the determination of return level $z_{p}$ is straightforward. The $p$ corresponds to the likelihood of $z_{p}$ being exceeded by the maximum negated PET in any 15-minute daytime interval. For example, a one-year return period corresponds to $p=1 /(12.09 \times 365 \times 4)=5.6653 \times 10^{-5}$.

Replacing parameter estimates into equation (6-4) gives the estimated return level of homogeneous GEV models as follows: 


$$
\hat{z}_{p}=\left\{\begin{array}{ll}
\hat{\mu}-\frac{\hat{\sigma}}{\hat{\xi}}\left[1-\{-\log (1-p)\}^{-\hat{\xi}}\right] & \text { for } \hat{\xi} \neq 0 \\
\hat{\mu}-\hat{\sigma} \log \{-\log (1-p)\} & \text { for } \hat{\xi}=0
\end{array} .\right.
$$

For the $\mathrm{r}$ largest order statistic generalization, the estimated return level of $\mathrm{k}^{\text {th }}$ order statistic requires a numerical inversion for a solution of $z_{p}$ that satisfies equation (6-48). Recall that when $k=1$, the distribution function of the $\mathrm{r}$ largest order statistic reduces to the GEV distribution. Moreover, only the largest negated PET in a block $(k=1)$ is of our concern and this is equivalent to the block maxima. This means that, as long as a block maximum is the only concern, the case of $k>1$ is irrelevant and the numerical inversion is not necessary. The return level of the $r$ largest order statistic models can be estimated in the same manner as in the GEV case. Replacing parameter estimates obtained from fitting the $r$ largest order statistic distributions into equation (6-56) yields the estimated return level associated with the block maxima $(k=1)$.

\subsubsection{Non-Stationary Models}

The estimation of the return level is more complicated in the non-stationary models as the distributions for intervals $i$ are no longer identical. Denoting $z_{p}$ as the return level corresponding to $1 / p$ return period and letting $N_{t}$ be the number of 15 -minute time blocks in the observation period $\mathrm{t}, z_{p}$ satisfied the equation

$$
\operatorname{Pr}\left\{\max \left(Z_{1}, \ldots, Z_{N_{t}}\right) \leq z_{p}\right\}=1-p
$$

By this definition, $p$ is the probability of the level $z_{p}$ being exceeded by the maximum negated PET during any period of length $t$ with similar conditions. By the independent assumption, equation (6-57) becomes

$$
\operatorname{Pr}\left\{Z_{1} \leq z_{p}\right\} \ldots \operatorname{Pr}\left\{Z_{N_{t}} \leq z_{p}\right\} \approx \prod_{i=1}^{N_{t}} p_{i}=1-p
$$

where 


$$
p_{i}= \begin{cases}G_{i}\left(z_{p}\right) & ; 1+\xi_{i}\left(\frac{z_{p}-\mu_{i}}{\sigma_{i}}\right)>0 \\ 1 & ; \text { otherwise }\end{cases}
$$

and $G_{i}(\cdot)$ corresponds to the distribution of block $i$ defined by equations (6-1) and (6-2) for the cases $\xi_{i} \neq 0$ and $\xi_{i}=0$ respectively. Note that $p_{i}=1$ includes the case of no PET in block $i$. Taking logarithms on both sides of equation (6-58),

$$
\sum_{i=1}^{N_{t}} \log p_{i}=\log (1-p)
$$

where

$$
\log p_{i}= \begin{cases}-\left[1+\xi_{i}\left(\frac{z_{p}-\mu_{i}}{\sigma_{i}}\right)\right]^{-1 / \xi_{i}} & ; 1+\xi_{i}\left(\frac{z_{p}-\mu_{i}}{\sigma_{i}}\right)>0 \text { and } \xi_{i} \neq 0 \\ -\exp \left[-\left(\frac{z_{p}-\mu_{i}}{\sigma_{i}}\right)\right] & ; 1+\xi_{i}\left(\frac{z_{p}-\mu_{i}}{\sigma_{i}}\right)>0 \text { and } \xi_{i}=0 . \\ 0 & ; \text { otherwise }\end{cases}
$$

The value of $p$ corresponding to a n-year return period is obtained by (the number of daytime periods of length $\mathrm{t}$ in $\mathrm{n}$ years $)^{-1}$. For example, the probability $p$ for a one-year return period is equal to $\left(\frac{12.09 \times 365 \times 1}{8}\right)^{-1}=(551.61)^{-1}=1.8129 \times 10^{-3}$. This means that there are approximately 551.61 blocks of eight-hour daytime period in one year. Solving (6-60) for a given $p$ using parameter estimates $\left(\hat{\mu}_{i}, \hat{\sigma}_{i}, \hat{\xi}_{i} ; i=1, \ldots, N_{t}\right)$ gives the estimated return level $\hat{z}_{p}$. Equation (6-60) cannot be solved analytically, but any standard numerical methods for non-linear equations can be applied. 


\subsection{QUANTIFICATION OF UNCERTAINTY}

Quantifying the precision of an estimator can usually be made by calculating a confidence interval. Certain techniques, such as the simulation approach, can provide a more complete picture of the property of estimators in terms of the empirical distributions. The information about the uncertainty of estimators tells us a range of values for which we can be "statistically confident" where the true parameter lies. Different techniques are suitable for different estimators, depending on the underlying assumptions and computational complexity. Methods used to quantify the uncertainty of model estimates in this study are summarized in this section. Then, selected methods for different model estimates are discussed.

\subsubsection{Inference Methods}

\subsubsection{Delta Method}

By the invariance property of the maximum likelihood estimates, if $\hat{\theta}_{0}$ is the maximum likelihood estimate of $\theta_{0}$ and $\phi=g(\theta)$ is a scalar function, then the maximum likelihood estimate of $\phi_{0}$ is given by $\hat{\phi}_{0}=g\left(\hat{\theta}_{0}\right)$.

The invariance property is also valid for d-dimensional parameter vector $\theta_{0}$ with approximate variance-covariance matrix $V_{\theta}$. Then if $\phi=g(\theta)$ is a scalar function, the maximum likelihood estimator of $\phi_{0}=g\left(\theta_{0}\right)$ satisfies $\hat{\phi}_{0}: N\left(\phi_{0}, V_{\phi}\right)$ where

$$
V_{\phi}=\nabla \phi^{T} V_{\theta} \nabla \phi
$$

with

$$
\nabla \phi=\left[\begin{array}{lll}
\frac{\partial \phi}{\partial \theta_{1}} & \cdots & \frac{\partial \phi}{\partial \theta_{d}}
\end{array}\right]^{T}
$$


evaluated at $\hat{\theta}_{0}$. This result is usually referred to as the delta method. The delta method enables the approximate normality of $\hat{\phi}_{0}$ to be used to obtain confidence intervals for $\phi_{0}$.

To illustrate the method, the maximum likelihood estimate of $z_{p}$ for $0<\mathrm{p}<1$, the $1 / \mathrm{p}$ return level, is obtained as

$$
\hat{z}_{p}= \begin{cases}\hat{\mu}-\frac{\hat{\sigma}}{\hat{\xi}}\left[1-y_{p}^{-\xi}\right] & \text { for } \hat{\xi} \neq 0 \\ \hat{\mu}-\hat{\sigma} \log y_{p} & \text { for } \xi=0\end{cases}
$$

where $y_{p}=-\log (1-p)$. Using the delta method, the variance of the $\hat{z}_{p}$ can be approximated as

$$
\operatorname{var}\left(\hat{z}_{p}\right) \approx \nabla z_{p}^{T} V \nabla z_{p}
$$

where $\mathrm{V}$ is the variance-covariance matrix of $(\hat{\mu}, \hat{\sigma}, \hat{\xi})$ and

$$
\nabla z_{p}^{T}=\left[\begin{array}{lll}
\frac{\partial z_{p}}{\partial \mu}, & \frac{\partial z_{p}}{\partial \sigma}, & \frac{\partial z_{p}}{\partial \xi}
\end{array}\right]
$$

\subsubsection{Profile Likelihood Function}

The profile log-likelihood of any individual parameter is obtained by fixing a parameter of interest and then maximizing the log-likelihood with respect to the remaining parameters. This is repeated for a specified range of the parameter of interest. The corresponding maximized values of the log-likelihood can be used to obtain approximate confidence intervals that are generally more accurate than those obtained from the delta method.

Let $x_{1}, \ldots, x_{n}$ be independent realizations from a parametric distribution and let $\hat{\theta}_{0}$ denote the maximum likelihood estimator of the d-dimensional model parameter $\theta_{0}=\left(\theta^{(1)}, \theta^{(2)}\right)$, where $\theta^{(1)}$ is a k-dimensional subset of $\theta_{0}$. Then, under suitable regularity conditions, for large $\mathrm{n}$ 


$$
D_{p}\left(\theta^{(1)}\right)=2\left\{1\left(\hat{\theta}_{0}\right)-1\left(\theta^{(1)}\right)\right\}: \chi_{k}^{2}
$$

The approximate inferences on the maximum likelihood estimator of $\theta^{(1)}$ can now be determined. For a single component $\theta_{i}, C_{\alpha}=\left\{\theta_{i}: D_{p}\left(\theta_{i}\right) \leq c_{\alpha}\right\}$ is a $(1-\alpha)$ confidence interval, where $c_{\alpha}$ is the $(1-\alpha)$ quantile of the $\chi_{1}^{2}$ distribution.

\subsubsection{Simulation}

A difficulty arises in the estimation of standard errors or confidence intervals of estimates of the non-stationary models since each independent realization is not identically distributed. Both the delta method and calculation of the profile likelihood are impractical. A crude approximation in this scenario can be obtained by simulation.

The value of interest in our analysis is usually a scalar function of model parameters. If the sampling distribution of the maximum likelihood estimator of the model parameters were known, we could simulate from this distribution and, for each simulated value, compute the quantity of interest to obtain the sampling distribution of the quantity of interest. Since the sampling distribution is generally unknown in practice, an alternative is to approximate this procedure by using the multivariate normal approximation of maximum likelihood estimates, under suitable regularity conditions.

More precisely, denoting the model parameters by $\theta$, and their maximum likelihood estimates by $\hat{\theta}$, the approximate sampling distribution to obtain $\theta_{1}^{*}, \ldots, \theta_{k}^{*}$, which constitute a sample from the approximate sampling distribution of the maximum likelihood estimator. For each $\theta_{j}^{*}$, compute the quantity of interest $g_{j}^{*}=g\left(\theta_{j}^{*}\right)$ which is a realization from the approximate sampling distribution of $g(\hat{\theta})$. Finally, the set $g_{1}^{*}, \ldots, g_{k}^{*}$ can be used to construct the density estimate of the distribution or to obtain approximate confidence intervals. 


\subsubsection{Guidelines for Method Selection}

Inferences on parameter estimates obtained by maximum likelihood estimations, under suitable regularity conditions, can be obtained from the inverse of the observed information matrix as described in Section 6.7.2.2.

Inferences on the return level can be made using both the delta method and the profile likelihood function with the exception of non-stationary models. The inferences from the delta method and the profile likelihood function on the return level are approximately the same for the low return period. However, the discrepancy may arise with the increasing return period, which is due in large to asymmetry in the profile log-likelihood surface. Such asymmetries are to be expected since the data provide increasingly weaker information about high levels of the process. The profile likelihood function should be preferred to the delta method where such discrepancy exists.

In the case of non-stationary models, inferences on the return level are obtained by the simulation method. Inferences by the delta method or the profile likelihood function are impractical due to the lack of an identically-distributed assumption for each independent realization.

Inferences on the crash frequency estimate are best obtained by the simulation approach. The crash frequency is a non-negative quantity and the delta method would simply use a symmetric pivot around the mean estimate to obtain a confidence interval, which can fall into the undesirable negative region. A profile likelihood method would require a reparameterization of an expression to determine the return level, such that the return period is a part of log-likelihood function to be maximized. Then, a profile likelihood plot can be obtained by maximizing the likelihood function with respect to a range of specified return periods. This procedure is not applicable in two cases: (a) the location has a block with no observed PET and (b) non-stationary cases. The profile likelihood requires a continuously differentiable function for the return level and the case of a block with no PET is equivalent to an infinite return level. The stepwise function is needed in this case and therefore the profile likelihood function cannot be constructed. In addition, nonstationary models create the same difficulty encountered in the case of inferences on the return level - non-homogeneous distributions. Therefore, the simulation method is the most appropriate method to draw inferences on the crash frequency estimate as it is unrestricted by the limitations encountered in other methods. 


\section{CHAPTER 7 MODEL DEVELOPMENT AND ESTIMATION RESULTS}

Following the proposed analytical approach outlined in the previous chapter, the PET modeling considerations are first discussed in this chapter. Then, the logic behind the model development and the criteria for model selection are explained. The results from a selected model are presented and discussed along with model interpretation. Important issues in modeling PETs with EVT are summarized. Suggested resolutions conclude the chapter.

\subsection{MODELING CONSIDERATIONS}

Several factors are taken into account in the procedure of extreme value modeling of PETs. These factors are discussed in the following sections.

\subsubsection{Sample Size}

A small sample size can lead to two major problems in the analysis procedure: (a) a large variance of model estimates and (b) a non-convergence of a maximum likelihood estimation algorithm. There are two different levels of sample size considerations in this study. The first one is the total number of PETs observed for each location and the second one is the number of data points that are used to estimate the models based on the selected extreme value sampling scheme. The total number of observed PETs depends on the length of the observation period and the frequency of crossing events at the location. Technically, the longer observation period is better but is not always possible due to time and resource constraints. The selected $r$ largest order statistic model allows us to include more data points for model estimation by increasing the $r$ values. However, the choice of a greater $r$ requires a careful examination as it may violate the asymptotic assumption upon which the model is established. 


\subsubsection{Data Aggregation}

The PET data were measured with reference to the location of their occurrences, which are referred to as conflict zones. Provided that a sample size is sufficient, the disaggregate modeling of the PET by conflict zones is preferable as it may provide more insight into the specific area of problems inside the intersection. This is, however, not practical in this study due to a limited sample size when PET data were disaggregated by conflict zones. A preliminary evaluation using PET data by conflict zones was found to give estimates of an extremely large variance and frequent cases of the non-converged maximum likelihood estimation. For these reasons, PET data from all conflict zones are combined for each site in further modeling of a distribution of PET extreme values.

\subsubsection{PET Classification}

Short PETs can be classified into two different categories according to the mechanism of the crossing events: (a) short PETs from regular crossings and (b) short PETs from queue spillbacks.

In the first case, the first vehicle traverses the virtual conflict spot at a normal speed. Unusually short PETs in this case are attributed to situations such as the first vehicle ran on red or the second vehicle started early upon or in anticipation of receiving the green light. This category of PETs regularly occurs over time depending on traffic signal settings and road users' reacting maneuvers in response to the traffic control and other road users.

The second type of short PETs occurs when queue spillbacks from the upstream traffic block the right-of-way of the vehicle from a conflicting approach. A vehicle in the queue typically traverses a conflict spot at an unusually low speed. In contrast to the first type, short PETs from queue spillbacks tend to recur during the congested period. The PET occurrence process is obviously non-random and tends to cluster for a certain period of time for the latter case.

Although the speed data were not collected for each vehicle, we are able to distinguish these two categories using the encroachment time (ET) as a speed proxy. ET was manually measured along with the PET for each crossing event. The relationship between the traversing vehicle speed and ET can be described as 


$$
E T \propto \frac{1}{\text { Speed }} .
$$

The second type of short PETs, however, cannot be omitted from the modeling since it would imply a fallacy that a congested intersection was safe and, consequently, the risk could be underestimated. On the other hand, the inclusion of this type into the models may lead to the risk overestimation due to the fact that the risk associated with these short PETs seems to be overrepresented. The dilemma posed by PETs from queue spillbacks triggers the need to carefully examine the following issues:

- How can we measure the degree of short PETs resulting from queue spillbacks?

- How can we use the above measure to address the dilemma posed by the short PETs from queue spillbacks?

For the first issue, the coefficient of variation (CV) of ET at each site can be computed in order to gauge the degree of short PETs from a queue spillback. The CV is defined by

$$
C V_{E T}=\frac{\sigma_{E T}}{\mu_{E T}} \approx \frac{s_{E T}}{\bar{x}_{E T}}
$$

where $\sigma_{E T}$ is the standard deviation of ET, estimated by the sample standard deviation $S_{E T}$ and $\mu_{E T}$ is the mean of ET, approximated by the sample mean $\bar{x}_{E T}$. Large value of $\mathrm{CV}_{\mathrm{ET}}$ indicates a potential problem of excessive number of short PETs from queue spillbacks.

Using the computed $\mathrm{CV}_{\mathrm{ET}}$, we can pinpoint the problematic sites and then filter out the PETs with high ET values prior to the model estimation to avoid the short PETs being overrepresented. There is no definite answer for how high the $\mathrm{CV}_{\mathrm{ET}}$ is deemed to be problematic and how the PET data should be filtered. Several ideas were implemented in order to find out the best approach to filter PET data using CV of ET as one of the criteria. Findings and results are discussed in the model development section. 


\subsubsection{Non-Stationarity Considerations}

Visual examinations of PET (Appendix C) and volume (Appendix D) variations over time reveal similar trends to an extent at several sites. This questions the assumption of homogeneity of the PET process. The corresponding variation patterns between PET and volume indicate a possibility of explaining the variability in the PET process using certain covariates. The exposure data, such as the frequency of PET counts and traffic volume, may be considered.

Non-stationary models generally demand more data and entail a relatively complicated parameter estimation. Therefore, it should be preferred to the homogeneous models only if it can substantially explain the variability in the process.

A list of potential covariates is summarized in Table 7-1. The basic principle underlying these possible covariates is that it should have a logical relationship with the PET process. The exposure data, such as PET counts and traffic volume, are obvious candidates. A PET count varies with the number of crossing events, which in turn also depends on the amount of traffic interactions at the intersections.

The conflicting volume, $V_{x}$, in Table 7-1 was defined earlier in equation (5-4) as

$$
V_{x}=\sqrt{\left(V_{N B} V_{E B}+V_{S B} V_{E B}+V_{N B} V_{W B}+V_{S B} V_{W B}\right)},
$$

where $V_{N B}, V_{S B}, V_{E B}$, and $V_{W B}$ are total approach volumes from northbound, southbound, eastbound, and westbound approaches respectively. The conflicting through-traffic volume $\left(V_{x t h}\right)$ is similar to the equation above, except that the through-traffic volumes from respective approaches are used instead of the total approach volumes; this was previously defined in equation (5-5) as

$$
V_{x t h}=\sqrt{\left(V_{\text {NBTH }} V_{E B T H}+V_{S B T H} V_{E B T H}+V_{N B T H} V_{W B T H}+V_{S B T H} V_{W B T H}\right)},
$$

where $V_{N B T H}, V_{S B T H}, V_{E B T H}$ and $V_{W B T H}$ are through-traffic volumes from northbound, southbound, eastbound, and westbound approaches respectively. 
These two variables aim to represent the opportunity for interactions at the intersection. The variables $V_{x, c z}$ and $V_{x t h, c z}$ use the product of the volumes at a conflict zone associated with each PET. The logarithm versions of certain covariates were also checked to determine if the model fits can be improved when adjusted for scaling effects. The remaining covariates in the list are self-explanatory.

Table 7-1: List of Covariates

\begin{tabular}{|c|c|}
\hline Acronym & Descriptions \\
\hline $\mathrm{V}_{\text {sum }}$ & Total intersection volume \\
\hline$V_{\text {sumth }}$ & Total through traffic volume \\
\hline$V_{x}$ & Conflicting volume \\
\hline$V_{x t h}$ & Conflicting through volume \\
\hline$V_{L T}$ & Total left-turn volume \\
\hline LTratio & Ratio of left-turn to total traffic volume \\
\hline $\mathrm{V}_{\text {sum }, \mathrm{cz}}$ & Total approach volume at a conflict zone \\
\hline $\mathrm{V}_{\text {sumth,cz }}$ & Total through-traffic volume at a conflict zone \\
\hline $\mathrm{V}_{\mathrm{x}, \mathrm{cz}}$ & Conflicting volume at a conflict zone \\
\hline $\mathrm{V}_{\mathrm{xth}, \mathrm{cz}}$ & Conflicting through volume at a conflict zone \\
\hline$f_{\text {PET<U }}$ & 15-minute counts of PET $<$ threshold $u$ \\
\hline $\log \left(V_{\text {sum }}\right)$ & Natural logarithm of $\mathrm{V}_{\text {sum }}$ \\
\hline $\log \left(V_{\text {sumth }}\right)$ & Natural logarithm of $\mathrm{V}_{\text {sumth }}$ \\
\hline $\log \left(V_{x}\right)$ & Natural logarithm of $V_{x}$ \\
\hline $\log \left(\mathrm{V}_{\mathrm{xth}}\right)$ & Natural logarithm of $V_{x t h}$ \\
\hline $\log \left(V_{L T}\right)$ & Natural logarithm of $V_{L T}$ \\
\hline $\log ($ LTratio) & Natural logarithm of LTratio \\
\hline $\log \left(V_{\text {sum }, \mathrm{cz}}\right)$ & Natural logarithm of $\mathrm{V}_{\text {sum,cz }}$ \\
\hline $\log \left(V_{\text {sumth }, c z}\right)$ & Natural logarithm of $\mathrm{V}_{\text {sumth,cz }}$ \\
\hline $\log \left(V_{x, c z}\right)$ & Natural logarithm of $\mathrm{V}_{\mathrm{x}, \mathrm{cz}}$ \\
\hline $\log \left(V_{x t h, c z}\right)$ & Natural logarithm of $V_{x t h, c z}$ \\
\hline
\end{tabular}

Both the GEV and the $r$ largest order statistic distributions have three model parameters: location $(\mu)$, scale $(\sigma)$, and shape $(\xi)$. The choices of the combination of covariates, the inverse-link structure (see Section 6.5.1), and the model parameters to be linked are important considerations in the non-stationary models of extreme values.

The identity and exponential functions are the two commonly-used inverse-link structures. The identity link is appropriate for location and shape parameters as it implies a linear unconstrained relationship between parameters and covariates. The exponential link is a natural choice for the 
scale parameter since it satisfies the condition of $\sigma>0$ in the GEV and the $\mathrm{r}$ largest order statistic distributions.

Table 7-2: Non-Stationary Models with Different Link Structures - Site 87933

\begin{tabular}{|c|c|c|c|c|c|c|c|c|c|c|c|c|c|c|c|c|c|}
\hline \multirow{2}{*}{$\begin{array}{l}\text { Site } 87933 \\
\text { Covariates }\end{array}$} & \multirow[b]{2}{*}{ Link } & \multicolumn{4}{|c|}{$\begin{array}{l}r \text { largest order } \\
\text { statistic model }\end{array}$} & \multicolumn{2}{|c|}{$\mu=\boldsymbol{X}^{T} \boldsymbol{\beta}$} & \multicolumn{2}{|c|}{$\sigma=e^{\left(\boldsymbol{X}^{T} \boldsymbol{\beta}\right)}$} & \multicolumn{2}{|c|}{$\xi=\boldsymbol{X}^{T} \boldsymbol{\beta}$} & \multicolumn{6}{|c|}{ Standard errors of parameter estimates } \\
\hline & & $\mathbf{r}$ & $\mathbf{n}$ & p & nllh & $\beta_{01}$ & $\beta_{11}$ & $\beta_{02}$ & $\beta_{12}$ & $\beta_{03}$ & $\beta_{13}$ & $\operatorname{se}\left(\beta_{01}\right)$ & $\operatorname{se}\left(\beta_{11}\right)$ & $\operatorname{se}\left(\beta_{02}\right)$ & $\operatorname{se}\left(\beta_{12}\right)$ & $\operatorname{se}\left(\beta_{03}\right)$ & $\operatorname{se}\left(\beta_{13}\right)$ \\
\hline None & NA & 2 & 62 & 3 & 74.706 & -4.674 & NA & 1.209 & NA & -0.237 & NA & 0.201 & NA & 0.104 & NA & 0.061 & NA \\
\hline $\mathrm{V}_{\text {sum }}$ & \multirow{12}{*}{$\begin{array}{l}\text { Identity link } \\
\text { in location } \\
\text { parameter }\end{array}$} & 2 & 62 & 4 & 71.007 & -7.083 & 0.010 & 1.132 & NA & -0.221 & NA & 0.857 & 0.003 & 0.098 & NA & 0.059 & NA \\
\hline$V_{\text {sumth }}$ & & 2 & 62 & 4 & 70.197 & -6.943 & 0.012 & 1.116 & NA & -0.221 & NA & 0.730 & 0.004 & 0.097 & NA & 0.058 & NA \\
\hline $\mathrm{V}_{\mathrm{x}}$ & & 2 & 62 & 4 & 70.904 & -7.671 & 0.033 & 1.127 & NA & -0.206 & NA & 1.032 & 0.011 & 0.099 & NA & 0.062 & NA \\
\hline$V_{x t h}$ & & 2 & 62 & 4 & 59.699 & -8.518 & 0.082 & 0.899 & NA & -0.180 & NA & 0.576 & 0.012 & 0.084 & NA & 0.081 & NA \\
\hline$V_{x, c z}$ & & 2 & 62 & 4 & 74.704 & -4.649 & 0.000 & 1.209 & NA & -0.238 & NA & 0.445 & 0.007 & 0.104 & NA & 0.062 & NA \\
\hline $\mathrm{V}_{\mathrm{xth}, \mathrm{cz}}$ & & 2 & 62 & 4 & 69.159 & -7.060 & 0.076 & 1.090 & NA & -0.230 & NA & 0.686 & 0.022 & 0.095 & NA & 0.064 & NA \\
\hline $\log \left(V_{\text {sum }}\right)$ & & 2 & 62 & 4 & 71.101 & -17.583 & 2.352 & 1.133 & NA & -0.226 & NA & 4.633 & 0.845 & 0.098 & NA & 0.059 & NA \\
\hline $\log \left(V_{\text {sumth }}\right)$ & & 2 & 62 & 4 & 70.337 & -16.003 & 2.172 & 1.119 & NA & -0.228 & NA & 3.689 & 0.709 & 0.097 & NA & 0.059 & NA \\
\hline $\log \left(V_{x}\right)$ & & 2 & 62 & 4 & 70.914 & -18.095 & 2.978 & 1.126 & NA & -0.209 & NA & 4.622 & 1.027 & 0.099 & NA & 0.062 & NA \\
\hline $\log \left(V_{x t h}\right)$ & & 2 & 62 & 4 & 59.672 & -18.567 & 3.639 & 0.900 & NA & -0.180 & NA & 2.123 & 0.559 & 0.084 & NA & 0.079 & NA \\
\hline $\log \left(V_{x, c z}\right)$ & & 2 & 62 & 4 & 74.696 & -4.895 & 0.057 & 1.209 & NA & -0.237 & NA & 1.589 & 0.402 & 0.104 & NA & 0.061 & NA \\
\hline $\log \left(V_{x t h, c z}\right)$ & & 2 & 62 & 4 & 69.362 & -12.073 & 2.172 & 1.096 & NA & -0.236 & NA & 2.155 & 0.634 & 0.095 & NA & 0.063 & NA \\
\hline $\mathrm{V}_{\text {sum }}$ & \multirow{12}{*}{$\begin{array}{c}\text { Exponential } \\
\text { link in scale } \\
\text { parameter }\end{array}$} & 2 & 62 & 4 & 74.506 & -4.620 & NA & 0.503 & -0.001 & -0.229 & NA & 0.217 & NA & 0.502 & 0.002 & 0.066 & NA \\
\hline$V_{\text {sumth }}$ & & 2 & 62 & 4 & 74.555 & -4.623 & NA & 0.419 & -0.001 & -0.228 & NA & 0.220 & NA & 0.428 & 0.002 & 0.067 & NA \\
\hline$V_{x}$ & & 2 & 62 & 4 & 73.996 & -4.578 & NA & 0.913 & -0.008 & -0.224 & NA & 0.211 & NA & 0.601 & 0.007 & 0.071 & NA \\
\hline$V_{x t h}$ & & 2 & 62 & 4 & 72.806 & -4.374 & NA & 1.377 & -0.026 & -0.058 & NA & 0.216 & NA & 0.536 & 0.011 & 0.130 & NA \\
\hline$V_{x, c z}$ & & 2 & 62 & 4 & 74.632 & -4.671 & NA & 0.268 & -0.001 & -0.245 & NA & 0.200 & NA & 0.226 & 0.004 & 0.065 & NA \\
\hline$V_{x t h, c z}$ & & 2 & 62 & 4 & 73.875 & -4.544 & NA & 0.850 & -0.022 & -0.190 & NA & 0.211 & NA & 0.508 & 0.016 & 0.076 & NA \\
\hline $\log \left(V_{\text {sum }}\right)$ & & 2 & 62 & 4 & 74.505 & -4.624 & NA & 1.894 & -0.312 & -0.227 & NA & 0.214 & NA & 2.677 & 0.488 & 0.066 & NA \\
\hline $\log \left(V_{\text {sumth }}\right)$ & & 2 & 62 & 4 & 74.514 & -4.623 & NA & 1.474 & -0.247 & -0.225 & NA & 0.215 & NA & 2.076 & 0.398 & 0.067 & NA \\
\hline $\log \left(V_{x}\right)$ & & 2 & 62 & 4 & 74.016 & -4.583 & NA & 3.480 & -0.735 & -0.221 & NA & 0.209 & NA & 2.732 & 0.608 & 0.071 & NA \\
\hline $\log \left(V_{x t h}\right)$ & & 2 & 62 & 4 & 72.051 & -4.402 & NA & 4.653 & -1.184 & -0.066 & NA & 0.201 & NA & 1.696 & 0.444 & 0.114 & NA \\
\hline $\log \left(V_{x, c z}\right)$ & & 2 & 62 & 4 & 74.688 & -4.671 & NA & 0.343 & -0.039 & -0.240 & NA & 0.201 & NA & 0.831 & 0.211 & 0.063 & NA \\
\hline$\underline{\log \left(V_{x t h, c z}\right)}$ & & 2 & 62 & 4 & 74.084 & -4.569 & NA & 1.905 & -0.507 & -0.199 & NA & 0.213 & NA & 1.523 & 0.447 & 0.073 & NA \\
\hline $\mathrm{V}_{\text {sum }}$ & \multirow{12}{*}{$\begin{array}{l}\text { Identity link } \\
\text { in shape } \\
\text { parameter }\end{array}$} & 2 & 62 & 4 & 74.468 & -4.686 & NA & 1.153 & NA & -0.774 & 0.002 & 0.202 & NA & 0.122 & NA & 0.813 & 0.003 \\
\hline$V_{\text {sumth }}$ & & 2 & 62 & 4 & 74.371 & -4.693 & NA & 1.137 & NA & -0.777 & 0.003 & 0.202 & NA & 0.125 & NA & 0.724 & 0.004 \\
\hline$V_{x}$ & & 2 & 62 & 4 & 74.508 & -4.669 & NA & 1.160 & NA & -0.886 & 0.007 & 0.200 & NA & 0.120 & NA & 1.029 & 0.012 \\
\hline$V_{\text {xth }}$ & & 2 & 62 & 4 & 70.019 & -4.608 & NA & 0.876 & NA & -2.024 & 0.036 & 0.158 & NA & 0.085 & NA & 0.585 & 0.012 \\
\hline$V_{x, c z}$ & & 2 & 62 & 4 & 73.825 & -4.685 & NA & 1.196 & NA & 0.051 & -0.007 & 0.186 & NA & 0.103 & NA & 0.190 & 0.004 \\
\hline$V_{\text {xth,cz }}$ & & 2 & 62 & 4 & 71.037 & -4.639 & NA & 0.955 & NA & -2.661 & 0.072 & 0.164 & NA & 0.084 & NA & 0.667 & 0.020 \\
\hline $\log \left(V_{\text {sum }}\right)$ & & 2 & 62 & 4 & 74.271 & -4.693 & NA & 1.136 & NA & -4.030 & 0.691 & 0.200 & NA & 0.118 & NA & 4.444 & 0.808 \\
\hline $\log \left(V_{\text {sumth }}\right)$ & & 2 & 62 & 4 & 74.143 & -4.699 & NA & 1.120 & NA & -3.551 & 0.633 & 0.200 & NA & 0.121 & NA & 3.629 & 0.692 \\
\hline $\log \left(V_{x}\right)$ & & 2 & 62 & 4 & 74.338 & -4.673 & NA & 1.143 & NA & -4.091 & 0.861 & 0.199 & NA & 0.117 & NA & 4.559 & 1.017 \\
\hline $\log \left(V_{x t h}\right)$ & & 2 & 62 & 4 & 69.900 & -4.603 & NA & 0.871 & NA & -6.460 & 1.599 & 0.157 & NA & 0.083 & NA & 1.969 & 0.510 \\
\hline $\log \left(V_{x, c z}\right)$ & & 2 & 62 & 4 & 74.116 & -4.685 & NA & 1.204 & NA & 0.901 & -0.304 & 0.191 & NA & 0.104 & NA & 1.040 & 0.279 \\
\hline $\log \left(V_{x t h, c z}\right)$ & & 2 & 62 & 4 & NA & NA & NA & NA & NA & NA & NA & NA & NA & NA & NA & NA & NA \\
\hline
\end{tabular}

In order to identify which model parameters should be linked, the following procedure was conducted. The basic homogeneous models were first fitted for all sites using the $r$ largest order statistic models as a base case. Then, a series of non-stationary models were estimated for each site with the identity link in location and shape parameters and the exponential link in scale parameter. Selected covariates from the list in Table 7-1 were used in model estimations. The 
findings were consistent from sites to sites; therefore partial estimation results from two arbitrarily selected sites -87933 and 97901 - are presented in Table 7-2 and Table 7-3.

Table 7-3: Non-Stationary Models with Different Link Structures - Site 97901

\begin{tabular}{|c|c|c|c|c|c|c|c|c|c|c|c|c|c|c|c|c|c|}
\hline \multirow{2}{*}{$\begin{array}{l}\text { Site } 97901 \\
\text { Covariates }\end{array}$} & \multirow[b]{2}{*}{ Link } & \multicolumn{4}{|c|}{$\begin{array}{l}r \text { largest order } \\
\text { statistic model }\end{array}$} & \multicolumn{2}{|c|}{$\mu=\boldsymbol{X}^{T} \boldsymbol{\beta}$} & \multicolumn{2}{|c|}{$\sigma=e^{\left(\boldsymbol{X}^{T} \boldsymbol{\beta}\right)}$} & \multicolumn{2}{|c|}{$\xi=\boldsymbol{X}^{T} \boldsymbol{\beta}$} & \multicolumn{6}{|c|}{ Standard errors of parameter estimates } \\
\hline & & $\mathbf{r}$ & $\mathbf{n}$ & $\mathbf{p}$ & nllh & $\beta_{01}$ & $\beta_{11}$ & $\beta_{02}$ & $\beta_{12}$ & $\beta_{03}$ & $\beta_{13}$ & $\operatorname{se}\left(\beta_{01}\right)$ & $\operatorname{se}\left(\beta_{11}\right)$ & $\operatorname{se}\left(\beta_{02}\right)$ & $\operatorname{se}\left(\beta_{12}\right)$ & $\operatorname{se}\left(\beta_{03}\right)$ & $\operatorname{se}\left(\beta_{13}\right)$ \\
\hline None & NA & 3 & 96 & 3 & 32.722 & -3.940 & NA & 0.655 & NA & -0.150 & NA & 0.102 & NA & 0.054 & NA & 0.081 & NA \\
\hline $\mathrm{V}_{\text {sum }}$ & \multirow{12}{*}{$\begin{array}{l}\text { Identity link } \\
\text { in location } \\
\text { parameter }\end{array}$} & 3 & 96 & 4 & 20.967 & -6.025 & 0.004 & 0.560 & NA & -0.109 & NA & 0.383 & 0.001 & 0.050 & NA & 0.109 & NA \\
\hline$V_{\text {sumth }}$ & & 3 & 96 & 4 & 21.522 & -5.953 & 0.005 & 0.564 & NA & -0.116 & NA & 0.381 & 0.001 & 0.050 & NA & 0.111 & NA \\
\hline $\mathrm{V}_{\mathrm{x}}$ & & 3 & 96 & 4 & 21.204 & -5.993 & 0.009 & 0.562 & NA & -0.113 & NA & 0.383 & 0.002 & 0.050 & NA & 0.108 & NA \\
\hline$V_{\text {xth }}$ & & 3 & 96 & 4 & 21.800 & -5.904 & 0.010 & 0.565 & NA & -0.122 & NA & 0.378 & 0.002 & 0.050 & NA & 0.110 & NA \\
\hline $\mathrm{V}_{\mathrm{x}, \mathrm{cz}}$ & & 3 & 96 & 4 & 22.124 & -5.663 & 0.014 & 0.564 & NA & -0.143 & NA & 0.342 & 0.003 & 0.049 & NA & 0.108 & NA \\
\hline$V_{x t h, c z}$ & & 3 & 96 & 4 & 22.644 & -5.490 & 0.015 & 0.567 & NA & -0.148 & NA & 0.315 & 0.003 & 0.049 & NA & 0.112 & NA \\
\hline $\log \left(V_{\text {sum }}\right)$ & & 3 & 96 & 4 & 21.013 & -15.965 & 1.962 & 0.563 & NA & -0.092 & NA & 2.247 & 0.370 & 0.052 & NA & 0.112 & NA \\
\hline $\log \left(V_{\text {sumth }}\right)$ & & 3 & 96 & 4 & 21.628 & -15.282 & 1.900 & 0.567 & NA & -0.098 & NA & 2.180 & 0.368 & 0.052 & NA & 0.114 & NA \\
\hline $\log \left(V_{x}\right)$ & & 3 & 96 & 4 & 21.236 & -14.457 & 1.938 & 0.564 & NA & -0.097 & NA & 1.990 & 0.370 & 0.051 & NA & 0.111 & NA \\
\hline $\log \left(V_{x t h}\right)$ & & 3 & 96 & 4 & 21.922 & -13.716 & 1.856 & 0.568 & NA & -0.104 & NA & 1.912 & 0.366 & 0.052 & NA & 0.113 & NA \\
\hline $\log \left(V_{x, c z}\right)$ & & 3 & 96 & 4 & 21.818 & -11.970 & 1.686 & 0.564 & NA & -0.128 & NA & 1.597 & 0.339 & 0.050 & NA & 0.110 & NA \\
\hline $\log \left(V_{x t h, c z}\right)$ & & 3 & 96 & 4 & 22.461 & -10.943 & 1.519 & 0.568 & NA & -0.132 & NA & 1.430 & 0.314 & 0.050 & NA & 0.114 & NA \\
\hline $\mathrm{V}_{\text {sum }}$ & \multirow{12}{*}{$\begin{array}{c}\text { Exponential } \\
\text { link in scale } \\
\text { parameter }\end{array}$} & 3 & 96 & 4 & 29.001 & -3.899 & NA & 1.000 & -0.003 & 0.111 & NA & 0.101 & NA & 0.405 & 0.001 & 0.130 & NA \\
\hline$V_{\text {sumth }}$ & & 3 & 96 & 4 & 29.316 & -3.900 & NA & 0.966 & -0.003 & 0.110 & NA & 0.102 & NA & 0.412 & 0.001 & 0.132 & NA \\
\hline $\mathrm{V}_{\mathrm{x}}$ & & 3 & 96 & 4 & 29.185 & -3.899 & NA & 0.960 & -0.006 & 0.103 & NA & 0.101 & NA & 0.404 & 0.002 & 0.128 & NA \\
\hline$V_{\text {xth }}$ & & 3 & 96 & 4 & 29.536 & -3.899 & NA & 0.915 & -0.007 & 0.099 & NA & 0.102 & NA & 0.412 & 0.002 & 0.130 & NA \\
\hline $\mathrm{V}_{\mathrm{x}, \mathrm{cz}}$ & & 3 & 96 & 4 & 30.346 & -3.892 & NA & 0.643 & -0.009 & 0.067 & NA & 0.103 & NA & 0.396 & 0.003 & 0.127 & NA \\
\hline $\mathrm{V}_{\mathrm{xth}, \mathrm{cz}}$ & & 3 & 96 & 4 & 30.780 & -3.891 & NA & 0.544 & -0.009 & 0.068 & NA & 0.104 & NA & 0.399 & 0.004 & 0.135 & NA \\
\hline $\log \left(V_{\text {sum }}\right)$ & & 3 & 96 & 4 & 28.766 & -3.903 & NA & 7.844 & -1.348 & 0.115 & NA & 0.101 & NA & 2.339 & 0.382 & 0.133 & NA \\
\hline $\log \left(V_{\text {sumth }}\right)$ & & 3 & 96 & 4 & 29.096 & -3.904 & NA & 7.448 & -1.316 & 0.114 & NA & 0.101 & NA & 2.306 & 0.386 & 0.136 & NA \\
\hline $\log \left(V_{x}\right)$ & & 3 & 96 & 4 & 28.944 & -3.902 & NA & 6.737 & -1.318 & 0.106 & NA & 0.101 & NA & 2.075 & 0.383 & 0.132 & NA \\
\hline $\log \left(V_{x t h}\right)$ & & 3 & 96 & 4 & 29.331 & -3.903 & NA & 6.297 & -1.273 & 0.103 & NA & 0.101 & NA & 2.043 & 0.388 & 0.134 & NA \\
\hline $\log \left(V_{x, c z}\right)$ & & 3 & 96 & 4 & 29.950 & -3.897 & NA & 4.397 & -1.008 & 0.067 & NA & 0.102 & NA & 1.681 & 0.351 & 0.125 & NA \\
\hline $\log \left(V_{x t h, c z}\right)$ & & 3 & 96 & 4 & 30.375 & -3.899 & NA & 3.748 & -0.899 & 0.067 & NA & 0.103 & NA & 1.589 & 0.342 & 0.131 & NA \\
\hline $\mathrm{V}_{\text {sum }}$ & \multirow{12}{*}{$\begin{array}{l}\text { Identity link } \\
\text { in shape } \\
\text { parameter }\end{array}$} & 3 & 96 & 4 & 28.351 & -4.002 & NA & 0.551 & NA & -1.753 & 0.004 & 0.093 & NA & 0.063 & NA & 0.523 & 0.001 \\
\hline$V_{\text {sumth }}$ & & 3 & 96 & 4 & 28.296 & -4.004 & NA & 0.551 & NA & -1.744 & 0.004 & 0.092 & NA & 0.061 & NA & 0.517 & 0.001 \\
\hline$V_{x}$ & & 3 & 96 & 4 & 28.414 & -4.004 & NA & 0.550 & NA & -1.737 & 0.008 & 0.093 & NA & 0.063 & NA & 0.518 & 0.002 \\
\hline$V_{\text {xth }}$ & & 3 & 96 & 4 & 28.352 & -4.005 & NA & 0.549 & NA & -1.727 & 0.009 & 0.092 & NA & 0.061 & NA & 0.511 & 0.003 \\
\hline $\mathrm{V}_{\mathrm{x}, \mathrm{cz}}$ & & 3 & 96 & 4 & 28.865 & -4.007 & NA & 0.549 & NA & -1.518 & 0.013 & 0.093 & NA & 0.061 & NA & 0.511 & 0.005 \\
\hline $\mathrm{V}_{\mathrm{xth}, \mathrm{cz}}$ & & 3 & 96 & 4 & 28.962 & -4.008 & NA & 0.547 & NA & -1.412 & 0.013 & 0.093 & NA & 0.061 & NA & 0.502 & 0.005 \\
\hline $\log \left(V_{\text {sum }}\right)$ & & 3 & 96 & 4 & 28.397 & -4.002 & NA & 0.551 & NA & -10.207 & 1.669 & 0.093 & NA & 0.064 & NA & 3.504 & 0.582 \\
\hline $\log \left(V_{\text {sumth }}\right)$ & & 3 & 96 & 4 & 28.362 & -4.003 & NA & 0.552 & NA & -9.862 & 1.655 & 0.093 & NA & 0.063 & NA & 3.355 & 0.573 \\
\hline $\log \left(V_{x}\right)$ & & 3 & 96 & 4 & 28.459 & -4.003 & NA & 0.550 & NA & -8.988 & 1.660 & 0.094 & NA & 0.065 & NA & 3.084 & 0.579 \\
\hline $\log \left(V_{x t h}\right)$ & & 3 & 96 & 4 & 28.427 & -4.005 & NA & 0.550 & NA & -8.642 & 1.643 & 0.093 & NA & 0.063 & NA & 2.936 & 0.569 \\
\hline $\log \left(V_{x, c z}\right)$ & & 3 & 96 & 4 & 28.891 & -4.005 & NA & 0.549 & NA & -6.686 & 1.398 & 0.094 & NA & 0.063 & NA & 2.598 & 0.556 \\
\hline $\log \left(V_{x t h, c z}\right)$ & & 3 & 96 & 4 & 29.001 & -4.009 & NA & 0.548 & NA & -5.862 & 1.264 & 0.094 & NA & 0.063 & NA & 2.364 & 0.523 \\
\hline
\end{tabular}

Abbreviation: $r=$ Number of largest negated PETs selected from each 15-minute block, $n=$ Total number of extremes used in the model estimation, $p=$ Number of parameters to be estimated, $\mathrm{nllh}=$ negative log-likelihood value at converge (NA if the algorithm fails to converge)

The significance of the covariates incorporated in the non-stationary models can be determined from the values of the negative log-likelihood at convergence in comparison to the base case homogeneous models. The greater reduction from the base case implies a better fit. From these two tables, the following conclusions can be made: 
- For the same set of covariates, the identity link in the location parameters gives the greatest reduction in negative log-likelihood value from the base case. This finding is also consistent with the results from other sites not presented herein.

- Marginal reductions in negative log-likelihood values are obtained from the links in the scale and shape parameters. This implies that non-stationarity in the scale and shape parameters can be excluded from further consideration in the model development. In addition, the shape parameter is usually left intact in practice unless there is factual evidence of the non-stationarity in the tail behavior of extreme value distributions (Coles, 2001).

- The logarithm versions of covariates do not give any significant improvement over the standard ones in terms of model goodness-of-fit.

There is a large catalogue of modeling covariates to be chosen in the non-stationary modeling framework. The test statistics described in Section 6.5.2 can be used to determine the statistical significance between nested models. The basic principles in building statistical models should be followed when selecting the best model; i.e. logical, simple, and parsimonious.

\subsection{MODEL DEVELOPMENT}

The $r$ largest order statistic distributions as described in 6.2.3 were fitted to the negated PET data. The model development procedure is discussed along with selected results. The issues discussed in the previous section were taken into account in order to improve the fit quality of the models. All the computational analysis is implemented in S-Plus environment (Insightful, 2001). Coles (2001) provides several functions as well as source codes to estimate extreme value models in SPlus. Everitt (2002) gives a good introduction to S-Plus for beginners and intermediate users. Venables and Ripley (2002) address the implementation in S-Plus more in-depth for advanced users and cover most of commonly-used statistical analyses.

\subsubsection{Criterion for $\mathbf{r}$ Selection}

The bias-variance tradeoff plays an important role in selecting a suitable $r$ in the order statistic models. First, we fitted the $r$ largest order statistic models to negated PET data at each site with $r$ varying from 1 to 3 to examine its effects on maximum likelihood estimates and confidence of 
estimates. For comparison purpose, basic homogeneous models were estimated in this case. The estimation results are shown in Table E-1.

The estimation results show that the location parameters change only slightly as the $r$ increases while the differences are relatively noticeable for the scale and shape parameters. The obvious trend is the decrease in the standard errors of estimates as $r$ increases. This is expected since more data points were used in the model estimations with greater $r$.

The greater $r$ may improve the confidence of estimates, but we have to ensure that the asymptotic assumption is not invalidated. Figure E-1 to Figure E-4 illustrate the probability and quantile plots for each order statistic $k$, where $r=3$. The departure from a unit diagonal line indicates a failure of the $\mathrm{k}^{\text {th }}$ order statistic. A visual inspection of these plots reveals that a departure from a unit diagonal is more likely as $\mathrm{k}$ increases. This finding is anticipated as the second largest and third largest order statistic may violate the asymptotic assumption, particularly at sites with small sample sizes. Note that the probability and quantile plots at certain sites, such as 97903, indicate a serious problem with the fit quality, even at $\mathrm{k}=1$. This situation requires a special treatment before model estimation since two classes of PETs (see Section 7.1.3) were observed. This problem is discussed later in this section.

Based on these plots, a general guideline for selecting $r$ is provided in Table 7-4. The $r=2$ is a reasonable choice in most cases with exception to the sites with a low frequency of PETs. In such cases, $r=1$ is recommended. The $r=3$ can be used for sites with a high frequency of PETs in order to increase the confidence of estimates.

Table 7-4: General Guideline for Selecting $r$

\begin{tabular}{|c|c|}
\hline Counts of PET $^{*} \leq 6 \mathrm{sec}$ & Recommended $\mathrm{r}$ \\
\hline$\leq 90$ & 1 \\
\hline $91-300$ & 2 \\
\hline$>300$ & 3 \\
\hline
\end{tabular}

* Counts per 8-hour observation period

\subsubsection{Homogeneous Models}

Following the criterion in Table 7-4, the homogeneous $r$ largest order statistic models were fitted to the negated PET extremes at each site. The estimation results are summarized in Table E- 2 . 
Although the data were collected for eight hours, or 32 15-minute intervals at each site, the number of extreme values is not necessarily equal to multiples of 32 because certain time blocks had no PET of eight seconds or less (observation threshold).

The estimated distribution is a three-parameter model in which the shape parameter estimate $\hat{\xi}$ determines the corresponding type of extreme value distributions. Examination of the standard errors of maximum likelihood estimates reveals that the standard error of $\hat{\xi}$ is relatively larger than the other two parameters at several sites. This phenomenon can be anticipated since extreme value distributions are most uncertain about tail behaviors and the nature of the model allows us to incorporate this uncertainty as a part of the estimation procedure, which is being reflected through the standard errors of the shape parameter estimates. The negative $\hat{\xi}$ across all the sites estimated implies that the tail behavior of the extreme negated PET has a strong tendency to follow the Weibull density. The Weibull density, or the extreme value type III, is defined on $\left(-\infty, z_{+}\right)$, which means that an upper bound is finite. An upper bound $z_{+}$can be computed based on the maximum likelihood estimates using equation (6-52). The condition $z_{+} \leq 0$ corresponds to the case where the expected risk of right-angle collisions is zero and, therefore, the expected crash frequency is zero. In addition, the strong case of the Weibull density across all the sites may indicate the problem of underestimation of safety levels.

Crash frequency estimates and return level estimates can be determined using the procedures described in Sections 6.8.2 and 6.8.3 respectively. Inferences on these estimates are also provided. Inferences for these model-based estimates can be made using a simulation-based inference method (see Section 6.9.1.3). The underestimation problem associated with the homogeneous models is confirmed when we consider the four-year crash frequency estimates shown in Table E-2. There are 12 out of 16 sites with zero estimates for a four-year crash frequency. Estimates of the remaining sites are less than two crashes per four years, except for site 97903 , for which the predicted crash frequency was 842 . The unusually high estimate at site 97903 is due in large to the excessive number of short PETs from queue spillbacks during congested periods. The probability and quantile plots, based on the fitted homogeneous models (see Figure E-5 and Figure E-6), indicate a lack-of-fit problem at several sites and particularly at site 97903. The excessive number of PETs caused by queue spillbacks triggers the need to refine 
the modeling approach such that the sites with high-frequency PETs are not overwhelmed by short PETs from traffic backup.

PET values can be classified whether they belong to normal operations or queue spillbacks, using ET data. Complete removal of short PETs from queue spillbacks is a logical flaw as it would simply eliminate the likelihood of experiencing a right-angle crash during a congested traffic condition. To handle this issue, we considered partial removal of the PET data at the sites with a high PET frequency based on the degree of variability of ET. This process is referred to as PET filtering.

The variability of ET, represented by a coefficient of variation $\left(\mathrm{CV}_{\mathrm{ET}}\right)$, approximates the impact that queue spillbacks have on the PET occurrences at an intersection. Based on the values of $\mathrm{CV}_{\mathrm{ET}}$, we can determine candidate sites for partial removal of PETs associated with queue spillbacks. The sites with low PET counts but having a high $\mathrm{CV}_{\mathrm{ET}}$ are not considered as candidates for PET removal because $\mathrm{CV}_{\mathrm{ET}}$ can be influenced by a few long ETs and the data removal can influence the estimation results drastically due to the small sample size. Diagnostic plots for the homogeneous models (Figure E-5 and Figure E-6) also indicate that the lack-of-fit is not a problem at sites with low PET counts.

For candidate sites, the PET data are removed when the corresponding ET is greater than the specified threshold; in other words, PETs are not used in the model estimation if

$$
E T>v_{E T, p}
$$

where $v_{E T, p}$ is the site-specific ET removal threshold. Two alternatives for specifying the ET removal threshold were considered. The first one is to use a normal probability threshold

$$
v_{E T, p}=\operatorname{mean}(E T)+z_{p} \operatorname{stdev}(E T)
$$

where $z_{p}$ is a $p \%$-quantile of a standard normal distribution. The mean and standard deviation of ET at each site are calculated using only ETs associated with PET $\leq 6$ seconds in order to be consistent with the criterion for selecting $r$. The second approach relies on the empirical quantile of ET, which is 


$$
v_{E T, p=i / n}=\left\{E T^{(i)}: \operatorname{Pr}\left(E T \leq E T^{(i)}\right)=i / n\right\}
$$

where $E T^{(i)}$ is the $\mathrm{i}^{\text {th }}$ value of the ET values in an ascending order. Both specification approaches were tested and it was found that the filtering of PET using the empirical quantile of ET as a threshold produces a better fit quality for estimated models. This can be explained by the fact that the actual underlying distributions of ET substantially depart from the normal density such that the threshold defined by (7-3) cannot effectively remove the PETs associated with queue spillbacks. The empirical quantile, on the other hand, is not affected by the skewness of the distribution and therefore is a preferred alternative in the PET filtering process.

A test was conducted to determine the appropriate quantile for a cutoff point and it was found that the 95\%-quantile of ET gives a reasonable improvement in the models' goodness-of-fit in most cases. This implies that only 5\% of PET observations are not considered in the modeling if necessary. Only site 97903 requires a lower threshold to improve the fit quality; however, this is consistent with its $\mathrm{CV}_{\mathrm{ET}}$ value, which is relatively higher than all the remaining sites. As a result, the suggested ET threshold specifications in the PET filtering process can be summarized as described in Table 7-5.

Table 7-5: Recommendation for PET Filtering

\begin{tabular}{|c|c|}
\hline Criteria & Suggested Filters \\
\hline PET counts $\leq 100$ and $\mathrm{CV}_{\mathrm{ET}} \leq 0.500$ & No PET is removed \\
\hline $0.500<\mathrm{CV}_{\mathrm{ET}} \leq 0.750$ & Remove PET where ET $>95 \%$ empirical quantile \\
\hline $\mathrm{CV}_{\mathrm{ET}}>0.750$ & Remove PET where ET $>80 \%$ empirical quantile \\
\hline \multicolumn{2}{|l|}{ Notes: } \\
\hline $\begin{array}{l}\text { 1) PET counts are based only on PET } \\
\text { 2) } \mathrm{CV}_{\mathrm{ET}} \text { is computed using only } \mathrm{ET} \text { a }\end{array}$ & $\begin{array}{l}6 \text { seconds during } 8 \text {-hour period of observation. } \\
\text { ociated with PET } \leq 6 \text { seconds. }\end{array}$ \\
\hline
\end{tabular}

Following the suggestions in Table 7-5, there are only four sites that require PET filtering: 87933, 97901, 97903, and 97905. The modeling results when PET filtering is considered are shown in Table E-3. Corresponding diagnostic plots for the sites with the PET filtered, shown in Figure E-7, indicate improved goodness-of-fit of the estimated models, particularly at site 97903. Hence, the PET filtering procedure is applied in all the subsequent modeling considerations. 
A quick examination of the return level and crash frequency estimates in Table 7-5 reveals that the underestimation problem still persists. This is not unexpected because the results only from the four sites with PET filtered have changed from the non-filtered case. This implies that further attempts to improve the models must be sought. If we consider the PET variation over time, together with the volume variation site by site (see Appendix C and Appendix D), we can observe the apparent association of temporal variations between these two sequences. This suggests that the variability in the PET process may be additionally explained by non-stationary covariates. Therefore, the non-stationarity of the PET process was examined within the framework of the $r$ largest order statistic models. The results from the homogeneous models in Table 7-5 are considered as a base case for the evaluation whether the model improvement, if any, is statistically significant.

\subsubsection{Non-Stationary Models}

Non-stationary models incorporate characteristics that change systematically over time. The apparent trends in PET variation plots suggest that the PET process is possibly better explained with the additional covariates. As discussed in Section 7.1.4, we examined the appropriate link structure with a number of covariates and the identity link in the location parameter $\mu$ outperforms the other alternatives in capturing the additional variability in the process. Several sets of covariates have been incorporated through the identity link in $\mu$. The findings and selected results are summarized in this section.

\subsubsection{Convergence of Model Estimation Algorithm}

The maximum likelihood estimation procedure still applies for the non-stationary models. In general, the optimal solutions of maximum likelihood estimates of the $r$ largest order statistic distributions can be found. However, the estimation procedure of the non-stationary models may not converge at times and the confidence of model estimates may be poor when a sample size is small.

After a series of model estimations with different combinations of covariates across all sites, it was found that the sites with low PET counts are more likely to encounter algorithm nonconvergence, particularly when non-stationarity is involved. For example, two sites that 
frequently involve model non-convergence are 87932 and 97940. Both sites have eight-hour counts of PET $\leq 6$ less than 30. Moreover, a small sample size leads to the problem of a large variance of estimates provided that a solution can be found. The magnitude of this problem increases during the model evaluation as a large variance can give rise to instability in crash frequency estimates and return level estimates. Therefore, it was decided to remove these sites having a low frequency of PETs from further modeling analysis. The frequency of PETs $\leq 6$ seconds at each site was used as a criterion to determine the candidates for removal and the value of 30 appears to be a natural threshold in this case.

In addition, the sites with a low frequency of PETs are less likely to experience an above-normal frequency of collisions from the correlation check in Section 5.5. The removal of potentially safe sites from further consideration was not a serious concern for the approach. The sites removed were $87923,87932,97922$, and 97940 . There were a total of 12 out of the 16 sites remaining in the subsequent analysis.

\subsubsection{Covariate Selection}

The amount of the variability explained by the addition of selected covariates can be evaluated in terms of the reduction in the negative log-likelihood values at convergence (see the test statistics described in Section 6.5.2. A number of models were estimated using the covariate list in Table 7-1 for all 12 sites, using the non-stationary $r$ largest order statistic distributions. This leads to the findings as follows:

- The additional variability explained by the volume-based covariates and the PET counts on a 15-minute interval basis were statistically significant.

- In terms of volume-based covariates, the conflicting volumes $\left(\mathrm{V}_{\mathrm{x}}\right.$ and $\left.\mathrm{V}_{\mathrm{xth}}\right)$ produced a better fit than the total volumes $\left(\mathrm{V}_{\text {sum }}\right.$ and $\left.\mathrm{V}_{\text {sumth }}\right)$.

- The conflict volumes $\left(\mathrm{V}_{\mathrm{x}}\right)$ and the conflicting through-volumes $\left(\mathrm{V}_{\mathrm{xth}}\right)$ gave more or less the same improvement with respect to the model goodness-of-fit but the conflicting through-volumes are preferred since they logically relate to the mechanism of right-angle collisions.

- Since each PET occurrence corresponds to a specific conflict zone, we checked as to whether conflicting volumes at a specific conflict zone $\left(\mathrm{V}_{\mathrm{x}, \mathrm{cz}}\right.$ and $\left.\mathrm{V}_{\mathrm{xth}, \mathrm{cz}}\right)$ can give a better 
model goodness-of-fit than intersection conflicting volumes $\left(\mathrm{V}_{\mathrm{x}}\right.$ and $\left.\mathrm{V}_{\mathrm{xth}}\right)$. It was found that model fits, using either covariate, did not significantly differ from their respective counterparts, i.e., $\mathrm{V}_{\mathrm{x}, \mathrm{cz}}$ versus $\mathrm{V}_{\mathrm{x}}$ and $\mathrm{V}_{\mathrm{xth}, \mathrm{cz}}$ versus $\mathrm{V}_{\mathrm{xth}}$.

- In addition to the volume-based covariates, 15-minute counts of PET shorter than a specified threshold $u\left(f_{\mathrm{PET}<\mathrm{u}}\right)$ constitute another variable tested to be consistently significant. A threshold of about 4.5 seconds was found to give statistically significant improvement to the model's goodness-of-fit. The corresponding covariate is denoted as

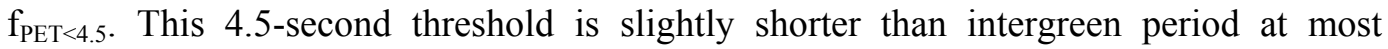
signalized intersections.

The volume-based covariates and the PET counts both represent the measure of traffic exposure that the intersections experienced during the observation period. The likelihood of right-angle collisions is zero if there is zero exposure, i.e., no traffic or no crossing interactions. This likelihood of collisions increases as the amount of exposure increases and this trend was captured by incorporating the volume and the PET counts as covariates linked with the location parameter.

\subsubsection{Modeling Results}

Non-stationary models are estimated using the same criteria for $r$ selection and PET filtering as in the case of homogeneous models. Table E-4 presents the modeling results when the covariate $V_{x t h}$ is linked with the location parameter as

$$
\mu_{t}=\beta_{0}+\beta_{1}\left(V_{x t h}\right)_{t}
$$

The corresponding diagnostic plots are displayed in Figure E-8 and Figure E-9. The intercept estimates $\hat{\beta}_{0}$ are consistently negative across all sites. The positive coefficient estimates $\hat{\beta}_{1}$ for most locations implies the tendency of increasing risk of collisions as the volume increases. This is because the location parameter tends to shift towards zero, which is the crash occurrence level by the definition of PET. The negative estimates $\hat{\beta}_{1}$ at sites 87905 and 87907 , however, are statistically insignificant at $95 \%$ confidence level based on the test statistics derived from the reductions in the negative log-likelihood value at convergence (see Section 7.3.1). 
The 15-minute PET count is another potential covariate for the non-stationary models. The link structure is similar to the previous case, which is

$$
\mu_{t}=\beta_{0}+\beta_{1}\left(f_{P E T<4.5}\right)_{t}
$$

where $f_{\mathrm{PET}<4.5}$ is the 15 -minute counts of PETs that are less than 4.5 seconds. The estimation results are shown in Table E-5.

The negative shape parameter estimates $\hat{\xi}$ still implies the Weibull density for all sites except for site 97901, where the positive $\hat{\xi}$ corresponds to the Fréchet distribution. The positive coefficient estimates $\hat{\beta}_{1}$ are consistent across all sites, which strongly support the notion that increasing exposure in terms of PET counts may increase the risk of right-angle collisions. The four-year crash frequency estimates from model (7-6) indicate a slight improvement in the underestimation problem.

Finally, we estimated the non-stationary $r$ largest order statistic models using both covariates in the link with the location parameter as

$$
\mu_{t}=\beta_{0}+\beta_{1}\left(V_{x t h}\right)_{t}+\beta_{2}\left(f_{P E T<4.5}\right)_{t}
$$

The estimation results are summarized in Table E- 6 and the corresponding diagnostics are plotted in Figure E-10 and Figure E-11. The coefficient estimates $\hat{\beta}_{1}$ are positive at sites where the addition of the covariate $\mathrm{V}_{\mathrm{xth}}$ alone is statistically significant. The coefficient estimates $\hat{\beta}_{2}$ for PET counts are consistently positive across all the 12 sites. This corresponds to the findings when only $\mathrm{f}_{\mathrm{PET}<4.5}$ is considered in the non-stationary models.

The model-based estimates of return level and crash frequency were also apparently improved. The return level estimates consist of both positive and negative values, whereas the crash frequency estimates are not overwhelmed by zero estimates. Simulation-based inferences on both estimates at each site are also provided.

The hypothesis testing was conducted to examine if the significance of covariates incorporated into the location parameter can be justified statistically using deviance statistics. 


\subsection{BEST FITTED MODELS}

\subsubsection{Analysis of Deviance}

The best fitted model for each site can be objectively determined using the analysis of deviance. First, denoting the modeling alternatives estimated earlier as follows:

- M1: Homogeneous $r$ largest order statistic distributions with $r$ selection and PET filtering as outlined in Table 7-4 and Table 7-5 respectively. This is considered as a base case.

- M2: Non-stationary $r$ largest order statistic distributions with $r$ selection and PET filtering as in M1. The link structure is $\mu_{t}=\beta_{0}+\beta_{1}\left(V_{x t h}\right)_{t}$.

- M3: Non-stationary $r$ largest order statistic distributions with $r$ selection and PET filtering as in M1. The link structure is $\mu_{t}=\beta_{0}+\beta_{1}\left(f_{P E T<4.5}\right)_{t}$.

- M4: Non-stationary $r$ largest order statistic distributions with $r$ selection and PET filtering as in M1. The link structure is $\mu_{t}=\beta_{0}+\beta_{1}\left(V_{x t h}\right)_{t}+\beta_{2}\left(f_{P E T<4.5}\right)_{t}$.

The deviance statistics to determine the significance of covariates between nested models are summarized in Table 7-6. The covariate " $\mathrm{V}_{\mathrm{xth}}$ " is statistically significant at the $95 \%$ confidence level if D1 $>\chi_{0.95, d f=1}^{2}$. Similarly, the covariate " $\mathrm{P}_{\mathrm{PET}<4.5}$ " is statistically significant at the $95 \%$ confidence level if D2 $>\chi_{0.95, d f=1}^{2}$. When both covariates are included in model M4, the statistic D3 can be compared against $\chi_{0.95, d f=2}^{2}$ to determine its signifance at the $95 \%$ confidence level.

The test statistic D4 can be used to determine if the inclusion of " $\mathrm{V}_{\mathrm{xth}}$ " into model M4 can

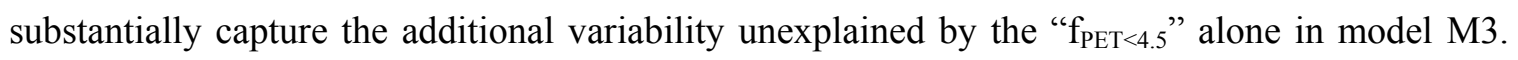
Model M4 is statistically significance at the $95 \%$ confidence level in comparison with model M3 if D4 $>\chi_{0.95, d f=1}^{2}$.

The values of D2 are relatively larger than D1 across all the sites observed, which indicates that

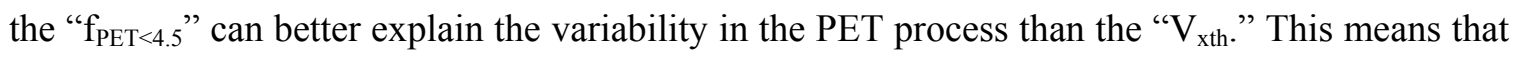
model M3 is preferred to M2 and both M3 and M2 are preferred to model M1. 
Table 7-6: Analysis of Deviance Statistics

\begin{tabular}{|c|c|c|c|c|c|}
\hline Model No. & M1 & M2 & M3 & M4 & \\
\hline Model Type & Homogeneous & Non-Stationary & Non-stationary & Non-stationary & \\
\hline Description & Base case & $\mu=\beta_{0}+\beta_{1} V_{x t h}$ & $\mu=\beta_{0}+\beta_{1} f_{\mathrm{PET}}<4.5$ & $\mu=\beta_{0}+\beta_{1} V_{x t h}+\beta_{2} f_{P E T<4.5}$ & \\
\hline Site & nllh1 & nIlh2 & nllh3 & nIlh4 & \\
\hline 87905 & 23.489 & 23.185 & 20.786 & 20.282 & \\
\hline 87906 & 68.169 & 60.496 & 54.072 & 51.811 & \\
\hline 87907 & 41.517 & 40.181 & NA & 29.406 & \\
\hline 87909 & 47.626 & 44.293 & 30.097 & 29.868 & \\
\hline 87915 & 54.008 & 46.660 & 40.067 & 38.515 & \\
\hline 87930 & 38.753 & 37.150 & NA & 27.855 & \\
\hline 87933 & 74.706 & 58.349 & 56.457 & 49.594 & \\
\hline 97901 & 32.722 & 15.025 & 4.844 & 4.143 & \\
\hline 97903 & 96.924 & 76.084 & 55.193 & 55.188 & \\
\hline 97905 & 57.003 & 60.818 & 52.086 & 52.049 & \\
\hline 97911 & 39.524 & NA & 33.030 & 31.968 & \\
\hline 97920 & 37.140 & 37.113 & 27.429 & 26.991 & \\
\hline \multicolumn{6}{|c|}{ Analysis of Deviance } \\
\hline \multirow{2}{*}{ Site } & $D 1 \sim X^{2}(1)$ & $D 2 \sim X^{2}(1)$ & D3 $\sim X^{2}(2)$ & $D 4 \sim X^{2}(1)$ & \multirow{2}{*}{$\begin{array}{c}\text { Selected } \\
\text { Model }\end{array}$} \\
\hline & 2(nllh1-nllh2) & 2(nIlh1-nIlh3) & 2(nIlh1-nIlh4) & 2(nIlh3-nllh4) & \\
\hline 87905 & 0.608 & 5.406 & 6.414 & 1.009 & M3 \\
\hline 87906 & 15.347 & 28.196 & 32.718 & 4.522 & M4 \\
\hline 87907 & 2.672 & NA & 24.222 & NA & M4 \\
\hline 87909 & 6.666 & 35.059 & 35.515 & 0.456 & M3 \\
\hline 87915 & 14.696 & 27.882 & 30.986 & 3.104 & M3 \\
\hline 87930 & 3.205 & NA & 21.795 & NA & M4 \\
\hline 87933 & 32.713 & 36.497 & 50.224 & 13.727 & M4 \\
\hline 97901 & 35.393 & 55.756 & 57.158 & 1.402 & M3 \\
\hline 97903 & 41.679 & 83.460 & 83.471 & 0.011 & M3 \\
\hline 97905 & Invalid & 9.833 & 9.907 & 0.074 & M3 \\
\hline 97911 & NA & 12.987 & 15.112 & 2.124 & M3 \\
\hline 97920 & 0.054 & 19.421 & 20.297 & 0.875 & M3 \\
\hline
\end{tabular}

Notes:

1) $X^{2}(0.95, d f=1)=3.841$

2) $X_{(0.95, \mathrm{df}=2)}^{2}=5.991$

3) NA is the case where no optimal mle can be found at convergence.

4) Invalid deviance is defined as the case where the deviance becomes negative. This can occur if the nllh of the full model is greater than the reduced one. This problem is likely to be caused by the local optimum of mle.

5) Base case is the homogeneous $r$ largest order statistic models fitted to filtered PET data where choice of $r$ is dependent on the 8-hour counts of PETs $\leq 6$ seconds

To summarize, using the deviance statistics shown in Table 7-6, the best fitted models can be determined as follows:

- $\quad$ Compare model M3 versus base case M1 using test statistic D2. If D2 > $\chi_{0.95, d f=1}^{2}=3.841$, then M3 is preferred to M1. In our analysis at all 12 sites, non-stationary model M3 outperformed model M1. 
- Compare model M4 versus model M3 using test statistic D4. If D4 > $\chi_{0.95, d f=1}^{2}=3.841$, then M4 is preferred to M3 or, equivalently, the inclusion of the " $\mathrm{V}_{\mathrm{xth}}$ " can substantially explain the variability in the PET process in addition to the covariate " $f_{\mathrm{PET}<4.5}$ " in model M3.

\subsubsection{Selected Models}

Following the model selection procedure described in the previous section, the final selected model for each site is listed in the last column of Table 7-6. The modeling results of the selected models are summarized in Table 7-7.

Both $\hat{\beta}_{1}$ and $\hat{\beta}_{2}$ are positive for all the sites estimated except for the $\hat{\beta}_{2}$ at site 87907 . This indicates a positive correlation for the risk of right-angle collisions with traffic volume and PETs. For site 87907, model M4 is selected because the maximum likelihood estimation algorithm cannot find the optimal solution at M3. The negative $\hat{\beta}_{2}$ at site 87907 is marginally significant and hence does not contradict the positive trend of estimates at the other sites.

At eight out of 12 sites, the volume covariate " $\mathrm{V}_{\mathrm{xth}}$ " is not statistically significant when added to model M3 where PET covariate " $\mathrm{f}_{\mathrm{PET}<4.5}$ " already existed. This is not unexpected since " $\mathrm{f}_{\mathrm{PET}<4.5}$ " is likely to correlate with " $\mathrm{V}_{\mathrm{xth}}$ ". However, PET is a better representation of opportunities for traffic interactions that can lead to right-angle collisions. A protected left-turn phase would decrease the risk of straight right-angle collisions, which is reflected through a longer PET value but not the traffic volume. The effect of increased traffic volume on the risk of right-angle collisions is more subtle. An increase in total traffic volume does not necessarily imply a greater risk of straight right-angle collisions if the left-turn traffic volume increases as well. At intersections controlled by actuated signals, PET values account for the manner in which the traffic control - phases and signal timings - responds to changes in traffic volume and directional split. Traffic volume, on the other hand, can only manifest the aggregate impact on the risk of right-angle collisions at best. 
Table 7-7: Modeling Results - Best Fitted Models

Model Descriptions:

Non-stationary $r$ largest order statistic models fitted to filtered PET data

M3: $\mu=\beta_{0}+\beta_{1} f_{\mathrm{PET}<4.5}$

M4: $\mu=\beta_{0}+\beta_{1} f_{P E T<4.5}+\beta_{2} V_{x t h}$

\begin{tabular}{|c|c|c|c|c|c|c|}
\hline Site & Model & $\begin{array}{c}\text { 8-Hour } \\
\text { Counts of } \\
\text { PET } \leq 6\end{array}$ & $\mathrm{CV}\left(\mathrm{ET}_{\mathrm{PET} \leq 6}\right)$ & Filter & $\mathbf{r}$ & $\mathbf{n}$ \\
\hline 87905 & M3 & 31 & 0.281 & None & 1 & 15.000 \\
\hline 87906 & M4 & 98 & 0.642 & None & 2 & 58.000 \\
\hline 87907 & M4 & 54 & 0.285 & None & 1 & 30.000 \\
\hline 87909 & M3 & 268 & 0.303 & None & 2 & 64.000 \\
\hline 87915 & M3 & 116 & 0.423 & None & 2 & 61.000 \\
\hline 87930 & M4 & 38 & 0.380 & None & 1 & 25.000 \\
\hline 87933 & M4 & 104 & 0.576 & $95 \%$-quantile & 2 & 62.000 \\
\hline 97901 & M3 & 311 & 0.538 & $95 \%$-quantile & 3 & 96.000 \\
\hline 97903 & M3 & 327 & 0.969 & $80 \%$-quantile & 3 & 96.000 \\
\hline 97905 & M3 & 106 & 0.537 & $95 \%$-quantile & 2 & 60.000 \\
\hline 97911 & M3 & 69 & 0.500 & None & 1 & 26.000 \\
\hline 97920 & M3 & 79 & 0.509 & None & 1 & 31.000 \\
\hline
\end{tabular}

\begin{tabular}{|c|c|c|c|c|c|c|c|c|c|c|c|c|}
\hline \multirow{2}{*}{ Site } & \multirow{2}{*}{ Model } & \multicolumn{11}{|c|}{ Maximum Likelihood Estimation Results } \\
\hline & & nllh & $\beta_{0}$ & $\beta_{1}$ & $\beta_{2}$ & $\sigma$ & $\xi$ & $\operatorname{se}\left(\beta_{0}\right)$ & $\operatorname{se}\left(\beta_{1}\right)$ & $\operatorname{se}\left(\beta_{2}\right)$ & $\operatorname{se}(\sigma)$ & $\operatorname{se}(\xi)$ \\
\hline 87905 & M3 & 20.786 & -5.835 & 0.523 & -- & 0.992 & -0.333 & 0.329 & 0.200 & -- & 0.210 & 0.199 \\
\hline 87906 & M4 & 51.811 & -6.709 & 0.685 & 0.014 & 0.802 & -0.272 & 0.580 & 0.149 & 0.006 & 0.073 & 0.090 \\
\hline 87907 & M4 & 29.406 & -5.126 & 1.017 & -0.004 & 0.881 & -0.830 & 0.594 & 0.207 & 0.005 & 0.180 & 0.200 \\
\hline 87909 & M3 & 30.097 & -4.759 & 0.308 & -- & 0.520 & -0.049 & 0.117 & 0.039 & -- & 0.055 & 0.122 \\
\hline 87915 & M3 & 40.067 & -5.222 & 0.586 & -- & 0.673 & -0.165 & 0.128 & 0.088 & -- & 0.060 & 0.056 \\
\hline 87930 & M4 & 27.855 & -6.767 & 1.495 & 0.026 & 0.774 & -0.360 & 1.175 & 0.330 & 0.032 & 0.145 & 0.225 \\
\hline 87933 & M4 & 49.594 & -7.736 & 0.524 & 0.053 & 0.743 & -0.230 & 0.538 & 0.118 & 0.013 & 0.069 & 0.086 \\
\hline 97901 & M3 & 4.844 & -4.773 & 0.299 & - & 0.471 & 0.032 & 0.101 & 0.033 & -- & 0.049 & 0.109 \\
\hline 97903 & M3 & 55.193 & -4.570 & 0.335 & -- & 0.794 & -0.110 & 0.162 & 0.040 & -- & 0.068 & 0.084 \\
\hline 97905 & M3 & 52.086 & -5.341 & 0.923 & -- & 0.798 & -0.454 & 0.209 & 0.196 & -- & 0.068 & 0.087 \\
\hline 97911 & M3 & 33.030 & -5.788 & 1.143 & -- & 0.953 & -0.456 & 0.289 & 0.243 & -- & 0.154 & 0.130 \\
\hline 97920 & M3 & 27.429 & -5.242 & 0.542 & -- & 0.614 & -0.350 & 0.142 & 0.104 & -- & 0.084 & 0.104 \\
\hline
\end{tabular}

\begin{tabular}{|c|c|c|c|c|c|c|c|c|c|c|c|c|c|}
\hline \multirow[b]{2}{*}{ Site } & \multirow{2}{*}{$\begin{array}{c}1 \text { 1-Year } \\
\text { Return Level } \\
\text { Estimate }\end{array}$} & \multirow{2}{*}{$\begin{array}{c}\text { 4-Year } \\
\text { Return } \\
\text { Level } \\
\text { Estimate }\end{array}$} & \multicolumn{11}{|c|}{ Simulation-based Inferences on 4-Year Return Level Estimates: Number of Simulation Runs = 5000} \\
\hline & & & Mean & SD & \multicolumn{9}{|c|}{$\%$-quantile of 4 -Year Return Level Estimates } \\
\hline 87905 & -0.603 & -0.468 & 0.157 & 2.970 & -2.306 & -2.006 & -1.623 & -1.397 & -0.291 & 1.260 & 1.767 & 3.036 & 5.021 \\
\hline 87906 & 0.074 & & & 0.686 & -0.771 & -0.621 & -0.433 & -0.301 & 0.293 & 0.977 & 1.168 & 1.528 & 1.842 \\
\hline 87907 & -2.447 & -2.443 & -2.412 & 0.364 & -3.098 & -3.002 & -2.878 & -2.791 & -2.422 & -2.028 & -1.937 & -1.802 & -1.663 \\
\hline 87930 & -0.859 & -0.772 & -0.160 & 2.999 & -1.545 & -1.397 & -1.251 & -1.141 & -0.628 & 0.225 & 0.699 & 2.182 & 4.756 \\
\hline 87933 & -0.257 & -0.093 & 0.129 & 0.750 & -0.909 & -0.787 & -0.651 & -0.545 & -0.002 & 0.772 & 1.038 & 1.500 & 1.927 \\
\hline 97901 & 1.345 & 2.248 & 4.891 & 8.156 & -1.639 & -1.340 & -0.898 & -0.491 & 2.383 & 10.107 & 13.315 & 19.185 & 26.870 \\
\hline 97903 & 1.809 & 2.2 & 2.822 & 2.497 & 0.124 & 0.315 & 0.597 & 0.824 & 2.215 & 4.824 & 5.731 & & 9.086 \\
\hline 97905 & -1.787 & -1.7 & -1.721 & 0.307 & -2.278 & -2.198 & -2.089 & -2.022 & & -1.419 & -1 & -1.2 & -1.059 \\
\hline 97911 & -0.386 & -0.3 & -0.2 & 0.600 & & -1.227 & -1.016 & -0.877 & & & & & 0.963 \\
\hline 97920 & -1.512 & & -1.385 & 0.421 & -2.151 & -2.041 & -1.902 & -1.805 & -1.404 & -0.960 & -0.844 & -0.673 & -0.508 \\
\hline \multirow[t]{2}{*}{ Site } & \multirow{2}{*}{$\begin{array}{l}\text { 4-Year Right- } \\
\text { Angle Crash } \\
\text { Counts } \\
\text { (Daytime) }\end{array}$} & \multirow{2}{*}{$\begin{array}{l}\text { 4-Yr Crash } \\
\text { Frequency } \\
\text { Estimate }\end{array}$} & \multirow{2}{*}{ Mean } & \multirow{2}{*}{ SD } & \multicolumn{9}{|c|}{$\%$-quantile of 4-Year Crash Frequency Estimates } \\
\hline & & & & & $2.5 \%$ & $5.0 \%$ & $10.0 \%$ & $15.0 \%$ & $50.0 \%$ & $85.0 \%$ & $90.0 \%$ & $95.0 \%$ & $97.5 \%$ \\
\hline 87905 & 1 & & & & 0.000 & 0.000 & 0.000 & 0.000 & 0.000 & 52.291 & 93.651 & 182.994 & 291.494 \\
\hline 87906 & 6 & 6.457 & 27.990 & 50.040 & 0.000 & 0.000 & 0.000 & 0.00 & 7.919 & 58. & 80. & 122.606 & 169.185 \\
\hline 87907 & 3 & 0.000 & 0.000 & 0.004 & 0.000 & 0.000 & 0.000 & 0.000 & 0.000 & 0.000 & 0.000 & 0.000 & 0.000 \\
\hline 87909 & 7 & 9.602 & 89.700 & 188.424 & 0.000 & 0.000 & 0.000 & 0.000 & 9.430 & 186.192 & 279.881 & 453.231 & 656.532 \\
\hline 87915 & 3 & 1.356 & 6.730 & 14.129 & 0.000 & 0.000 & 0.000 & 0.002 & 1.546 & 13.047 & 18.629 & 30.137 & 44.471 \\
\hline 87930 & 0 & 0.000 & 10.327 & 53.219 & 0.000 & 0.000 & 0.000 & 0.000 & 0.000 & 3.729 & 11.987 & 46.170 & 112.077 \\
\hline 87933 & 1 & 0.376 & 13.394 & 31.181 & 0.000 & 0.000 & 0.000 & 0.000 & 0.934 & 26.436 & 40.065 & 68.561 & 99.819 \\
\hline 97901 & 18 & 35.555 & 141.123 & 243.439 & 0.000 & 0.000 & 0.000 & 0.035 & 35.619 & 307.316 & 424.430 & 636.468 & 849.675 \\
\hline 97903 & 7 & 386.951 & 560.530 & 534.540 & 3.922 & 14.740 & 44.111 & 80.840 & 404.764 & 1079.948 & 1295.943 & 1638.861 & 1981.178 \\
\hline 97905 & 7 & 0.000 & 0.000 & 0.014 & 0.000 & 0.000 & 0.000 & 0.000 & 0.000 & 0.000 & 0.000 & 0.000 & 0.000 \\
\hline
\end{tabular}

Abbreviations: $\mathrm{n}=$ Number of extremes used in the model estimation, $\mathrm{nllh}=$ Negative log-likelihood value at model convergence Note: NA is the case where no optimal mle can be found at convergence. 
The crash frequency estimates appear to be in a reasonable range, except for site 97903, for which the range of estimates is unreasonably large. This is probably due to the special condition at this site, where there are frequent short PETs from traffic backups during peak hours. The proposed non-stationary model at this site improves the model's goodness-of-fit significantly but still fails to provide a reasonable crash frequency estimate. The method deficiencies will be discussed in detail in the next chapter.

\subsubsection{Quantifying Uncertainty of Estimates}

The inferences on the estimates of four-year return level and four-year right-angle crash frequency are provided in terms of empirical quantiles of estimates in Table 7-7. Considerations for inference methods were discussed earlier in Section 6.9. For the best fitted models, only the simulation-based inference method is plausible since the modeled distributions of PET are nonhomogeneous over time. In addition, the simulation-based method is a generic approach that can be applied to the homogeneous case as well. The simulation-based method relies on the standard asymptotic results of maximum likelihood estimates, which follow multivariate normal distribution under suitable regularity conditions. The disadvantage of this approach is that it is computationally intensive and time-consuming.

To determine the confidence interval from the result table, a $90 \%$ confidence interval of the fouryear crash frequency estimate can be determined by reading the 5\%-and $95 \%$-quantiles from the table as the lower and upper bounds respectively. For example, the $90 \%$ confidence interval of the four-year crash frequency estimate at site 87915 is $(0,30)$. Similarly, the $90 \%$ confidence interval of the four-year return level estimate at the same site is $(-0.749,1.264)$.

It should be noted that the empirical distributions of both the crash frequency and the return level estimates are skewed in general and therefore the empirical means will not coincide with the model-based estimates. Medians, or 50\%-quantiles, are the suitable approximations to the modelbased estimates. A quick check on the accuracy of the simulated distributions can be done by comparing the empirical medians versus the model-based estimates. These two figures should be close. A large discrepancy may be remedied by simply increasing the number of iterations in the simulation. 
The confidence intervals obtained for both crash frequency and return level estimates are still very large however. This can be explained by several factors, such as a small sample size, a too short observation period, and the model settings. We will evaluate the fitted models as well as the problem of large uncertainty of the estimates in the next chapter. 


\section{CHAPTER 8 MODEL EVALUATION AND SIMULATION STUDY}

We presented the extreme value approach for modeling safety using the PET data in previous chapters. The standard approach for safety estimation requires the constant risk assumption for a group of similar locations. Furthermore, the standard approach is reactive since considerable waiting time is needed for sufficient accident occurrences. This renders the traditional approach impractical for safety estimation of a new transportation system. The proposed EVT approach, on the contrary, no longer requires historical crash data and individualizes the risk spatially (location-specific) and temporally (time-specific) through the variability of the surrogate safety measure called the post-encroachment times. The proposed method is proactive in that it does not require crash data in the model calibration.

The EVT approach enables the extrapolation of the observed levels of PETs to the rarely observed collision level. The difficulties encountered in typical applications of the EVT are the validity of the extrapolation. In the extreme value modeling of natural phenomena such as sea level, wind speed, and earthquake, the validation task is virtually implausible because the prediction horizon is usually distant from the observed levels and the events of interest may have never existed in the past. In our applications, the validation task is feasible since the extreme event of interest is the right-angle collision in which its expected frequency of occurrences can be reasonably assumed to follow historical crash data. Two precautions are worth mentioning here about the validation data. The locations must not have any major upgrade or renovation that could influence the safety levels of intersections since the time period in which the crash data are retrieved. Second, there must be no major change in the land-use pattern in the vicinity of the studied intersections because significant changes in traffic patterns can have a major impact on driver behavior and consequently the safety levels of intersections. It should be stressed that these precautions impose limitations on the validation procedure, but not on the EVT validity. 
In this chapter we will check the relationship between the model estimates and the observed counts of right-angle collisions. Then, we will evaluate the proposed extreme value modeling approach for several model settings using a simulation experiment.

\subsection{VALIDATION OF EXTREME VALUE MODELING RESULTS}

Two safety estimates determined from the extreme value models are the crash frequency estimates and the extreme return level estimates of negated PETs. The former can be validated by direct comparison with the actual crashes. The extreme return level, on the other hand, must be validated using its correlation with the observed counts. Direct comparison is not plausible for this latter case because crash frequency and return level estimates are not convertible straightforwardly.

The performance of the traditional regression approach cannot be compared with that of extreme value models directly because the observed crash counts, as a response variable, must be known and incorporated in the multivariate regression analysis. The regressional analysis appears to perform well since the observed counts were already a part of the estimation procedure. On the contrary, the observed crash counts were not used at all in the calibration of the extreme value models. The extreme behaviors of PETs determine the estimates of crash frequency. The extreme value approach satisfies the objective of study in the sense that the safety estimation approach is independent of historical crash data and satisfies the postulate of risk varying across locations.

The proposed method measures safety during the period of PET observation. To enable comparison with the safety obtained through the crash-based approach, the PET observation period should be representative of the longer period of recorded crashes.

\subsubsection{Crash Frequency Estimates}

\subsubsection{Direct Comparison}

The model-based crash frequency estimates are computed by the functions described in Section 6.8.2 evaluated at maximum likelihood estimates. Let us first consider the uncertainty of the model estimates of crash frequency as summarized in Table 8-1. The confidence intervals of the 
crash frequency estimates are very large at both the $90 \%$ and $95 \%$ confidence intervals. The fouryear observed counts of right-angle crashes at nine out of 12 sites (75\%) fall within the range of the $95 \%$ confidence intervals. The corresponding coverage for the $90 \%$ confidence intervals is eight out of 12 sites (67\%). Three sites that fall outside the range of the $95 \%$ confidence intervals had 1, 4, and 7 crashes during the four-year period. The range of predicted estimates for these three sites is very tight, which is about 0 to 0.002 . The implication is that the models tend to underestimate the expected number of collisions at the sites with relatively infrequent PET occurrences. Due to the rarity of sufficiently short PETs, these sites may require much longer observation periods in order to observe PET values that can influence the crash frequency estimate. We will further examine this estimate behavior through a simulation experiment (see Section 8.2).

Table 8-1: Model-based Estimates of Crash Frequency and Inferences

\begin{tabular}{|c|c|c|c|c|c|c|c|c|c|c|}
\hline \multirow{3}{*}{ Site } & \multirow{3}{*}{$\begin{array}{l}\text { 4-Year Right- } \\
\text { Angle Crash } \\
\text { Counts } \\
\text { (Daytime) }\end{array}$} & \multirow{3}{*}{$\begin{array}{c}\text { Sample } \\
\text { Size }\end{array}$} & \multirow{3}{*}{$\begin{array}{c}\text { 4-Yr Crash } \\
\text { Frequency } \\
\text { Estimate }\end{array}$} & \multicolumn{7}{|c|}{$\begin{array}{c}\text { Summary Statistics of Model-based 4-year Crash } \\
\text { Frequency Estimates }{ }^{(2)}\end{array}$} \\
\hline & & & & \multirow{2}{*}{ Mean } & \multirow{2}{*}{ SD } & \multirow{2}{*}{ CV } & \multicolumn{2}{|c|}{$90 \%$ C.I. } & \multicolumn{2}{|c|}{ 95\% C.I. } \\
\hline & & & & & & & Lower & Upper & Lower & Upper \\
\hline 87905 & 1 & 15 & 0.000 & 32.351 & 94.552 & 2.923 & 0.000 & 182.994 & 0.000 & 291.494 \\
\hline 87906 & 6 & 58 & 6.457 & 27.990 & 50.040 & 1.788 & 0.000 & 122.606 & 0.000 & 169.185 \\
\hline 87907 & 3 & 30 & 0.000 & 0.000 & 0.004 & $-{ }^{(1)}$ & 0.000 & 0.000 & 0.000 & 0.000 \\
\hline 87909 & 7 & 64 & 9.602 & 89.700 & 188.424 & 2.101 & 0.000 & 453.231 & 0.000 & 656.532 \\
\hline 87915 & 3 & 61 & 1.356 & 6.730 & 14.129 & 2.099 & 0.000 & 30.137 & 0.000 & 44.471 \\
\hline 87930 & 0 & 25 & 0.000 & 10.327 & 53.219 & 5.153 & 0.000 & 46.170 & 0.000 & 112.077 \\
\hline 87933 & 1 & 62 & 0.376 & 13.394 & 31.181 & 2.328 & 0.000 & 68.561 & 0.000 & 99.819 \\
\hline 97901 & 18 & 96 & 35.555 & 141.123 & 243.439 & 1.725 & 0.000 & 636.468 & 0.000 & 849.675 \\
\hline 97903 & 7 & 96 & 386.951 & 560.530 & 534.540 & 0.954 & 14.740 & 1638.861 & 3.922 & 1981.178 \\
\hline 97905 & 7 & 60 & 0.000 & 0.000 & 0.014 & $-{ }^{(1)}$ & 0.000 & 0.000 & 0.000 & 0.000 \\
\hline 97911 & 4 & 26 & 0.000 & 24.918 & 79.493 & 3.190 & 0.000 & 145.110 & 0.000 & 253.468 \\
\hline 97920 & 1 & 31 & 0.000 & 0.015 & 0.306 & $-{ }^{(1)}$ & 0.000 & 0.000 & 0.000 & 0.002 \\
\hline
\end{tabular}

Notes:

(1) CV cannot fairly represents the degree of variability in the estimates where the mean is close to zero.

(2) The modeling results are based on the best fitted models.

An alternative way to quantify the quality of the estimates is in terms of the coefficient of variations $(\mathrm{CV})$. The $\mathrm{CV}$ is computed by dividing the stardard deviation (SD) by the mean. The mean and SD are obtained by the simulation-based inference method where multivariate normal maximum likelihood estimates are used to compute crash frequency estimates. The majority of the sites ( 8 sites) have CVs ranging from about 1 to 3 . Only site 87930 has a CV that is fairly larger than the others (about 5). Note that there are three sites with the means close to zero and thus CVs cannot fairly represent the uncertainty of the estimates in this case. The range of CVs 
from 1 to 3 is somewhat better compared to the CVs of the 15 -minute traffic conflict counts in the study by Glauz and Migletz (1980) in which they reported CVs ranging from 2 to 6 for crosstraffic conflict types. Though it is not a direct comparison, the repeatability of the estimates here obviously fares better than the traditional traffic conflict counts.

Next, the plot of observed crash counts versus the model-based estimates of crash frequency for all the sites is shown in Figure 8-1. Because of the highly overestimated crash frequency at site 97903, 11 of 12 data points are clustering near the ordinate when all data points are presented on the same scale. To address the scaling issue, we excluded the data point of site 97903 in Figure 8-2 to better illustrate the relationship between observed crash counts and model estimates.

Historical Crash Counts versus Model Estimates

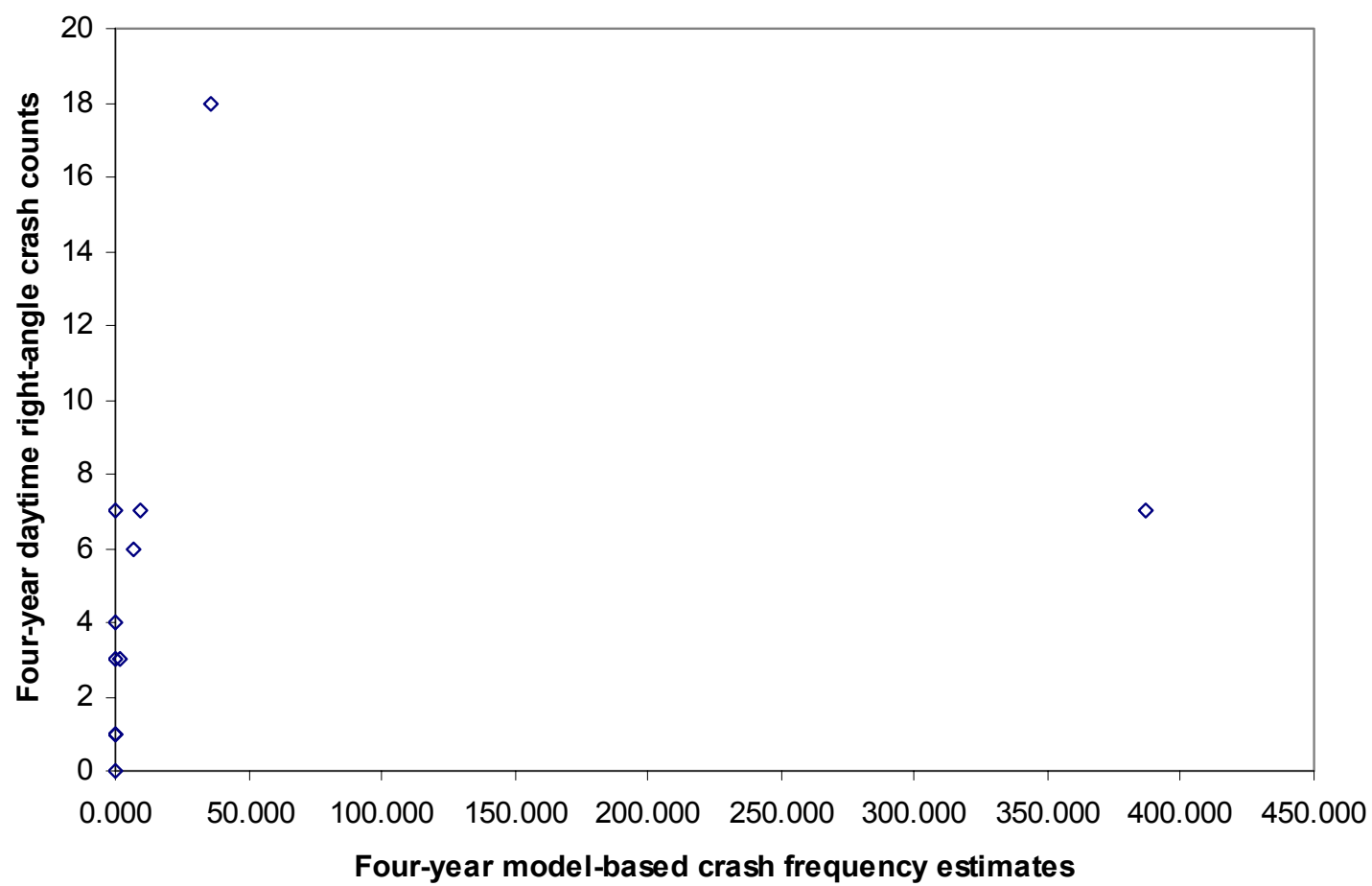

Figure 8-1: Model-based Crash Estimates versus Actual Crash Counts

A unit diagonal line in Figure 8-2 represents the case of perfect agreement between the observed counts and the model-based estimates. The inspection of this figure reveals that the models are likely to underestimate the observed counts at locations with a low crash frequency as indicated by the cluster of data points on the left of the unit diagonal line. In contrast, the models have a 
tendency to overestimate observed counts where short PETs from congestion comprise a significant portion of all PETs observed. This is particularly the case for site 97903 where the method falls short of a reasonable prediction.

Historical Crash Counts versus Model Estimates (without site 97903)

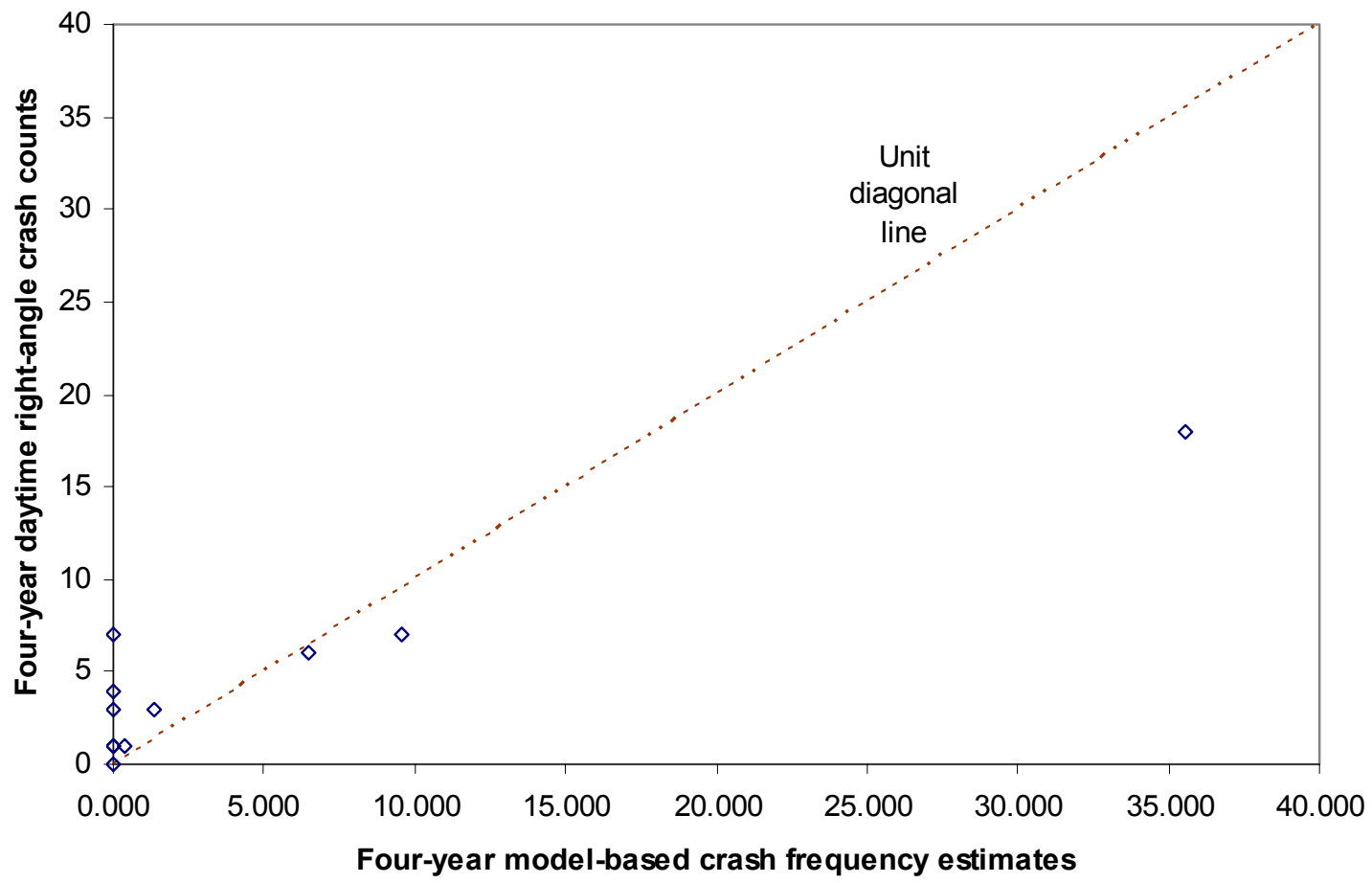

Figure 8-2: Model-based Crash Estimates versus Actual Crash Counts (without 97903)

The positive correlation between the observed counts and the model-based estimates is very promising nevertheless. Without site 97903, the Pearson correlation between these two is 0.931 . This is quite intuitive as the higher model-based estimates imply the higher observed crash counts.

\subsubsection{Root-Mean-Square Error (RMSE)}

If we assume that the observed counts of accidents at each site are the best estimates of the true safety level, then the model performance can be evaluated in terms of the residual variation between the model estimates and the observed counts. The model performance of the estimated extreme value models for a group of selected sites can be measured by the root-mean-square error 
(RMSE), which measures the spread of the residuals. The smaller RMSE therefore implies better model performance. RMSE can be computed as

$$
R M S E=\sqrt{\frac{1}{n} \sum_{i=1}^{n}\left(c_{i}-\hat{c}_{i}\right)^{2}},
$$

where $c_{i}$ and $\hat{c}_{i}$ are the actual counts and the estimated counts of right-angle collisions at location $i$ respectively.

The RMSE of crash frequency estimates based on all the sites except for problematic site 97903 is 5.99 crashes, which is fairly reasonable in comparison to the corresponding mean of the observed counts at 4.64 crashes. The RMSE is an aggregate measure which tells us how close the model estimates are to the observed crash counts; i.e., RMSE tells us if the approach gives the biased estimates. From the eight-hour observation period, it is not feasible to conclude if the models give biased estimates based on the RMSE at this point because the presence of the model bias, if any, can be obscured by compound effects from a large variance of the model estimates.

\subsubsection{Poisson Confidence Intervals}

The observed counts of right-angle collisions at each location are in fact just one observation of the underlying count process, which means that the true level of safety of a location is unknown. However, one can fairly assume that the observed counts for a number of years can approximately represent the safety level of a location. We would like to know how our modelbased estimates fare in the prediction task once the variability of accident counts is considered. This can be done by establishing the confidence intervals based on the observed counts and then checking if the model estimates lie in this range on a site-by-site basis.

The assumption of the variability of accident counts is needed to establish confidence intervals. Nicholson (1985) studied this issue and proposed three different distributions to represent the variability of accident counts. To choose the appropriate distribution, one needs to determine the temporal variation of accident counts as determined by the variance-to-mean ratio. If the variance is close to the mean, then the popular Poisson assumption is suitable. If the historical counts are fairly regular, then the binomial distribution is the most appropriate. On the other hand, if a 
pattern of counts at a location is very irregular, then the negative binomial distribution should be preferred.

Nicholson (1985) determined the temporal variability based on a series of accident counts with durations from 5 to 30 years. We do not have that amount of historical crash data herein to conduct the same test. However, it is fair to say that a particular type of collision, especially rightangle crashes, at intersections is quite an irregular process and therefore unlikely to follow the binomial distribution. The negative binomial choice, though seemingly appropriate, requires the estimated variance-to-mean ratio. In addition, the negative binomial distribution is a less conservative choice as it gives wider confidence intervals that can easily favor our model estimates. Hence, the Poisson variability is assumed for the observed counts of right-angle crashes. Note that we are addressing this from the viewpoint of classical statistics. The true accident frequency $(\lambda)$ of a Poisson population is fixed but unknown. The random quantity is in the confidence intervals, not the parameter $\lambda$.

\section{Poisson Interval Estimator}

Nicholson (1987) showed that the Poisson confidence interval can be used for the observed crash counts for a particular location. It has been shown that the $\chi^{2}$ integral and the cumulative sum of the terms of the Poisson distribution are different forms of the same mathematical function. We will outline the proof briefly in this section. Further details can be found in Casella and Berger (2002) for instance.

First, recall the relationships among Poisson, gamma, and chi-square. If $X$ is a gamma $(\alpha, \beta)$ random variable, where $\alpha$ is an integer, then for any $x$,

$$
\operatorname{Pr}\{X \leq x\}=\operatorname{Pr}\{Y \geq \alpha\}
$$

where $Y$ : Poisson $\left(\frac{x}{\beta}\right)$.

If we set $\alpha=v / 2$, where $v$ is an integer, and $\beta=2$, then the gamma pdf becomes 


$$
f(x \mid v)=\frac{1}{\Gamma(v / 2) 2^{v / 2}} x^{(v / 2)-1} e^{-x / 2}, 0<x<\infty
$$

which is the chi squared pdf with $v$ degrees of freedom.

Let $C_{1}, \ldots, C_{n}$ be random crash counts in $n$ years from a Poisson population with the true mean annual crash occurrences equal to $\lambda$ and define $Y=\sum C_{i} . Y$ is sufficient for $\lambda$ and $Y$ : Poisson $(n \lambda)$. If $Y=y_{0}$ is the observed counts during $n$ years, we are led to solve for $\lambda$ in the equation (8-4).

$$
\sum_{k=0}^{y_{0}} e^{-n \lambda} \frac{(n \lambda)^{k}}{k !}=\frac{\alpha}{2} \text { and } \sum_{k=y_{0}}^{\infty} e^{-n \lambda} \frac{(n \lambda)^{k}}{k !}=\frac{\alpha}{2} .
$$

Using the relationships above, we can write

$$
\frac{\alpha}{2}=\sum_{k=0}^{y_{0}} e^{-n \lambda} \frac{(n \lambda)^{k}}{k !}=\operatorname{Pr}\left\{Y \leq y_{0} \mid \lambda\right\}=\operatorname{Pr}\left\{\chi_{2\left(y_{0}+1\right)}^{2}>2 n \lambda\right\},
$$

where $\chi_{2\left(y_{0}+1\right)}^{2}$ is a chi squared random variable with $2\left(y_{0}+1\right)$ degrees of freedom. Thus, the solution to the above equation is to take

$$
\lambda=\frac{1}{2 n} \chi_{2\left(y_{0}+1\right), \alpha / 2}^{2}
$$

Similarly, applying the identity to the other equation in (8-4) yields

$$
\frac{\alpha}{2}=\sum_{k=y_{0}}^{\infty} e^{-n \lambda} \frac{(n \lambda)^{k}}{k !}=\operatorname{Pr}\left\{Y \geq y_{0} \mid \lambda\right\}=\operatorname{Pr}\left\{\chi_{2 y_{0}}^{2}<2 n \lambda\right\}
$$

With some algebraic manipulation, the $1-\alpha$ confidence interval for $\lambda$ can be obtained as

$$
\left\{\lambda: \frac{1}{2 n} \chi_{2 y_{0}, 1-\alpha / 2}^{2} \leq \lambda \leq \frac{1}{2 n} \chi_{2\left(y_{0}+1\right), \alpha / 2}^{2}\right\} .
$$


At $y_{0}=0$, we define $\chi_{0,1-\alpha / 2}^{2}=0$.

For example, we observed seven counts of daytime right-angle collisions during a four-year period at intersection 97905. Therefore, using equation (8-5), the $95 \%$ confidence interval for expected annual crash frequency is given by

$$
\frac{1}{8} \chi_{14,0.975}^{2} \leq \lambda \leq \frac{1}{8} \chi_{16,0.025}^{2}
$$

which is $[0.704,3.606]$ per year or $[2.814,14.423]$ per four years.

\section{Comparison Results}

Figure 8-3 presents the Poisson confidence intervals of expected counts at each site and the corresponding model-based estimates. The confidence of model estimates (see Table 8-1) is not included in this figure because it is too wide to visualize any meaningful comparison. If we include the confidence intervals of model estimates, the Poisson confidence intervals based on the observed crash counts will become a subset of the confidence intervals of model estimates. The comparison results would be inconclusive at best.

However, it is interesting to see how the most credible value in that wide range of confidence interval of model estimates, i.e., the predicted crash frequency, will compare against the Poisson confidence intervals based on the observed crash counts. We assume that the true accident rate is unknown, but the Poisson confidence intervals can be established based on the observed counts. It is postulated that the observed crash count is a Poisson realization with a mean equal to the true accident rate.

At the $95 \%$ confidence level, the model-based estimates are in the range of Poisson confidence intervals at seven out of 12 sites (see Figure 8-3). The corresponding figure increases to eight out of 12 sites at the $99 \%$ confidence level (see Figure 8-4). 


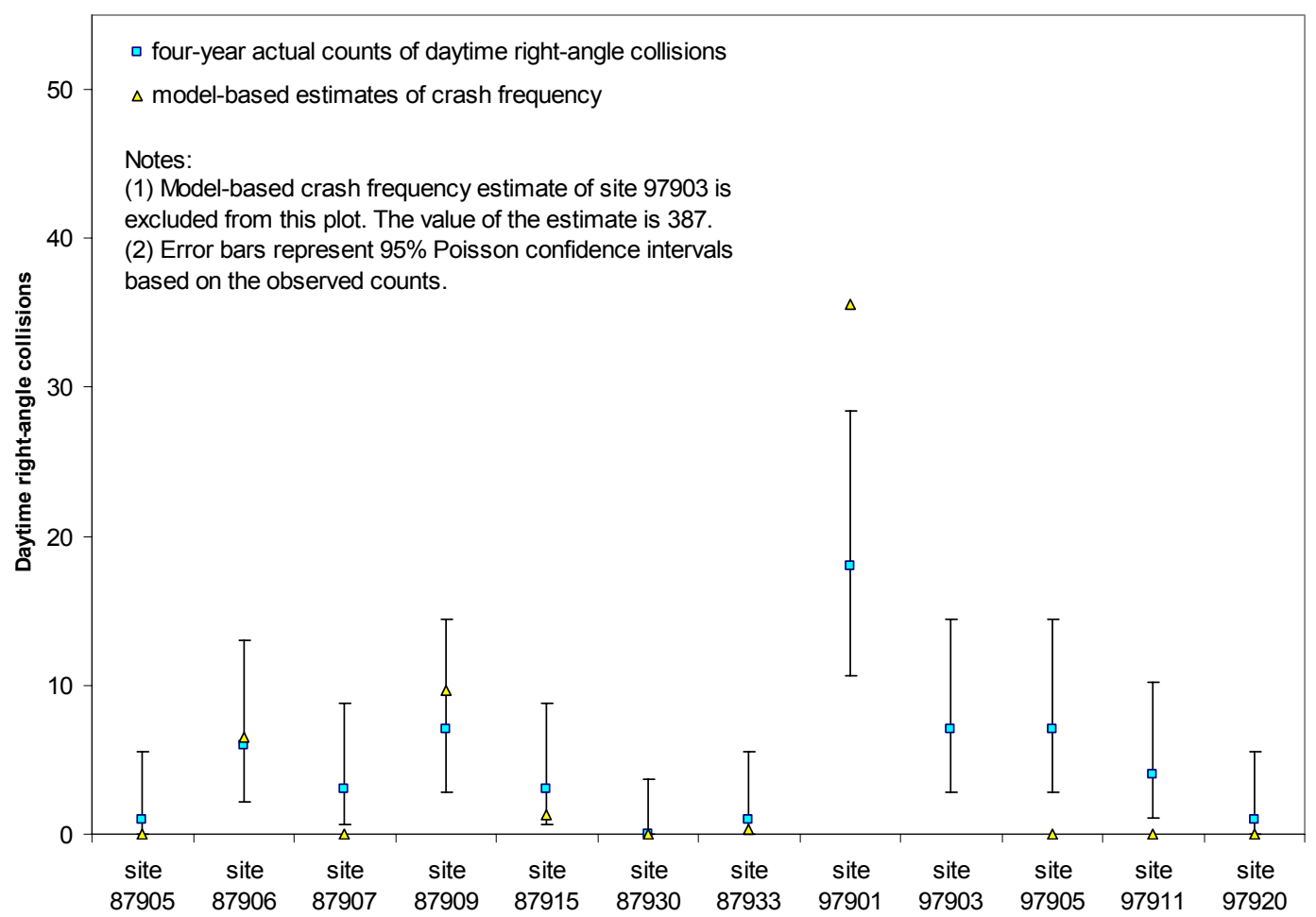

Figure 8-3: Model Estimates versus Crash-based 95\% Poisson Confidence Intervals

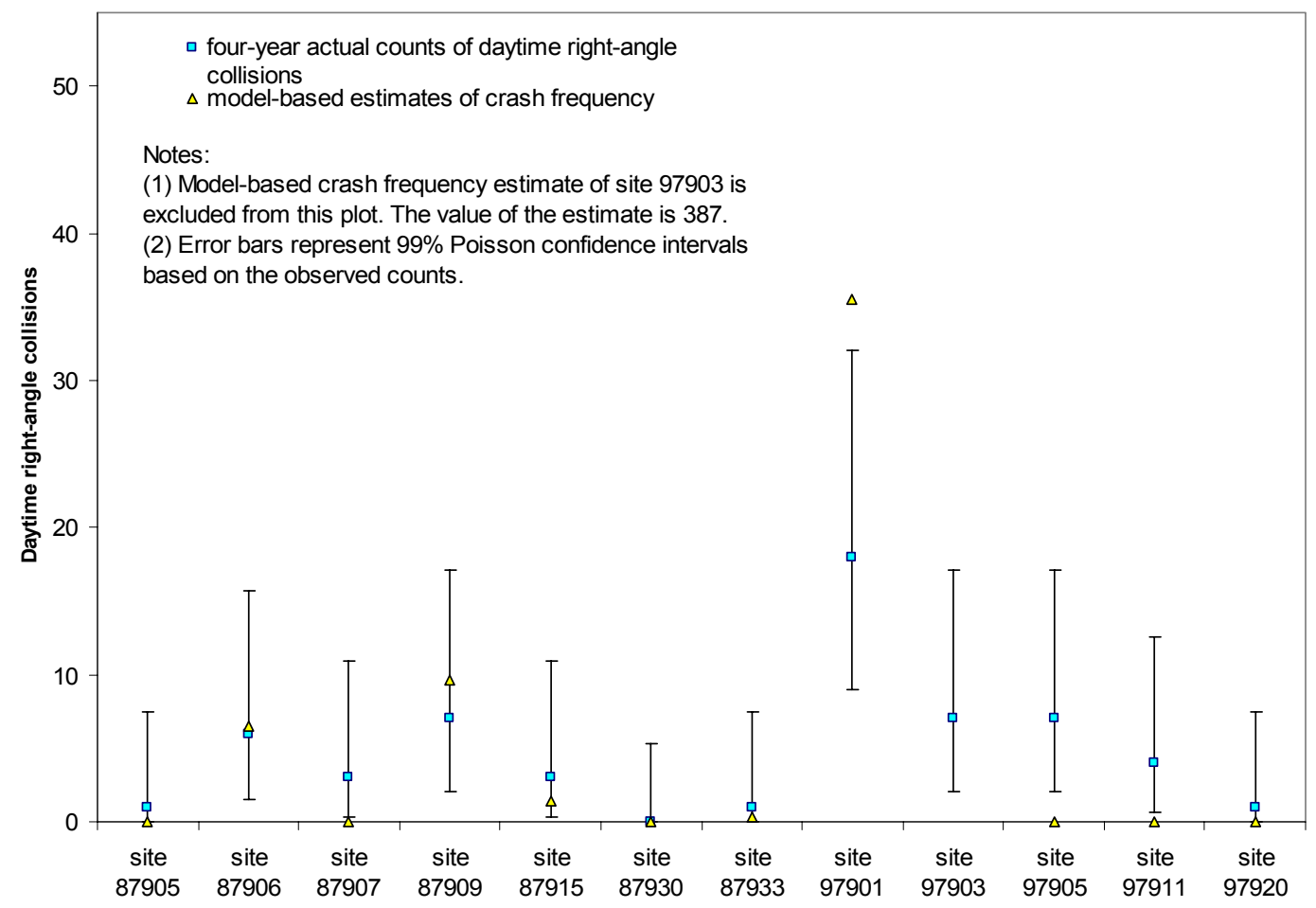

Figure 8-4: Model Estimates versus Crash-based 99\% Poisson Confidence Intervals 
The results were examined case by case. The overestimation and underestimation across the majority of locations appear to be influenced by the randomness in both model-based estimates and observed counts. However, the highly overestimated result at site 97903 and the apparent underestimation at site 97905 are possibly linked to additional factors other than the random variability of estimates.

The overestimation at site 97903 appears to be more serious than the other locations as the extreme value model consistently gives an unreasonably high crash frequency estimate. Undoubtedly, this problem is due in part to the inherent uncertainty of a short-term observation of PET extremes. However, site 97903 is unique in several respects in comparison to other sites. The eight-hour counts of PET are the second highest among all studied sites and there were a number of extremely short PETs observed from queue spillbacks during congested periods at this location. The coefficient of variation of ET is greater than 0.9 while all the others range from 0.4 to 0.7 . This leads to our concern that the proposed extreme value approach may not be able to estimate the crash counts properly at the intersections with excessive queue spillbacks. The main reason underlying this issue is possibly related to the violation of the assumption of the underlying distribution of the PET process. The PET from a normal crossing situation may have a different underlying distribution from PET caused by queue spillbacks. For example, a onesecond PET associated with a red-light violation definitely carries a greater risk than a onesecond PET from queue spillbacks. The current modeling approach assumes both cases of PET arise from the same distribution and therefore treats both cases equally. A more advanced extreme value modeling approach for this special condition may be necessary to account for a mixture of two underlying distribution processes and properly combine these different processes of risk to give single estimate of safety level. Such an approach is beyond the scope of this study at this point.

The model-based crash frequency estimate of site 97905, on the contrary, tends to give zero estimates of crash counts. The visual examination of the trend of observed PETs during the afternoon peak hour (4:30-5:30PM) shows that PETs become noticeably longer during this period. This contradicts the volume variation trend, which gradually increases during that same period. We researched the weather conditions during the observed period and surprisingly we found that it was raining from 4:00PM - 6:00PM on that date, which likely explains the longer PET values during that period. Drivers probably adjusted their driving behaviors to compensate for their extra perceived risk during bad weather conditions. The effect of this risk compensation 
was reflected through the PET values. They may overcompensate the risk such that the PETs during that hour become longer than usual when shorter PETs are in fact expected. When long PETs are unexpectedly observed instead of short PETs, the risk becomes lower than expected, thus leading to an underestimation problem at this site. This finding is quite interesting on its own because this may indicate the potential of PET to serve as a measure for risk perception of road users. Nevertheless, more data are needed before we can strongly support this argument.

\subsubsection{Extreme PET Return Level Estimates}

In a similar manner to that used for crash frequency estimates, the successful validation of return level estimates can be confirmed by its correlation with observed crash counts. However, a direct comparison is not plausible since the return level and the crash frequency estimates are not directly convertible. The extreme return level is an expected level of negated PET being exceeded on average once for a given return period.

\subsubsection{Uncertainty of Estimates}

A summary of the four-year return level estimates and inferences based on the best fitted extreme value models for all the selected 12 sites is presented Table 8-2. The comparison of standard deviations of the return level estimates versus those of the crash frequency estimates reveals that the return level estimates give much tighter confidence intervals as well as smaller standard errors at most sites. In this sense, the return level estimate appears to be a more robust safety indicator derived from the proposed models comparing to the crash frequency estimate.

\subsubsection{Correlation Check}

The plots of one-year and four-year return level estimates versus observed counts of right-angle collisions are given in Figure 8-5. These two plots indicate similar positive correlations. The Pearson correlation between the one-year return level estimates and the observed crash counts is 0.564 . With the four-year return level estimates, the Pearson correlation is slightly better at 0.631 . These show that both return level estimates exhibit a good agreement with the observed crash counts. The correlation with crash data is quite robust to the choice of a time horizon (one year or 
four years) used in the prediction. Hence, the four-year return period is preferred in this case as it gives a slightly better correlation and it is also consistent with a duration considered in the crash frequency estimate.

Table 8-2: Model-based Estimates of Return Level of Negated PETs and Inferences

\begin{tabular}{|c|c|c|c|c|c|c|c|c|}
\hline \multirow{3}{*}{ Site } & \multirow{3}{*}{$\begin{array}{l}\text { 4-Year Right-Angle } \\
\text { Crash Counts } \\
\text { (Daytime) }\end{array}$} & \multirow{3}{*}{$\begin{array}{l}\text { 4-Year Return } \\
\text { Level Estimate }\end{array}$} & \multicolumn{6}{|c|}{$\begin{array}{c}\text { Summary Statistics of Model-based Estimates of } \\
\text { 4-year Return Level of Negated PET }{ }^{(2)}\end{array}$} \\
\hline & & & \multirow{2}{*}{ Mean } & \multirow{2}{*}{$S D^{(1)}$} & \multicolumn{2}{|c|}{$90 \%$ C.I. } & \multicolumn{2}{|c|}{ 95\% C.I. } \\
\hline & & & & & Lower & Upper & Lower & Upper \\
\hline 87905 & 1 & -0.468 & 0.157 & 2.970 & -2.006 & 3.036 & -2.306 & 5.021 \\
\hline 87906 & 6 & 0.239 & 0.352 & 0.686 & -0.621 & 1.528 & -0.771 & 1.842 \\
\hline 87907 & 3 & -2.443 & -2.412 & 0.364 & -3.002 & -1.802 & -3.098 & -1.663 \\
\hline 87909 & 7 & 0.751 & 2.427 & 5.597 & -1.351 & 11.481 & -1.578 & 16.425 \\
\hline 87915 & 3 & 0.058 & 0.151 & 0.640 & -0.749 & 1.264 & -0.892 & 1.583 \\
\hline 87930 & 0 & -0.772 & -0.160 & 2.999 & -1.397 & 2.182 & -1.545 & 4.756 \\
\hline 87933 & 1 & -0.093 & 0.129 & 0.750 & -0.787 & 1.500 & -0.909 & 1.927 \\
\hline 97901 & 18 & 2.248 & 4.891 & 8.156 & -1.340 & 19.185 & -1.639 & 26.870 \\
\hline 97903 & 7 & 2.214 & 2.822 & 2.497 & 0.315 & 7.614 & 0.124 & 9.086 \\
\hline 97905 & 7 & -1.765 & -1.721 & 0.307 & -2.198 & -1.200 & -2.278 & -1.059 \\
\hline 97911 & 4 & -0.331 & -0.277 & 0.600 & -1.227 & 0.726 & -1.394 & 0.963 \\
\hline 97920 & 1 & -1.438 & -1.385 & 0.421 & -2.041 & -0.673 & -2.151 & -0.508 \\
\hline
\end{tabular}

Notes:

(1) SD is preferred to CV since CV cannot fairly represent the degree of variability in the estimates as the means are very small in many cases.

(2) The modeling results are based on the best fitted models.
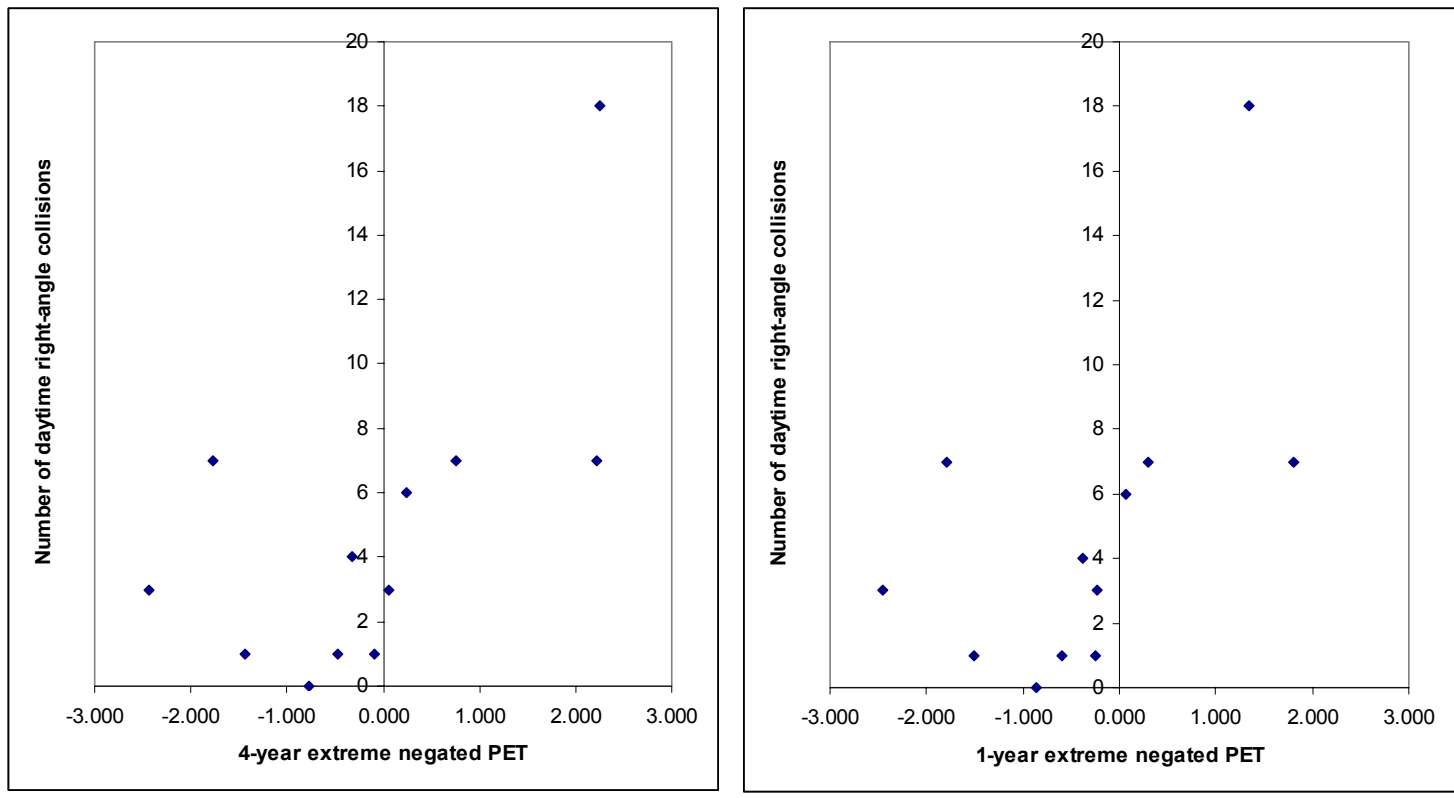

Figure 8-5: Actual Counts of Collisions versus Extreme Return Level Estimates 
In contrast to the crash frequency estimate, the extreme return level estimate does not suffer from the overestimation problem at site 97903. From a sequence of validation plots with the other model settings (see Appendix F), it is apparent that the return level estimates are consistently in the expected range in comparison to the crash frequency estimates of the same site.

Note that the return level is determined in terms of negated PET, not the PET itself. The higher return level estimates imply riskier locations. Based on the best fitted models, the relationship between crash frequency and return level estimates is shown in Figure 8-6. The data point from site 97903 is excluded in the right figure to better illustrate the trend visually. From this figure, we can observe that the crash frequency estimates are zero or close to zero where the four-year return level estimates are less than zero, which is expected because the negative four-year return level estimate implies that the likelihood of right-angle collisions is extremely low during the four-year period. Recall that the negated PET $>0$ characterizes the event of collision. For the entire four-year period, the probability of a right-angle collision would be extremely small if the expected maximum negated PET is still less than zero. When we compute the return level by increasing the return period to infinity, the negative maximum return level basically implies the zero expected risk of collision for a location.
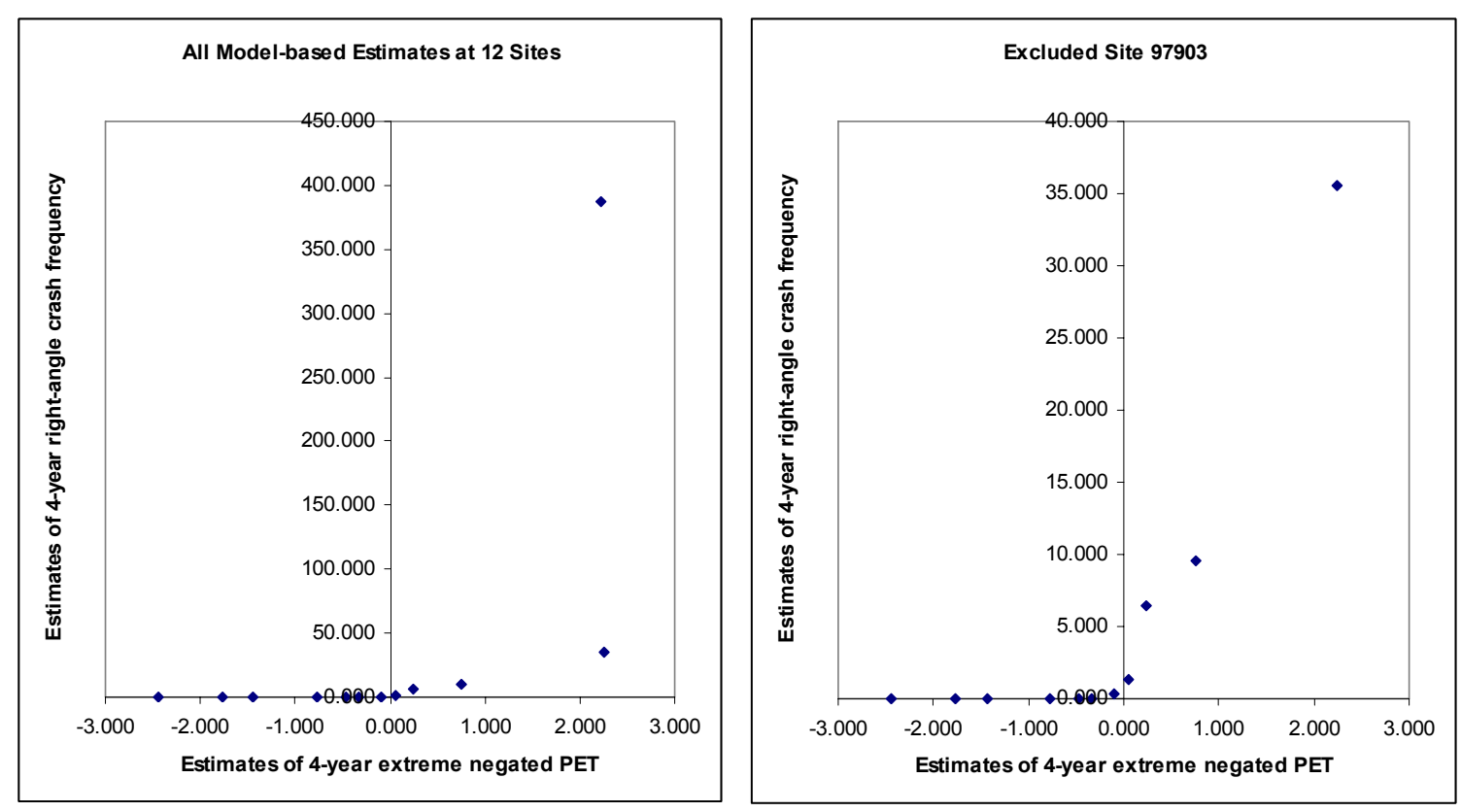

Figure 8-6: Estimates of Crash Frequency versus Estimates of Return Level 


\subsubsection{Summary of Validation Results}

Both return level and crash frequency estimates of the extreme value models exhibit a promising relationship with the widely-used safety indicator, i.e., historical crash data. However, there are obvious pros and cons of each estimate that are worth special consideration. The validation results and findings regarding the crash frequency estimates can be summarized as follows:

- The crash frequency estimate is a safety indicator whose interpretation is directly understandable for policymakers as well as highway engineers.

- Crash frequency estimates exhibit a potential relationship with observed crash counts. Excluding the data point of site 97903, the Pearson correlation between actual counts and model estimates is 0.931 . The crash frequency estimates correspond well with the observed counts at locations with high counts of right-angle collisions.

- The confidence interval of a PET-based crash frequency estimate is still very wide, which is due in large to an insufficient sample of extreme values from an eight-hour observation period. The data requirement to obtain a reasonable confidence interval will be examined later in this chapter.

- A crash frequency estimate suffers from the overestimation problem where a number of short PETs are caused by queue spillbacks.

- A crash frequency estimate is non-negative, which tends to cluster at zero for relatively safe intersections. Hence, it may not well discriminate varying levels of safety across locations with low-crash and moderate-crash counts.

The validation results and findings of the estimates of return levels of negated PETs can be concluded as follows:

- Similar to crash frequency estimates, return level estimates exhibit a good agreement with observed crash counts. In addition, the return level estimate does not suffer from the overestimation problem, particularly at site 97903 . The Pearson correlation using all data points is 0.631 for a four-year prediction horizon.

- Return level estimates are not restricted to only non-negative values as in the case of crash frequency estimates. Therefore, they fare better in discriminating varying safety levels at safe or moderately safe locations.

- The standard errors of return level estimates are relatively much smaller than those obtained for crash frequency estimates. 
- Safety interpretation of return level estimates is, however, less intuitive than for crash frequency estimates. In addition, return level estimates cannot be converted into crash frequency estimates.

- The safety implication based on return level estimates of negated PETs is similar to crash frequency estimates; a higher return level associated with a greater risk of collisions.

- General guidelines for safety interpretation of return levels of a negated PET are as follows: (a) if the four-year return level for a particular location is less than zero, the risk of right-angle collisions is likely to be low, but additional evaluation is recommended before it can be confirmed; and (b) if the four-year return level is greater than zero, there is an above-normal risk of right-angle collisions and a comprehensive safety evaluation is recommended.

The current extreme value approach using the block maxima distributions appears to produce promising results, as exhibited by the correlations between model estimates and observed crash counts. However, the major shortcoming of the approach is the poor confidence of estimates, which may be attributable to an insufficient sample of PET extreme values from an eight-hour observation period. To examine this issue in greater detail, we studied the effects of model settings including the block length, the choice of $r$ value, and the observation period on the confidence of model estimates. Analyzing these effects with field observation would be extremely data-intensive and thus impractical at this point. Instead, we propose a simulationbased analysis which takes into account the field data and historical crash counts to generate the PET observations. The method and findings are presented in the next section.

\subsection{SIMULATION STUDY OF MODEL CHARACTERISTICS}

A simulation-based analysis of the PET sampling scheme is proposed to evaluate the impact of model settings on the confidence of model estimates. The comparison benchmark for an acceptable confidence interval of crash frequency estimates is the crash-based confidence interval. This simulation-based approach assumes that the complete and accurate information about the PET distribution is known for a given site. Provided that the assumption of the continuum of events is valid, the tail behavior of this distribution reflects the actual frequency of right-angle collisions. The analytical framework resulting from this notion is to construct a continuous distribution that can approximate PET variability in the field and simultaneously give 
an accurate estimate of crash frequency. Then, based on the fitted distribution, we can simulate the PET observations over time for a sampling scheme of interest to investigate its impact on the confidence of model estimates.

The approach to establishing the parametric distribution from observed PETs at selected sites is first described. Then, we describe the PET simulation procedure based on the selected distribution. It is important to note that the proposed simulation approach does not account for the temporal dependency and non-stationarity that may exist in a series of empirical PET data. Consequently, only the homogeneous extreme value models are considered for the simulated PET. The dependency in a series of observations requires no special treatment in the block maxima extreme value models since the effect of dependency is automatically handled in the parameter estimates. The possible non-stationarity in the PET occurrence process, though not considered in the simulation, should have minimal impact in this analysis. It is important to bear in mind that the goal of this task is to understand the effect of model settings and the observation period on the confidence of estimates. The non-stationarity may influence the accuracy of model estimates in the field condition but the impact on the confidence of estimates is unlikely to be consequential. This is more or less confirmed once we describe the simulation results. The variability observed from the model-based estimates based on one-day simulated PETs (eight hours per day) is somewhat similar to what we experienced with the field data.

\subsubsection{Constructing Underlying Parametric Distribution}

Denoting $X_{1}, X_{2}, \ldots$ as independent realizations of negated PETs and letting $X_{i}: F$ where $F$ is a parametric distribution underlying PETs at a specific site. Distribution $F$ must satisfy

$$
\operatorname{Pr}\left\{X_{i}>0 \mid X_{i}>u\right\}=p_{c}
$$

where $u$ is a PET modeling threshold and $p_{c}$ is the likelihood of right-angle collisions given that the threshold $u$ is exceeded. The PET modeling threshold $u$ represents the lower limit of negated PET upon which the distribution $F$ is to be established. Notice that the modeling threshold $u$ is not necessarily the same as the observation threshold. In fact, the modeling threshold is intentionally chosen to be lower than the observation threshold (eight seconds) to ensure that all the PETs in a range of interest were measured. In our study, we consider $u=-6$ 
(or 6 seconds) as our modeling threshold for negated PETs based on visual examinations of PET histograms of several sites.

Condition (8-6) ensures that the area under the tail region of the distribution $F$ can accurately reflect the specified number of crashes. The likelihood $p_{c}$ can be determined from the historical crash data as

$$
p_{c}=\frac{c_{n_{y}} /\left(n_{y} \times 12.09 \times 365\right)}{f_{u, t_{o b s}} / t_{o b s}},
$$

where $c_{n_{y}}$ is the observed counts daytime right-angle collisions during $n_{y}$ years and $f_{u, t_{\text {obs }}}$ is the counts of negated PETs that are less than threshold $u(u=-6)$ during the observation period of $t_{\text {obs }}$ hours.

Let $\theta$ be a parameter vector of the distribution $F$. Then, the parameter estimates can be determined by maximizing the likelihood function

$$
L\left(\theta \mid x_{1}, \ldots, x_{n}\right)=\prod_{i=1}^{n} f\left(x_{i}\right)
$$

subject to the constraint (8-6) where $x_{1}, \ldots, x_{n}$ are the observed negated PETs equal to or greater than $u$. Computationally, the constraint $(8-6)$ is relaxed to ensure the convergence of the algorithm for maximum likelihood estimation, which becomes

$$
\left(\log \hat{p}_{c}-\log p_{c}\right)^{2}<k
$$

where $\hat{p}_{c}$ is the conditional likelihood of collisions evaluated at maximum likelihood estimates $\hat{\theta}$ and $\mathrm{k}$ is the tolerance level. The specification of $\mathrm{k}$ is a trial-and-error process which is dependent on an assumed distribution $F$ and observed PET data. 


\subsubsection{Fitting Parametric Distributions}

We selected the PET data from site 97901 as an example case to determine the appropriate distributional function. Visual examination of an empirical histogram of negated PET data of this site (see Figure C-35) indicates that the PET modeling threshold at six seconds appears to be suitable. The PET values less than six seconds are then negated and fitted to the following distributions. The negated PET modeling threshold is $u=-6$.

\section{Generalized Extreme Value Distribution}

Recall the GEV distribution function from equation (6-1),

$$
G(z)=\exp \left\{-\left[1+\xi\left(\frac{z-\mu}{\sigma}\right)\right]^{-1 / \xi}\right\}
$$

defined on the set $\{z: 1+\xi(z-\mu) / \sigma>0\}$, where the parameters satisfy $-\infty<\mu<\infty, \sigma>0$ and $-\infty<\xi<\infty$. The log-likelihood function of GEV distribution was given in equations (6-36) and (6-38) for $\xi \neq 0$ and $\xi=0$ respectively.

The maximum likelihood estimates $(\hat{\mu}, \hat{\sigma}, \hat{\xi})$ must maximize the log-likelihood function while subject to (8-9) where the estimated conditional likelihood of collisions $\hat{p}_{c}$ in (8-9) can be obtained by

$$
\hat{p}_{c}=\frac{1-\hat{G}(0)}{1-\hat{G}(u)}
$$

evaluated at $(\hat{\mu}, \hat{\sigma}, \hat{\xi})$.

Setting $\mathrm{k}=0.01$ and maximizing the constraint log-likelihood function give the fitted GEV density in Figure 8-7. There appears to be a lack-of-fit problem at the range of negated PETs from -6 to -5 seconds. The histogram of negated PETs observed at site 97901 is also shown for comparison. 


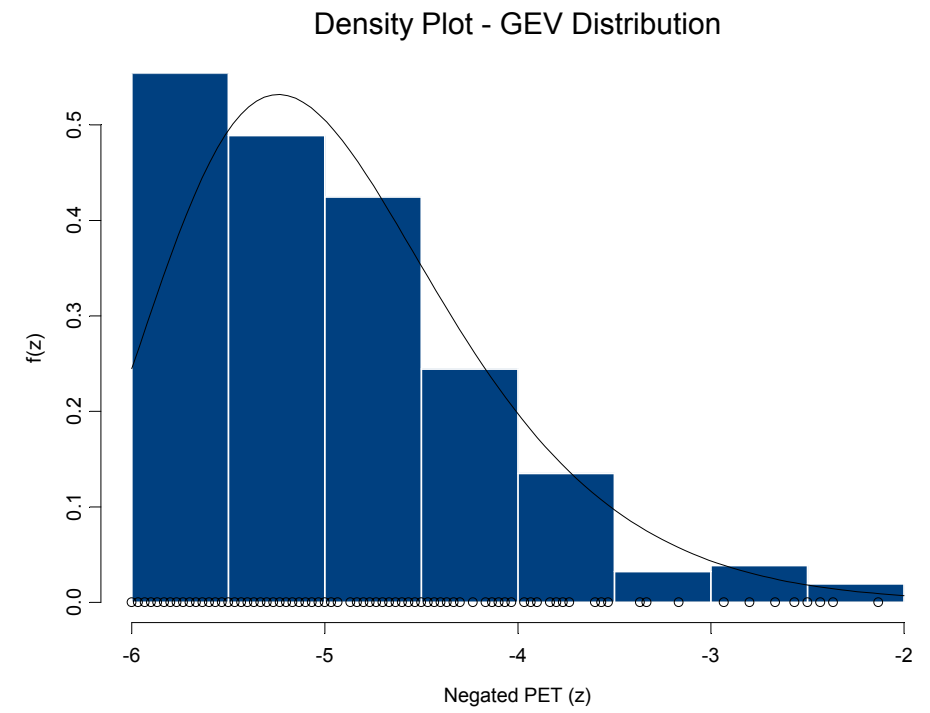

Figure 8-7: Fitted Constraint GEV Density - Site 97901

\section{Exponential Distribution}

Exponential density has a continuous decreasing trend, which is similar to the empirical histogram of the negated PETs observed from the field. In addition, an exponential distribution is the only distribution in a family of continuous distributions that has the memoryless property; this implies that, for a modeling threshold of negated PETs $u$,

$$
\operatorname{Pr}\{X>0 \mid X>u\}=\operatorname{Pr}\{X>-u\}
$$

The distribution function of the exponential distribution is

$$
F(x)=1-e^{-\frac{(x-\mu)}{\beta}} ; x-\mu \geq 0, \beta>0 .
$$

The corresponding log-likelihood function is

$$
1(\mu, \beta)=n \log \left(\frac{1}{\beta}\right)+\sum_{i=1}^{n}\left(\frac{x_{i}-\mu}{\beta}\right)
$$

By (8-11), the conditional likelihood of collisions can be estimated by 


$$
\hat{p}_{c}=F(-u)
$$

evaluated at $(\hat{\mu}, \hat{\beta})$.

Maximizing log-likelihood (8-13) subject to condition (8-9) with k set at 0.01 yields the fitted exponential density as depicted in Figure 8-8. Although the trend is correct, the lack-of-fit problem is still obvious for the entire range of fitted values.

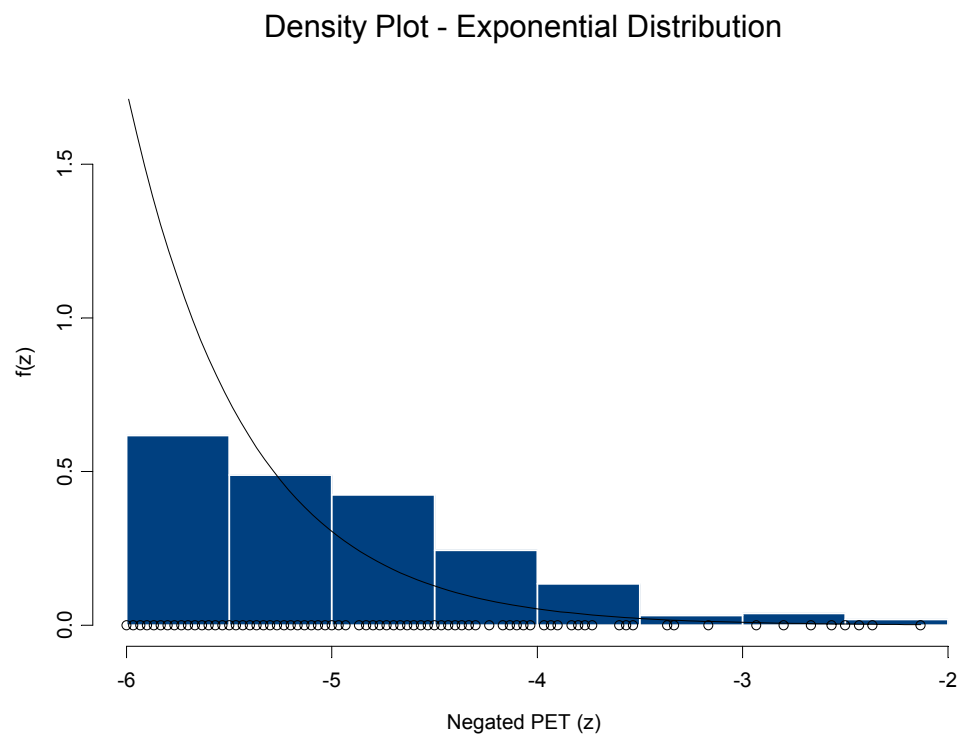

Figure 8-8: Fitted Constraint Exponential Density - Site 97901

\section{Generalized Pareto Distribution}

We discussed the generalized Pareto distribution briefly in Section 6.3.1. In the extreme value applications, this distribution was fitted to extremes defined by the threshold excesses of a prespecified high threshold. We fit the negated PET to this distribution at a threshold of $u=-6$. A high threshold is not a concern herein because PET extremes will not be directly taken from the fitted distribution. Instead, we will be taking only the block maxima from a set of simulated PET values. Note that we are searching for a distribution that can reasonably approximate the variability in PET data for a given site. A good fitted distribution will allow us to reliably generate PET observations that adequately replicate field data. 
Rewrite equation (6-10), the generalized Pareto for threshold excesses $Y=X-u$ where $X$ is negated PETs is

$$
H(y)=1-\left(1+\frac{\xi y}{\sigma}\right)^{-1 / \xi}
$$

defined on $\{y: y>0$ and $(1+\xi \mathrm{y} / \sigma)>0\}$. For threshold excesses $y_{1}, \ldots, y_{n}$, the log-likelihood function can be derived as

$$
1(\sigma, \xi)=-n \log \sigma-(1+1 / \xi) \sum_{i=1}^{n} \log \left(1+\xi y_{i} / \sigma\right)
$$

Correspondingly, the conditional likelihood $p_{c}$ can be estimated by

$$
\hat{p}_{c}=\left[1+\frac{\xi u}{\sigma}\right]^{-1 / \xi},
$$

evaluated at $(\hat{\sigma}, \hat{\xi})$. Maximizing (8-16) subject to constraint (8-9) at $\mathrm{k}=0.005$ gives the fitted generalized Pareto density as shown in Figure 8-9.

Density Plot - Generalized Pareto Distribution

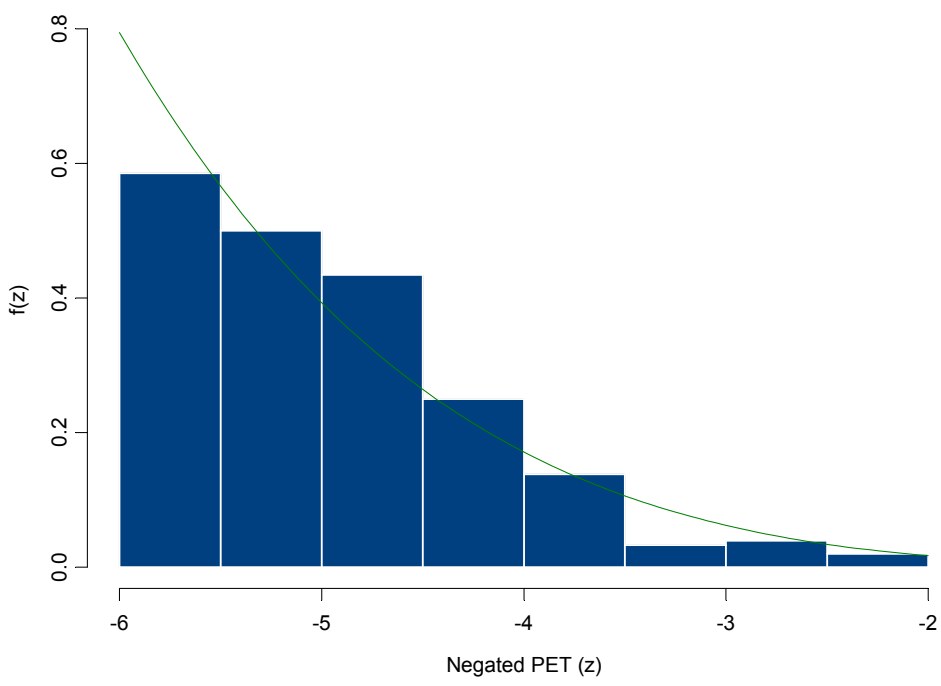

Figure 8-9: Fitted Constraint Generalized Pareto Distribution - Site 97901 


\subsubsection{Comparison of Distributional Assumptions}

The distributions fitted to site 97901 are summarized in Table 8-3. The comparion of negative log-likelihood at convergence indicates that the generalized Pareto distribution gives the best fit among selected distributions.

Table 8-3: Comparison of Fitted Distributions

Site 97901, modeling threshold $=6$ sec, observed crash counts $=18$

\begin{tabular}{|c|c|c|c|c|c|c|}
\hline \multirow{2}{*}{$\begin{array}{c}\text { Distribution } \\
\text { GEV }\end{array}$} & \multicolumn{3}{|c|}{ Parameter Estimates } & \multirow{2}{*}{$\begin{array}{c}\begin{array}{c}\text { Estimated } \\
\text { Conditional } \\
\text { Probability of } \\
\text { Crashes }\left(\mathbf{p}_{\mathrm{c}}\right)\end{array} \\
2.5132 \mathrm{E}-05\end{array}$} & \multirow{2}{*}{$\begin{array}{c}\text { Estimated 4- } \\
\text { Year Crash } \\
\text { Frequency }\end{array}$} & \multirow{2}{*}{$\begin{array}{c}\begin{array}{c}\text { Negative } \\
\text { Log- } \\
\text { likelihood }\end{array} \\
341.20\end{array}$} \\
\hline & $\begin{array}{c}0.6933 \\
(\mu)\end{array}$ & $\begin{array}{c}0.6933 \\
(\sigma)\end{array}$ & $\begin{array}{c}-0.0668 \\
(\xi) \\
\end{array}$ & & & \\
\hline Exponential & $\begin{array}{c}-6.0000 \\
(\mu) \\
\end{array}$ & $\begin{array}{c}0.5742 \\
(\beta) \\
\end{array}$ & & $2.8986 \mathrm{E}-05$ & 19.89 & 734.02 \\
\hline $\begin{array}{l}\text { Generalized } \\
\text { Pareto (GP) }\end{array}$ & & $\begin{array}{c}1.2588 \\
(\sigma) \\
\end{array}$ & $\begin{array}{c}-0.1775 \\
(\xi) \\
\end{array}$ & $2.6430 \mathrm{E}-05$ & 17.73 & 311.38 \\
\hline
\end{tabular}

Visual examination of Figure 8-9 also reveals a good agreement between the fitted density and the empirical histogram of the negated PET. The fitted generalized Pareto distribution is therefore selected as a parameteric model for negated PETs greater than the specified threshold.

\subsubsection{PET Simulation Procedure}

Now we have devised the procedure to determine and estimate the distribution of PETs such that the likelihood of a collision satisfies the observed crash counts. Let us consider crossing events and a PET observation process to help understand the simulation procedure. A PET is the result of a crossing event which usually takes place during the change of signal phasings. If we assume that there exists at least one through-vehicle from each approach in every phase, then we always have at least one PET for every cycle. The approximate number of PETs is therefore dependent on the average number of cycles per unit time at a signalized intersection.

It should be noted, nevertheless, that not all PET values are recorded in the PET measurement. Due to the fact that a large PET is basically caused by a normal crossing event, a PET is recorded only when it is shorter than a specified threshold. The total number of observable PETs is relatively large compared to the total number of recorded PETs. In other words, the likelihood of 
having PET recorded is equal to the likelihood of a PET being less than a reasonably low threshold. The number of PETs collected per unit time is the same as the number of PETs less than the specified threshold. If we treat the chance of having a PET recorded as a Bernoulli process, then the number of collected PETs per unit time follows the binomial distribution. When this likelihood is sufficiently small, a Poisson approximation of a binomial distribution is applicable. Hence, for a sufficiently large block length, we can assume that the number of recorded PETs per block is Poisson-distributed with the mean equal to the average count of PETs less than the specified threshold.

The next issue is how to simulate PETs that are greater than the specified threshold. This range of PETs obviously cannot be approximated by the generalized Pareto distribution. A general solution depends on the size of the block length being considered. If the block length is sufficiently large, the likelihood of PET extremes (block maxima of negated PET) falling into this range is negligible. Therefore, this range of PETs can be ignored for a sufficiently large block length. Another scenario is when a site has low PET counts and consequently the block length may not be large enough. In such a case, we cannot ensure that the PET extremes will always fall within the range of the fitted generalized Pareto distribution. A uniform distribution is employed to generate PET observations within the range of one to two seconds greater than the threshold to supplement the PET values in the range that is not included in the fitted generalized Pareto distribution.

The step-by-step procedure to simulate PETs can be described as follows:

1. Specify the number of blocks to be generated. Without loss of generality, let us assume that observation period is fixed at eight hours a day. Therefore, the observation period can be referred to in the unit of days for the sake of convenience.

2. Generate the number of PETs per block using Poisson distribution with a mean equal to the average block count of PETs less than the specified threshold.

3. If a block length is sufficiently large, generate each PET observation using the fitted generalized Pareto distribution.

4. If a block length is short, generate each PET observation using a two-step process. The likelihood of falling within the range of large PETs (greater than the threshold) is estimated by the actual proportion of the field data. Generate a large PET with the uniform distribution and a short PET with the fitted generalized Pareto distribution. The 
chance of having a large PET is determined by the estimated probability of negated PETs greater than the modeling threshold.

\subsubsection{Selected Sites and Estimated Distributions}

The simulation experiment is conducted for two selected sites: 87906 and 97901 . Site 87906 had six right-angle collisions during the daytime from 1997 to 2000. Site 97901 had 18 collisions for the same period. Selected sites aim to represent two opposite situations: (a) low crash counts and (b) high crash counts. The parameter estimation for site 97901 was explained earlier in Section 8.2.1 to exemplify the procedure. Applying the same procedure to the observed PETs at site 87906, the parameter estimates can be determined in a similar manner. The generalized Pareto distribution apparently gives a reasonable goodness-of-fit for both locations. The parameter estimates of both sites are summarized in Table 8-4.

Table 8-4: Summary of Parameter Estimates at Selected Sites

\begin{tabular}{|c|c|c|c|c|c|c|}
\hline $\begin{array}{l}\text { Selected } \\
\text { Sites }\end{array}$ & $\begin{array}{c}\text { PET } \\
\text { Modeling } \\
\text { Threshold } \\
\text { (sec) }\end{array}$ & $\begin{array}{l}\text { Observed } \\
\text { Counts per } \\
4 \text { years }\end{array}$ & $\begin{array}{l}\text { Parameter } \\
\text { Estimates }\end{array}$ & $\begin{array}{l}\text { Estimated } \\
\text { Conditional } \\
\text { Probability of } \\
\text { Crashes }\left(p_{c}\right)\end{array}$ & $\begin{array}{l}\text { Estimated 4- } \\
\text { Year Crash } \\
\text { Frequency }\end{array}$ & $\begin{array}{l}\text { Negative } \\
\text { Log- } \\
\text { likelihood }\end{array}$ \\
\hline 87906 & 6.0 & 6 & $\begin{array}{cc}1.2670 & -0.1788 \\
(\sigma) & (\xi)\end{array}$ & 2.7742E-05 & 5.81 & 100.41 \\
\hline 97901 & 6.0 & 18 & $\begin{array}{cc}1.2588 & -0.1775 \\
(\sigma) & (\xi)\end{array}$ & 2.6430E-05 & 17.73 & 311.38 \\
\hline
\end{tabular}

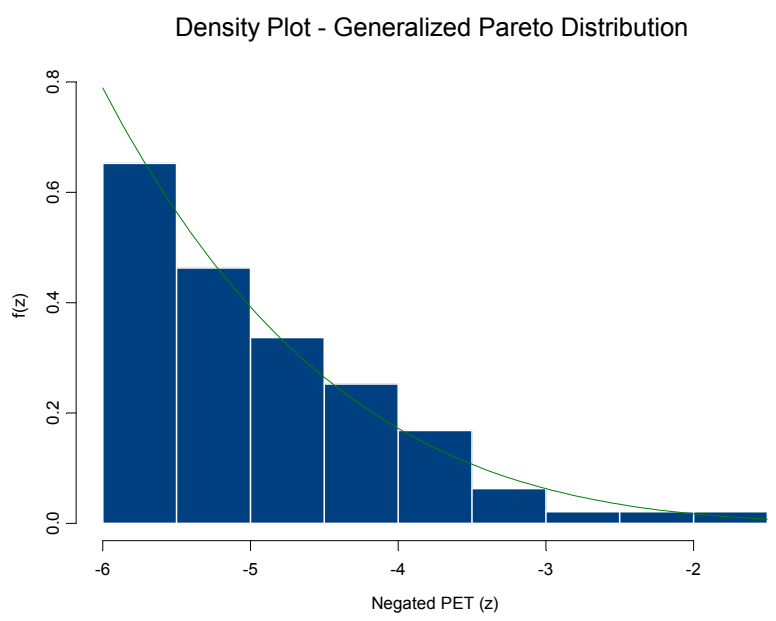

Figure 8-10: Fitted Constraint Generalized Pareto Distribution - Site 87906 
Notice that the estimated conditional probabilities of crashes at both sites are very close. This is a coincidence because the ratio of PET counts and the ratios of observed right-angle crash counts between both sites are fairly close (about 3 times). The plot of the fitted constraint generalized Pareto distribution versus the empirical observations at site 87906 is given in Figure 8-10. A similar plot for site 97901 was shown earlier in Figure 8-9.

\subsubsection{Simulation Experiment and Results}

We simulate the PETs from the fitted distributions at both sites and then apply the proposed extreme value modeling approach to determine the crash frequency estimates and return level estimates of negated PET. To be consistent with the validation part, a four-year prediction horizon is applied in all subsequent analysis.

Without the loss of generality, let us assume that the PET data will be collected for eight hours per day for simplicity. The number of observation days is referred to instead of the number of hours in the simulation results. The number of observation days, model choice (number of block maxima), and block length are varied in simulation settings to investigate its effect on the confidence of model estimates.

As a result, three settings are considered in this simulation experiment for both sites, which are:

- Experiment 1: the $r$ largest order statistic model at $r=1$ (GEV equivalent) and a 15minute block length

- Experiment 2: the $r$ largest order statistic model at $r=1$ (GEV equivalent) and a one-hour block length

- Experiment 3: the $\mathrm{r}$ largest order statistic model at $\mathrm{r}$ chosen as proposed in Table 7-4 and a 15-minute block length. In other words, $r=2$ for site 87906 and $r=3$ for site 97901 .

For each experiment, we increase the number of observation days from one day to 500 days (or 100 days if the model execution time is unreasonably long). For each set of simulated series of PETs, we estimate the extreme value model and compute the estimates of crash frequency and return level of negated PETs. Therefore, one experiment produces two graphical plots: (a) crash frequency estimates versus observation days and (b) return level estimates versus observation days. This is equivalent to six figures for one site (three experiment setups for two types of 
estimate). Two selected sites are considered in this study and thus lead to a total of 12 plots of simulation results as shown in Appendix G.

The most natural benchmark for the confidence of crash frequency estimates is the Poisson confidence intervals based on observed crash counts. The 95\% and 99\% Poisson confidence intervals are provided for all the plots related to crash frequency estimates to illustrate the desirable level of uncertainty.

The return level benchmark is taken at the crash occurrence level which is equivalent to a zero negated PET value. In Section 8.1.2, we noted that the return level estimate of a negated PET greater than zero indicates a potential risk of right-angle collisions. Therefore, the desirable confidence of return level estimates is achieved when the chance of a negative return level being incorrectly predicted is very slim.

\subsubsection{Effects of Block Length}

Consideration of a suitable choice of block length is governed by the bias-variance tradeoff. As the consequence of a violation of the asymptotic assumption underlying the extreme value distributions, a too short block length can give rise to biased estimates. A large block length, on the other hand, will generate fewer PET extremes, which in turn can give parameter estimates with a large variance. The eight-hour field observation poses a constraint on us to favor a smaller block length for two practical reasons: (a) to increase the confidence of model estimates and (b) to ensure an adequate sample size for the convergence of maximum likelihood estimation. This restriction is no longer an issue in a simulated environment. We estimate the GEV models $(r=1)$ using two different block lengths at selected sites to evaluate its impact on modeling results.

To examine the effects of block length, the plots of estimated crash frequency using a 15-minute versus one-hour block length are compared. The comparison of the effect of block lengths on the crash frequency estimates of site 87906 is shown in Figure 8-11. The number of observation days, from 10 to 500, is shown in the plots. On the left figure, the tendency to underestimate the crash frequency is noticeable when a 15-minute block length is selected. The bias disappears when the block length is increased to one hour on the right figure. However, the elimination of the model bias was achieved at the expense of a large uncertainty of model estimates. 

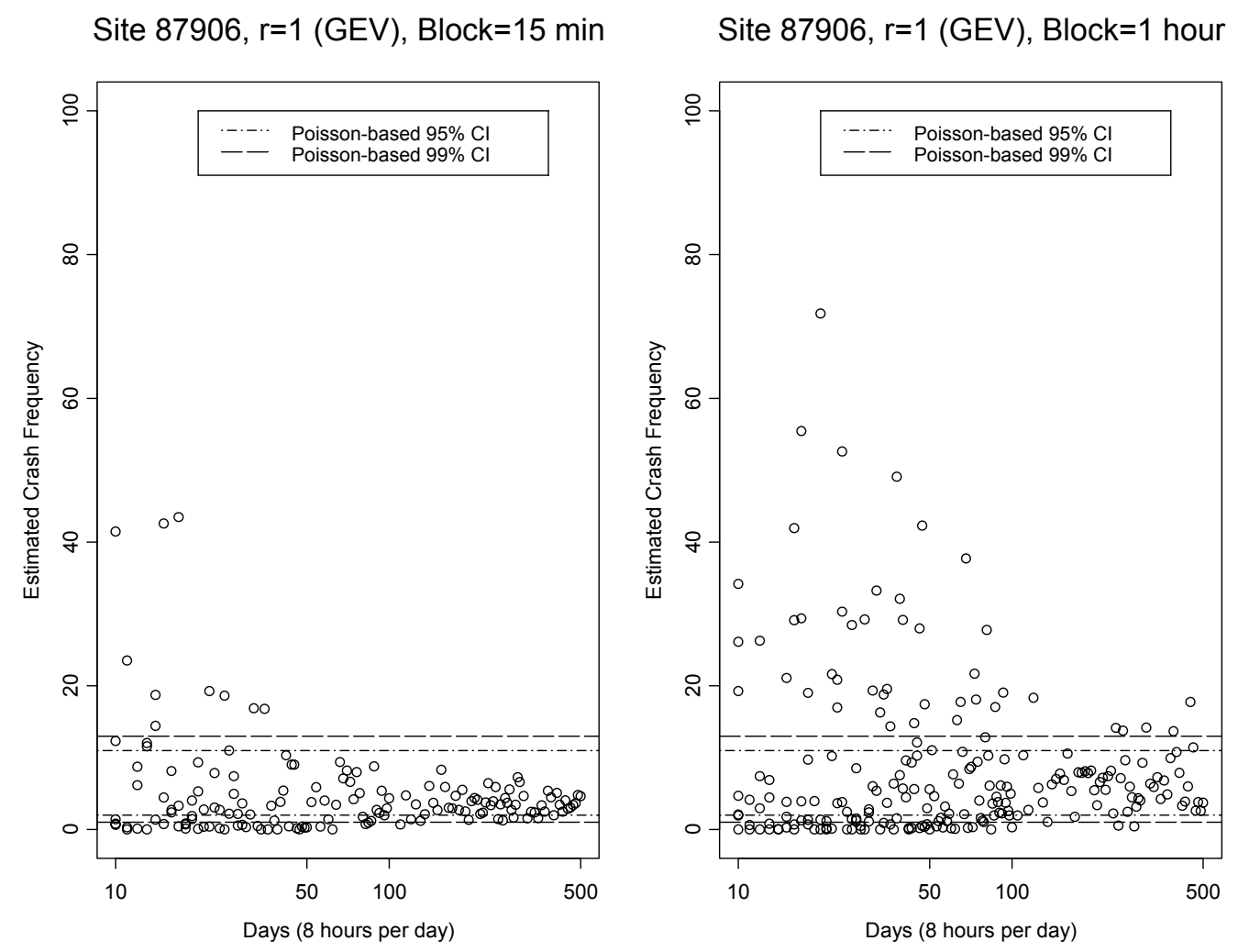

Figure 8-11: Effect of Block Length on Crash Frequency Estimates - Site 87906

The total number of PET equal to or less than eight seconds at site 87906 is 190 for the eight-hour period, which is equivalent to about six per block on average when a 15-minute block is being used. With a one-hour block length, the average number of PET per block is about 24.

Let us look at a similar comparison at site 97901 in Figure 8-12. The bias is not a problem at either 15-minute or one-hour blocks at site 97901. However, the estimates suffer from poor confidence when a one-hour block was chosen. The observed number of PET equal to or less than eight seconds at this site is 573 for the eight-hour period. That is an average of 18 per 15-minute block or 72 per one-hour block. The 15-minute block length is apparently a suitable choice for site 97901. 
Site 97901, $r=1$ (GEV), Block=15 min

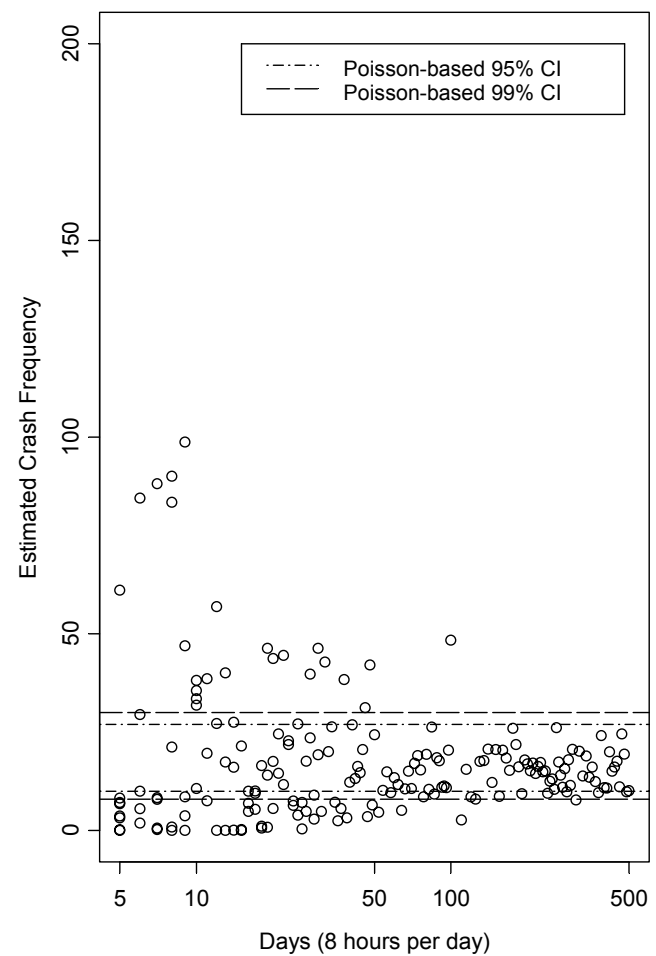

Site 97901, r=1 (GEV), Block=1 hour

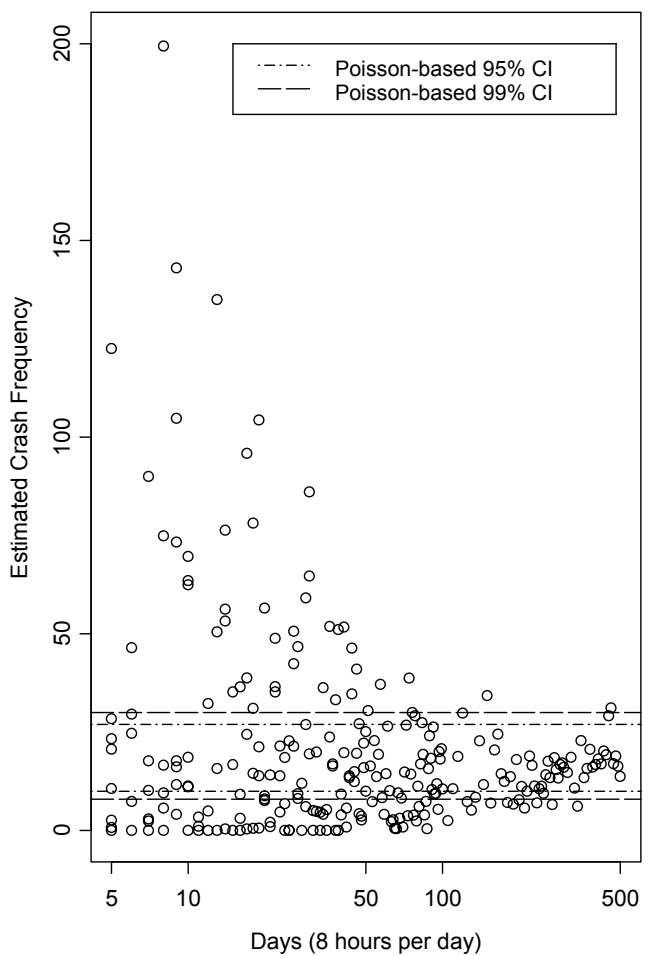

Figure 8-12: Effect of Block Length on Crash Frequency Estimates - Site 97901

Site 87906, RL Models: r=1 (GEV), 30-minute blocks

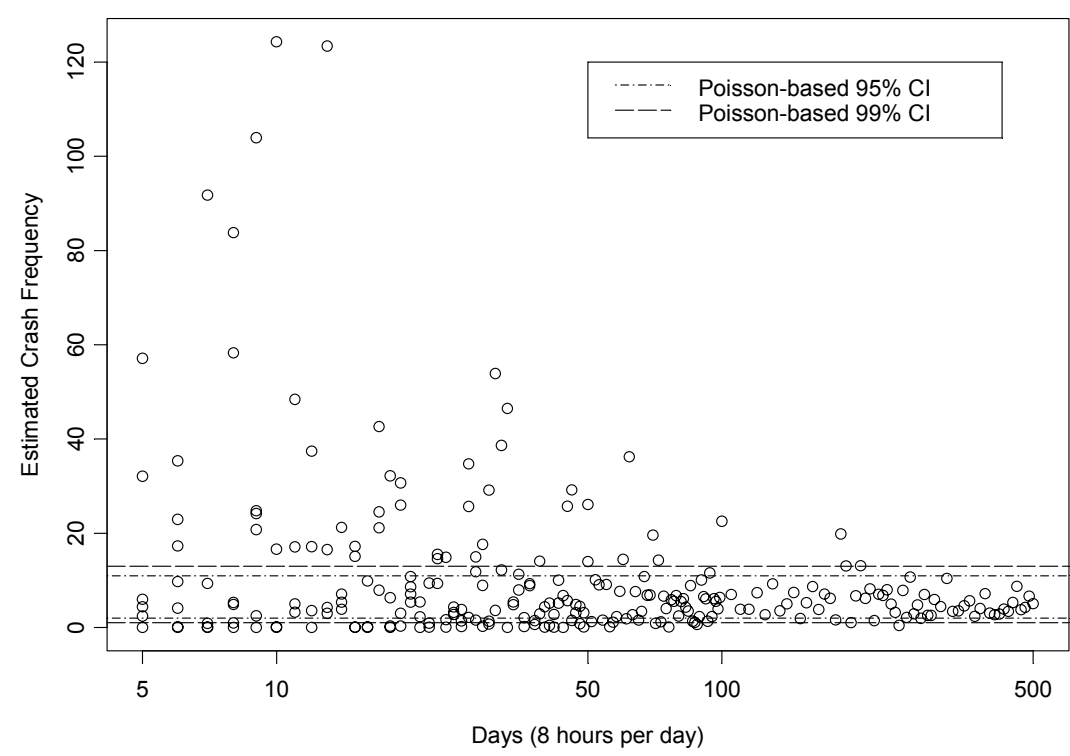

Figure 8-13: Estimated Crash Frequency at Site 87906 Using $r=1$ and 30-minute Blocks 
This has led us to the suggestion that, for a long observation period, a suitable choice of block length should depend on the average number of PETs per block. It is observed that an average of six per block is inadequate while an average of 18 per block is sufficient. To find the optimal average number of PETs per block, we ran the simulation test again for site 87906 at 30-minute blocks to examine if the model bias can be corrected. The 30-minute block length at site 87906 gives an average of 12 PETs per block. The crash frequency estimates are plotted correspondingly in Figure 8-13.

It can be seen from the plot that the bias problem at site 87906 is eliminated at a 30 -minute block length. This is equivalent to an average of 12 PETs per block. Based on the simulation experiment on the effects of block length, general guidelines for a selection of block length can be given as follows:

1. For a short-term observation period (one to five days), a small block length (usually 15 minutes) is preferred in favor of better confidence of model estimates and algorithm convergence. To illustrate, poor algorithm convergence is inevitable when only eight extreme values are observed in one day using a one-hour block and a model has at least three parameters to be estimated.

2. For a long-term observation period (longer than five days), a suitable block length can be selected based on the average number of PET observations per block. To ensure that the asymptotic assumption is satisfied, the average number of PET observations per block should be at least 10. The selection among typical block lengths of 15 minutes, 30 minutes, and one hour are likely to be sufficient in most cases.

In reality, the true measure of safety is unknown and thus it is not possible to check if the safety estimates of the extreme value models are biased by comparison. However, we can rely on the model diagnostic plots to determine a possible violation of the asymptotic assumption, which may signify a biased estimate. We illustrate this diagnostic check with site 87906 where a 15 -minute block length appears to give a biased estimate. Let us assume that the PET data are collected for 10 days and then fitted to GEV models using a 15-minute block length versus a one-hour block length. The diagnostic plots of both cases are shown in Figure 8-14 and Figure 8-15 respectively. 
Probability Plot

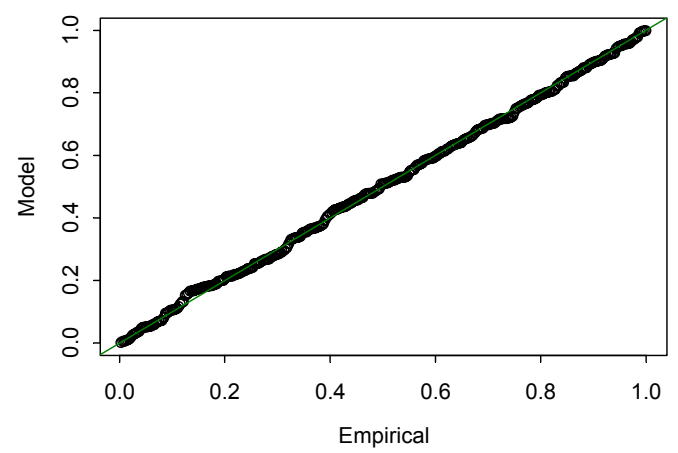

Return Level Plot

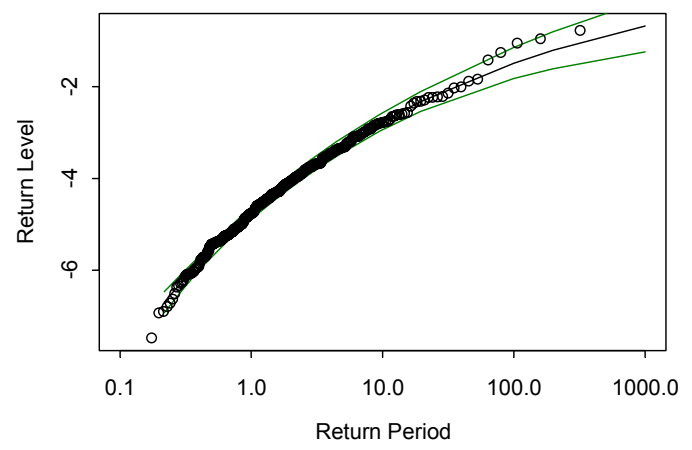

Quantile Plot

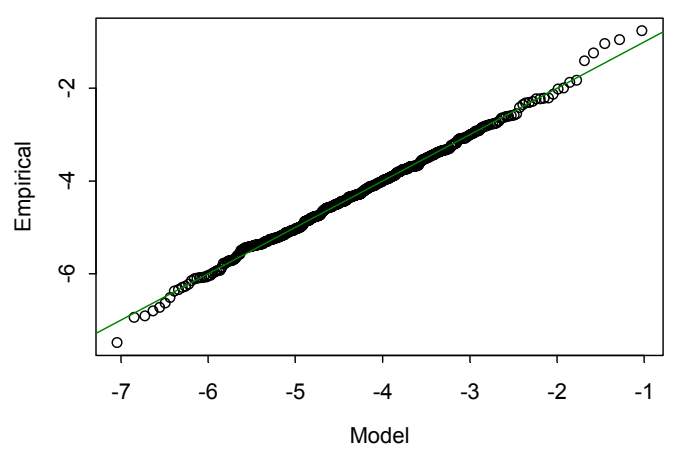

Density Plot

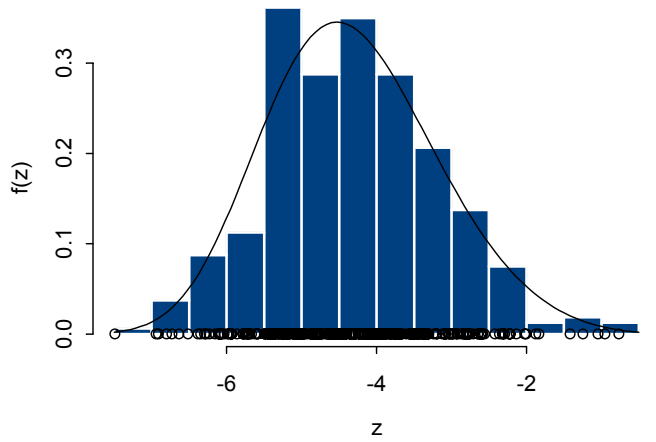

Figure 8-14: Diagnostic Plots of Model Fitted to 10 Days of Simulated PET at Site 87906 (block length $=15$ minutes and $r=1$ )

The quantile and return level plots in Figure 8-14 show a potential violation of the asymptotic assumption as indicated by a few data points at the extreme ends of both plots. On the other hand, at a 10-day observation period, no apparent violation is observed for a one-hour block length in Figure 8-15. Hence, it is possible to point out a potential model bias in practice even if the true safety level is unknown, provided the data from a long-term observation are available. Nevertheless, this is not the case for a short-term observation because the sources of imperfect diagnostic plots are often compounded between an asymptotic bias and a large variance of parameter estimates. 
Probability Plot

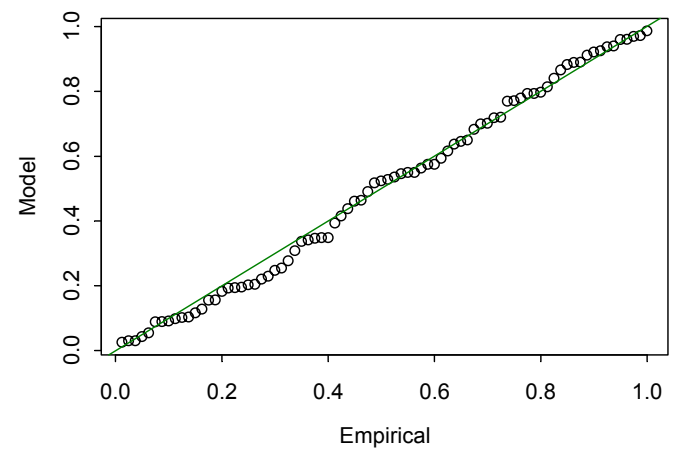

Return Level Plot

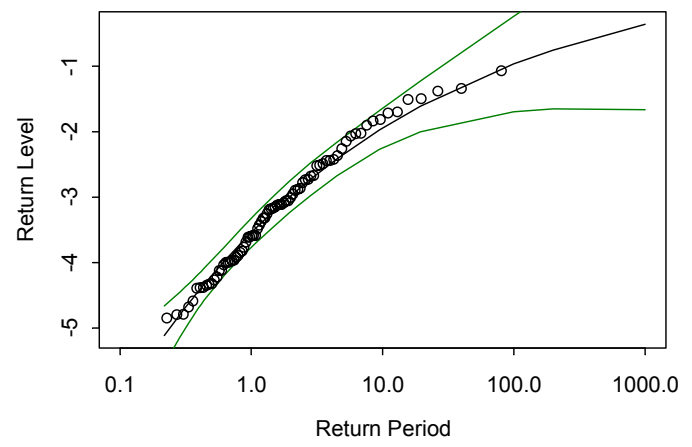

Quantile Plot

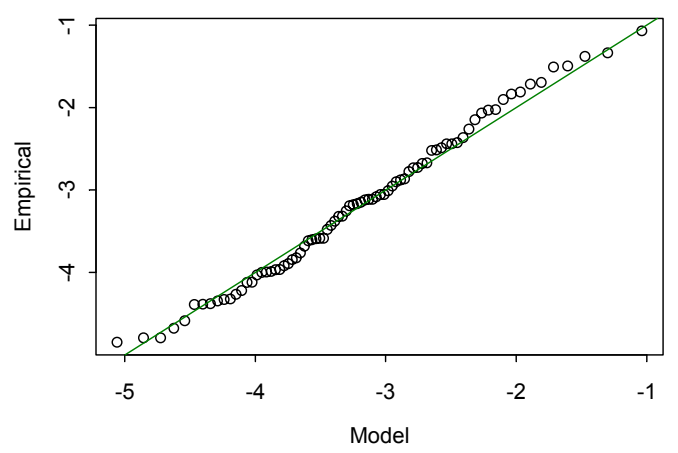

Density Plot

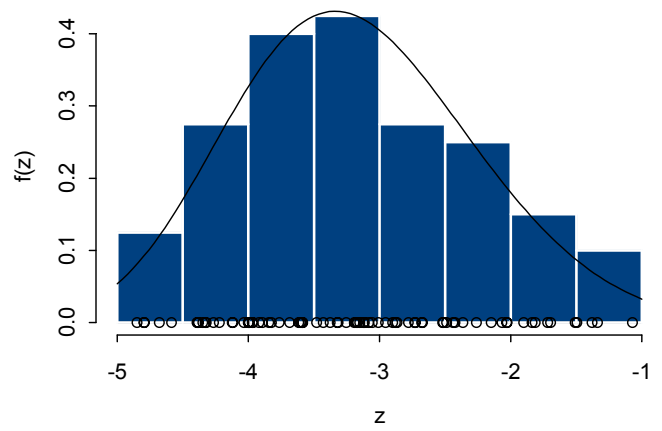

Figure 8-15: Diagnostic Plots of Model Fitted to 10 Days of Simulated PET at Site 87906 (block length $=1$ hour and $r=1$ )

\subsubsection{Effects of Number of Block Maxima}

The proposed $\mathrm{r}$ largest order statistic models for PET extremes is a generalization of the GEV distribution such that the model can incorporate more than just one block maximum in the estimation. The choice of $r$, or the number of block maxima of negated PETs, depends on the data set. For the field data, we selected $r$ based on the number of PETs observed during the eight-hour period. The selected $r$ ranges from 1 to 3 . In this simulation study, $r$ is set at 2 and 3 for 87906 and 97901 respectively. Theoretically, a larger $r$, or using more extremes per block, is expected to increase the confidence of the model estimates. However, we do not know the degree to which the confidence of estimates is improved. Therefore, this simulation study can help reveal the effect of $r$ selection on this particular aspect. 
In doing so, we compare the plots of crash frequency estimates using $r=1$ (equivalent to GEV distribution) versus $r=2$ at site 87906. The comparison plots are presented in Figure 8-16 and Figure 8-17. The first figure shows the crash frequency estimates obtained from one to 10 days of simulated PETs while the second figure displays estimates obtained from 10 to 100 days. We separate the plots for the same site into two figures because the range of crash frequency estimates is too wide to appropriately represent them all on the same scale.
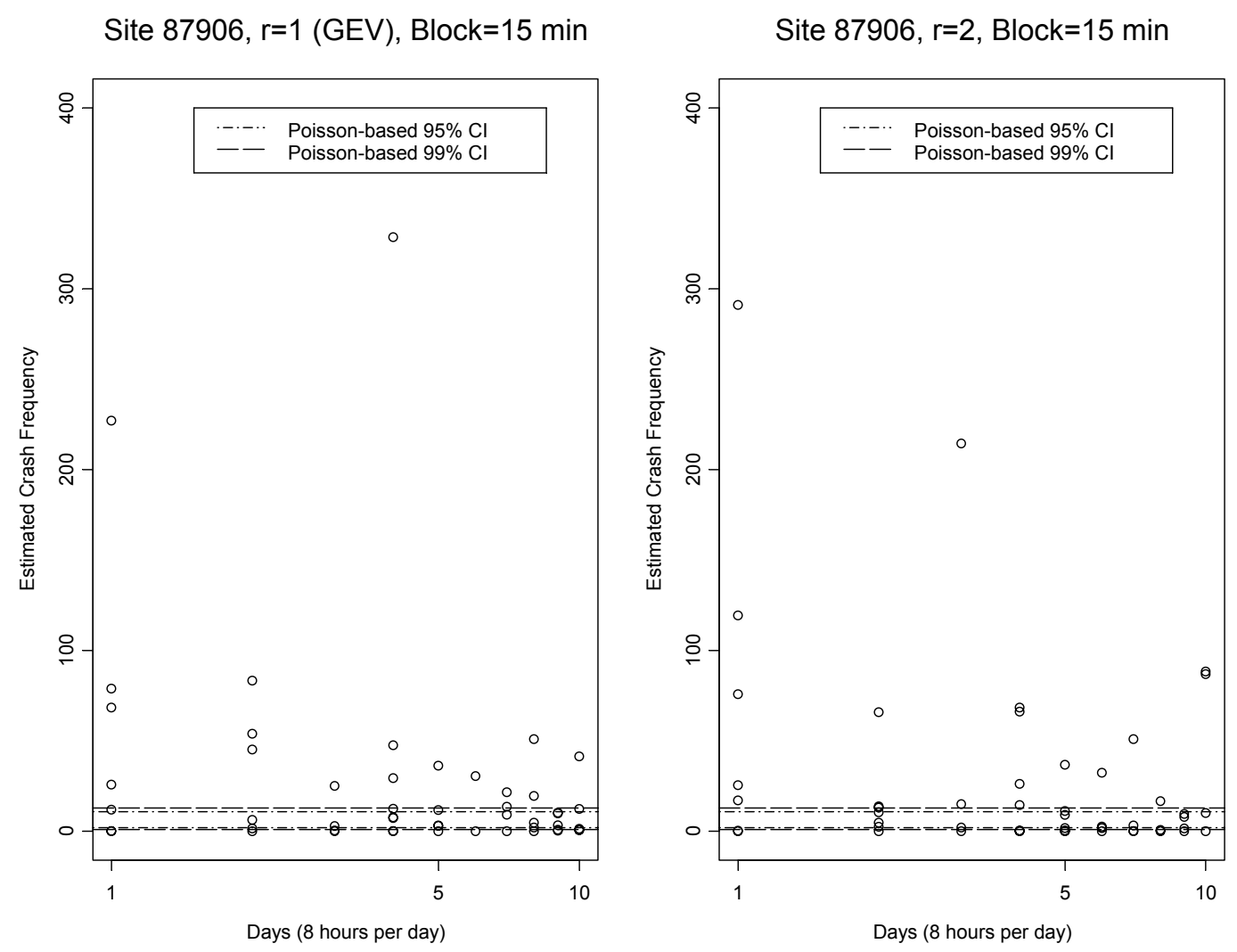

\section{Figure 8-16: Effect of Choice of r on Crash Frequency Estimates - Site 87906 (Observation $=1$ to 10 days)}

From both figures at site 87906 , the improvement in the confidence of the estimates is marginal for the range of one to 10 days and is more noticeable for the range of 10 to 100 days. This is not unexpected because site 87906 has a low average number of PET per 15-minute block, which means that there is a good chance that some blocks may have just one PET occurrence. Therefore, the setting of $r=2$ only slightly increases the sample size of PET extremes for one to 10 days of observation, which is reflected through marginal improvement of the confidence of the estimates in this case. 

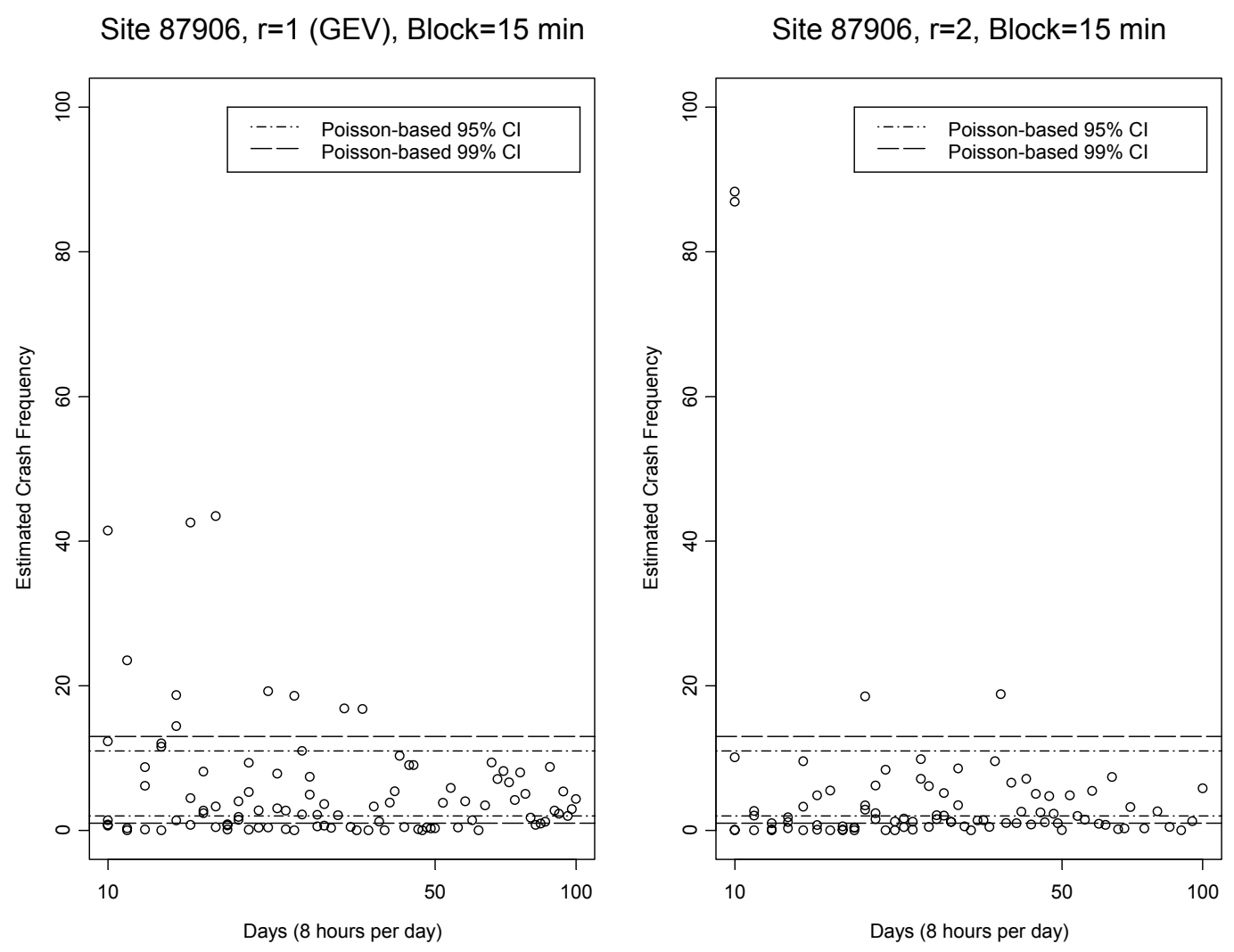

\section{Figure 8-17: Effect of Choice of r on Crash Frequency Estimates - Site 87906 (Observation $=10$ to 100 days)}

Similar comparison is carried out for site 97901 as shown in Figure 8-18 and Figure 8-19. For this site, we compare $r=1$ versus $r=3$ since the number of observed PETs on average is much higher than those of site 87906 .

It can be seen in Figure 8-18 that the confidence of the crash frequency estimates is apparently better for $r=3$ where the observation period is short. The fitted models can seriously overestimate the crash frequency at $r=1$ when only one or two days of observation is available. However, when the observation period is longer, improvement of the confidence of the estimates through the increase in $r$ is very marginal (see Figure 8-19). 

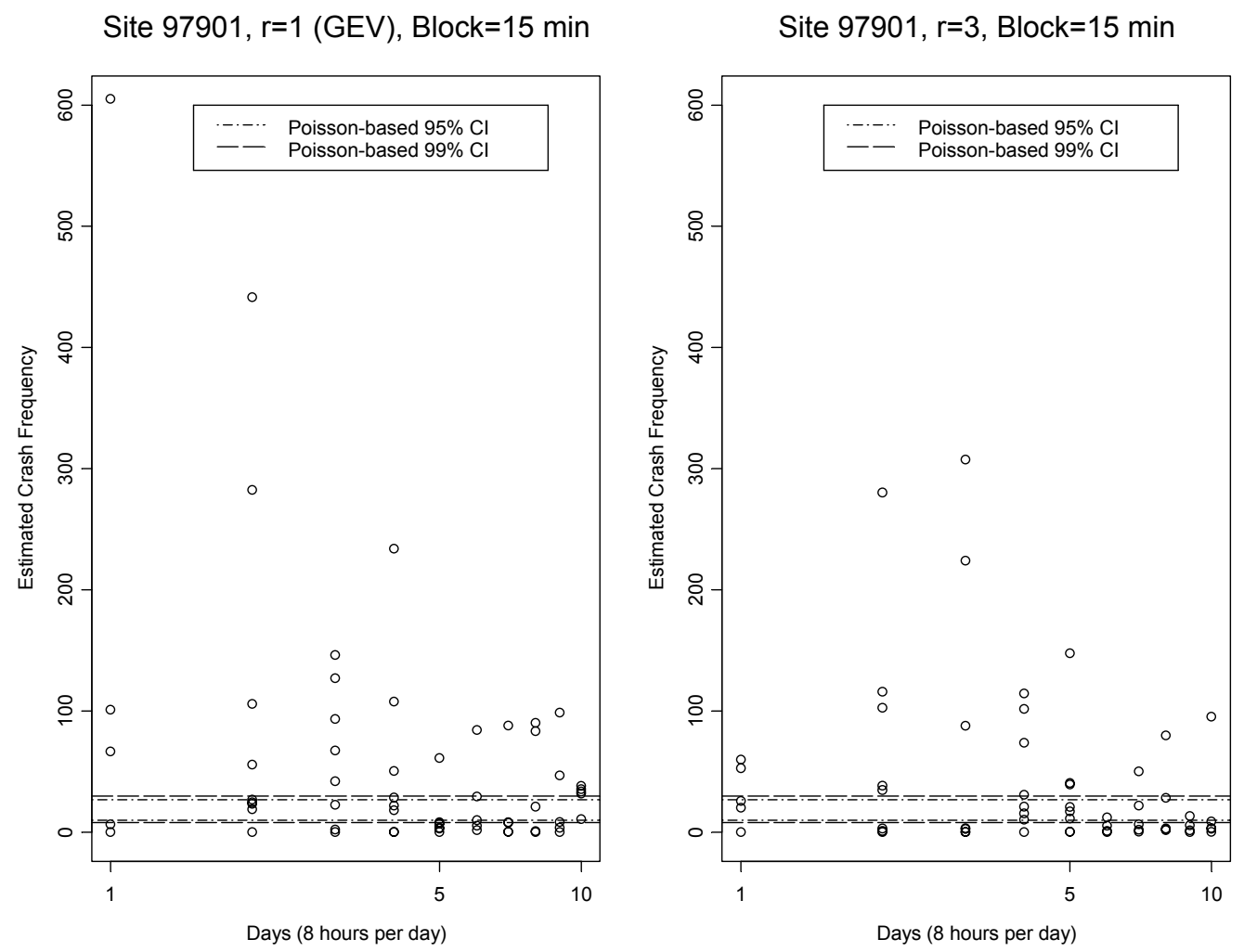

\section{Figure 8-18: Effect of Choice of r on Crash Frequency Estimates - Site 97901 (Observation $=1$ to 10 Days)}

From the examination of simulated results at both sites, the effect of the number of extremes selected per block on the confidence of the model estimates can be summarized as follows:

- For a short observation period (less than five days), a r value should be selected as large as possible subject to model adequacy. The benefits of a large $r$ value for a short observation period are twofold: (a) to ensure the convergence of maximum likelihood estimation and (b) to increase the confidence of model estimates.

- For a long observation period (five days or more), the benefit of increased confidence of the estimates is no longer substantial and the model convergence is no longer an issue. Therefore, there is no reason to favor a large $r$ value in this case. The proposed $r$ largest order statistic model can be reduced to the GEV model $(r=1)$ when the sample size is sufficiently large.

- From a computational aspect, the GEV model is also preferred to the $r$ largest order statistic model for a large sample size. The computational time for the $r$ largest order 
statistic model seems to increase exponentially with the sample size of PET extremes. To illustrate, the execution time of the case of $r=3$ for site 97901 takes about six hours for one day to 100 days of simulated PETs while this same data set will take merely 30 minutes with the GEV models.
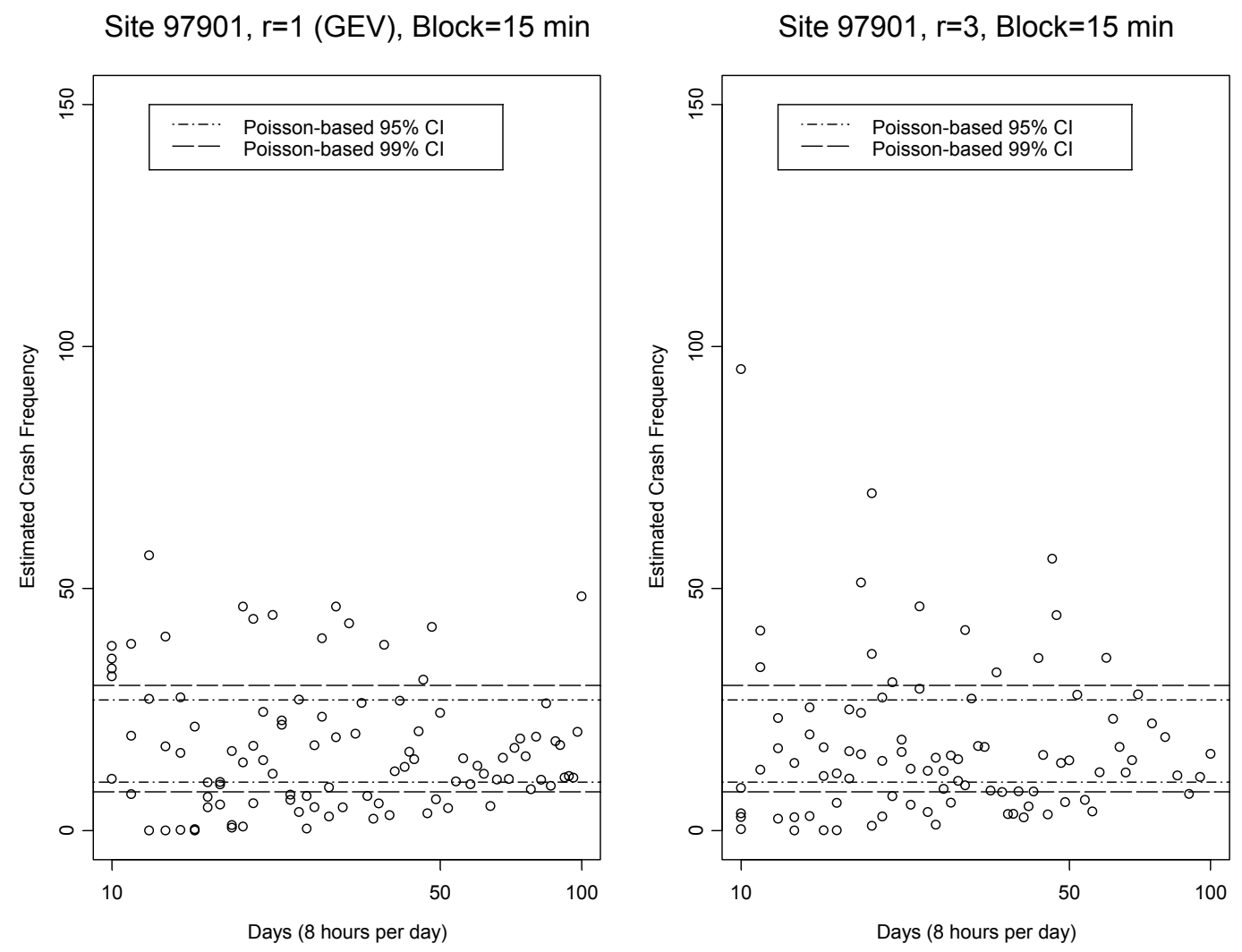

\section{Figure 8-19: Effect of Choice of r on Crash Frequency Estimates - Site 97901 (Observation $=10$ to 100 days)}

\subsubsection{Optimal Observation Period}

The last issue to be examined is the optimal observation period. Let us first consider the return level estimates of negated PETs in Figure 8-20. We use $r=1$ or GEV models as a basis for comparison since we have shown earlier that there is no significant gain in terms of confidence of the estimates from large $r$ values once the number of observation days is sufficiently large (five days or more). 
The horizontal line indicates a zero value, which is equivalent to the crash occurrence level. A higher return level implies a greater risk of right-angle collisions. There is no direct relationship between crash frequency and return level estimates. However, from the results in Section 8.1.2, we found that the intersections with positive return level estimates are more likely to experience high counts of right-angle crashes. Therefore, by means of a return level estimate, the suitable observation period is defined as the length of observation that allows us to confidently confirm whether the extreme return level of negated PETs is positive. As a result, the examination of Figure 8-20 indicates the following:

- For a high-crash-count location such as site 97901, we can confidently assure that the return level estimate is positive at 15 days of observation or more.

- For a moderate-crash-count location such as site 87906, we can also assert with a high degree of confidence that the return level estimate is positive at 15 days of observation or longer.
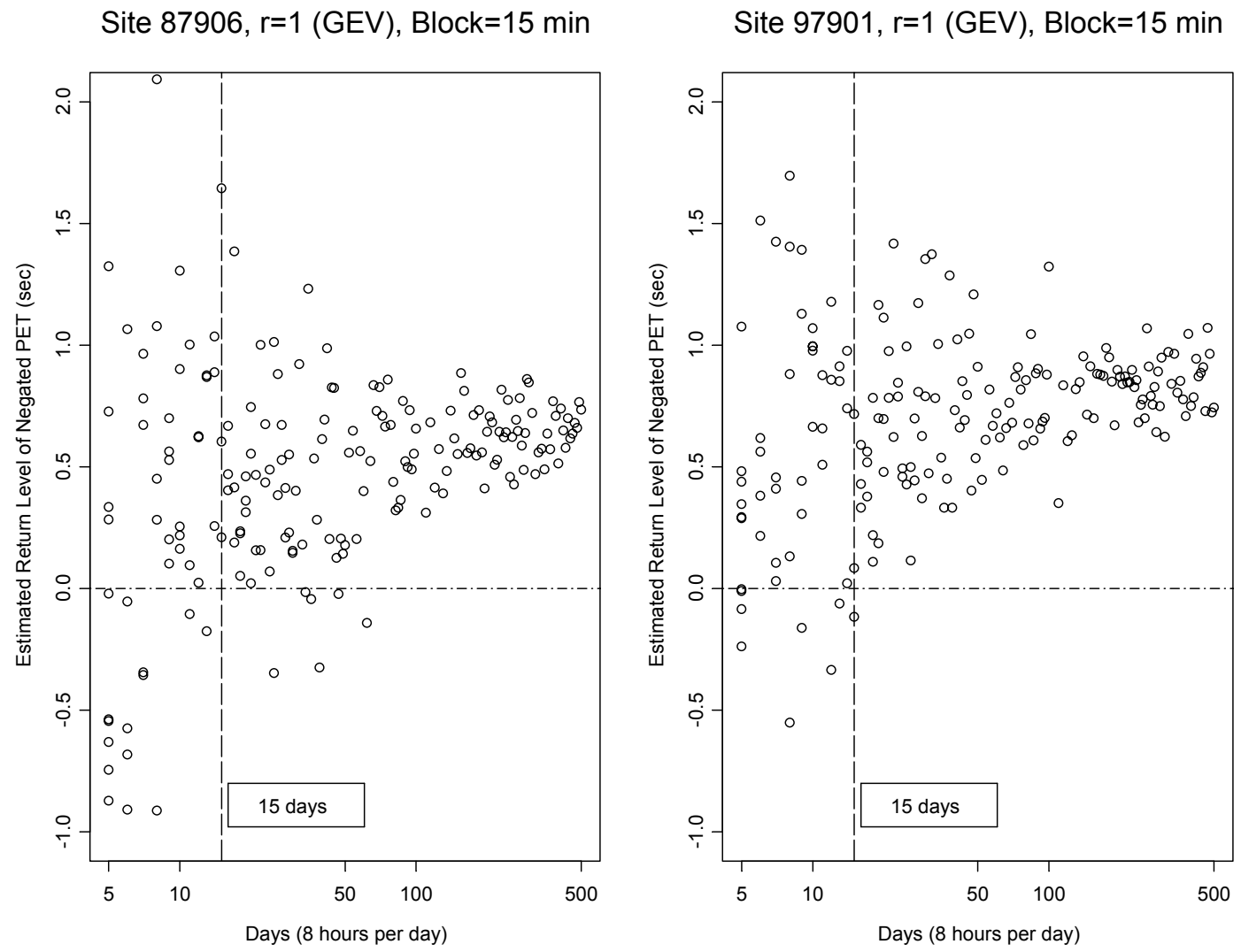

Figure 8-20: Comparison of Return Level Estimates 
This basically implies that by the means of return level estimates with a minimum of 15 days of PET observation, we can tell whether the intersection warrants a comprehensive safety evaluation program without the need for historical crash data. If the return level estimate based on 15 days of observed PET is positive, the intersection is justified for further evaluation due to the excessive risk of right-angle collisions. The negative estimate, however, may not immediately confirm that the intersection is safe but it is very likely that the risk of right-angle collisions at that intersection is below average.

Once we can affirm that the intersection is at excessive risk for right-angle collisions, the next question is how long the observation period has to be in order to predict the crash frequency with a satisfactory level of confidence. The most natural benchmark for a comparison of confidence levels is the crash-based Poisson confidence intervals. The GEV models with 15-minute and 30minute block lengths are estimated for sites 97901 and 87906 respectively. The comparison of crash frequency estimates with corresponding crash-based Poisson confidence intervals is presented in Figure 8-21.
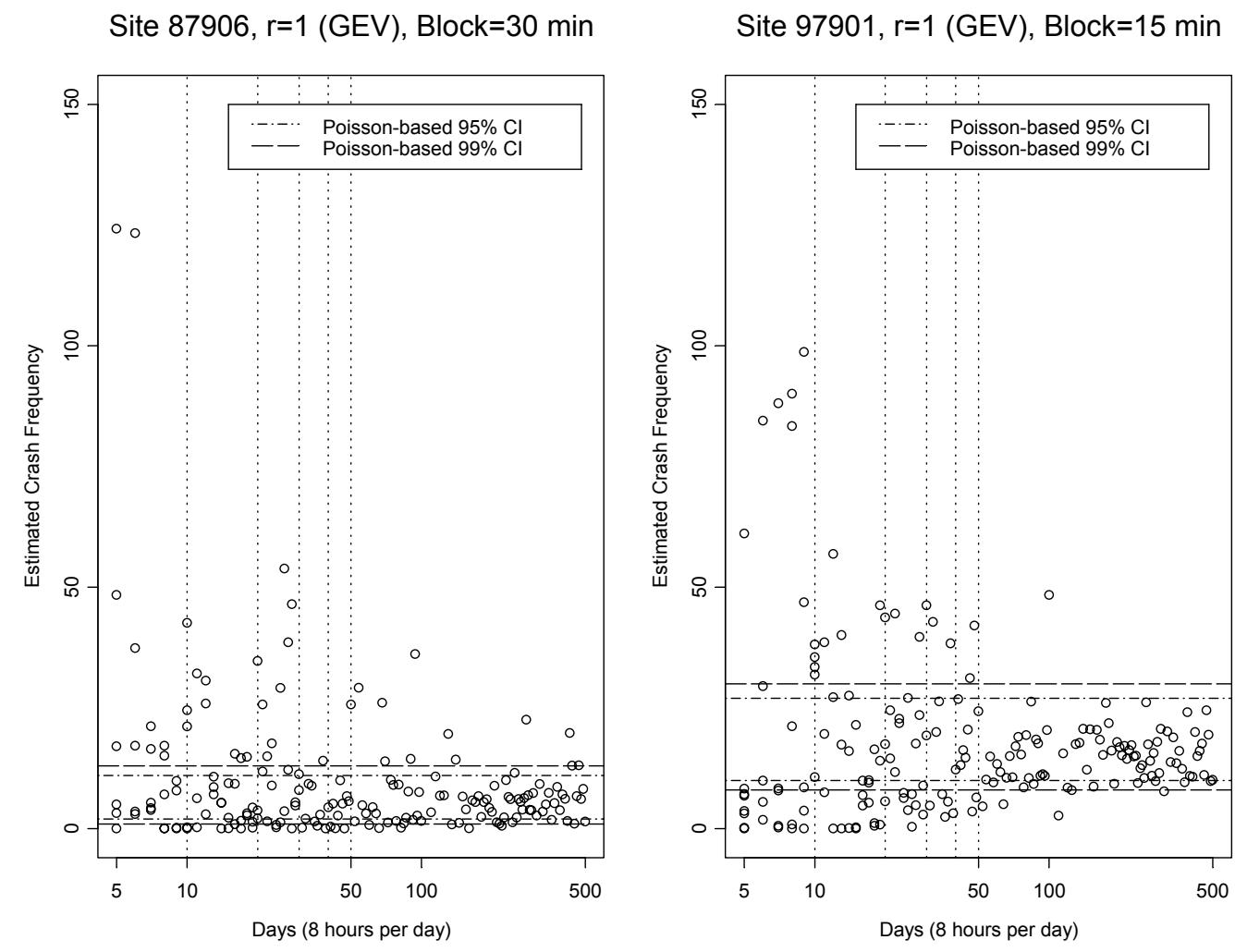

Figure 8-21: Comparison of Crash Frequency Estimates 
From the plots of both sites, we can observe that the estimates converge rapidly from five days to 50 days and then continue to converge gradually thereafter. Therefore, the recommended observation period in order to predict the right-angle crash frequency with a level of confidence close to what we can obtain with four years of crash data is approximately 50 days. There is no substantial gain in terms of the confidence of the estimates once the observation period has reached 50 days. In fact, a shorter observation period, such as 30 days, appears to be justified for a moderate-crash-count location such as site 87906. Therefore, in practice, the first target for the observation period can be set at 30 days; then if situations warrant such, increase the observation period to 50 days. For example, the observation period should be increased to 50 days if the prediction of crash frequency is still off the mark using a 30-day period, i.e., unrealistically high or unusually low. 


\section{CHAPTER 9 CLOSURE}

A summary of the research findings, conclusions, and recommendations for future research are presented in this final chapter. In addition, we also provide a simple method for an evaluation of the risk of right-angle collisions at signalized intersections in Appendix B.

\subsection{RESEARCH SUMMARY}

We studied the traffic crossing events leading to right-angle collisions at signalized intersections. We measured the post-encroachment time (PET) and the encroachment time (ET) at 18 selected intersections for eight hours each. The PET is defined as the time between the moment the last vehicle leaves a conflict spot and the moment the first conflicting vehicle enters a conflict spot. The PET is a proposed surrogate measure of safety to evaluate the risk of right-angle collisions caused by two crossing straight vehicles. A shorter PET value implies a greater risk of collisions. Under the postulate of a continuum of events, a collision occurs when the PET takes a value of zero or less.

Different PET measurement techniques were tested, including the automated method, the semiautomated method, and the manual method. The automated and semi-automated methods used the Autoscope ${ }^{\circledR}$ to help detect and measure the events. However, the methods still produced significant rates of false and missed detections; therefore, the manual method was chosen for all subsequent measurement of PETs. 15-minute directional traffic volume counts were also collected during the eight-hour observation period for all selected sites using Jamar ${ }^{\circledR}$ counters.

The validity of PET as a safety indicator was first examined using Poisson and negative binomial regression methods. Using observed crash counts as a response variable, three categories of regression models were calibrated: (a) PET-based models, (b) volume-based models, and (c) combined models. The models were estimated for two different levels in each category: (a) site level and (b) conflict zone level. In the regression analyses, the counts of short PETs and the 
products of crossing flows were found to have the most explanatory power in explaining the variability of observed right-angle crash counts.

In addition, the regression results revealed that a PET is capable of discriminating varying safety levels within a location (conflict zone models) as well as across locations (site models). A PET carries safety information unexplained by traffic volume alone. The implication is that a PET can either supplement or complement the traffic volume data in a safety evaluation. There is statistical evidence of benefits from having both PET data and traffic volume data available.

We propose the extreme value theory approach to model a behavior of post-encroachment times. Note that negated PET values are modeled instead of actual PET values for the sake of mathematical convenience. There are two alternative definitions of PET extremes: (a) time-based, which is the largest negated PET observed in an interval, and (b) event-based, which is the negated PET exceeding a pre-specified threshold.

The first approach was chosen for the analysis for the following reasons: (a) the possible dependence in a series of extreme values is automatically handled in the parameter estimation; (b) the point process characterization of event-based PET extremes, on the other hand, requires a declustering scheme which remains quite subjective; and (c) a threshold specification for eventbased extremes is quite arbitrary, neither a mean residual life plot nor an assessment of parameter stability can completely eliminate subjectivity in determining appropriate thresholds.

Time-based negated PET extremes were fitted to a generalization of a block maximum distribution (GEV) as the non-stationary $r$ largest order statistic model. The $r$ largest order statistic model enables us to incorporate more than one extreme from each time interval in order to increase the confidence of parameter estimates. The selection of the $r$ value was based on eight-hour counts of short PETs. The PET can be classified into two categories: (a) those associated with normal crossing events and (b) those associated with queue spillbacks. The encroachment time (ET) was used to help discriminate these two catogories. A coefficient of variation of the ET helped determine intersections that had excessive numbers of short PETs from queue spillbacks.

The non-stationarity of PET process was substantially explained by the traffic volume and the frequency of PET occurrences. These non-stationary covariates were incorporated into the location parameter of the model using the identity link. There were 12 intersections with a 
sufficient number of PET extremes for model estimations and eight of them required only the frequency of PET occurrences as a sole covariate. For the remaining four intersections, the addition of traffic volume improved the goodness-of-fit of the models at the $95 \%$ confidence level.

The risk of collision can be derived from the estimated extreme value models and then converted to the crash frequency estimate. The risk of right-angle collision during any time interval is the probability of a negated PET being greater than zero. Since the risk is the expected frequency of collisions during a short interval, summation of the risk over a long period $\mathrm{T}$ gives the expected number of collisions during period $\mathrm{T}$.

The second safety estimate, not as straightforward as the risk of collision, is the extreme return level. The extreme return level is the maximum negated PET level, which is expected to be exceeded once every return period $1 / \mathrm{p}$. The higher return level of negated PETs implies a greater hazard of right-angle collisions. There is a positive correlation between the return level and the risk (crash frequency) estimates; however, these two quantities are neither convertible nor interchangeable. The advantages of the risk estimates are: (a) intuitive and simple to understand, (b) convertible to crash frequency, and (c) easy to validate with observed crash counts by direct comparison. However, the risk and the crash frequency estimate are a non-negative quantity which may tend to cluster near zero. For this reason, the crash frequency estimate may not well discriminate a spectrum of safety levels among a group of low-risk intersections. The extreme return level, on the contrary, is a better behaving estimate. However, the concerns with the use of extreme return level are: (a) it is not convertible to crash frequency; (b) validity check by direct comparison is not possible; (c) the estimation procedure is computationally cumbersome; and (d) the interpretation is less intuitive than the risk and crash frequency estimates.

The modeling of PET behavior using the extreme value statistics relaxes several assumptions imposed in the traditional regression analysis. The immediate advantages of the extreme value approach can be summarized as follows:

- The risk is estimated for each location separately. We know that there are no two exactly identical intersections. The extreme value approach no longer requires the assumption of the constant risk across a group of similar locations in order to apply the results 
elsewhere. The extreme value approach estimates the risk based upon the behavior of the PET extremes observed during specific conditions in time and space.

- The calibration of the extreme value models does not require historical crash data. In the traditional approach, the calibration of regression models of surrogate measures such as conflict counts requires historical crash counts. In the extreme value approach, we estimate the crash frequency based on the mechanism of PET occurrences where the collision is defined by zero PET or less.

- The extreme value modeling of PETs is a proactive safety evaluation approach in that we no longer need to wait for crashes to take place before we can confirm the safety level of an intersection. This is particularly true for a new or recently modernized intersection with no historical crash data available.

- The extreme value approach enables a simultaneous estimation of risk and exposure for a particular intersection. The exposure is the number of time intervals having conditions similar to the period of observation. In comparison, a traffic conflict study is a two-step procedure in that conflict counts for individual locations are being treated as exposure while the risk is subsequently calibrated for a group of studied locations. This estimated risk is then assumed to be invariant for similar locations for the result to be applicable elsewhere.

In the method validation, we compared the safety estimates obtained from the best fitted extreme value models with the observed crash counts. Crash frequency estimates were examined by both direct comparison and correlation check. The validity of the return level estimates can only be confirmed by correlation.

The observed right-angle crash counts at intersections are widely accepted as the objective measure of safety. The validation results indicate the existence of a relationship between both types of safety estimates and observed crash counts. Poisson confidence intervals based on observed crash counts were established to account for the variability of crash-based safety estimates. The confidence intervals of crash frequency estimates are still very large however. For an eight-hour observation period, the crash-based Poisson confidence intervals would be a subset of the PET-based confidence intervals at most locations. However, there is evidence of a possible relationship between model-based estimates and observed crash counts as follows: 
- The extreme value approach yields a crash frequency estimate in a reasonable range. Similar unpublished attempts in the past had discouraging results.

- The Pearson correlation between crash frequency estimates and observed crash counts is very high (0.931) without intersection 97903 where the estimate was unreasonably large. A similar trend was also observed between the return level estimates and observed crash counts $($ Pearson correlation $=0.631)$.

- The expected crash frequency estimates fall within the $95 \%$ crash-based Poisson confidence intervals at eight out of 12 intersections. This figure increases to nine out of 12 intersections when the $99 \%$ Poisson confidence intervals are considered.

Three intersections that fall outside the Poisson confidence intervals are 97901, 97903, and 97905. Although this situation can be easily explained once the variability of the estimates is accounted for, a closer examination of the three intersections uncovers certain compelling findings as follows:

- Intersections 97901 and 97903 experienced a number of extremely short PETs caused by queue spillbacks. This may be the main cause of the overestimation of crash risk due to the violation of the assumption of a single underlying distribution of the PET process. PETs of normal crossing situations may have a different underlying distribution from PETs caused by a queue spillback.

- There was a heavy rain during the evening peak at intersection 97905. The longer PETs observed during this peak hour contradicts with the observed increasing trend of traffic volume. This may indicate the driver risk compensation behavior during perceived difficult driving conditions.

Safety estimates derived from the extreme value models based on a short observation period still have a large variance and it is therefore difficult to check for the presence of bias in the estimates. To study the variance of the estimates, we conducted a semi-empirical simulation experiment to examine impacts of model settings.

While the PET data in the field represent the frequency of observed crossing behaviors, the observed crash counts represent the extreme portion of the process, which is rarely observable within a reasonable amount of time. From the postulate of event continuum, we can estimate the distribution that applied to the entire spectrum of PETs observable in the field, as well as the 
crash frequency. The maximum likelihood method subject to the constraint on the conditional probability of right-angle collisions was used to fit the generalized Pareto distribution to the collected PET data. The fitted generalized Pareto distribution was used to generate PET values in the simulation study. The findings from the simulation results can be summarized as follows:

- A too short time interval (block length) can yield biased estimates which can be eliminated only at the expense of a large variance of estimates.

- For a short observation period (one to five days), a small block length (e.g., 15 minutes) is preferred in order to decrease the variance of estimates and ensure the convergence of maximum likelihood estimation.

- For an observation period longer than five days, a suitable block length should have at least 10 observed PETs (that are less than eight seconds) on average to avoid violation of the asymptotic assumption. Typical time intervals of 15 minutes, 30 minutes, and one hour are likely to be sufficient for most intersections.

- For a short observation period, the $r$ value in the $r$ largest order statistic model should be selected as large as possible subject to model adequacy to ensure the convergence of parameter estimation and to increase the confidence of model estimates.

- For a long observation period (five days or more), the increase in confidence of the estimates is no longer substantial and the convergence of parameter estimation is no longer an issue. The $r$ largest order statistic model can be reduced to the GEV model or equivalently $r=1$.

- From a computational aspect, the GEV model is also preferred to the $r$ largest order statistic model for a large sample size. Computational time for the $r$ largest order statistic model seems to increase exponentially with a sample size of PET extremes.

- Based on the return level estimate, we can confirm whether the risk of right-angle collisions at a location warrants further safety evaluation once the observation period has reached 15 days.

- An observation period of 50 days is needed to have confidence intervals of PET-based crash frequency estimates as tight as crash-based confidence intervals (four years). There is no substantial gain in the confidence thereafter. The implication is that we can cut down the waiting time of four years in the crash-based safety evaluation to only 50 days in the PET-based method. This is equivalent to a time-saving ratio of approximately 1:30. The required observation period may be shortened if collisions are more frequent. This 
could be a location with frequent right-angle crashes or other types of collision that are more regular than right-angle crashes. For example, the required observation period at intersection 97901 may be shortened from four years of crash data to 30 days of PET observation, or equivalently, the corresponding time-saving ratio of about 1:50.

Although not directly related, this optimal period offers some insight into the reason why a oneday or even a one-week conflict count program of the widely-used traffic conflict technique yielded mixed findings in the past in terms of its relationship with observed crash counts. As illustrated in the simulation study, the extreme nature of PETs, which is also applicable to traffic conflicts, requires a sufficiently long period of data collection in order to have unbiased and lowvariance safety estimates.

The current limitations of the proposed extreme value approach for modeling PET extremes can be summarized as follows:

- The method tends to overestimate the expected crash frequency at intersections that have a number of short PETs caused by queue spillbacks.

- The method is very data-intensive. A short-term observation for a few days is unlikely to yield low-variance safety estimates. Weeks of continuous monitoring at a location are indispensable to obtaining desirable safety estimates. A manual measurement method, despite its accuracy and reliability, is impractical at the moment.

- Analysis of extreme value statistics is not a standard implementation in commonly-used statistical and econometric analysis software. The method proposed in this research was implemented in S language which can be executed on S-Plus ${ }^{\circledR}$, a specialized statistical computing platform. The current version of the implementation may not be readily accessible for highway safety practitioners.

\subsection{CONCLUSIONS}

A risk of collision can be estimated using traffic observables during crash-free operations. Observed traffic characteristics must satistify the postulate of event continuum; in other words, the selected characteristic must be continuous and take values during crash-free operations as well as crash occurrences. An extreme value theory approach is well suited for safety estimation using traffic observables. 
In this research, the PET between through-crossing traffic is a valid measure of the risk of rightangle collisions. A risk of right-angle collisions can be estimated using PETs observed at invidual locations for a specific condition. A series of negated PETs should be discretized into fixed time intervals, and the block maxima are known to follow the generalized extreme value (GEV) distribution. This extreme value modeling approach is preferred to the threshold excess models since the dependence of extremes is automatically handled in the maximum likelihood estimation and no arbitrary threshold specification is required.

A GEV model may yield large-variance and biased estimates and the following issues therefore must be taken into account in the modeling of PET extremes:

- Bias-variance trade-off must be considered in choosing a suitable block length.

- The $\mathrm{r}$ largest order statistic model is preferred when only a few days of PET observations are available.

- Non-stationarity in a series of observations must be addressed if the conditions during the observation period are changing. For example, a temporal variation of traffic volume is a potential covariate in the link structure of the location parameter of the model.

Our proposed risk estimation method is a novel idea in extreme value theory applications. A return level is a typical value of interest in applications of EVT. For example, a structural engineer may be interested in the expected maximum wind speed in the next 100 years, which corresponds to a 100-year return level of wind speed. In our application, we are interested in the area under the distribution curve where the observed value exceeds a fixed return level. This fixed return level defines a boundary between crash and non-crash events in our method and the risk of collision is defined as the area under the distribution where the negated PET is greater than zero. The estimated risk can be converted to the estimated crash frequency using exposure that is representative of conditions during PET observation.

Evaluation results of the proposed extreme value theory approach indicate the existence of a relationship between model estimates and crash data but more data are required to confirm this result due to a large variance of crash frequency estimates at present. A simulation study revealed that a large variance of estimates is primarily due to a short observation period. Several weeks to a few months of PET observation would be required to obtain a desirable confidence level of the estimates. Any systematic bias can be eliminated by increasing the length of time interval, 
provided that the sample size is sufficiently large. Otherwise, a model bias is likely to be obscured by uncertainty of the estimates.

The method proposed in this study is neither limited to only right-angle crashes nor a specific measure such as post-encroachment times. The proposed method can be applied to other traffic characteristics as well as other types of collision. To apply this method, the key considerations can be summarized into the following steps:

1. Traffic characteristics leading to the collision type of interest must be defined.

2. A valid traffic characteristic must be observable and possess a continuous characteristic that can represent varying risk levels during crash-free operations as well as characterize a collision at extremes.

3. A boundary between crash and non-crash events may need to be redefined, depending on the traffic characteristic being considered.

4. The proposed risk estimation method, using the $\mathrm{r}$ largest order statistic model, can be applied to extremes of the observed traffic characteristic. General model considerations still apply, which include a bias-variance trade-off in choosing the block length, a choice of $r$ value, and non-stationarity.

\subsection{FUTURE RESEARCH}

Several weeks to a few months of PET observation are required to obtain a desirable confidence level of the estimates. Cost and resources needed for a monitoring program using human observers would be prohibitive and thus impractical. A more efficient and cost-effective automated measurement method is definitely vital to overcome the implementation barrier. Despite the current increasing capability of the video image processing (VIP) technology, the present challenges of accurate and efficient measurement of a traffic characteristic such as PET using VIP include:

- Different lighting conditions over the course of a day

- Long shadows from trucks, nearby obstacles, and physical infrastructure in the vicinity

- Camera vibrations

- Varying vehicle travel paths

- Partial occlusions in the field of view 
- Lack of an efficient algorithm for real-time processing.

Taking into account the aforementioned difficulties, more efficient and accurate measurement technique may aim toward physical detection instead. A hardware-oriented system to detect the physical presence of vehicles around conflict spots may offer a more promising capability to achieve the desired accuracy and efficiency.

Once the appropriate automated data collection system is available, we can re-examine the extreme value approach based on a long-term observation period. Field data are still needed to support the simulation results in this study. Applications for other types of collisions and locations can be considered as well.

The current extreme value approach does not properly represent the extreme behavior of PETs at intersections with excessive queue spillbacks during congested traffic conditions. One possible hypothesis for this problem is the different underlying distributions of PETs from normal crossing situations versus queue spillbacks. The risks of collisions associated with these two situations could differ yet share the same PET value. Despite the fact that the extremal type theorem is independent of an underlying distribution, the mixture of underlying distributional assumptions may require a special treatment of extremes. Further research on the extreme value method for risk estimation could advance on the following aspects:

- An effective approach to discriminate different categories of PETs

- A method that allows a mixture of underlying distributional assumptions

Estimates of unsafety at a location can be improved by combining PET-based and crash-based safety information. Let us consider PET-based and crash-based safety information as two separate entities. We, in fact, do not know which measure can give a closer approximation of a true measure of safety. But, we do know that both measures contribute some information about the hazards of a location. A method to combine these two entities should be researched and validated once safety information obtained from a surrogate safety measure can be obtained at a satisfactory level of confidence. Empirical Bayesian combination is one possible alternative that may allow us to proceed from the Poisson confidence intervals based solely on observed crash counts to the narrower Poisson credible intervals in which the PET-based safety information is incorporated. 
In addition, the empirical PET observation reveals that changes in drivers' behavior during certain driving conditions may be reflected through the observed PETs. This may lead to an innovative approach to study a risk perception of road users under different driving conditions. This evaluation requires a data set that comprises PETs observed for a variety of intersection features and driving conditions. A classification analysis or a cluster analysis as described in Johnson and Wichern (2002), for example, may be an appropriate tool for the study. 


\section{LIST OF REFERENCES}

Abbas, M. M. and J. A. Bonneson (2003). "Detection Placement and Configuration Guidelines for Video Image Vehicle Detection Systems." Proceedings of $82^{\text {nd }}$ Annual Meeting (CD$R O M)$, TRB, National Research Council, January 12-16.

Abbess, C., D. Jarrett, and C. C. Wright (1981). "Accidents at Blackspots: Estimating the Effectiveness of Remedial Treatment, with Special Reference to the Regression-to-Mean Effect." Traffic Engineering and Control, Vol. 22, No. 10, pp. 535-542.

Abdel-Aty, M. A. and A. E. Radwan (2000). "Modeling Traffic Accident Occurrence and Involvement." Accident Analysis and Prevention, Vol. 32, No. 5, pp. 633-642.

Affum, J. K. and M. Ap Taylor (1996). "Predictive Models for Road Accidents at Signalised Intersection." IATSS Research, Vol. 20, No. 2.

Akcelik, R. (1994). "Estimation of Green Times and Cycle Time for Vehicle-Actuated Signals." Transportation Research Record 1457, TRB, National Research Council, Washington, D.C., pp. 63-72.

Allen, B. L., B. T. Shin, and P. J. Cooper (1978). "Analysis of Traffic Conflict Collisions." Transportation Research Record 667, TRB, National Research Council, Washington D.C., pp. 67-74.

Al-Masaeid, H. R. (1997). "Performance of Safety Evaluation Methods." Journal of Transportation Engineering, ASCE, Vol. 123, No. 5, pp. 364-369.

Al-Masaeid, H. R. and K. C. Sinha (1994). "Analysis of Accident Reduction Potentials of Pavement markings." Journal of Transportation Engineering, ASCE, Vol. 120, No. 5, pp. 723-736.

Amundsen, F. H. and C. Hyden (1977). Proceeding of the First Workshop on Traffic Conflicts, Institute of Transport Economics, Oslo/Lund Institute of Technology, Oslo, Norway.

Belanger, C. (1994). "Estimation of Safety of Four-Legged Unsignalized Intersections." Transportation Research Record 1467, TRB, National Research Council, Washington, D.C., pp. 23-29.

Benioff B., F. C. Dock, and C. Carson (1980). "A Study of Clearance Intervals, Flashing Operation and Left-Turn Phasing at Traffic Signals. Vol 2: Clearance Intervals." Report No. FHWA-RD-78-47, Federal Highway Administration, Washington, D.C., May. 
Casella, G. and R. L. Berger (2001). Statistical Inference. $2^{\text {nd }}$ Ed., Duxbury.

Chin, H. C. and M. A. Quddus (2003). "Applying the Random Effect Negative Binomial Model to Examine Traffic Accident Occurrence at Signalized Intersections." Accident Analysis and Prevention, Vol. 35, No. 2, pp. 253-259.

Chin, H. C., S. T. Quek, and R. L. Cheu (1991). "Traffic Conflicts in Expressway Merging." Journal of Transportation Engineering, ASCE, Vol. 117, No. 6, pp. 633-643.

Chin, H. C., S. T. Quek, and R. L. Cheu (1992). "Quantitative Examination of Traffic Conflicts." Transportation Research Record 1376, TRB, National Research Council, Washington D.C., pp. 67-74.

Chin, H. C. and S. T. Quek (1997). "Measurement of Traffic Conflicts." Safety Science, Vol. 26, No. 3, pp. 169-185.

Coifman, B., D. Beymer, P. McLauchlan, and J. Malik (1998). "A Real-Time Computer Vision System for Vehicle Tracking and Traffic Surveillance." Transportation Research Part C, Pergamon, pp. 271-288.

Coles, S. (2001). An Introduction to Statistical Modeling of Extreme Values. Springer-Verlag, London, UK.

Courage, K. G. and R. Akcelik (1994). "A Computational Framework for Modeling Traffic Actuated Controller Operations." Working Paper NCHRP 3-48-1, Transportation Research Center, University of Florida, Gainesville, May.

Dobson, A. J. (2002). An Introduction to Generalized Linear Models. $2^{\text {nd }}$ Ed., Chapman \& Hall/CRC.

Econolite Control Products, Inc. (2000). Product Descriptions, Data Sheets, Specification, and Application Notes for Autoscope ${ }^{\circledR}$ 2004LE Machine Vision Processor. http://www.econolite.com/product/autoscope/2004LE.htm.

Everitt, B. S. (2002). A Handbook of Statistical Analyses Using S-Plus. $2^{\text {nd }}$ Ed., Chapman \& Hall/CRC.

FHWA (1981). "Highway Safety Evaluation - Procedural Guide." FHWA Report, FHWA-TS-81219, Federal Highway Administration, November.

Galambos, J., J. Lechner, and E. Simiu (1994). "Extreme Value Theory and Applications." Proceedings of the Conference on Extreme Value Theory and Applications, Volume 3, NIST Special Publication 860, Gaithersburg, Maryland.

Garder, P. E., D. Lord, B. N. Persaud, and R. A. Retting (2001). "Safety Effect of Roundabout Conversions in the United States: Empirical Bayes Observational Before-After Study." Transportation Research Record 1751, Transportation Research Board, Washington D.C., pp. 1-8. 
Gettman, D. and L. Head (2003). "Surrogate Safety Measures from Traffic Simulation Models." Final Report FHWA-RD-03-050, Office of Safety Research and Development, FHWA, December.

Glauz, W. D., K. M. Bauer, and D. J. Migletz (1985). "Expected Traffic Conflict Rates and Their Use in Predicting Accidents." Transportation Research Record 1026, Transportation Research Board, National Research Council, Washington D. C., pp. 1-12.

Glauz, W. D. and D. J. Migletz (1980). "Application of Traffic Conflict Analysis at Intersections." NCHRP Report 219, Transportation Research Board, National Research Council, Washington D. C.

Glennon, J. C., W. D. Glauz, M. C. Sharp, and B. A. Thornson (1977). "Critique of Traffic Conflict Techniques." Transportation Research Record 630, Transportation Research Board, National Research Council, Washington D. C., pp. 32-38.

Grenard, J., A. P. Tarko, and D. Bullock (2001). "Evaluation of Selected Video Detection Systems." Report FHWA/IN/JTRP-2000/5, Purdue University, August.

Harlow, C. and Y. Wang (2001). “Automated Accident Detection System." Transportation Research Record 1746, TRB, National Research Council, Washington, D.C., pp. 90-93.

Hauer, E. (1982). "Traffic Conflicts and Exposure." Accident Analysis and Prevention, Vol. 14, No. 5, pp. 359-364.

Hauer, E. (1995). “On Exposure and Accident Rate." Traffic Engineering and Control, Vol. 36, No. 3, pp. 134-138.

Hauer, E. (1997). Observational Before-After Studies in Road Safety. $1^{\text {st }}$ Ed., Elsevier Science Ltd.

Hauer, E. and P. Garder (1986). "Research into the Validity of the Traffic Conflict Technique." Accident Analysis and Prevention, Vol. 18, No. 6, pp. 471-481.

Hauer, E. and B. N. Persaud (1983). "A Common Bias in Before and After Accident Comparisons and Its Elimination." Transportation Research Record 905, Transportation Research Board, Washington, D.C., pp. 164-174.

Hauer, E. and B. N. Persaud (1987). "How to Estimate the Safety of Rail-Highway Grade Crossings and the Safety Effect of Warning Devices." Transportation Research Record 1114, Transportation Research Board, Washington D.C., pp. 131-140.

Hauer, E., J. C. N. Ng, and J. Lovell (1989). "Estimation of Safety at Signalised Intersections." Transportation Research Record 1185, TRB, National Research Council, Washington, D.C., pp. 48-61.

Hayward, J. C. (1972). "Near Miss Determination through the Use of a Scale of Danger." Report TTSC 7115, The Pennsylvania State University. 
Horst, van der (1990). "A Time-Based Analysis of Road User Behavior at Intersections." Proceedings of Third Workshop of International Cooperation on Theories and Concepts in Traffic Safety, Cracow, Poland, pp. 91-107.

Huang, X. P. and P. D. Pant (1994). "Simulation-Neural Network Model for Evaluating Dilemma Zone Problems at High-Speed Signalized Intersections." Transportation Research Record 1456, TRB, National Research Council, Washington, D.C., pp. 34-42.

Hulscher, F. R. (1984). "The Problem of Stopping Drivers after the Termination of the Green Signal at Traffic Lights." Traffic Engineering and Control, pp. 110-116.

Hulscher, F. R. and A. G. Sims (1974). "Use of Vehicle Detectors for Traffic Control." Traffic Engineering and Control, Vol. 15, No. 19, pp. 866-869, November.

Insightful Corporation (2001). S-Plus 6 for Windows - Programmer's Guide. Insightful Corporation, Seattle, Washington.

Johnson, R. A. and D. W. Wichern (2002). Applied Multivariate Statistical Analysis. $5^{\text {th }}$ Ed., Prentice-Hall, Inc.

Kamijo, S., Y. Matsushita, K. Ikeuchi, and M. Sakauchi (2000). "Occlusion Robust Vehicle Tracking for Behavior Analysis Utilizing Spatio-Temporal Markov Random Field Model." Proceedings of IEEE Conference on Intelligent Transportation Systems, ITSC, Dearborn, MI, October 1-3, pp. 340-345.

Karlaftis, M. G. and I. Golias (2002). "Effects of Road Geometry and Traffic Volumes on Rural Roadway Accident Rates." Accident Analysis and Prevention, Vol. 34, No. 3, pp. 357365.

Katamine, N. M. and I. M. Hamarneh (1998). "Use of the Traffic Conflict Technique to Identify Hazardous Intersections." Road \& Transport Research, Vol. 7, No. 3, September, pp. 1735 .

Kloeden, C. N., A. J. McLean, V. M. Moore, and G. Ponte (1997). "Traveling Speed and the Risk of Crash Involvement." NHMRC Road Accident Research Unit, The University of Adelaide.

Koller, D., J. Weber, and J. Malik (1994). "Robust Multiple Car Tracking with Occlusion Reasoning." Technical Report UCB/CSD-93-780, October, 1993 and California PATH Working Paper UCB-ITS-PWP-94-01 (ISSN 1055-1417), January.

Leadbetter, M. R., G. Lindgren, and H. Rootzén (1983). Extremes and Related Properties of Random Sequences and Series. Springer Verlag, New York.

Lin, P.-S. and K. G. Courage (1996). "Phase Time Prediction for Traffic-Actuated Intersections." Transportation Research Record 1555, TRB, National Research Council, Washington, D.C., pp. 17-22.

Luttinen, R. T. (1992). "Statistical Properties of Vehicle Headways." Transportation Research Record 1365, TRB, National Research Council, Washington, D.C., pp. 92-98. 
Mahalel, D. and J. Prashker (1987). "A Behavioral Approach to Risk Estimation of Rear-End Collisions at Signalized Intersections." Transportation Research Record 1114, TRB, National Research Council, Washington, D.C., pp. 96-102.

Mahalel, D. and D. Zaidel (1986). "A Probabilistic Approach for Determining the Change Interval." Transportation Research Record 1069, TRB, National Research Council, Washington, D.C., pp. 39-45.

Mahalel, D., D. Zaidel, and T. Klein (1985). "Driver's Decision Process on Termination of the Green Light." Accident Analysis and Prevention, Vol. 17, No. 5, pp. 373-380.

Maher, M. J. and I. Summersgill (1996). "A Comprehensive Methodology for the Fitting of Predictive Accident Models." Accident Analysis and Prevention, Vol. 28, No. 3, pp. 281296.

Malik, J., S. Russell, J. Weber, T. Huang and D. Koller (1994). "A Machine Vision Based Surveillance System for California Roads.” Path Project MOU-83 Final Report.

Masaki, I. (1998). "Machine-Vision Systems for Intelligent Transportation Systems." IEEE Intelligent Systems, Vol. 13, No. 6, pp. 24-31.

May, A. D. (1990). Traffic Flow Fundamentals. Prentice-Hall, Inc., Englewood Cliffs, N.J., 1990.

Minderhoud, M. M. and P. H. L. Bovy (2001). "Extended Time-to-Collision Measures for Road Traffic Safety Assessment." Accident Analysis and Prevention, Vol. 33, No. 1, pp. 89-97.

Mizun, H., H. Yasaud, M. Itoh, S. Yamamoto, and S. Mori (2002). "Measurement of the Driver's Blinking and Estimation of Their Awareness Using the Image Processing." Proceedings of 9th World Congress on Intelligent Transport Systems, Chicago, Illinois, October 1417.

Nestor, Inc. (2001). "Traffic Monitoring and Detection." Nestor Traffic Systems, Inc., http://www.nestor.com/nts/trafficvision/tv_set2.htm.

Neter, J., M. H. Kutner, C. J. Nachtsheim, and W. Wasserman (1996). Applied Linear Statistical Models. $4^{\text {th }}$ Ed., McGraw-Hill.

Nicholson, A. J. (1985). "The Variability of Accident Counts." Accident Analysis and Prevention, Vol. 17, No. 1, pp. 47-56.

Nicholson, A. J. (1987). "The Estimation of Accident Rates and Countermeasure Effectiveness." Traffic Engineering and Control, Vol. 28, No. 10, pp. 518-523.

Ozawa, S. (1999). "Image Processing for Intelligent Transport Systems.” IEICE Transactions on Information and Systems, Vol. E82-D, No. 3, pp. 629-636.

Parker, M. R. and C. V. Zegeer (1989a). "Traffic Conflict Technique for Safety and Operation: Engineers Guide.” Report FHWA-IP-88-026, FHWA, U.S. Department of Transportation. 
Parker, M. R. and C. V. Zegeer (1989b). "Traffic Conflict Technique for Safety and Operation Observers Manual." Report FHWA-IP-88-027, FHWA, U.S. Department of Transportation.

Persaud, B. and T. Nguyen (1998). "Disaggregate Safety Performance Models for Signalized Intersections on Ontario Provincial Roads." Transportation Research Record 1998, TRB, National Research Council, Washington, D.C., pp. 113-120.

Poch, M. and F. Mannering (1996). "Negative Binomial Analysis of Intersection-Accident Frequencies." Journal of Transportation Engineering, ASCE, Vol. 122, No. 2, pp. 105113.

Porter, B. E., T. D. Berry, and J. Harlow (1999). "A Nationwide Survey of Red Light Running: Measuring Driver Behaviors for the 'Stop Red Light Running' Program." Report, DaimlerChrysler Corporation.

Reiss, R.-D. and M. Thomas (1997). Statistical Analysis of Extreme Values. Birkhauser Verlag, Basel, Switzerland.

Retting, R. A., J. F. Chapline, and A. F. Williams (2002). "Changes in Crash Risk Following Retiming of Traffic Signal Change Intervals." Accident Analysis and Prevention, Vol. 34, No. 2, pp. 215-220.

Salman, N. K. and K. J. Al-Maita (1995). "Safety Evaluation at Three-Leg Unsignalized Intersections by Traffic Conflict Technique." Transportation Research Record 1485, Transportation Research Board, National Research Council, Washington D.C., pp. 177185.

Shankar V. N., J. C. Milton, and F. L. Mannering (1997). "Modeling Accident Frequencies as Zero-Altered Probability Processes: An Empirical Inquiry." Accident Analysis and Prevention, Vol. 29, No. 6, pp. 829-837.

Shoarian-Sattari, K. and D. Powell (1987). "Measured Vehicle Flow Parameters as Predictors in Road Traffic Accident Studies." Traffic Engineering and Control, Vol. 28, No. 6, pp. 328-335.

Smith, R. L. (1985). "Maximum Likelihood Estimation in a Class of Non-Regular Cases." Biometrika, Vol. 72, pp. 67-90.

Tarko, A. P. and B. R. DeSalle (2002). "Internet-Supported Evaluation of Highway Safety." Final Report FHWA/IN/JTRP-2002/9, Grant No. SPR-2483, July.

Tarko, A. P. and N. Lakshmikanth (2002). "Monitoring Red Light Running Using Tripwire Video Detection Systems." Proceedings of $81^{\text {st }}$ Annual Meeting (CD-ROM), TRB, National Research Council, January.

US DOT (1998). “Strategic Plan 1999-2004.” Office of Inspector General, U.S. Department of Transportation, October 1. 
Venables, W. N. and B. D. Ripley (2002). Modern Applied Statistics with S. $4^{\text {th }}$ Ed., Springer, New York.

Vuong, Q. (1989). "Likelihood Ratio Tests for Model Selection and Non-Nested Hypotheses." Econometrica, Vol. 57, March, pp. 307-333.

Washington, S., M. Karlaftis, and F. Mannering (2003). Statistical and Econometric Methods for Transportation Data Analysis. CRC Press, Boca Raton, FL.

WESTAT (2002). "Relationship Between Crashed and Safety Surrogate Measures: A Review of the Recent Literature." FHWA Contract DTFH61-01-C-00180, Draft Report, ITS Enhanced Data Collection for Safety Evaluation, November 20. 


\section{APPENDIX A PROOF OF THEOREMS}

This appendix summarizes the proof of theorem pertinent to the analytical models proposed in this research.

\section{A.1 EXTREMAL TYPES THEOREM}

Formal proof of the extremal types theorem is somewhat complicated mathematically - see Leadbetter et al. (1983) for example. In this section, we will outline the important steps of this proof. To begin with, let us make the following definition:

Definition A.1: A distribution $\mathrm{G}$ is said to be max-stable if, for every $n=2,3, \ldots$ there are constants $\alpha_{n}>0$ and $\beta_{n}$ such that

$$
G^{n}\left(\alpha_{n} z+\beta_{n}\right)=G(z)
$$

Since $G^{n}$ is the distribution function of $M_{n}=\max \left\{X_{1}, \ldots, X_{n}\right\}$, where the $X_{i}$ are independent variables each having distribution function $G$, max-stability is a property satisfied by distributions for which the operation of taking sample maxima leads to an identical distribution, apart from a change of scale and location.

Theorem A.1: A distribution is max-stable if, and only if, it is a generalized extreme value distribution.

It is relatively straightforward to show that all members of the GEV family are indeed max-stable. The converse requires advanced knowledge of functional analysis - see Leadbetter et al. (1983).

Theorem A.1 is used directly in the proof of the extremal types theorem. The idea is to consider $M_{n k}$, the maximum random variable in a sequence of $n \times k$ variables for some large value of $n$. This can be regarded as the maximum of a single sequence of length $n \times k$, or as the maximum of $k$ maxima, each of which is the maximum of $n$ observations. More precisely, suppose the limit distribution of $\left(M_{n}-b_{n}\right) / a_{n}$ is $G$. So, for large enough $n$, 


$$
\operatorname{Pr}\left\{\left(M_{n}-b_{n}\right) / a_{n} \leq z\right\} \approx G(z) .
$$

Hence, for any integer $k$, since $n k$ is large,

$$
\operatorname{Pr}\left\{\left(M_{n k}-b_{n k}\right) / a_{n k} \leq z\right\} \approx G(z)
$$

But, since $M_{n k}$ is the maximum of $k$ variables having the same distribution as $M_{n}$,

$$
\operatorname{Pr}\left\{\left(M_{n k}-b_{n}\right) / a_{n} \leq z\right\}=\left[\operatorname{Pr}\left\{\left(M_{n}-b_{n}\right) / a_{n} \leq z\right\}\right]^{k}
$$

Hence, by (A-3) and (A-4) respectively,

$$
\operatorname{Pr}\left\{M_{n k} \leq z\right\} \approx G\left(\frac{z-b_{n k}}{a_{n k}}\right)
$$

and

$$
\operatorname{Pr}\left\{M_{n k} \leq z\right\} \approx G^{k}\left(\frac{z-b_{n}}{a_{n}}\right) .
$$

Therefore, $G$ and $G^{k}$ are identical apart from location and scale coefficients. It follows that $G$ is max-stable and therefore a member of the GEV family by theorem A.1. 


\section{APPENDIX B EVALUATION OF RISK OF RIGHT-ANGLE COLLISIONS}

A simple estimation method of risk of right-angle collisions at signalized intersections using short-term observation of post-encroachment times (PETs) is described. Before we begin the description of the method, let us introduce important definitions relevant to the method:

- Conflict zone is an area defined by the intersection of two crossing traffic flows. One intersection may have up to four conflict zones - see Figure B-1.

- Conflict spot is an intersection area of two crossing vehicle paths in a conflict zone - see example in Figure B-2.

- Post-encroachment time (PET) is the time it takes from the end of the right-of-way infringement of the first vehicle for the second vehicle to reach the conflict spot, measured from the rear bumper of the first vehicle to the front bumper of the second vehicle - see Figure B-3.

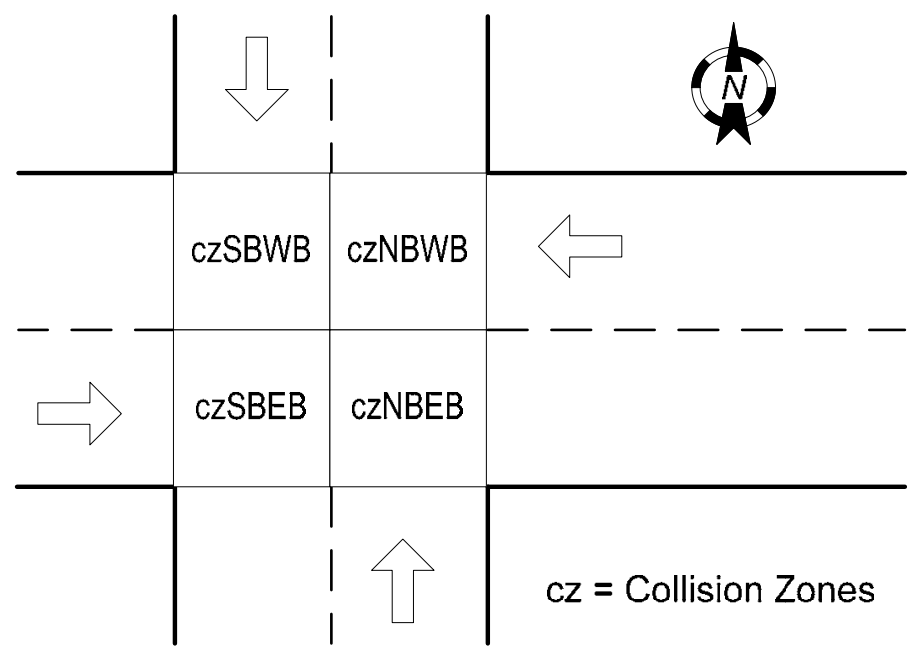

Collision zones are defined by a pair of conflicting vehicle streams

Figure B-1: Collision/Conflict Zone Notation 


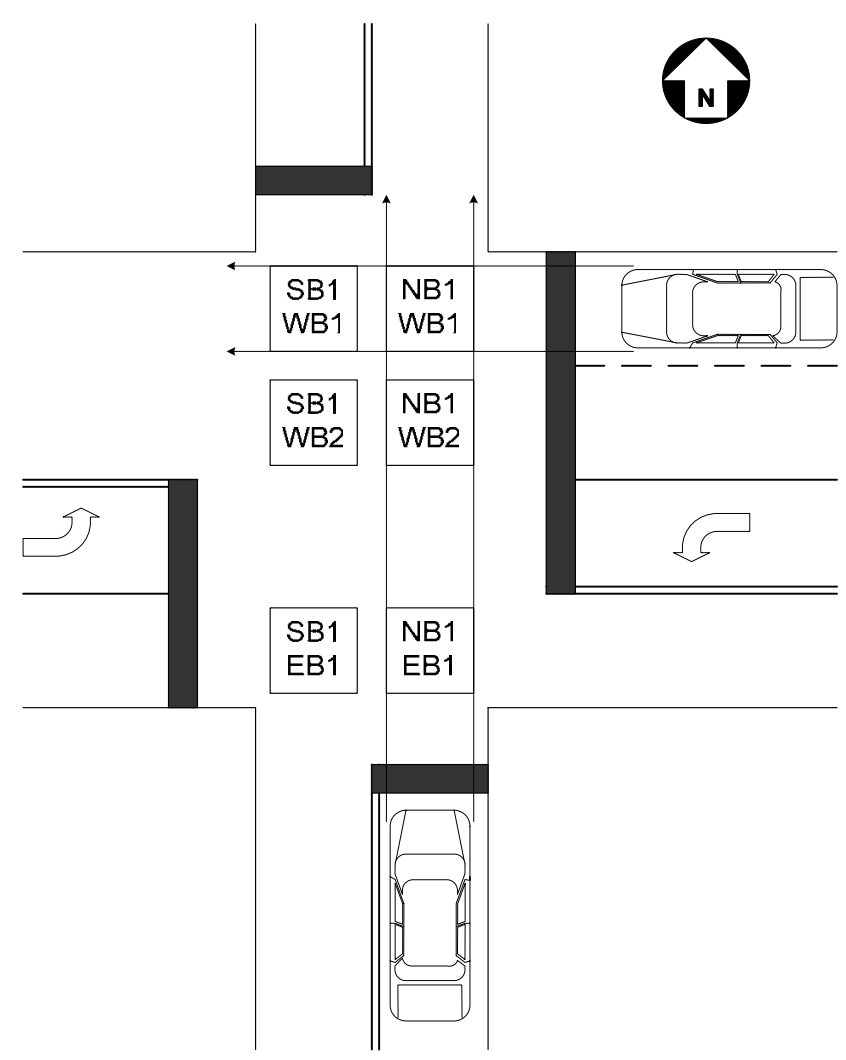

Figure B-2: Example of Conflict Spot Designation
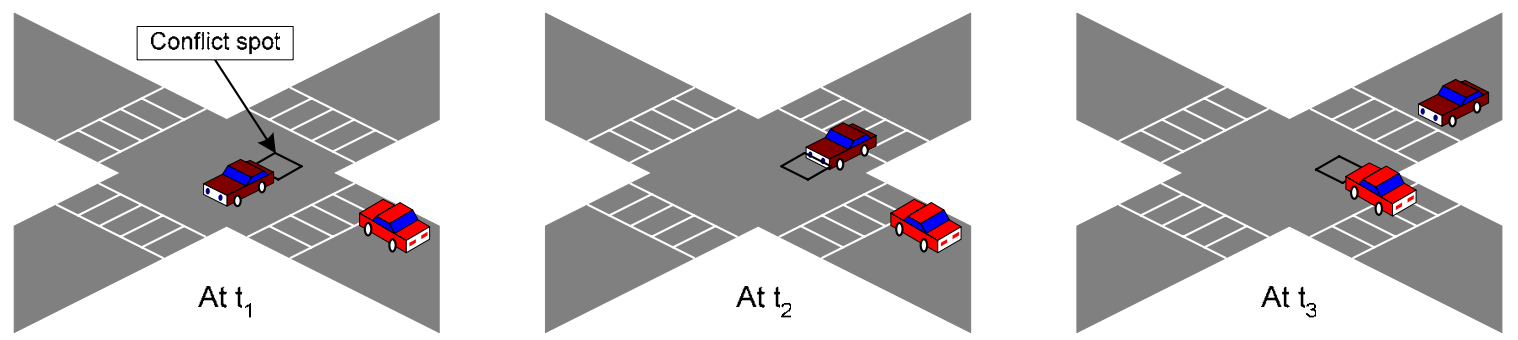

Encroachment Time, $\mathrm{ET}=\mathrm{t}_{2}-\mathrm{t}_{1} ;$ Post-encroachment Time, PET $=\mathrm{t}_{3}-\mathrm{t}_{2} ;$ Gap time, $\mathrm{GT}=\mathrm{t}_{3}-\mathrm{t}_{1}=\mathrm{ET}+\mathrm{PET}$

Figure B-3: Illustration of Post-Encroachment Time

PETs reflect driver behaviors in response to intersection control, intersection geometry, and other drivers and these make PET a desirable safety indicator. Long-term observation of PET, if practical automated measurement method is available in the future, may lead to rapid crash frequency estimation without the need to rely on a trend from a group of similar locations. 


\section{B.1 METHOD OVERVIEW}

We will illustrate step-by-step the procedure for collecting the PETs between two crossing traffic streams and estimating the frequency of right-angle collisions. The proposed method is simple in the sense that (a) only counts of PET are required and (b) the estimation of crash frequency is straightforward. The method estimates annual frequency of daytime right-angle crashes and is extended to enable estimation of the annual frequency of all right-angle collisions regardless of the time of day.

The method procedure can be summarized in the following steps:

- Data collection - collect the frequency of short PETs between crossing traffic flows at a conflict zone or at the entire intersection.

- Crash frequency estimation - estimate the crash frequency using the equations provided.

- Interpretation of results - interpret the results to determine if there is excessive risk of right-angle collisions at the studied location.

In the data collection, PETs shorter than 6.5 seconds are counted during an 8-hour period. The hardware and software requirements for data collection are discussed in the next section.

\section{B.2 EQUIPMENT}

Hardware and software requirements are given at two different levels: (a) desirable and (b) minimum. To obtain the best results, the desirable requirement should be followed, particularly for complex intersections - heavy traffic volume and more than one through lane at intersection approaches. The minimum requirement is acceptable if one of the two following conditions is met:

1. A safety estimation at a relatively simple intersection with single through lanes and light traffic volume, or

2. A safety estimation of a single conflict zone.

\section{Desirable Equipment}

The following equipment is desirable: 
- One computer with 512 MB RAM and Pentium 4 processor at least

- Available harddrive storage space of about 100GB for 8-hour data ( 12GB per hour)

- A computer must be installed with a graphic card that can digitize videos at $30 \mathrm{fps}$. For example, Pinnacle DV 500 plus was used in this project.

- Clear plastic tapes (affix the tape to a computer monitor and then use a marker to draw virtual conflict spots).

- Video viewing software with capability of accessing the video frame-by-frame (e.g. Adobe Premiere)

- Video camcorder installed at 30-40 feet height or above at a corner of an intersection or a corner of a conflict zone being studied

- Camcorder installation platform. A pole nearby the intersection corner or a mobile traffic lab similar to what we used in this study is acceptable.

- Jamar ${ }^{\circledR}$ traffic counters - for PET counts instead of vehicle counts

\section{Minimum Equipment}

The minimum specification requires no special hardware or software. The following tools are necessary in the data collection:

- Stop watches

- Chalk to mark the conflict spot on the pavement

- Jamar ${ }^{\circledR}$ traffic counters - for PET counts instead of vehicle counts

\section{B.3 DATA COLLECTION PROCEDURE}

PET shorter than 6.5 seconds can be counted in two different ways depending on available equipment. If the desirable equipment is obtainable, PET can be counted post-processed from the recorded video clips in the office. This would require no more than one human observer for the entire intersection. The minimum equipment relies on on-site observation of PETs less than 6.5 seconds and this would require at least 2 human observers for one intersection. Recommended period of observation is 8 hours during daytime with coverage of morning and afternoon peak periods for one hour each. 


\section{Counting Short PETs from Video Clips}

If the desirable specification is attainable, the post-processing of recorded videos to count PETs shorter than 6.5 seconds will require only one human observer. PET counts can be obtained accurately because an observer can always review a portion of video clips in question. The steps for the entire procedure can be summarized as follows:

1. Set up the video camcorder such that the field of view covers the intersection region as much as possible. Always zoom-in to obtain the best coverage of conflict spots and leave out the unused portion of the intersection.

2. Record traffic movements for at least 8 hours. Recommended periods are 7.00AM to 10:00AM, 11:00AM to 2:00PM, and 3:00PM to 6:00PM. This will give 9 hours of recordings to ensure that at least 8-hour observation criterion is met. The 3-hour duration can be recorded in one S-VHS tape media. The suggested periods also cover morning and afternoon peak periods.

3. Back to the office, recorded videos should be digitized at $30 \mathrm{fps}$ for fast frame-by-frame access during video clip reviewing process.

4. Open the digitized video clips in video viewing software such as Adobe Premiere. Then, affix a clear plastic tape at the intersection whereabouts on the computer screen.

5. Play the video clips and observe the traffic movements. Focus on the areas where two traffic flows are crossing. Mark the virtual conflict spots on the screen and keep adjusting as necessary. Reviewer may pause the video to help locate exact paths of vehicles traversing a conflict spot. The virtual conflict spots on the screen should be similar to Figure B-4.

6. A group of conflict spots belong to the same conflict zone if the conflicts are generated by the same pair of crossing traffic flows. Label all the conflict spots and their corresponding conflict zones. For example, from the Figure B-5, the crossing conflicts between EB through traffic (2 lanes) and SB through traffic (1 lane) create 2 conflict spots (SB1EB1 and SB1EB2) in one conflict zone (czSBEB).

7. Count the number of PETs shorter than 6.5 seconds for each conflict spot. Video time stamp with frame reference in the Adobe Premiere can be used in the PET measurement. Jamar ${ }^{\circledR}$ counter may be applied to the counting task by assigning different conflict zones to different movement numbers. 
8. Record the collected data, which are the number of PETs shorter than 6.5 seconds for each conflict zone.

During the counting procedure, an observer may notice that sufficiently short PETs usually take place during the intergreen period, which is between the moment that the green light is terminating on one approach and the moment that the green light is beginning on another approach. Therefore, the portion of video clips outside these intergreen periods may be partially skipped by the reviewer. This can help shorten the post-processing time of the video materials.

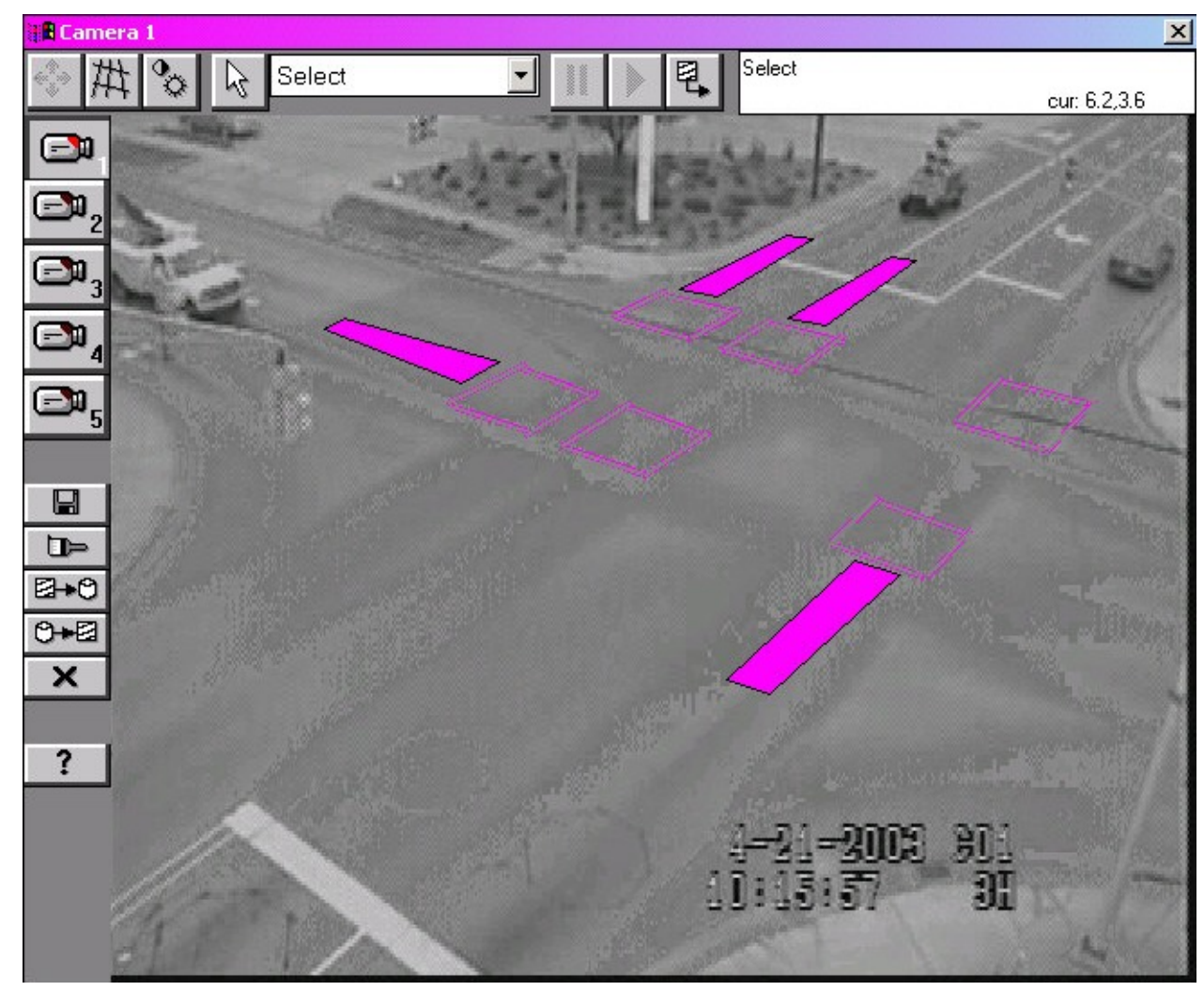

Figure B-4: Example of Conflict Spots at Site 97905

In addition, the crash frequency can be estimated for individual conflict zones. The implication is that PET counts can be limited to individual conflict zones of safety concern. The counts of short PETs at specific conflict zones can be carried out much faster than the counts for the entire intersection. On-site PET counts may be a viable alternative if (a) an intersection is not too large and traffic volume is light or (b) no more than two conflict zones are being studied. The on-site observation is a simplified observation procedure which we will explain in the subsequent section. 


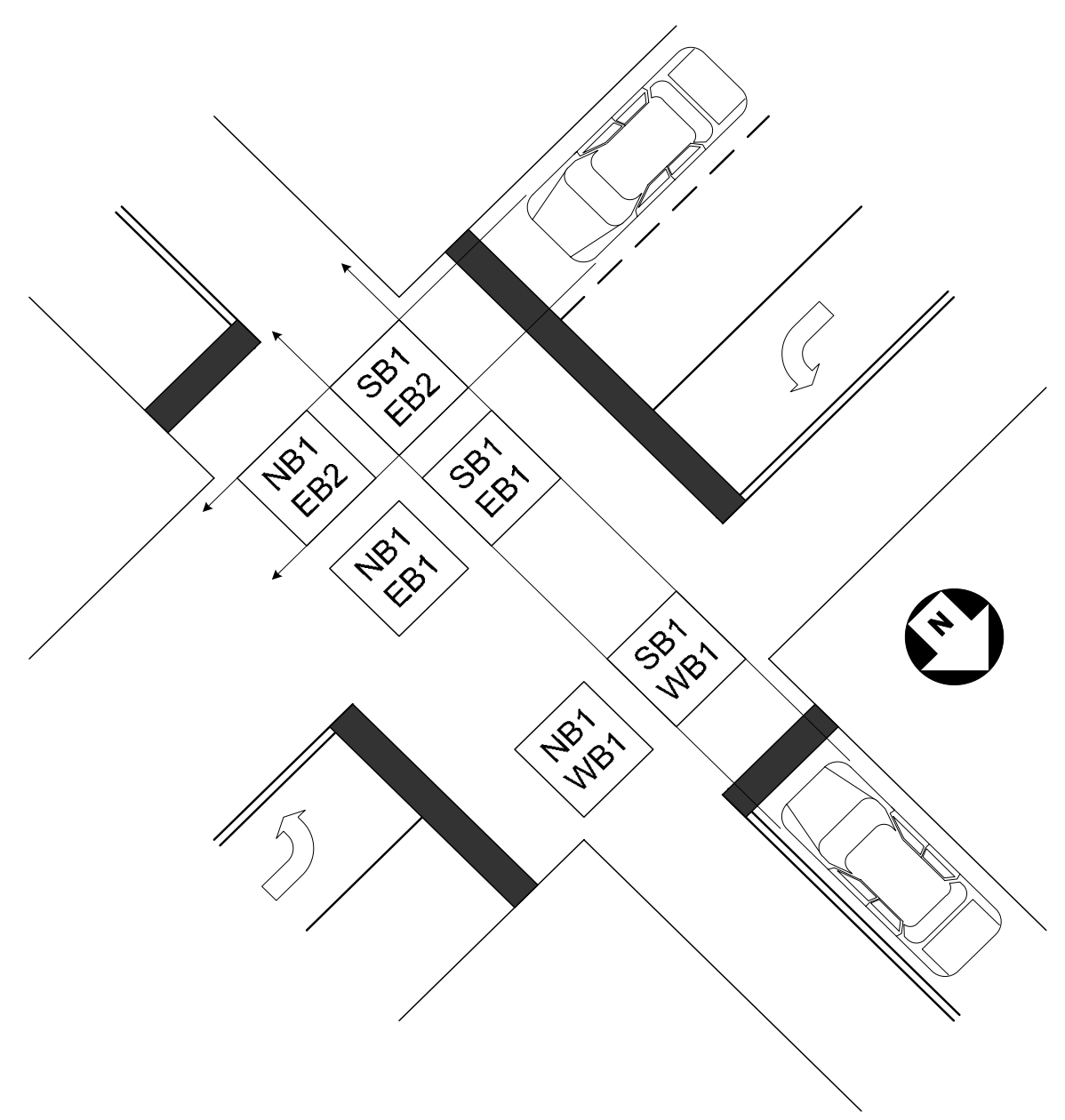

Figure B-5: Labeling of Conflict Spots

\section{On-Site Observation}

The on-site observation is a simplified method which requires neither special hardware nor postprocessing efforts. This method requires one observer for each conflict zone observed. One observer may be able to handle PET counts for two conflict zones if traffic volume is light enough. This method is not recommended if there are more than four conflict spots to be observed. The data collection procedure can be summarized in the following steps:

1. Mark the pavement for approximate position of conflict spots. This can be done quickly during light-traffic conditions but safety precautions must be carefully exercised in this step. An observer should put on a safety vest and traffic cones should be placed to alert the oncoming traffic. 
2. Using a stop watch, measure the time between the moment that the last vehicle leaves a conflict spot $\left(\mathrm{t}_{2}\right)$ and the moment that the first vehicle from a crossing flow enters a conflict spot $\left(\mathrm{t}_{3}\right)$ - see PET illustration in Figure B-3. Stop watch with time lapse feature can be helpful for this task. Instead of using start/stop buttons, start/lapse buttons can be used to time the departure/entry events. If the last vehicle anticipated from the approach is actually not the last one, an observer can still time the next one using lapse feature instead.

3. During the waiting period for the next intergreen, review the stop watch and determine if the measured PET is shorter than 6.5 seconds $\left(t_{3}-t_{2}\right)$.

4. Count only the PET value that is shorter than 6.5 seconds. Jamar ${ }^{\circledR}$ traffic counter can facilitate the counting task.

5. Reset the stop watch and watch for the crossing traffic at the assigned conflict spots during the next intergreen period.

6. Repeat the steps 1 to 5 until the PETs are counted for 8 hours. Example of recommended periods is $7.00 \mathrm{AM}$ to $9: 30 \mathrm{AM}, 11: 00 \mathrm{AM}$ to $2: 00 \mathrm{PM}$, and 3:30PM to $6: 00 \mathrm{PM}$.

7. Record the collected data, which are the number of PETs shorter than 6.5 seconds for each conflict zone.

Although the on-site observation is relatively simpler and consumes less time than the previous one, the disadvantages of the method should be noted as follows:

- PET counts may not be as accurate as counting from video clips due to subjective judgment required in identifying the exact $t_{2}$ and $t_{3}$.

- More human observers are required for the same task. One observer should be allocated to one conflict spot for best results and no more than two simultaneously.

- The method is prone to error if applied to a large intersection with heavy traffic volume.

\section{B.4 ESTIMATION OF RIGHT-ANGLE CRASH FREQUENCY}

Based on the Poisson and negative binomial regression of PET-based models (see Section 5.5.2), the frequency of daytime right-angle collisions can be estimated by simply substituting the 8-hour counts of PET shorter than 6.5 seconds into the given equations. Two levels of PET-based models were calibrated - site and conflict zone. If the PET counts are aggregated from all conflict zones, 
the site equation (B-1) should be applied. If the PET counts are disaggregated by conflict zones, the conflict zone equation (B-2) should be applied.

In case where PET counts are available for all conflict zones, the summation will give the PET counts for the entire intersection. We checked the results using both equations and they were comparable for the same intersection. This implies that if PET counts are disaggregated by conflict zones, either site or conflict zone can be applied.

From the regression results in Table 5-8, the annual frequency of daytime right-angle collisions can be estimated as

$$
a=0.25 \cdot \exp \left(0.6894+0.0043 f_{P E T}\right),
$$

where $a$ is the estimated annual frequency of daytime right-angle collisions at the intersection and $f_{P E T}$ is the 8-hour counts of PET shorter than 6.5 seconds for the entire intersection (all conflict zones).

Similarly, from the regression results in Table 5-9, the annual frequency of daytime right-angle collisions for individual conflict zone can be estimated as

$$
a_{c z}=0.25 \cdot \exp \left(-0.3908+0.0117 f_{P E T, c z}\right)
$$

where $a_{c z}$ is the estimated annual frequency of daytime right-angle collisions for individual conflict zone and $f_{P E T, c z}$ is the 8-hour count of PET shorter than 6.5 seconds at individual conflict zone.

Table B-1 to Table B-3 show examples of crash frequency estimation results for three selected intersections using equations (B-1) and (B-2).

\section{Example B-1}

PET count at intersection 87907 is equal to 68 (summing up the counts from all conflict zones). The expected annual daytime right-angle crash frequency can be estimated for this intersection using equation (B-1) as 


$$
a=0.25 \times \exp (0.6894+0.0043 \times 68)=0.6684 \text {. }
$$

This means that the expected number of daytime right-angle collisions is 0.6684 per year or approximately 2.7 crashes for 4-year period.

Table B-1: Example of Estimation of Right-Angle Crash Frequency at Site 87907

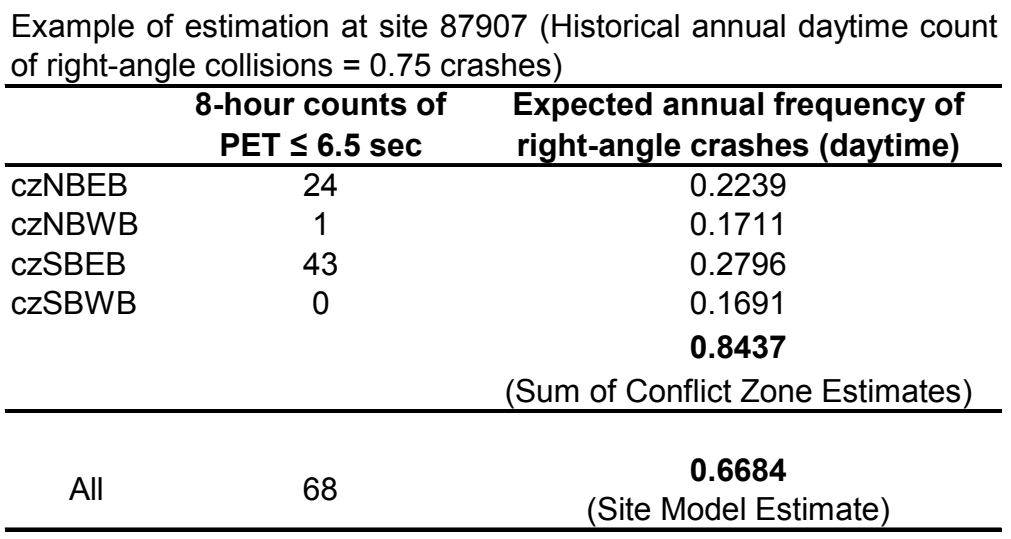

Table B-2: Example of Estimation of Right-Angle Crash Frequency at Site 87909

Example of estimation at site 87909 (Historical annual daytime count of right-angle collisions $=1.75$ crashes)

\begin{tabular}{lcc}
\hline & $\begin{array}{c}\text { 8-hour counts of } \\
\text { PET } \leq \mathbf{6 . 5 ~ s e c}\end{array}$ & $\begin{array}{c}\text { Expected annual frequency of } \\
\text { right-angle crashes (daytime) }\end{array}$ \\
\hline CZNBEB & 97 & 0.5256 \\
CZNBWB & 103 & 0.5638 \\
czSBEB & 76 & 0.4112 \\
CZSBWB & 81 & 0.4360 \\
& & $\mathbf{1 . 9 3 6 7}$ \\
& & (Sum of Conflict Zone Estimates) \\
\hline \multirow{2}{*}{ All } & 357 & $\mathbf{2 . 3 3 2 7}$ \\
& & (Site Model Estimate) \\
\hline
\end{tabular}

\section{Example B-2}

PET count at the conflict zone "czNBWB" of the intersection 87909 is equal to 103 . The expected annual daytime right-angle crash frequency for this conflict zone can be estimated using equation (B-2) as

$$
a_{c z}=0.25 \times \exp (-0.3908+0.0117 \times 103)=0.5638
$$


This means that the expected number of daytime right-angle collisions at this conflict zone is 0.5638 per year or approximately 2.25 crashes per 4 years. Similar calculation was applied to other conflict zones. Summation of all conflict zone estimates gives the annual daytime rightangle crash frequency at this intersection, which is 1.94 per year or equivalently 7.76 per 4 years.

Table B-3: Example of Estimation of Right-Angle Crash Frequency at Site 97901

\begin{tabular}{|c|c|c|}
\hline & $\begin{array}{l}\text { 8-hour counts of } \\
\text { PET } \leq 6.5 \mathrm{sec}\end{array}$ & $\begin{array}{l}\text { Expected annual frequency of } \\
\text { right-angle crashes (daytime) }\end{array}$ \\
\hline czNBEB & 120 & 0.6878 \\
\hline czNBWB & 64 & 0.3574 \\
\hline czSBEB & 128 & 0.7552 \\
\hline \multirow[t]{3}{*}{ czSBWB } & 101 & 0.5508 \\
\hline & & 2.3513 \\
\hline & & (Sum of Conflict Zone Estimates) \\
\hline All & 413 & $\begin{array}{c}\mathbf{2 . 9 7 1 9} \\
\text { (Site Model Estimate) }\end{array}$ \\
\hline
\end{tabular}

\section{Crash Frequency Estimate Regardless of Time of Day}

The estimated daytime annual crash frequency can be extended to include all conditions regardless of time of day. We discussed earlier in Section 5.5.2.1 that the trends between PET counts and observed right-angle crash counts are similar for various conditions during crash occurrences - see Figure 5-2. The implication is that a short period of PET observation may be sufficiently representative for the entire period of crash counting. Crash counting period includes periods with conditions different from those in the PET observation period. From this observation, the annual crash frequency regardless of time of day can be approximated by multiplying the annual daytime crash frequency with the ratio of all observed right-angle crash counts to observed daytime right-angle crash counts. This leads to

$$
\hat{c}_{\text {all }}=\frac{128}{71} \hat{c}_{d a y}=1.803 \hat{c}_{d a y}
$$

where $\hat{c}_{\text {all }}$ is the estimated right-angle crash frequency regardless of time of day and $\hat{c}_{d a y}$ is the estimated right-angle crash frequency during daytime only. 


\section{Example B-3}

Let us consider the data from the intersection 97901. From Table B-3, the estimated annual daytime frequency of right-angle collisions at the intersection 97901 is 2.972 crashes or 11.888 per 4 years. Therefore, using equation (B-3), the frequency of right-angle collisions for the entire year can be approximated as

$$
\hat{c}_{\text {all }}=(1.803)(2.972)=5.359
$$

Equivalently, the estimated annual frequency of right-angle collisions at the intersection 97901 is 21.434 per 4 years.

\section{Estimation of Annual Left-Turn Right-Angle Crash Frequency}

Left-turn right-angle collisions as shown in Figure B-6 may be frequent at signalized intersections with a protected left-turn phase and heavy left-turn traffic volume. Due to a similarity of crash occurrence mechanism, the equations given earlier may be applied to the estimation of annual daytime frequency of left-turn right-angle collisions as well. However, the results should be used with cautions because the equations were not calibrated specifically for this case. The estimates are intended to give only approximate safety levels of this type of collision at studied conflict zones or intersections.
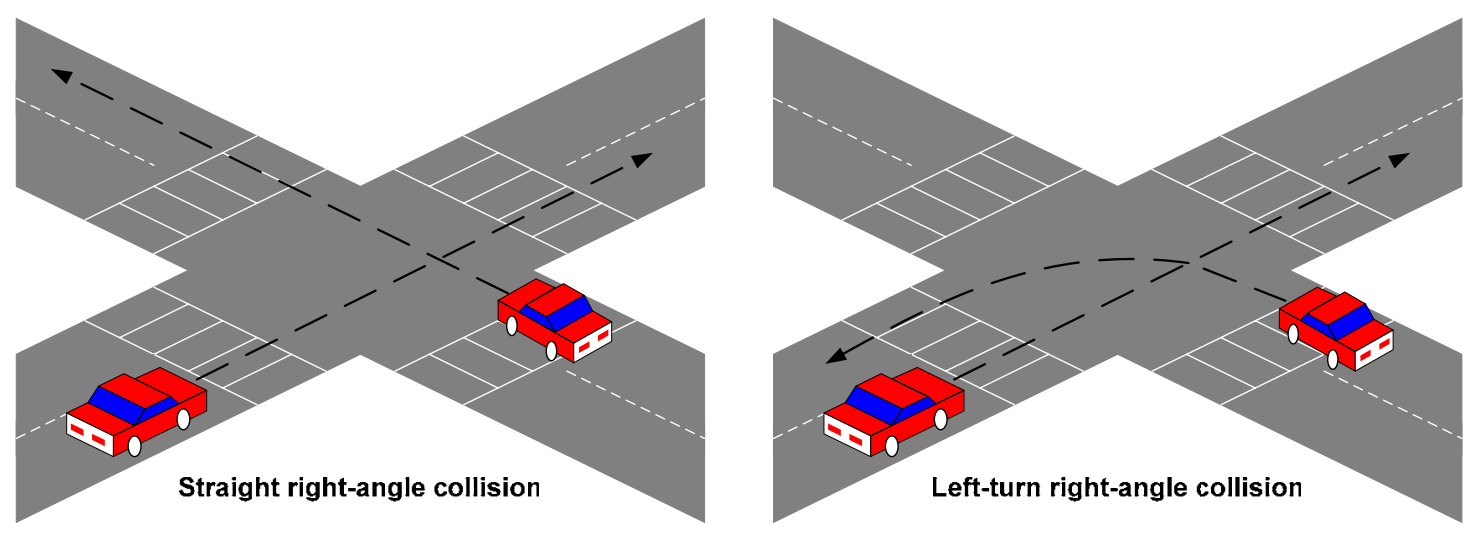

Figure B-6: Illustration of a Left-Turn Right-Angle Collision

To apply the given equations, PETs shorter than 6.5 seconds between left-turn and through vehicles must be counted for 8 hours instead of PETs between through vehicles. Then, substituting PET count into equation (B-1) gives an estimate of annual daytime crash frequency 
of left-turn right-angle collisions at a studied intersection. Similarly, substituting PET count at individual conflict zone into equation (B-2) gives a conflict zone estimate of annual daytime crash frequency of left-turn right-angle collisions.

The next section will provide a guideline to evaluate if the predicted right-angle crash frequency at a studied intersection or individual conflict zone is indicating a safety problem which may call for comprehensive safety evaluation and/or preventive countermeasures.

\section{B.5 INTERPRETATION OF RESULTS}

We established a guideline to help evaluate if the estimated right-angle collisions are of safety concern using empirical PET counts across all the observed conflict zones in this study. By substituting the counts of PETs shorter than 6.5 seconds from the 61 observed conflict zones into equation (B-2), empirical cumulative distribution curve for annual daytime right-angle crash frequency of individual conflict zones was established as shown in Figure B-7.

Histogram and Cumulative Empirical Distribution

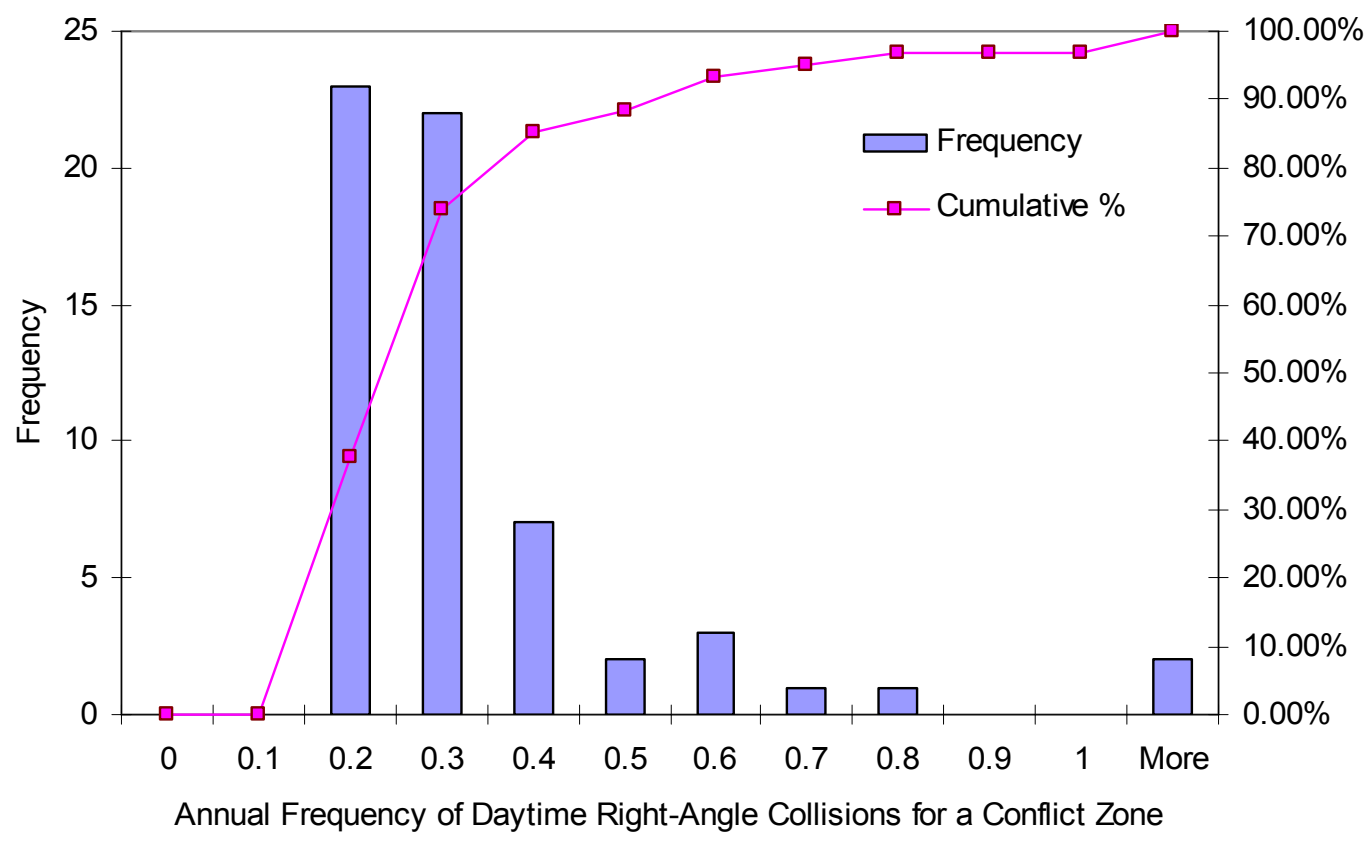

Figure B-7: Empirical Cumulative Distribution of Annual Daytime Right-Angle Crash Frequency of Individual Conflict Zones 
The level of crash frequency estimates that define excessive risk of right-angle collisions depends on the criteria set by highway policy/decision makers.

For example, a location can be considered as having excessive risk of right-angle collision if the right-angle crash frequency at that location is higher than $85 \%$ of all locations. This corresponds to the $85^{\text {th }}$ percentile value in Figure B-7, which gives the reading of 0.3924 daytime crashes per year for individual conflict zone or $1.803(0.3924)=0.7075$ crashes per year regardless of time of day. Typically, there are four conflict zones in an intersection unless one or more of crossing roads is one-way. Therefore, the corresponding number for the entire intersection is equal to $4(0.7075)=2.830$ right-angle crashes per year.

Table B-4 summarizes the annual crash frequency estimates at $85^{\text {th }}$ and $90^{\text {th }}$ percentiles for individual conflict zone as well as an intersection.

Table B-4: Annual Right-Angle Crash Frequency Estimates at $85^{\text {th }}$ and $90^{\text {th }}$ Percentiles

\begin{tabular}{cccc}
\hline & \multirow{2}{*}{ Conditions } & \multicolumn{2}{c}{ Percentile } \\
\cline { 3 - 4 } $\mathbf{8 5}^{\text {th }}$ & $\mathbf{9 0}^{\text {th }}$ \\
\hline \multirow{2}{*}{ Conflict zone } & Daytime only & 0.3924 & 0.5256 \\
& All & 0.7076 & 0.9477 \\
Intersection & Daytime only & 1.5697 & 2.1026 \\
(4 conflict zones) & All & 2.8302 & 3.7910 \\
\hline
\end{tabular}

\section{Example B-4}

As a continuation from Example B-2, the estimated annual right-angle crash frequency for a conflict zone "czNBWB" at the intersection 87909 is 0.5638 for daytime condition. This value is greater than $85^{\text {th }}$ and $90^{\text {th }}$ percentiles in Table B-4, which are 0.3924 and 0.5256 per year respectively. Therefore, the risk of right-angle collisions at this conflict zone is higher than most locations and may warrant further safety evaluation.

\section{Example B-5}

As a continuation from Example B-3, the estimated annual right-angle crash frequency regardless of time of day at the intersection 97901 is 5.359 crashes. This value is greater than $85^{\text {th }}$ and $90^{\text {th }}$ 
percentiles in Table B-4, which are 2.8302 and 3.7910 per year respectively. Therefore, there is an excessive risk of right-angle collisions at this intersection and comprehensive safety evaluation may be considered. 


\section{APPENDIX C POST-ENCROACHMENT TIME}

Plots of PET variations over time and histograms of the negated PET values at the selected 18 signalized intersections are shown in this appendix. 
PET variation over time at site 87905

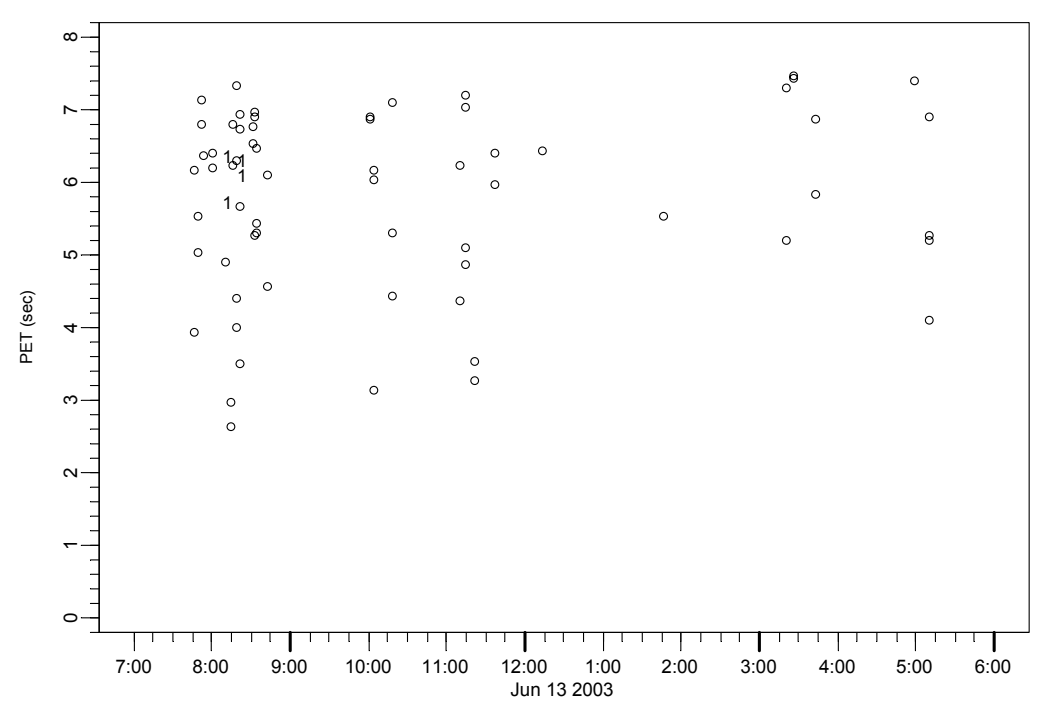

Figure C-1: PET Variation over Time at Site 87905

PET variation over time at site 87905 : czNBEB

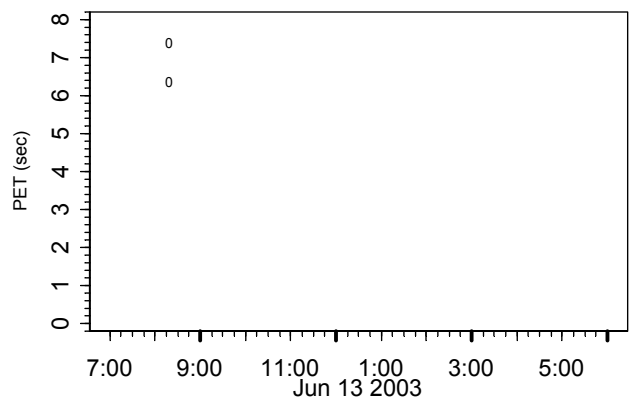

PET variation over time at site 87905 : czSBEB

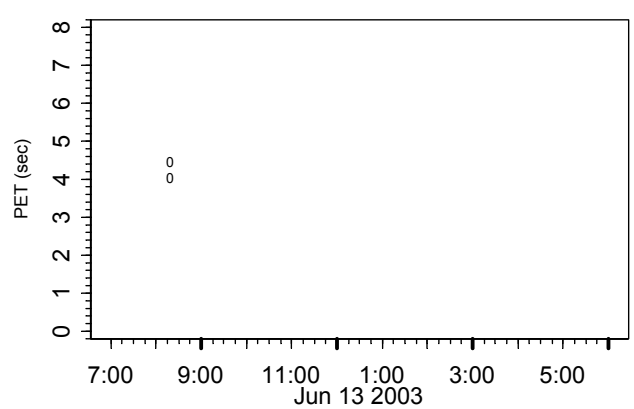

PET variation over time at site 87905 : czNBWB

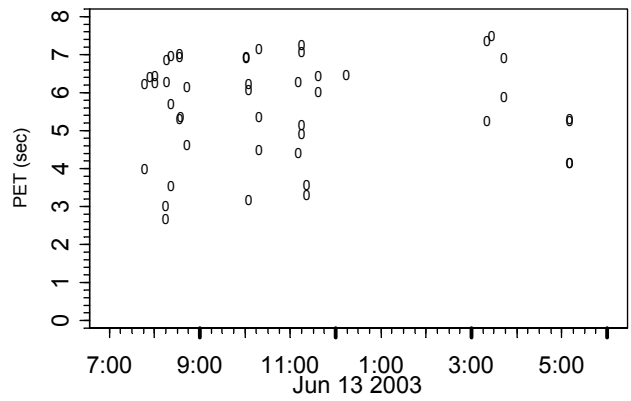

PET variation over time at site 87905 : czSBWB

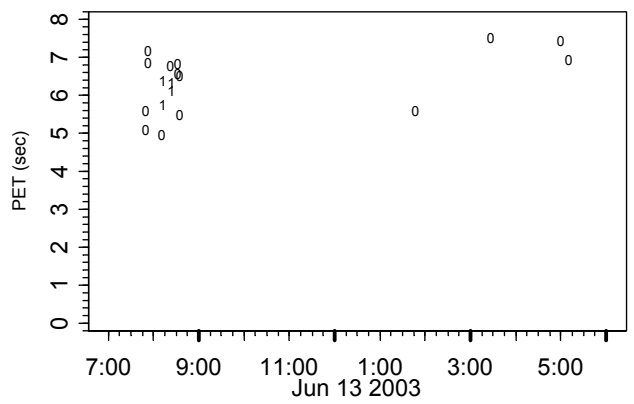

Figure C-2: PET Variation over Time by Conflict Zones at Site 87905 
PET variation over time at site 87906

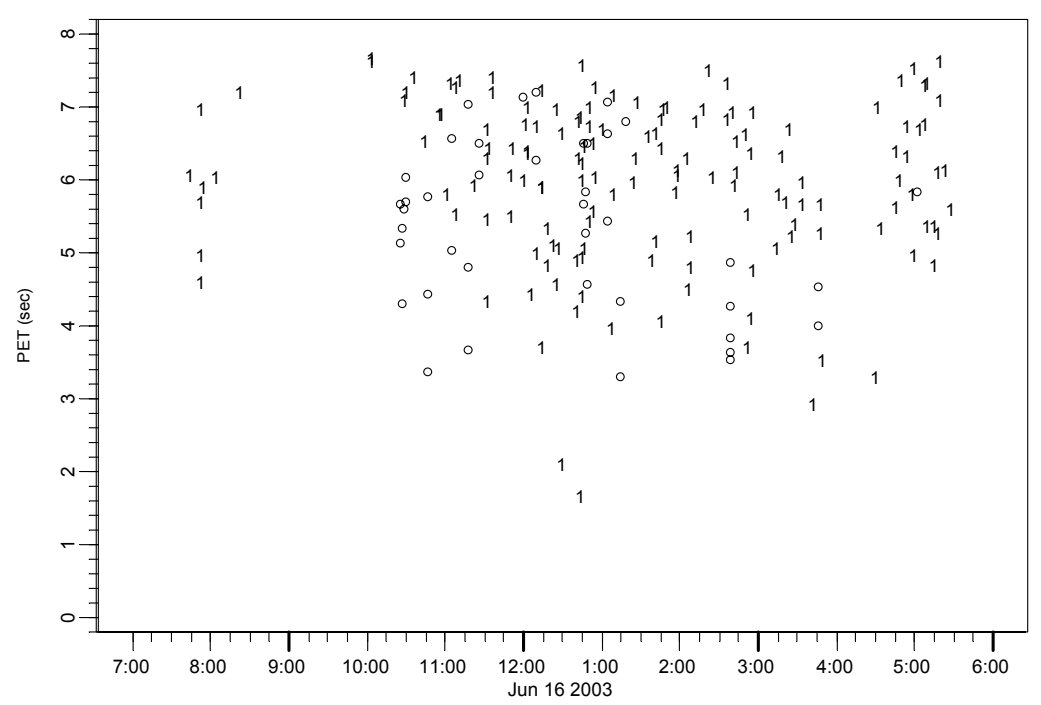

Figure C-3: PET Variation over Time at Site 87906

PET variation over time at site 87906 : czNBEB

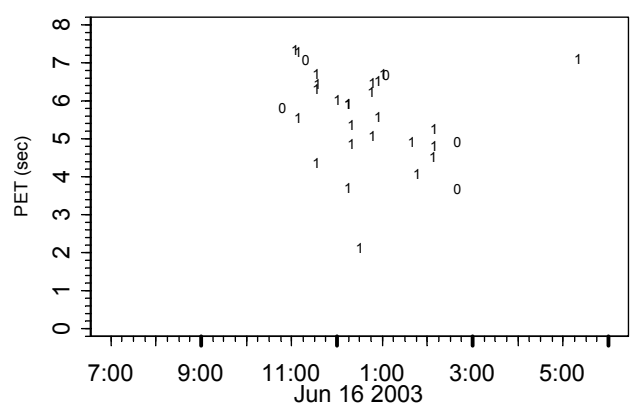

PET variation over time at site 87906 : czSBEB

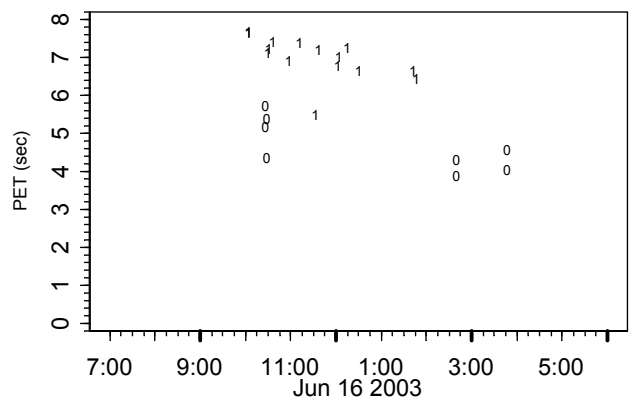

PET variation over time at site $87906:$ czNBWB

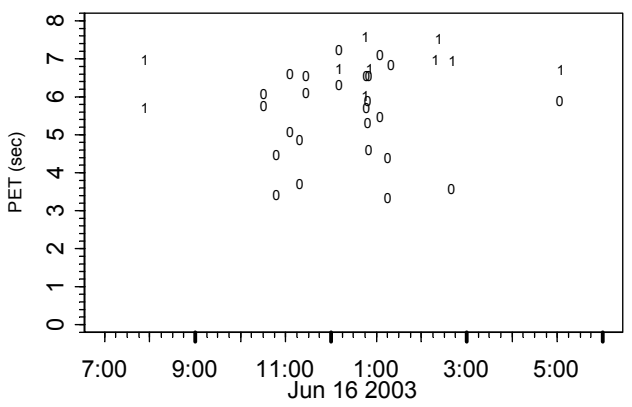

PET variation over time at site 87906 : czSBWB

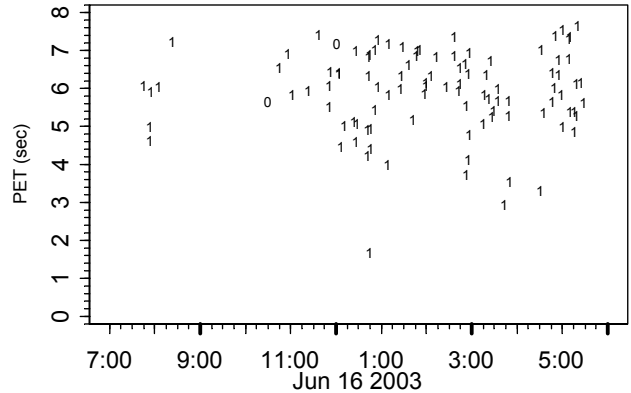

Figure C-4: PET Variation over Time by Conflict Zones at Site 87906 
PET variation over time at site 87907

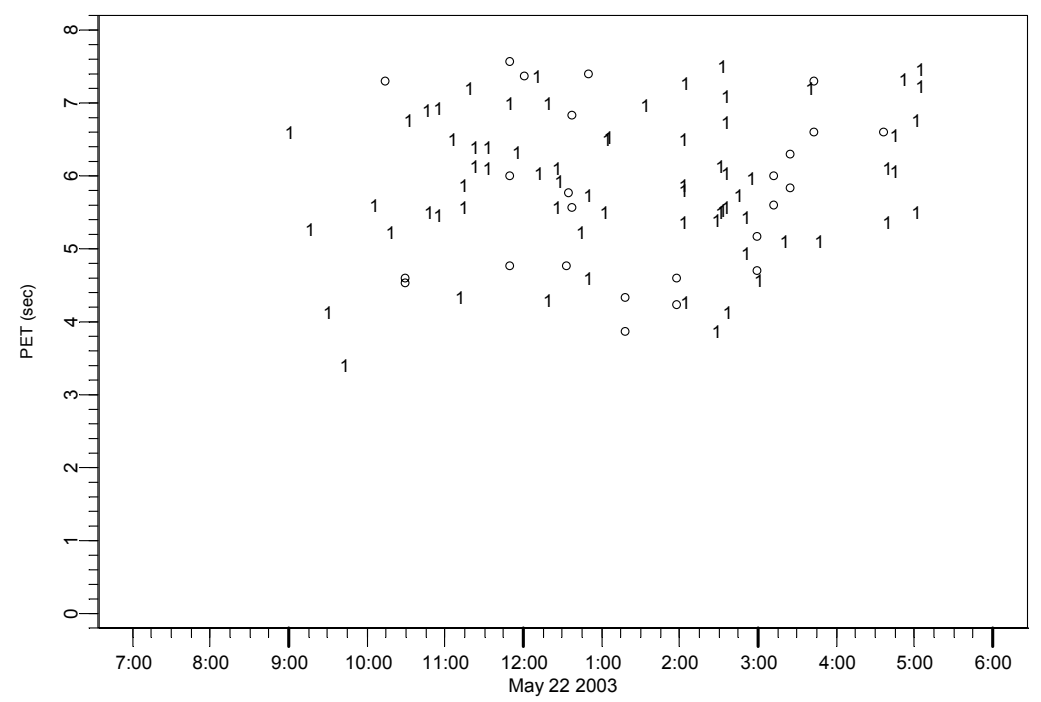

Figure C-5: PET Variation over Time at Site 87907
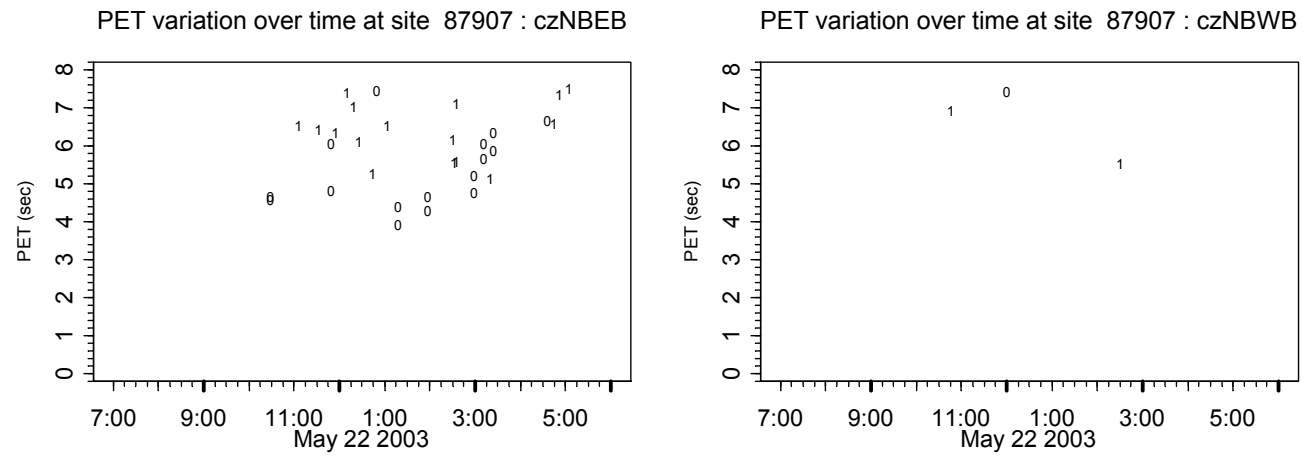

PET variation over time at site 87907 : czSBEB

PET variation over time at site 87907 : czSBWB
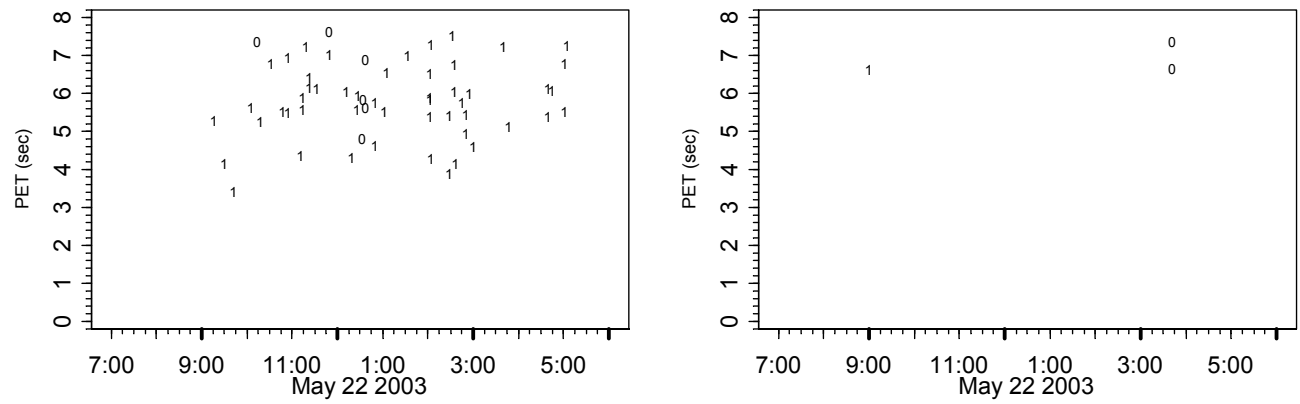

Figure C-6: PET Variation over Time by Conflict Zones at Site 87907 
PET variation over time at site 87909

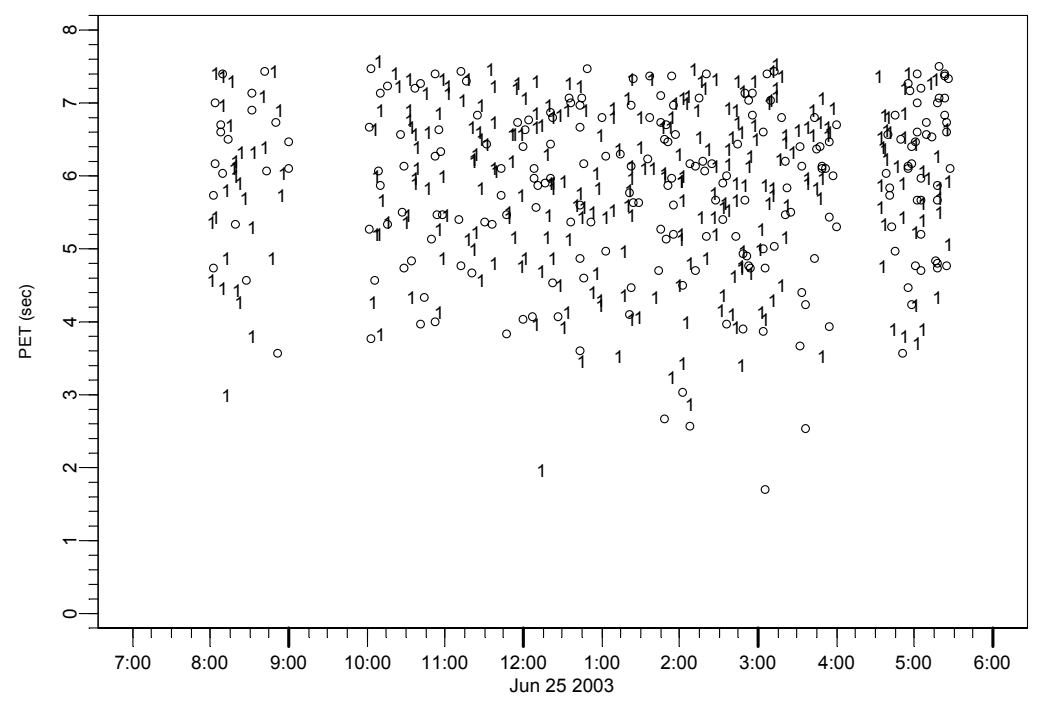

Figure C-7: PET Variation over Time at Site 87909

PET variation over time at site 87909 : czNBEB

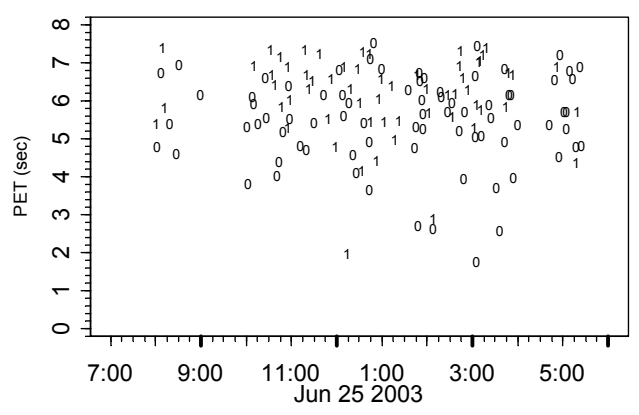

PET variation over time at site 87909 : czSBEB

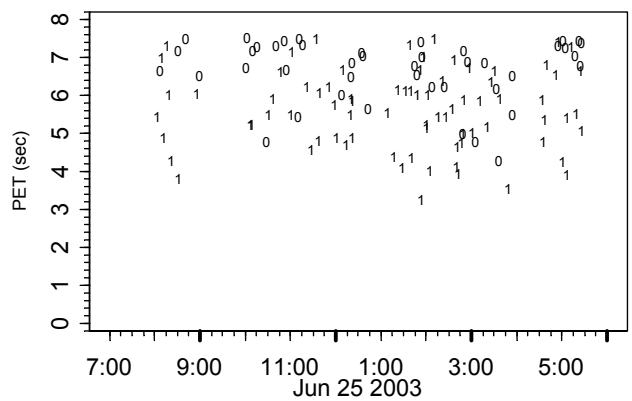

PET variation over time at site 87909 : czNBWB

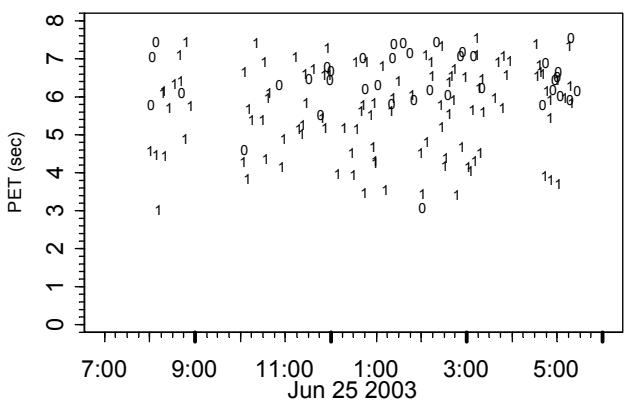

PET variation over time at site 87909 : czSBWB

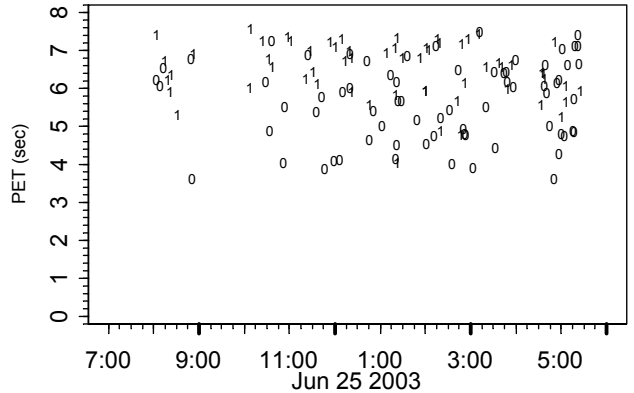

Figure C-8: PET Variation over Time by Conflict Zones at Site 87909 
PET variation over time at site 87915

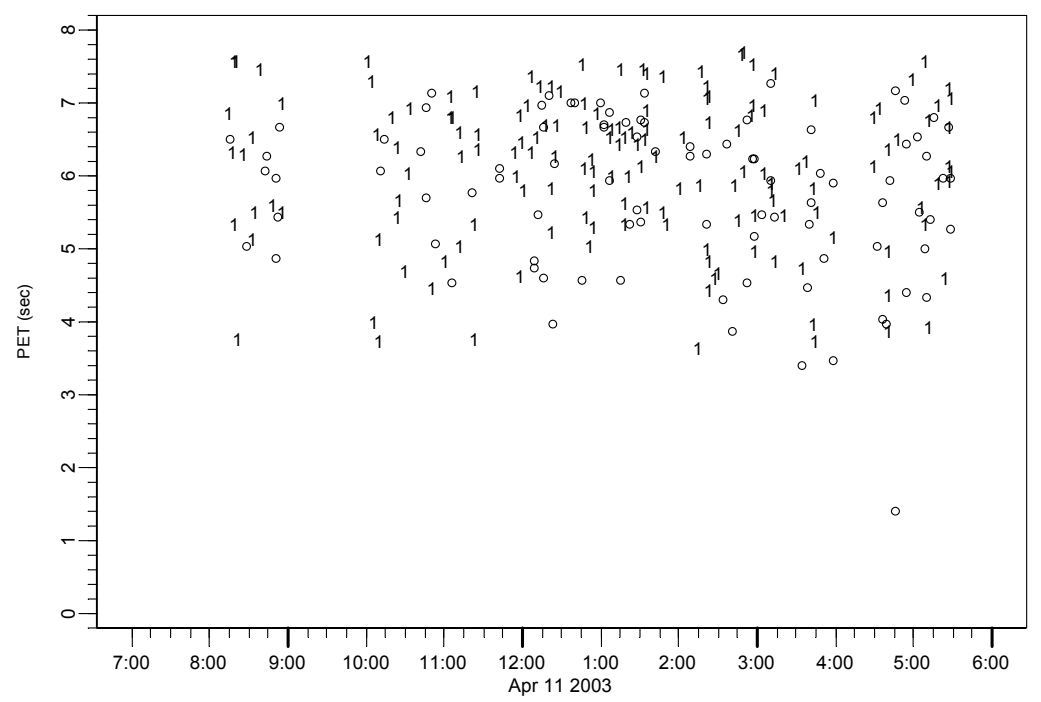

Figure C-9: PET Variation over Time at Site 87915

PET variation over time at site 87915 : czNBEB

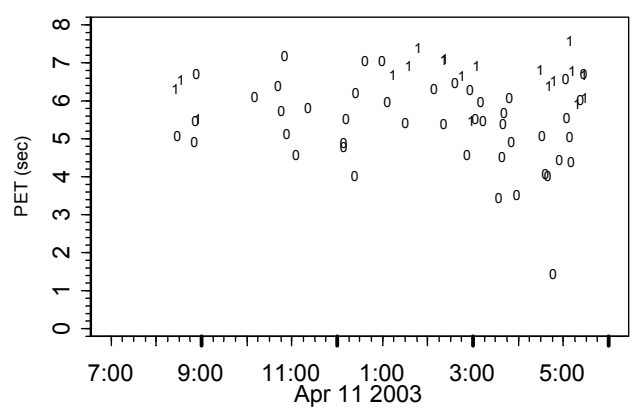

PET variation over time at site 87915 : czSBEB

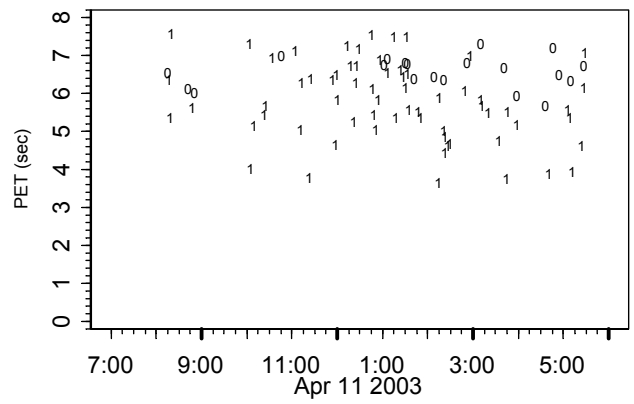

PET variation over time at site 87915 : czNBWB

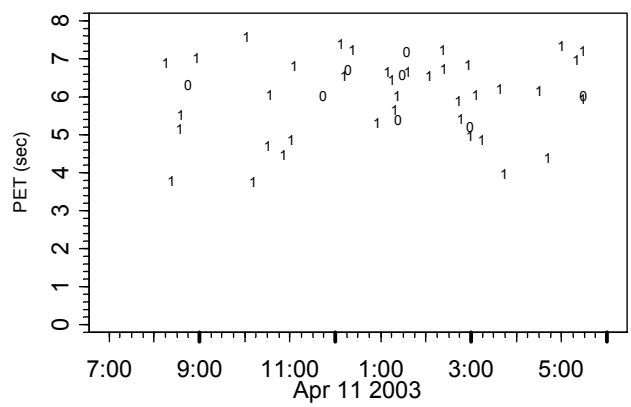

PET variation over time at site 87915 : czSBWB

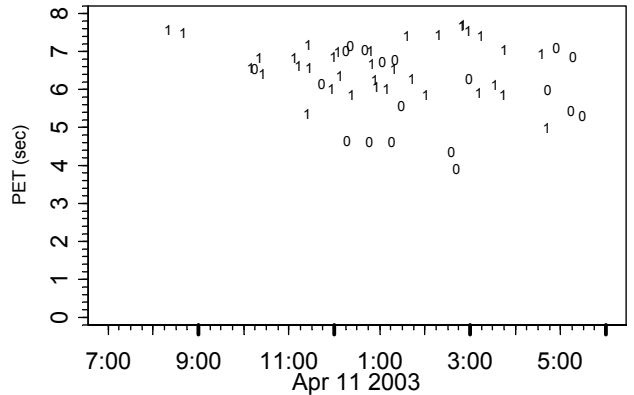

Figure C-10: PET Variation over Time by Conflict Zones at Site 87915 
PET variation over time at site 87923

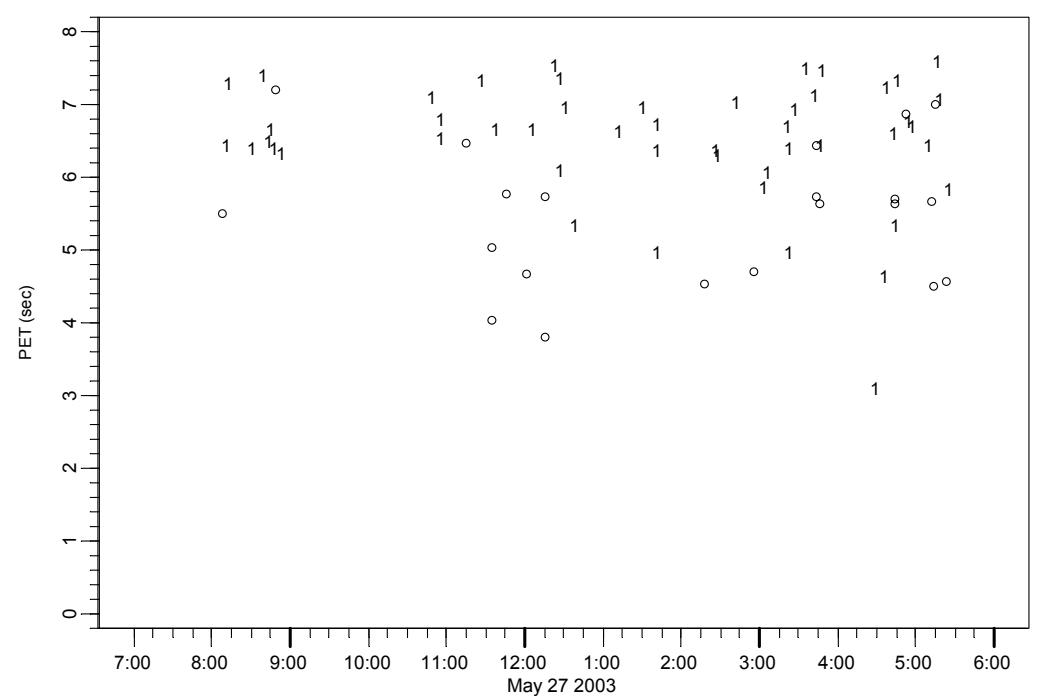

Figure C-11: PET Variation over Time at Site 87923

PET variation over time at site 87923 : czNBEB

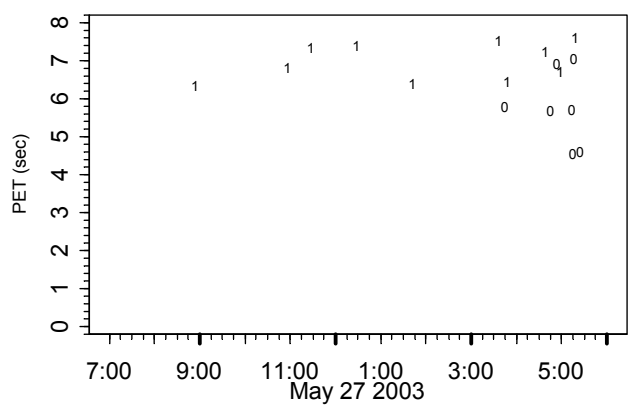

PET variation over time at site 87923 : czSBEB

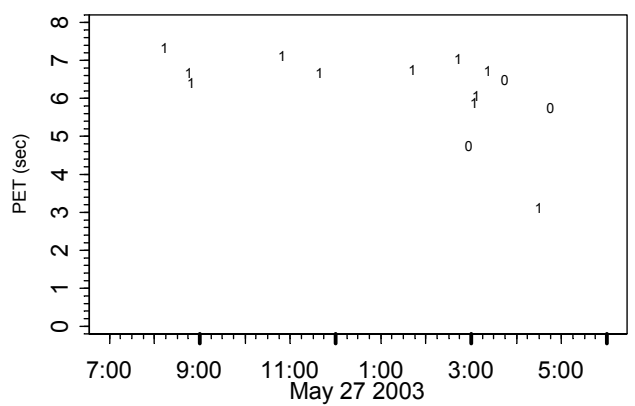

PET variation over time at site 87923 : czNBWB

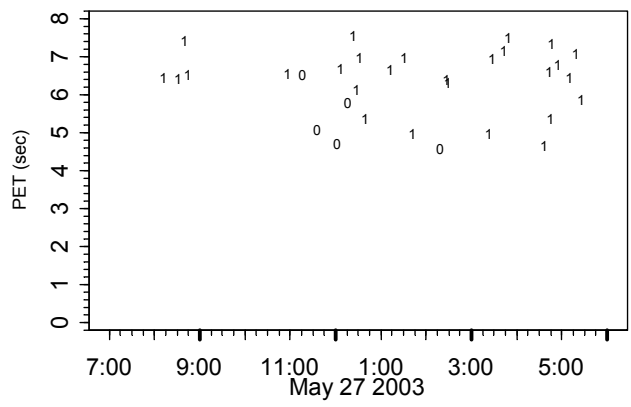

PET variation over time at site 87923 : czSBWB

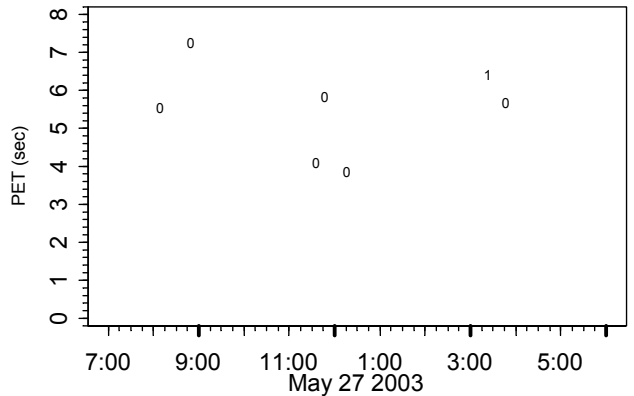

Figure C-12: PET Variation over Time by Conflict Zones at Site 87923 
PET variation over time at site 87930

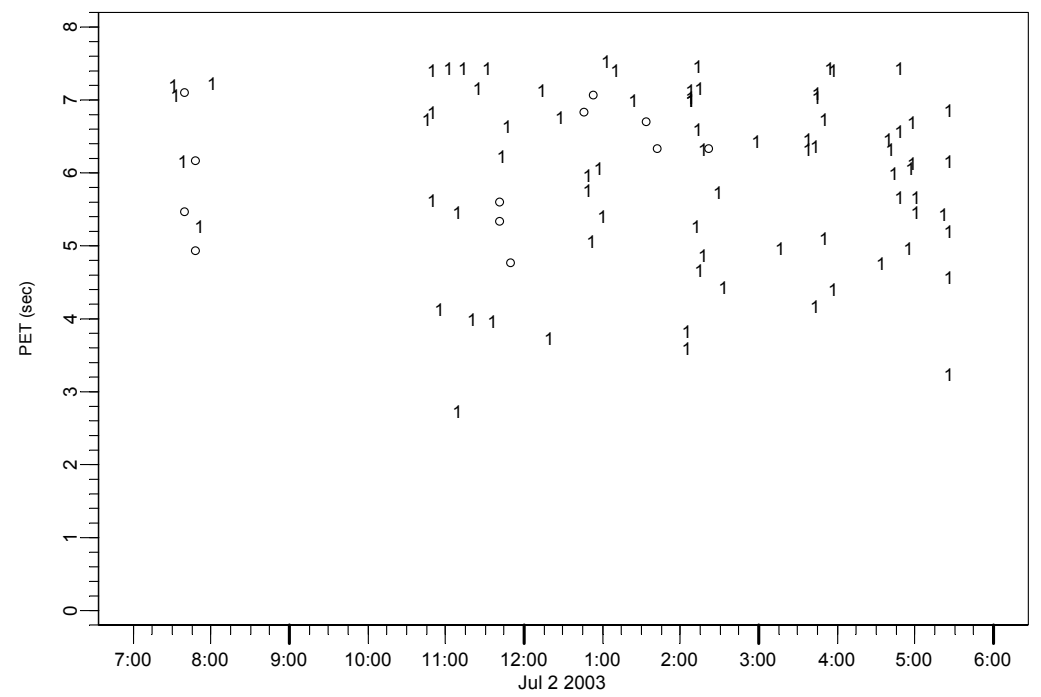

Figure C-13: PET Variation over Time at Site 87930

PET variation over time at site $87930:$ czNBEB

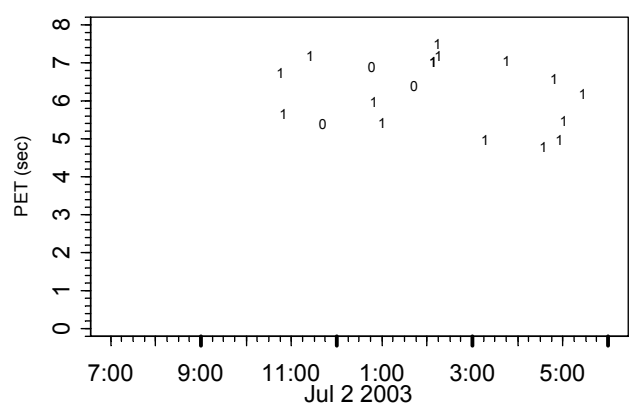

PET variation over time at site 87930 : czSBEB

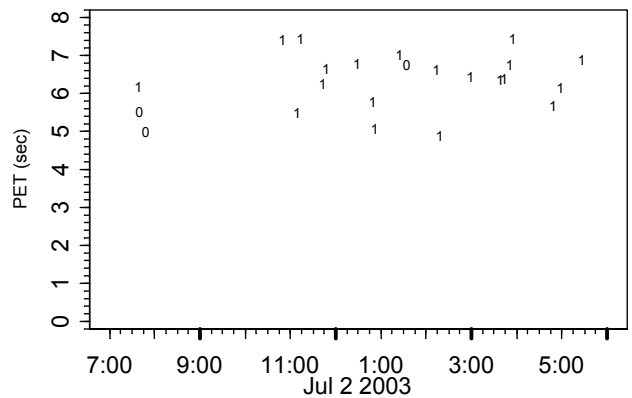

PET variation over time at site $87930:$ czNBWB

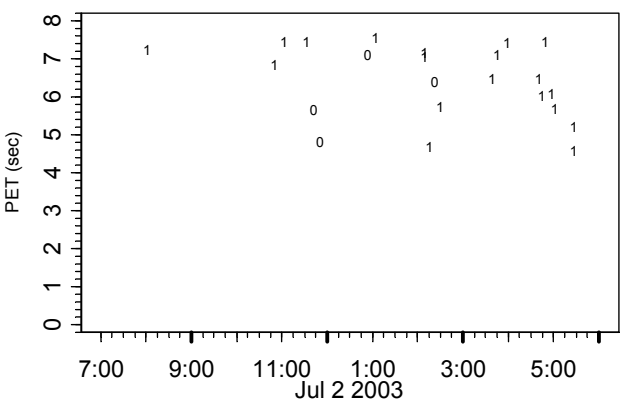

PET variation over time at site 87930 : czSBWB

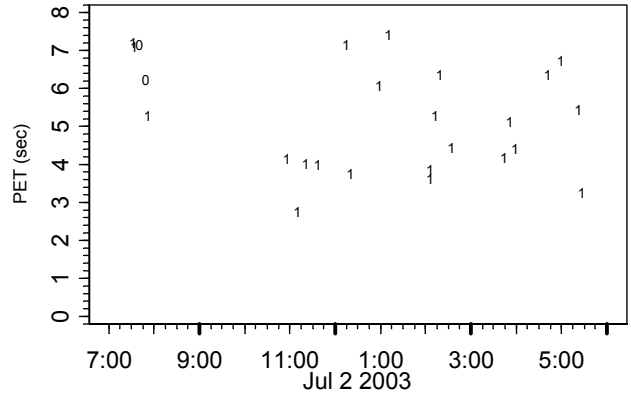

Figure C-14: PET Variation over Time by Conflict Zones at Site 87930 


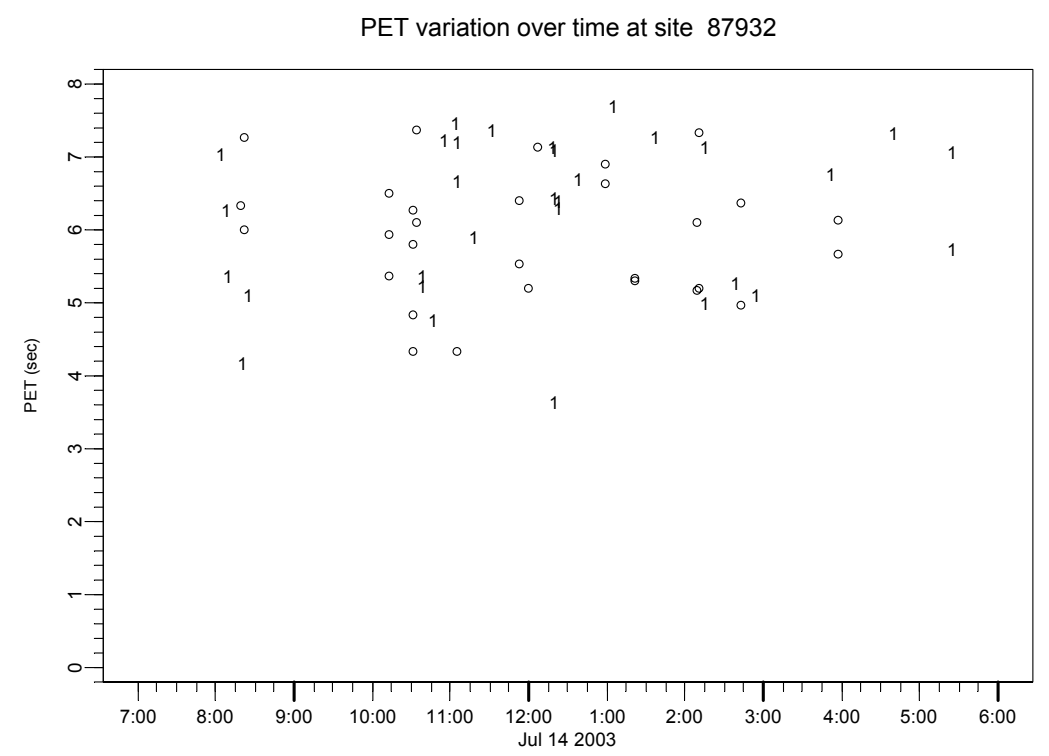

Figure C-15: PET Variation over Time at Site 87932

PET variation over time at site 87932 : czNBEB

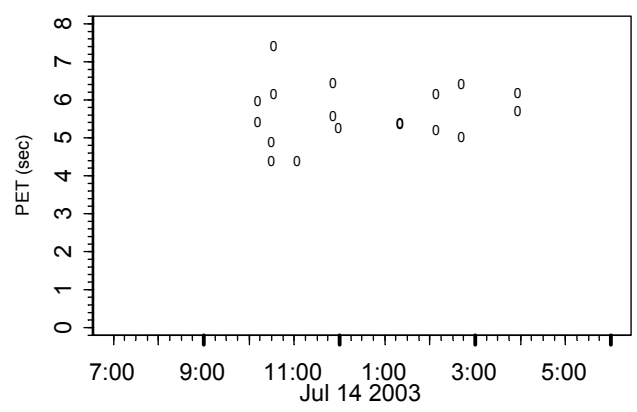

PET variation over time at site 87932 : czSBEB

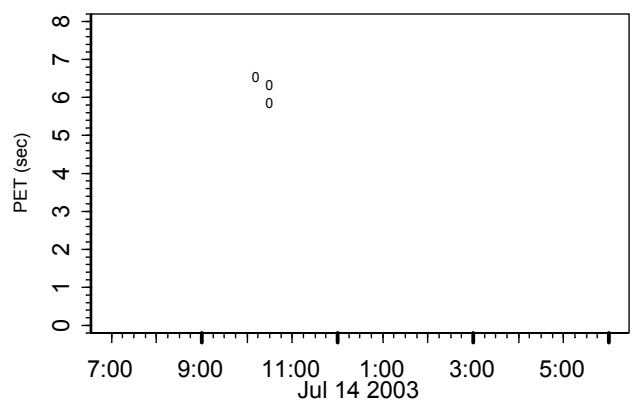

PET variation over time at site 87932 : czNBWB

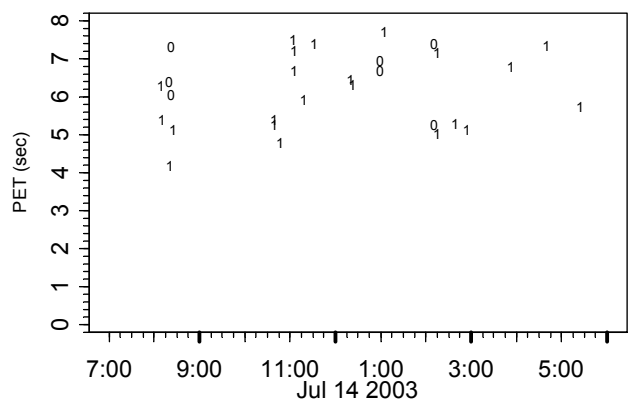

PET variation over time at site 87932 : czSBWB

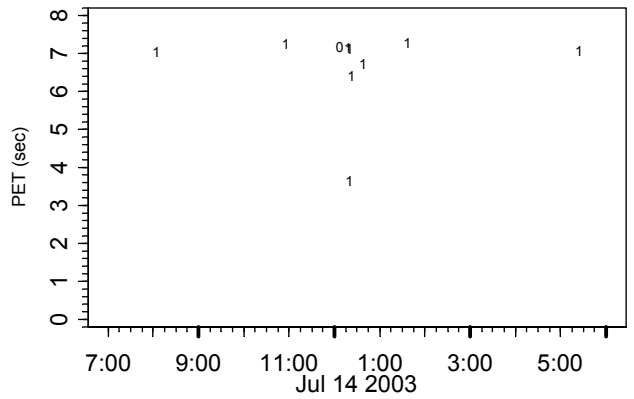

Figure C-16: PET Variation over Time by Conflict Zones at Site 87932 
PET variation over time at site 87933

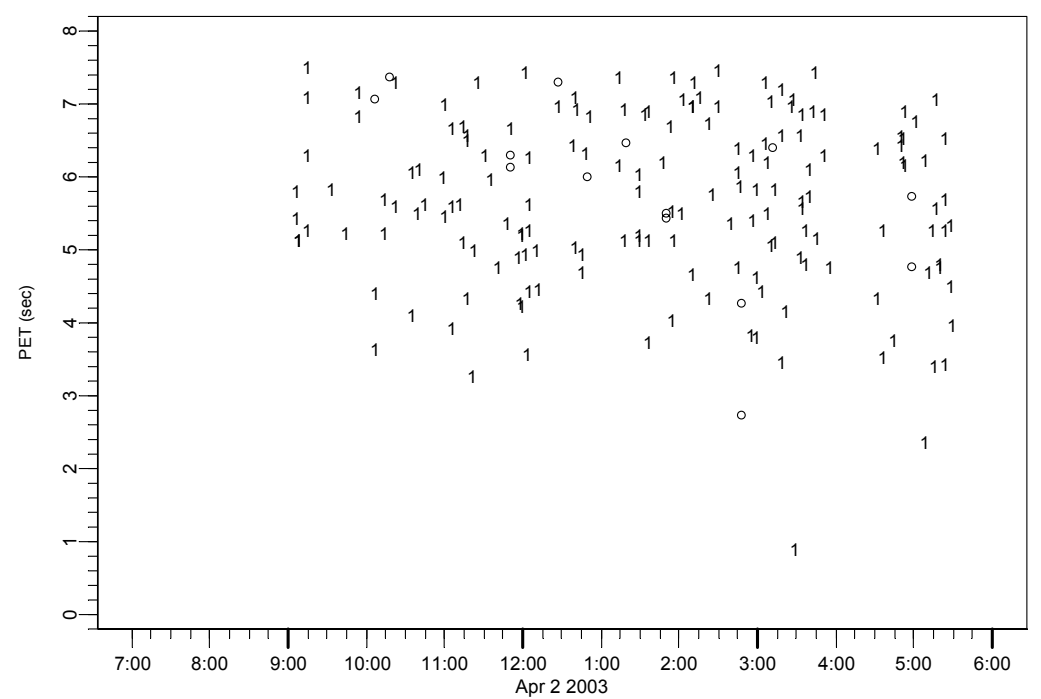

Figure C-17: PET Variation over Time at Site 87933

PET variation over time at site 87933 : czNBEB

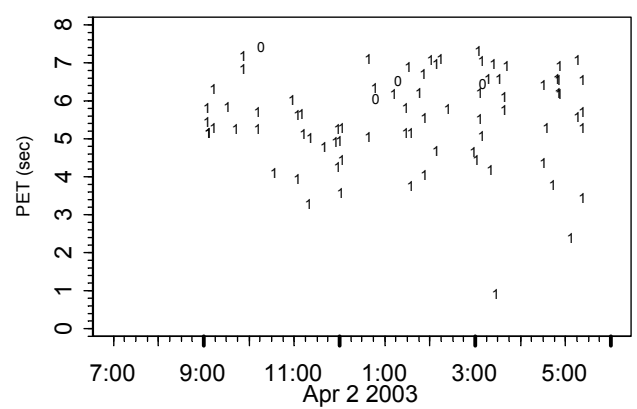

PET variation over time at site 87933 : czSBEB

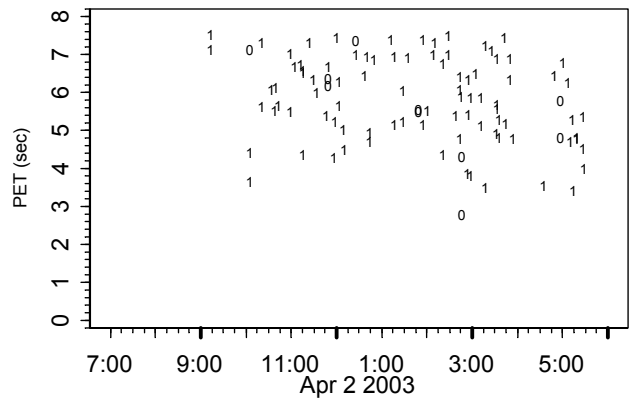

PET variation over time at site $87933:$ czNBWB

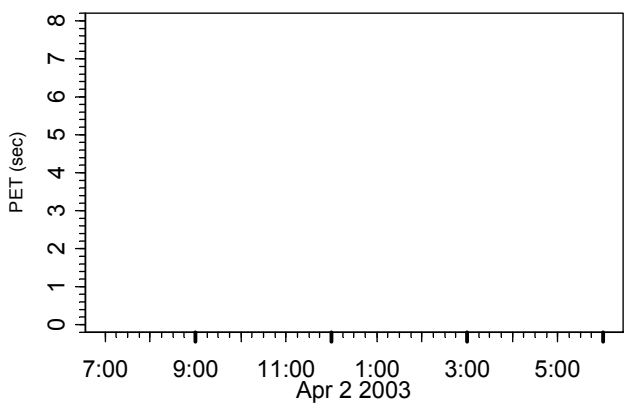

PET variation over time at site 87933 : czSBWB

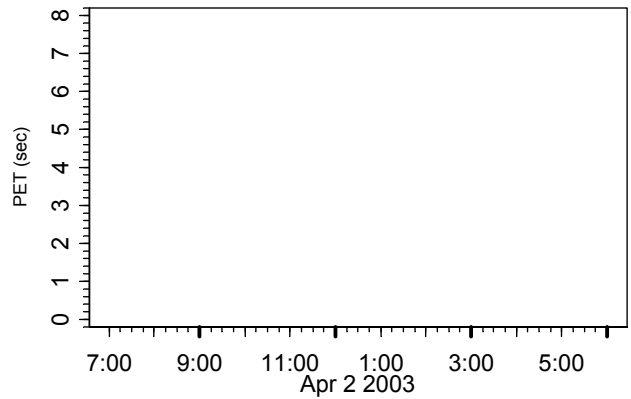

Figure C-18: PET Variation over Time by Conflict Zones at Site 87933 
PET variation over time at site 97901

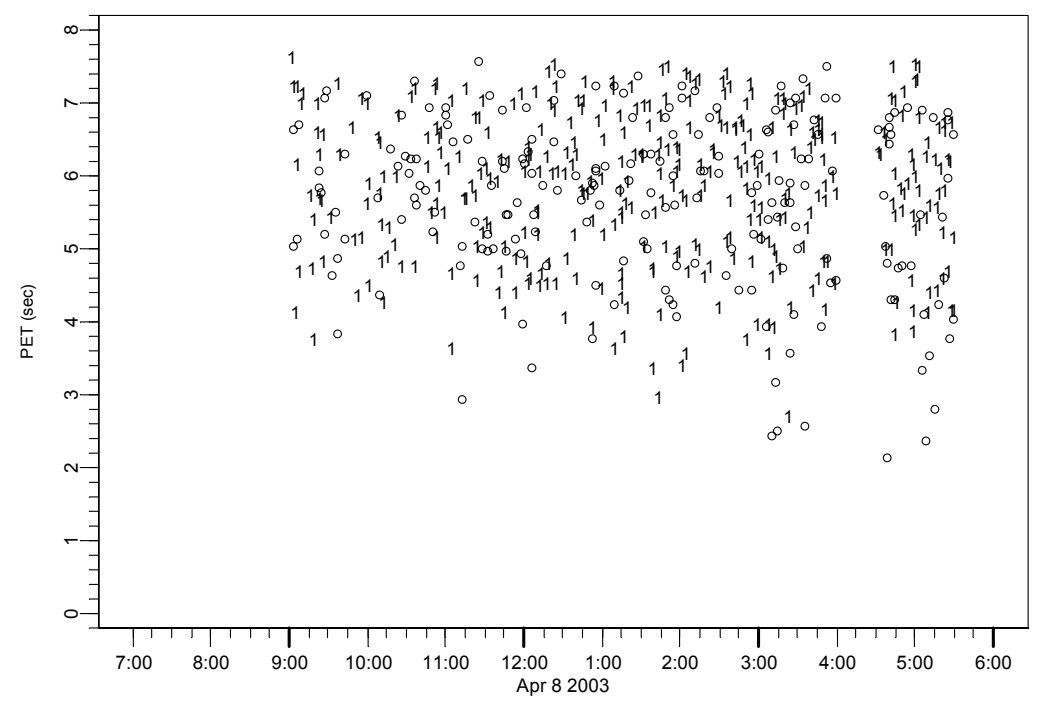

Figure C-19: PET Variation over Time at Site 97901

PET variation over time at site 97901 : czNBEB

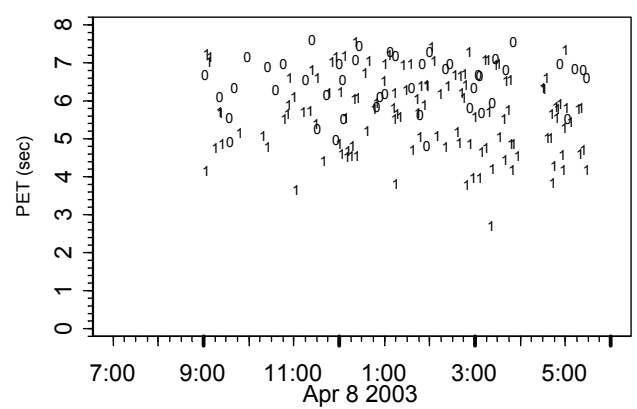

PET variation over time at site 97901 : czSBEB

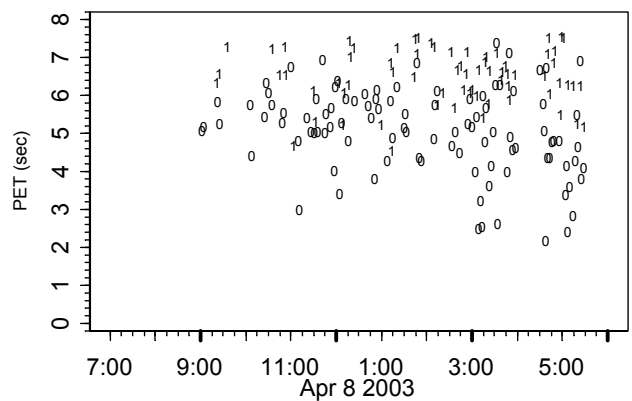

PET variation over time at site 97901 : CzNBWB

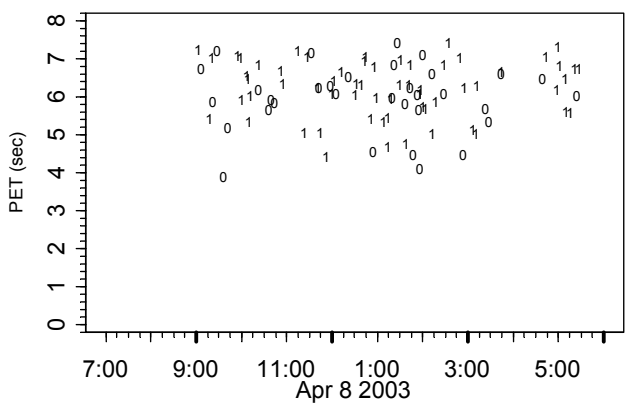

PET variation over time at site 97901 : czSBWB

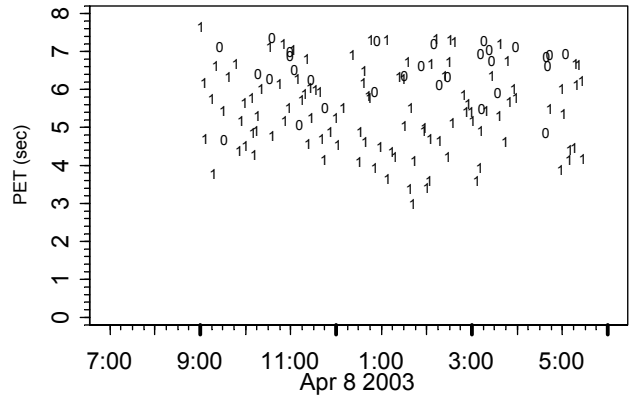

Figure C-20: PET Variation over Time by Conflict Zones at Site 97901 
PET variation over time at site 97903

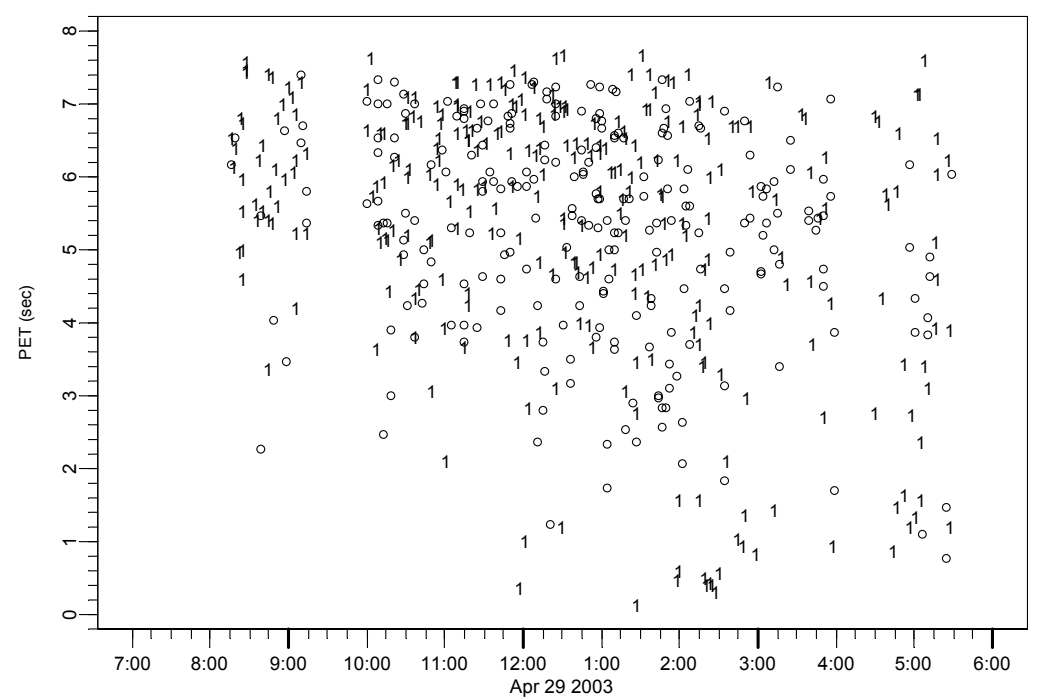

Figure C-21: PET Variation over Time at Site 97903

PET variation over time at site 97903 : czNBEB

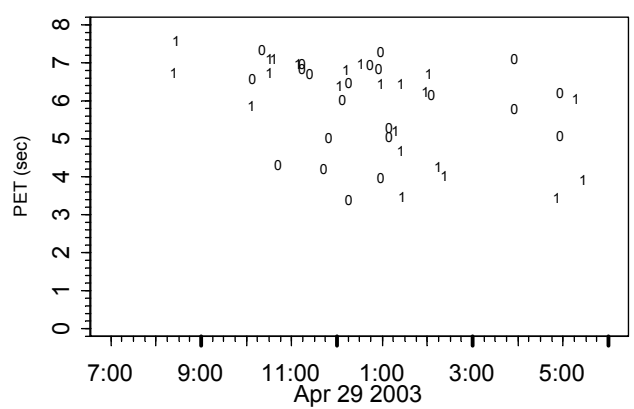

PET variation over time at site 97903 : czSBEB

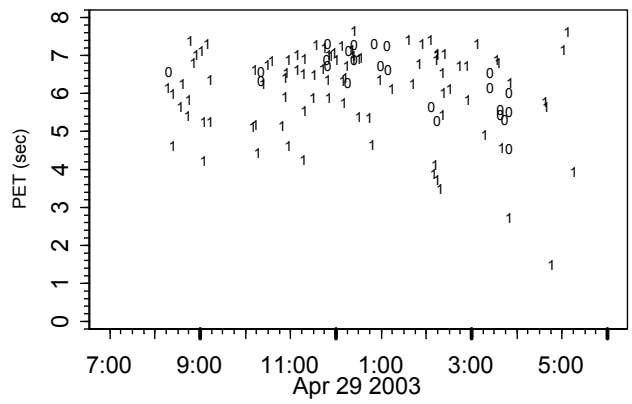

PET variation over time at site 97903 : czNBWB

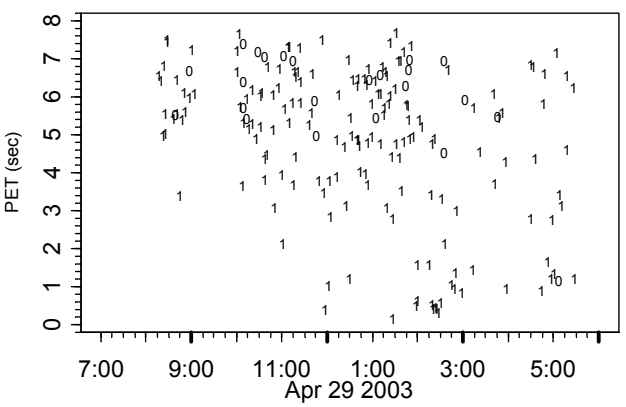

PET variation over time at site 97903 : czSBWB

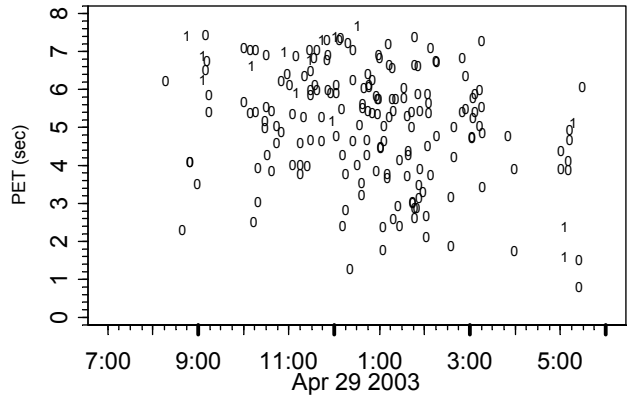

Figure C-22: PET Variation over Time by Conflict Zones at Site 97903 


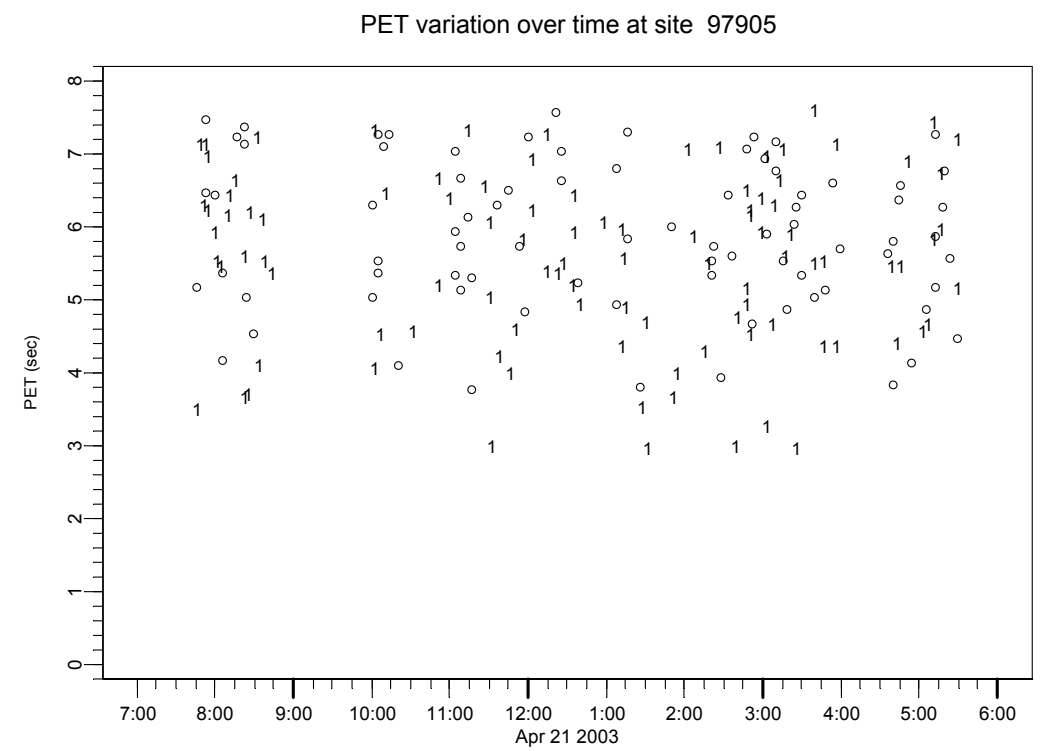

Figure C-23: PET Variation over Time at Site 97905

PET variation over time at site 97905 : czNBEB

PET variation over time at site $97905:$ czNBWB
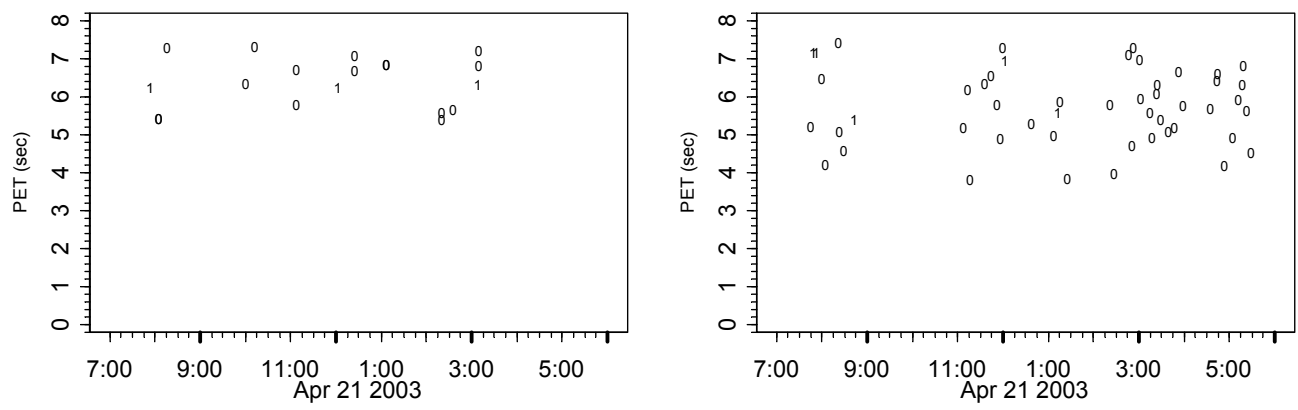

PET variation over time at site 97905 : czSBEB

PET variation over time at site 97905 : czSBWB
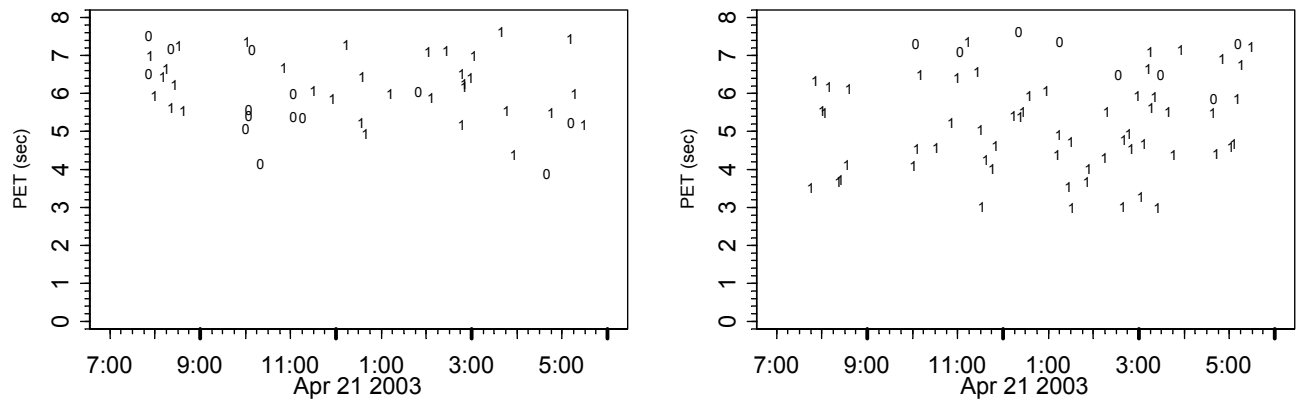

Figure C-24: PET Variation over Time by Conflict Zones at Site 97905 
PET variation over time at site 97911

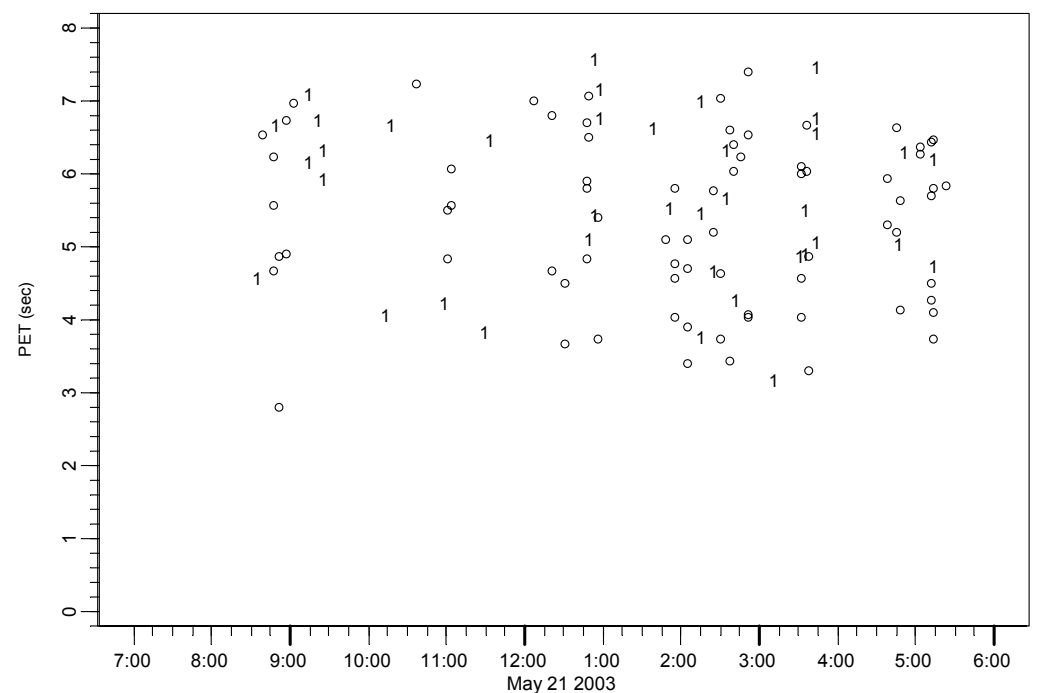

Figure C-25: PET Variation over Time at Site 97911

PET variation over time at site 97911 : czNBEB

PET variation over time at site 97911 : czNBWB
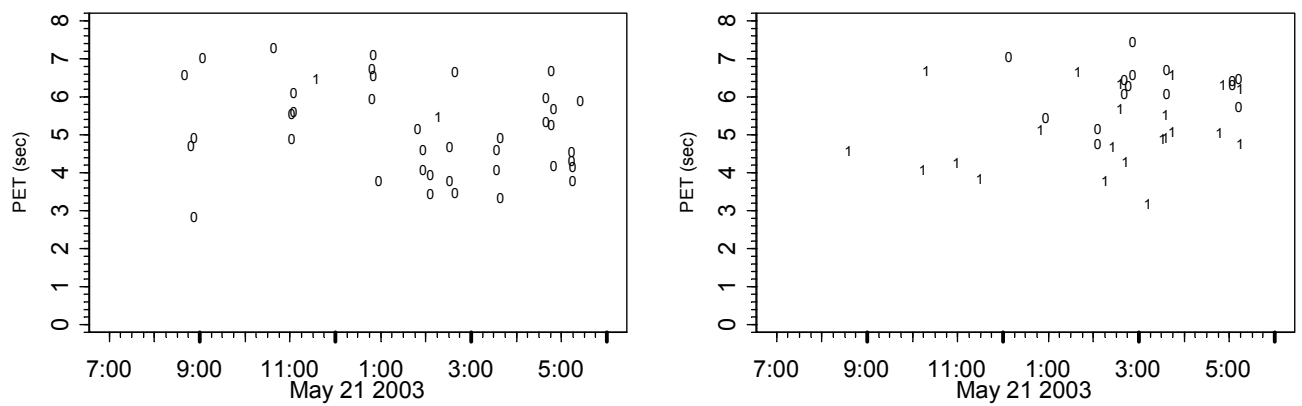

PET variation over time at site 97911 : czSBEB

PET variation over time at site 97911 : czSBWB
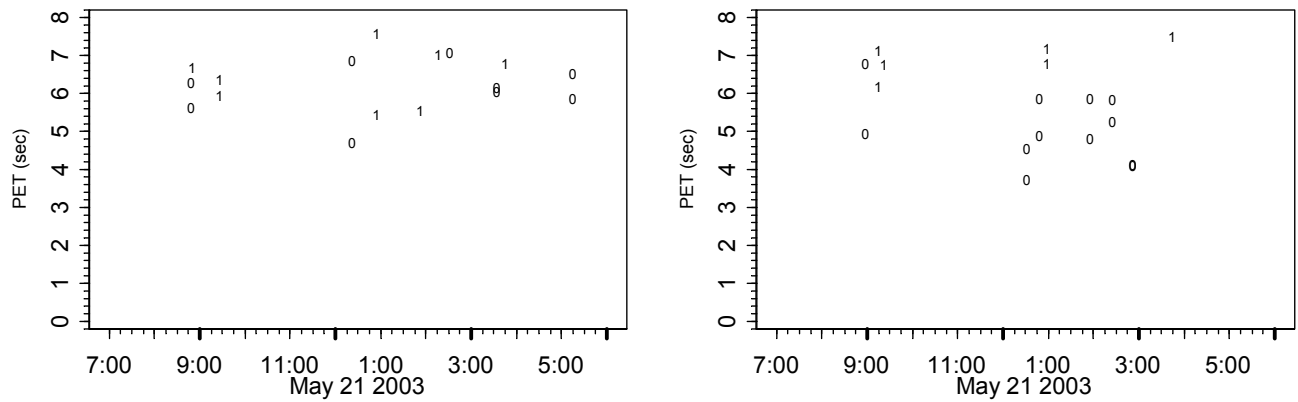

Figure C-26: PET Variation over Time by Conflict Zones at Site 97911 
PET variation over time at site 97920

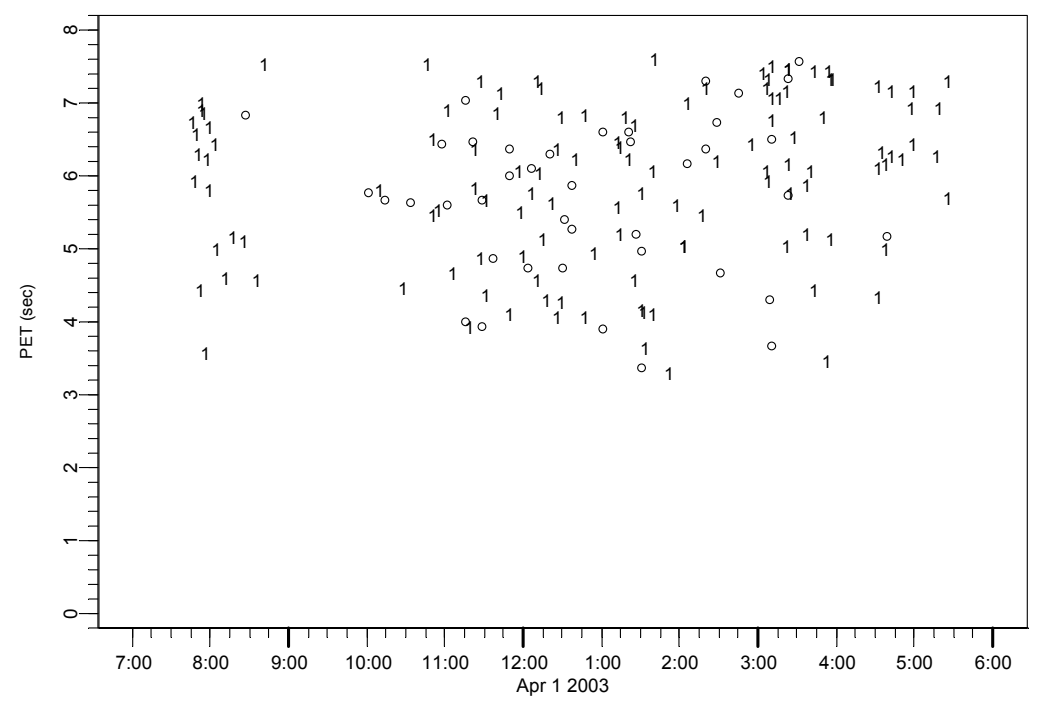

Figure C-27: PET Variation over Time at Site 97920

PET variation over time at site 97920 : czNBEB

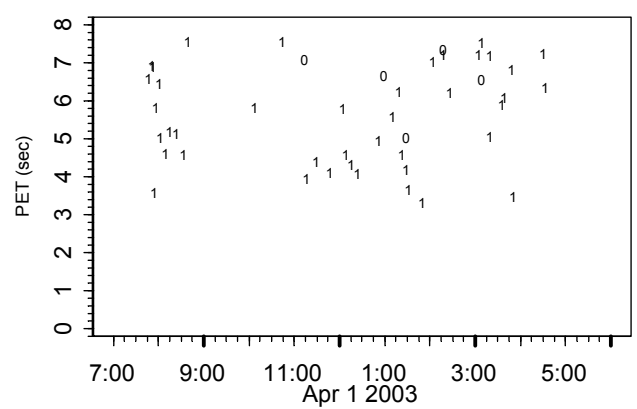

PET variation over time at site 97920 : czSBEB

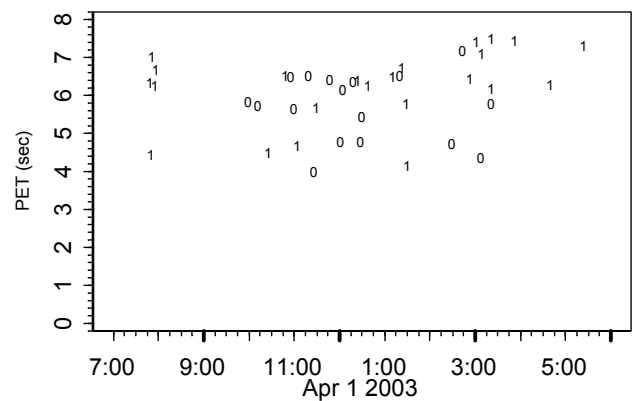

PET variation over time at site 97920 : czNBWB

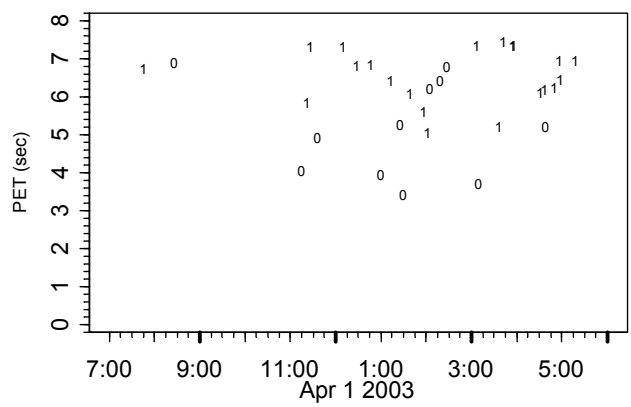

PET variation over time at site 97920 : czSBWB

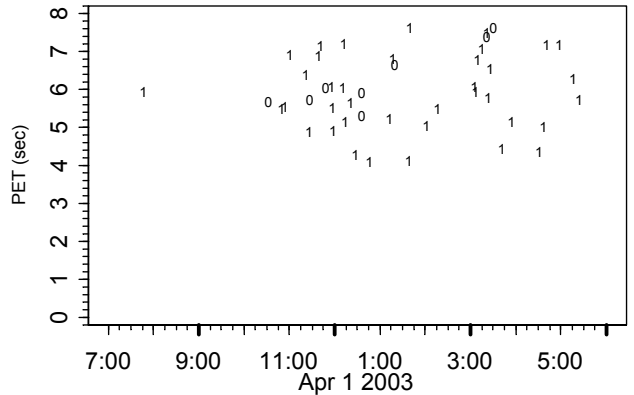

Figure C-28: PET Variation over Time by Conflict Zones at Site 97920 
PET variation over time at site 97922

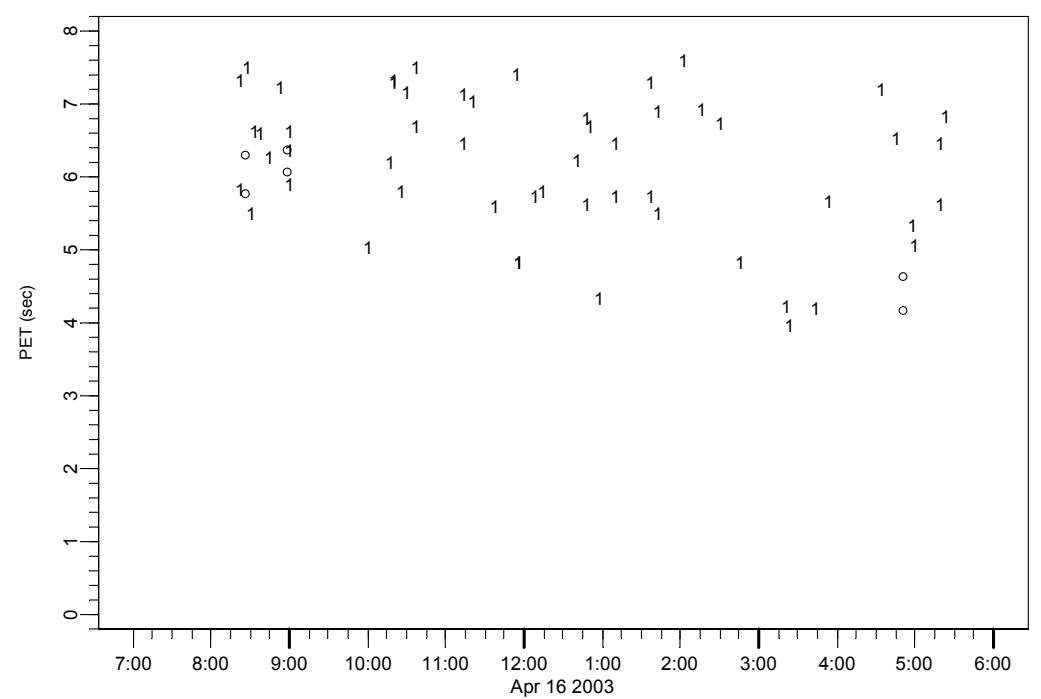

Figure C-29: PET Variation over Time at Site 97922

PET variation over time at site 97922 : czNBEB

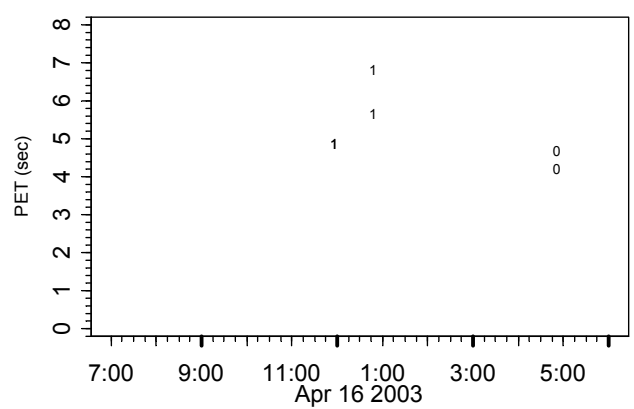

PET variation over time at site 97922 : czSBEB

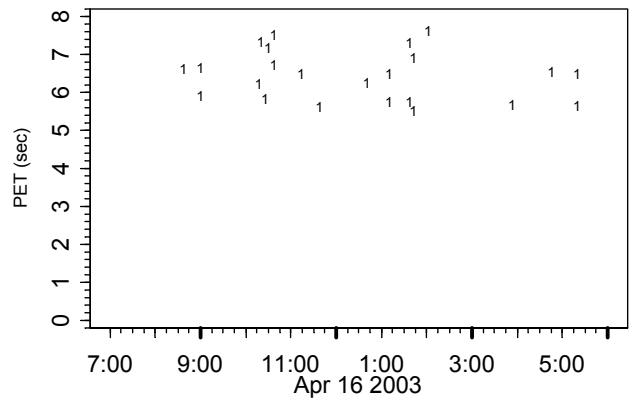

PET variation over time at site $97922:$ czNBWB

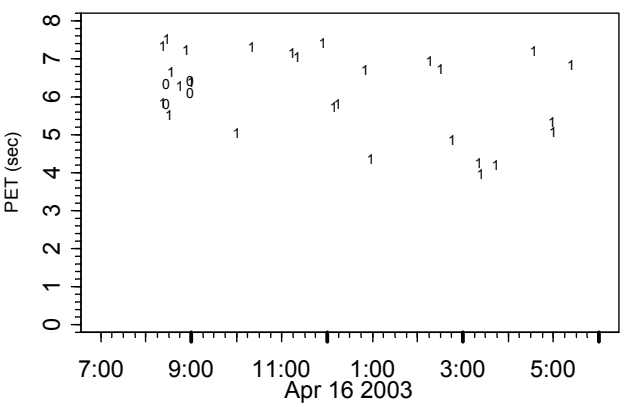

PET variation over time at site 97922 : czSBWB

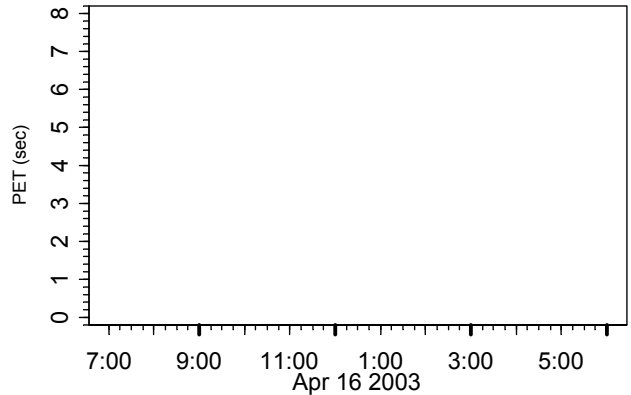

Figure C-30: PET Variation over Time by Conflict Zones at Site 97922 


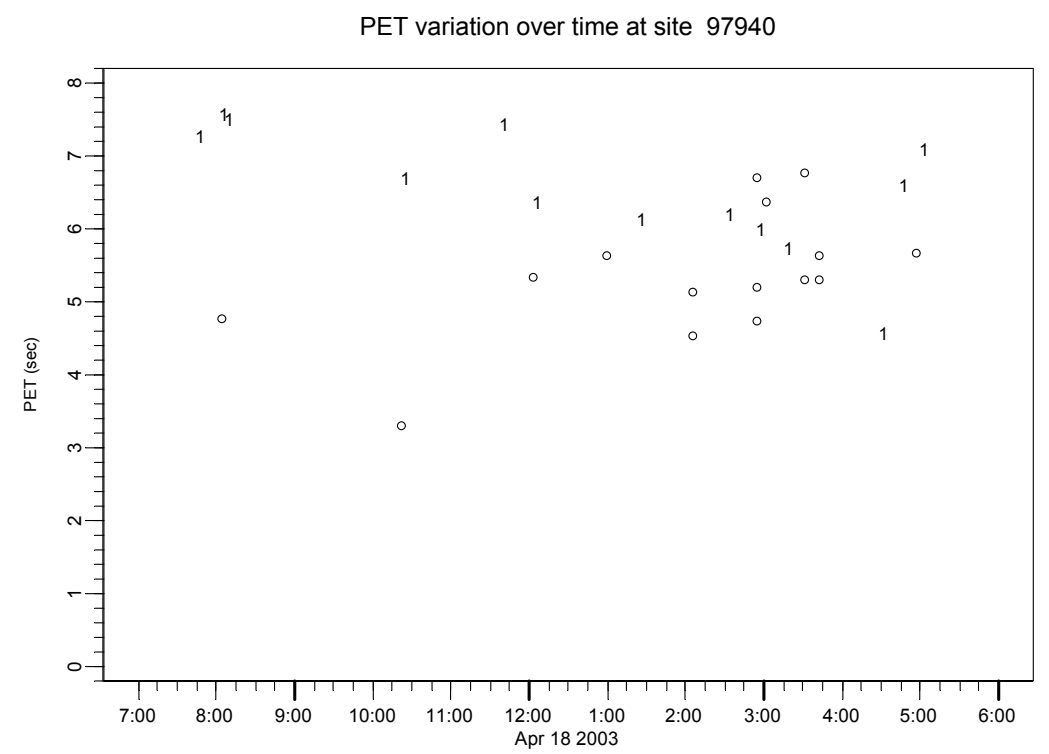

Figure C-31: PET Variation over Time at Site 97940
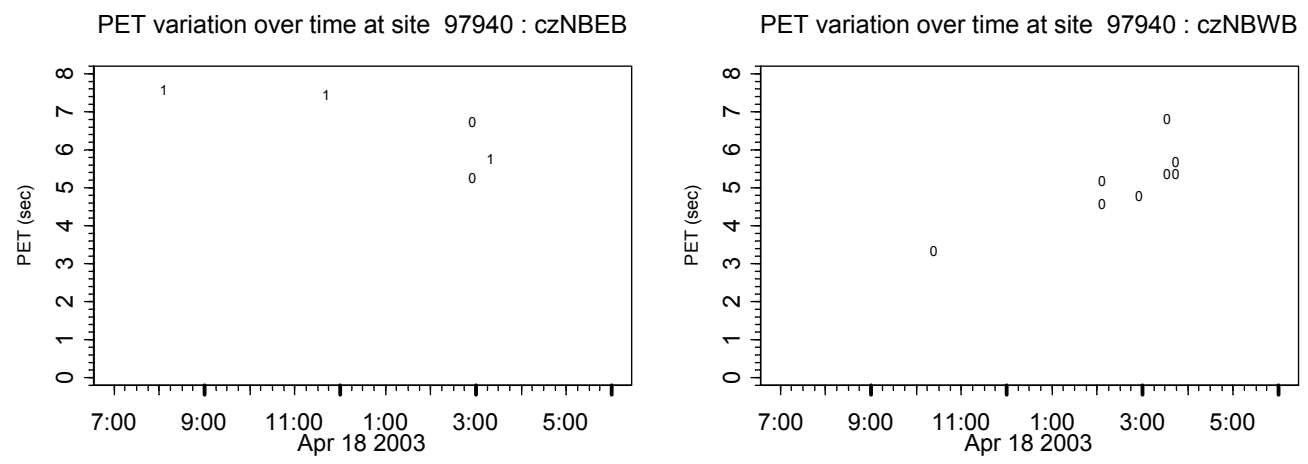

PET variation over time at site 97940 : czSBEB

PET variation over time at site 97940 : czSBWB
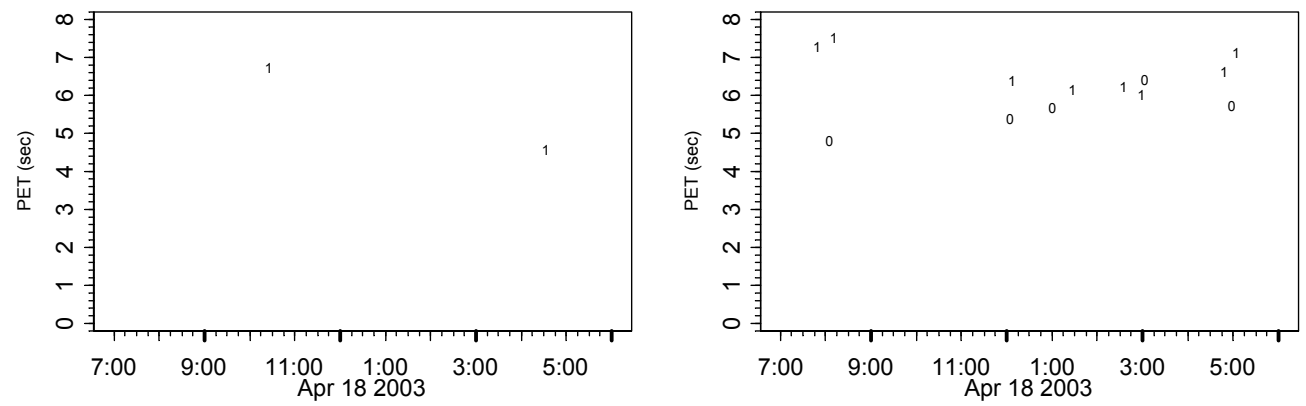

Figure C-32: PET Variation over Time by Conflict Zones at Site 97940 

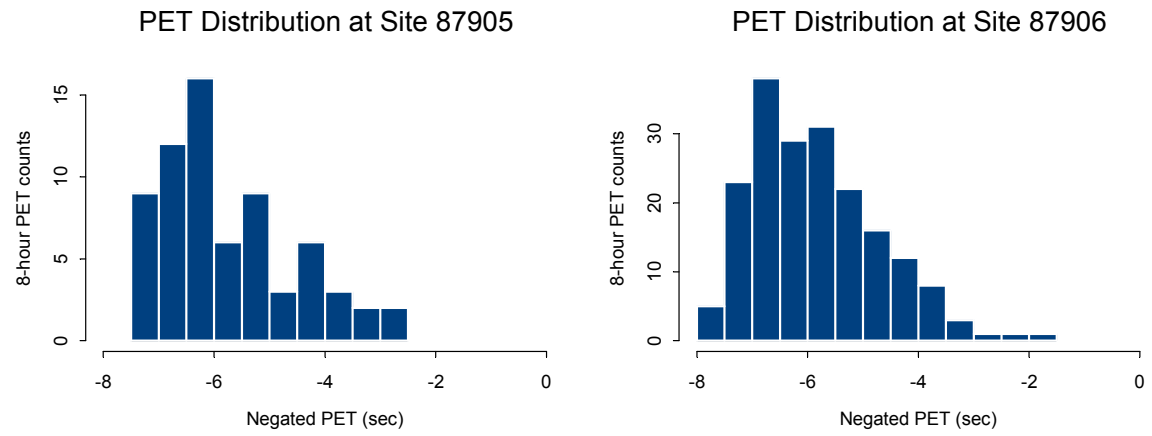

PET Distribution at Site 87907

PET Distribution at Site 87909
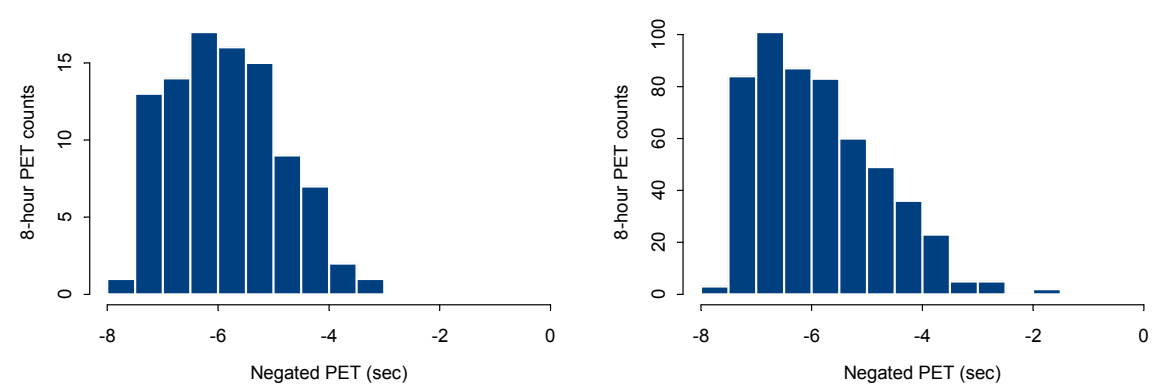

Figure C-33: Distributions of Negated PETs (1)

PET Distribution at Site 87915

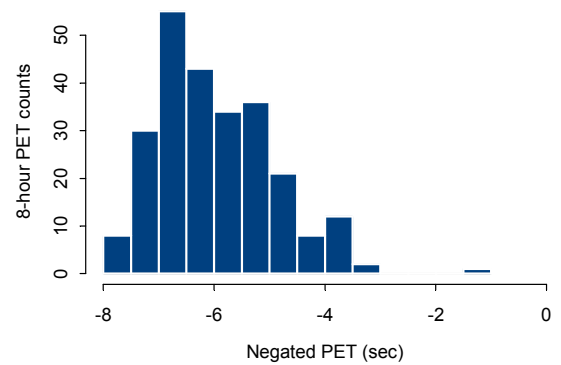

PET Distribution at Site 87930

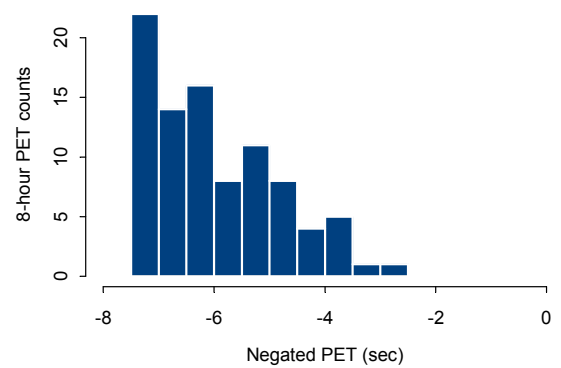

PET Distribution at Site 87923

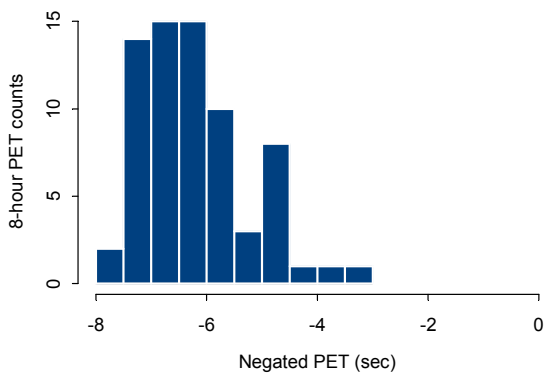

PET Distribution at Site 87932

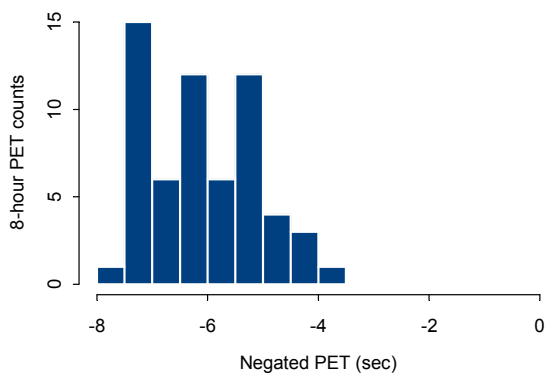

Figure C-34: Distributions of Negated PETs (2) 
PET Distribution at Site 87933

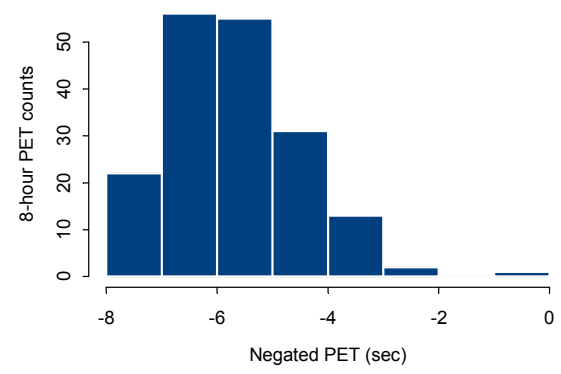

PET Distribution at Site 97903

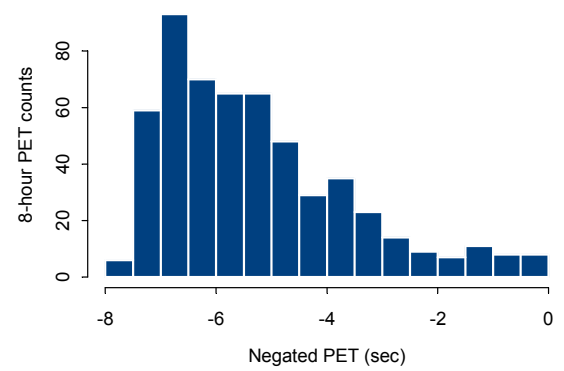

PET Distribution at Site 97901

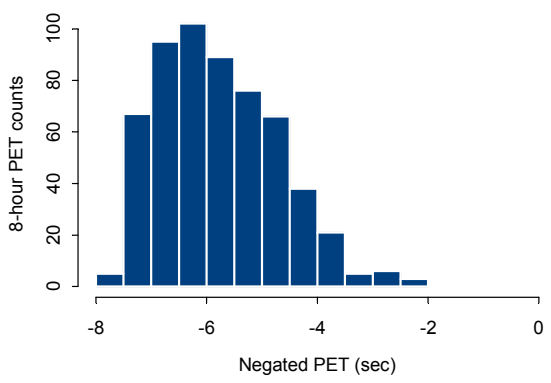

PET Distribution at Site 97905

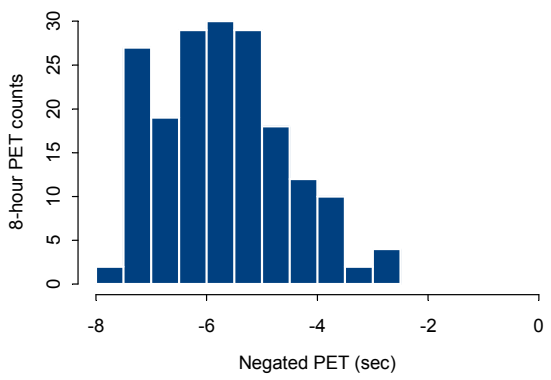

Figure C-35: Distributions of Negated PETs (3)

PET Distribution at Site 97911

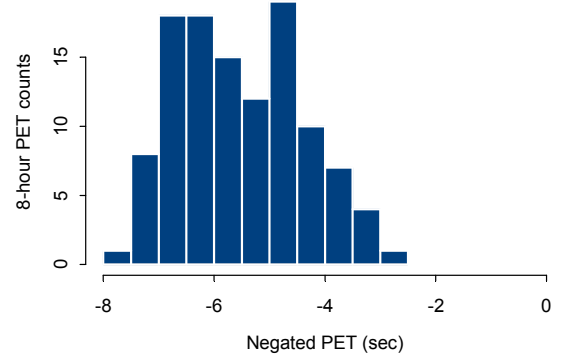

PET Distribution at Site 97922

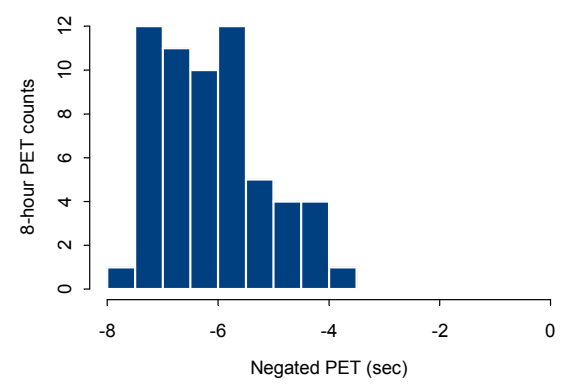

PET Distribution at Site 97920

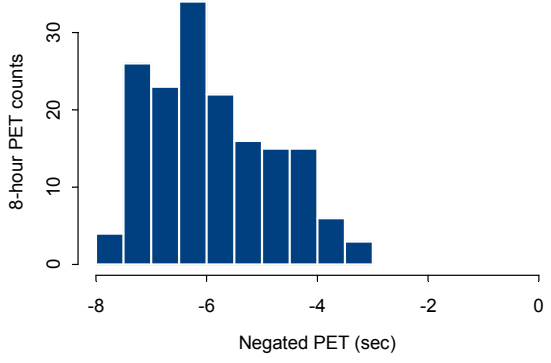

PET Distribution at Site 97940

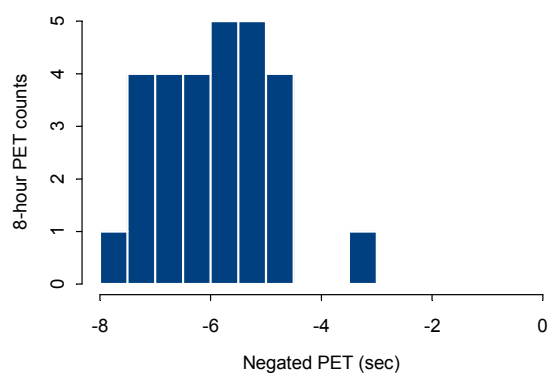

Figure C-36: Distributions of Negated PETs (4) 


\section{APPENDIX D TRAFFIC VOLUME}

In this appendix, 15-minute traffic volume variations for total entering volumes and conflicting volumes are plotted for all the studied sites. A gradual increasing trend of traffic volume can observed for most locations. 

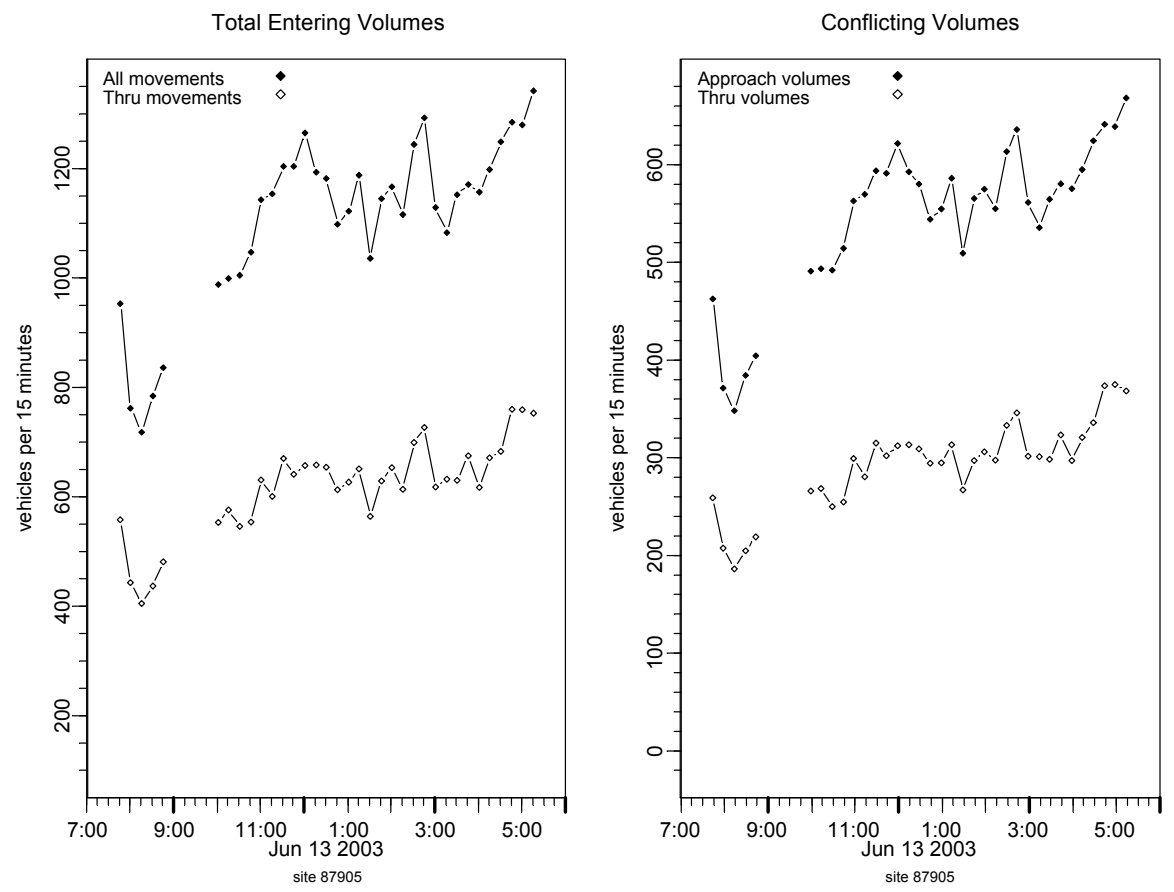

Figure D-1: 15-minute Volume Variation over Time at Site 87905
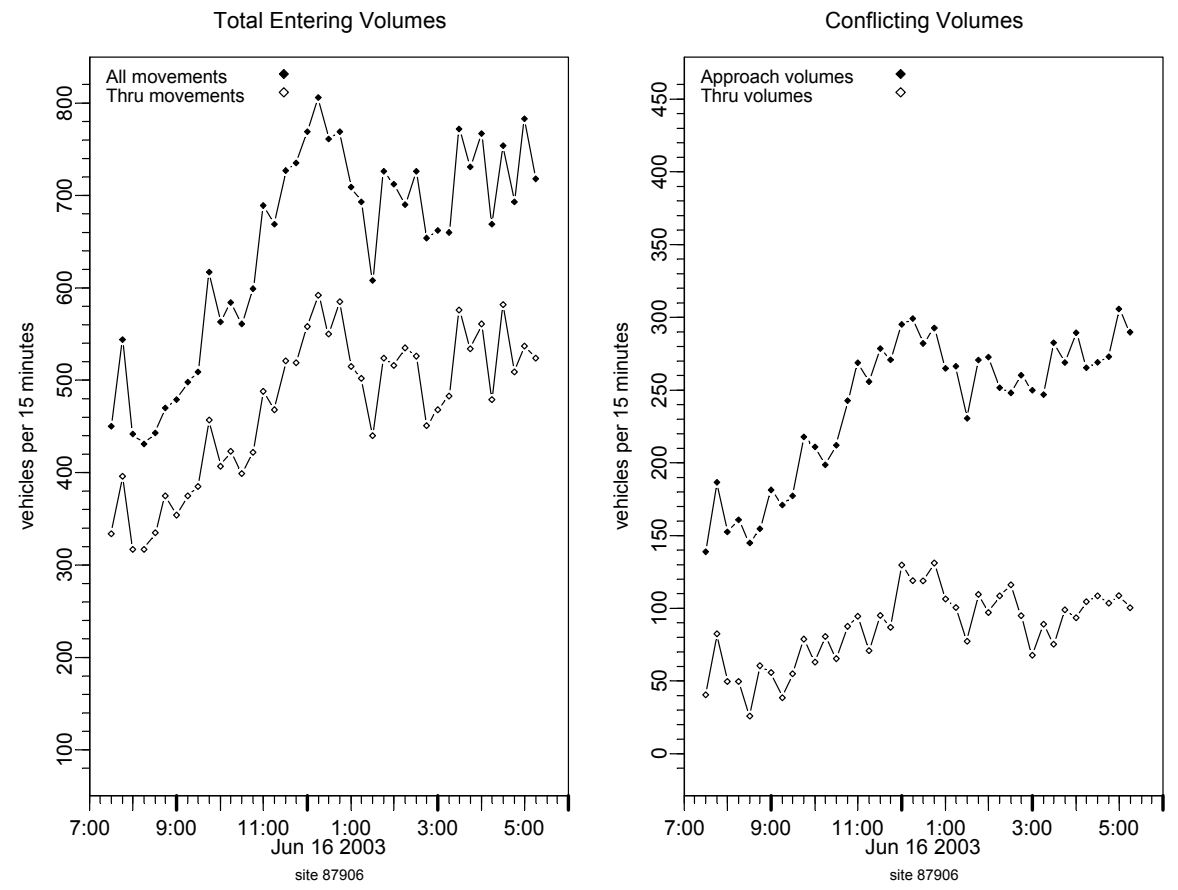

Figure D-2: 15-minute Volume Variation over Time at Site 87906 

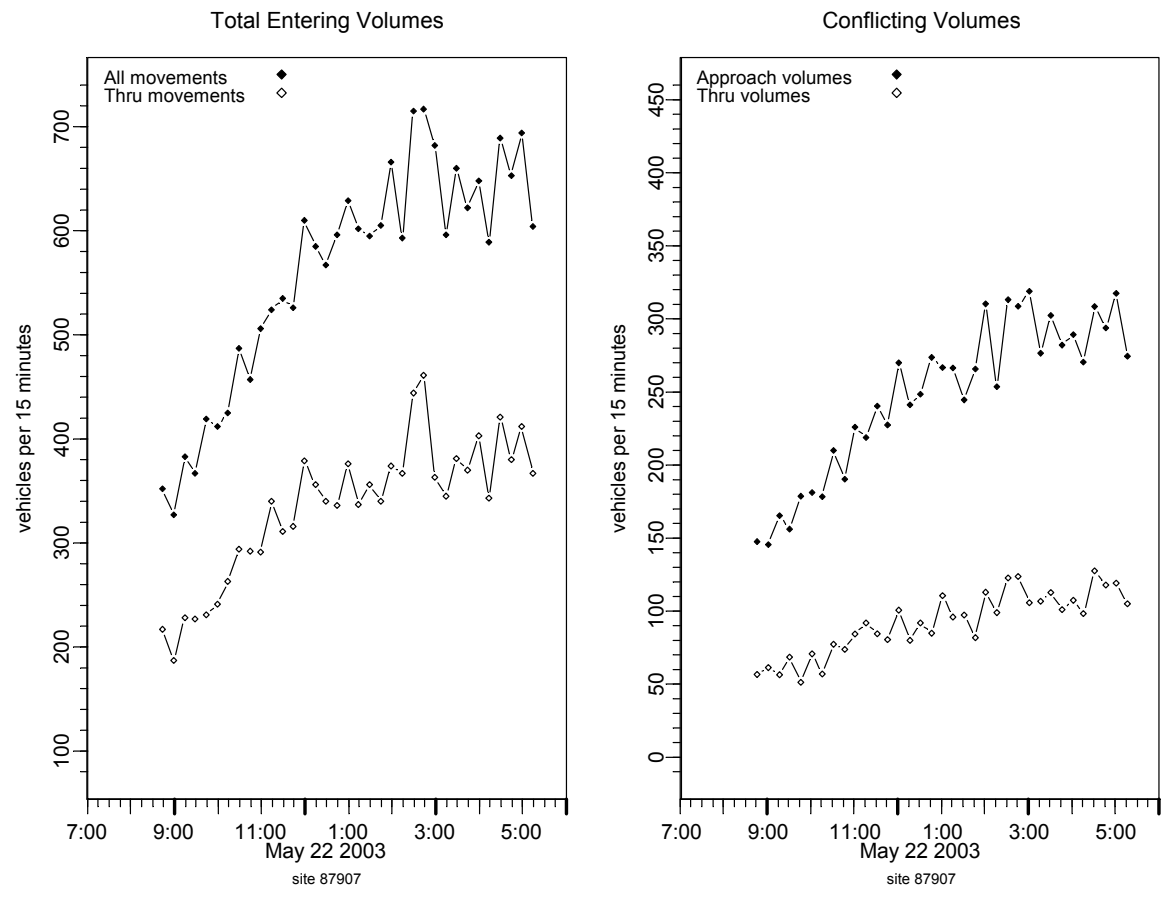

Figure D-3: 15-minute Volume Variation over Time at Site 87907
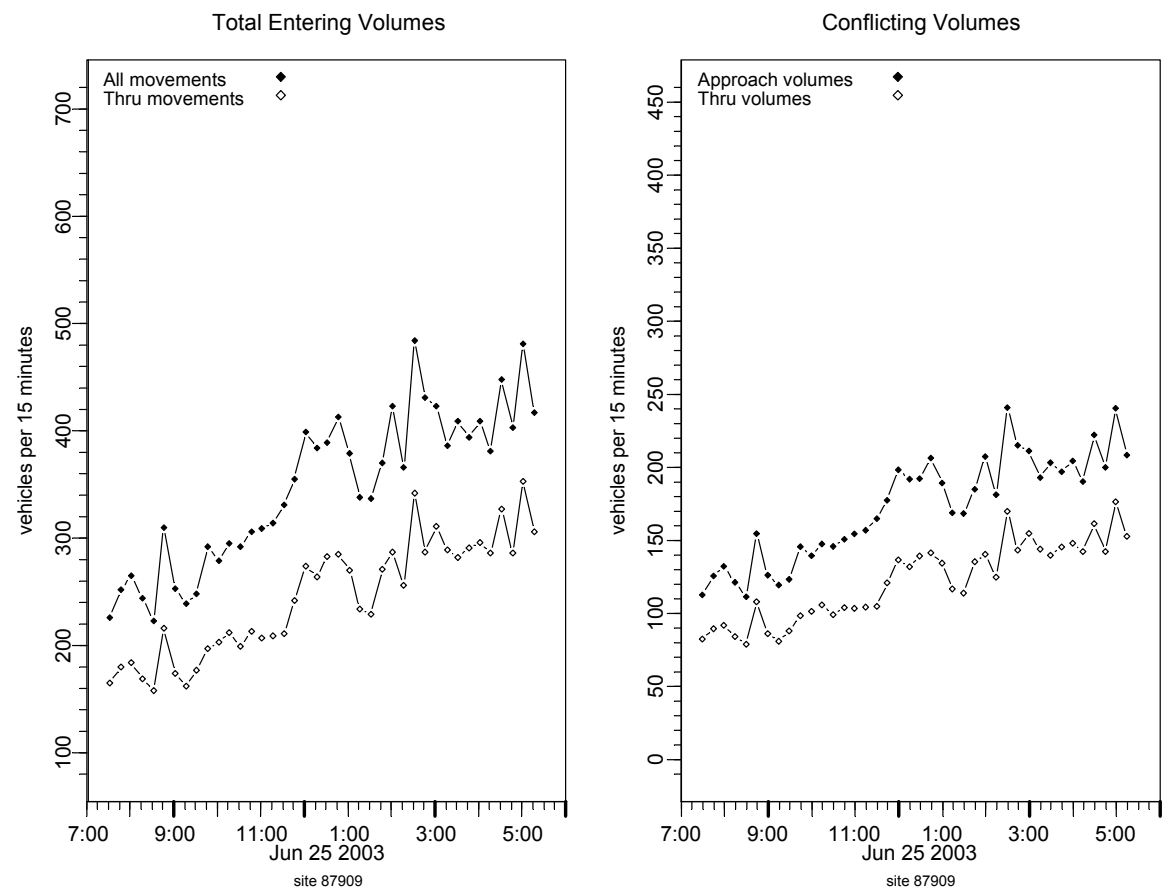

Figure D-4: 15-minute Volume Variation over Time at Site 87909 

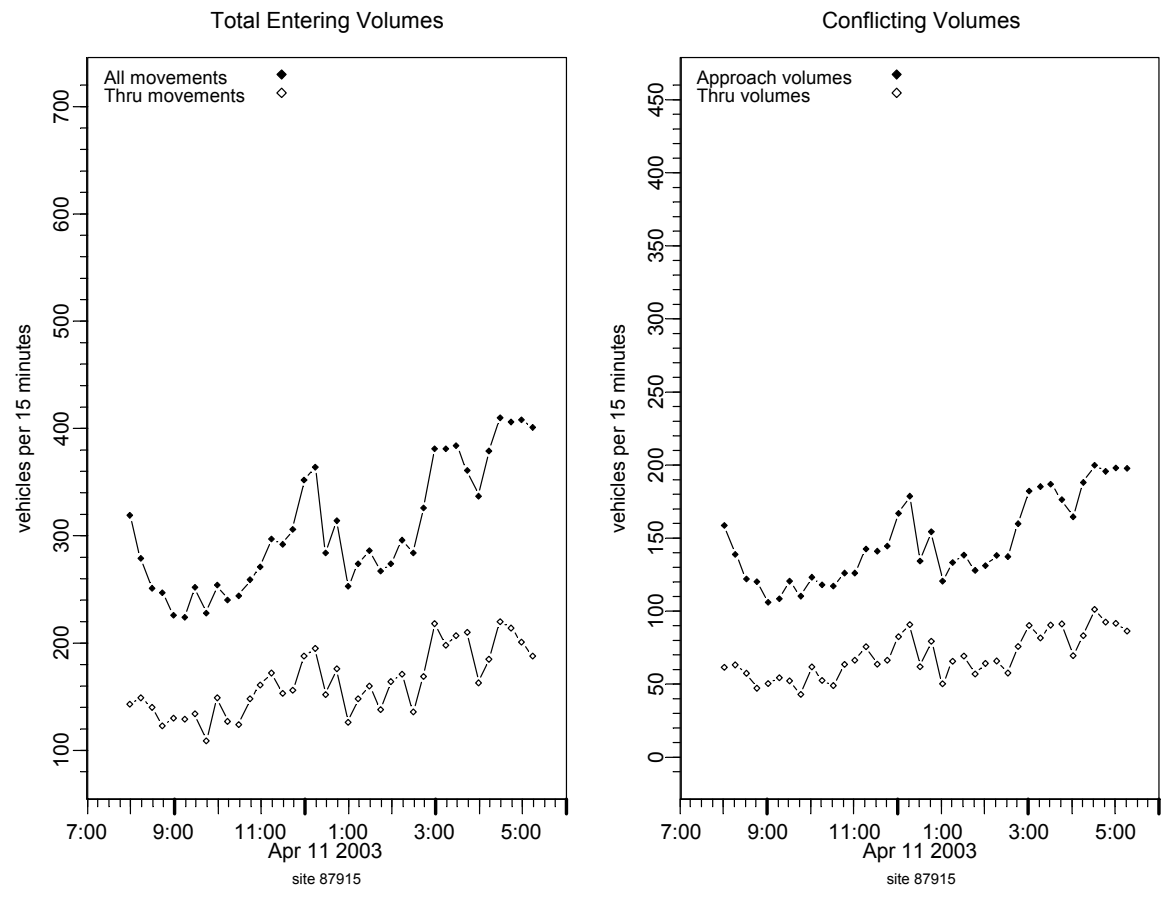

Figure D-5: 15-minute Volume Variation over Time at Site 87915
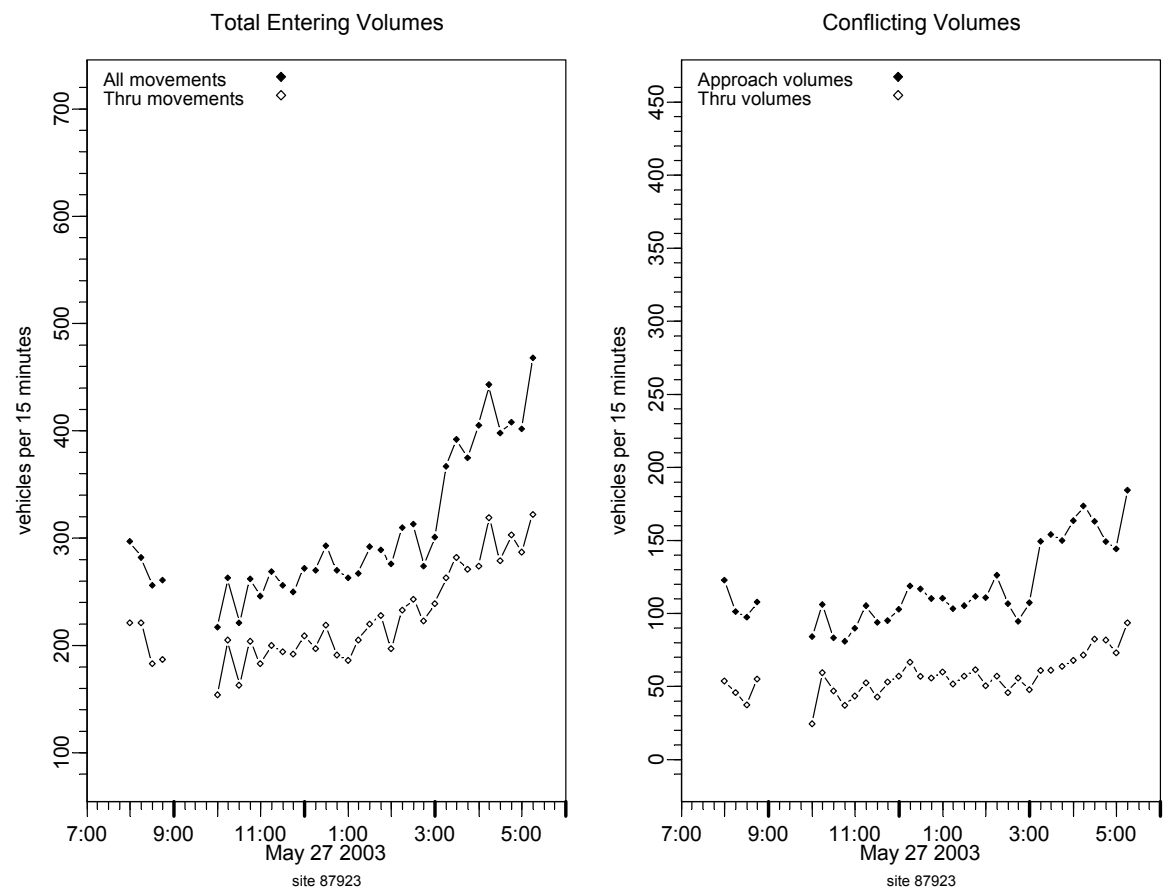

Figure D-6: 15-minute Volume Variation over Time at Site 87923 

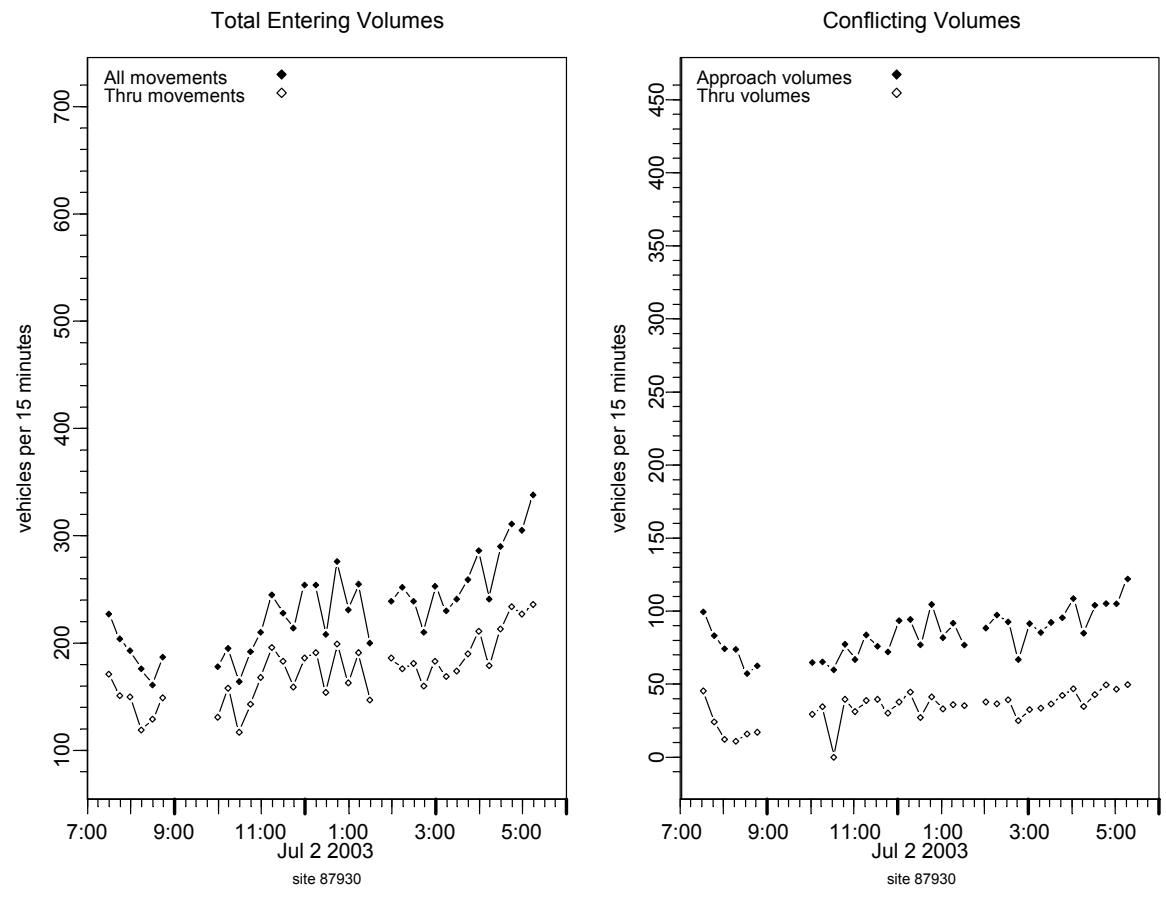

Figure D-7: 15-minute Volume Variation over Time at Site 87930
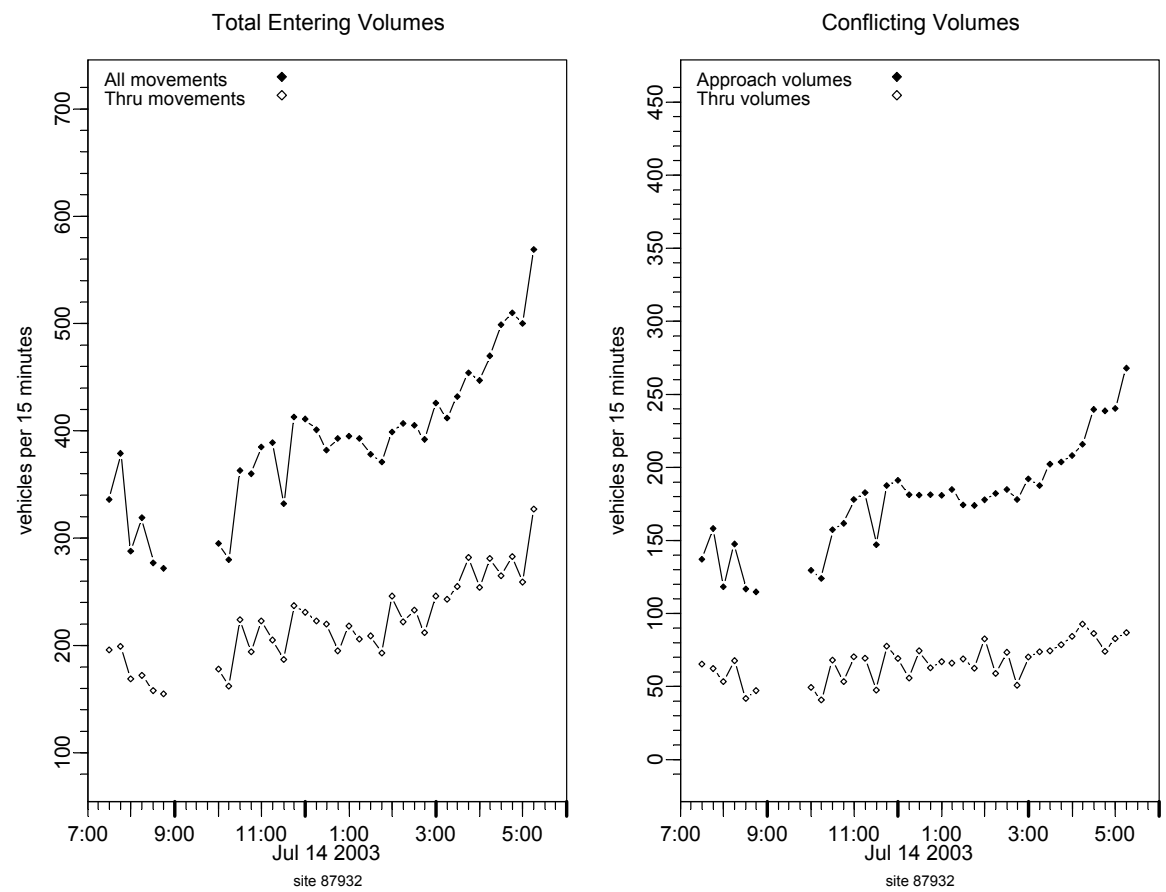

Figure D-8: 15-minute Volume Variation over Time at Site 87932 

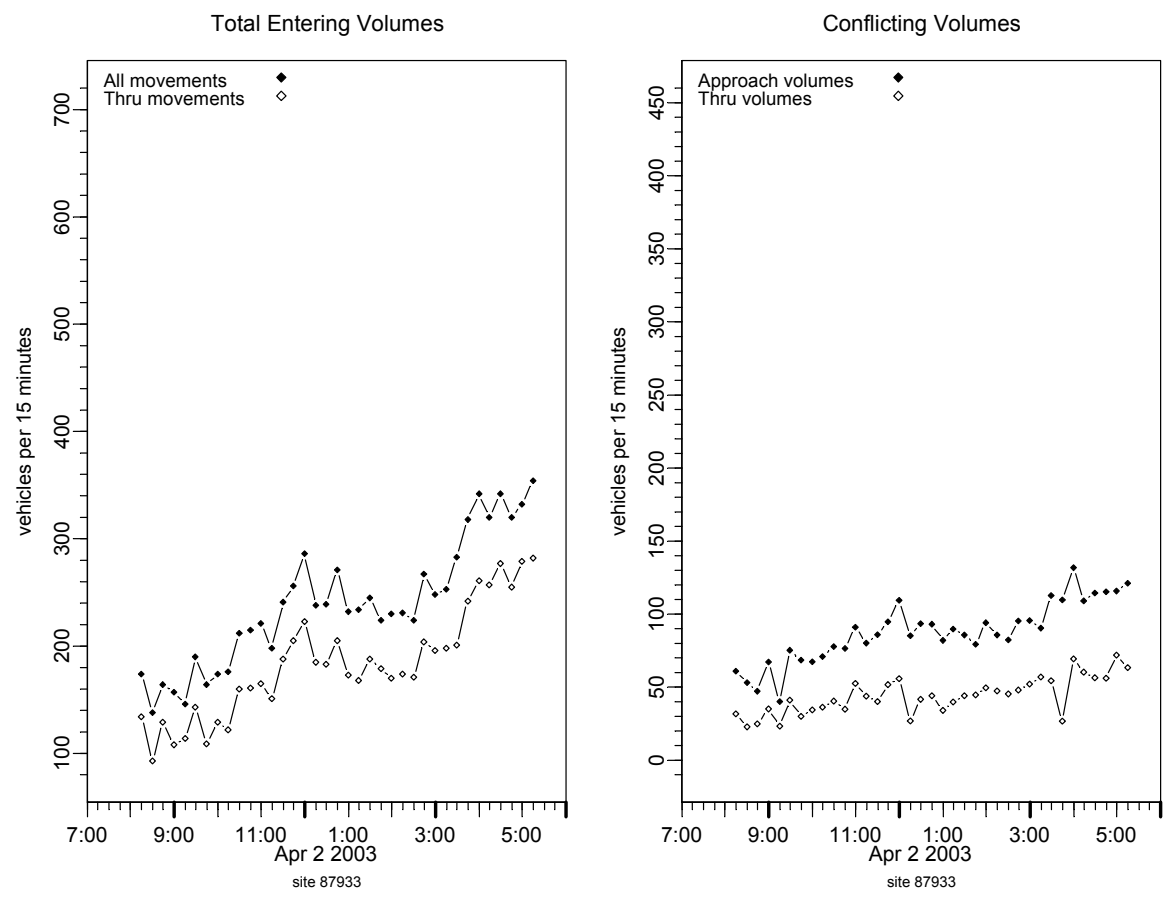

Figure D-9: 15-minute Volume Variation over Time at Site 87933
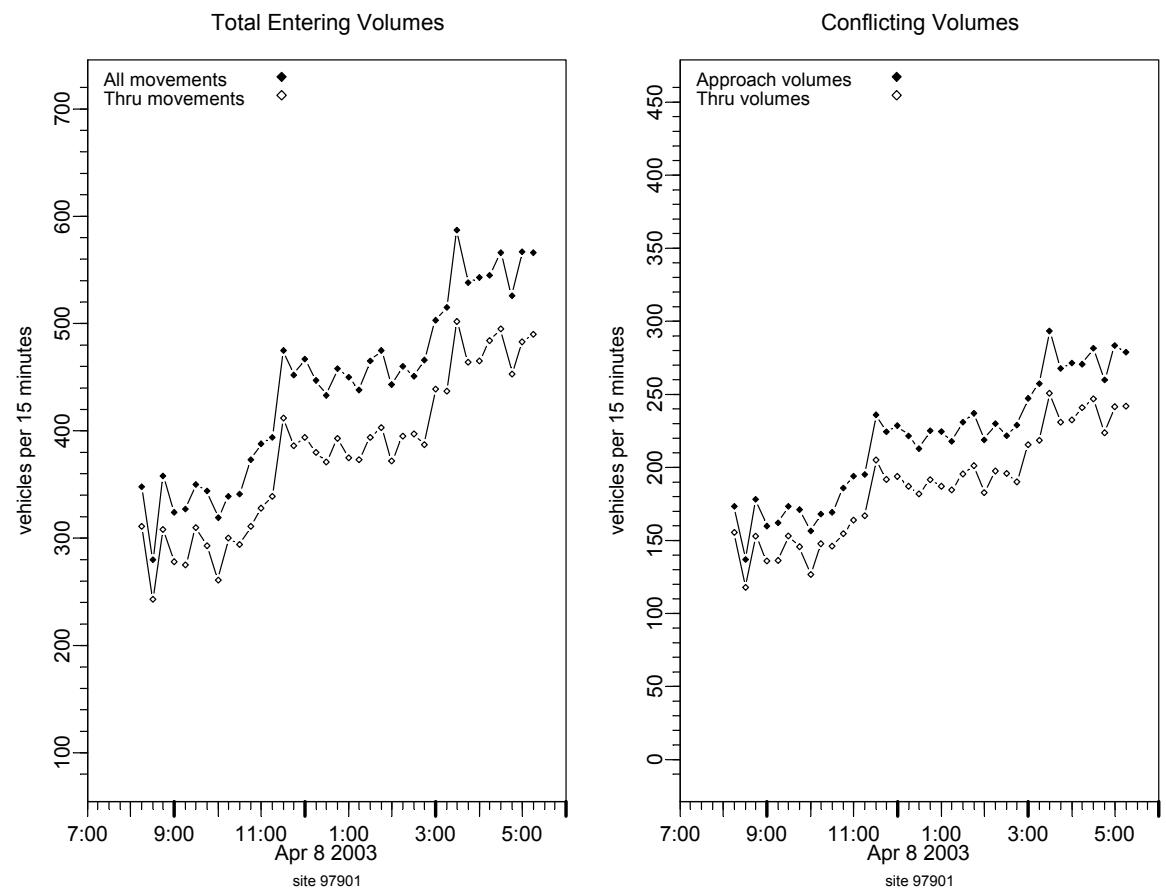

Figure D-10: 15-minute Volume Variation over Time at Site 97901 

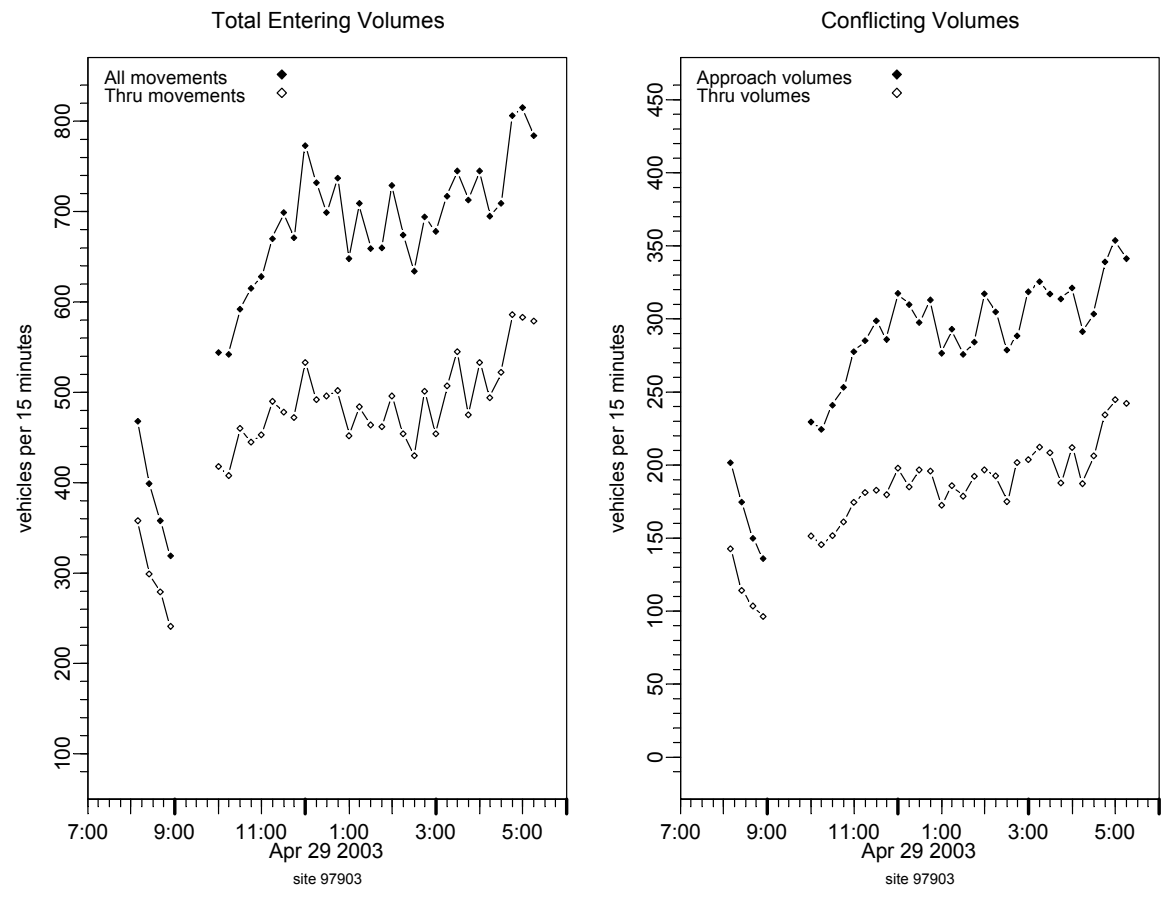

Figure D-11: 15-minute Volume Variation over Time at Site 97903
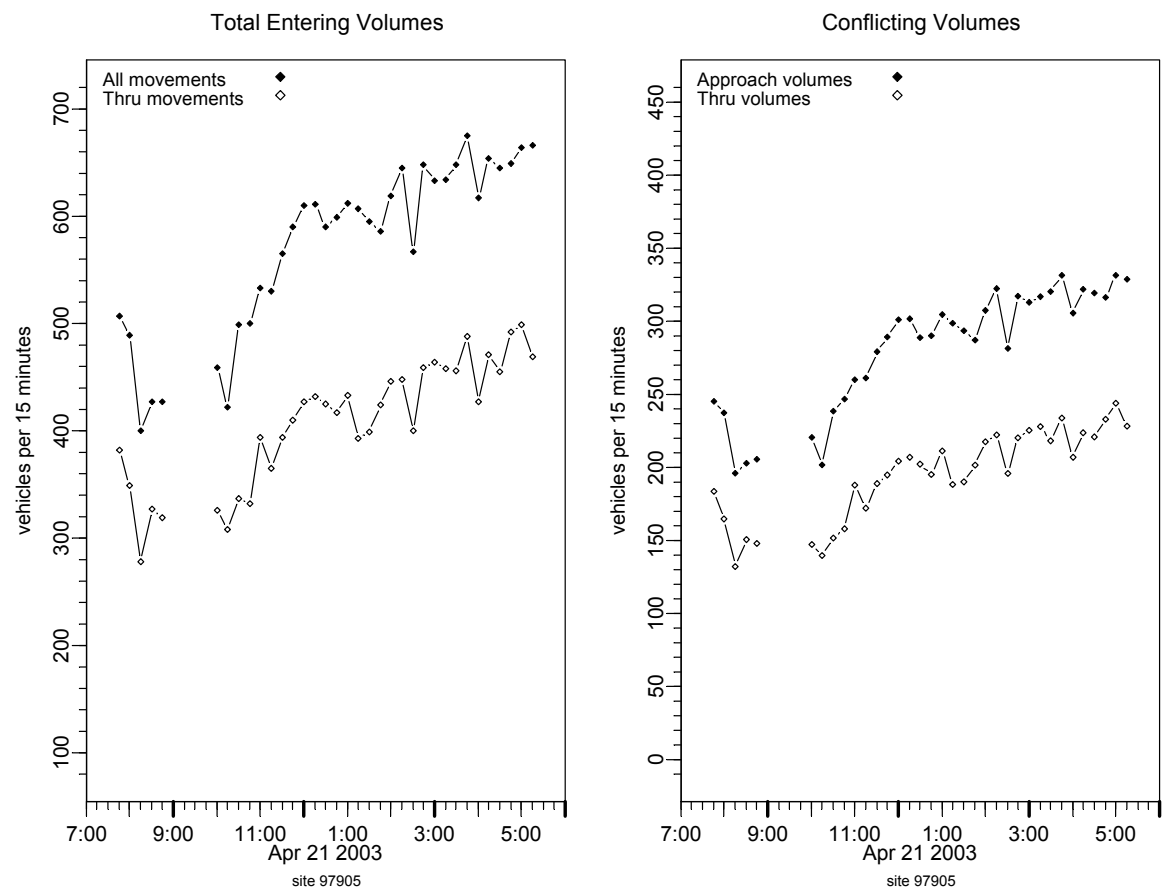

Figure D-12: 15-minute Volume Variation over Time at Site 97905 

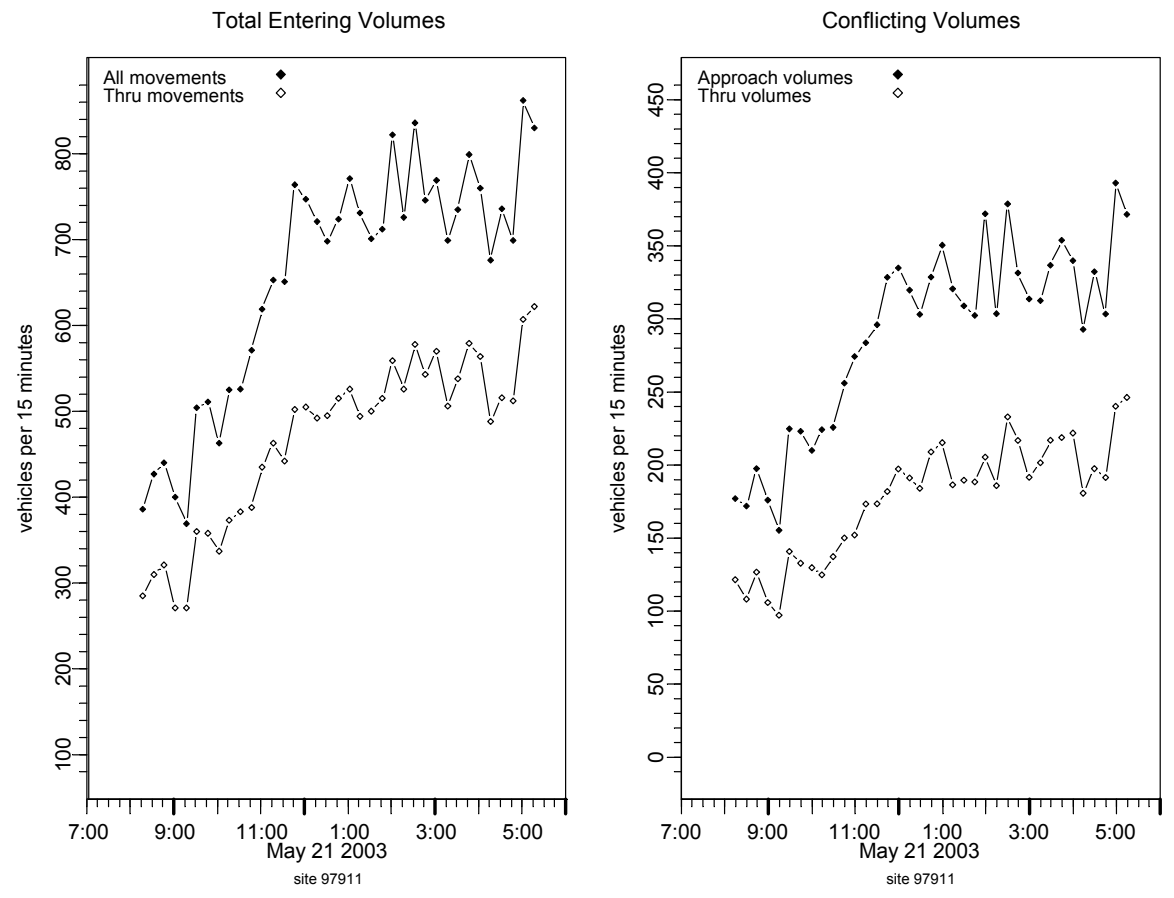

Figure D-13: 15-minute Volume Variation over Time at Site 97911
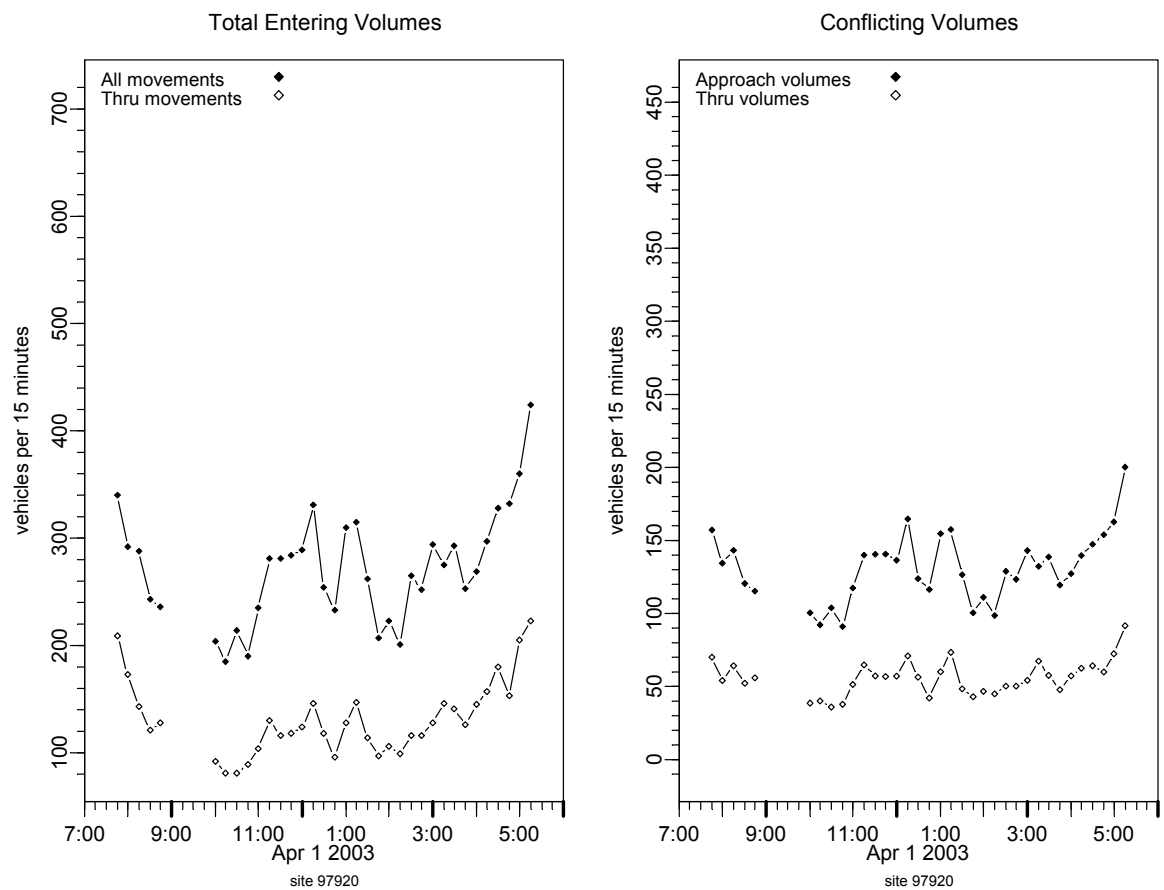

Figure D-14: 15-minute Volume Variation over Time at Site 97920 

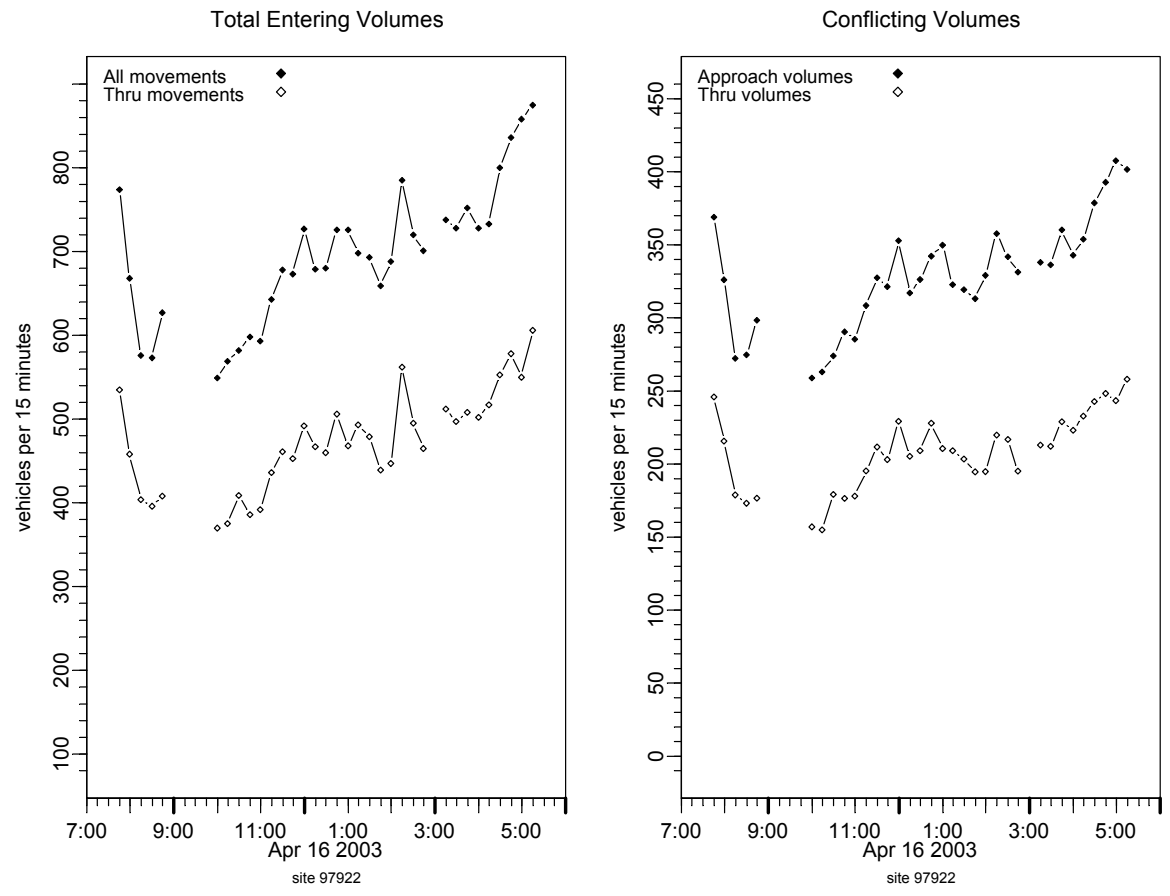

Figure D-15: 15-minute Volume Variation over Time at Site 97922
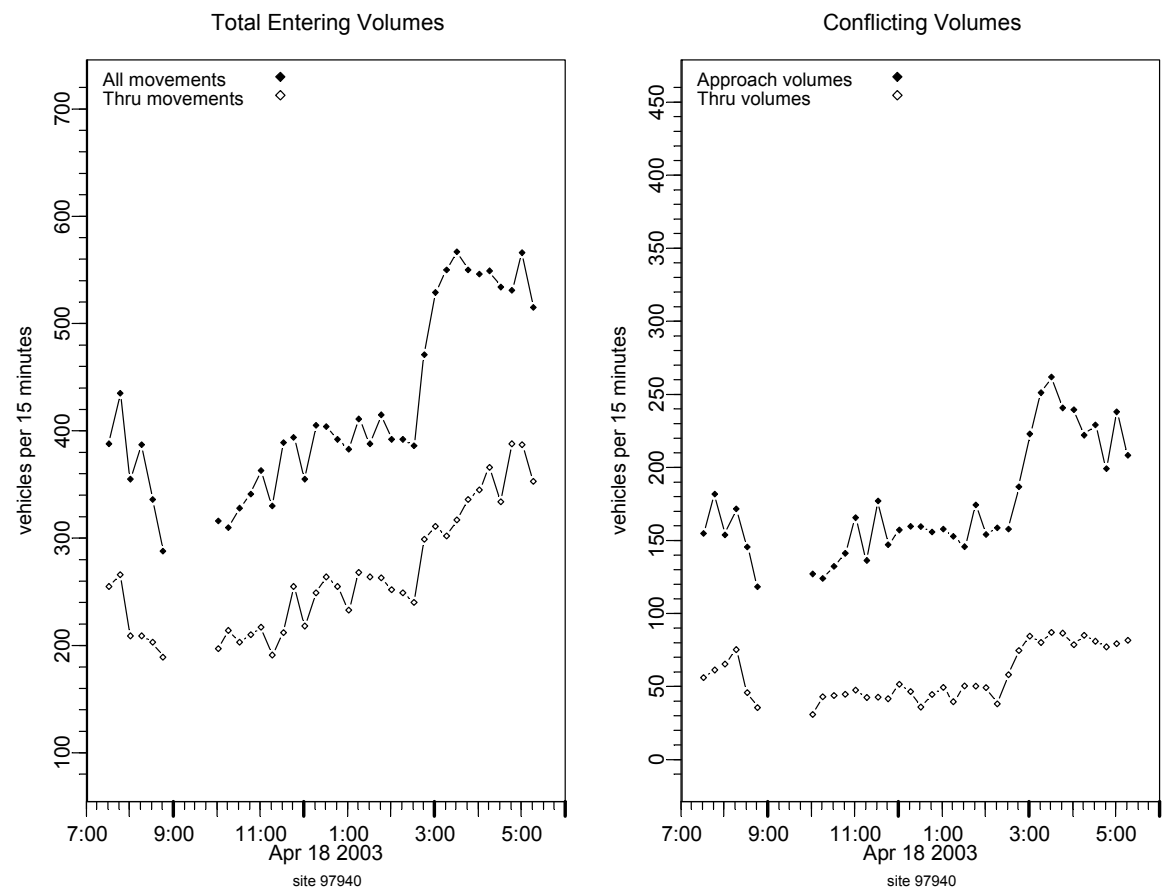

Figure D-16: 15-minute Volume Variation over Time at Site 97940 


\section{APPENDIX E ESTIMATION RESULTS AND MODEL DIAGNOSTICS}

Model estimation results and related model diagnostics of the GEV models and the $r$ largest order statistic models are documented in this appendix. All the computational procedure was implemented in S-Plus ${ }^{\circledR}$. 
Table E-1: Estimation Results of All PET Data Fitted to Homogeneous Models

\begin{tabular}{|c|c|c|c|c|c|c|c|c|c|c|}
\hline \multirow{2}{*}{ Site } & \multirow{2}{*}{$\begin{array}{c}\text { 8-Hour } \\
\text { Counts of } \\
\text { PET } \leq 8\end{array}$} & \multirow{2}{*}{$\begin{array}{l}\text { 8-Hour } \\
\text { Counts of } \\
\text { PET } \leq 6\end{array}$} & \multicolumn{8}{|c|}{ Homogeneous, All PETs, $r=1$} \\
\hline & & & $\mathbf{n}$ & nllh & $\mu$ & $\sigma$ & $\xi$ & $\operatorname{se}(\mu)$ & $\operatorname{se}(\sigma)$ & $\operatorname{se}(\xi)$ \\
\hline 87905 & 68 & 31 & 15 & 23.489 & -5.384 & 1.257 & -0.444 & 0.375 & 0.300 & 0.262 \\
\hline 87906 & 190 & 98 & 30 & 50.465 & -4.844 & 1.362 & -0.345 & 0.270 & 0.187 & 0.101 \\
\hline 87907 & 95 & 54 & 30 & 41.517 & -5.380 & 1.094 & -0.498 & 0.217 & 0.166 & 0.116 \\
\hline 87909 & 538 & 268 & 32 & 34.928 & -4.058 & 0.619 & -0.017 & 0.125 & 0.091 & 0.144 \\
\hline 87915 & 250 & 116 & 31 & 44.764 & -4.910 & 1.000 & -0.191 & 0.194 & 0.127 & 0.075 \\
\hline 87923 & 70 & 24 & 23 & 31.322 & -5.755 & 0.867 & -0.139 & 0.207 & 0.150 & 0.173 \\
\hline 87930 & 90 & 38 & 25 & 38.753 & -5.233 & 1.240 & -0.422 & 0.271 & 0.197 & 0.124 \\
\hline 87932 & 60 & 27 & 23 & 33.724 & -5.867 & 1.152 & -0.442 & 0.262 & 0.195 & 0.134 \\
\hline 87933 & 180 & 104 & 31 & 49.918 & -4.866 & 1.162 & -0.181 & 0.227 & 0.152 & 0.088 \\
\hline 97901 & 573 & 311 & 32 & 35.340 & -4.025 & 0.667 & -0.135 & 0.135 & 0.098 & 0.154 \\
\hline 97903 & 550 & 327 & 32 & 51.957 & -2.235 & 1.561 & -0.711 & 0.300 & 0.273 & 0.149 \\
\hline 97905 & 182 & 106 & 32 & 37.810 & -4.407 & 0.892 & -0.510 & 0.179 & 0.144 & 0.167 \\
\hline 97911 & 113 & 69 & 26 & 39.524 & -4.764 & 1.415 & -0.701 & 0.298 & 0.253 & 0.136 \\
\hline 97920 & 164 & 79 & 31 & 37.140 & -4.838 & 0.897 & -0.495 & 0.183 & 0.149 & 0.174 \\
\hline 97922 & 60 & 26 & 25 & 34.389 & -6.011 & 0.979 & -0.336 & 0.225 & 0.169 & 0.183 \\
\hline 97940 & 28 & 15 & 16 & 23.723 & -6.012 & 1.043 & -0.244 & 0.291 & 0.207 & 0.176 \\
\hline \multirow{2}{*}{ Site } & \multirow{2}{*}{$\begin{array}{c}\text { 8-Hour } \\
\text { Counts of } \\
\text { PET } \leq 8\end{array}$} & \multirow{2}{*}{$\begin{array}{l}\text { 8-Hour } \\
\text { Counts of } \\
\text { PET } \leq 6\end{array}$} & \multicolumn{8}{|c|}{ Homogeneous, All PETs, $r=2$} \\
\hline & & & $\mathbf{n}$ & nllh & $\mu$ & $\sigma$ & $\xi$ & $\operatorname{se}(\mu)$ & $\operatorname{se}(\sigma)$ & $\operatorname{se}(\xi)$ \\
\hline 87905 & 68 & 31 & 27 & 35.288 & -5.318 & 1.109 & -0.355 & 0.318 & 0.149 & 0.199 \\
\hline 87906 & 190 & 98 & 58 & 68.169 & -4.685 & 1.098 & -0.269 & 0.186 & 0.096 & 0.069 \\
\hline 87907 & 95 & 54 & 54 & 59.259 & -5.329 & 0.951 & -0.431 & 0.172 & 0.085 & 0.085 \\
\hline 87909 & 538 & 268 & 64 & 47.626 & -3.981 & 0.738 & -0.185 & 0.122 & 0.067 & 0.082 \\
\hline 87915 & 250 & 116 & 61 & 54.008 & -4.745 & 0.863 & -0.161 & 0.143 & 0.078 & 0.061 \\
\hline 87923 & 70 & 24 & 42 & 42.450 & -5.929 & 0.798 & -0.022 & 0.174 & 0.103 & 0.152 \\
\hline 87930 & 90 & 38 & 44 & 59.628 & -5.379 & 1.106 & -0.316 & 0.224 & 0.116 & 0.110 \\
\hline 87932 & 60 & 27 & 39 & 48.789 & -5.902 & 0.978 & -0.338 & 0.212 & 0.108 & 0.115 \\
\hline 87933 & 180 & 104 & 62 & 74.706 & -4.674 & 1.209 & -0.237 & 0.201 & 0.104 & 0.061 \\
\hline 97901 & 573 & 311 & 64 & 43.007 & -3.931 & 0.690 & -0.207 & 0.116 & 0.062 & 0.096 \\
\hline 97903 & 550 & 327 & 64 & 81.998 & -2.074 & 1.284 & -0.600 & 0.233 & 0.115 & 0.126 \\
\hline 97905 & 182 & 106 & 60 & 57.003 & -4.407 & 0.875 & -0.509 & 0.153 & 0.075 & 0.096 \\
\hline 97911 & 113 & 69 & 44 & 57.452 & -4.542 & 1.112 & -0.614 & 0.222 & 0.112 & 0.096 \\
\hline 97920 & 164 & 79 & 59 & 56.589 & -4.728 & 0.896 & -0.570 & 0.156 & 0.080 & 0.094 \\
\hline 97922 & 60 & 26 & 39 & 40.148 & -5.794 & 0.808 & -0.307 & 0.196 & 0.082 & 0.127 \\
\hline 97940 & 28 & 15 & 23 & 30.485 & -6.120 & 0.849 & -0.157 & 0.248 & 0.123 & 0.139 \\
\hline \multirow{2}{*}{ Site } & \multirow{2}{*}{$\begin{array}{c}\text { 8-Hour } \\
\text { Counts of } \\
\text { PET } \leq 8\end{array}$} & \multirow{2}{*}{$\begin{array}{c}\text { 8-Hour } \\
\text { Counts of } \\
\text { PET } \leq 6\end{array}$} & \multicolumn{8}{|c|}{ Homogeneous, All PETs, $r=3$} \\
\hline & & & $\mathbf{n}$ & nllh & $\mu$ & $\sigma$ & $\xi$ & $\operatorname{se}(\mu)$ & $\operatorname{se}(\sigma)$ & $\operatorname{se}(\xi)$ \\
\hline 87905 & 68 & 31 & 36 & 38.253 & -5.349 & 0.958 & -0.278 & 0.267 & 0.101 & 0.138 \\
\hline 87906 & 190 & 98 & 85 & 80.426 & -4.664 & 1.069 & -0.269 & 0.174 & 0.076 & 0.060 \\
\hline 87907 & 95 & 54 & 71 & 62.381 & -5.307 & 0.861 & -0.396 & 0.162 & 0.058 & 0.067 \\
\hline 87909 & 538 & 268 & 96 & 50.472 & -3.898 & 0.776 & -0.256 & 0.120 & 0.054 & 0.058 \\
\hline 87915 & 250 & 116 & 89 & 67.051 & -4.717 & 0.930 & -0.212 & 0.146 & 0.066 & 0.046 \\
\hline 87923 & 70 & 24 & 56 & 45.816 & -6.001 & 0.780 & -0.087 & 0.167 & 0.078 & 0.103 \\
\hline 87930 & 90 & 38 & 58 & 67.583 & -5.468 & 0.993 & -0.257 & 0.214 & 0.085 & 0.096 \\
\hline 87932 & 60 & 27 & 48 & 50.848 & -6.008 & 0.820 & -0.238 & 0.200 & 0.078 & 0.107 \\
\hline 87933 & 180 & 104 & 88 & 89.059 & -4.591 & 1.173 & -0.250 & 0.188 & 0.081 & 0.049 \\
\hline 97901 & 573 & 311 & 96 & 32.722 & -3.940 & 0.655 & -0.150 & 0.102 & 0.054 & 0.081 \\
\hline 97903 & 550 & 327 & 96 & 96.924 & -1.983 & 1.160 & -0.529 & 0.204 & 0.083 & 0.111 \\
\hline 97905 & 182 & 106 & 86 & 72.463 & -4.420 & 0.888 & -0.524 & 0.155 & 0.058 & 0.080 \\
\hline 97911 & 113 & 69 & 57 & 63.555 & -4.454 & 0.953 & -0.552 & 0.205 & 0.070 & 0.082 \\
\hline 97920 & 164 & 79 & 84 & 71.685 & -4.697 & 0.887 & -0.584 & 0.157 & 0.061 & 0.083 \\
\hline 97922 & 60 & 26 & 49 & 43.389 & -5.972 & 0.746 & -0.230 & 0.210 & 0.067 & 0.117 \\
\hline 97940 & 28 & 15 & 26 & 26.307 & -6.278 & 0.660 & -0.062 & 0.244 & 0.086 & 0.130 \\
\hline
\end{tabular}

Notes:

(1) $n=$ Number of extreme values used in the model estimation

(2) $n$ llh = Negative log-likelihood value at model convergence 

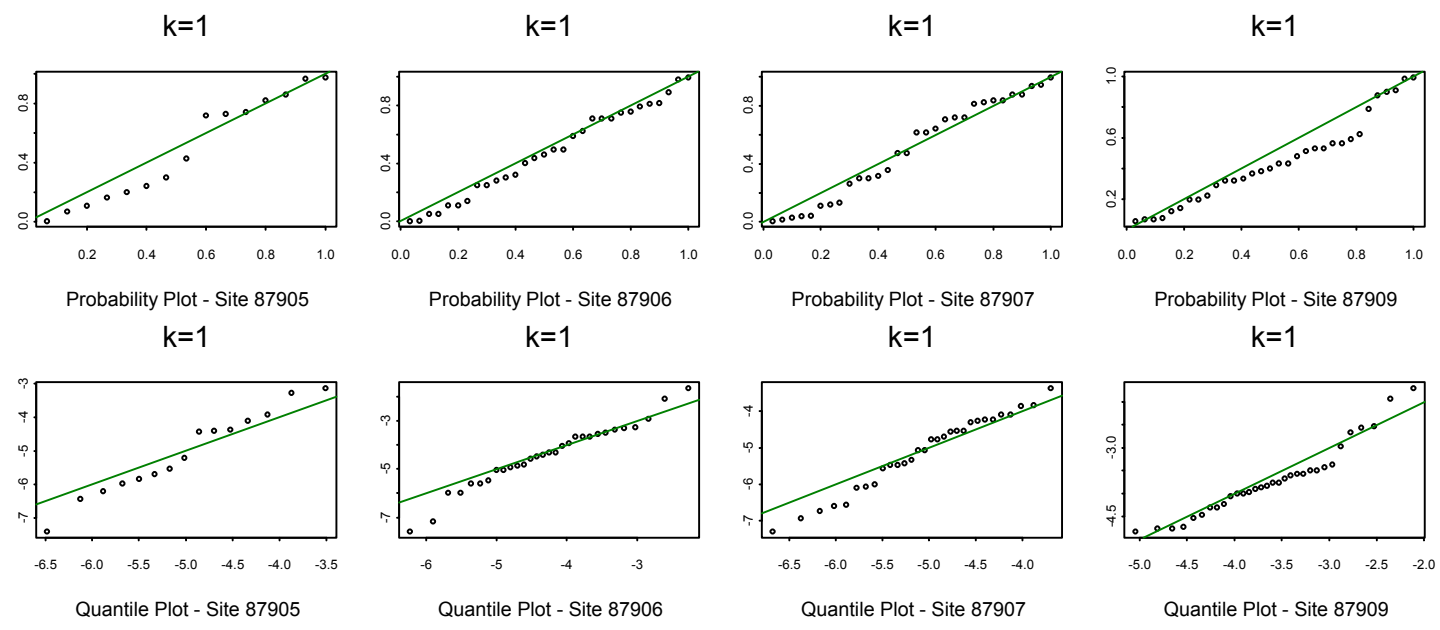

$\mathrm{k}=2$

$\mathrm{k}=2$

$\mathrm{k}=2$

$\mathrm{k}=2$
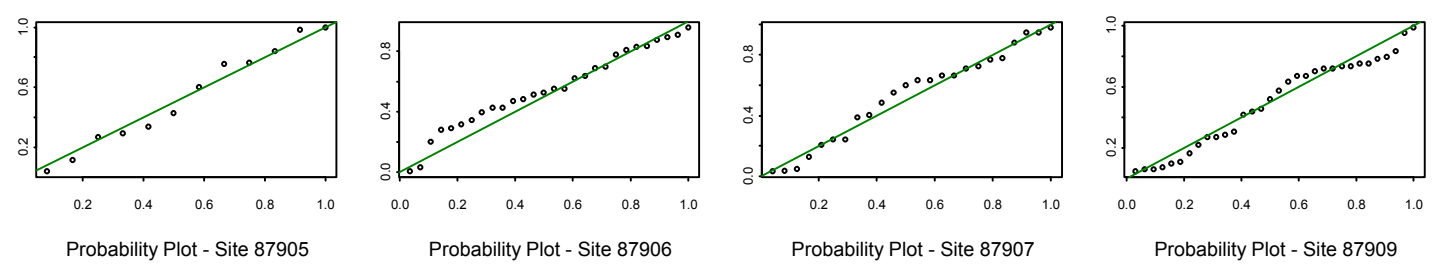

$\mathrm{k}=2$

$\mathrm{k}=2$
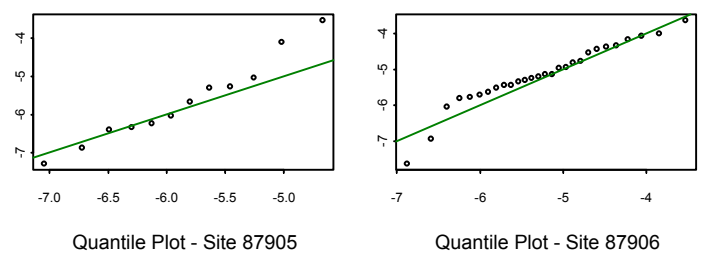

$k=2$

$\mathrm{k}=2$
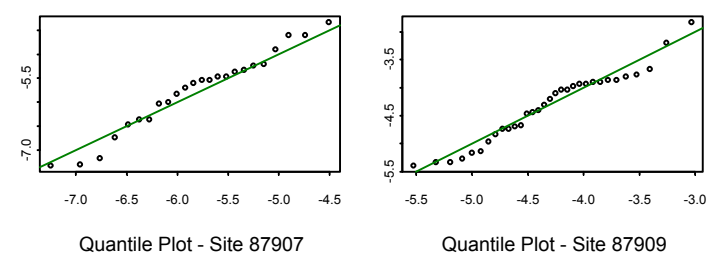

$\mathrm{k}=3$

$$
k=3
$$
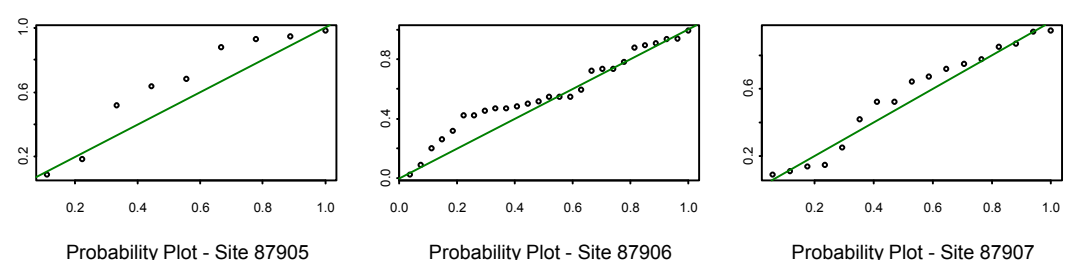

$\mathrm{k}=3$

$\mathrm{k}=3$
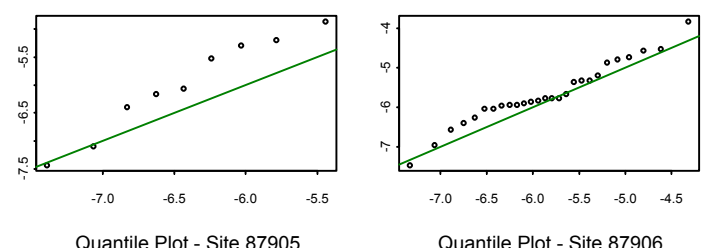

Probability Plot - Site 87907

$$
k=3
$$
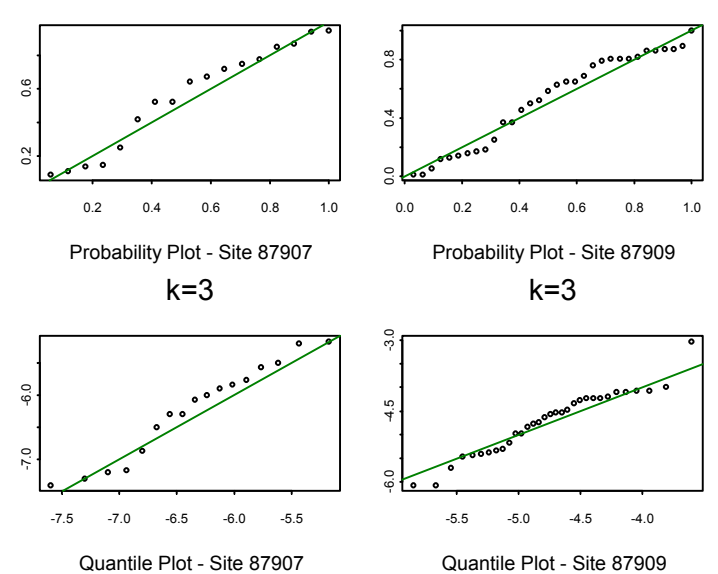

$\mathrm{k}=3$

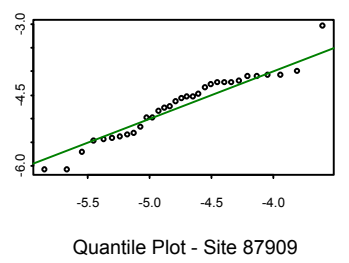

Figure E-1: Diagnostics of Order Statistics for $r$ Selection (1) 

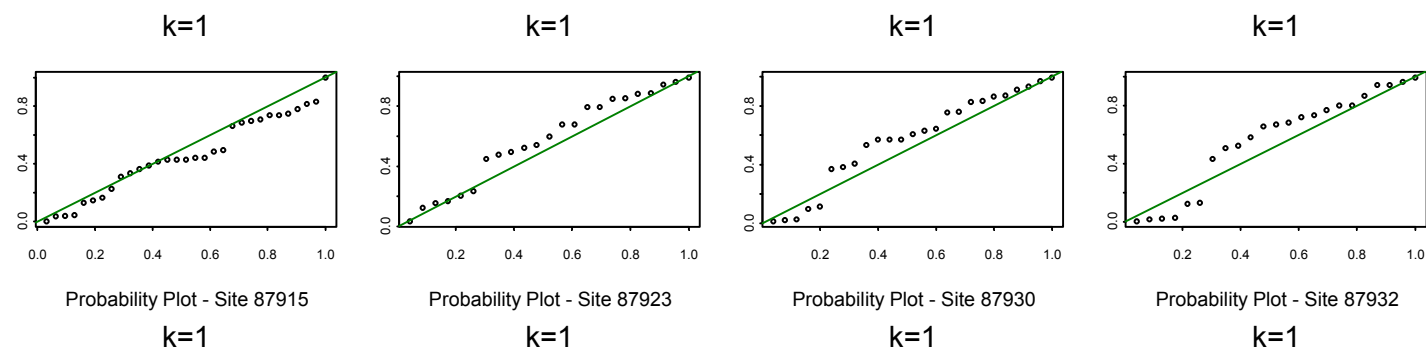

$\mathrm{k}=1$
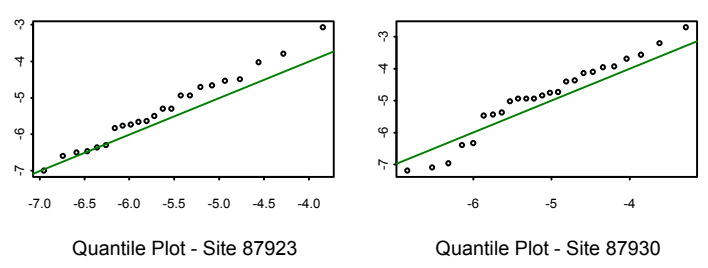

Probability Plot - Site 87932

$\mathrm{k}=1$

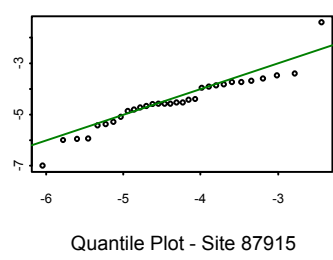

$\mathrm{k}=2$

$\mathrm{k}=2$

Quantile Plot - Site 87930

$\mathrm{k}=2$

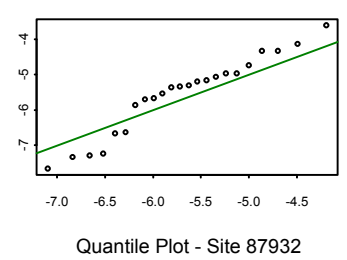

$\mathrm{k}=2$
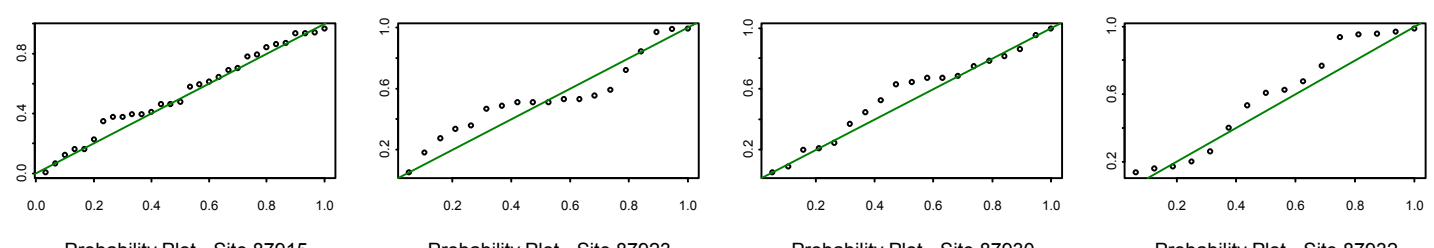

Probability Plot - Site 87915

$\mathrm{k}=2$

Probability Plot - Site 87923

$\mathrm{k}=2$

Probability Plot - Site 87930

$\mathrm{k}=2$

Probability Plot - Site 87932
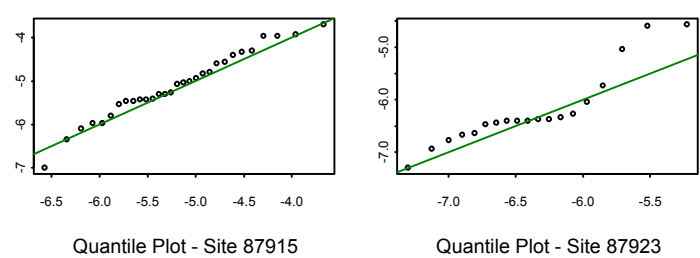

$\mathrm{k}=3$

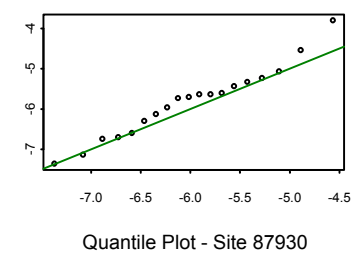

$\mathrm{k}=3$
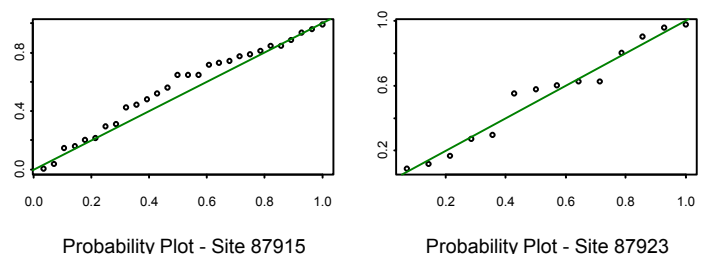

Probability Plot - Site 87923

$\mathrm{k}=3$

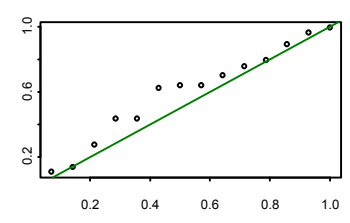

Probability Plot - Site 87930 $\mathrm{k}=3$

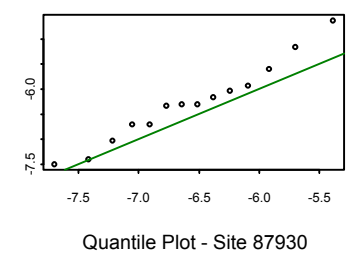

$\mathrm{k}=2$

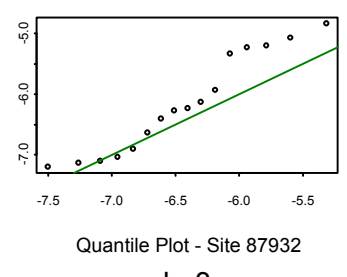

$\mathrm{k}=3$

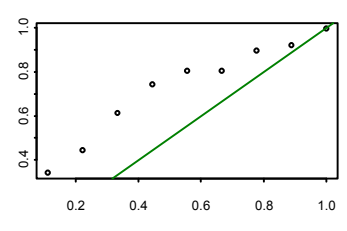

Probability Plot - Site 87932 $\mathrm{k}=3$
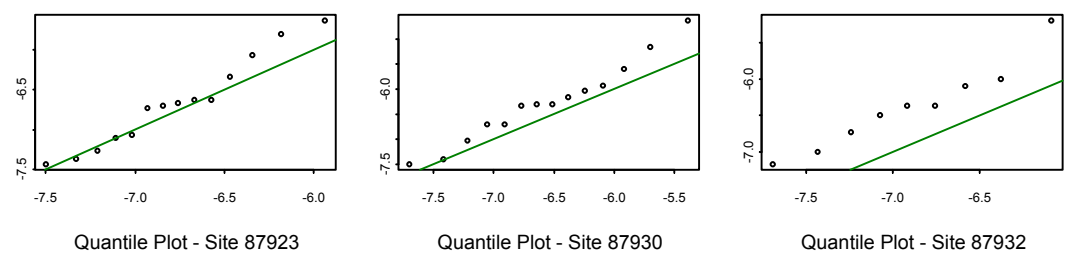

Figure E-2: Diagnostics of Order Statistics for r Selection (2) 

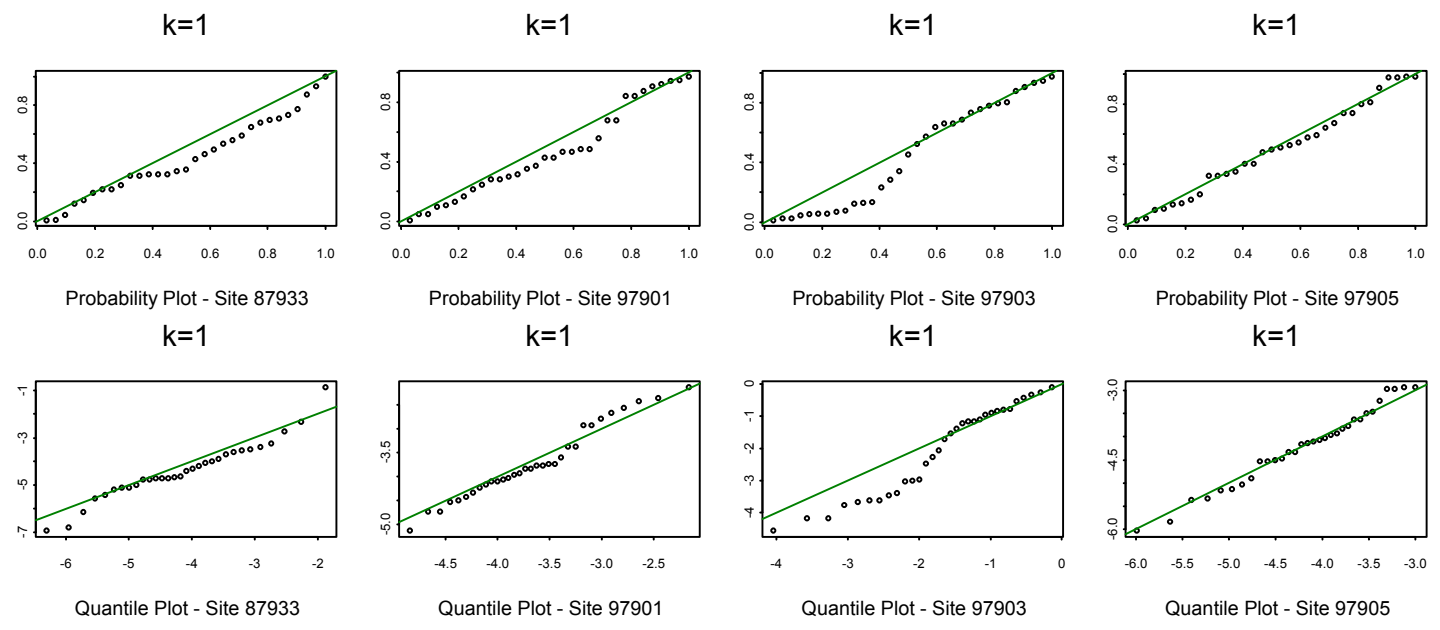

$\mathrm{k}=2$

$\mathrm{k}=2$
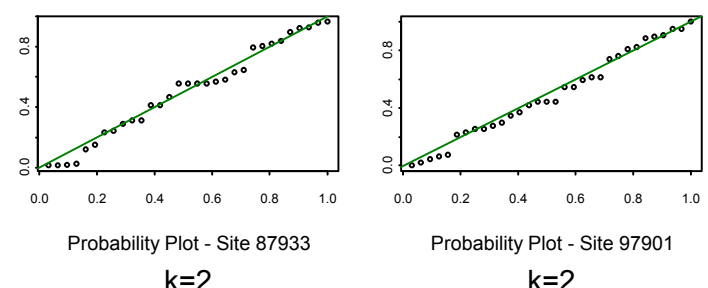

$\mathrm{k}=2$

$\mathrm{k}=2$
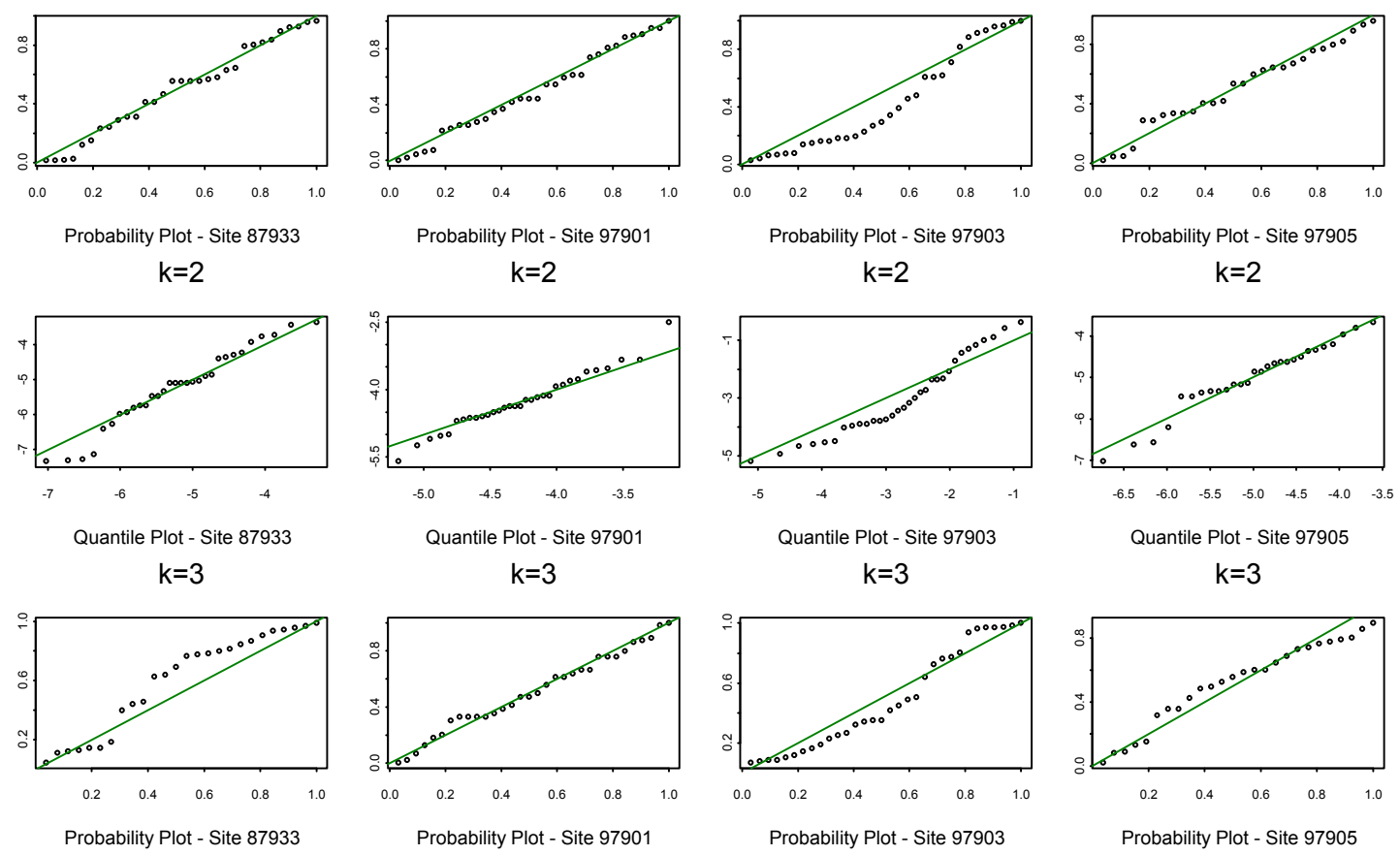

Probability Plot - Site 87933

$\mathrm{k}=3$

$\mathrm{k}=3$

$$
k=3
$$

Probability Plot - Site 97905
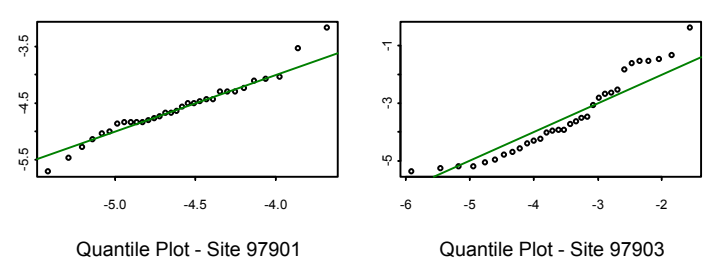

$\mathrm{k}=3$

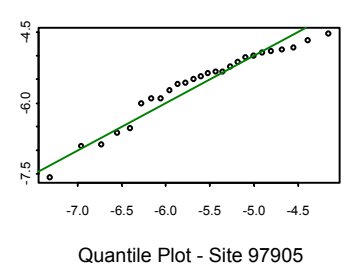

Figure E-3: Diagnostics of Order Statistics for r Selection (3) 


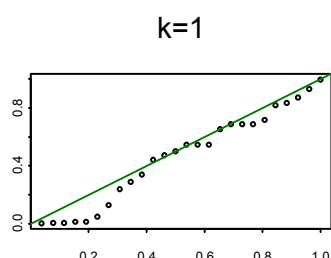

Probability Plot - Site 97911

$k=1$

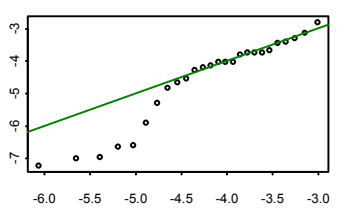

Quantile Plot - Site 97911

$\mathrm{k}=2$

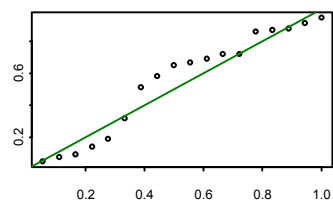

Probability Plot - Site 97911

$k=2$

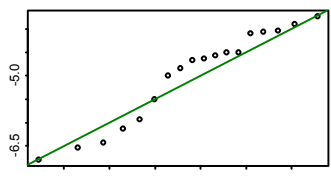

$\begin{array}{llllll}5 & -6.0 & -5.5 & -50 & -4.5 & -4.0\end{array}$

Quantile Plot - Site 97911

$\mathrm{k}=3$

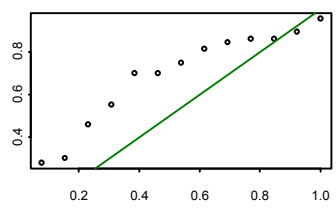

Probability Plot - Site 97911

$\mathrm{k}=3$

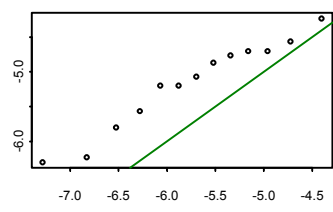

Quantile Plot - Site 97911 $k=1$

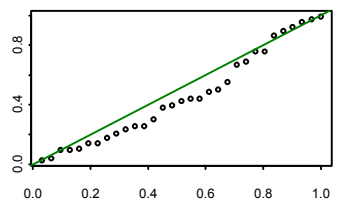

Probability Plot - Site 97920

$k=1$

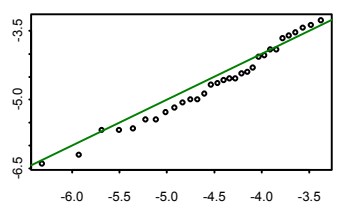

Quantile Plot - Site 97920

$\mathrm{k}=2$

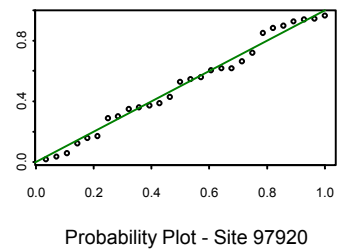

$\mathrm{k}=2$

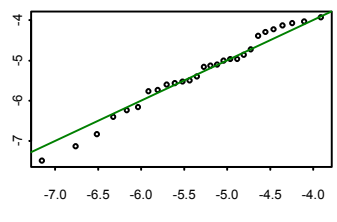

Quantile Plot - Site 97920

$\mathrm{k}=3$

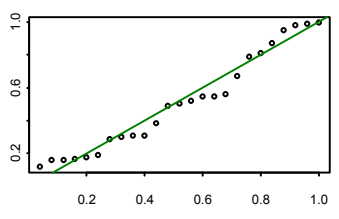

Probability Plot - Site 97920

$\mathrm{k}=3$

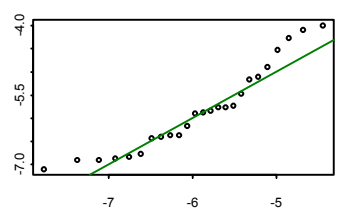

Quantile Plot - Site 97920 $\mathrm{k}=1$

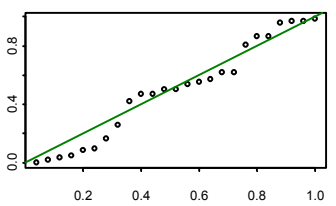

Probability Plot - Site 97922

$\mathrm{k}=1$

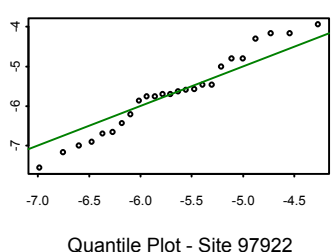

$\mathrm{k}=2$

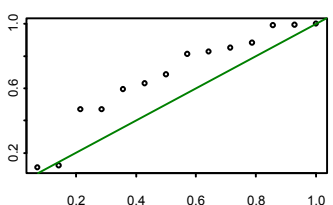

Probability Plot - Site 97922

$\mathrm{k}=2$

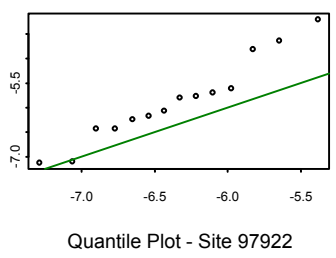

$\mathrm{k}=3$

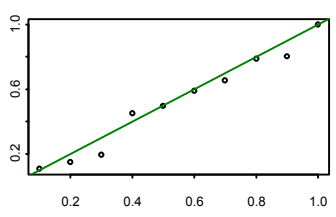

Probability Plot - Site 97922 $\mathrm{k}=3$

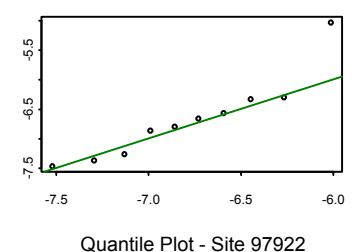

$k=1$

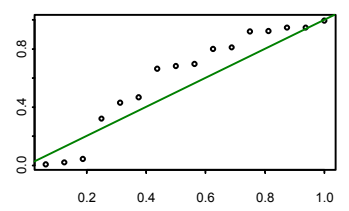

Probability Plot - Site 97940

$k=1$

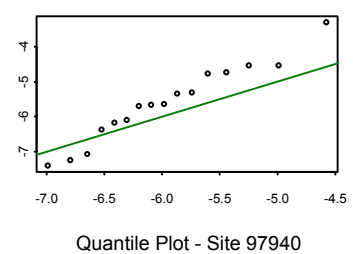

$\mathrm{k}=2$

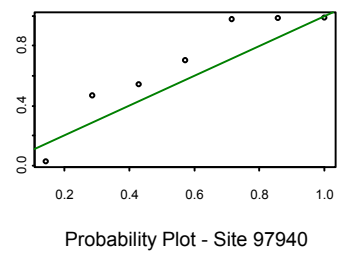

$\mathrm{k}=2$

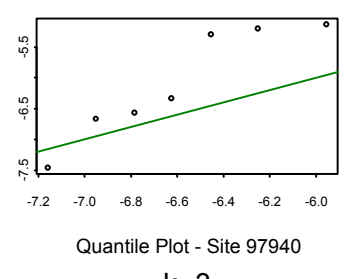

$k=3$

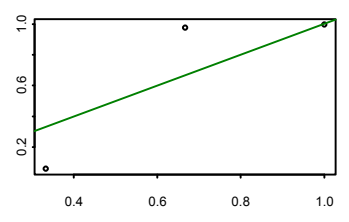

Probability Plot - Site 97940 $\mathrm{k}=3$

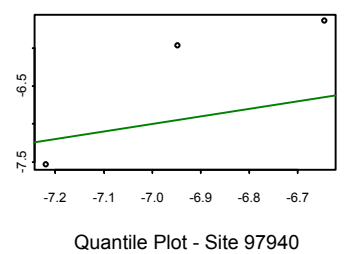

Figure E-4: Diagnostics of Order Statistics for r Selection (4) 
Table E-2: Results - Homogeneous Models Using All PET Data

Model Descriptions: all PET data fitted to the homogeneous $r$ largest order statistic model

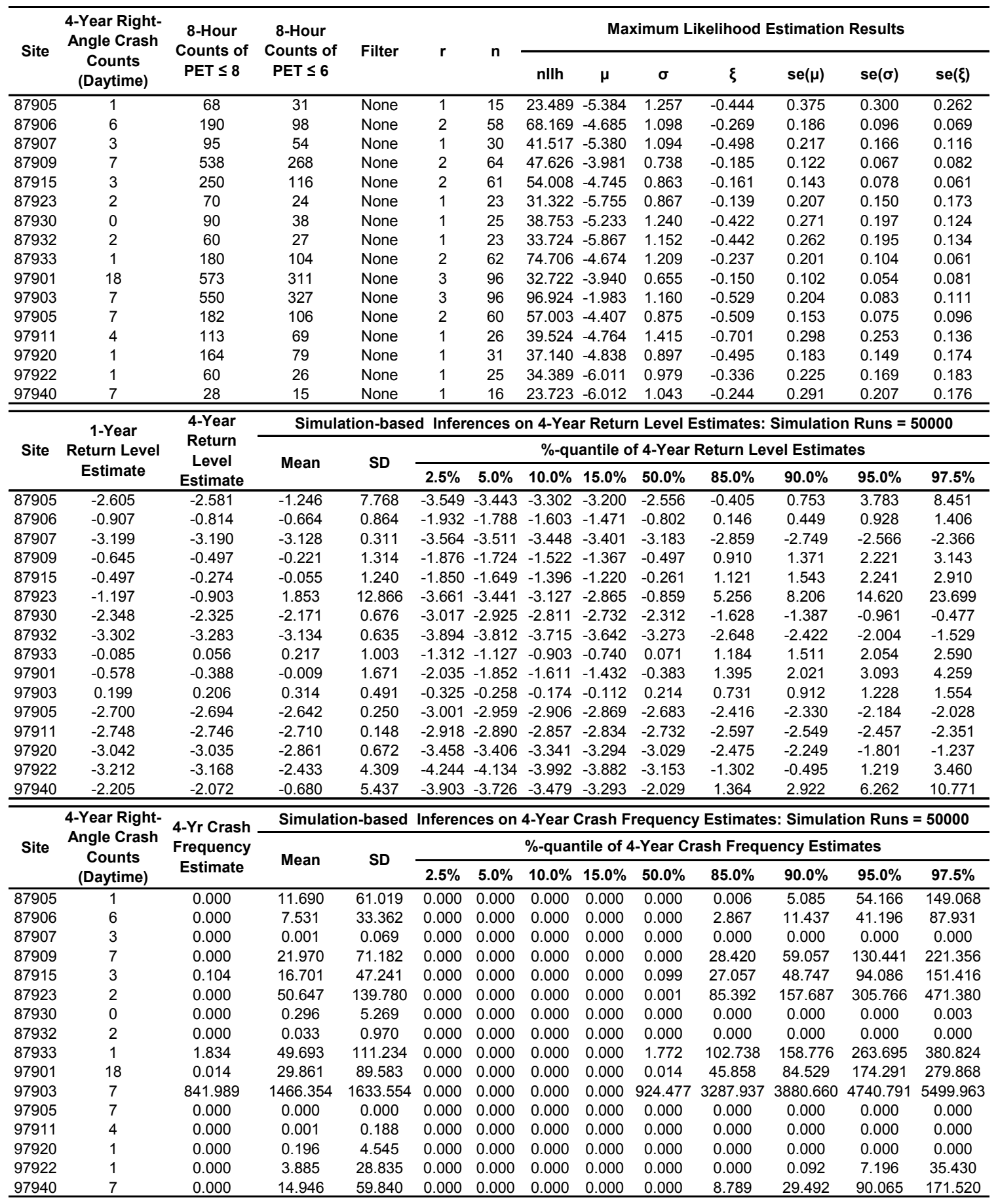

Abbreviations: $n=$ Number of extreme values used in the model estimation, $n$ llh $=$ Negative log-likelihood value at model convergence 
Table E-3: Results - Homogeneous Models Using Filtered PETs

Model Descriptions: Filtered PET data fitted to the homogeneous $r$ largest order statistic models

\begin{tabular}{|c|c|c|c|c|c|c|c|c|c|c|c|c|c|}
\hline \multirow{2}{*}{ Site } & \multirow{2}{*}{$\begin{array}{l}\text { 4-Year Right- } \\
\text { Angle Crash } \\
\text { Counts } \\
\text { (Daytime) }\end{array}$} & \multirow{2}{*}{$\begin{array}{l}\text { 8-Hour } \\
\text { Counts of } \\
\text { PET } \leq 6\end{array}$} & \multirow{2}{*}{$\mathrm{CV}\left(\mathrm{ET}_{\mathrm{PET} \leq 6}\right)$} & \multirow{2}{*}{ Filter } & \multirow{2}{*}{$\mathbf{r}$} & \multirow{2}{*}{$\mathbf{n}$} & \multicolumn{7}{|c|}{ Maximum Likelihood Estimation Results } \\
\hline & & & & & & & nllh & $\boldsymbol{\mu}$ & $\boldsymbol{\sigma}$ & $\xi$ & $\operatorname{se}(\mu)$ & $\operatorname{se}(\sigma)$ & $\operatorname{se}(\xi)$ \\
\hline 87905 & 1 & 31 & 0.281 & None & 1 & 15 & 23.489 & -5.384 & 1.257 & -0.444 & 0.375 & 0.300 & 0.262 \\
\hline 87906 & 6 & 98 & 0.642 & None & 2 & 58 & 68.169 & -4.685 & 1.098 & -0.269 & 0.186 & 0.096 & 0.069 \\
\hline 87907 & 3 & 54 & 0.285 & None & 1 & 30 & 41.517 & -5.380 & 1.094 & -0.498 & 0.217 & 0.166 & 0.116 \\
\hline 87909 & 7 & 268 & 0.303 & None & 2 & 64 & 47.626 & -3.981 & 0.738 & -0.185 & 0.122 & 0.067 & 0.082 \\
\hline 87915 & 3 & 116 & 0.423 & None & 2 & 61 & 54.008 & -4.745 & 0.863 & -0.161 & 0.143 & 0.078 & 0.061 \\
\hline 87923 & 2 & 24 & 0.725 & None & 1 & 23 & 31.322 & -5.755 & 0.867 & -0.139 & 0.207 & 0.150 & 0.173 \\
\hline 87930 & 0 & 38 & 0.380 & None & 1 & 25 & 38.753 & -5.233 & 1.240 & -0.422 & 0.271 & 0.197 & 0.124 \\
\hline 87932 & 2 & 27 & 0.216 & None & 1 & 23 & 33.724 & -5.867 & 1.152 & -0.442 & 0.262 & 0.195 & 0.134 \\
\hline 87933 & 1 & 104 & 0.576 & $95 \%$-quantile & 2 & 62 & 74.706 & -4.674 & 1.209 & -0.237 & 0.201 & 0.104 & 0.061 \\
\hline 97901 & 18 & 311 & 0.538 & $95 \%$-quantile & 3 & 96 & 32.722 & -3.940 & 0.655 & -0.150 & 0.102 & 0.054 & 0.081 \\
\hline 97903 & 7 & 327 & 0.969 & $80 \%$-quantile & 3 & 96 & 96.924 & -1.983 & 1.160 & -0.529 & 0.204 & 0.083 & 0.111 \\
\hline 97905 & 7 & 106 & 0.537 & $95 \%$-quantile & 2 & 60 & 57.003 & -4.407 & 0.875 & -0.509 & 0.153 & 0.075 & 0.096 \\
\hline 97911 & 4 & 69 & 0.500 & None & 1 & 26 & 39.524 & -4.764 & 1.415 & -0.701 & 0.298 & 0.253 & 0.136 \\
\hline 97920 & 1 & 79 & 0.509 & None & 1 & 31 & 37.140 & -4.838 & 0.897 & -0.495 & 0.183 & 0.149 & 0.174 \\
\hline 97922 & 1 & 26 & 0.445 & None & 1 & 25 & 34.389 & -6.011 & 0.979 & -0.336 & 0.225 & 0.169 & 0.183 \\
\hline 97940 & 7 & 15 & 0.649 & None & 1 & 16 & 23.723 & -6.012 & 1.043 & -0.244 & 0.291 & 0.207 & 0.176 \\
\hline \multirow{3}{*}{ Site } & \multirow{3}{*}{$\begin{array}{c}\text { 1-Year Return } \\
\text { Level } \\
\text { Estimate }\end{array}$} & \multirow{3}{*}{$\begin{array}{c}\text { 4-Year } \\
\text { Return } \\
\text { Level } \\
\text { Estimate }\end{array}$} & \multicolumn{11}{|c|}{ Simulation-based Inferences on 4-Year Return Level Estimates: Simulation Runs $=\mathbf{5 0 0 0 0}$} \\
\hline & & & \multirow{2}{*}{ Mean } & \multirow{2}{*}{ SD } & & & $\%$-quan & ntile of 4 & 4-Year R & Return Le & vel Estim & nates & \\
\hline & & & & & $2.5 \%$ & $5.0 \%$ & $10.0 \%$ & $15.0 \%$ & $50.0 \%$ & $85.0 \%$ & $90.0 \%$ & $95.0 \%$ & $97.5 \%$ \\
\hline 87905 & -2.605 & -2.581 & -1.136 & 13.666 & -3.558 & -3.445 & -3.305 & -3.200 & -2.551 & -0.434 & 0.731 & 3.722 & 8.419 \\
\hline 87906 & -0.907 & -0.814 & -0.669 & 0.858 & -1.939 & -1.788 & -1.608 & -1.476 & -0.808 & 0.148 & 0.433 & 0.906 & 1.379 \\
\hline 87907 & -3.199 & -3.190 & -3.125 & 0.311 & -3.559 & -3.506 & -3.444 & -3.399 & -3.181 & -2.856 & -2.751 & -2.556 & -2.355 \\
\hline 87909 & -0.645 & -0.497 & -0.224 & 1.302 & -1.892 & -1.733 & -1.528 & -1.371 & -0.500 & 0.930 & 1.402 & 2.238 & 3.115 \\
\hline 87915 & -0.497 & -0.274 & -0.056 & 1.237 & -1.860 & -1.659 & -1.402 & -1.220 & -0.258 & 1.118 & 1.548 & 2.255 & 2.933 \\
\hline 87923 & -1.197 & -0.903 & 1.939 & 12.388 & -3.660 & -3.444 & -3.129 & -2.872 & -0.851 & 5.288 & 8.297 & 14.994 & 24.217 \\
\hline 87930 & -2.348 & -2.325 & -2.169 & 0.689 & -3.014 & -2.926 & -2.814 & -2.734 & -2.314 & -1.614 & -1.382 & -0.950 & -0.476 \\
\hline 87932 & -3.302 & -3.283 & -3.129 & 0.638 & -3.890 & -3.807 & -3.707 & -3.638 & -3.266 & -2.636 & -2.415 & -1.997 & -1.545 \\
\hline 87933 & 0.009 & 0.166 & 0.347 & 1.128 & -1.344 & -1.146 & -0.903 & -0.721 & 0.171 & 1.431 & 1.807 & 2.430 & 3.030 \\
\hline 97901 & -1.064 & -0.914 & -0.683 & 1.152 & -2.208 & -2.053 & -1.865 & -1.718 & -0.919 & 0.359 & 0.759 & 1.463 & 2.229 \\
\hline 97903 & 0.231 & 0.327 & 0.577 & 1.230 & -1.039 & -0.882 & -0.671 & -0.518 & 0.333 & 1.669 & 2.100 & 2.845 & 3.626 \\
\hline 97905 & -2.773 & -2.769 & -2.732 & 0.184 & -3.011 & -2.976 & -2.934 & -2.904 & -2.758 & -2.563 & -2.503 & -2.400 & -2.295 \\
\hline 97911 & -2.748 & -2.746 & -2.712 & 0.146 & -2.921 & -2.892 & -2.858 & -2.834 & -2.733 & -2.600 & -2.554 & -2.465 & -2.356 \\
\hline 97920 & -3.042 & -3.035 & -2.858 & 0.676 & -3.454 & -3.402 & -3.340 & -3.294 & -3.025 & -2.468 & -2.240 & -1.804 & -1.245 \\
\hline 97922 & -3.212 & -3.168 & -2.442 & 2.828 & -4.245 & -4.137 & -3.995 & -3.886 & -3.157 & -1.291 & -0.450 & 1.325 & 3.864 \\
\hline 97940 & -2.205 & -2.072 & -0.688 & 5.459 & -3.911 & -3.730 & -3.490 & -3.298 & -2.042 & 1.325 & 2.921 & 6.368 & 10.769 \\
\hline & 4-Year Right- & 4-Yr Crash & Simulati & Minased & monos & $\mathrm{Sa}_{0}$ & anout & iाequ & dincy & stimates. & . Sinturate & On Puns - & 50000 \\
\hline Site & Angle Crash & Frequency & & & & & o-quantil & le of $4-Y$ & Year Cra & ash Frequ & uency Est & timates & \\
\hline & (Daytime) & Estimate & & SD & $2.5 \%$ & $5.0 \%$ & $10.0 \%$ & $15.0 \%$ & $50.0 \%$ & $85.0 \%$ & $90.0 \%$ & $95.0 \%$ & $97.5 \%$ \\
\hline 87905 & 1 & 0.000 & 11.865 & 62.852 & 0.000 & 0.000 & 0.000 & 0.000 & 0.000 & 0.008 & 5.045 & 52.767 & 148.853 \\
\hline 87906 & 6 & 0.000 & 7.513 & 33.950 & 0.000 & 0.000 & 0.000 & 0.000 & 0.000 & 3.121 & 11.750 & 40.209 & 85.685 \\
\hline 87907 & 3 & 0.000 & 0.000 & 0.029 & 0.000 & 0.000 & 0.000 & 0.000 & 0.000 & 0.000 & 0.000 & 0.000 & 0.000 \\
\hline 87909 & 7 & 0.000 & 21.458 & 69.074 & 0.000 & 0.000 & 0.000 & 0.000 & 0.000 & 28.671 & 58.424 & 127.400 & 213.971 \\
\hline 87915 & 3 & 0.104 & 16.550 & 46.700 & 0.000 & 0.000 & 0.000 & 0.000 & 0.104 & 27.544 & 47.939 & 92.506 & 150.440 \\
\hline 87923 & 2 & 0.000 & 50.188 & 138.426 & 0.000 & 0.000 & 0.000 & 0.000 & 0.000 & 83.259 & 157.217 & 306.090 & 462.923 \\
\hline 87930 & 0 & 0.000 & 0.240 & 4.182 & 0.000 & 0.000 & 0.000 & 0.000 & 0.000 & 0.000 & 0.000 & 0.000 & 0.001 \\
\hline 87932 & 2 & 0.000 & 0.059 & 2.652 & 0.000 & 0.000 & 0.000 & 0.000 & 0.000 & 0.000 & 0.000 & 0.000 & 0.000 \\
\hline 87933 & 1 & 4.272 & 63.340 & 132.951 & 0.000 & 0.000 & 0.000 & 0.000 & 4.638 & 136.194 & 201.134 & 324.633 & 458.338 \\
\hline 97901 & 18 & 0.000 & 7.307 & 31.324 & 0.000 & 0.000 & 0.000 & 0.000 & 0.000 & 4.580 & 12.649 & 39.676 & 80.931 \\
\hline 97903 & 7 & 32.941 & 229.702 & 406.030 & 0.000 & 0.000 & 0.000 & 0.000 & 33.101 & 528.019 & 739.195 & 1092.225 & 1432.845 \\
\hline 97905 & 7 & 0.000 & 0.000 & 0.000 & 0.000 & 0.000 & 0.000 & 0.000 & 0.000 & 0.000 & 0.000 & 0.000 & 0.000 \\
\hline 97911 & 4 & 0.000 & 0.000 & 0.003 & 0.000 & 0.000 & 0.000 & 0.000 & 0.000 & 0.000 & 0.000 & 0.000 & 0.000 \\
\hline 97920 & 1 & 0.000 & 0.181 & 5.421 & 0.000 & 0.000 & 0.000 & 0.000 & 0.000 & 0.000 & 0.000 & 0.000 & 0.000 \\
\hline 97922 & 1 & 0.000 & 3.968 & 29.099 & 0.000 & 0.000 & 0.000 & 0.000 & 0.000 & 0.000 & 0.087 & 8.003 & 37.088 \\
\hline 97940 & 7 & 0.000 & 14.465 & 57.185 & 0.000 & 0.000 & 0.000 & 0.000 & 0.000 & 8.356 & 27.830 & 86.715 & 170.038 \\
\hline
\end{tabular}

Abbreviations: $n=$ Number of extremes used in the model estimation, $\mathrm{nllh}=$ Negative log-likelihood value at model convergence 

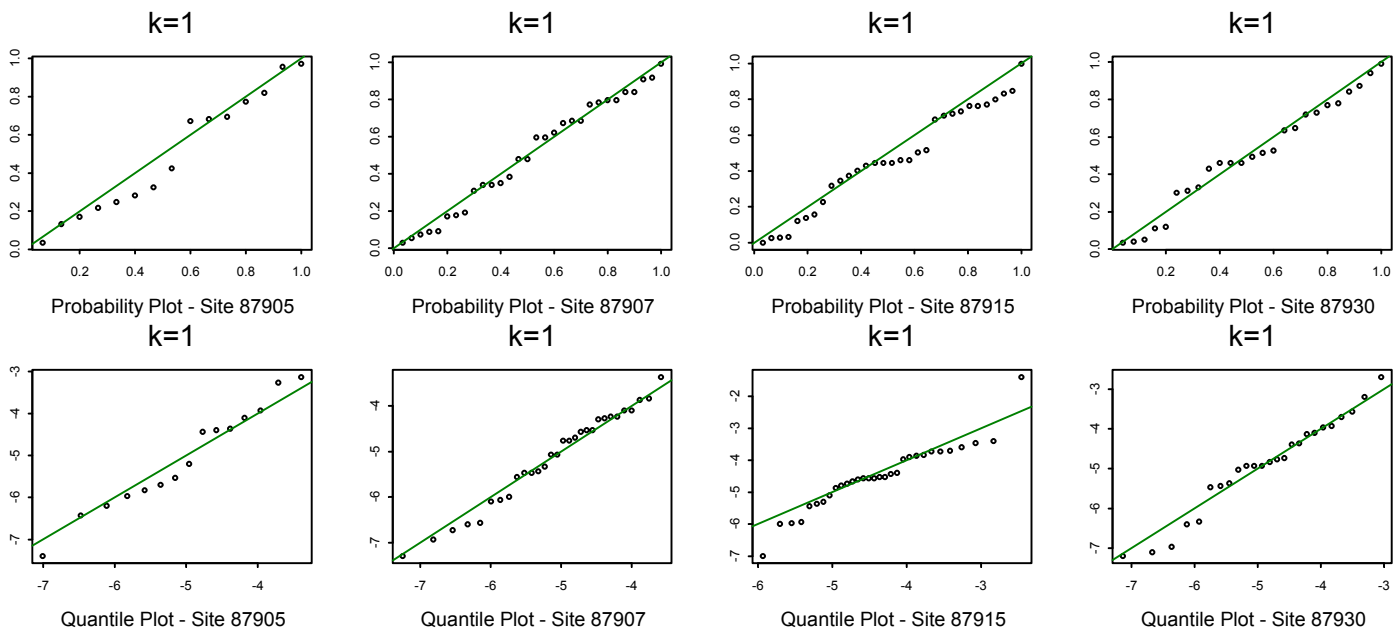

$k=1$

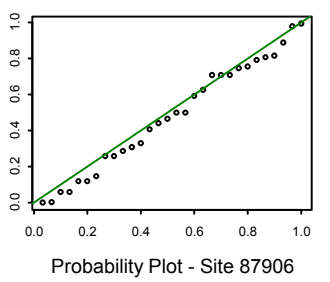

$\mathrm{k}=1$

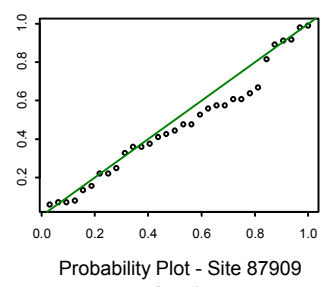

$\mathrm{k}=2$

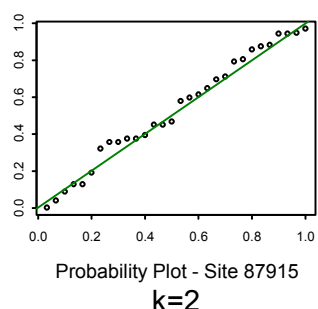

$\mathrm{k}=1$
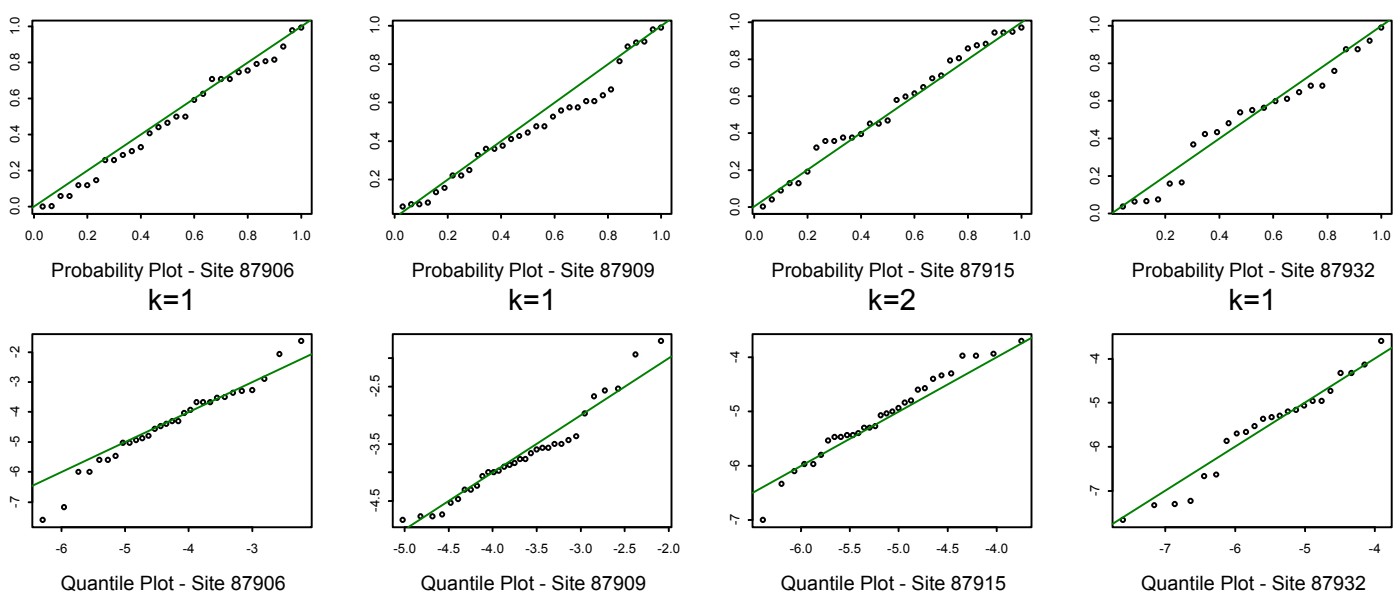

$\mathrm{k}=1$

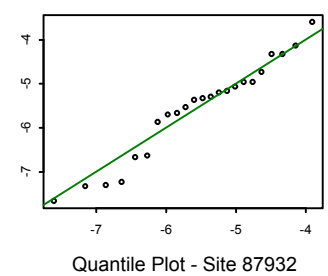

$\mathrm{k}=1$
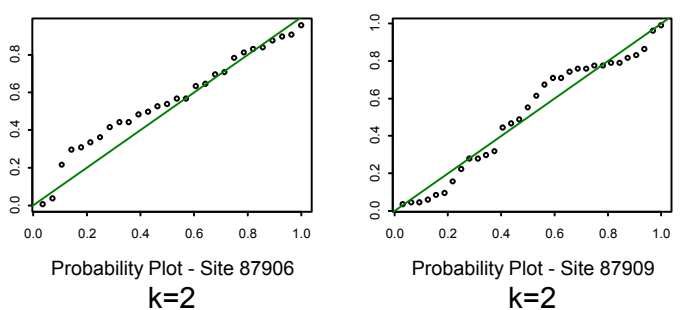

$\mathrm{k}=1$
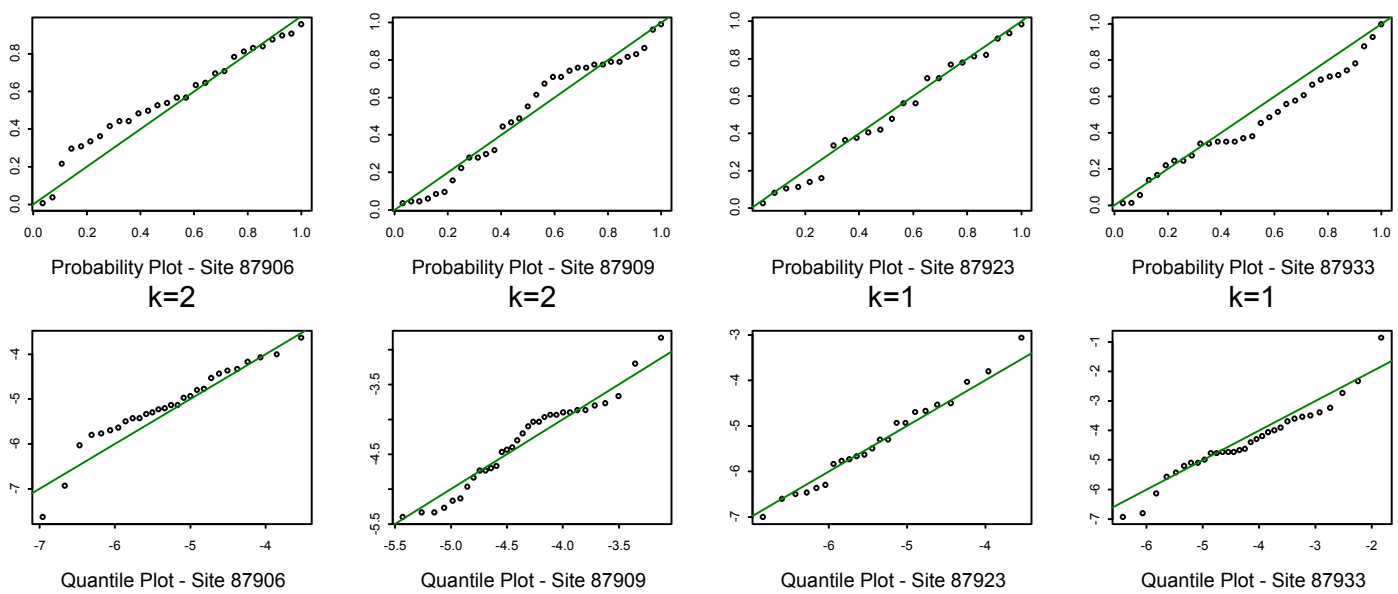

Figure E-5: Selected Diagnostics for Homogeneous Models Using All PET Data (1) 

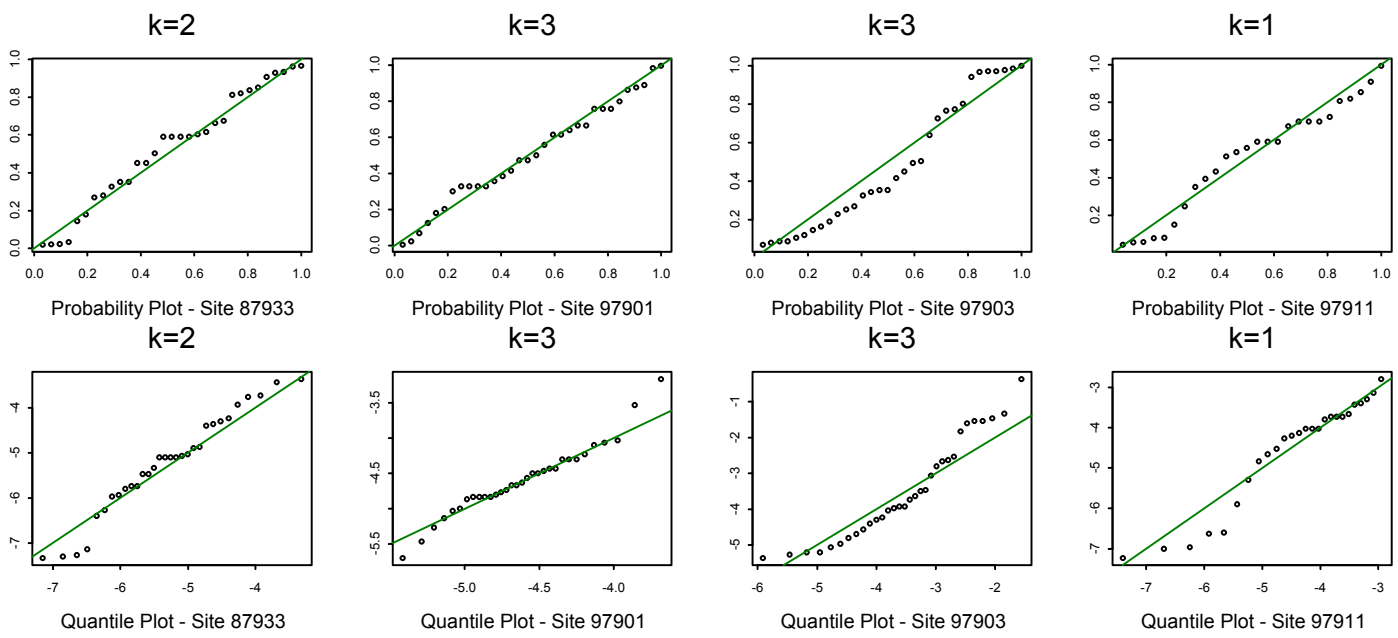

$k=1$

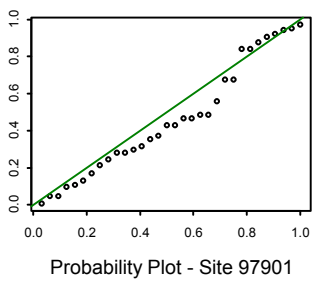

$\mathrm{k}=1$

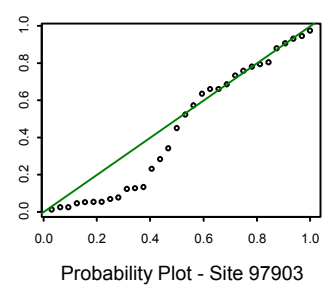

$\mathrm{k}=1$
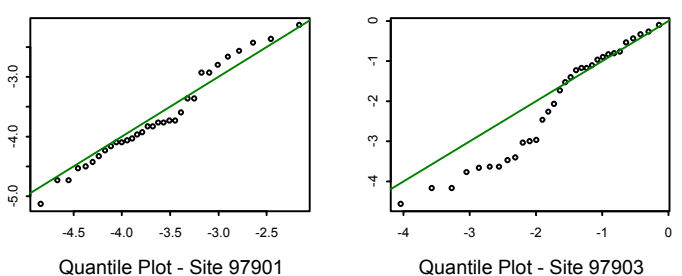

$\mathrm{k}=2$

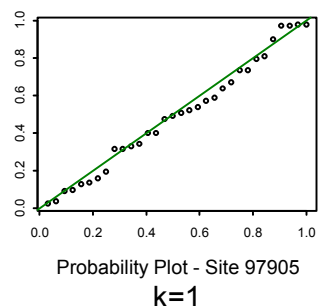

$\mathrm{k}=1$

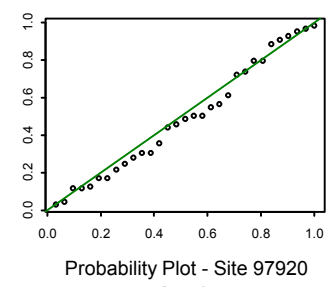

$\mathrm{k}=1$
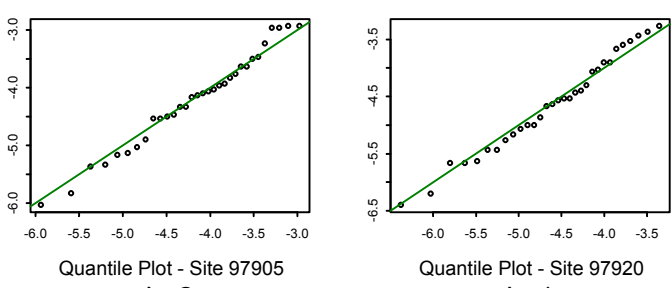

$\mathrm{k}=2$
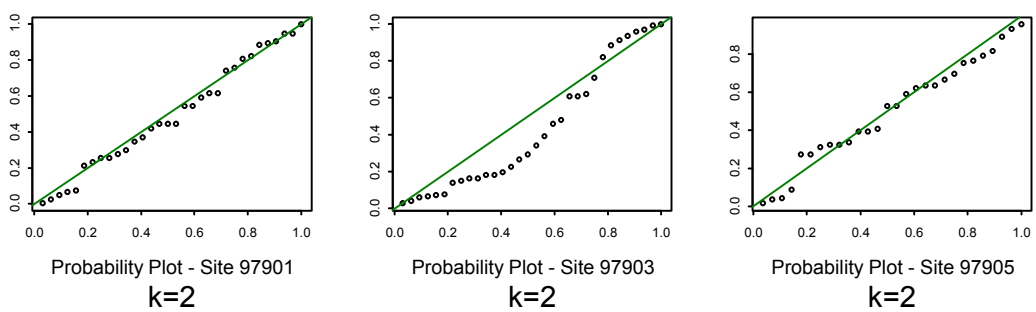

$\mathrm{k}=1$
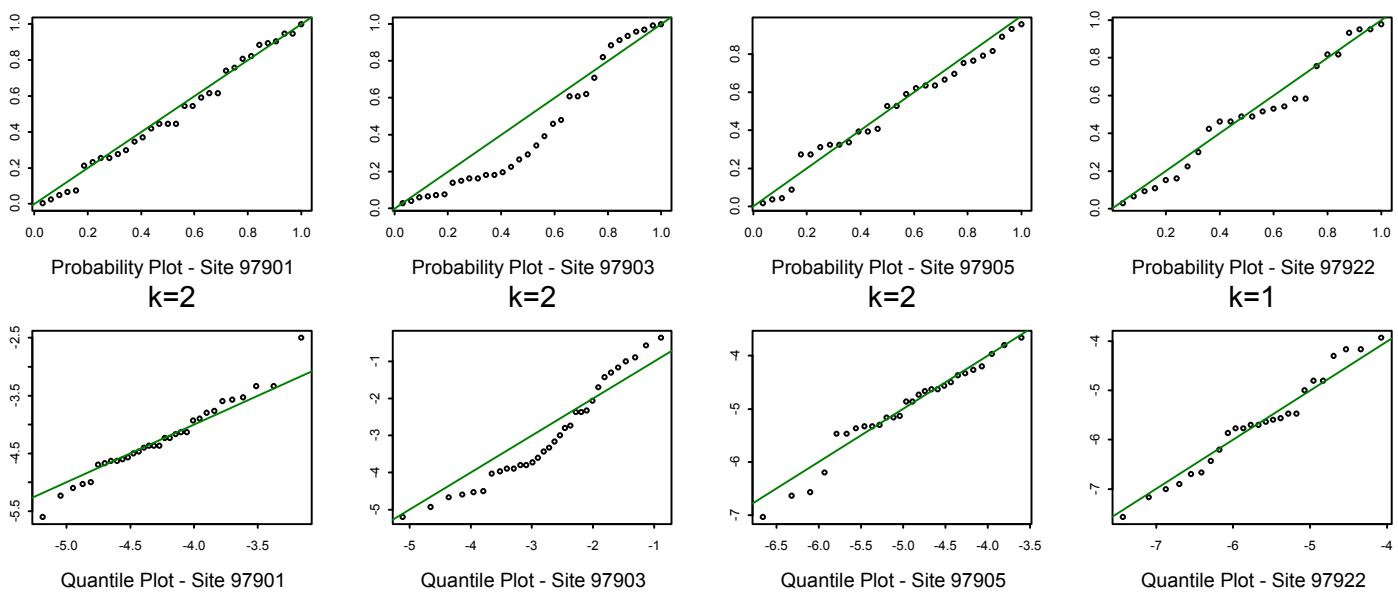

Figure E-6: Selected Diagnostics for Homogeneous Models Using All PET Data (2) 

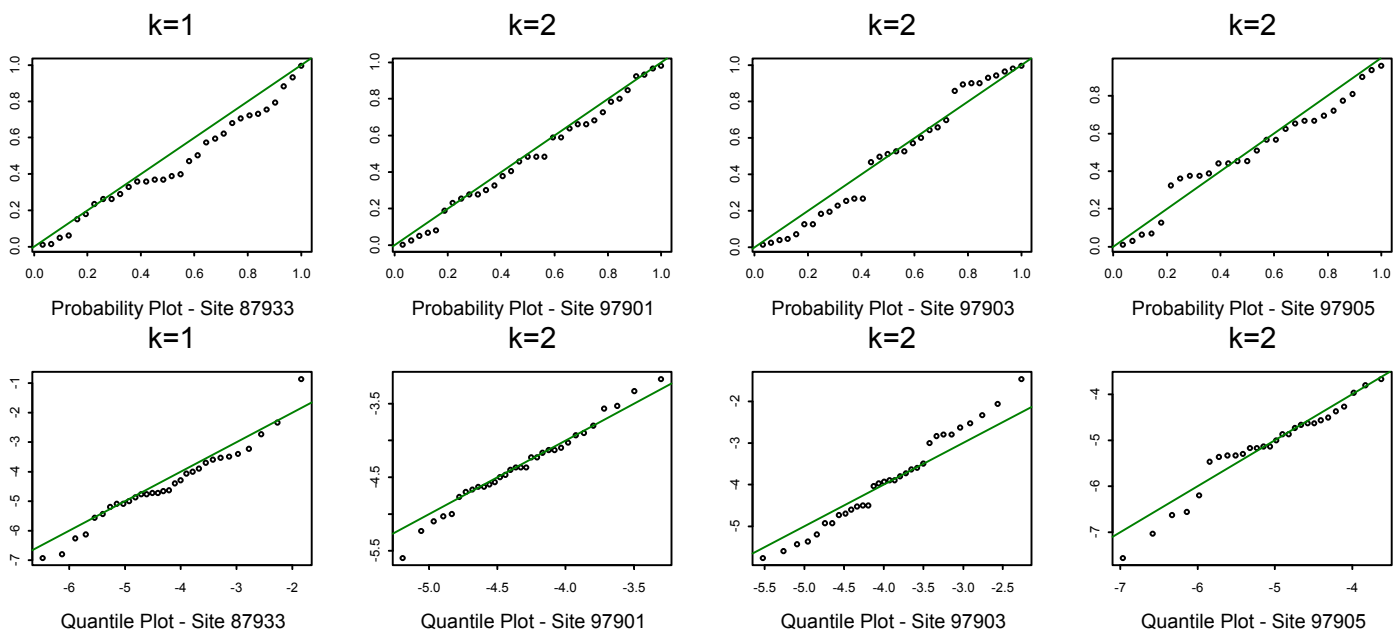

$$
k=2
$$

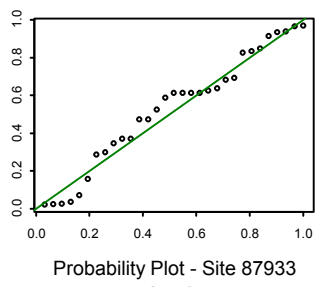

$\mathrm{k}=2$

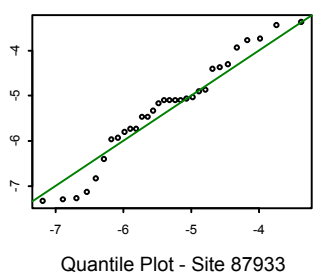

$\mathrm{k}=1$

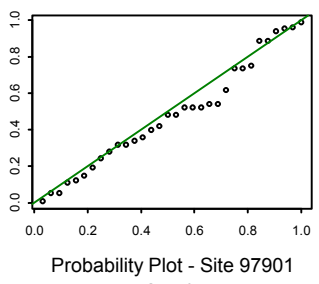

$k=1$
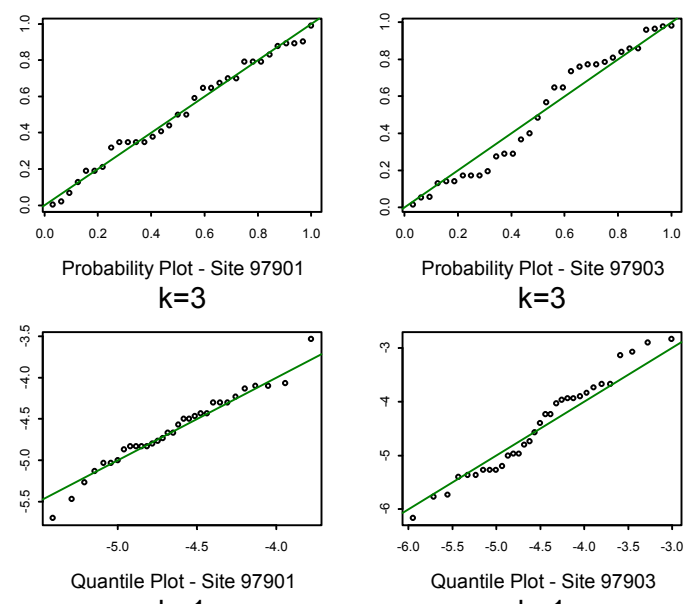

$\mathrm{k}=1$
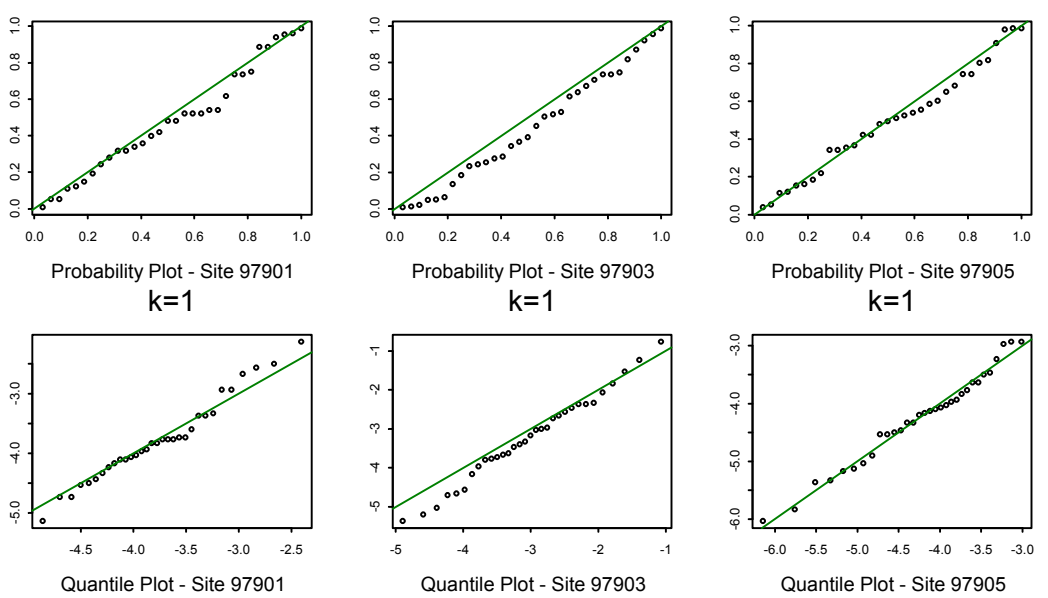

Figure E-7: Diagnostics for Sites with PETs Filtered - Homogeneous Models 
Table E-4: Results - Non-Stationary Models Using “ $V_{x t h}$ ”

Model Descriptions: Filtered PET data fitted to non-stationary $r$ largest order statistic models, $\mu=\beta_{0}+\beta_{1} V_{x t h}$

\begin{tabular}{|c|c|c|c|c|c|c|c|c|c|c|c|c|c|}
\hline \multirow{2}{*}{ Site } & \multirow{2}{*}{$\begin{array}{l}\text { 4-Year Right- } \\
\text { Angle Crash } \\
\text { Counts } \\
\text { (Daytime) }\end{array}$} & \multirow{2}{*}{$\begin{array}{l}\text { 8-Hour } \\
\text { Counts of } \\
\text { PET } \leq 6\end{array}$} & \multirow{2}{*}{$\mathrm{CV}\left(\mathrm{ET}_{\mathrm{PET} \leq 6}\right)$} & \multirow{2}{*}{ Filter } & \multirow{2}{*}{$\mathbf{r}$} & \multirow{2}{*}{$\mathbf{n}$} & \multicolumn{7}{|c|}{ Maximum Likelihood Estimation Results } \\
\hline & & & & & & & nllh & $\beta_{0}$ & $\beta_{1}$ & $\sigma$ & $\xi$ & $\operatorname{se}\left(\beta_{0}\right)$ & $\operatorname{se}\left(\beta_{1}\right)$ \\
\hline 87905 & 1 & 31 & 0.281 & None & 1 & 15 & 23.185 & -4.056 & -0.005 & 1.175 & -0.369 & 1.663 & 0.006 \\
\hline 87906 & 6 & 98 & 0.642 & None & 2 & 58 & 60.496 & -7.420 & 0.029 & 0.955 & -0.290 & 0.668 & 0.007 \\
\hline 87907 & 3 & 54 & 0.285 & None & 1 & 30 & 40.181 & -4.106 & -0.012 & 1.167 & -0.701 & 0.513 & 0.004 \\
\hline 87909 & 7 & 268 & 0.303 & None & 2 & 64 & 44.293 & -5.360 & 0.011 & 0.692 & -0.184 & 0.523 & 0.004 \\
\hline 87915 & 3 & 116 & 0.423 & None & 2 & 61 & 46.660 & -7.025 & 0.032 & 0.747 & -0.167 & 0.551 & 0.008 \\
\hline 87930 & 0 & 38 & 0.380 & None & 1 & 25 & 37.150 & -7.343 & 0.056 & 1.079 & -0.278 & 1.068 & 0.028 \\
\hline 87933 & 1 & 104 & 0.576 & $95 \%$-quantile & 2 & 62 & 58.349 & -8.668 & 0.084 & 0.877 & -0.156 & 0.552 & 0.012 \\
\hline 97901 & 18 & 311 & 0.538 & $95 \%$-quantile & 3 & 96 & 15.025 & -5.867 & 0.009 & 0.530 & -0.092 & 0.339 & 0.002 \\
\hline 97903 & 7 & 327 & 0.969 & $80 \%$-quantile & 3 & 96 & 76.084 & -4.444 & 0.006 & 0.982 & -0.334 & 0.888 & 0.005 \\
\hline 97905 & 7 & 106 & 0.537 & $95 \%$-quantile & 2 & 60 & 60.818 & -4.482 & 0.000 & 0.934 & -0.551 & 0.824 & 0.004 \\
\hline 97911 & 4 & 69 & 0.500 & NA & 1 & 26 & NA & NA & NA & NA & NA & NA & NA \\
\hline 97920 & 1 & 79 & 0.509 & None & 1 & 31 & 37.113 & -4.982 & 0.002 & 0.889 & -0.480 & 0.666 & 0.011 \\
\hline \multirow{3}{*}{ Site } & \multirow{3}{*}{$\begin{array}{l}\text { 1-Year Return } \\
\text { Level Estimate }\end{array}$} & trai & \multicolumn{11}{|c|}{ Simulation-based Inferences on 4-Year Return Level Estimates: Simulation Runs = 1000} \\
\hline & & $\begin{array}{c}\text { Return } \\
\text { Level }\end{array}$ & \multirow{2}{*}{ Mean } & \multirow{2}{*}{ SD } & \multicolumn{9}{|c|}{ \%-quantile of 4-Year Return Level Estimates } \\
\hline & & Estimate & & & $2.5 \%$ & $5.0 \%$ & $10.0 \%$ & $15.0 \%$ & $50.0 \%$ & $85.0 \%$ & $90.0 \%$ & $95.0 \%$ & $97.5 \%$ \\
\hline 87905 & -2.033 & -1.943 & 7.686 & 68.411 & -3.420 & -3.221 & -3.017 & -2.863 & -1.586 & 4.073 & 10.931 & 35.108 & 80.263 \\
\hline 87906 & -0.835 & -0.692 & -0.551 & 0.758 & -1.701 & -1.537 & -1.379 & -1.254 & -0.665 & 0.150 & 0.347 & 0.823 & 1.491 \\
\hline 87907 & -3.164 & -3.15 & -3.057 & 0.274 & -3.396 & -3.357 & -3.308 & -3.268 & -3.100 & -2.887 & -2.799 & -2.620 & -2.332 \\
\hline 87909 & -0.650 & -0.471 & -0.195 & 1.253 & -1.670 & -1.542 & -1.380 & -1.248 & -0.494 & 0.934 & 1.425 & 2.089 & 2.756 \\
\hline 87915 & -0.709 & -0.473 & -0.248 & 1.088 & -1.733 & -1.603 & -1.378 & -1.215 & -0.461 & 0.761 & 1.153 & 1.835 & 2.268 \\
\hline 87930 & -1.231 & -1.076 & -0.883 & 1.175 & -2.583 & -2.357 & -2.087 & -1.937 & -1.101 & 0.199 & 0.514 & 1.186 & 1.918 \\
\hline 87933 & 0.926 & 1.321 & 1.672 & 1.656 & -0.211 & -0.050 & 0.214 & 0.421 & 1.346 & 2.838 & 3.249 & 4.478 & 5.712 \\
\hline 97901 & -0.490 & -0.188 & 0.577 & 2.599 & -2.017 & -1.807 & -1.594 & -1.366 & -0.167 & 2.362 & 3.512 & 5.802 & 7.879 \\
\hline 97903 & -0.306 & -0.214 & 0.065 & 0.886 & -0.957 & -0.863 & -0.721 & -0.629 & -0.152 & 0.809 & 1.070 & 1.707 & 2.188 \\
\hline 97905 & -2.774 & -2.769 & -2.558 & 0.247 & -2.934 & -2.896 & -2.828 & -2.785 & -2.596 & -2.321 & -2.228 & -2.093 & -1.987 \\
\hline 97911 & NA & NA & NA & NA & NA & NA & NA & NA & NA & NA & NA & NA & NA \\
\hline 97920 & -2.977 & -2.947 & -2.623 & 0.650 & -3.342 & -3.269 & -3.200 & -3.144 & -2.778 & -2.158 & -1.895 & -1.360 & -0.985 \\
\hline \multirow{3}{*}{ Site } & \multirow{3}{*}{$\begin{array}{l}\text { 4-Year Right- } \\
\text { Angle Crash } \\
\text { Counts } \\
\text { (Daytime) }\end{array}$} & \multirow{3}{*}{$\begin{array}{c}\text { 4-Yr Crash } \\
\text { Frequency } \\
\text { Estimate }\end{array}$} & \multicolumn{11}{|c|}{ Simulation-based Inferences on 4-Year Crash Frequency Estimates: Simulation Runs $=\mathbf{3 0 0 0 0}$} \\
\hline & & & & & \multicolumn{9}{|c|}{$\%$-quantile of 4-Year Crash Frequency Estimates } \\
\hline & & & Thedn & D & $2.5 \%$ & $5.0 \%$ & $10.0 \%$ & $15.0 \%$ & $50.0 \%$ & $85.0 \%$ & $90.0 \%$ & $95.0 \%$ & $97.5 \%$ \\
\hline 87905 & 1 & 0.000 & 65.272 & 198.960 & 0.000 & 0.000 & 0.000 & 0.000 & 0.000 & 84.368 & 197.193 & 438.182 & 701.172 \\
\hline 87906 & 6 & 0.000 & 4.959 & 23.803 & 0.000 & 0.000 & 0.000 & 0.000 & 0.000 & 2.539 & 7.333 & 24.310 & 53.203 \\
\hline 87907 & 3 & 0.000 & 0.056 & 2.299 & 0.000 & 0.000 & 0.000 & 0.000 & 0.000 & 0.000 & 0.000 & 0.000 & 0.000 \\
\hline 87909 & 7 & 0.003 & 21.195 & 70.247 & 0.000 & 0.000 & 0.000 & 0.000 & 0.005 & 26.349 & 55.268 & 123.004 & 208.597 \\
\hline 87915 & 3 & 0.022 & 8.063 & 27.406 & 0.000 & 0.000 & 0.000 & 0.000 & 0.027 & 10.049 & 19.740 & 45.538 & 79.566 \\
\hline 87930 & 0 & 0.000 & 8.109 & 36.386 & 0.000 & 0.000 & 0.000 & 0.000 & 0.000 & 3.563 & 12.526 & 43.739 & 91.244 \\
\hline 87933 & 1 & 51.191 & 111.064 & 147.502 & 0.003 & 0.574 & 3.631 & 7.789 & 58.632 & 219.886 & 285.139 & 403.383 & 529.867 \\
\hline 97901 & 18 & 0.388 & 37.055 & 99.897 & 0.000 & 0.000 & 0.000 & 0.000 & 0.456 & 62.297 & 108.223 & 213.081 & 337.238 \\
\hline 97903 & 7 & 0.000 & 83.038 & 246.568 & 0.000 & 0.000 & 0.000 & 0.000 & 0.001 & 121.483 & 240.233 & 508.123 & 826.529 \\
\hline 97905 & 7 & 0.000 & 0.000 & 0.000 & 0.000 & 0.000 & 0.000 & 0.000 & 0.000 & 0.000 & 0.000 & 0.000 & 0.000 \\
\hline 97911 & 4 & NA & NA & NA & NA & NA & NA & NA & NA & NA & NA & NA & NA \\
\hline 97920 & 1 & 0.000 & 0.375 & 6.769 & 0.000 & 0.000 & 0.000 & 0.000 & 0.000 & 0.000 & 0.000 & 0.000 & 0.004 \\
\hline
\end{tabular}

Abbreviations: $\mathrm{n}=$ Number of extreme values used in the model estimation, $\mathrm{nllh}=$ Negative log-likelihood value at model convergence

Notes: NA is the case where there is no optimal mle can be found at convergence 
Table E-5: Results - Non-Stationary Models Using “ $f_{\mathrm{PET}<4.5}$ "

Model Descriptions: Filtered PET data fitted to the non-stationary $r$ largest order statistic models, $\mu=\beta_{0}+\beta_{1} f_{P E T<4.5}$

\begin{tabular}{|c|c|c|c|c|c|c|c|c|c|c|c|c|c|c|c|}
\hline \multirow{2}{*}{ Site } & \multirow{2}{*}{$\begin{array}{l}\text { 4-Year Right- } \\
\text { Angle Crash } \\
\text { Counts } \\
\text { (Daytime) }\end{array}$} & \multirow{2}{*}{$\begin{array}{l}\text { 8-Hour } \\
\text { Counts of } \\
\text { PET } \leq 6\end{array}$} & \multirow{2}{*}{$\mathrm{CV}\left(\mathrm{ET}_{\mathrm{PET} \leq 6}\right)$} & \multirow{2}{*}{ Filter } & \multirow{2}{*}{$r$} & \multirow{2}{*}{$\mathbf{n}$} & \multicolumn{9}{|c|}{ Maximum Likelihood Estimation Results } \\
\hline & & & & & & & nllh & $\beta_{0}$ & $\beta_{1}$ & $\sigma$ & $\xi$ & $\operatorname{se}\left(\beta_{0}\right)$ & $\operatorname{se}\left(\beta_{1}\right)$ & $\operatorname{se}(\sigma)$ & $\operatorname{se}(\xi)$ \\
\hline 87905 & 1 & 31 & 0.281 & None & 1 & 15 & 20.786 & -5.835 & 0.523 & 0.992 & -0.333 & 0.329 & 0.200 & 0.210 & 0.199 \\
\hline 87906 & 6 & 98 & 0.642 & None & 2 & 58 & 54.072 & -5.508 & 0.850 & 0.837 & -0.289 & 0.181 & 0.141 & 0.075 & 0.086 \\
\hline 87907 & 3 & 54 & 0.285 & NA & 1 & 30 & NA & NA & NA & NA & NA & NA & NA & NA & NA \\
\hline 87909 & 7 & 268 & 0.303 & None & 2 & 64 & 30.097 & -4.759 & 0.308 & 0.520 & -0.049 & 0.117 & 0.039 & 0.055 & 0.122 \\
\hline 87915 & 3 & 116 & 0.423 & None & 2 & 61 & 40.067 & -5.222 & 0.586 & 0.673 & -0.165 & 0.128 & 0.088 & 0.060 & 0.056 \\
\hline 87930 & 0 & 38 & 0.380 & NA & 1 & 25 & NA & NA & NA & NA & NA & NA & NA & NA & NA \\
\hline 87933 & 1 & 104 & 0.576 & 95\%-quantile & 2 & 62 & 56.457 & -5.548 & 0.825 & 0.861 & -0.322 & 0.169 & 0.126 & 0.075 & 0.089 \\
\hline 97901 & 18 & 311 & 0.538 & 95\%-quantile & 3 & 96 & 4.844 & -4.773 & 0.299 & 0.471 & 0.032 & 0.101 & 0.033 & 0.049 & 0.109 \\
\hline 97903 & 7 & 327 & 0.969 & $80 \%$-quantile & 3 & 96 & 55.193 & -4.570 & 0.335 & 0.794 & -0.110 & 0.162 & 0.040 & 0.068 & 0.084 \\
\hline 97905 & 7 & 106 & 0.537 & 95\%-quantile & 2 & 60 & 52.086 & -5.341 & 0.923 & 0.798 & -0.454 & 0.209 & 0.196 & 0.068 & 0.087 \\
\hline 97911 & 4 & 69 & 0.500 & None & 1 & 26 & 33.030 & -5.788 & 1.143 & 0.953 & -0.456 & 0.289 & 0.243 & 0.154 & 0.130 \\
\hline 97920 & 1 & 79 & 0.509 & None & 1 & 31 & 27.429 & -5.242 & 0.542 & 0.614 & -0.350 & 0.142 & 0.104 & 0.084 & 0.104 \\
\hline
\end{tabular}

\begin{tabular}{|c|c|c|c|c|c|c|c|c|c|c|c|c|c|}
\hline \multirow{3}{*}{ Site } & \multirow{3}{*}{$\begin{array}{c}\text { 1-Year Return } \\
\text { Level } \\
\text { Estimate }\end{array}$} & \multirow{3}{*}{$\begin{array}{c}\text { 4-Year } \\
\text { Return } \\
\text { Level } \\
\text { Estimate }\end{array}$} & \multicolumn{11}{|c|}{ Simulation-based Inferences on 4-Year Return Level Estimates: Simulation Runs = 2000} \\
\hline & & & \multirow{2}{*}{ Mean } & \multirow{2}{*}{ SD } & \multicolumn{9}{|c|}{ \%-quantile of 4-Year Return Level Estimates } \\
\hline & & & & & $2.5 \%$ & $5.0 \%$ & $10.0 \%$ & $15.0 \%$ & $50.0 \%$ & $85.0 \%$ & $90.0 \%$ & $95.0 \%$ & $97.5 \%$ \\
\hline 87905 & -0.603 & -0.468 & -0.009 & 1.912 & -2.343 & -2.054 & $\begin{array}{l}-1.726 \\
\end{array}$ & -1.472 & -0.296 & 1.172 & 1.707 & 2.889 & 4.845 \\
\hline 87906 & 0.317 & 0.471 & 0.581 & 0.577 & -0.422 & -0.283 & -0.106 & 0.017 & 0.555 & 1.108 & 1.268 & 1.514 & 1.789 \\
\hline 87907 & NA & NA & NA & NA & NA & NA & NA & NA & NA & NA & NA & NA & NA \\
\hline 87909 & 0.300 & 0.751 & 2.458 & 5.528 & -1.517 & -1.343 & -1.048 & -0.795 & 0.774 & 5.360 & 7.153 & 11.916 & 15.713 \\
\hline 87915 & -0.229 & 0.058 & 0.151 & 0.628 & -0.845 & -0.725 & -0.564 & -0.452 & 0.081 & 0.728 & 0.926 & 1.239 & 1.549 \\
\hline 87930 & NA & NA & NA & NA & NA & NA & NA & NA & NA & NA & NA & NA & NA \\
\hline 87933 & 0.071 & 0.196 & 0.280 & 0.390 & -0.428 & -0.299 & -0.185 & -0.102 & 0.254 & 0.653 & 0.766 & 0.937 & 1.112 \\
\hline 97901 & 1.345 & 2.248 & 4.440 & 7.539 & -1.739 & -1.348 & -0.921 & -0.491 & 2.147 & 8.826 & 11.573 & 17.889 & 23.981 \\
\hline 97903 & 1.809 & 2.214 & 2.822 & 2.497 & 0.124 & 0.315 & 0.597 & 0.824 & 2.215 & 4.824 & 5.731 & 7.614 & 9.086 \\
\hline 97905 & -1.787 & -1.765 & -1.725 & 0.307 & -2.292 & -2.202 & -2.096 & -2.026 & -1.740 & -1.414 & -1.336 & -1.204 & -1.074 \\
\hline 97911 & -0.386 & -0.331 & -0.266 & 0.590 & -1.378 & -1.203 & -1.015 & -0.878 & -0.2 & 0.321 & 0.477 & 0.719 & 0.951 \\
\hline 97920 & -1.512 & -1.438 & -1.388 & 0.426 & -2.154 & -2.032 & -1.892 & -1.812 & -1.408 & -0.973 & -0.863 & -0.655 & -0.525 \\
\hline \multirow{3}{*}{ Site } & \multirow{3}{*}{$\begin{array}{l}\text { 4-Year Right- } \\
\text { Angle Crash } \\
\text { Counts } \\
\text { (Daytime) }\end{array}$} & \multirow{3}{*}{$\begin{array}{c}\text { 4-Yr Crash } \\
\text { Frequency } \\
\text { Estimate }\end{array}$} & \multicolumn{11}{|c|}{ Simulation-based Inferences on 4-Year Crash Frequency Estimates: Simulation Runs = 10000} \\
\hline & & & \multirow{2}{*}{ Mean } & \multirow{2}{*}{ SD } & \multicolumn{9}{|c|}{$\%$-quantile of 4-Year Crash Frequency Estimates } \\
\hline & & & & & $2.5 \%$ & $5.0 \%$ & $10.0 \%$ & $15.0 \%$ & $50.0 \%$ & $85.0 \%$ & $90.0 \%$ & $95.0 \%$ & $97.5 \%$ \\
\hline 87905 & 1 & 0.000 & 32.623 & 94.918 & 0.000 & 0.000 & 0.000 & 0.000 & 0.000 & 55.071 & 93.164 & 183.819 & 293.777 \\
\hline 87906 & 6 & 23.948 & 58.581 & 87.285 & 0.000 & 0.000 & 0.087 & 1.089 & 27.364 & 118.438 & 152.334 & 218.123 & 287.078 \\
\hline 87907 & 3 & NA & NA & NA & NA & NA & NA & NA & NA & NA & NA & NA & NA \\
\hline 87909 & 7 & 9.602 & 90.762 & 188.225 & 0.000 & 0.000 & 0.000 & 0.000 & 9.577 & 191.071 & 280.929 & 461.408 & 654.942 \\
\hline 87915 & 3 & 1.356 & 6.615 & 13.439 & 0.000 & 0.000 & 0.000 & 0.003 & 1.591 & 12.707 & 18.268 & 29.996 & 43.266 \\
\hline 87930 & 0 & NA & NA & NA & NA & NA & NA & NA & NA & NA & NA & NA & NA \\
\hline 87933 & 1 & 7.075 & 26.219 & 46.187 & 0.000 & 0.000 & 0.000 & 0.056 & 9.878 & 50.048 & 67.961 & 107.692 & 152.203 \\
\hline 97901 & 18 & 35.555 & 141.540 & 238.901 & 0.000 & 0.000 & 0.000 & 0.032 & 35.964 & 308.877 & 421.787 & 654.739 & 852.504 \\
\hline 97903 & 7 & 386.951 & 560.530 & 534.540 & 3.922 & 14.740 & 44.111 & 80.840 & 404.764 & 1079.948 & 1295.943 & 1638.861 & 1981.178 \\
\hline 97905 & 7 & 0.000 & 0.000 & 0.016 & 0.000 & 0.000 & 0.000 & 0.000 & 0.000 & 0.000 & 0.000 & 0.000 & 0.000 \\
\hline 97911 & 4 & 0.000 & 24.995 & 80.134 & 0.000 & 0.000 & 0.000 & 0.000 & 0.000 & 33.594 & 69.970 & 145.638 & 254.280 \\
\hline 97920 & 1 & 0.000 & 0.016 & 0.444 & 0.000 & 0.000 & 0.000 & 0.000 & 0.000 & 0.000 & 0.000 & 0.000 & 0.003 \\
\hline
\end{tabular}

Abbreviations: $n=$ Number of extremes used in the model estimation, $n$ llh $=$ Negative log-likelihood value at model convergence

Notes: NA is the case where there is no optimal mle can be found at convergence 
Table E-6: Results - Non-Stationary Models Using " $V_{x t h}$ " and " $f_{P E T<4.5}$ "

Model Descriptions: Filtered PET data fitted to non-stationary $r$ largest order statistic models, $\mu=\beta_{0}+\beta_{1} V_{x t h}+\beta_{2} f_{P E T<4.5}$

\begin{tabular}{|c|c|c|c|c|c|c|c|c|c|c|c|c|c|}
\hline \multirow{2}{*}{ Site } & \multirow{2}{*}{$\begin{array}{l}\text { 4-Year Right- } \\
\text { Angle Crash } \\
\text { Counts } \\
\text { (Daytime) }\end{array}$} & \multirow{2}{*}{$\begin{array}{l}\text { 8-Hour } \\
\text { Counts of } \\
\text { PET } \leq 6\end{array}$} & \multirow{2}{*}{$\mathrm{CV}\left(\mathrm{ET}_{\mathrm{PET} \leq 6}\right)$} & \multirow{2}{*}{ Filter } & \multirow{2}{*}{$\mathbf{r}$} & \multirow{2}{*}{$\mathrm{n}$} & \multicolumn{7}{|c|}{ Maximum Likelihood Estimation Result } \\
\hline & & & & & & & nllh & $\beta_{0}$ & $\beta_{1}$ & $\beta_{2}$ & $\sigma$ & $\xi$ & $\operatorname{se}\left(\beta_{0}\right)$ \\
\hline 87905 & 1 & 31 & 0.281 & None & 1 & 15.000 & 20.282 & -4.435 & -0.005 & 0.519 & 0.965 & -0.345 & 1.431 \\
\hline 87906 & 6 & 98 & 0.642 & None & 2 & 58.000 & 51.811 & -6.709 & 0.014 & 0.685 & 0.802 & -0.272 & 0.580 \\
\hline 87907 & 3 & 54 & 0.285 & None & 1 & 30.000 & 29.406 & -5.126 & -0.004 & 1.017 & 0.881 & -0.830 & 0.594 \\
\hline 87909 & 7 & 268 & 0.303 & None & 2 & 64.000 & 29.868 & -4.982 & 0.002 & 0.294 & 0.517 & -0.041 & 0.346 \\
\hline 87915 & 3 & 116 & 0.423 & None & 2 & 61.000 & 38.515 & -6.149 & 0.014 & 0.468 & 0.650 & -0.167 & 0.526 \\
\hline 87930 & 0 & 38 & 0.380 & None & 1 & 25.000 & 27.855 & -6.767 & 0.026 & 1.495 & 0.774 & -0.360 & 1.175 \\
\hline 87933 & 1 & 104 & 0.576 & 95\%-quantile & 2 & 62.000 & 49.594 & -7.736 & 0.053 & 0.524 & 0.743 & -0.230 & 0.538 \\
\hline 97901 & 18 & 311 & 0.538 & 95\%-quantile & 3 & 96.000 & 4.143 & -5.113 & 0.002 & 0.259 & 0.466 & 0.034 & 0.299 \\
\hline 97903 & 7 & 327 & 0.969 & $80 \%$-quantile & 3 & 96.000 & 55.188 & -4.518 & 0.000 & 0.335 & 0.794 & -0.107 & 0.520 \\
\hline 97905 & 7 & 106 & 0.537 & 95\%-quantile & 2 & 60.000 & 52.049 & -5.564 & 0.001 & 0.931 & 0.799 & -0.463 & 0.843 \\
\hline 97911 & 4 & 69 & 0.500 & None & 1 & 26.000 & 31.968 & -4.776 & -0.006 & 1.307 & 1.013 & -0.642 & 0.570 \\
\hline 97920 & 1 & 79 & 0.509 & None & 1 & 31.000 & 26.991 & -4.618 & -0.011 & 0.587 & 0.634 & -0.440 & 0.805 \\
\hline \multirow{3}{*}{ Site } & \multirow{3}{*}{$\begin{array}{c}\text { 1-Year } \\
\text { Return Level } \\
\text { Estimate }\end{array}$} & \multirow{3}{*}{$\begin{array}{c}\text { 4-Year } \\
\text { Return } \\
\text { Level } \\
\text { Estimate }\end{array}$} & \multicolumn{11}{|c|}{ Simulation-based Inferences on 4-Year Return Level Estimates: Simulation Runs $=\mathbf{3 0 0 0}$} \\
\hline & & & Monn & $s D$ & \multicolumn{9}{|c|}{$\%$-quantile of 4-Year Return Level Estimates } \\
\hline & & & Treain & S & $2.5 \%$ & $5.0 \%$ & $10.0 \%$ & $15.0 \%$ & $50.0 \%$ & $85.0 \%$ & $90.0 \%$ & $95.0 \%$ & $97.5 \%$ \\
\hline 87905 & -0.621 & -0.500 & 0.385 & 3.874 & -2.046 & -1.777 & -1.485 & -1.259 & -0.221 & 1.207 & 1.828 & 3.566 & 6.980 \\
\hline 87906 & 0.074 & 0.239 & 0.351 & 0.681 & -0.772 & -0.600 & -0.408 & -0.291 & 0.274 & 0.961 & 1.198 & 1.572 & 1.901 \\
\hline 87907 & -2.447 & -2.443 & -2.410 & 0.372 & -3.113 & -3.012 & -2.889 & -2.796 & -2.423 & -2.019 & -1.929 & -1.795 & -1.672 \\
\hline 87909 & 0.397 & 0.877 & 2.812 & 7.629 & -1.525 & -1.324 & -1.027 & -0.798 & 0.893 & 5.673 & 8.171 & 12.039 & 19.371 \\
\hline 87915 & -0.306 & -0.031 & 0.055 & 0.616 & -0.986 & -0.819 & -0.646 & -0.527 & -0.021 & 0.636 & 0.844 & 1.162 & 1.453 \\
\hline 87930 & -0.859 & -0.772 & -0.061 & 3.814 & -1.531 & -1.401 & -1.270 & -1.157 & -0.621 & 0.331 & 0.948 & 2.691 & 5.024 \\
\hline 87933 & -0.257 & -0.093 & & 0.757 & -0.919 & -0.779 & -0.638 & -0.541 & -0.006 & 0.786 & 1.091 & 1.532 & 2.072 \\
\hline 97901 & 1.362 & 2.284 & 4.899 & 8.305 & -1.607 & -1.340 & -0.878 & -0.557 & 2.240 & 10.327 & 13.098 & 20.074 & 27.312 \\
\hline 97903 & 1.863 & 2.279 & 3.036 & 2.742 & 0.121 & 0.350 & 0.666 & 0.902 & 2.291 & 5.207 & 6.269 & 8.099 & 10.240 \\
\hline 97905 & -1.788 & -1.762 & -1.621 & 0.328 & -2.203 & -2.097 & -2.003 & -1.940 & -1.651 & -1.300 & -1.201 & -1.043 & -0.926 \\
\hline 97911 & -0.710 & -0.694 & -0.648 & 0.551 & -1.702 & -1.545 & -1.350 & -1.223 & -0.652 & -0.069 & 0.063 & 0.257 & 0.421 \\
\hline 97920 & -1.665 & -1.624 & -1.412 & 0.770 & -2.270 & -2.167 & -2.029 & -1.932 & -1.526 & -1.010 & -0.817 & -0.483 & 0.102 \\
\hline \multirow{3}{*}{ Site } & \multirow{3}{*}{$\begin{array}{l}\text { 4-Year Right- } \\
\text { Angle Crash } \\
\text { Counts } \\
\text { (Daytime) }\end{array}$} & \multirow{3}{*}{$\begin{array}{l}\text { 4-Yr Crash } \\
\text { Frequency } \\
\text { Estimate }\end{array}$} & \multicolumn{2}{|c|}{ Simulation-based I } & netence & 19 & Tear cia & 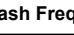 & 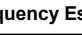 & . Simates. & & rents & \\
\hline & & & & & \multicolumn{9}{|c|}{$\%$-quantile of 4-Year Crash Frequency Estimates } \\
\hline & & & & & $2.5 \%$ & $5.0 \%$ & $10.0 \%$ & $15.0 \%$ & $50.0 \%$ & $85.0 \%$ & $90.0 \%$ & $95.0 \%$ & $97.5 \%$ \\
\hline 87905 & 1 & 0.000 & 42.741 & 129.454 & 0.000 & 0.000 & 0.000 & 0.000 & 0.001 & 64.403 & 114.692 & 233.083 & 405.855 \\
\hline 87906 & 6 & 6.457 & 27.990 & 50.040 & 0.000 & 0.000 & 0.000 & 0.000 & 7.919 & 58.435 & 80.501 & 122.606 & 169.185 \\
\hline 87907 & 3 & 0.000 & & 0.004 & 0.000 & 0.000 & 0.000 & 0.000 & 0.000 & 0.000 & 0.000 & 0.000 & 0.000 \\
\hline 87909 & 7 & 11.909 & 105.248 & 212.143 & 0.000 & 0.000 & 0.000 & 0.000 & 13.156 & 222.170 & 330.568 & 529.597 & 751.881 \\
\hline 87915 & 3 & 0.837 & 5.266 & 11.858 & 0.000 & 0.000 & 0.000 & 0.000 & 0.978 & 9.865 & 14.548 & 24.972 & 36.669 \\
\hline 87930 & 0 & 0.000 & 10.327 & 53.219 & 0.000 & 0.000 & 0.000 & 0.000 & 0.000 & 3.729 & 11.987 & 46.170 & 112.077 \\
\hline 87933 & 1 & 0.376 & 13.394 & 31.181 & 0.000 & 0.000 & 0.000 & 0.000 & 0.934 & 26.436 & 40.065 & 68.561 & 99.819 \\
\hline 97901 & 18 & 35.470 & 137.917 & 237.838 & 0.000 & 0.000 & 0.000 & 0.054 & 35.749 & 303.820 & 409.583 & 621.523 & 832.699 \\
\hline 97903 & 7 & 401.621 & 593.618 & 587.115 & 3.002 & 13.697 & 43.231 & 77.810 & 418.618 & 1160.041 & 1386.899 & 1779.118 & 2138.191 \\
\hline 97905 & 7 & 0.000 & 0.002 & 0.131 & 0.000 & 0.000 & 0.000 & 0.000 & 0.000 & 0.000 & 0.000 & 0.000 & 0.000 \\
\hline 97911 & 4 & 0.000 & 20.587 & 97.380 & 0.000 & 0.000 & 0.000 & 0.000 & 0.000 & 0.000 & 8.721 & 107.848 & 282.629 \\
\hline 97920 & 1 & 0.000 & 0.899 & 12.239 & 0.000 & 0.000 & 0.000 & 0.000 & 0.000 & 0.000 & 0.000 & 0.095 & 1.847 \\
\hline
\end{tabular}

Abbreviations: $\mathrm{n}=$ Number of extreme values used in the model estimation, $\mathrm{nllh}=$ Negative log-likelihood value at model convergence

Notes: NA is the case where there is no optimal mle can be found at convergence 
$\mathrm{k}=1$
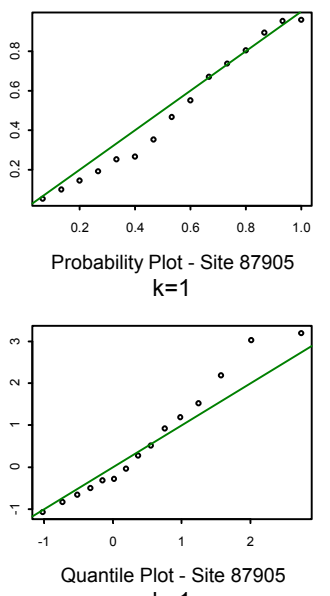

$\mathrm{k}=1$
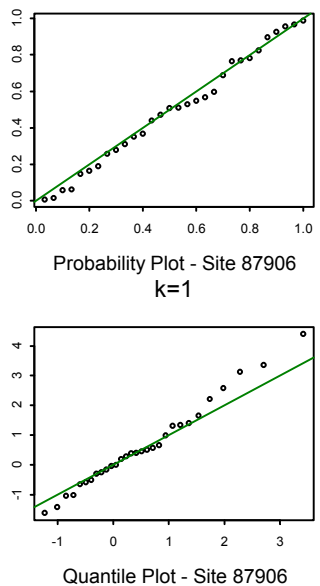

$\mathrm{k}=2$
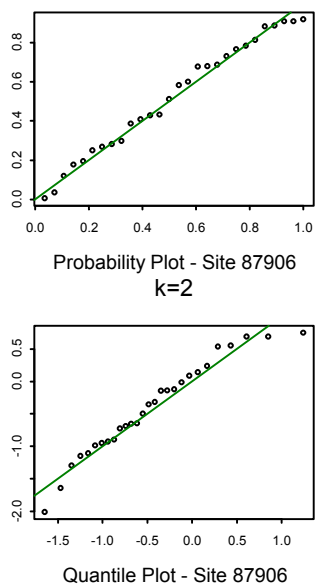

$k=1$

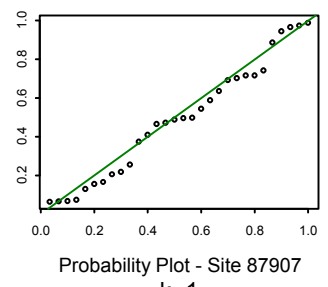

$k=1$

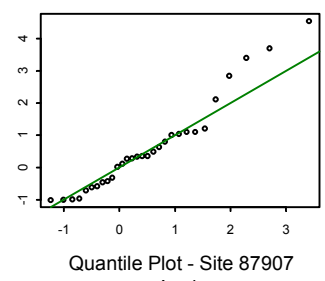

$\mathrm{k}=1$

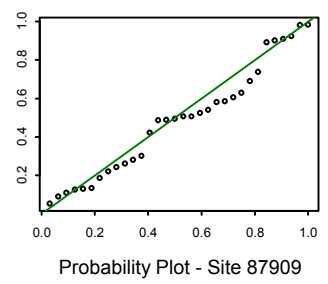

$k=1$

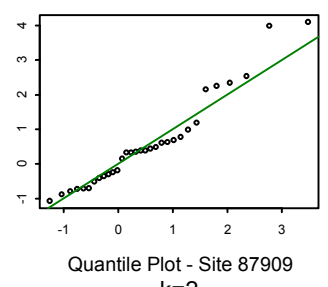

$\mathrm{k}=2$

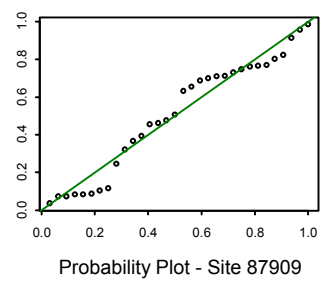

$\mathrm{k}=2$

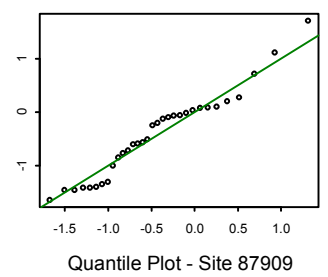

$k=1$
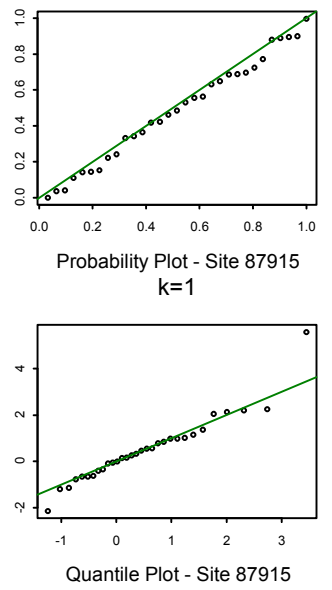

$\mathrm{k}=2$
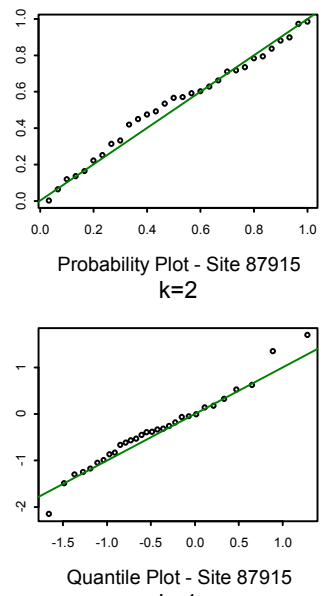

$k=1$
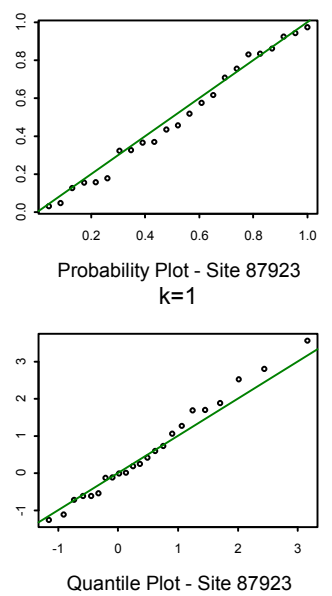

$\mathrm{k}=1$

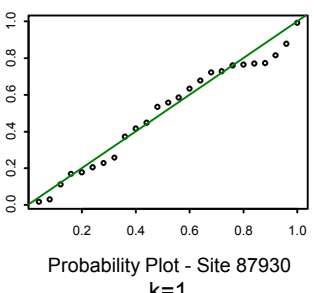

$\mathrm{k}=1$

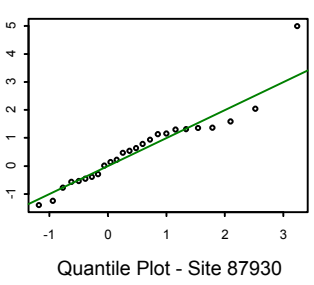

$k=1$

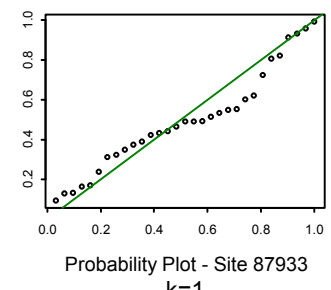

$k=1$

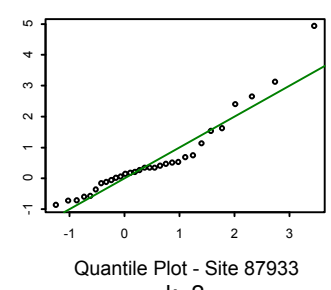

$\mathrm{k}=2$

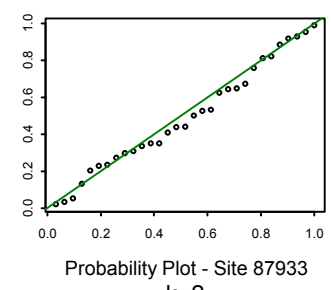

$\mathrm{k}=2$

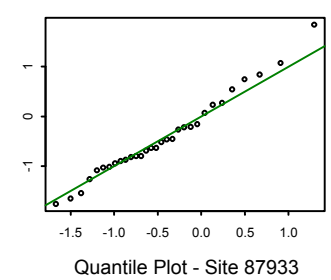

Figure E-8: Diagnostics - Non-Stationary Models Using “ $V_{x t h}$ " (1) 
$\mathrm{k}=1$
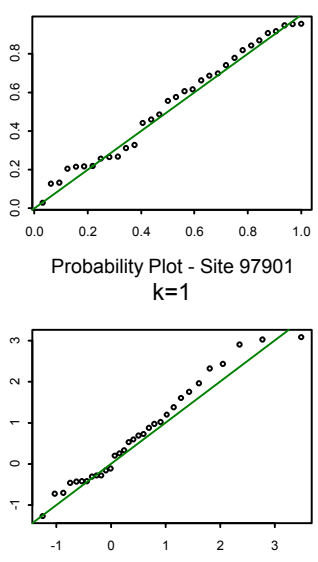

Quantile Plot - Site 97901

$\mathrm{k}=2$

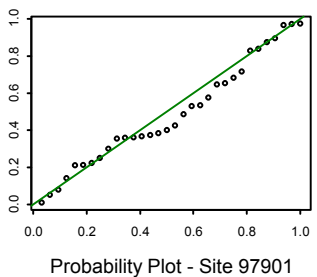

$\mathrm{k}=2$

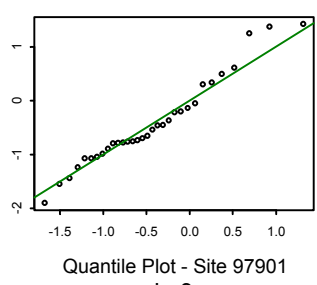

$\mathrm{k}=3$
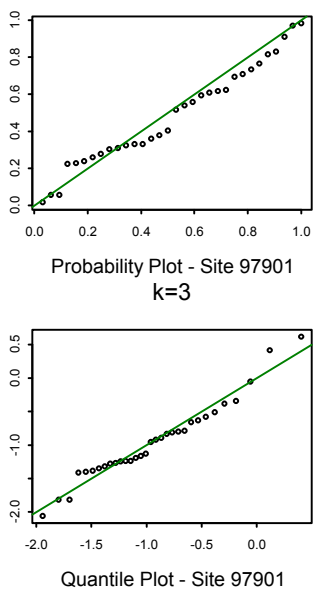

$k=1$

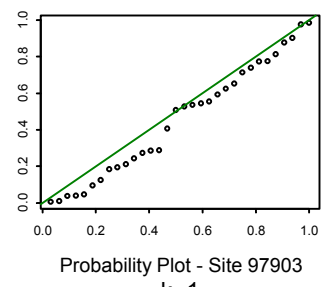

$k=1$

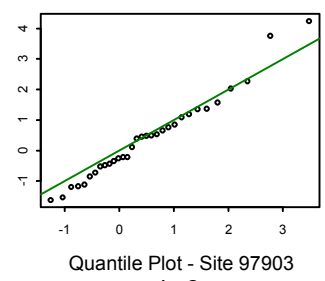

$\mathrm{k}=2$

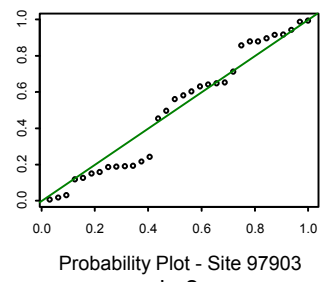

$k=2$

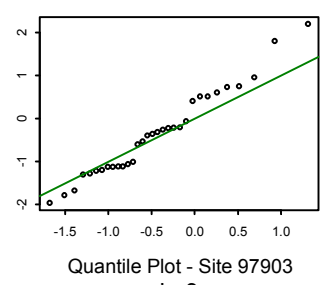

$\mathrm{k}=3$

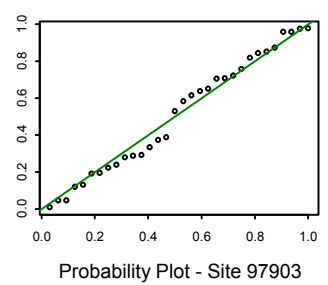

$k=3$

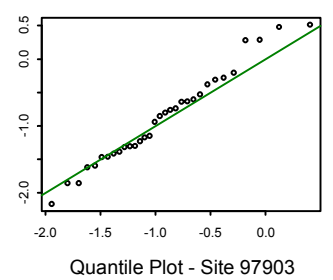

$k=1$

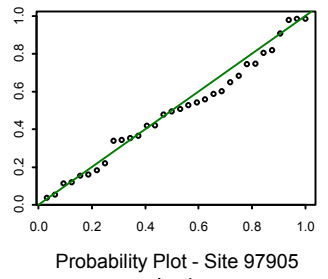

$k=1$

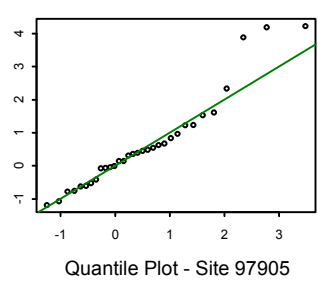

$\mathrm{k}=2$
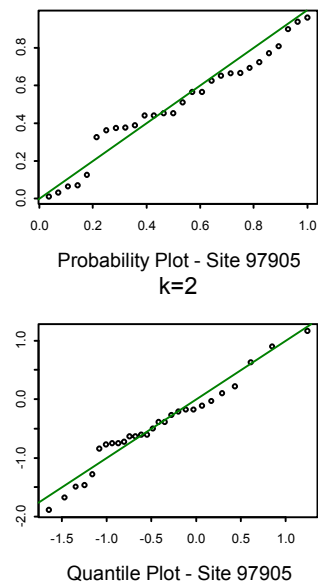

$k=1$

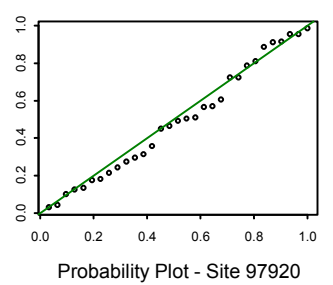

$k=1$

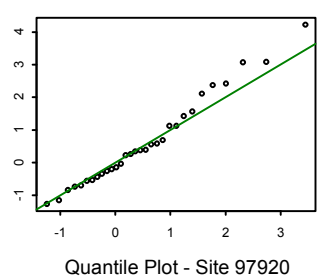

$\mathrm{k}=1$

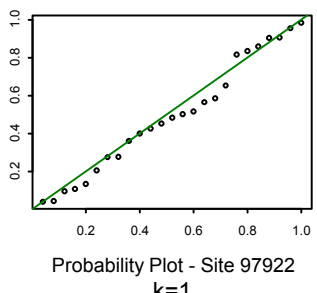

$\mathrm{k}=1$

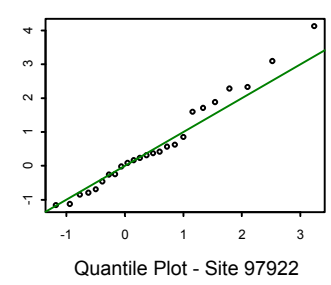

$\mathrm{k}=1$

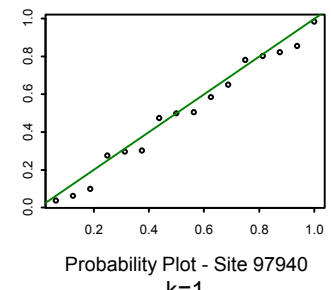

$k=1$

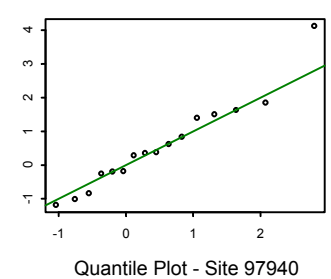

Figure E-9: Diagnostics - Non-Stationary Models Using “ $V_{x t h}$ ” (2) 
$\mathrm{k}=1$
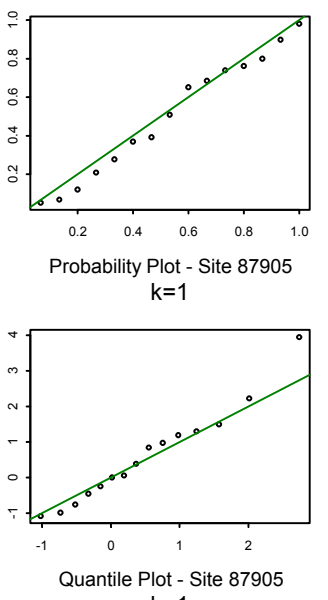

$\mathrm{k}=1$
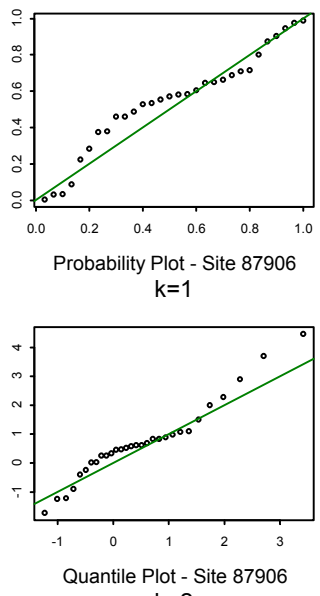

$k=2$
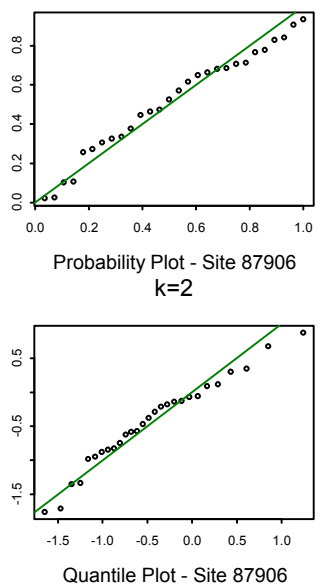

$k=1$

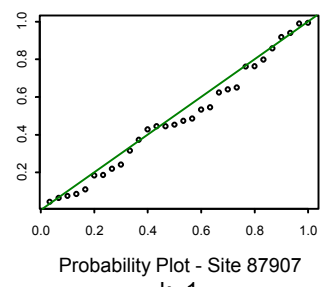

$k=1$

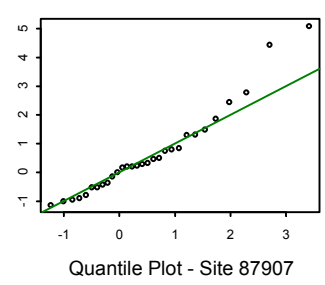

$k=1$

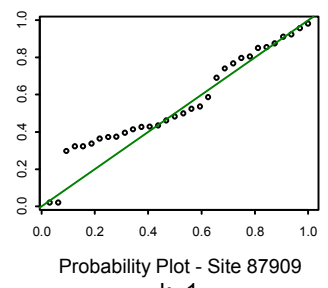

$k=1$

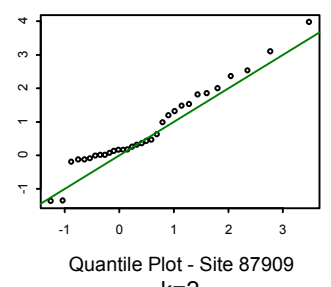

$\mathrm{k}=2$

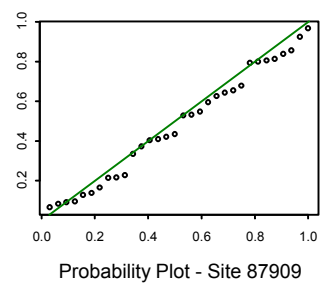

$k=2$

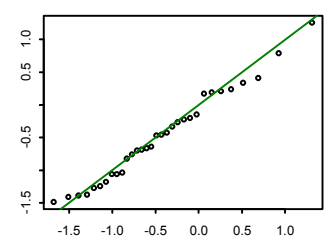

Quantile Plot - Site 87909 $k=1$
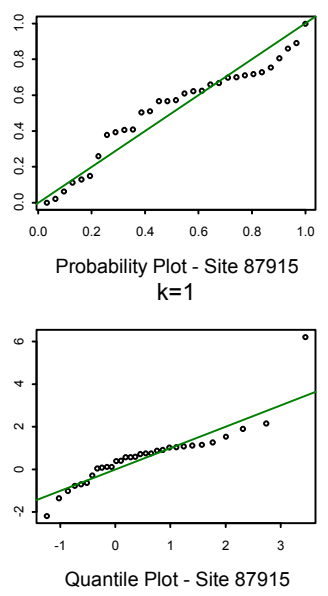

$\mathrm{k}=2$
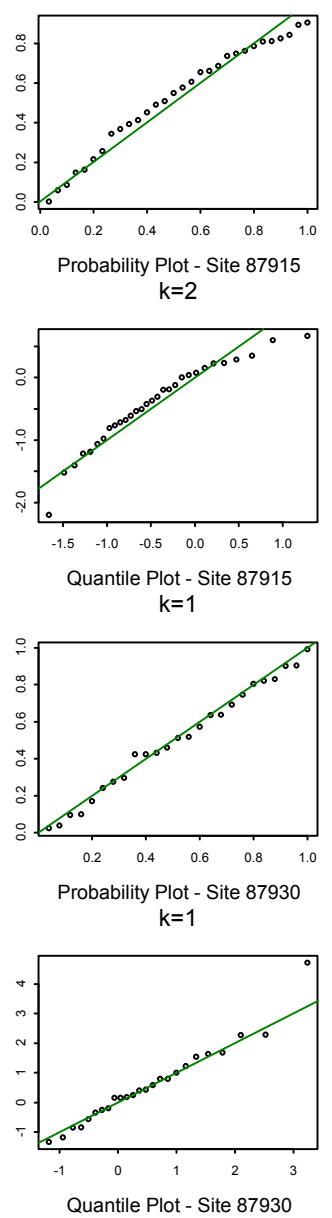

$\mathrm{k}=1$

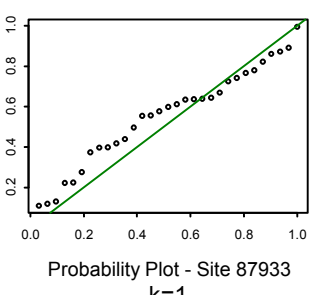

$\mathrm{k}=1$

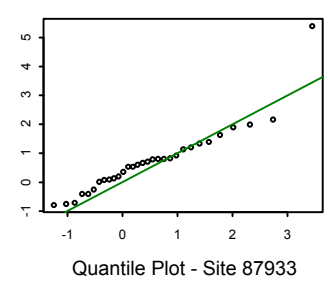

$\mathrm{k}=2$

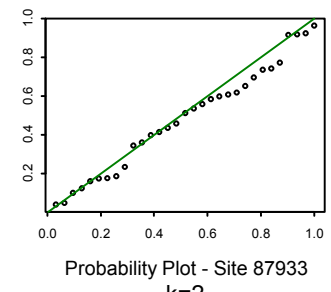

$\mathrm{k}=2$

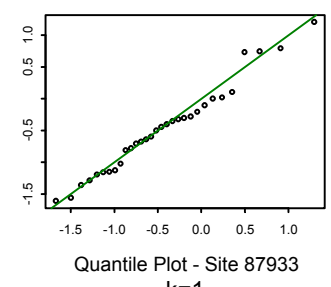

$\mathrm{k}=1$

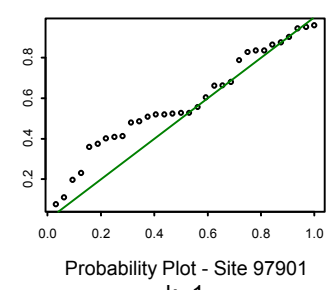

$\mathrm{k}=1$

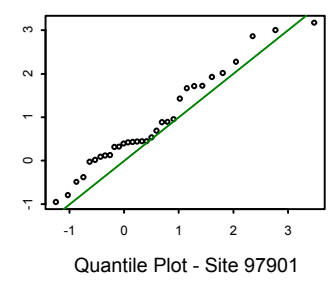

Figure E-10: Diagnostics - Non-Stationary Models Using “ $V_{x t h}$ " and “ $f_{\text {PET }<4.5 \text { " (1) }}$ 
$\mathrm{k}=2$
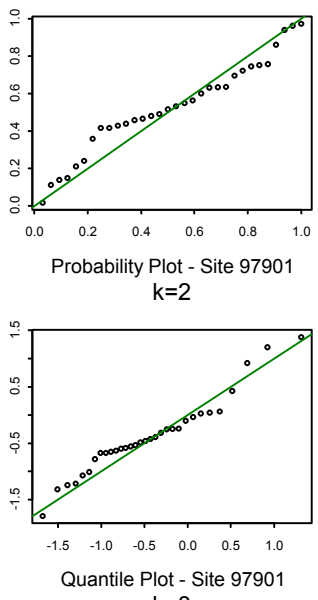

$\mathrm{k}=3$
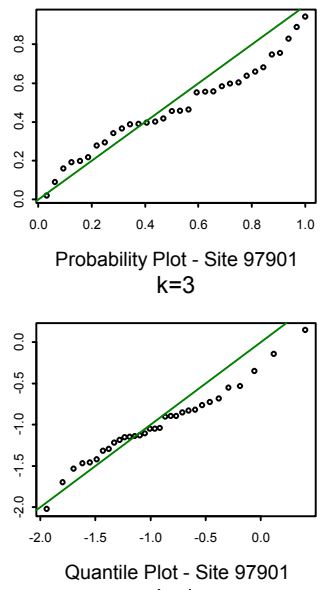

$\mathrm{k}=1$
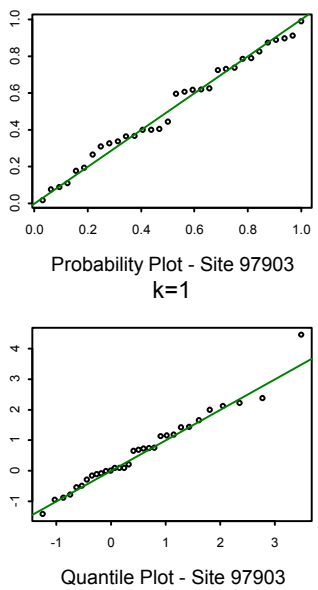

$k=2$

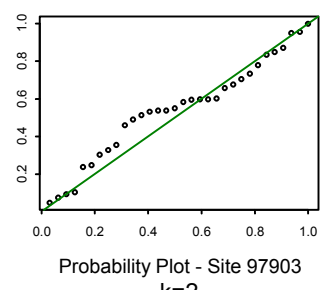

$k=2$

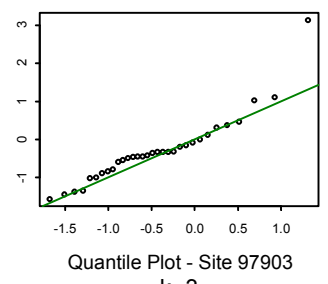

$\mathrm{k}=3$

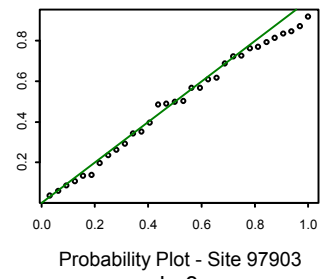

$k=3$

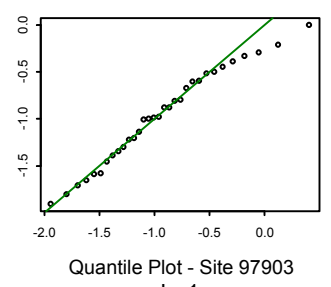

$\mathrm{k}=1$

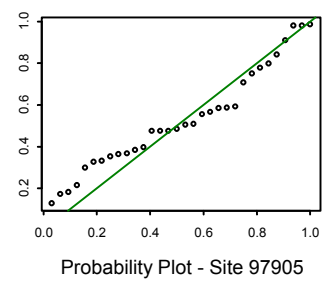

$k=1$

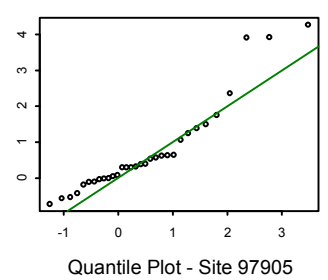

$k=2$
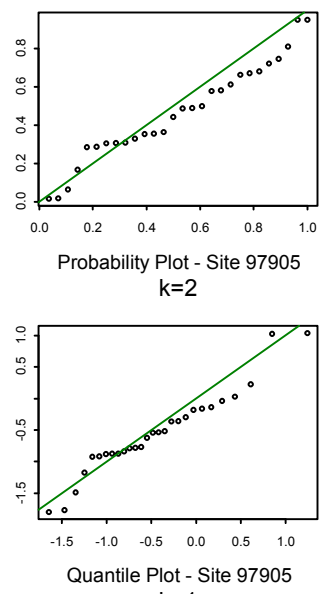

$\mathrm{k}=1$

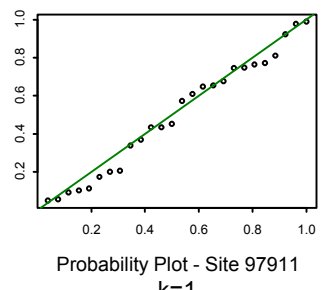

$\mathrm{k}=1$

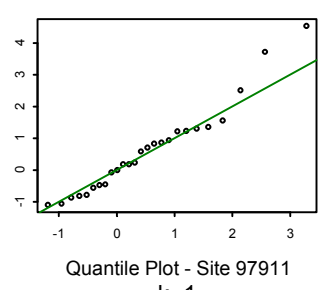

$\mathrm{k}=1$
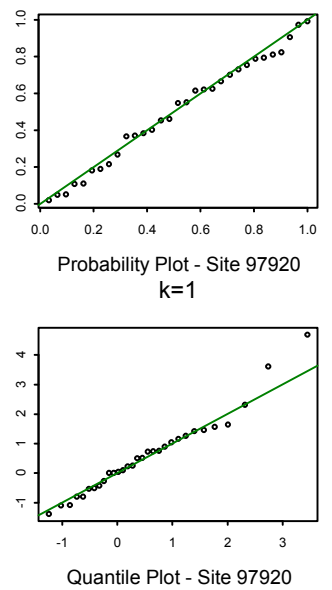

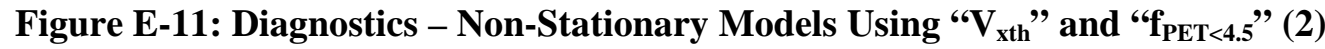




\section{APPENDIX F VALIDATION PLOTS}

Sequences of validation plots showing return level estimates versus observed crash counts, crash frequency estimates versus observed crash counts, and crash frequency estimates versus return level estimates are given in this appendix. The plots are shown for several model settings that were considered in the model development process. 

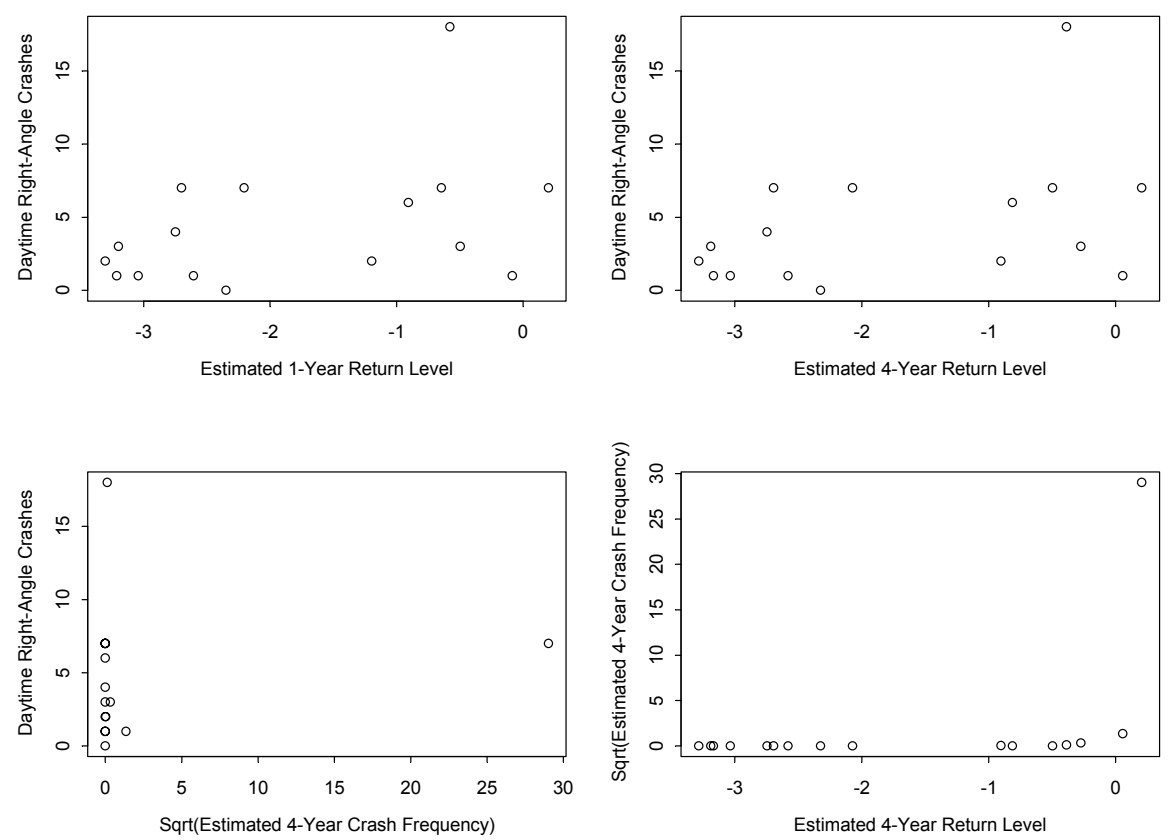

Figure F-1: Validation Plots - Homogeneous Models Using All PETs
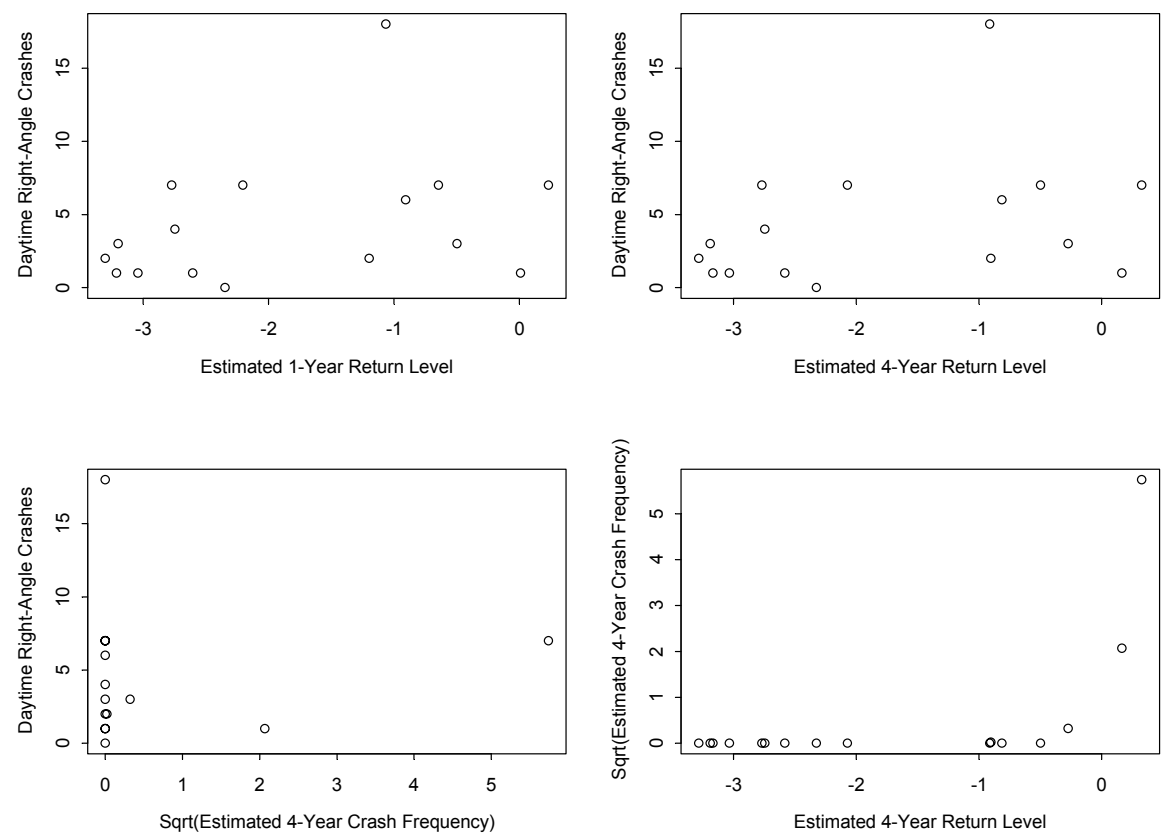

Figure F-2: Validation Plots - Homogeneous Models Using Filtered PETs 

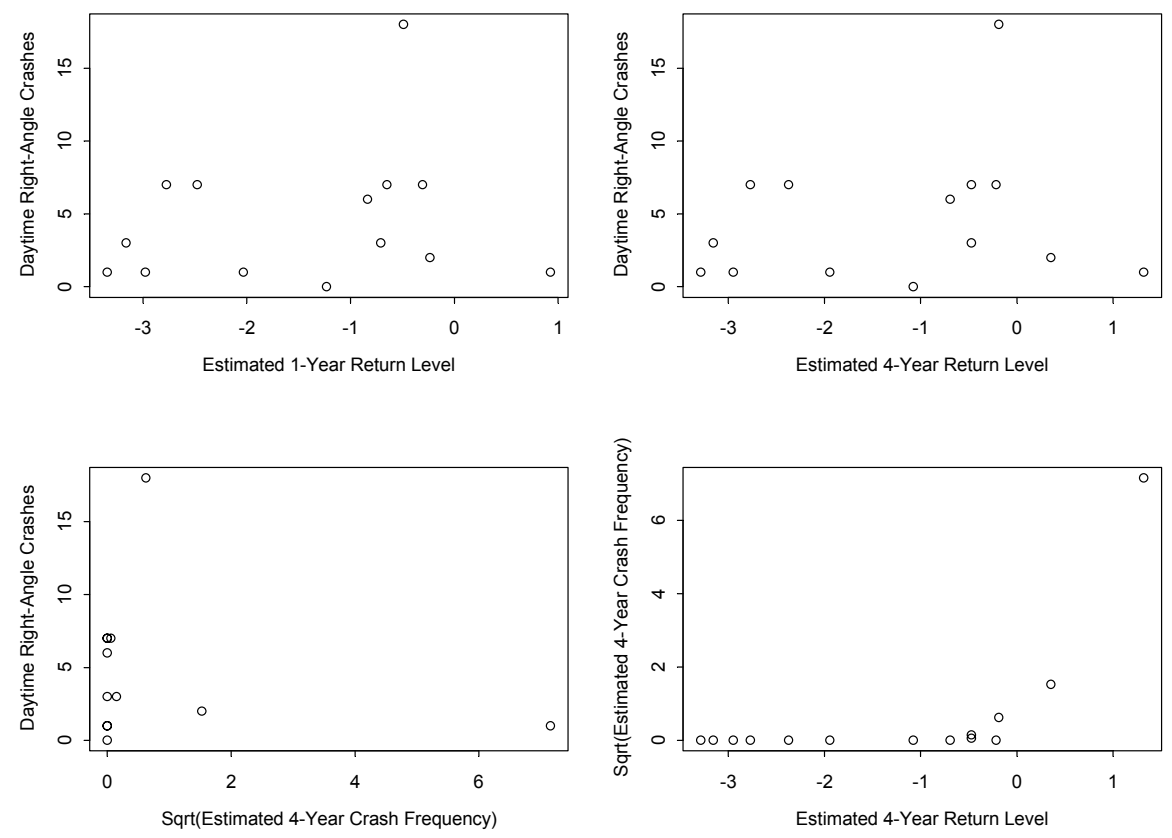

Figure F-3: Validation Plots - Non-Stationary Models Using " $\mathrm{V}_{\mathrm{xth}}$ "
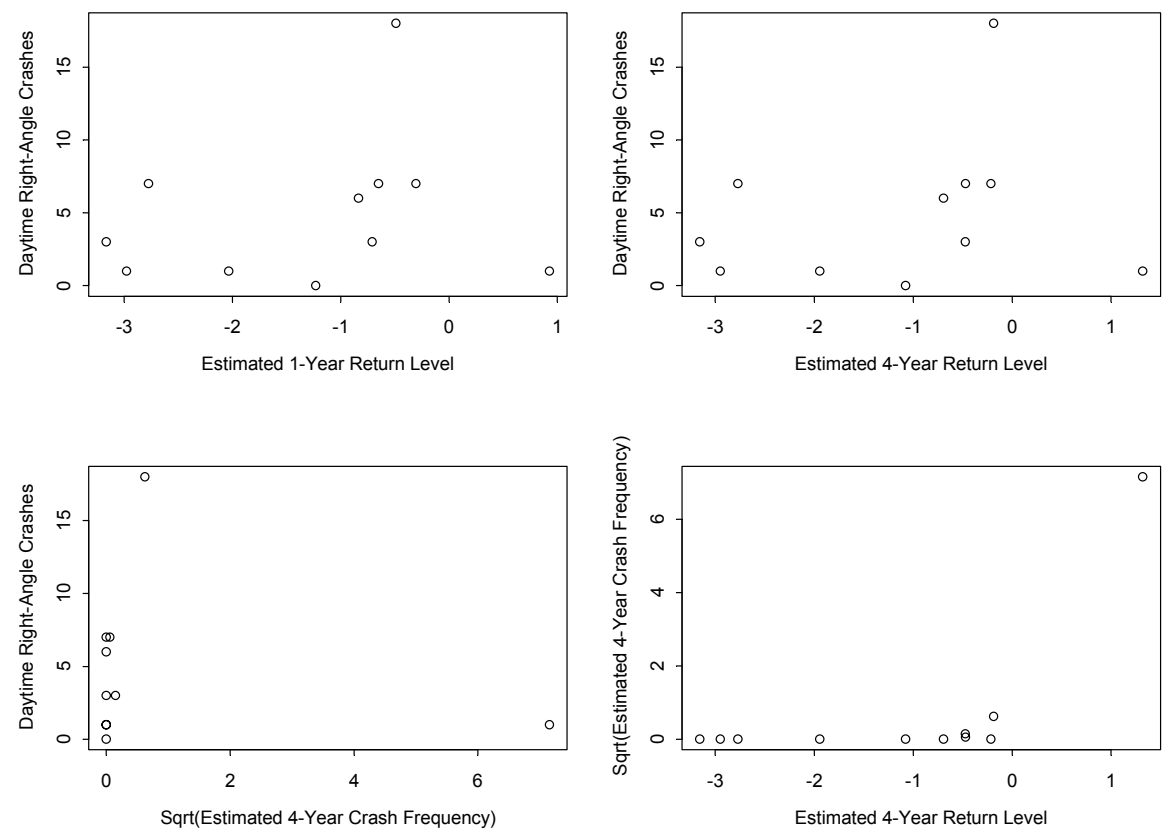

Figure F-4: Validation Plots of 12 Sites - Non-Stationary Models Using " $V_{x t h}$ " 

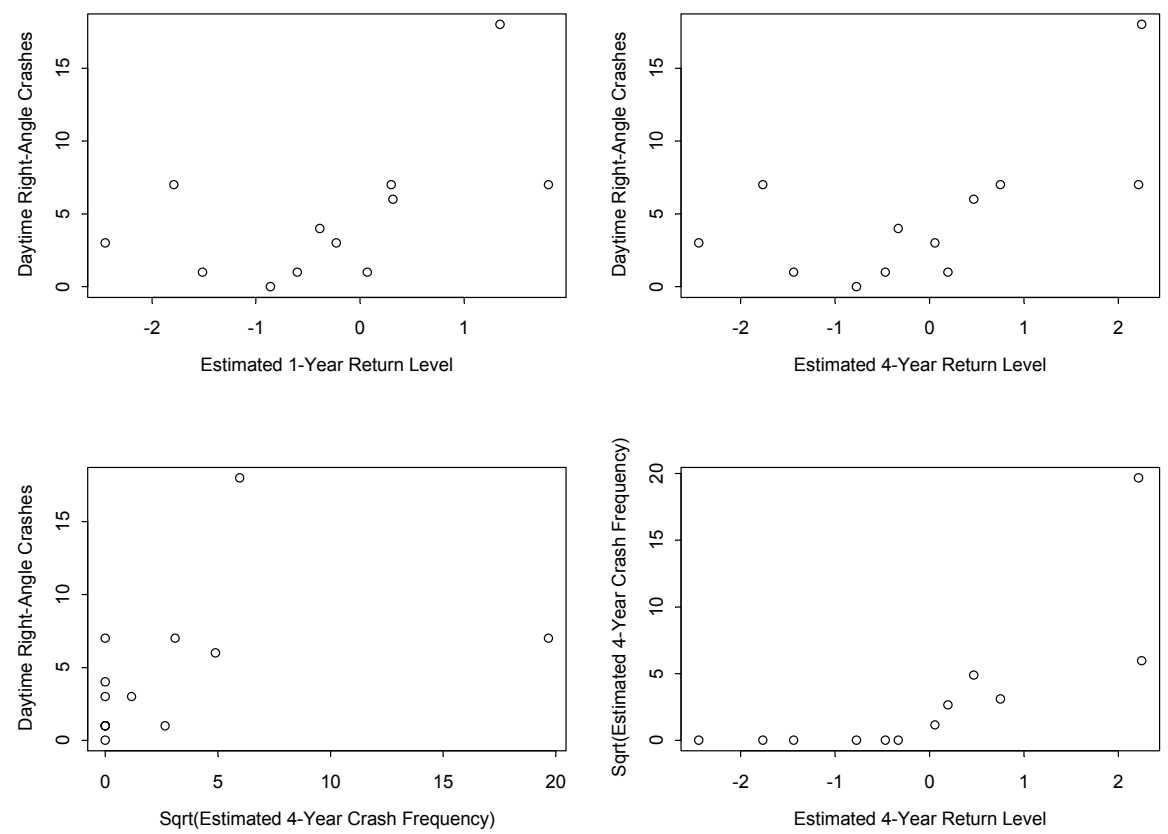

Figure F-5: Validation Plots of 12 Sites - Non-Stationary Models Using “ $\mathbf{f}_{\text {PET }<4.5}$ "
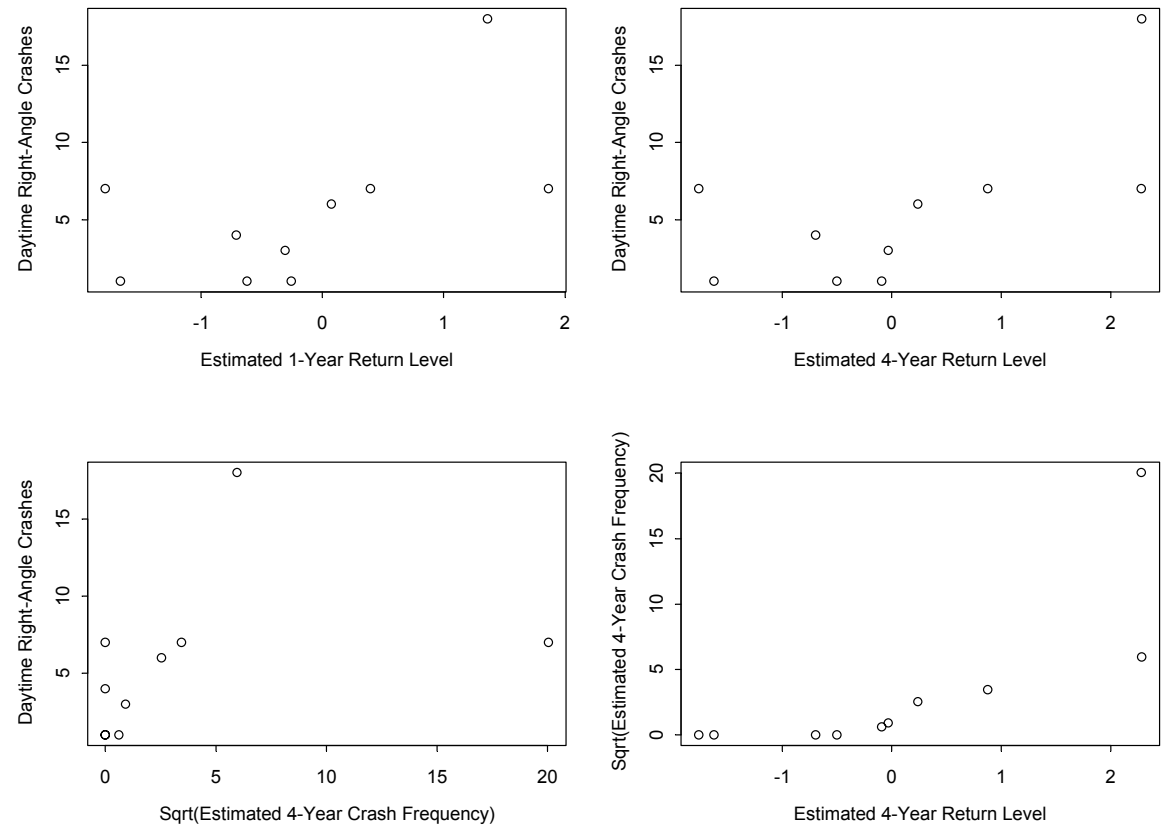

Figure F-6: Validation Plots of 12 Sites - Non-Stationary Models Using Both Covariates 


\section{APPENDIX G SIMULATION EXPERIMENT}

This appendix documents the results of simulation experiment for two selected sites - 87906 and 97901. The examined settings are a block length, a choice or $r$ value, and length of observation period. 
Site 87906, RL Models: r=1 (GEV), 15-minute blocks

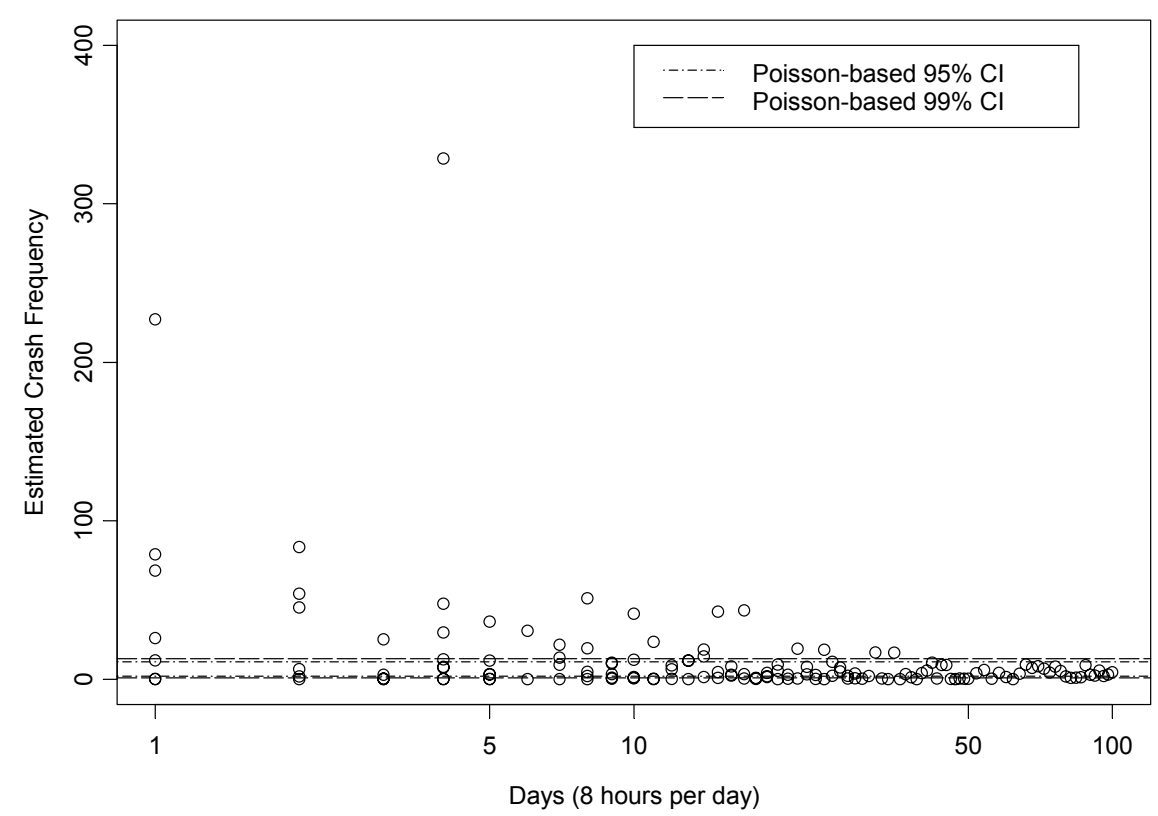

Figure G-1: Estimated Crash Frequency at Site 87906 using $r=1$ and 15-minute Blocks

Site 87906, RL Models: r=1 (GEV), 15-minute blocks

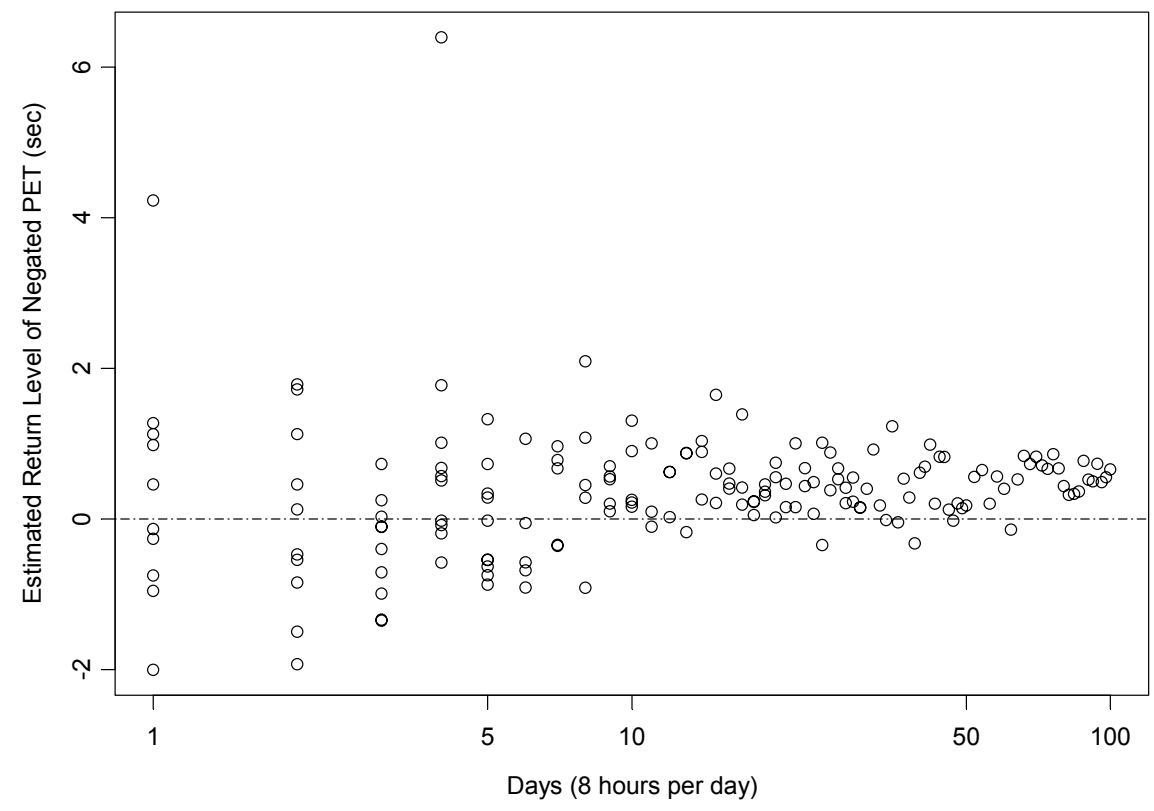

Figure G-2: Estimated Return Level at Site 87906 using r = 1 and 15-minute Blocks 
Site 87906, RL Models: r=1 (GEV), 1-hour blocks

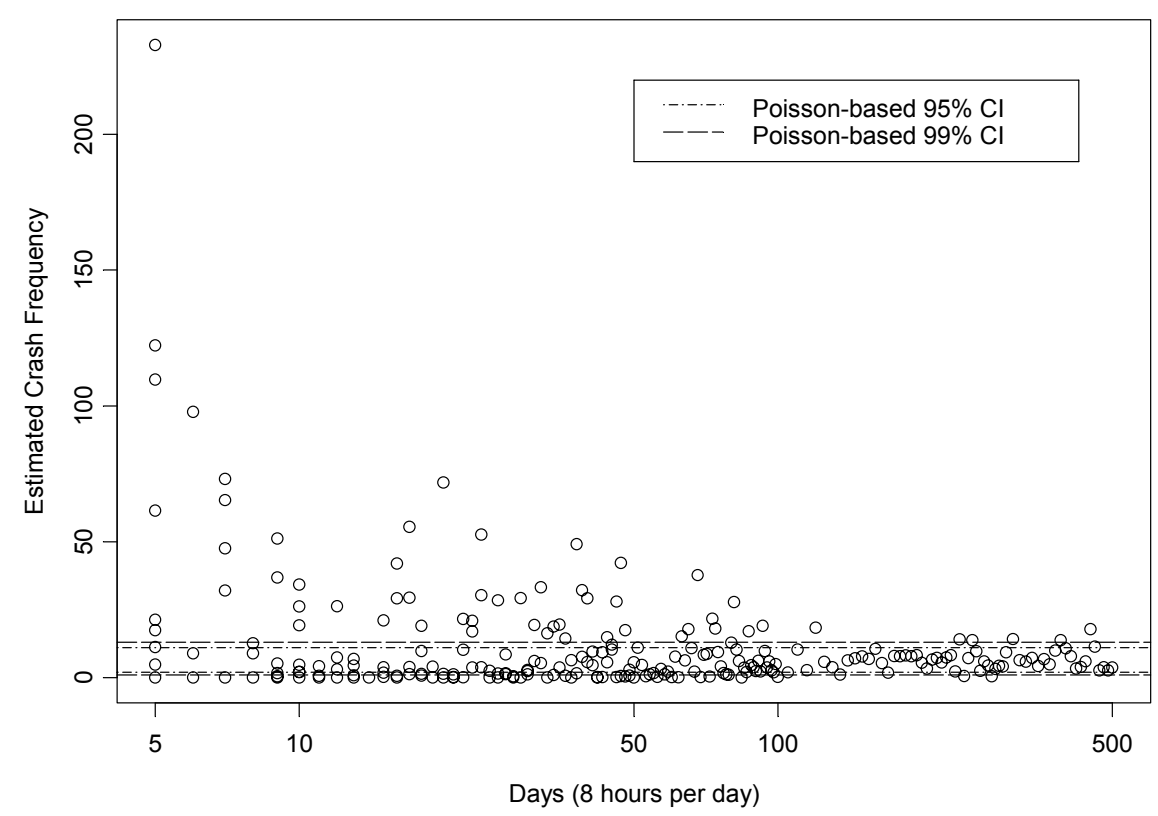

Figure G-3: Estimated Crash Frequency at Site 87906 using r = 1 and 1-hour Blocks

Site 87906, RL Models: r=1 (GEV), 1-hour blocks

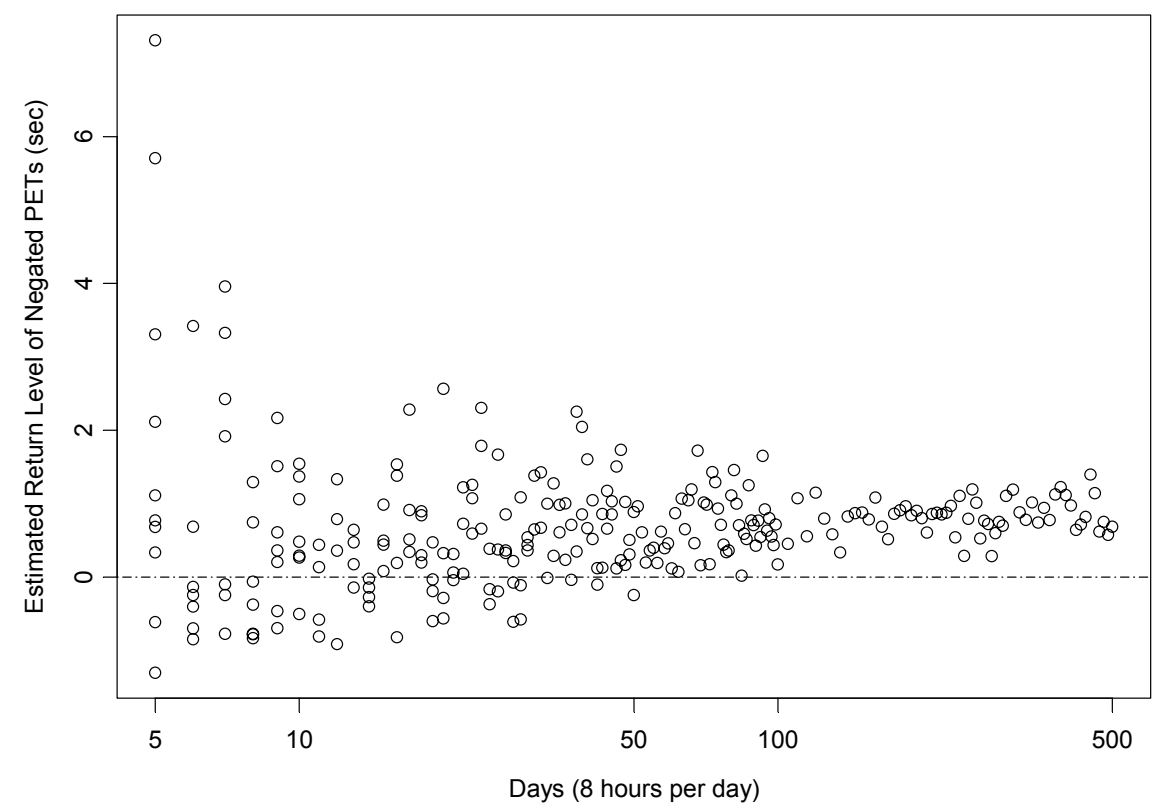

Figure G-4: Estimated Return Level at Site 87906 using r = 1 and 1-hour Blocks 
Site 87906, RL Models: $r=2,15-$ minute blocks

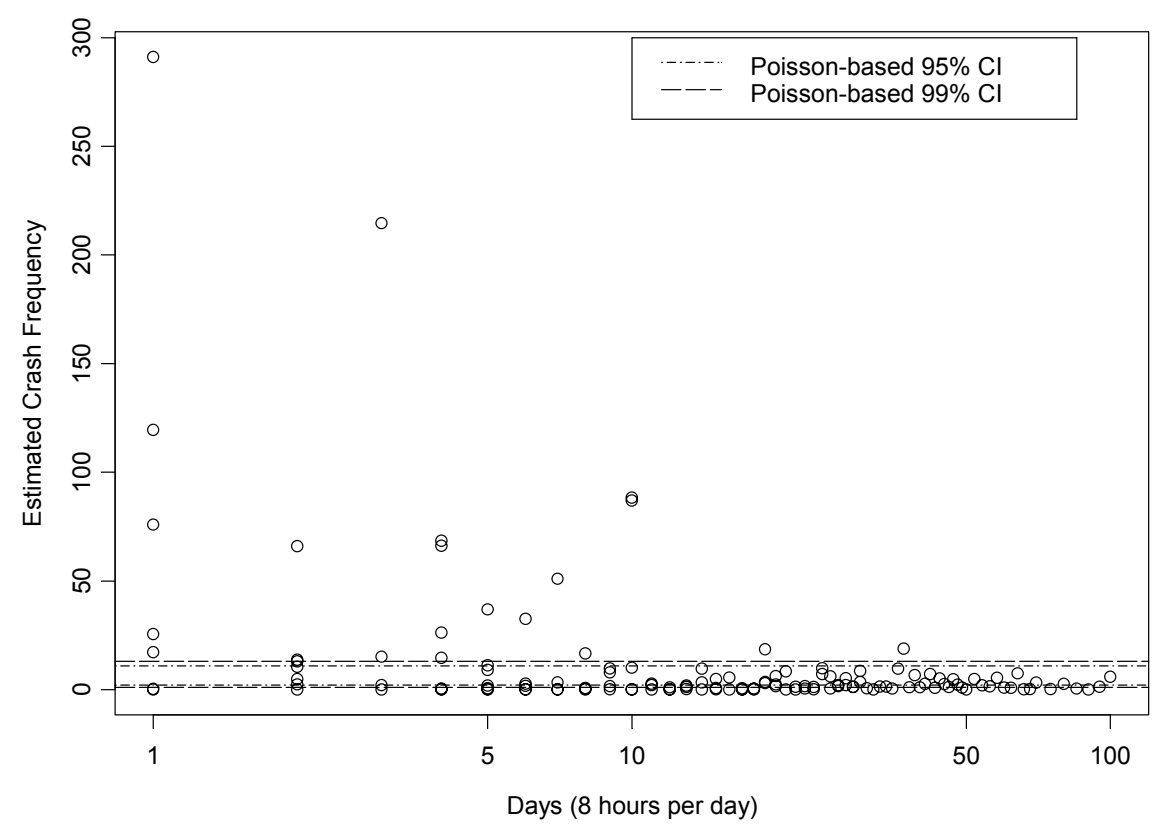

Figure G-5: Estimated Crash Frequency at Site 87906 using $r=2$ and 15-minute Blocks

Site 87906, RL Models: $r=2,15-$ minute blocks

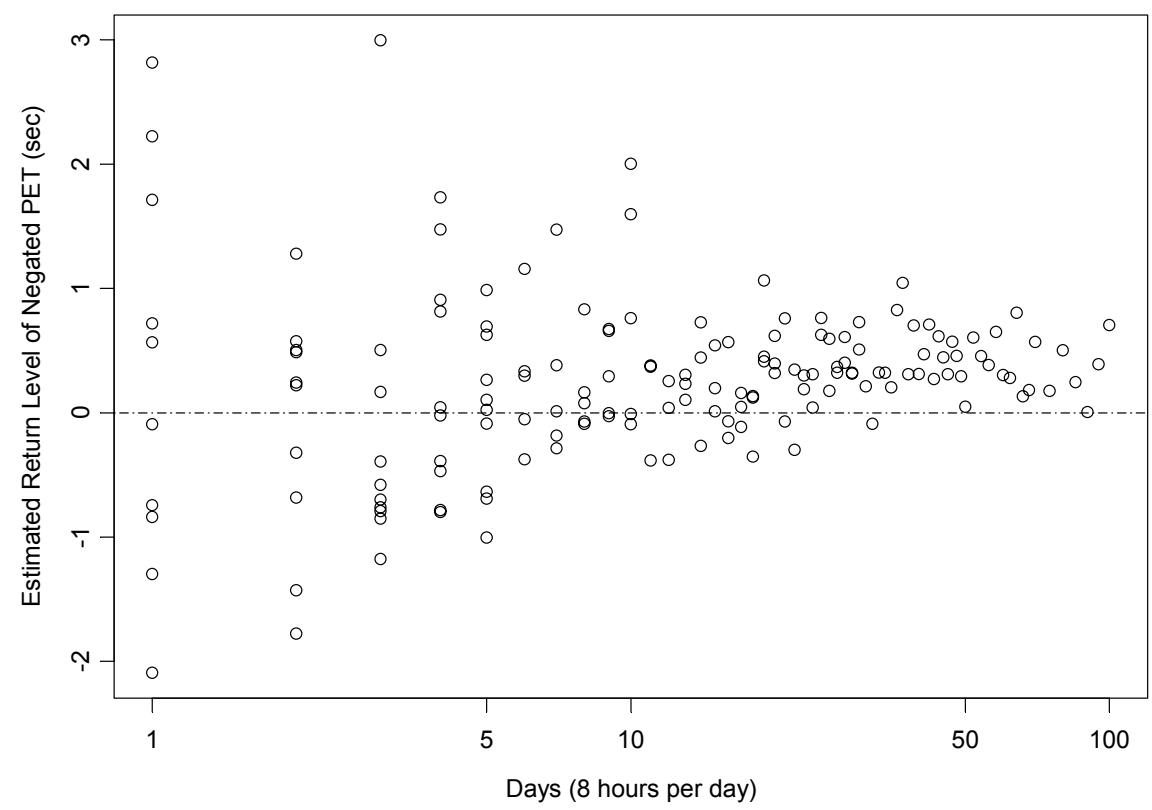

Figure G-6: Estimated Return Level at Site 87906 using r = 2 and 15-minute Blocks 
Site 97901, RL Models: r=1 (GEV), 15-minute blocks

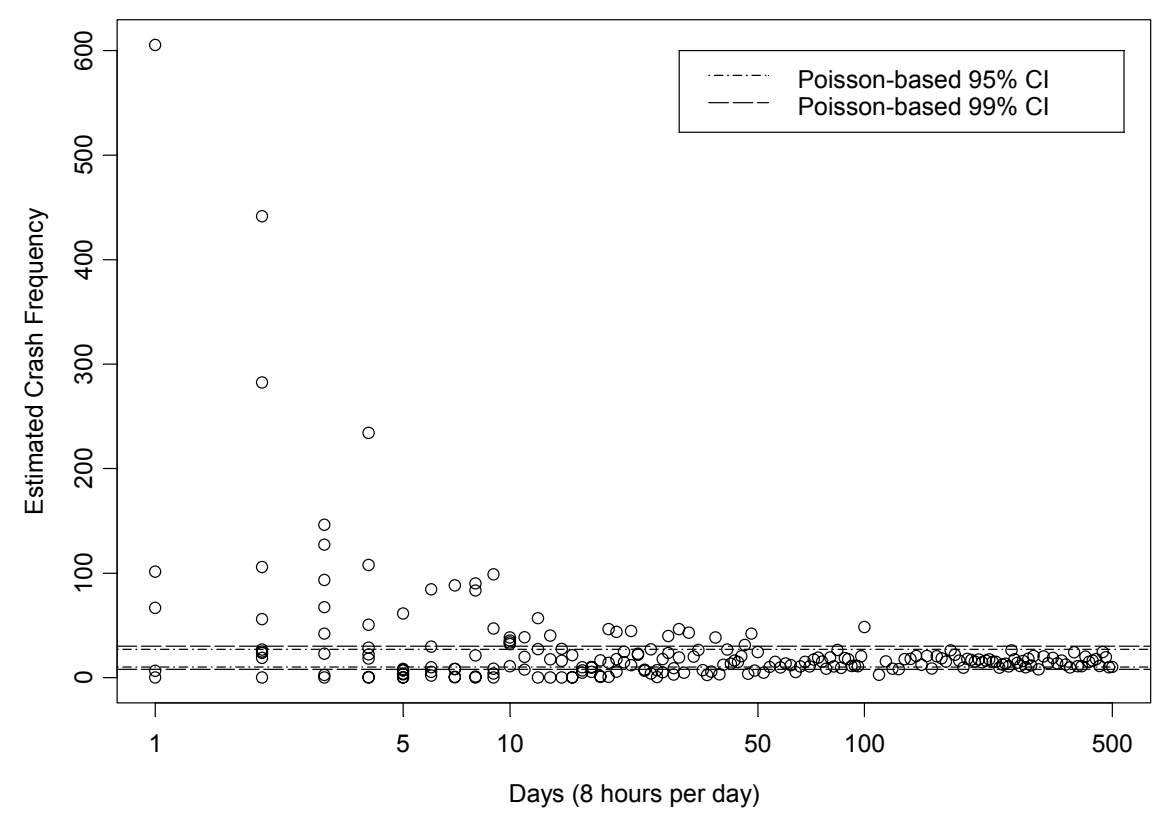

Figure G-7: Estimated Crash Frequency at Site 97901 using $r=1$ and 15-minute Blocks

Site 97901, RL Models: r=1 (GEV), 15-minute blocks

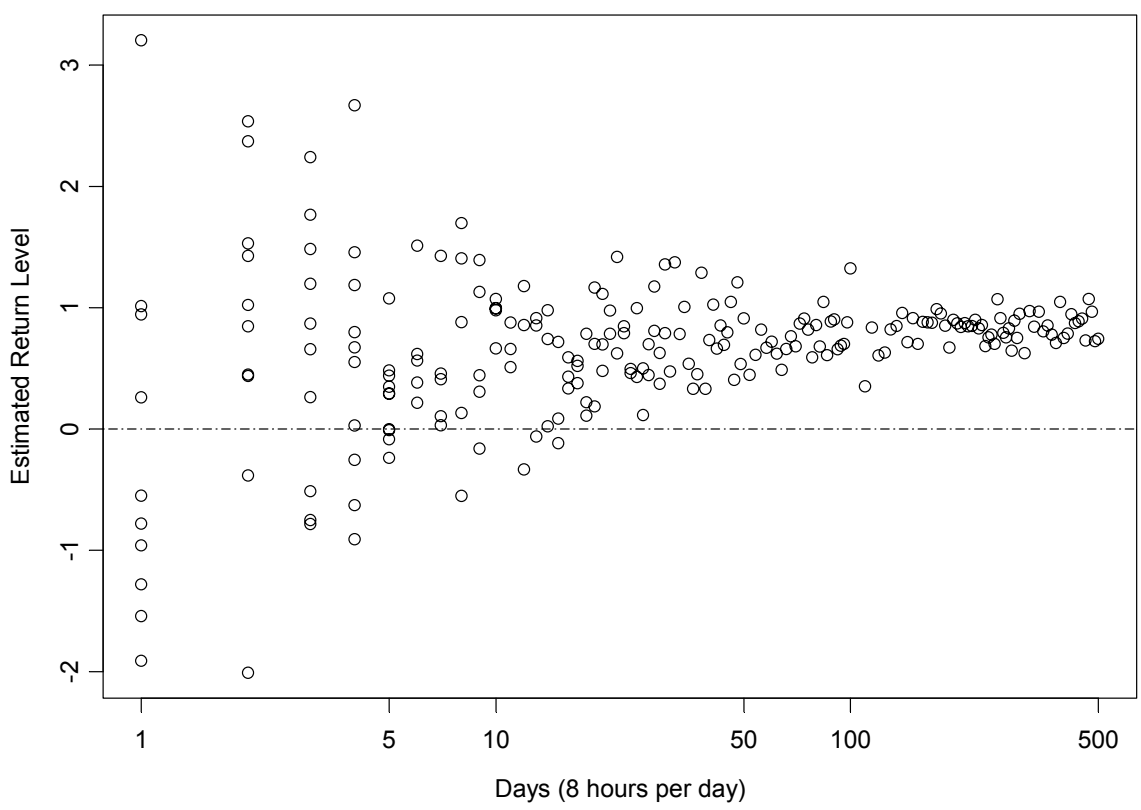

Figure G-8: Estimated Return Level at Site 97901 using r = 1 and 15-minute Blocks 
Site 97901, RL Models: r=1 (GEV), 1-hour blocks

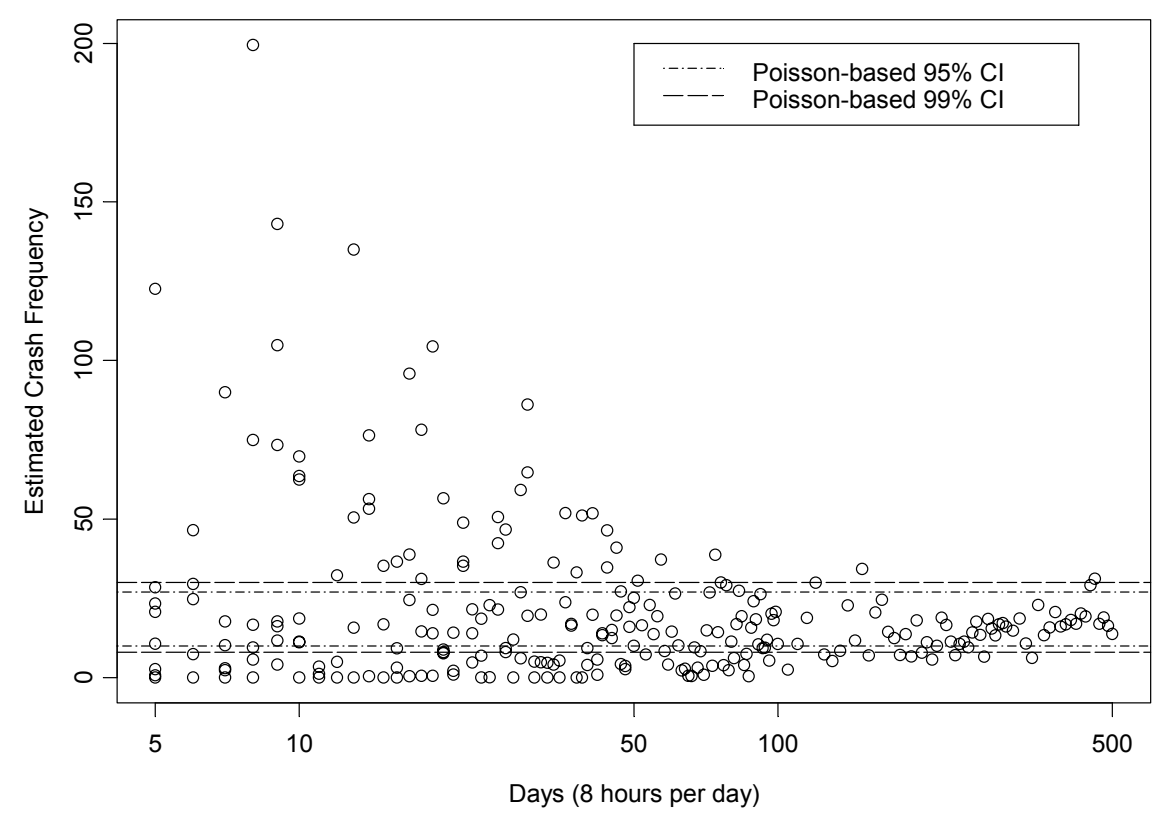

Figure G-9: Estimated Crash Frequency at Site 97901 using r $=1$ and 1-hour Blocks

Site 97901, RL Models: r=1 (GEV), 1-hour blocks

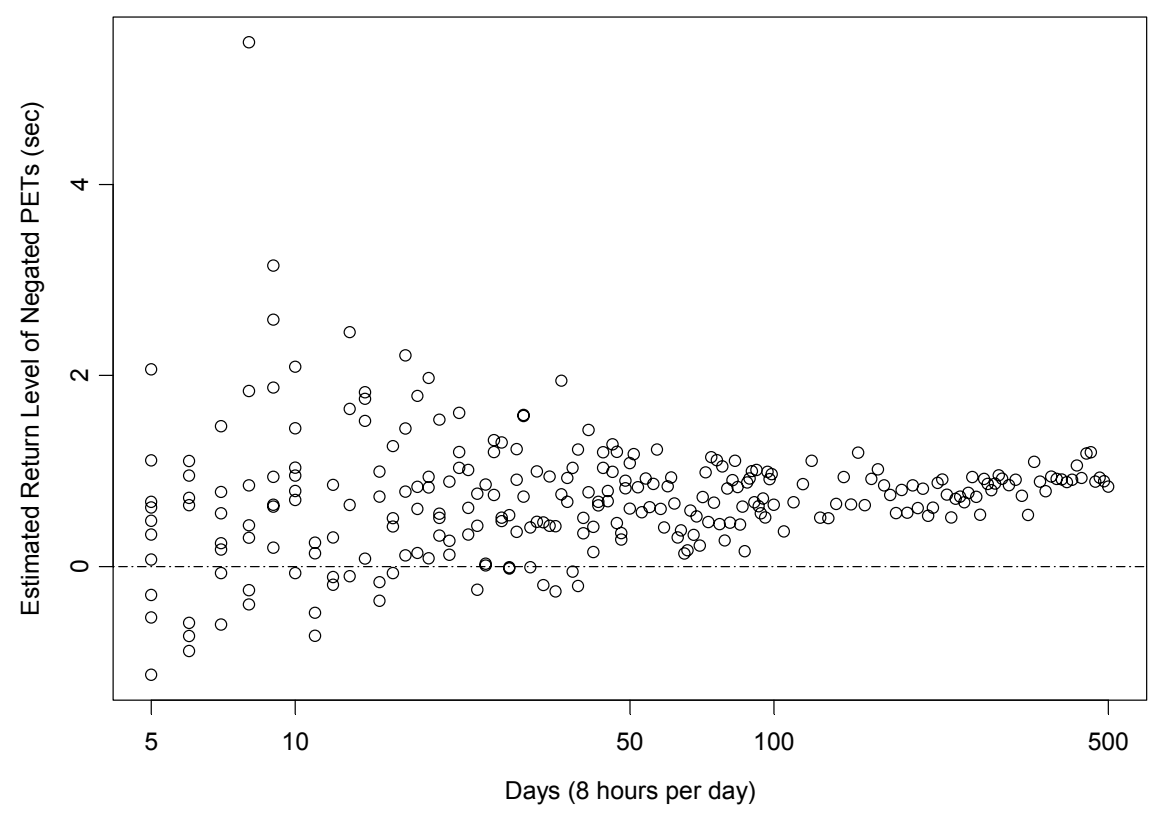

Figure G-10: Estimated Return Level at Site 97901 using $r=1$ and 1-hour Blocks 
Site 97901, RL Models: $r=3,15-$ minute blocks

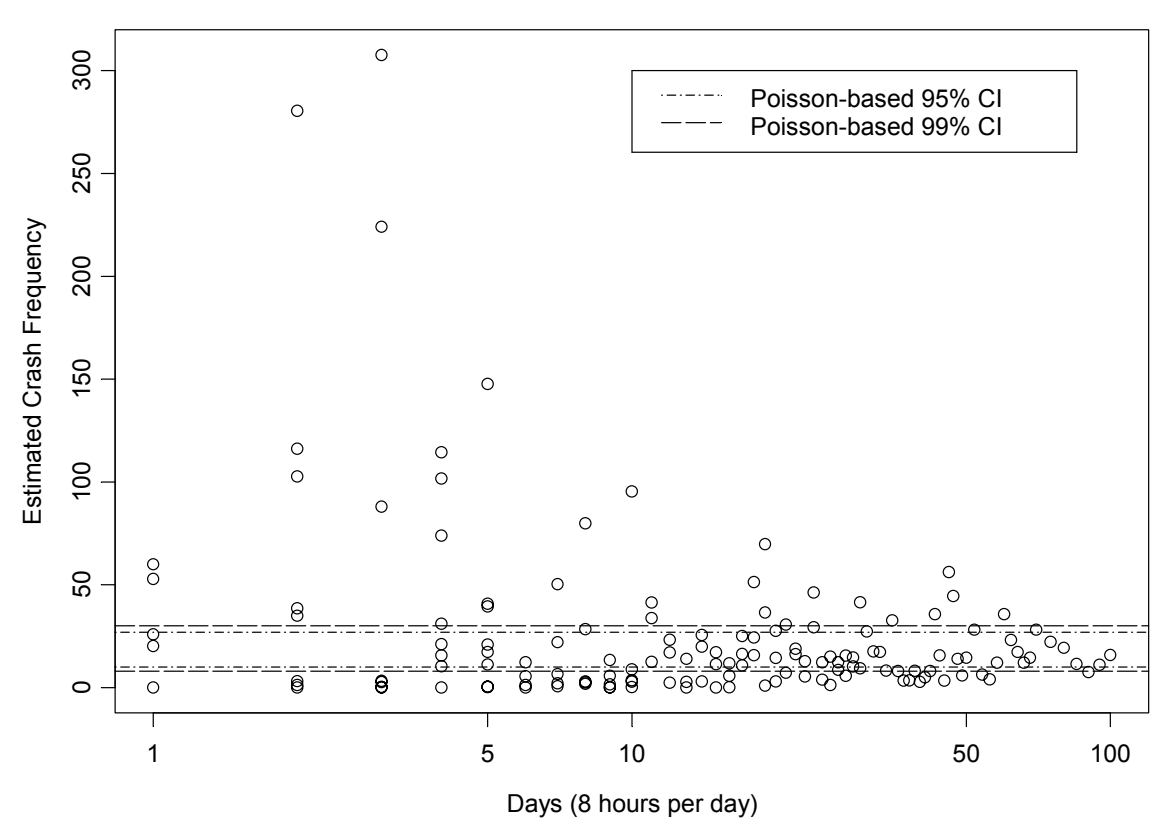

Figure G-11: Estimated Crash Frequency at Site 97901 using r $=3$ and 15-minute Blocks

Site 97901, RL Models: r=3, 15-minute blocks

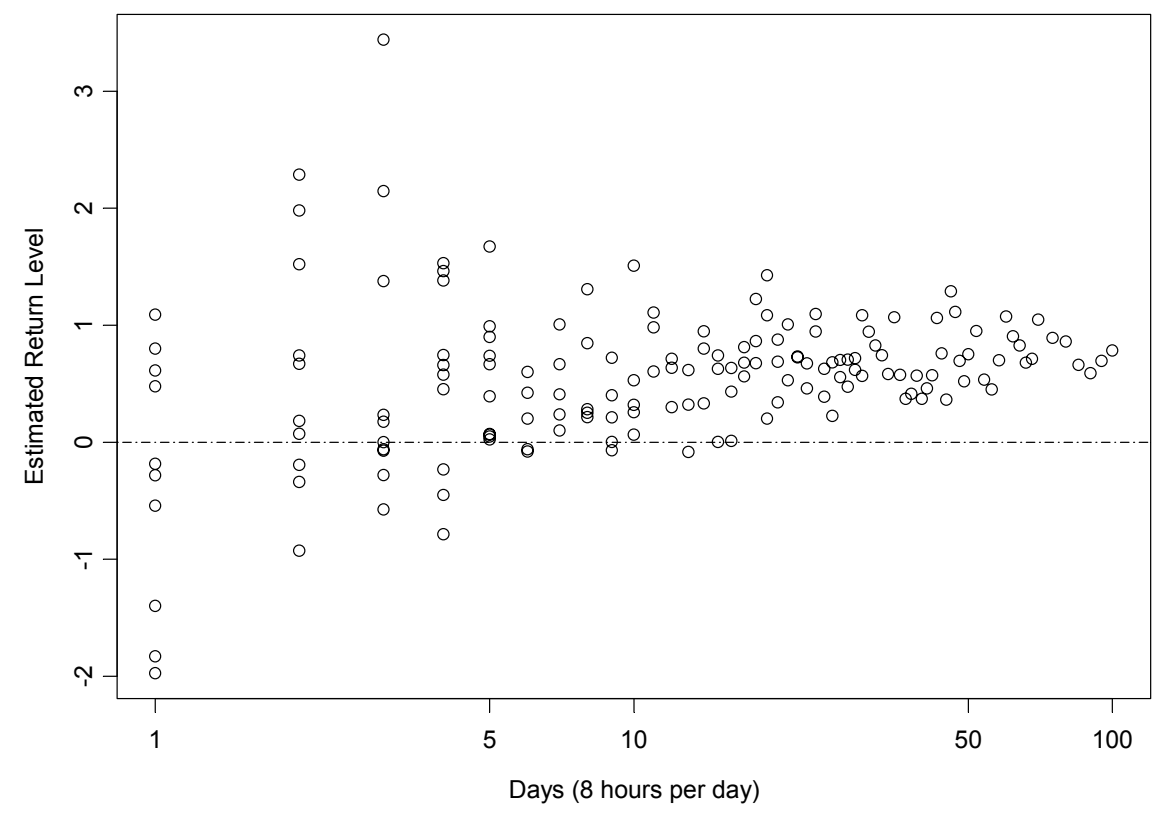

Figure G-12: Estimated Return Level at Site 97901 using r = 3 and 15-minute Blocks 


\section{APPENDIX H IMAGE PROCESSING SOFTWARE FOR CONFLICT DETECTION}

This appendix describes the development of image-based processing software for PET measurement. The developed software employs the optical flow calculation algorithm. The installation and usage instructions of the software are also provided in this appendix.

We would like to acknowledge the effort of Zbigniew Bublinski, Zbigniew Mikrut, Piotr Pawlik, and Andrzej Adamski for their contribution in the development of this software. 


\section{H.1 INTRODUCTION}

The aim of the project is to build a software package capable of automated measurement of selected traffic characteristics at the monitored intersections. The system input consists of video files (*avi), compressed with the "dvcodec" (Video for Windows) compressor. The digital video clips are recorded in the NTSC standard:

- frame rate: $29.97 / 30$ frames per second

- resolution: $720 \times 480$ pixels

- color depth: 24 bits (RGB)

The software should be able to process images, recorded as *.avi video clips, detect passing vehicles, and record their arrival directions and times of their presence in predefined crossroad areas, called conflict spots. Results from the analysis should be written to a text file in order to allow further statistical analysis.

The main challenges that still remained unsolved include:

- occasional blending of the analyzed objects (vehicles) in the background,

- variable weather and lighting conditions,

- occlusion of objects by other objects, related to the perspective projection, which should be considered both in the context of mutual occlusion of the analyzed vehicles and the occlusion of vehicles by other fixed objects, visible in the scene (e.g. line supports, lampposts, billboards, traffic lights, etc.),

- vibrations of the camera recording the video clips,

- transient changes of the video signal level caused by automated camera accommodation to the actual average lighting level of the image.

Some of the above mentioned problems have been solved in a satisfactory way. The applied method of preliminary image segmentation, based on the calculated optical flow (see page 346), allows detection of moving objects blended with the background and it makes the results insensitive to the presence of shadows cast by fixed objects in the scene. Image post-processing carried out after the segmentation (see page 348 ) reduces other negative impacts. The problems 
still left unsolved include shadows cast by vehicles, in some cases occlusion of vehicles by other vehicles, and vehicles stopped for a prolonged period.

\section{Programming Environment}

According to the specifications prepared by the School of Civil Engineering, Purdue University, the software was expected to run off-line under the Windows ${ }^{\mathrm{TM}}$ system. The authors assumed that a user would like to review the results of the main processing stage, i.e., the mutual coincidence of objects recognized as vehicles with predefined conflict spots. It was decided that the software package VirtualDub ${ }^{1}$ (Lee, 2003) would be used and appended by a set of filters. After reading in the video file and linking appropriate filters, the VirtualDub program works in a frame server mode to allow off-line continuous processing, pausing the video, reviewing frame-by-frame, skipping to another frame, and simultaneous displaying of both the original and processed video images.

The filters executing the processing algorithms, described in the next chapter, were programmed using the Microsoft Visual C++ Studio 6.0 environment. This is the programming environment recommended by the author of VirtualDub (Lee, 2003).

\section{Processing Speed}

The advanced image processing algorithms are extremely time-consuming, and the segmentation technique applied in the project, based on the optical flow calculations, was not widely used until recently because of the huge computation effort required. Therefore, in order to complete the calculation in a reasonable time the authors took the following steps:

- The resolution of the analyzed image was reduced twice: from $720 \times 480$ to $360 \times 240$ pixels.

- The color information has been skipped, i.e., the image with three RGB color components was transformed to grayscale image.

\footnotetext{
${ }^{1}$ the software is available under the GNU General Public License.
} 
- Additionally, the action of some of the analysis algorithms was limited to the specific scene area (Region of Interest, ROI), which covers the central part of the crossroad together with the entrance and exit traffic lanes (see Figure H-1).

\section{Configuration Elements}

A program user can create up to three configuration files to adapt the intersection geometry and to locate conflict spots for detection purposes.

Using a graphic application (e.g. Jasc Paint Shop Pro, see Section H.7), the user should properly mark the configuration elements, like the stop lines and conflict spots and should indicate (also by "painting") the objects occluding the ROI. In such a way, two graphic bitmap files are created, the names of which must be inserted in the text configuration file. An exemplary placement of the elements required for vehicle detection is shown in Figure H-1, which was obtained as a screendump of one of the VirtualDub windows. The borders of the Region of Interest are determined automatically by analysis of the locations of stop lines. Examples of creation of both types of configuration files can be found in Section H.7.

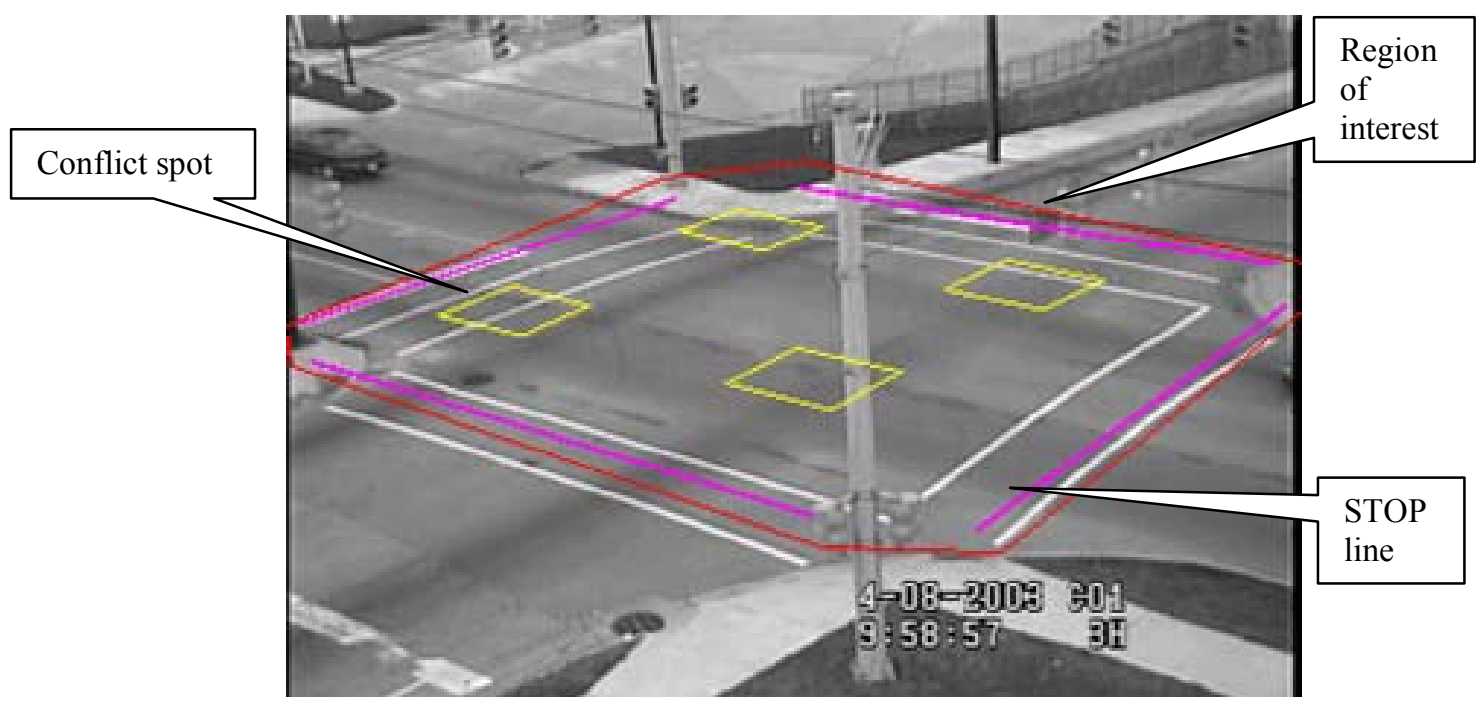

Figure H-1: Conflict spots, STOP lines and Region of Interest on the Screen Capture of the Intersection 97901 (SR-26 @ 18 ${ }^{\text {th }}$ St)

Every configuration element must be described in the text file in strict conformance with specific rules (see Section H.6). Additionally, the file should contain other information necessary for 
correct program operation, including the recording start time (coded by the user from the first video frame), the names of graphic configuration files, and the name of the output text file (containing the results of the analysis). The file may also contain (as a comment) additional information required by the user for proper identification of video material. That information will be copied to the output text file.

\section{Running the Software}

A detailed program installation description and the user's manual (with examples) can be found in Section H.5. The manual operation of the program comprises the following tasks:

- $\quad$ starting the VirtualDub program

- $\quad$ opening the digital video clip file (*.avi)

- loading the filters executing the analysis algorithm

- $\quad$ starting the processing

During the program operation the source image and the graphic interpretation of the analysis results are concurrently displayed. The program creates two files: (a) an error file, in which all of the warnings and possible errors are logged and (b) a preliminary output file (LOG) with the detection area numbers and the corresponding entrance/exit times for all detected vehicles. After completing the analysis by the filters, the LOG file is re-ordered. The re-ordered file is processed by another program which calculates the values of interest and saves them into the final output file.

\section{H.2 METHODS AND ALGORITHMS}

The developed algorithm of image analysis calculates the so-called motion field (optical flow). The result of the calculations is an image containing translation information for each pixel in the time period of the predefined number of frames (two frames for the illustrated case). The method is sensitive even to the smallest motion, provided that the surrounded pixels do not form large and homogenous areas.

The optical flow produces a binary image well-suited for segmentation. The segmentation is done only inside ROI. Small objects are eliminated. During the processing, the current colors are 
replaced by the colors of the objects obtained in the previous processing step. Correction algorithms that merge and divide objects are also executed.

The next step is inspection of the STOP lines and conflict spots: the occupancy times are recorded for every object detected. If certain conditions are fulfilled, the record, including information for the detected object, is saved into the output file.

After completing the video processing, the results are analyzed by a separate program (see Section H.5). The target situation that qualifies for recording is the consecutive passage through the conflict spot of two vehicles traveling in perpendicular directions within a predefined time interval. The user can manually check the files produced by VirtualDub with Excel or other spreadsheet applications.

The algorithm described above will be discussed in the following series of sections.

\section{Optical Flow}

Optical flow (also called optic flow, visual flow, image velocity) is a vector field. This field contains information required for transforming one video image into a second video image by executing a transformation, consisting of translation of the first image areas according to the vectors determined previously (Horn and Schunck, 1993). The basic conditions required for application of optical flow to video segmentation are (Beauchemin and Barron, 1995):

- uniform lighting - required for the assumption that the object location with respect to the light source does not affect its actual look - the lighting of the object surface does not change,

- ideal light diffusion from the object surface - required for the assumption that the object location with respect to the light sensor and light source also does not affect the object's actual look - the image of the object is the same in various projection planes,

- projection 3D -> 2D is parallel - it does not introduce the perspective effects.

For the case of a real scene analysis, the fulfillment of all these conditions is highly improbable. However, it is assumed that the conditions may be fulfilled locally for a three-dimensional scene, and what follows may be also fulfilled locally in the image plane. 
The condition of constant brightness of the considered pixel can be written as the following formula:

$$
\frac{d I(\stackrel{1}{x}, t)}{d t}=0
$$

where $I(\tilde{x}, t)$ is the function of image intensity in the point $\tilde{x}$ and at the time $t$.

The assumption of constant point intensity follows directly from the definition of the optical flow:

$$
I(\stackrel{1}{x}, t)=I(\stackrel{1}{x}+\delta \stackrel{1}{x}, t+\delta t)
$$

where $\delta^{1} x$ is a translation of a given image point in the time interval $\delta t$. After appropriate transformations the basic flow equation can be obtained in the form:

$$
\nabla I \cdot \stackrel{I}{v}+I_{t}=0
$$

in which $\stackrel{\mathrm{r}}{v}=\frac{\delta x}{\delta t}=(u, v)$ denotes the optical speed, $I_{t}$ is the first order derivative of the intensity function $I(\tilde{x}, t)$, and $\nabla I=\left(I_{x}, I_{y}\right)$ is the space gradient of intensity.

Taking into account the works (Barron et al., 1994; Liu et al., 1998; Galvin et al., 1998), in which their authors have carried out comparative tests of a wide spectrum of methods dedicated to calculations of optical flow and taking into account the application's requirements, it was decided that in the first stage the utility of two first-order gradient methods should be tested: local LucasKanade method (Lucas and Kanade, 1981) and global Horn-Schunck method (Horn and Schunck, 1981; Horn and Schunck, 1993). These two methods have been compared in the paper (Mikrut and Palczynski, 2003). After analysis of the results, it was decided that the Horn-Schunck method would be applied in the described algorithm and that the time-space derivatives would be approximated by the respective first order finite differences.

In the Horn-Schunck method, the basic condition (H-1) has been appended by an extra assumption that neighboring points, belonging to one moving object, exhibit rather close speed values and the motion field changes smoothly in the whole image area. Field discontinuities are observed only in areas where different objects overlap. The measure of smoothness of the optical 
flow field is provided by Laplacian values for both of its spatial components, and the authors of the paper (Horn and Schunck, 1981) propose the following approximation:

$$
\begin{aligned}
& \nabla^{2} u=\frac{\partial^{2} u}{\partial x^{2}}+\frac{\partial^{2} u}{\partial y^{2}} \approx \bar{u}-u \\
& \nabla^{2} v=\frac{\partial^{2} v}{\partial x^{2}}+\frac{\partial^{2} v}{\partial y^{2}} \approx \bar{v}-v
\end{aligned}
$$

where $\bar{u} \mathrm{i} \bar{v}$ are values averaged over the point's neighborhood.

Finally the functional that should be minimized takes the form:

$$
\vartheta^{2}=\iint_{D}\left\{\left(\nabla I \cdot \stackrel{\mathrm{r}}{v}+I_{t}\right)^{2}+\alpha^{2}\left[\left(\nabla^{2} u\right)^{2}+\left(\nabla^{2} v\right)^{2}\right]\right\} d x d y
$$

where $\alpha$ is a weight coefficient in condition (H-4) and $\mathrm{D}$ is the image area.

Applying the rules of variational calculus Horn and Schunck obtained an expression minimizing the value of functional (H-5) thus determining the values of optical speed $(u, v)$.

Due to the size of the resulting system of equations, Horn and Schunck suggest the application of the iterative Gauss-Seidel method for its solution. Therefore, the accuracy of the optical flow field calculation depends on the number of completed iterations.

In the paper (Mikrut and Palczynski, 2003), the authors assessed the effect of algorithm parameters on the obtained results. It was found that the algorithm provides acceptable results for $\alpha=33$ after nine iterations. The binarization criterion was taken as the condition of the absolute value of the optical flow exceeding a fixed value of 0.2 . The values listed above were used in the videodetector algorithm.

\section{Segmentation Process}

The segmentation of the binary image, obtained from binarization of the optical flow, was carried out by the classical "flood-fill" method, vastly described in literature - see Pratt (1988) for an example. The objects with surface area less than 100 pixels were regarded as noise and removed. 
A further stage of the algorithm is aimed at the identification of vehicles in consecutive frames of the video clip. At that stage the vehicles are represented as labeled objects. Unfortunately, such a representation does not take into account the following problems:

- merging of representations of two or more vehicles into one object,

- discontinuities of vehicle representation (division into two or more objects),

- occlusion of vehicles by other vehicles or the fixed elements in the scene.

Therefore, an additional (virtual) vehicle representation was introduced: a record containing, first of all, a unique object identifier and other fields describing the vehicle (surface area, envelope rectangle) or its motion (e.g. the recent positions of its center of gravity).

The vehicle identification consists of the correct attribution of objects in the scene to the virtual vehicle representation. The attribution is carried out by analysis of two consecutive video frames and the list of records.

It is assumed that every object in the first video frame represents exactly one vehicle. The basic parameters of the vehicle representation are calculated, i.e., its area and the rectangle circumscribed about the object (envelope rectangle). Because occasionally one vehicle can be represented by several objects, it seems reasonable to merge the description of all such objects into one record, which is done by appending small objects to the considerably bigger ones if their respective envelope rectangles overlap. At that moment the first unique identifiers are assigned to the records, describing individual vehicles. In further stages the segmented image will be reindexed, with identifiers that should be unique and cannot coincide with the segmentation indices, so the first identifier therefore should be greater than the highest possible index. It seems that the value of 150 (used in the present algorithm implementation) should be sufficient, which is several times higher than the usual number of objects detected in the scene.

After the attribution of identifiers, other consecutive parameters describing the vehicles (e.g. gravity center) are determined and the above mentioned re-indexing is carried out, ending the first algorithm step.

The second image frame (and the following ones) is analyzed with respect to the information obtained in the previous algorithm step. The beginning of the analysis is identical with the beginning of the first step: an auxiliary list of records is created, under the assumption that one 
record refers to exactly one vehicle. The records are filled with information regarding the objects and then the main part of the analysis takes place, namely, the synchronization of the auxiliary list with the list obtained in the previous step (further on called the main list). The synchronization comprises the assignment of records from the main list to records of the auxiliary list, such that both records describe the same vehicle (in two consecutive video frames). During the synchronization procedure the possibility of the following cases occurring should be taken into account (Figure H-2):

- one record of the auxiliary list refers to one record of the main list

- two or more records of the auxiliary list refer to one record of the main list

- one record of the auxiliary list refers to two or more records of the main list

- many records of the auxiliary list refer to many records in the main list

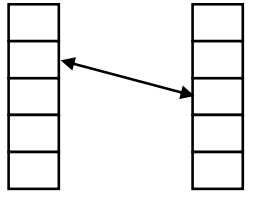

a)

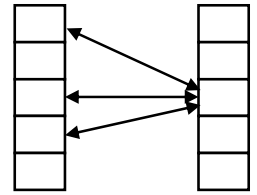

b)

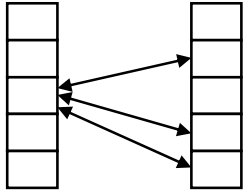

c)

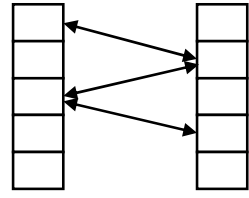

d)

Figure H-2: Possible Relations between the Main and Auxiliary List

The term "record reference" should be understood as the object property of sharing a common area in two consecutive frames.

The first case is the simplest. The record in the auxiliary list is assigned a unique identifier, copied from the respective record of the main list, i.e., the vehicle is represented by exactly one object in the scene.

In the second case, division of one object into several objects occurs - see Figure H-2(b). All of the records in the auxiliary list are assigned an identifier taken from the respective record of the main list. This picture describes a situation when, after segmentation, one vehicle is represented by several objects. However, occasionally a new vehicle shows up in the location occupied by another vehicle in the previous frame. Therefore, in the final stage ( post-processing of the main list,described below), an additional analysis takes place, i.e., analysis of the locations of gravity 
centers for objects tagged by one identifier and in the case when some predefined limit is exceeded the abnormally "distant" object is assigned a new identifier.

The third case denotes unintended merging of representations of several vehicles into one object. In order to retain control over the motion of every vehicle, an additional segmentation of the object takes place, by copying the overlapping part of individual vehicles and execution of a morphological operation called "dilation" on the common area. The "dilation" operation exhibits directional features, where each sub-object grows in the direction of its present motion, defined by the translation vector of the gravity center location. In the actual algorithm version the "dilation" operation is performed in three directions. The direction closest to the vehicle's motion is determined, together with two neighboring directions, and only these directions are used in the "dilation" operation. If the vehicle has not been moving (the translation of the gravity center location is a zero vector) then a standard eight-directional "dilation" is executed. After completing the directional dilation, one more standard dilation operation is performed in order to append the remaining shared parts of the object. At this point, a question emerges about how the dilation operation is taken into account in the record lists. In the auxiliary list there is only one respective record (according to the general rule: one record - one vehicle) and it is assigned the identifier of the object which shares the biggest common part with the represented object. The remaining vehicles taking part in the dilation are represented by records from the main list and these records are assigned the identifier of the object to which they have been appended.

The fourth case, many to many - see Figure H-2(d), rarely occurs. At the moment, it is not taken into the analysis because the resources required for its proper analysis considerably outweigh the frequency of its occurrence.

After synchronization of the relationships between the records in both lists, the procedure looks for the unmatched records in the auxiliary list, i.e., the records which do not have their counterparts in the main list. They are treated as representations of new vehicles and they are assigned consecutive new identifiers.

On the other hand, the records in the main list which do not have their counterparts in the auxiliary list represent the vehicles that have left the ROI or have stopped. They are properly labeled and after a predefined time period removed from the list. 
The aim of the list synchronization stage is the assignment of proper, unique identifiers to the records from the auxiliary list. After that step the current frame is re-indexed using the assigned identifiers. As the last step, the main list is updated. The records taking part in the dilation operation (appended to other objects) and the records referring to vehicles that have stopped or left the ROI are left in the main list. The remaining records are replaced by records from the auxiliary list.

After that stage, post-processing of the new main list is required. First of all, several records with the same identifier may be found (resulting from object division into several smaller objects). In such a case, the distances between the objects represented by those records are checked for possible detection of new vehicles. The records describing too distant objects also are assigned new identifiers. The remaining records are merged into one record, which should describe all the remaining objects. Secondly, the changes in objects taking parts in the dilation operation should be taken into account. For both of the above mentioned reasons, the parameters are recalculated for all the objects in the scene, taking into account their new indices (identifiers).

After completing that stage, the next frame is loaded for the analysis and the whole process is repeated.

\section{Occupancy of Conflict Spots}

The image file containing the conflict spots and the stop lines is used for detection of the presence of analyzed vehicles in these zones. A vehicle is present in the zone if at least one of the points belonging to the moving vehicles in the image area coincides with one of the points belonging to the conflict spots. Presence time is recorded and later used to identify the accurate entrance and exit times for the conflict zone. In the field condition, it was quite frequent that a single vehicle simultaneously occupied more than one conflict spot because of varying vehicle travel paths but static conflict spots. To address this situation, we assume that a conflict occurs if the vehicle occludes at least $1 / 3$ of the conflict spot area. The condition has to be fulfilled only with respect to the conflict spots. The stop lines do not require such a treatment because of their different shapes and roles in the process of analysis. 
Output (LOG) File

The fact of a vehicle presented in the conflict spot is recorded if, in the LOG file, at least one of the two conditions is fulfilled:

1) the vehicle has crossed at least two stop lines, and at least one of the occupancy times (difference between the exit and entrance) is non-zero, or,

2) the vehicle has crossed at least one stop line and at least one conflict spot and at least one of the occupancy times of the stop lines and at least one of the occupancy times of the conflict spots are non-zero.

The information about each passage contains:

- recorded time (frame number in the analyzed video),

- vehicle number,

and for each stop line and conflict zone with non-zero occupancy time:

- $\quad$ spot number,

- entry time,

- exit time.

The data for a passage are written in a simple line and separated with semicolons.

Postprocessing of Output (LOG) File

The data saved into the LOG file are further processed with the program TRAFFIC.EXE (see Section H.5). Important stages of the processing include:

- consolidation of all of the information concerning the same vehicle, thereby, one record is obtained for each vehicle,

- output data writing, whereby, a file is created containing data which are similar to the output (LOG) file, but the data concerning a given vehicle are written only once and the zones are ordered with respect to the entrance time to the zone. This allows a fast determination of the vehicle trajectory and enables a verification of the obtained results by comparison with the results of reviewing the video clip. The data are written to a file 
with the filename specified in the text configuration file (CONF) by the SORT parameter (see Section H.5),

- determination of the direction from which the vehicle has arrived, which is done by finding the zone for which the entrance time is the least. If the zone found is not a stop line but rather a conflict spot, then the direction determination is not possible and such data are rejected and are not included in further stages of the analysis,

- determination of the direction of the vehicle leaving the ROI, which is done by finding the zone with the latest exit time. If the zone found is not a stop line but a conflict spot such a direction determination is not possible. However, such data are not rejected because there is always a chance that the missing information can be completed in further stages of the analysis,

- identification of the motion trajectory, which makes use of the information contained in the configuration file, specifying the zone numbers and the order of their crossing (see Section H.6). A satisfactory matching of the actual vehicle trajectory to a model route specified in the configuration file enables a proper determination of the exit direction,

- merging of vehicles, whereby an attempt takes place to merge neighboring and incomplete records. The aim is to merge the record pairs from which the first contains information concerning the initial stage of the passage (the entrance into the crossroad (stop line), passages through the first conflict spots) and the next concerns the final stage of the passage (the last conflict spots and the stop line (exit from the crossroad)). The condition allowing the merge is the consistence of the order of zones crossed with the model route, determined from the information about the direction from which the vehicle has arrived. Additionally, in the merged records one shared zone (spot) can be found,

- output data writing, whereby a file is created containing data similar to the data in the file created earlier (SORT), which contained reduced and sorted data but now the data concerning a vehicle are appended by information about its entrance and exit directions and the respective zones are ordered according to their entrance times. The file is appended with vehicle records, for which the vehicle trajectory is consistent with the routes contained in the CONF file (see Sections H.5 and H.6). This file is used only for testing purposes - there is no possibility to specify its filename in the configuration file, the data are always written to a file named TEST.TXT,

- final calculations and report generation, during which the reduced, appended, and merged data are used for detection of conflict events and determination of their parameters, and 
later for generation of the final report of the predefined form. The name of the created report file is specified by the REPORT parameter (see Sections H.5 and H.6),

- additional information output, which occurs before the end of processing, contains additional information concerning the data analysis that is output to the screen. The information includes the number of records contained in the LOG file, the number of objects (vehicles) after the information reduction, the number of vehicles for which the determination of entrance and exit directions was not possible, the number of vehicles for which the motion trajectory matching was completed, and the number of successful merges of incomplete objects. The last information output to the screen is the number of detected conflict events.

\section{H.3 RESULTS}

The results obtained from analysis of seven video clips (eight hours) are summarized in Table H-1 and Table H-2. All of the details can be found in GapData_res2.xls file (see Section H.8). The particular items are defined as follows:

- actual conflicts: the number of conflicts detected by the human operator (eight seconds or less),

- reported conflicts: the number of reported measurements from the post-processing of the VirtualDub filter event data file. All the reported values of 8.2 seconds or less were counted,

- detected conflicts: the number of actual conflicts that can be detected by matching the occurrence times and conflict spots of the actual and reported conflicts,

- false detections: the number of reported conflicts that could not be matched with the actual conflicts,

- detection rate: the ratio of the number of detected conflicts to actual conflicts,

- false detection rate: the ratio of the number of false detections to reported conflicts,

- gap time (GT): the time between the moment when the first vehicle enters into the conflict spot and the moment when the second vehicle enters into the conflict spot (front bumper to front bumper),

- GT mean error: mean of the differences between actual and detected GTs,

- GT std error: the standard deviation of differences between the actual and detected GTs, 
- post enchroachment time (PET): the time between the moment when the first vehicle leaves the conflict spot and the moment when the second vehicle enters into the conflict spot (rear bumper to front bumper),

- PET mean error: mean value of the differences between the actual and detected PETs,

- PET std error: the standard deviation of differences between the actual and detected PETs.

In Table H-1, the detection performance is summarized. The false detections are defined as all the reported detections that cannot be matched with the actual ones. False detection rates appear to be excessive at certain intersections. After a manual check of false detections one-by-one, a number of false detections can be discounted since they are correctly reported but the actual values are greater than the threshold and therefore cannot be matched. The detection performance after a careful revision of false detections is given in Table $\mathrm{H}-2$.

Table H-1: Detection Performance

\begin{tabular}{|c|c|c|c|c|c|c|c|c|c|c|}
\hline \multirow[b]{2}{*}{ Intersection } & \multicolumn{6}{|c|}{ Detection Performance } & \multicolumn{4}{|c|}{ Measurement Errors (sec) } \\
\hline & $\begin{array}{c}\text { Actual }(\leq \\
8 \mathrm{~s})\end{array}$ & $\begin{array}{l}\text { Reported } \\
(\leq 8.2 \mathrm{~s})\end{array}$ & Detected & False & $\begin{array}{l}\text { Detection } \\
\text { Rate (\%) }\end{array}$ & $\begin{array}{c}\text { False Rate } \\
(\%)\end{array}$ & \multicolumn{2}{|c|}{ GT } & \multicolumn{2}{|c|}{ PET } \\
\hline 87907 & 10 & 11 & 8 & 3 & 80.00 & 27.27 & 0.048 & 0.903 & -0.135 & 0.912 \\
\hline 87933 & 30 & 39 & 29 & 10 & 96.67 & 25.64 & -0.050 & 0.197 & -0.188 & 0.187 \\
\hline 97901_10 & 49 & 76 & 44 & 32 & 89.80 & 42.11 & -0.026 & 0.278 & -0.124 & 0.263 \\
\hline 97901_16 & 92 & 88 & 83 & 5 & 90.22 & 5.68 & -0.115 & 0.449 & -0.174 & 0.642 \\
\hline
\end{tabular}

Table H-2: Detection Performance after Manual Check of False Detections

\begin{tabular}{|c|c|c|c|c|c|c|c|c|c|c|}
\hline \multirow{3}{*}{ Intersection } & \multicolumn{6}{|c|}{ Detection Performance } & \multicolumn{4}{|c|}{ Measurement Errors (sec) } \\
\hline & \multirow{2}{*}{$\begin{array}{c}\text { Actual ( } \leq \\
8 s)\end{array}$} & \multirow{2}{*}{$\begin{array}{c}\text { Reported } \\
(\leq 8.2 \mathrm{~s})\end{array}$} & \multirow{2}{*}{ Detected } & \multirow{2}{*}{ False $^{(1,2)}$} & \multirow{2}{*}{$\begin{array}{l}\text { Detection } \\
\text { Rate (\%) }\end{array}$} & \multirow{2}{*}{$\begin{array}{c}\text { False Rate } \\
(\%)\end{array}$} & \multicolumn{2}{|c|}{ GT } & \multicolumn{2}{|c|}{ PET } \\
\hline & & & & & & & Mean & Std Error & Mean & Std Error \\
\hline 87907 & 10 & 11 & 8 & 1 & 80.00 & 9.09 & 0.048 & 0.903 & -0.135 & 0.912 \\
\hline 87923 & 19 & 45 & 18 & 21 & 94.74 & 46.67 & -0.010 & 0.701 & 0.044 & 1.030 \\
\hline 87933 & 30 & 39 & 29 & 9 & 96.67 & 23.08 & -0.050 & 0.197 & -0.188 & 0.187 \\
\hline 97901_10 & 49 & 76 & 44 & 20 & 89.80 & 26.32 & -0.026 & 0.278 & -0.124 & 0.263 \\
\hline 97901_16 & 92 & 88 & 83 & 1 & 90.22 & 1.14 & -0.115 & 0.449 & -0.174 & 0.642 \\
\hline 97905 & 39 & 35 & 33 & 1 & 84.62 & 2.86 & 0.043 & 0.546 & -0.158 & 0.405 \\
\hline 97920 & 20 & 21 & 20 & 0 & 100.00 & 0.00 & 0.010 & 0.210 & -0.056 & 0.238 \\
\hline
\end{tabular}

1. False detections include the following scenarios:

(a) A reported conflict spot is different from the ground truth

(b) A vehicle path is covering two conflict spots and therefore two conflicts are reported

2. False detections are not counted if the actual value is $>8 \mathrm{~s}$ but the difference between reported and actual values is $<0.5 \mathrm{~s}$

At the moment, three sources of recoverable errors can be pointed out as follows:

- camera vibrations, 
- positioning (and areas) of the conflict spots,

- occlusions caused by the poles.

The camera vibrations are the main source of the high false detection rates at 87923 and 97901_10. Another source of problems is double detections for one single PET event when the vehicle covers a portion of two conflict spots simultaneously. The vehicle traveling path can be slightly varied while the conflict spots are fixed in the field of view. This happened frequently at 87923, 87933, and 97905.

In the case of occlusion occurrence, the present version of the detection algorithm often fails when two (or more) vehicles are concurrently passing the occluded zone (pole). In order to avoid such situations the image obtained from the camera should be similar to intersection 97920 rather than intersection 87923 .

\section{H.4 SUMMARY AND CONCLUSIONS}

The result of this project is a unique software package, offering both the identification of vehicles in motion and visualization of the process. The latter is possible due to the application of the VirtualDub program (Lee, 2003). The identification procedure is based on the calculations of optical flow (see page 346) and the algorithms of merging and division of labeled objects described in Section 2.2. Each object is assigned a unique number (color), which provides a good basis for developing a full tracking procedure (in further development stages). At the present stage of the project, the study includes the analysis of time periods when the detected objects overlap with the defined conflict spots and stop lines. The obtained information is used to verify vehicle routes and then generate output files with the analysis results (see pages 353-353). The adjustment of the program operation to the actual scene geometry is done by the user. The user must provide the configuration files: one text file and two bitmap graphic files, specifying the locations of conflict spots, stop lines, and scene elements obscuring the view (see Sections H.6 and H.7).

The developed software package, to a large degree, meets the requirements specified to the AGH UST team by the Purdue University group. In comparison to the specified detection rate of $95 \%$ and false rate of $5 \%$, the achieved values were about $90 \%$ and $11 \%$ respectively. The main error sources were, attributed to the camera vibrations, insufficient precision of the specification of 
conflict spots, and the imperfections that are still present in the algorithm. Numerical evaluation of error rates for individual video clips can be found in Table H-1 and Table H-2.

The precision of evaluation of the GT and PET times should be also mentioned (see Table H-1). The maximum values of average errors do not exceed $0.2 \mathrm{sec}$ for all of the video clips, the standard deviations do not exceed $1.03 \mathrm{sec}$ in comparison to the specified values of $1.0 \mathrm{sec}$ and $3.0 \mathrm{sec}$ respectively.

In the current version of the "traffic.vdf" filter (v1.3), only partial time optimization has been completed by suitable selection of compiler options and some other factors. Some extra acceleration can be achieved by optimizing the optical flow calculation algorithm and the process of secondary segmentation (morphological operations). The processing times for individual video clips are listed in Table H-3. The analysis of video clips takes 4.6 to 5.6 times longer than their actual duration time on PC Pentium III / $800 \mathrm{MHz}$ processor and $256 \mathrm{MB}$ of RAM. It can be seen in Table H-3 that the processing time is sensitive to neither the traffic intensity nor the number of elements obscuring the view. It seems that the processing time is affected by the object size: the larger is the object (higher zoom factor), the longer is the processing time. When faster processor (Intel $2.4 \mathrm{GHz}$ ) is used, the processing time is shortened to the range between 1.67 and 1.79 (for VirtualDub working in batch mode - see Table H-3).

Table H-3: Processing Times of Video Clips (in multiples of real-time duration)

\begin{tabular}{cccccccc}
\hline Intersection & $\begin{array}{c}\text { Pentium III } \\
800 \mathrm{MHz}\end{array}$ & $\begin{array}{c}\text { AMD 2.4 GHz } \\
\text { normal (display) }\end{array}$ & $\begin{array}{c}\text { Intel 2.4 GHz } \\
\text { normal (display) }\end{array}$ & $\begin{array}{c}\text { Batch } \\
\text { mode }\end{array}$ & $\begin{array}{c}\text { Zoom } \\
\text { Traffic } \\
\text { intensity }\end{array}$ & Occlusion \\
\hline 87907 & 4.59 & 2.59 & 2.0 & 1.76 & small heavy & + \\
87923 & 5.15 & - & - & 1.70 & medium medium & + \\
87933 & 5.65 & 2.62 & 1.98 & 1.79 & large medium & - \\
$97901 \_10$ & 5.17 & - & - & 1.74 & medium medium & + \\
$97901 \_16$ & 5.43 & - & - & 1.67 & medium medium & + \\
97905 & 5.49 & - & - & 1.74 & medium heavy & - \\
97920 & 5.32 & 2.63 & 1.94 & 1.74 & medium medium & $\sim$ \\
\hline
\end{tabular}

The main sources of errors should be considered for the improved version of the software. The software should neutralize camera vibrations, which is the source of false detections. The improvements in the configuration of conflict spots can be achieved by the program user. The further refinement of the image analysis algorithm may include: 
- improvement of the object merging/division algorithm,

- tracking of stopped vehicles within the intersection,

- implementation of full-vehicle tracking with the calculation of motion trajectory,

- object analysis methods other than the optical flow calculations may be incorporated, i.e., the subtraction of dynamically updated background from the current video frame; unfortunately, this implementation is likely to increase the processing time considerably.

\section{H.5 INSTRUCTIONS FOR SOFTWARE INSTALLATION AND USAGE}

The software has two components:

- The filter for the VirtualDub (VD) application, which executes the processing algorithms described in Section H.2 (optical flow), performs visualization of the final segmentation and writes the file with the "raw" results (the second mode of the filter operation is the visualization of the video clip with overlaid detection areas, stop lines and ROI).

- The program to post-process the data obtained from the VD filter, generate the list of vehicles, and then calculate the GT and PET for the detection areas.

The inputs required for both components are read from the following configuration files:

- A text file which contains (a) the information on the other files, (b) descriptions of conflict spots and STOP lines, and (c) other information such as the video clip identifier, the recording time, etc. (see Section H.6 for examples).

- Two binary graphic files (*.bmp) which define the positions of conflict spots, stop lines, and the occluded areas (see Section H.7).

\section{Installation}

The first step consists of installation of the VirtualDub program and the "dvcodec" decompressor (Video for Windows) $)^{2}$. These two components provide a playback interface for video clips.

\footnotetext{
${ }^{2}$ Both the VD archive (version 1.5.1) and a working codec version dvdemocodecv2.1.exe are provided in the software CD (see Appendix D).
} 
Installation of the VD program comprises the archive decompression and the creation of the desktop shortcut if required. The "traffic.vdf" filter should be copied to subdirectory "Plugins" in the VD installation directory. The application for post-processing the results obtained from the VD program (traffic.exe) should be copied to the subdirectory, where the output files and text configuration files will be stored. All the configuration files (both text and graphic files) for the video clip being processed should be copied to the video clip directory.

\section{Video Clip Analysis}

The present procedure describes a method for running the VirtualDub program with the required filters. It is assumed that the video clip and the corresponding configuration files are placed in the same directory. The order of the described operations is essential - the video clip must be read before defining the required filters.

The user may automate some of the operations using the VD program scripts or VD batch mode. In the list below, the basic "manual" method is described:

- Start the VirtualDub program.

- $\quad$ Read in the video clip to be analyzed.

- Decrease by half the window for the source clip (ClickR the left window, choose " $1 / 2$ size" option).

- Select "Filters" from the "Video" menu: an empty window appears.

- Click "Add": a list of internal VD filters appears.

- Choose "2:1" reduction, click OK.

- Click "Add..." once more.

- Find "Traffic analysis" filter".

- Select this filter and click OK. A configuration window will pop up (see Figure H-3). Type in the name of the text configuration file ("97901_conf.txt" for the example in the Figure H-3) and choose the appropriate options: (a) if the user does not check any fields,

\footnotetext{
${ }^{3}$ If there is no such a filter load it manually (copy "traffic.vdf" to video clip directory): click "Load": the contents of the video clip location directory is shown, choose "Traffic.vdf": an additional filter entitled "traffic analysis" appears.
} 
the software will start with the run-time visualization of the image processing on the right window, (b) if the user checks the "Turn off visualization of analysis" field, the run-time visualization will be turned off, which will slightly improve the processing time, or (c) if the user checks the "Only visualization of stops and spots" field, the video clip will not be analyzed and in the right window the source clip will be displayed with configuration elements overlaid (useful for manual review or manual measurement).

- click $\mathrm{OK}$ in the configuration window and then $\mathrm{OK}$ in the filter selection window.

- In the main window of the VD program, start the analysis (and/or visualization) with the control buttons ("output playback" or "key next" - step operation). The screen appearance during the analysis is shown in Figure H-5.

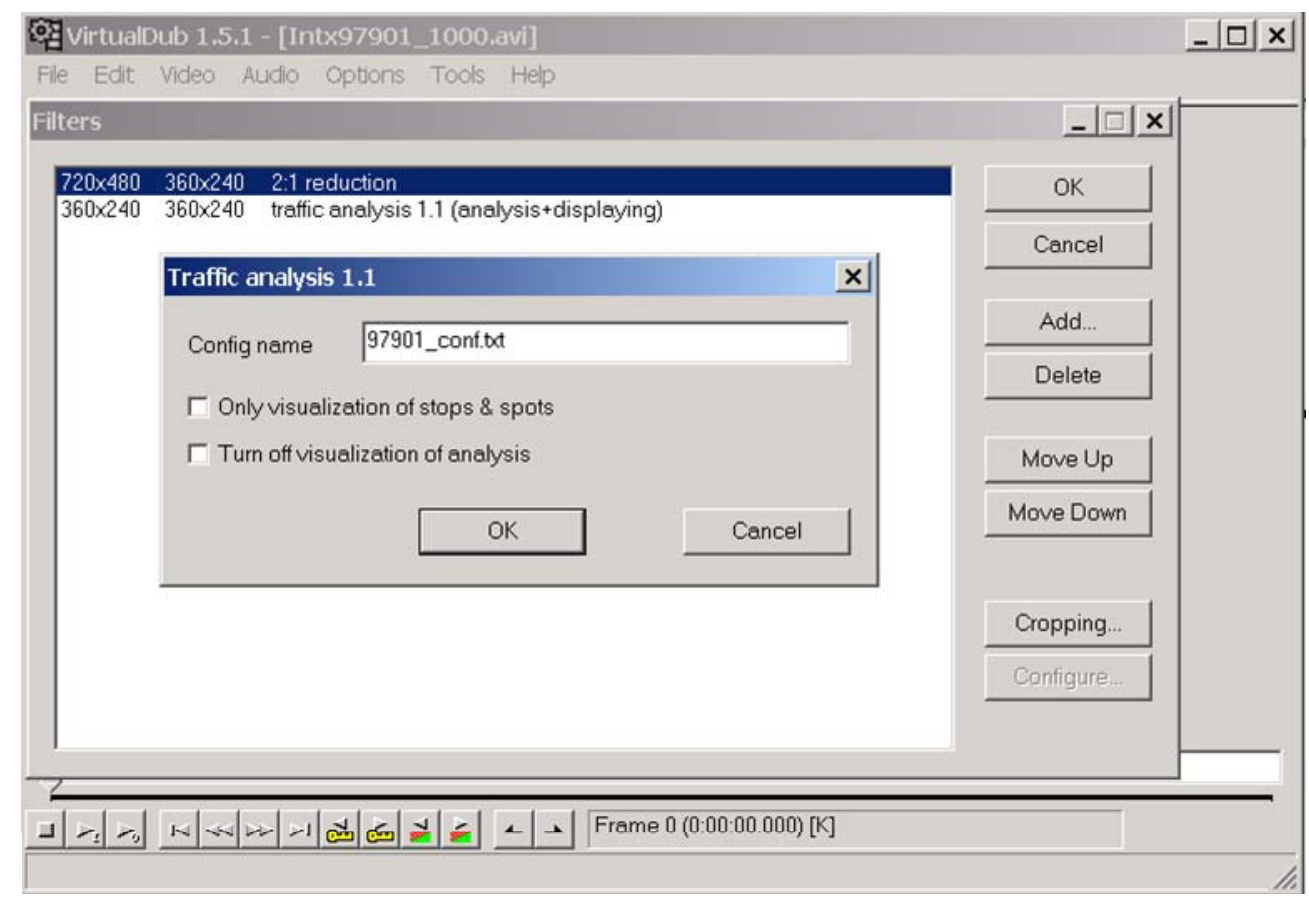

Figure H-3: Configuration Window of the “Traffic.vdf” Filter

Attention: the "traffic.vdf" filter generates a file containing the output data, with a filename consistent with the name given in the text configuration file. If the VirtualDub program is stopped and then restarted, the error message will be displayed (see Figure H-4) because it attempts to create the file that already exists. The user has to remove the file in person or comment out the specific line of code in the text configuration file (the line with the LOG name see Section H.6) so that the program will not try to re-create the file. 


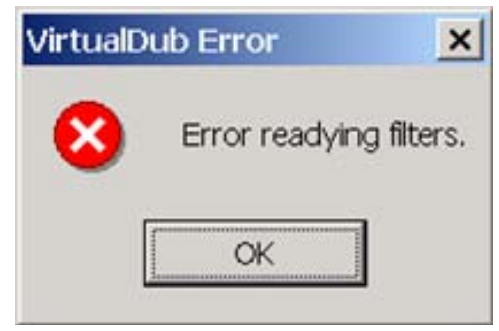

Figure H-4: Dialogue Box of Error Message

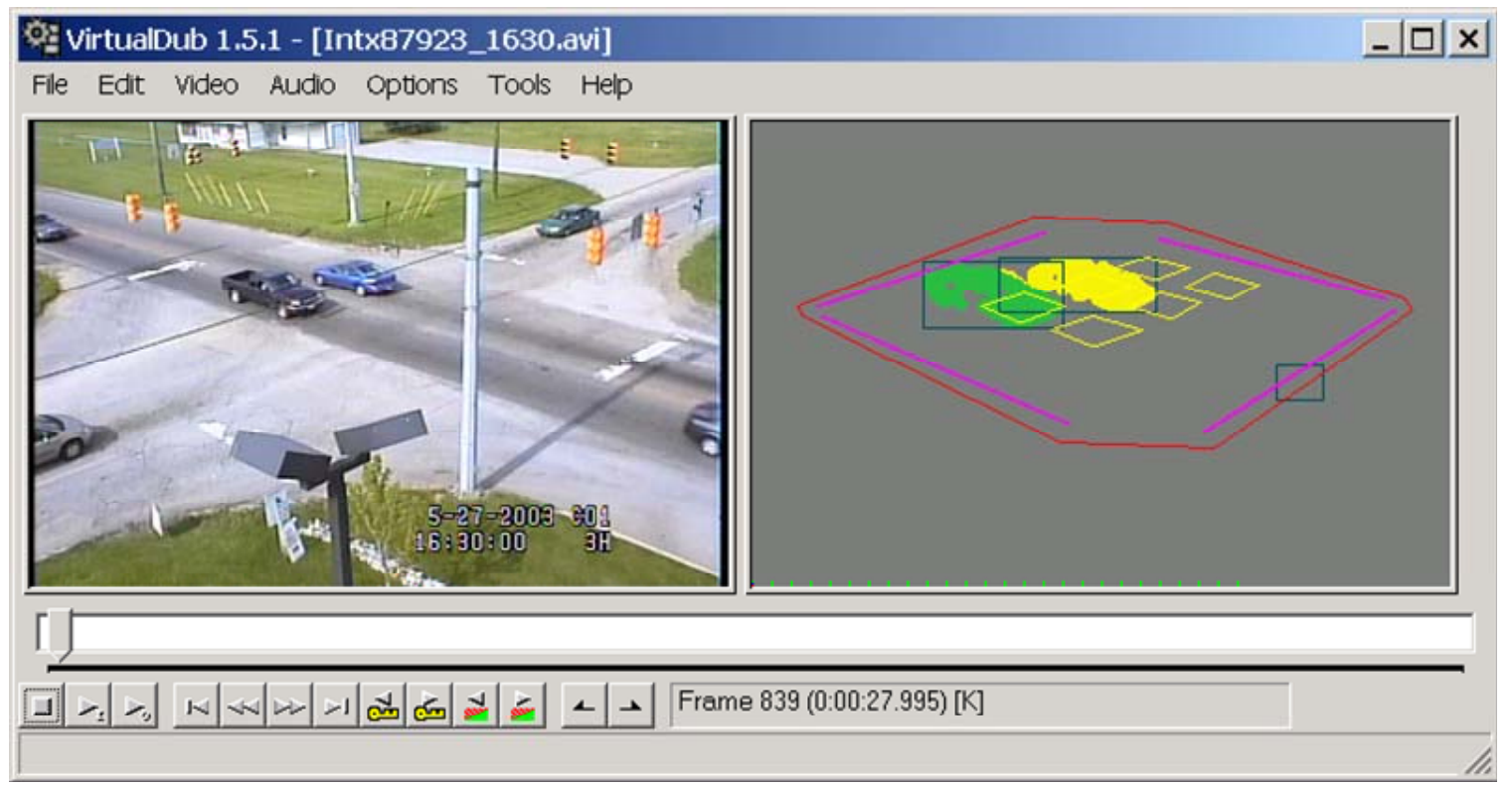

Left: source image, right: segmented objects (yellow and green ones) inside ROI (red). STOP lines and collision spots are drawn in violet and yellow respectively.

Figure H-5: Image Analysis by VirtualDub and “Traffic.vdf”

After completing the video clip analysis, we need to remove the excessive elements from the file and generate the conflict list in a format readable by a spreadsheet application (semicolon delimiter). The program is executed from the command line by typing:

\section{traffic <conf $>$}

where $<$ conf $>$ is the name of the text configuration file. The "Traffic.exe" program reads from that file the information regarding the stop lines, conflict directions, starting time, filenames for the input and output files and records added by the user. 


\section{Input and Output Files}

The "Traffic.vdf" filter and the "Traffic.exe" program read in and create files according to the following scheme (see Figure H-6 - file labeling are consistent with the names discussed in Section H.6).

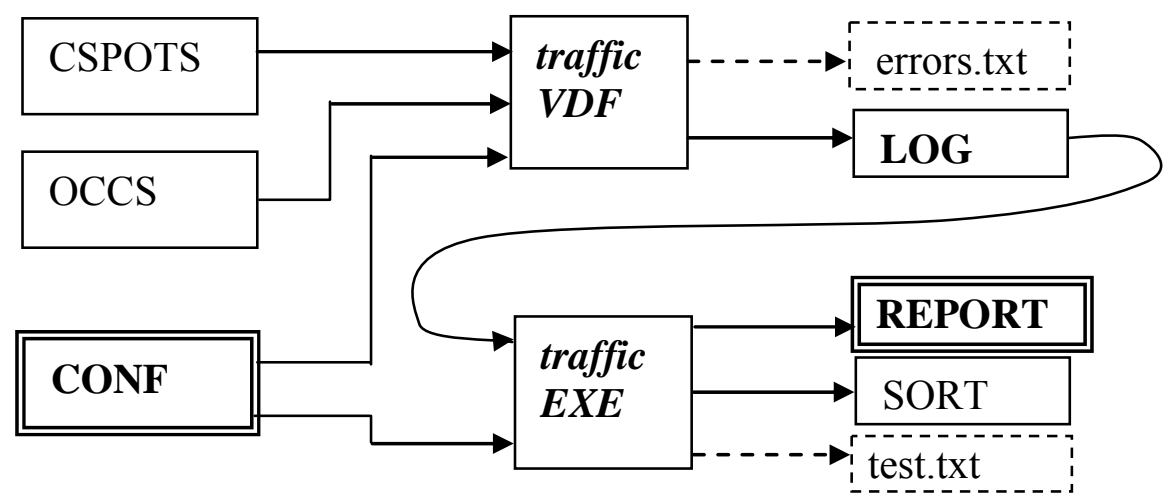

Figure H-6: Software Structure

The key file, required for correct operation of both programs, is the text configuration file (CONF). Its detailed structure is described in Section H.6. In addition, the "Traffic.vdf" filter can read the bitmap files CSPOTS and OCCS (see Appendix C). These graphic files contain the conflict spots, stop lines (CSPOTS), and occluded areas (OCCS). The "Traffic.vdf" filter generates the LOG file, which contains records for all the objects detected in a given frame. In cases when processing errors are encountered, the error messages are appended to a file "errors.txt"

The "Traffic.exe" program reads in the data from the CONF file and then processes the file with a filename specified in the LOG line. The output of this program is the REPORT file, in which all the events associated with detected conflicts are recorded. The SORT file is a result of the LOG file filtering: it contains records of consecutive objects registered. Additionally one more file with a fixed name "Test.txt" is created. It contains records of all the objects moving straight.

The LOG, SORT and "Test.txt" files have similar record structures. Each record belongs to one object. Consecutive data items are separated by semicolons and denote in sequence:

1. end time of the recording (in frames)

2. object number

3. code of the first stop line or spot

4. entry time for the first stop line or spot 
5. departure time for the first stop line or spot

n. code of the $n$-th stop line or spot

$\mathrm{n}+1$. entry time for the $\mathrm{n}$-th stop line or spot

$\mathrm{n}+2$. departure time for the $\mathrm{n}$-th stop line or spot

The "Test.txt" file has a similar structure with the number of entry areas and departure areas recorded next to the object number (in square brackets).

\section{Batch Mode}

Batch mode (File/Job control) is the fastest computing method (see Table H-3) because there are no delays caused by video clip input/output display. To use it effectively, one should understand the concept of Sylia scripting (Lee, 2003). Below we present a simplified example obtained from "trials and errors": the automatically generated script has been manually changed to match our needs.

Let us assume that, in the directory J:187907_1000, the following files exist:

- 2.avi and 3.avi - video clips to be analyzed

- 87907_conf_2.txt and 87907_conf_3.txt - text configuration files for 2.avi and 3.avi respectively

- Graphic configuration files as specified in text configuration files

- There should be no LOG files specified in text configuration files

After executing the steps in "Video Clip Analysis", the user should select "File/Save as *.avi" menu option. The window entitled "Save as *.avi" appears: the user should specify a file name (in this case " $2 x ")$, mark the box pointed by white arrow (see Figure H-7) and click "Save". At this moment the first job is appended to "VirtualDub.jobs" file. 


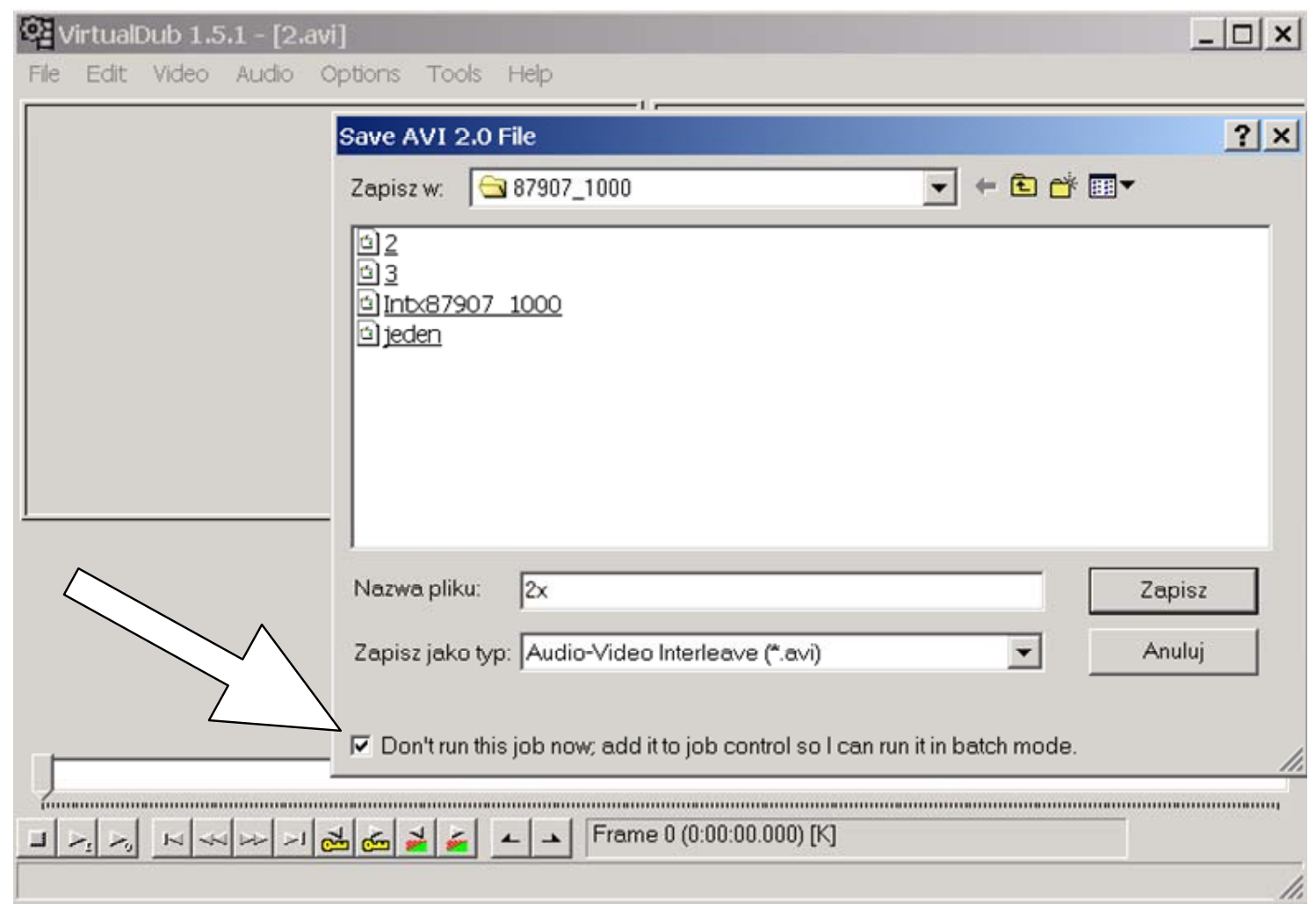

Figure H-7: Writing the Script to be Executed in Batch Mode

The same steps should be repeated for the second video clip to be analyzed.

Now it is the time to close VirtualDub program and change the name of "VirtualDub.jobs" file (eg. to "VD_example.jobs"). This slightly modified file (changes are underlined and in bold) is presented in Box H.1.

There are at least three modifications (in every job):

- The full path to the CONF file and two quotation marks are added to the line describing the "traffic.vdf" filter parameters

- The instruction for AVI saving is commented (using two slashes //)

- The instruction to start VirtualDub in "preview" mode is added 
Box H.1. The modified file „VD_example.jobs” (changes are underlined and in bold).

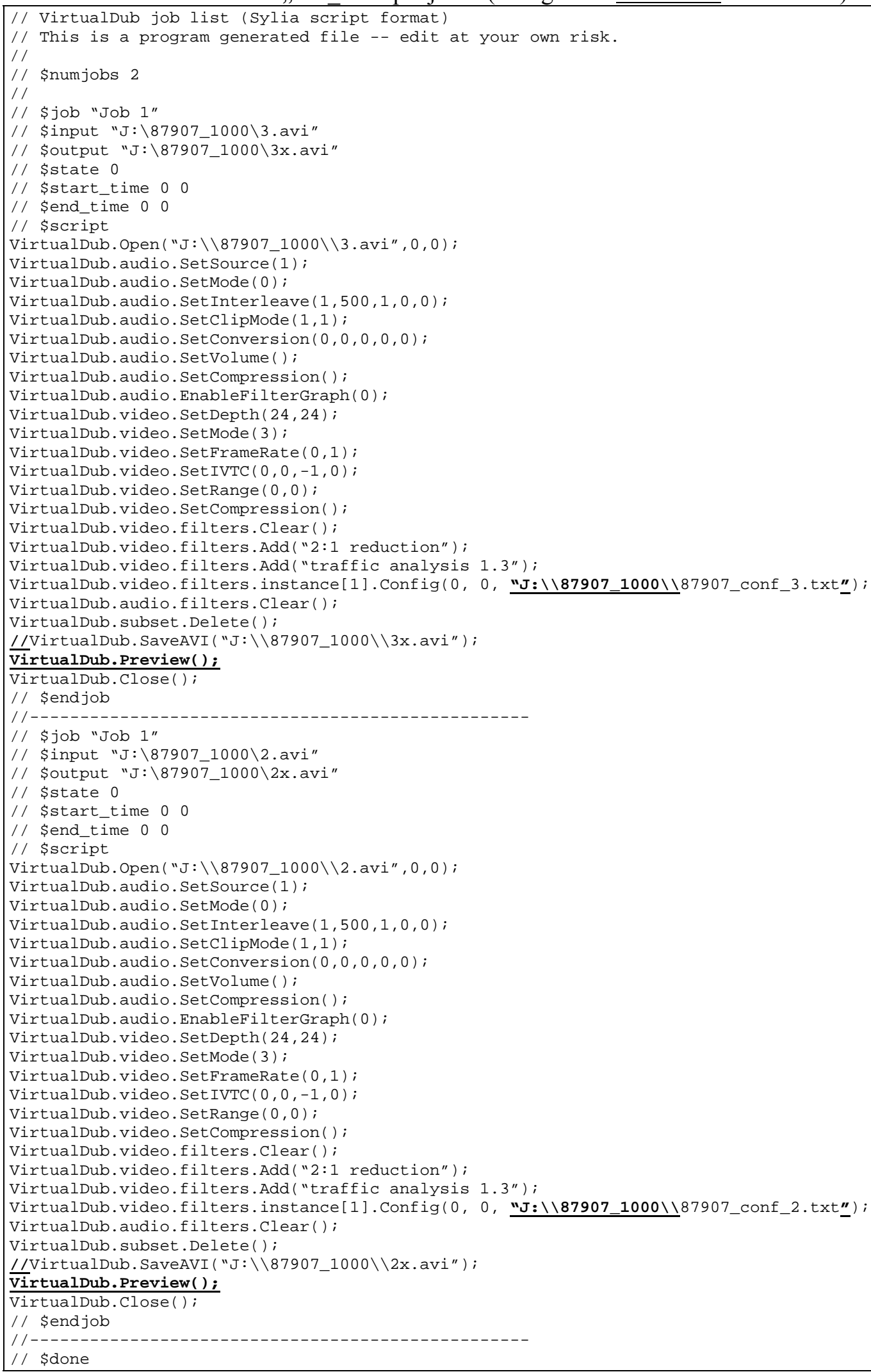


The last step is to carefully check if all the files specified in CONFs exist and restart VirtualDub. To enter the batch mode, one should choose the "File/Job control" option from the main menu, load (File/ Load job list...) the appropriate *.jobs file (in our case "VD_example.jobs), mark the first job, and press START. In Figure H-8, the larger window (in background) shows particular jobs and their status. The small foreground window provides information about the progress of the job being executed (in this case the second one). When all of the jobs are executed, the small window disappears.

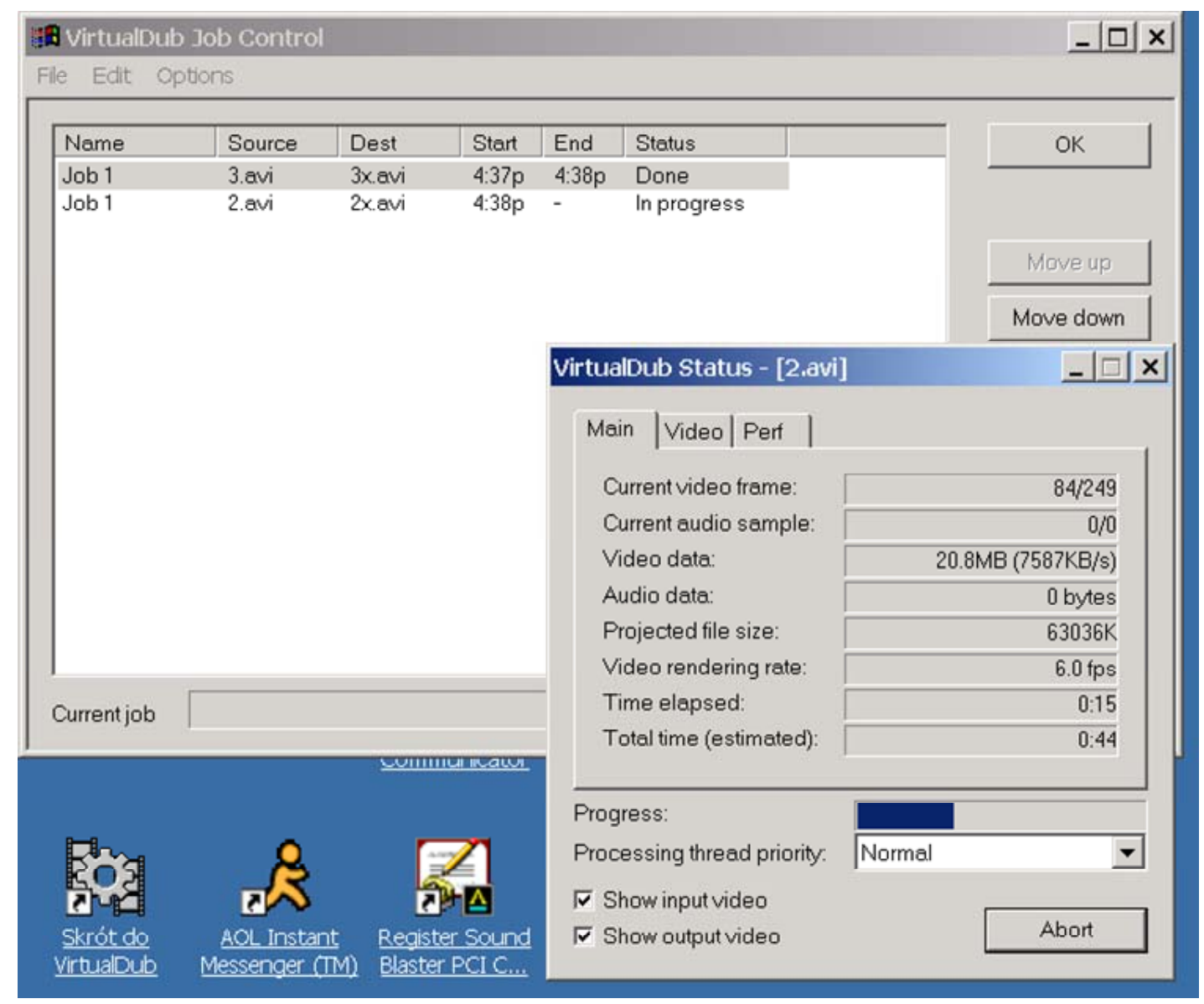

Figure H-8: Executing Jobs in “Batch” Mode

\section{H.6 DESCRIPTIONS OF THE CONFIGURATION FILE}

The configuration file must contain all of the information required for the execution of the VirtualDub filter (traffic.vdf) and post-processing program (traffic.exe). Some parameters are 
shared by both applications. Some information is merely copied from the configuration file to the output files.

The configuration data can be divided into two classes:

- Information comments - the lines begin with $\angle \#>$ character. These comments will be directly transferred to the output files with the lines begin with $<;>$ character.

- Data inputs are in one of the following forms:

$<$ data_name $>=<$ data_value $>$

or

<grey_level $>=<$ object_name $>$

or

$<$ data_name $>=<$ data_list $>$

In order to preserve data consistency, the data regarding stop lines and conflict spots should be ordered with respect to the gray level, which specifies the respective data values in the graphic files (see Boxes H.2 and H.3, Figure H-9, and Section H.7).

- The configuration of individual parameters is given for video clip 97901 as an example (see Box H.2). Each individual line is numbered to the left for subsequent explanations.

- Lines 1-5 are basic comments that will be written out to the output files (see item 2). The next two lines $(6,7)$ and the lines 16,21 , and 26 are comments added to provide additional information (see item 1). The next data block contains lines starting from line 8 and ending with line 15 . The lines can be inserted in an arbitrary order as long as the correct data format is preserved.

\section{Names and their meaning:}

TIME - Starting time of the video clip recording

FRAMES - Frame rate; it usually takes the values of 30 or 29.97 frames per second (fps) in the NTSC format 
MINDIFF - The conflict detection threshold [s]: maximum GT time that will be taken into account in the output report

CSPOTS - Name of the graphic configuration file, in which the locations of the stop lines and conflict spots are defined (see Section H.7)

OCCS - Name of the graphic file defining the objects obscuring the view (see Section H.7)

LOG - Name of the file containing the raw output: this is a file produced by the VirtualDub filter "traffic.vdf", which is later processed using the "traffic.exe" program.

SORT - Name of the file containing the data on consecutive objects (this is a file that has been filtered and sorted using the "traffic.exe" program).

REPORT - Name of the file containing the data of all conflicts detected (the file is generated by the "traffic.exe" program).

Lines 17-20 (stop lines) define the locations of intersection entry and departure. Lines 22-25 define the conflict spots defining the locations of crossroad entrances/departures and the conflict spots (lines 22-25). These lines are of the following format

$$
<\text { gray_level }>=<\text { data_name }>\text {. }
$$

The naming conventions for the conflict spots have been specified by the Purdue University team. The stop lines are defined by a conventional geographic orientation attributed to the entering vehicle: 1 denotes the vehicle entry/departure along the main road, while 2 denotes the respective vehicle entry/departure along the side street.

The last set of inputs contains definitions of possible vehicle paths (see lines 27-30). The syntax of line input is as follows:

$<$ traffic direction $><$ traffic lane number in the given direction $>=<$ stop line code $>$, $<$ number of conflict spots $>,<$ code of the first conflict spot $>, \ldots,<$ code of the $n$-th conflict spot $>$

The configuration specified in Box H.2 is consistent with Figure H-10 in Section H.7. A more complicated example has been presented in Box H.3 and Figure H-9. 
Box H.2: Configuration file for video clip 97901

\begin{tabular}{|c|c|}
\hline 1 & ; SITE CODE: \\
\hline 2 & ; MAIN STREET: \\
\hline 3 & ; SIDE STREET: \\
\hline 4 & ; DATE: $\quad$ Apr 8, 2003 \\
\hline 5 & ; TIME : $\quad 9: 58: 56$ \\
\hline 6 & \# ANY OTHER USEFUL DATA \\
\hline 7 & \# \\
\hline 8 & TIME=9:58:56 \\
\hline 9 & FRAMES=29.970 \\
\hline 10 & MINDIFF=8. 2 \\
\hline 11 & CSPOTS=97901_AREAS . BMP \\
\hline 12 & OCCS=97901_OCC . BMP \\
\hline 13 & LOG=97901_10_log. TXT \\
\hline 14 & SORT=97901_10_srt . TXT \\
\hline 15 & REPORT=97901_10_REP . TXT \\
\hline 16 & \# STOP lines \\
\hline 17 & $1=W 1$ \\
\hline 18 & 2=E1 \\
\hline 19 & $3=\mathrm{N} 2$ \\
\hline 20 & $4=S 2$ \\
\hline 21 & \# Conflict spots \\
\hline 22 & $11=S B 1 W B 1$ \\
\hline 23 & 12=NB1WB1 \\
\hline 24 & 13=SB1EB1 \\
\hline 25 & 14=NB1EB1 \\
\hline 26 & \# routes \\
\hline 27 & $N 1=3,2,14,12$ \\
\hline 28 & $S 1=4,2,11,13$ \\
\hline 29 & $E 1=2,2,13,14$ \\
\hline 30 & $W 1=1,2,12,11$ \\
\hline
\end{tabular}


Box H.3: Configuration file for video clip 87923

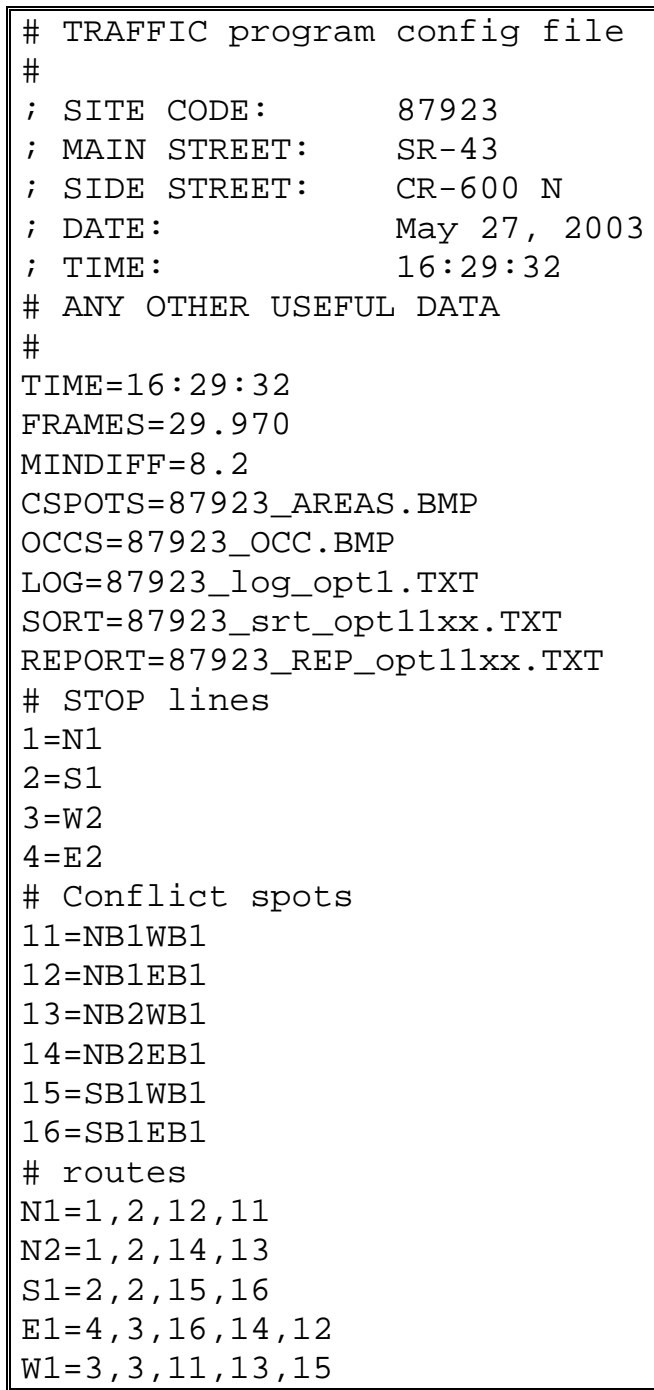




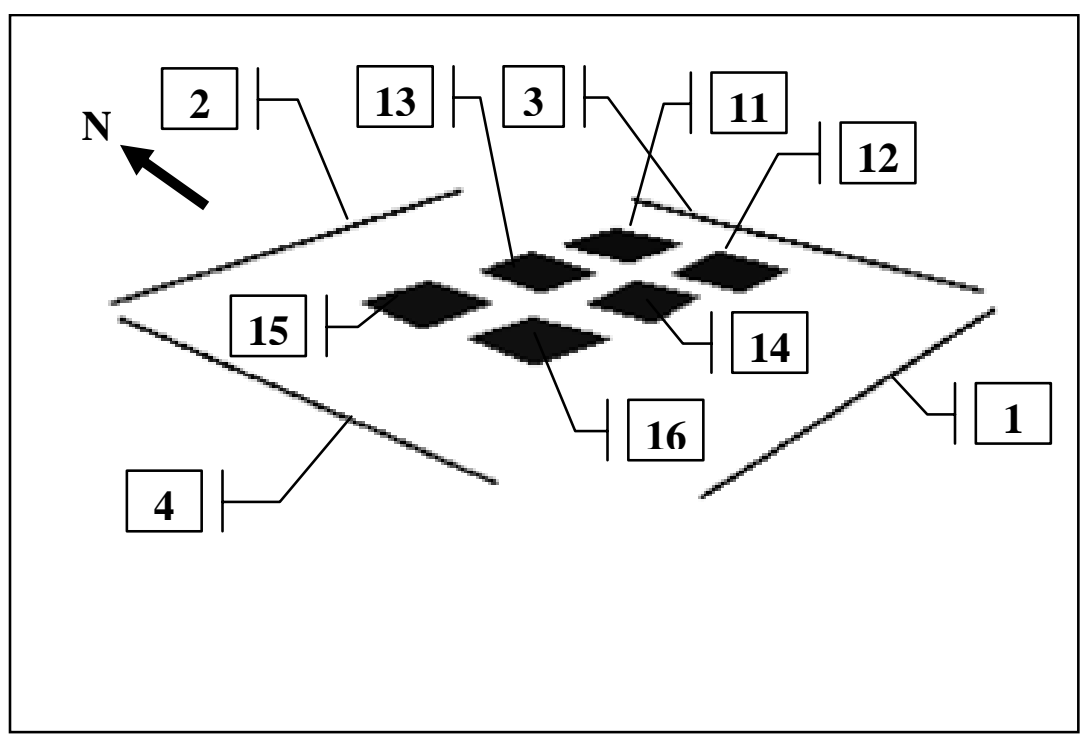

Figure H-9: Graphic Configuration File for Clip 87923 - “routes” Section in Box H.3

\section{H.7 CREATING GRAPHIC CONFIGURATION FILES}

To configure software to detect vehicles in the intersection area, two graphic configuration files have to be created: (a) the graphic file defining conflict spots and stop lines and (b) the graphic file defining occlusion areas (optional). The sizes of these two bitmap files should exactly match the size of the screen capture of the video where the vehicles are to be detected (at present, the preferred image size is $=360 \times 240$ pixels).

\section{Conflict Spots}

This configuration file is required for proper vehicle detections. The user should define two types of elements: the stop lines and the conflict spots. The elements of different types should be marked with different shades of gray (see below).

Stop lines are used to determine the directions from which the vehicles enter and depart the intersection. Therefore, the stop lines should be stretched across the whole width of the road and should be situated closer to the intersection than the stop bars (see Figure H-10). The positions of stop lines also define the Region of Interest (ROI).

The conventions used are: 
- The stop lines should be defined by gray levels from 1 to 9 . Note that the 1 and 2 values are reserved for the main road. The stop lines should be drawn with solid lines, with 1 pixel width.

- The gray levels of the conflict spots should start from the level 11 and be assigned in sequence.

- The configuration elements should be drawn on a white background (level 255).

- The picture should be saved as a bitmap file with the 8-bit color depth ( 256 colors).

- Each element should be correctly described in the text configuration file (Section H.6).
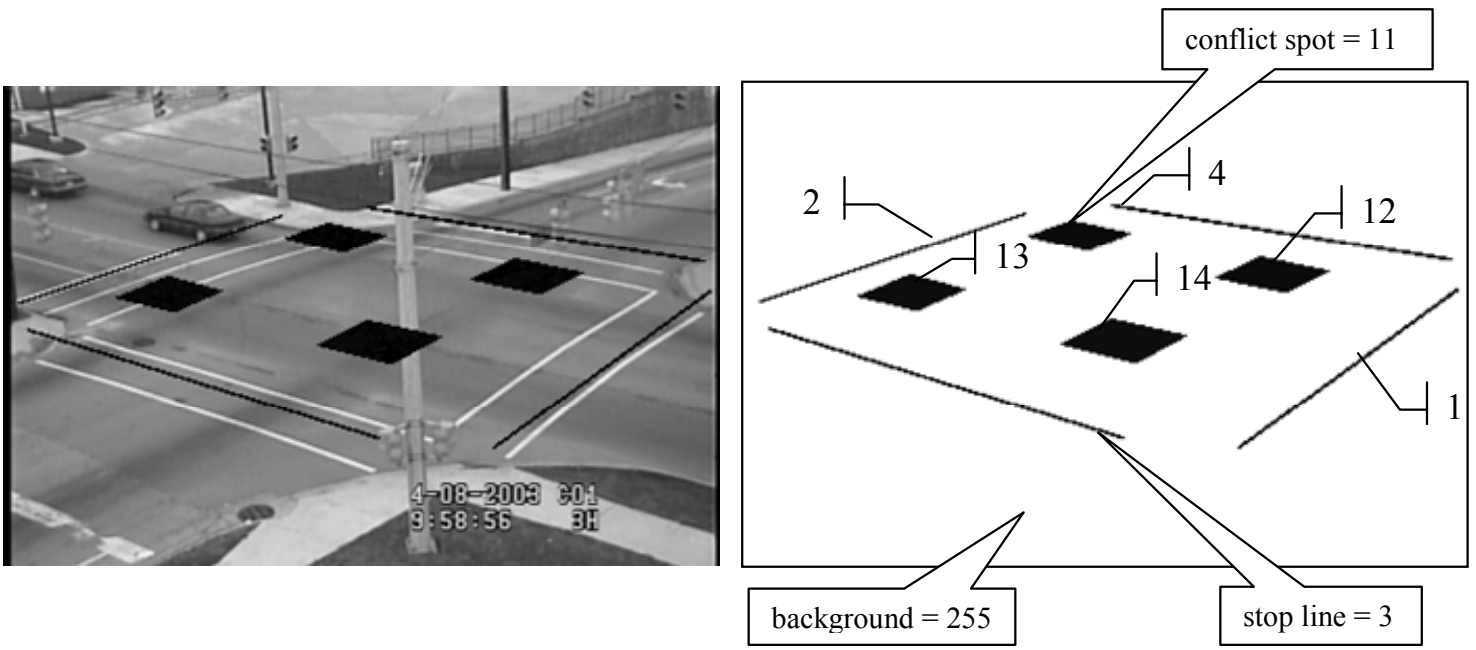

Figure H-10: Overlaid Configuration Elements at Clip 97901 (Left) and Configuration File (.bmp) with Gray Levels (Right)

\section{Using the Jasc Paint Shop Pro to Create Graphic Configuration Files}

The Jasc Paint Shop Pro (PSP) application is easy-to-use photo-editing software, well-suited for the creation of graphic configuration files for our application. The list below describes the stepby-step procedure for PSP Version 8:

1. Open image file $(360 \times 240$ pixels $)$ and zoom it $(\mathrm{Num}+)$

- if you are using PSP for the first time find in the "materials" palette "swatches" and add appropriate grey levels (eg. from $\mathrm{RGB}=111$ to 666 and from $\mathrm{RGB}=11,11,11$ to $18,18,18$ ) 
2. Add new vector layer (Layers/New Vector Layer). The defined areas can be re-edited later.

3. Choose Pen Tool and set the parameters (using the toolbar above the image, see Figure H-11):

- $\quad$ select Contigous, Drawing Mode, Line style $=$ Solid, Width=1 pixel, do not set Antialias.

4. For Pen Tool: select New, set the same color (greylevel, $R=G=B$ ) both for the foreground and for background (in Materials/Swatches clickL to set Foreground, clickR to set Background, or clickL on Foreground/Background and set RGB values, clickL OK),

5. draw the stop line: ClickL at the beginning of the line, release the button and ClickL at the end point (you can move these points and change color for the selected object)

6. check the color of the object using Dropper Tool

7. To draw further objects go to 4

8. When all of the lines and spots are drawn, save the image as "*.pspimage" (you can edit objects in future)

9. Save image once more using different name

10. Select background as an active layer

11. Select Flood Fill Tool, set foreground=background $=255$

- fill background layer (ClickL on the image): you should see "almost black" configuration elements on white background (see Figure H-10 right)

12. Look at Image/Image information: pixel depth/colors should be $8 / 256$.

- If it not the case apply: Image/Greyscale

13. Save this image as bitmap

14. Write info on gray levels and objects' names in the text file (see Section H.6). 


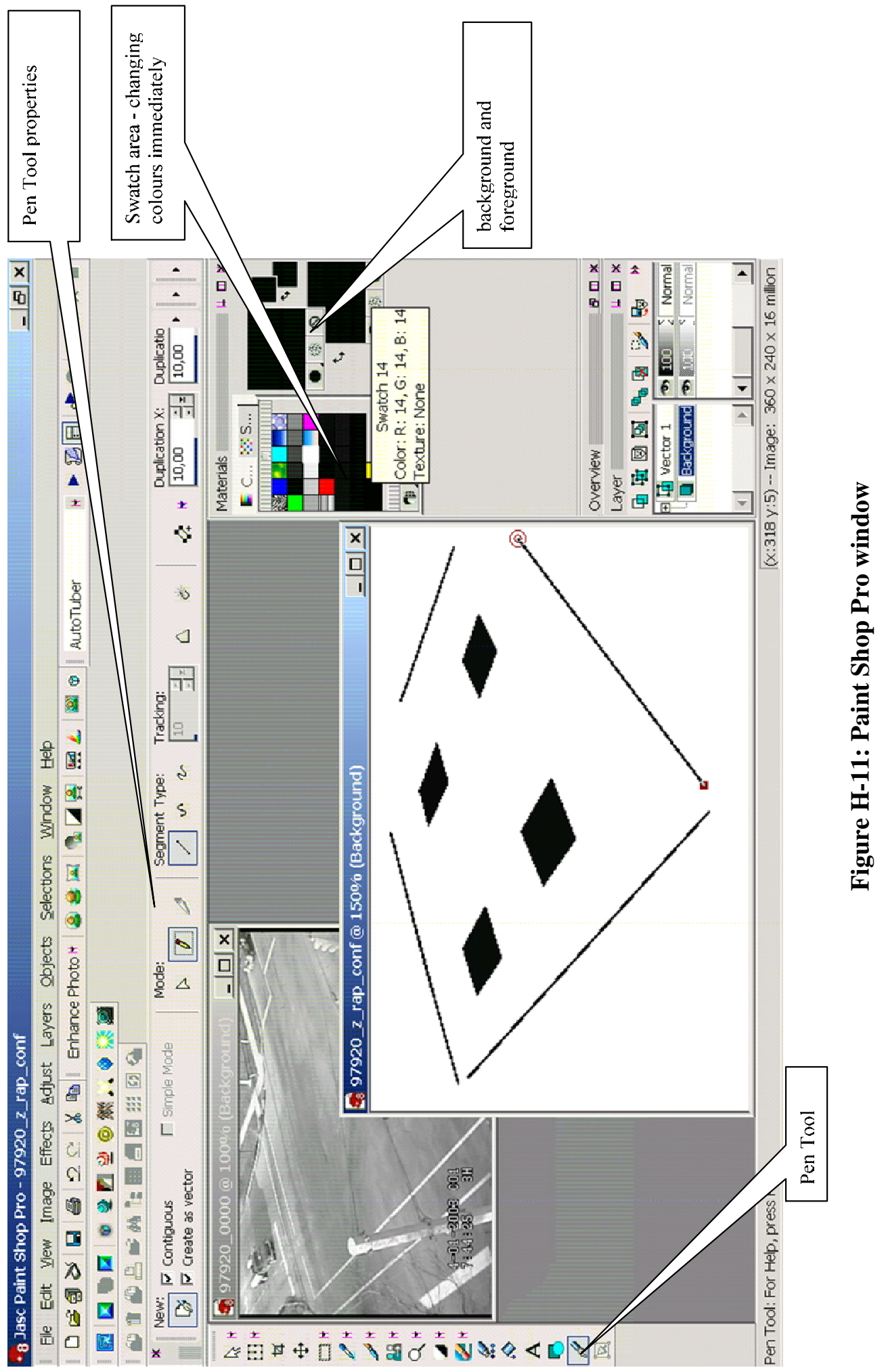




\section{Occluded Areas}

In the second bitmap file the user should mark the areas (objects) which may obscure the observed vehicles (see Figure H-12). The list of elements includes:

- line supports, lamp-posts

- $\quad$ big traffic lights

- road signs ordering the traffic direction from a given traffic lane, hanging over the road

- $\quad$ other objects (see the file for the video clip for the 87923 crossroad)

The list of occluding objects, for which the locations should be marked, should contain objects, which can effectively obscure the moving vehicles or the presence of which may result in division of the monitored objects into parts.
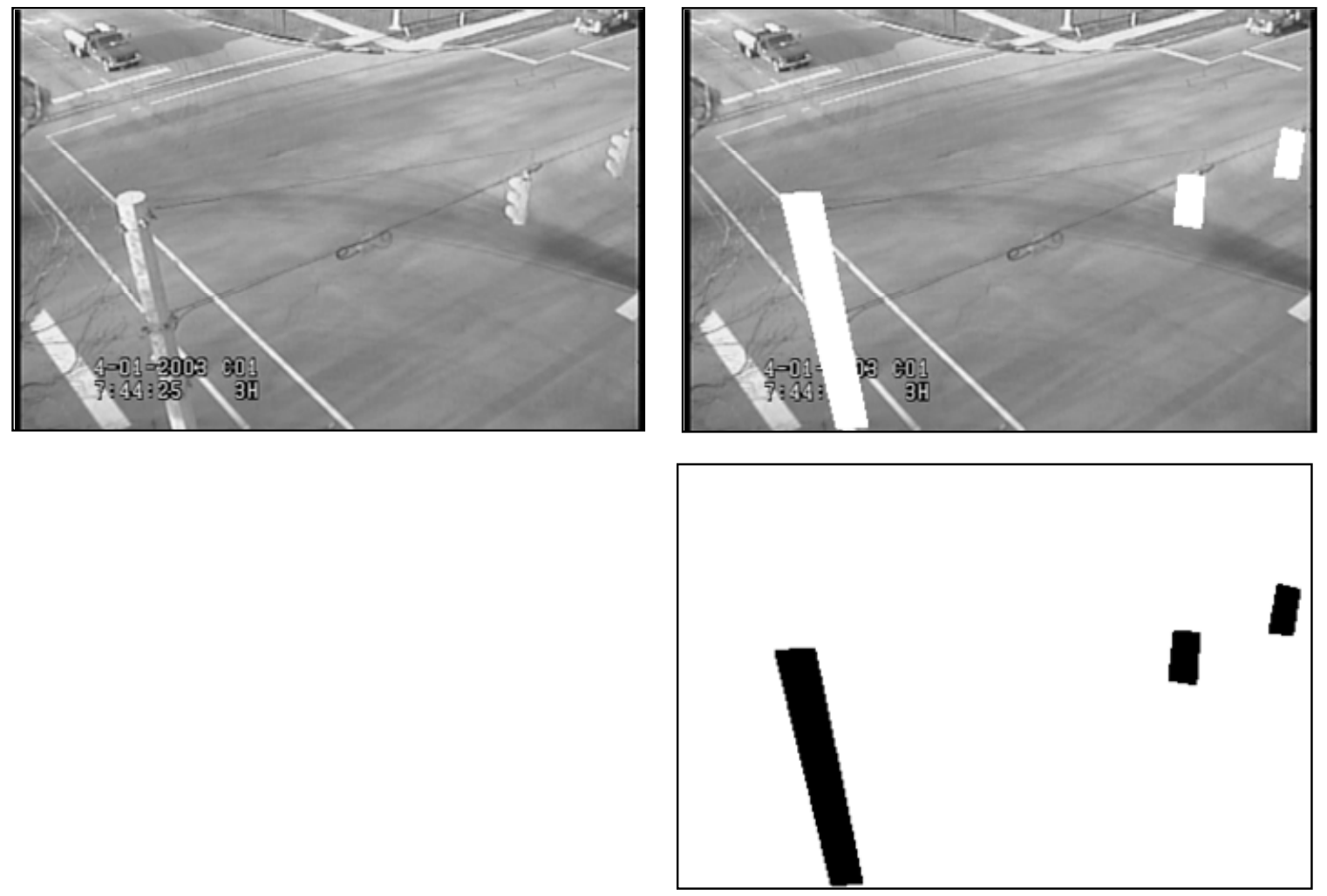

Figure H-12: Consecutive Stages of Generation of the Graphic File with the Occluded Areas (Bottom Right - Inverted Image) 
The drawing can be realized using various software packages for creation of graphic files, e.g., the Paint Shop Pro program described earlier. The method for objects generation is similar to drawing the conflict spots, only the applied gray levels are different, both for the objects and the background.

\section{Conventions Used}

- The occluding objects should be drawn in white $(\mathrm{RGB}=255,255,255)$ and should be somewhat bigger than the images of actual objects.

- The background of the final image should be set to $0(\mathrm{RGB}=0,0,0)$.

- $\quad$ The image should be saved as a bitmap, with color depth equal to 256 (8 bits). 


\section{H.8 SOFTWARE CONTENTS}

The root directory contains the following subdirectories and files:

\section{Software:}

traffic13.vdf: VirtualDub Filter ver. 1.3

Traffic1 1.exe: program which generates the final REPORT on detected conflicts (ver. 1.1)

traffic.zip: the two above mentioned files zipped

dvdemocodecv2.1.zip: demo (version 2.1) of the DV codec

VirtualDub-1.5.1.zip: VirtualDub program (ver. 1.5.1)

\section{doc_res:}

Orep.doc: the main part of report

app_ae.doc: Section H.5

app_be.doc: Section H.6

app_ce.doc: Section H.7

app_de.doc: Section H.8 (this file)

GapData_res2.xls: results of processing compared to the ground data, summary of recognition rates

Configuration and result files for all the analysed video clips (see example for the video clip 87907 below)

87907

\begin{tabular}{|c|c|}
\hline 87907_areas.bmp & $\begin{array}{l}\text { graphic conf. file - definition of conflict spots } \\
\text { and STOP lines }\end{array}$ \\
\hline 87907_conf.txt & text configuration file \\
\hline 87907_LOG_opt.zip & result file from „traffic.vdf" \\
\hline 87907_occ.bmp & $\begin{array}{l}\text { graphic conf. file - definition of occluding } \\
\text { elements }\end{array}$ \\
\hline 87907_REP_TR3_3A.TXT & $\begin{array}{l}\text { REPORT file, generated by „traffic.exe” } \\
\text { program (detected conflicts) }\end{array}$ \\
\hline 87907_SRT3_3A.TXT & $\begin{array}{l}\text { SORT file, generated by ,traffic.exe” program } \\
\text { (records of all the detected vehicles) }\end{array}$ \\
\hline 87907_occ.pspimagesam & $\begin{array}{l}87907 \text { occ.bmp but in } \\
\text { vector graphics (generated by Jasc }\end{array}$ \\
\hline 7907_areas.pspimage & same as 87907 areas.bmp but in \\
\hline
\end{tabular}




\section{H.9 SUPPLEMENT}

\section{Supplement 1: Dealing with the Camera Vibrations}

In Table H-2 of the main (previous) report, the authors point out the most important sources of errors generated by the program for analysis of crossroad conflicts. By comparison of the program output results with the respective ground data, camera vibrations have been found to be the main reason of interferences causing the so-called "false detections." The authors of the program have appended the algorithm, described in Section H.2, with an additional filter, which makes the main traffic analysis algorithm (in particular the optical flow part) insensitive to small vibrations of the image.

The main principle of the algorithm for analysis of video clips remains unchanged, as well as the form and sequence of processing, the output files, and the program configuration files. The changes affect the final results (Section H.3) and the program setup and reference manual (Section H.5). For the user's convenience, Supplement 1 has been appended with Section H.5, now called Section S1.H.5. The previous sections were appended by a supplement so that for the cases when the camera is fastened steady the original software version, described in the main report, may be still safely used.

\section{S1.1 Algorithm}

The action of the algorithm is based on the well-tested and widely applied method of tracking a fixed object in the scene. The object is selected and indicated by the user. In the cases when the object location is changed, it is assumed that the camera has been moved. The digital image is then shifted in the direction consistent with the apparent object motion. After such a correction the object should be restored to the original location.

That simple method required the implementation, studies and elaboration of many details. In particular it turned out that in some cases the image movements were of subpixel nature. Therefore the image size was enlarged by a factor of two, using the filter of the VirtualDub program. In some (very rare) cases, probably because of cumulating errors of the subpixel image shifts, the image was not returned to the original, stable position, but exhibited a steady 
migration. Another problem was created by lighting changes, which made the adjustments rather difficult.

A simplified flowchart of the consecutive steps of the algorithm is presented in Box S1.1. The standard size of the video clip picture was $720 \times 480$ pixels. In such a picture the user defines a rectangle, in which the selected fixed object is located. The rules of the object selection are described in Section H.10. Exemplary objects, defined for various video clips have been described in Table H-4. The object is enveloped by a fixed width frame. In that area the matching operation is carried out (see Section 1 in Box S1.1). The pattern is shifted within these borders. After each shift a special matching function $\mathrm{M}(\mathrm{x}, \mathrm{y})$ is calculated, which takes the form:

$$
M(x, y)=\sum_{k=0}^{m-1} \sum_{l=0}^{n-1}|d(x+k, y+l)-o(k, l)|
$$

where $\mathrm{O}$ is the pattern (object) area (mxn pixels), D the area in which the matching is looked for, $k$ and $l$ are the pattern coordinates, $x$ and $y$ are $\mathrm{D}$ area coordinates. In the next stage the minimum value of the matching function is found, the coordinates for which the minimum takes place are determined and finally the whole image is shifted. Then another filter (included in the VirtualDub distribution) executes the image shrinking by a factor of four.

The update and supervision of the total_shift variable prevents the image migration phenomenon, described above. In the case when a threshold value is exceeded the unshifted current picture is sent to the next filter, appended by a special marker.

Each time the pattern is collected from the present image (see Section 6 in Box S1.1). It increases the matching precision, because it eliminates the effect of lighting changes, introduced by the time of the day or weather changes.

Applying the camera vibration removal filter resulted in small modifications of the main traffic analysis filter. The elements implemented in the filter included management of frames, on which the additional marker is placed. Such a frame is saved as a base image for calculation of the optical flow in the consecutive step. No calculations are carried out for the frame itself. Additionally the program part selecting every third frame for the analysis has been removed. The decimation setting is specified by the user directly in the VirtualDub program (see Section H.10). The setting is valid for all the filters. 
Box S1.1. Consecutive steps of the algorithm of camera vibration removal.

1. Set:

limits of the window containing the object

limits of the matching window

2. Set:

total_shift $=0$

collect the first frame

remember the pattern

3. collect the image

4. match the pattern

5. update the total_shift variable

6. if total_shift $>$ threshold

generate a marker for the image

remember the pattern

total_shift $=0$

go to 7

else

shift the image

remember the pattern

go to 7

7. send the picture to the next filter go to 3

Table H-4: Coordinates of Objects Selected for the Camera Stabilization (for 720x480)

\begin{tabular}{|l||c|c|c|c|c|l|}
\hline Video clip & $\mathrm{x} 1$ & $\mathrm{y} 1$ & $\mathrm{x} 2$ & $\mathrm{y} 2$ & Size $(\mathrm{X} \times \mathrm{Y})$ & Remarks \\
\hline 87907 & 402 & 17 & 448 & 32 & $46 \times 15$ & top of a pole \\
\hline 87923 & 250 & 442 & 285 & 462 & $35 \times 20$ & info board near a pole \\
\hline 87933 & 605 & 110 & 635 & 127 & $30 \times 17$ & traffic lights on a pole \\
\hline $\left.97901 \_10 \mathrm{a}\right)$ & 393 & 449 & 438 & 470 & $45 \times 21$ & base of a pole \\
\hline $\left.97901 \_10 \mathrm{~b}\right)$ & 247 & 43 & 299 & 69 & $52 \times 26$ & traffic lights (far) \\
\hline $\left.97901 \_10 \mathrm{c}\right)$ & 380 & 50 & 410 & 63 & $30 \times 13$ & top of a pole \\
\hline 97905 & 631 & 453 & 667 & 470 & $36 \times 17$ & base of a pole \\
\hline 97920 & 110 & 204 & 157 & 226 & $47 \times 22$ & top of a pole \\
\hline
\end{tabular}

\section{S1.2 Results}

The results obtained from analysis of seven video clips (eight hours) are summarized in Table H-5 and Table H-6. All of the details can be found in GapData_res5.xls file (see S1.H.8). The specific items are defined as follows: 
- actual conflicts: the number of conflicts detected by the human operator ( 8 seconds or less),

- reported conflicts: the number of reported measurements from the post-processing of the VirtualDub filter event data file. All the reported values of 8.2 seconds or less were counted,

- detected conflicts: the number of actual conflicts that can be detected by matching the occurrence times and conflict zones of the actual and reported conflicts,

- false detections: a number of reported conflicts that could not be matched with the actual conflicts,

- detection rate: the ratio of number of detected conflicts to actual conflicts,

- false detection rate: the ratio of number of false detections to reported conflicts,

- gap time (GT): the time between the moment when the first vehicle enters into the conflict spot and the moment when the second vehicle enters into the conflict spot (front bumper to front bumper),

- GT mean error: mean of the differences between actual and detected GTs,

- GT std error: the standard deviation of differences between the actual and detected GTs,

- post enchroachment time (PET): the time between the moment when the first vehicle leaves the conflict spot and the moment when the second vehicle enters into the conflict spot (rear bumper to front bumper),

- PET mean error: mean value of the differences between the actual and detected PETs,

- PET std error: the standard deviation of differences between the actual and detected PETs.

Table H-5 is a counterpart of the Table H-2, presented in the main report. Application of the "unshake" filter and proper modifications in the "traffic analysis" filter have completely removed the "false detection" cases, caused by the camera vibrations. The other detection results were more or less the same as before: for video clips 87907 and 97905 the number of detected situations increased (by a total of three cases), while for video clip 97901, the number has decreased by one case. Three of the four false detections in video clip 87923 were generated by an atypical, particularly tall vehicle, which happened to cover an additional stop line. 
Table H-5: Results Obtained after Applying Vibration Removal Algorithm

\begin{tabular}{|c|c|c|c|c|c|c|c|c|c|c|}
\hline & \multicolumn{4}{|c|}{ conflicts } & \multicolumn{2}{|c|}{ detection (\%) } & \multicolumn{2}{|c|}{ measurement [s] } & \multirow[b]{2}{*}{ PET_mean } & \multirow[b]{2}{*}{ PET_std } \\
\hline Intersection & actual & report. & detect. & false & rate & false rate & GT_mean & GT_std & & \\
\hline 87907 & 10 & 12 & 10 & 0 & 100,00 & 0,00 & $-0,023$ & 0,862 & $-0,176$ & 0,864 \\
\hline 87923 & 19 & 36 & 18 & 4 & 94,74 & 11,11 & 0,051 & 0,503 & $-0,023$ & 0,571 \\
\hline 87933 & 30 & 40 & 29 & 0 & 96,67 & 0,00 & $-0,046$ & 0,164 & $-0,195$ & 0,203 \\
\hline 97901_10a & 49 & 56 & 44 & 0 & 89,80 & 0,00 & $-0,035$ & 0,248 & $-0,133$ & 0,234 \\
\hline 97901_16 & 92 & 87 & 82 & 0 & 89,13 & 0,00 & $-0,079$ & 0,446 & $-0,145$ & 0,547 \\
\hline 97905 & 39 & 36 & 34 & 0 & 87,18 & 0,00 & 0,033 & 0,541 & $-0,173$ & 0,409 \\
\hline 97920 & 20 & 21 & 20 & 0 & 100,00 & 0,00 & 0,010 & 0,210 & $-0,056$ & 0,238 \\
\hline \multicolumn{5}{|l|}{ average: } & 93,93 & 1,59 & 0,040 & 0,425 & 0,129 & 0,438 \\
\hline $\max$ & & & & & & & 0,079 & 0,862 & 0,195 & 0,864 \\
\hline
\end{tabular}

Detailed analysis of the video clips revealed the existence of events undetected by the human operator as well as marginal situations, for which the algorithm of hazardous events detection acted properly. The list of such situations is presented in Table H-6 in the part called "algorithm detections." The events undetected by the human operator and detected by the algorithm are located in the "OK" column. In the column labeled as " $\sim 8[\mathrm{~s}]$ " the authors have summed up all of the situations detected by the algorithm near the acceptable time limit (for all of the video clips the registration time between consecutive events has been taken as equal to $8.2[\mathrm{~s}])$. The situations in which a vehicle covered (e.g., as a result of the perspective projection) the neighboring conflict spot are listed in the "n.spot" (neighbor spot) column. In the "Total" column all three situations discussed above have been added.

Table H-6: Performance Results of the Algorithm

\begin{tabular}{|c|c|c|c|c|c|c|c|c|c|c|}
\hline & \multicolumn{4}{|c|}{ conflicts } & \multicolumn{2}{|c|}{ detection (\%) } & \multicolumn{4}{|c|}{ algorithm detections } \\
\hline Intersection & actual & report. & detect. & false & rate & false rate & $\sim 8[\mathrm{~s}]$ & OK. & n. spot & Total \\
\hline 87907 & 12 & 12 & 12 & 0 & 100,00 & 0,00 & 0 & 0 & 2 & 2 \\
\hline 87923 & 33 & 36 & 32 & 4 & 96,97 & 11,11 & 2 & 4 & 8 & 14 \\
\hline 87933 & 41 & 40 & 40 & 0 & 97,56 & 0,00 & 0 & 2 & 9 & 11 \\
\hline 97901_10a & 61 & 56 & 56 & 0 & 91,80 & 0,00 & 5 & 7 & 0 & 12 \\
\hline 97901_16 & 97 & 87 & 87 & 0 & 89,69 & 0,00 & 4 & 1 & 0 & 5 \\
\hline 97905 & 41 & 36 & 36 & 0 & 87,50 & 0,00 & 0 & 2 & 0 & 2 \\
\hline 97920 & 21 & 21 & 21 & 0 & 100,00 & 0,00 & 1 & 0 & 0 & 1 \\
\hline \multirow[t]{2}{*}{ average: } & & & & & 94,79 & 1,59 & & & & \\
\hline & & & & & & total: & 12 & 15 & 19 & 46 \\
\hline
\end{tabular}


From the algorithm's point of view the discussed situations cannot be regarded as errors. Therefore the number of situations from the "total" column was added to the respective fields in the "actual" and "detected" columns. The results obtained by such action are even closer to the real values.

\section{S1.3 Summary and Conclusions}

In Supplement 1 the authors describe a way to eliminate false detections caused by small camera vibrations. As a consequence, a special algorithm, described in Section S1.1, has been constructed, implemented, and tested. The results of conflict detection obtained after the elimination of camera vibrations are listed in Table H-5 and Table H-6. For these tables the detection rate and false detection rate were calculated using two methods: as the average over all detected events in all video clips and as an "average of the averages," calculated separately for each individual video clip. For the results listed in Table H-5 the respective results of such calculations were: $91.5 \%$ and $93.9 \%$ for the detection rate and $1.38 \%$ and $1.59 \%$ respectively for the false detection rate. It was noticed that the algorithm works correctly in some cases that remain undetected for the human operator and for some marginal cases. Therefore, such situations have been identified and are listed in Table H-6 and the respective table columns have been supplemented. For the corrected results the detection rate has increased to $92.78 \%$ and $94.79 \%$ respectively. Thus, the main goal of the software modification has been achieved: as a result of elimination of false detections caused by camera vibrations, the false detection rate has went down from about $11 \%$ to about $1.5 \%$.

The obtained value is much lower than the recommended number of 5\%. Also, the detection rate, after taking into account the marginal situations, is closer to the assumed $95 \%$.

The software development has also affected its complexity level. The user now has to load more filters to the VirtualDub program, as well as find and properly define the fixed scene element, which will be tracked by one of the filters. The respective procedure for the new version of the program has been described in Section H.10. Some of the operations can be automated by using scripts (see S1.H.5.4).

The result of the increased program complexity level is also the extension of analysis time. In Table H-7 the actual processing times have been compared with the respective times obtained 
previously. The times have been given in the relative form, i.e., as the ratio of the analysis time to the actual duration of the video clip. The tests were carried out on a PC with a Pentium IV 2.4GHz processor in batch mode, working under the MS Windows 2000 operating system.

Table H-7: Relative Video Clips Processing Times in the Batch Mode between Previous and New Versions of the Software

\begin{tabular}{|c|c|c|c|c|c|}
\hline Video clip & time & length & previously & now & fixed element area [pixels] \\
\hline 87907 & $1: 01: 32$ & 110653 & 1.76 & 2.30 & 690 \\
\hline 87923 & $1: 01: 32$ & 110663 & 1.70 & 2.25 & 700 \\
\hline 87933 & $1: 01: 50$ & 111189 & 1.79 & 2.15 & 510 \\
\hline 97901 10 a) & \multirow{3}{*}{$1: 02: 19$} & \multirow{3}{*}{112083} & \multirow{3}{*}{1.74} & 2.39 & 945 \\
\hline $97901 \_10$ b) & & & & 2.48 & 1352 \\
\hline 97901 10 c) & & & & 2.08 & 390 \\
\hline 97901 & $1: 02: 00$ & 111499 & 1.67 & 2.23 & 945 \\
\hline 97905 & $2: 02: 01$ & 219433 & 1.74 & 2.11 & 612 \\
\hline 97920 & $1: 01: 44$ & 111034 & 1.74 & 2.27 & 1034 \\
\hline
\end{tabular}

Note: The 97901_10 video clip has been analyzed three times with various fixed elements selected (* camera vibrations which affected the results obtained by using the previous software version).

For the 97901_10 video clip three additional tests have been carried out, comprising the execution of the algorithm for three various fixed elements to be tracked. The respective objects have been defined and described in Table H-4. In Figure H-13, a frame is presented, in which the rectangles enveloping the selected objects have been marked.

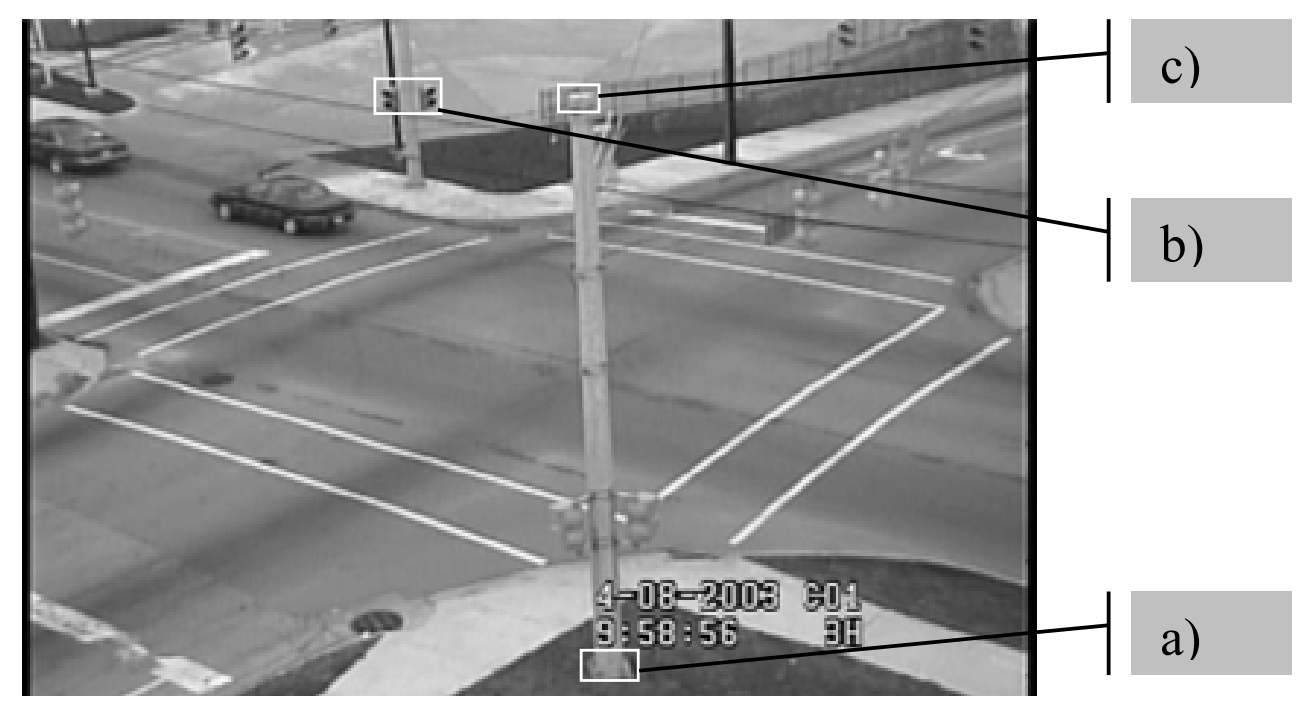

Figure H-13: Locations of the Selected Reference Points for the Vibration Removal Algorithm (97901_10) 
The results obtained from these three tests have been presented in Table H-8 and Table H-9 similarly as in the Table H-5 and Table H-6. For all the experiments the statistical values of GT and PET times are rather close to each other. The same can be said about conflict events: the changes of both detections and false detections are minimal.

Table H-8: Results Obtained Using 3 Different Tracked Objects (Clip 97901_10)

\begin{tabular}{|l|cccc|cc|cccc|}
\hline & \multicolumn{5}{|c|}{ conflicts } & \multicolumn{2}{c|}{ detection (\%) } & \multicolumn{2}{c|}{ measurement [s] } \\
\cline { 1 - 4 } Intersection & \multirow{2}{*}{ actual } & report. & detect. false & rate & $\begin{array}{c}\text { ralse } \\
\text { rate }\end{array}$ & GT_mean & GT_std & PET_mean PET_std \\
\hline 97901_10a & 49 & 56 & 44 & 0 & 89,80 & 0,00 & $-0,035$ & 0,248 & $-0,133$ & 0,234 \\
97901_10b & 49 & 59 & 45 & 1 & 91,84 & 1,69 & $-0,035$ & 0,250 & $-0,139$ & 0,235 \\
97901_10c & 49 & 59 & 45 & 0 & 91,84 & 0,00 & $-0,040$ & 0,246 & $-0,139$ & 0,231 \\
\hline
\end{tabular}

Table H-9: Results as in Table H-6 Using 3 Different Tracked Objects (Clip 97901_10)

\begin{tabular}{|c|c|c|c|c|c|c|c|c|c|c|}
\hline \multirow[b]{2}{*}{ Intersection } & \multicolumn{4}{|c|}{ conflicts } & \multicolumn{2}{|c|}{ detection (\%) } & \multicolumn{4}{|c|}{ algorithm detections } \\
\hline & actual & report. & detect. & false & rate & false rate & $\sim 8[\mathrm{~s}]$ & OK. & n. spot & total \\
\hline 97901_10a & 61 & 56 & 56 & 0 & 91,80 & 0,00 & 5 & 7 & 0 & 12 \\
\hline 97901_10b & 62 & 59 & 58 & 1 & 93,55 & 1,69 & 5 & 8 & 0 & 13 \\
\hline 97901_10c & 63 & 59 & 59 & 0 & 93,44 & 0,00 & 6 & 8 & 0 & 14 \\
\hline
\end{tabular}

The prospects of further development of the software were described in the main report in Section 4 and mainly pertain to the modification of the video clip analysis algorithm. In relation to the mentioned problems regarding the proper way of defining the conflict spots, the construction of a dedicated tool to assist the user also should be considered. After the preliminary analysis of a video clip section, a special filter would be able to indicate the areas of maximum traffic, providing a good basis for precise location of the required conflict spots.

\section{H.10 ADDRESSING CAMERA VIBRATION}

The necessary software consists of three items:

- The first filter for the VirtualDub (VD) application which executes the "unshake" procedure (see S1.1).

- The second filter for VD implements processing algorithms described in Section H.2 (optical flow and segmentation), then performs visualisation of the final segmentation and writes the file with the "raw" results (the second mode of the filter operation is the visualization of the video clip with overlayed detection areas, stop lines and ROI). 
- The application filtering the data obtained from the VD filter, generating the vehicles list and then calculating the GT and PET times for the detection areas, occluded (covered) by consecutive vehicles, moving along directions that are perpendicular to each other.

For the first filter the user should define a rectangle bordering the object to be observed.

The input data for programs 2 and 3 are read from shared configuration files:

- the text file, which contains the information on the other files, used or created by particular applications, descriptions of conflict spots and stop lines, as well as other data, allowing identification of the video clip, the recording time etc. (detailed description and example can be found in Section H.6),

- Two binary graphic files (*.bmp), defining the location of individual conflict spots and stop lines, and defining the occluded areas (see Section H.7).

\section{S1.H.5.1 Installation}

The first step consists of installation of the VirtualDub program ${ }^{4}$ and the "dvcodec" decompressor (Video for Windows) ${ }^{5}$, required for playing the video clips. Installation of the VD program comprises the archive decompression and creation of the respective desktop shortcut if required. The "unshake.vdf" and "traffic20.vdf" filters should be copied to subdirectory "Plugins" in the VD installation directory. The application interpreting the results obtained from VD program (traffic.exe) should be copied to the subdirectory, where the output files and text configuration files will be stored. All of the configuration files (both text and graphic files), related to the video clip being processed, should be additionally copied to the video clip location.

\section{S1.H.5.2 Video clip analysis}

The present procedure describes a method for running the VirtualDub program with the required filters. It is assumed that the video clip and the respective configuration files are located in one

\footnotetext{
${ }^{4}$ the required VD version is 1.5 .10 .

${ }^{5}$ both the VD archive (version 1.5.10) and a working codec version dvdemocodecv2.1.exe have been stored in the accompanying software package.
} 
directory. The order of the described operations is essential - it is particularly important to read in the video clip first, and only then define the required filters.

In the analyzed video clip, a fixed scene element should be found, which can be used in the vibration removal algorithm. The element should be:

- small,

- contrasted,

- characterized by a complex outline (not too simple),

- located outside the Region of Interest,

- located at least 10 pixels from the picture border,

- free from obscuring by any other scene elements.

For such an element a rectangle should be defined (enveloping the object and at least two pixels of its neighborhood) by saving the coordinates of its corners (x1, y1) and (x2, y2). These points respectively define the upper left and lower right corners of the rectangle. It is assumed that the origin of the coordinate system $(x=0, y=0)$ is located in the upper left corner of the picture. The coordinates are defined for the respective picture (clip) resolution, i.e., 720x480 pixels, by using any bitmap graphic manipulation program.

The user may attempt to automate some of the operations, using the VD program scripts or VD batch mode (see S1.H.5.4). In the list below the basic, "manual" method is described:

- start the VirtualDub (ver. 1.5.10) program,

- $\quad$ read in the video clip to be analyzed,

- decrease by half the window used for playing the source clip (ClickR the left window, choose " $50 \%$ " option),

- $\quad$ select "Filters" from the "Video" menu: an empty window appears,

- click "Add": a list of available VD filters appears. Choose:

resize, specify the expansion parameters (1440 960) and select the interpolation method (filter mode = bilinear, see Figure $\mathbf{H}-14$ ),

unshake, specify the previously determined corners of the rectangle enveloping the object (see Figure H-15), 
resize, specify the parameters $(360240)$ and select the interpolation method (filter mode $=$ bilinear),

$>$ traffic $2.0^{6}$, specify the name of the configuration file for the analyzed clip (see Figure H-16). Type in the name of the text configuration file (in the example in the Figure H-16 it is the "97901_10c_conf.txt" file) and choose the appropriate options: (a) if the user does not mark any of the respective fields it results in starting the analysis with visualization of its results in the right window of the VD program, (b) if the user marks the "Turn off visualization of analysis" field the results of the analysis will not be shown (the program runs a bit faster), and (c) if the user marks the "Only visualization of stops and spots" field the video clip will not be analyzed, and in the right window the source clip will be played with configuration elements overlaid (useful for results revision or generation of the ground data)

$>$ click $\mathrm{OK}$ in the configuration window and then $\mathrm{OK}$ in the filter selection window,

- from the Video/Frame rate menu mark decimate by 3 field,

- in the main window of the VD program start the analysis (and/or visualization) using the respective button ("output playback" or "key next" - step operation). The screen appearance during the analysis is shown in Figure H-18.

\footnotetext{
${ }^{6}$ traffic2.0 is a new version of a previous filter traffic1.3, adjusted to the needs of the unshake filter
} 


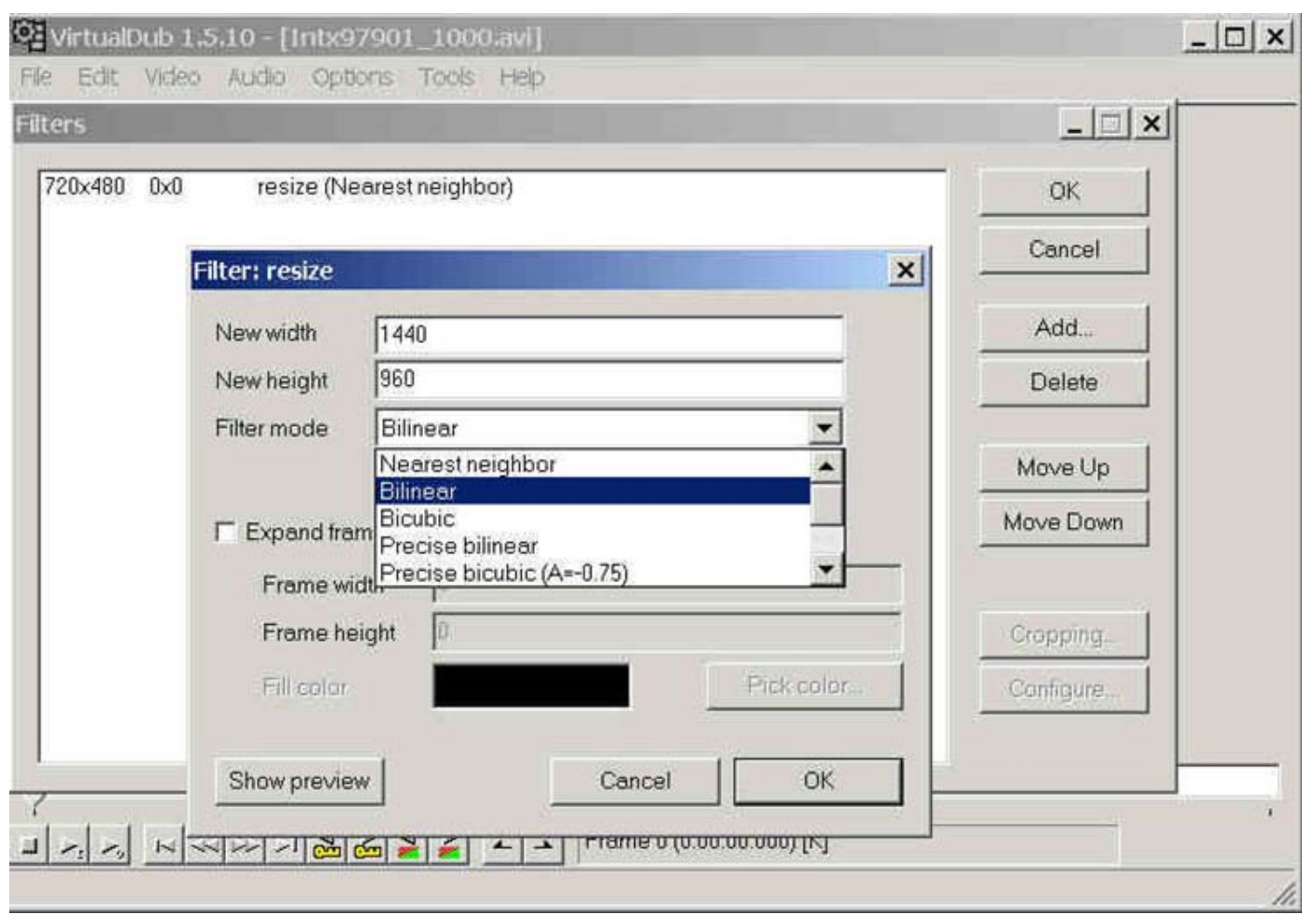

Figure H-14: Configuration of the First Filter (“resize”)

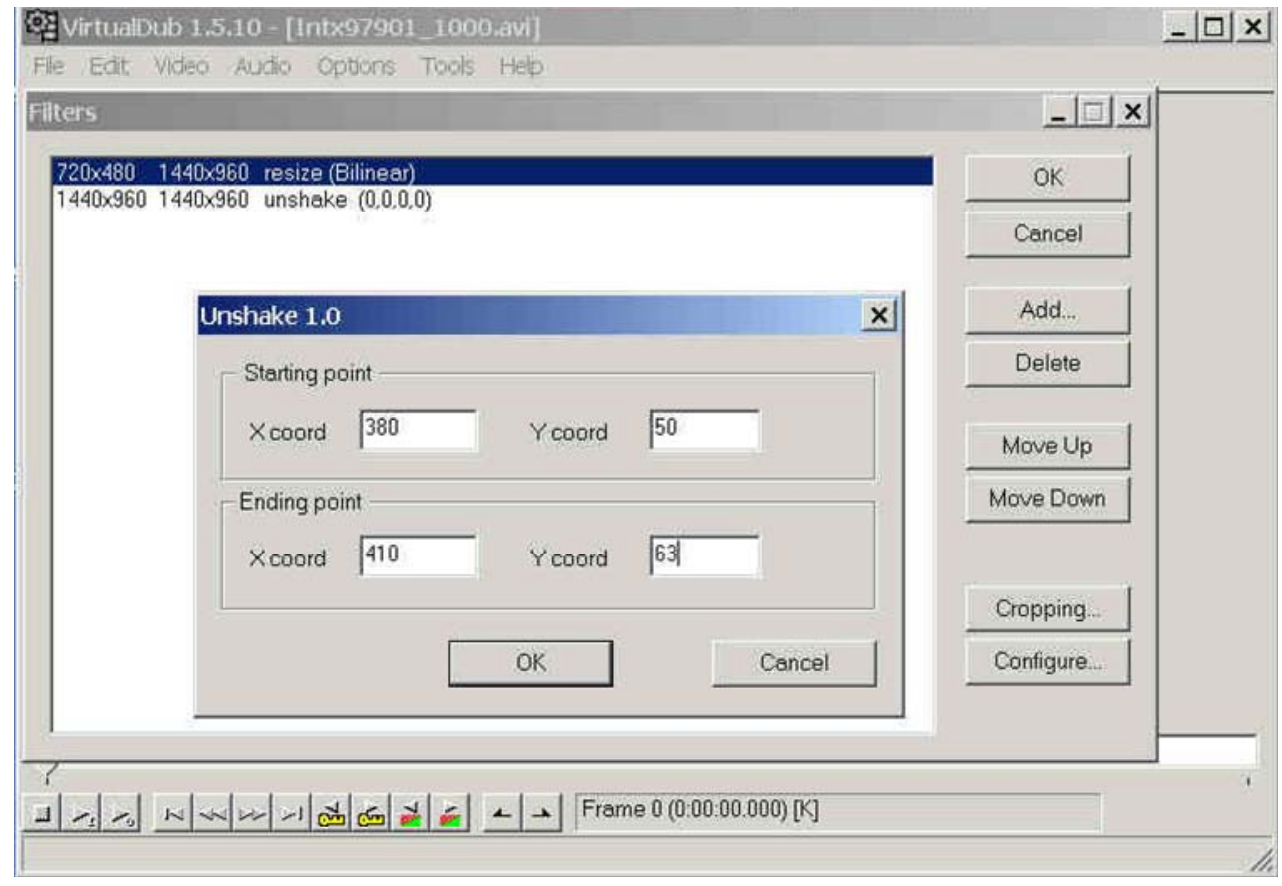

Figure H-15: The "unshaken” Filter - Specifying the Coordinates of the Object Selected 


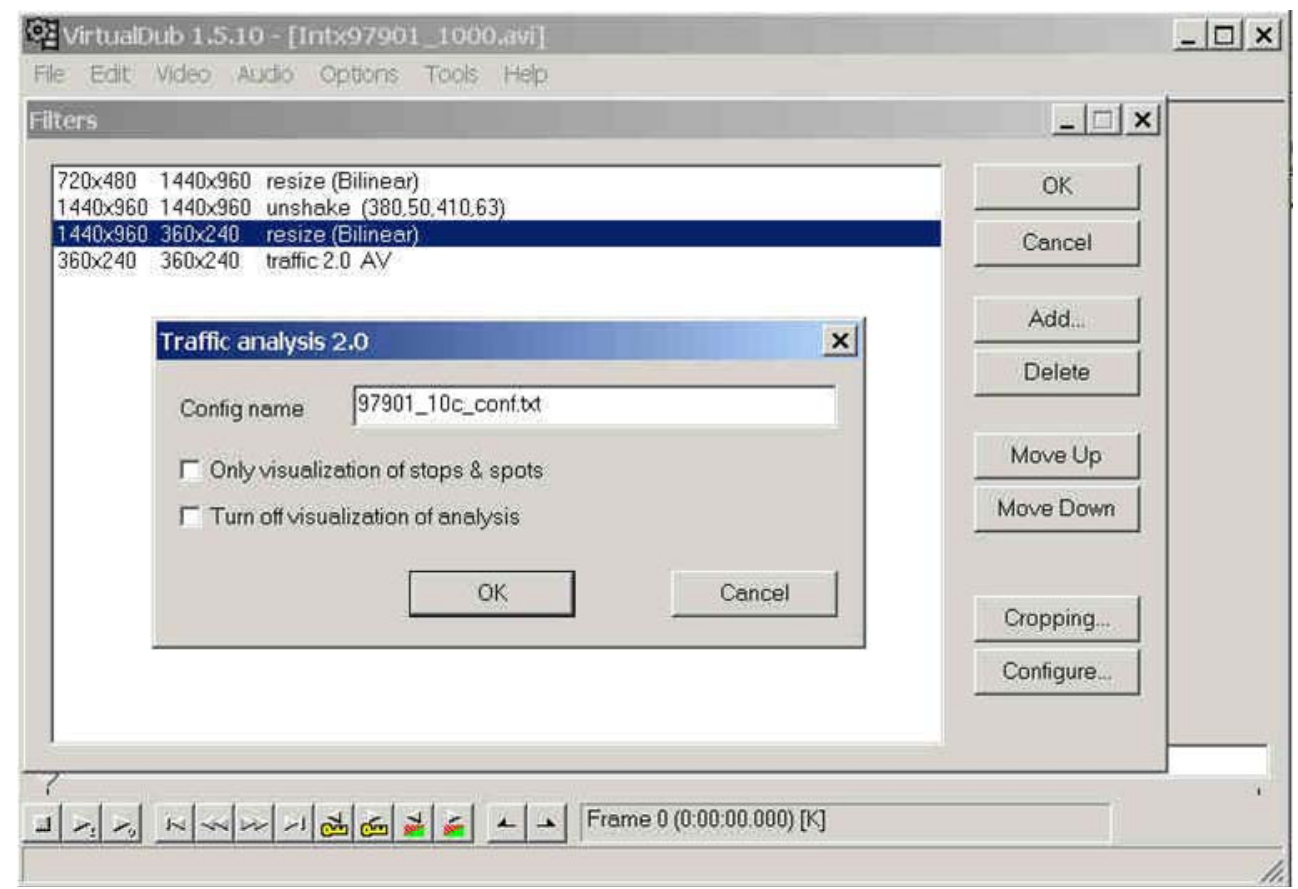

Figure H-16: Configuration Window for the "traffic 2.0" Filter

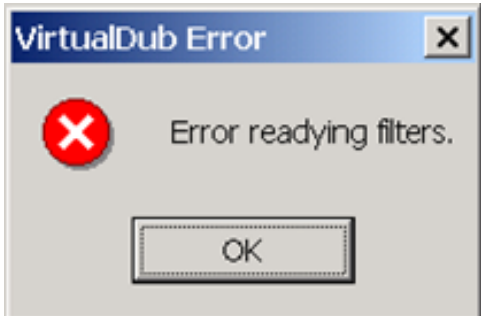

Figure H-17: Error Window

ATTENTION: The "traffic.vdf" filter generates a file containing the output data, with a filename consistent with the name declared in the text configuration file. If the VirtualDub program is stopped and then restarted, it generates an error message (see Figure H-17) caused by the fact that the respective file cannot be created as it already exists. The user has to remove the file or comment out the respective line in the text configuration file (the line with the LOG name - see Section H.6): after that step, the program will not perform the file creation.

After completing the video clip analysis the next program should be run, which removes the excessive elements from the file and generates the conflict list in a format that can be processed 
using a spreadsheet application (the number fields are separated by semicolons). The program is started from the command line by typing:

\section{traffic $<$ conf $>$}

where $<$ conf $>$ is the name of the text configuration file. The "Traffic.exe" program reads from that file information concerning the stop lines, conflict directions, starting time, filenames for the input and output files and records added by the user.

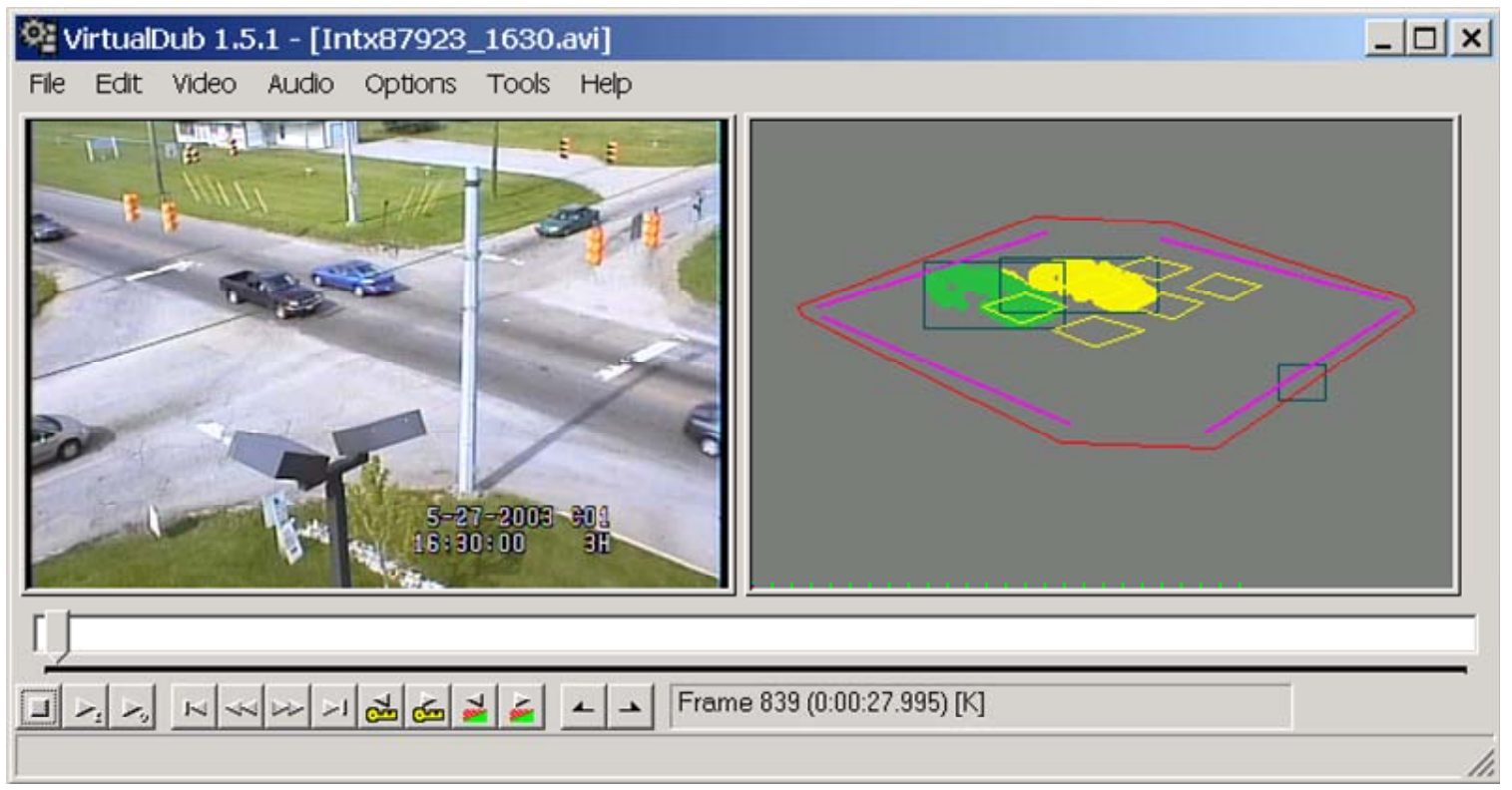

Left: source image, right: segmented objects (yellow and green ones) inside ROI (red). STOP lines and conflict spots are drawn in violet and yellow respectively.

Figure H-18: Image Analysis by VirtualDub and User-Defined Filters

\section{S1.H.5.3 Input and output files}

The "Traffic20.vdf" filter and the "Traffic.exe" program read in and create files according to the following scheme (see Figure H-19 - file labeling consistent with the names discussed in Section H.6). 


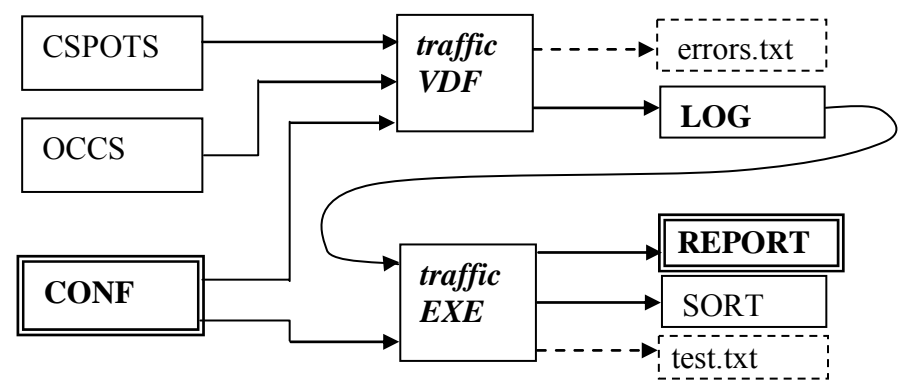

Figure H-19: Files and Programs

The key file, required for correct operation of both programs, is the text configuration file (CONF). Its detailed structure is described in Section H.6. In addition to that file, the "Traffic20.vdf" filter can read the bitmap files CSPOTS and OCCS (see Section H.7). In those graphic files the conflict spots and stop lines (CSPOTS), as well as occluded areas (OCCS), are defined. The "Traffic20.vdf" filter also generates the LOG file, which contains records for all of the objects detected in a given frame. In cases when processing errors are encountered the respective error messages are appended to a file with a fixed name "errors.txt."

The "Traffic.exe" program reads in the data from the CONF file and then processes the file with a filename specified in the LOG line. The main result of its action is the REPORT file, in which all the events associated with detected conflicts are recorded. The SORT file is a result of the LOG file filtering: it contains records of consecutive objects registered. Additionally, one more file with a fixed name "Test.txt" is created. It contains records of all the objects moving straight.

The LOG, SORT, and "Test.txt" files exhibit similar record structures. Each record is attributed to one object. Consecutive data items are separated by semicolons and denote in sequence:

1. end time of the recording (in frames)

2. object number

3. code of the first Sstop line or spot

4. entrance time for the first stop line or spot

5. departure time for the first stop line or spot

n. code of the $n$-th stop line or spot

$\mathrm{n}+1$. entrance time for the $\mathrm{n}$-th stop line or spot

$\mathrm{n}+2$. departure time for the $\mathrm{n}$-th stop line or spot

The "Test.txt" file exhibits a similar structure, with the only difference that after the object number (in square brackets) the numbers of entrance area and departure area are written. 


\section{S1.H.5.4 Batch mode}

Batch mode (File/ Job control) is the fastest computing method (see Table H-3) because there are no delays caused by video clip input/output display. To use it effectively one should understand the concept of Sylia scripting (Lee, 2003). Below we present a very simplified example obtained from "trials and errors": the automatically generated script has been manually changed to match our needs.

Let us assume that in the directory h: \digitalvideo the following files exist:

- intx97901_1000.avi - video clip to be analyzed,

- 97901_10c_conf.txt - text configuration files for intx97901_1000.avi,

- graphic configuration files defined in text configuration files.

There should be no LOG files defined in text configuration files.

After executing steps 1-6 (see S1.H.5.2) the user should select "File/ Save as AVI" menu option. The window entitled "Save AVI 2.0 File" appears: the user should write a file name (in this case “2x"), mark the box pointed by white arrow (see Figure H-20) and click "Save". At this moment the first job is appended to "VirtualDub.jobs" file.

If needed - the same steps should be repeated for the second video clip to be analyzed.

Now it is the time to close VirtualDub program and change the name of "VirtualDub.jobs" file (eg. to "vibra97901c.jobs"). This slightly modified file (changes are underlined and in bold) is presented in Box S1.A1.

The three manual modifications listed below remain unchanged - in relation to the previous script version:

- the full path to the CONF file and two quotation marks are added to the line describing the "traffic20.vdf" filter parameters,

- the instruction responsible for AVI saving is commented (using two slashes //),

- the instruction starting VirtualDub in "preview" mode is added. 


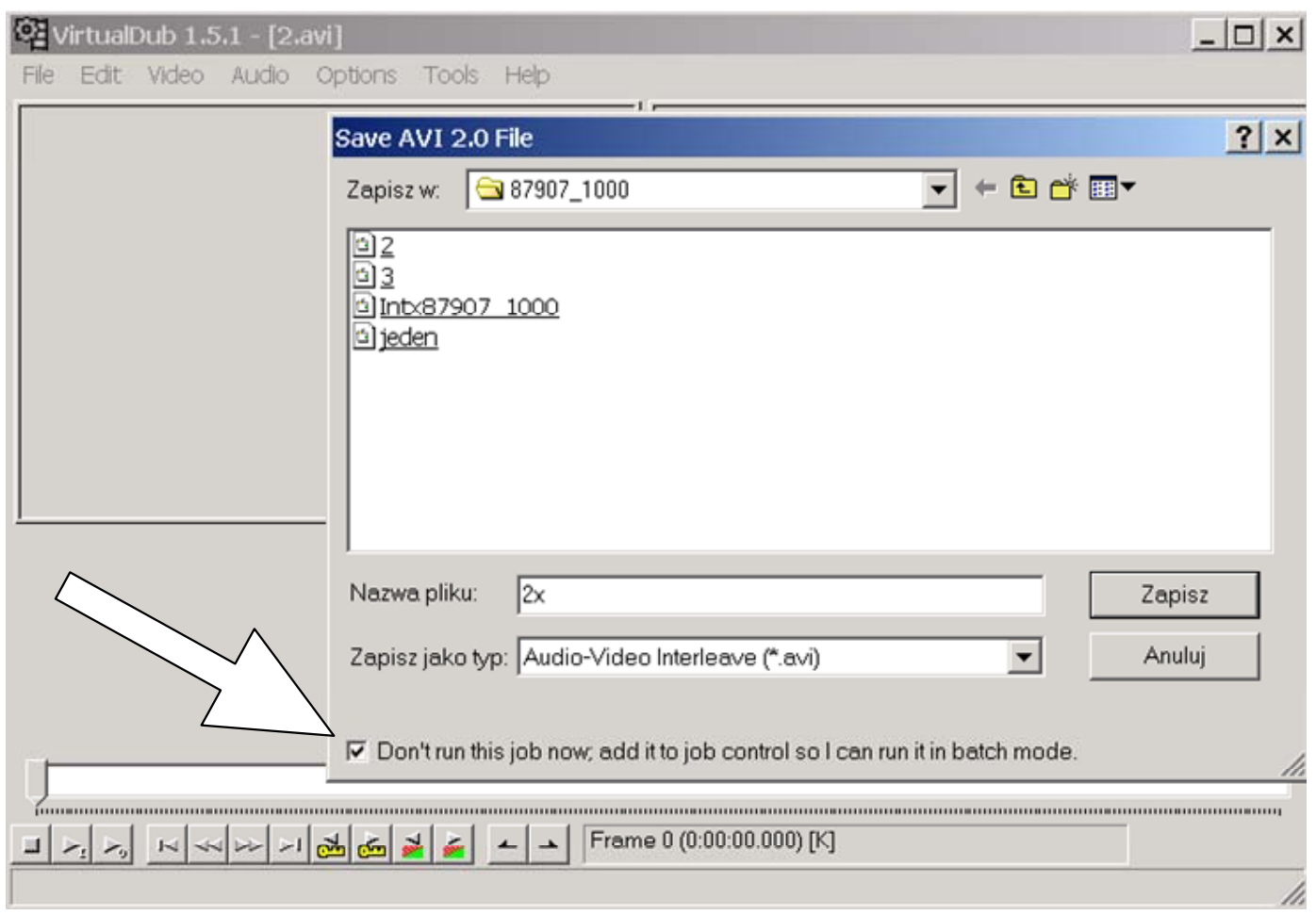

Figure H-20: Writing the Script to be Executed in Batch Mode

Changes introduced in relation to the previous version of the script can be divided into two categories:

- changes, that has been introduced by the software modification, as a consequence of the camera vibration removal (they are labeled by a consecutive number and a "+" character)

- changes introduced by use of newer version of the VirtualDub program (they have been labeled by a consecutive number and the "*” character).

Changes introduced as a consequence of the software modification (1) are:

- $1+$ the second argument is now equal to 3. It is responsible for the decimation, i.e., the generation of every third frame taken from the video clip,

- $2+$ and the following line form a definition of the first filter, the task of which is to increase the frame size twice, using the "bilinear" approximation,

- $3+$ and the following line are responsible for adding the "unshake" filter and defining in the next line the rectangle enveloping the object to be tracked, 
- $4+$ and the following line form a definition of the filter decreasing the frame size to 360x240 pixels (method: "bilinear"),

- 5+ and the following line denote addition of the "traffic" filter in 2.0 version, adjusted to the needs of the "unshake" filter.

Changes introduced by the use of the VirtualDub program (2) in version 1.5.10:

- $1^{*}$ : is a line changed automatically (the word "Delete" has been replaced by the word "Clear"),

- $2^{*}$ : is a new line, determining the range of the video clip analysis (in frames).

The last step is to carefully check if all of the files mentioned in CONFs exist and start VirtualDub again. To enter the batch mode, one should choose "File/ Job control" option from the main menu, load (File/ Load job list...) the appropriate *.jobs file (in our case "vibra97901c.jobs), mark the first job and press START. In Figure H-21, the larger window (in background) shows the particular jobs and their status. The small foreground window provides information on the progress of the job under execution. When all of the jobs are finished, the small window disappears. 
Box S1.A1. The modified file "vibra97901c.jobs" (manual changes are underlined boldface)

// VirtualDub job list (Sylia script format)

// This is a program generated file -- edit at your own risk.

$/ /$

// \$numjobs 1

$/ /$

// \$job "Job 1"

// \$input "d:\digitalvideo\intx97901_1000.avi"

// \$output "D:\DigitalVideo\2x.avi"

// \$state 0

// \$start_time 00

$/ /$ \$end_time 00

// \$script

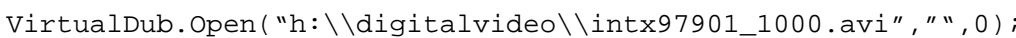

VirtualDub.audio.SetSource(1);

VirtualDub.audio.SetMode $(\odot)$;

VirtualDub audio.Set Interleave $(1,500,1,0,0)$;

VirtualDub . audio.SetClipMode $(1,1)$;

VirtualDub audio. SetConversion $(\odot, \odot, \odot, \odot, \odot)$;

VirtualDub.audio.SetVolume( );

VirtualDub. audio. SetCompression( );

VirtualDub audio. EnableFilterGraph $(\odot)$;

VirtualDub.video.SetDepth $(24,24)$;

VirtualDub.video.SetMode(3);

$1+$ VirtualDub.video.SetFrameRate $(0,3)$;

VirtualDub.video.SetIVTC $(0,0,-1,0)$;

VirtualDub.video.SetRange $(0,0)$;

VirtualDub.video.SetCompression( );

VirtualDub.video.filters.Clear();

2+ VirtualDub.video.filters.Add("resize");

VirtualDub.video.filters.instance [0].Config $(1440,960,1)$;

$3+$ VirtualDub.video.filters.Add("unshake ");

VirtualDub.video.filters.instance [1] . Config $(380,50,410,63)$;

4+ VirtualDub.video.filters.Add("resize");

VirtualDub.video.filters.instance [2] .Config(360, 240,1);

$5+$ VirtualDub.video.filters.Add("traffic 2.0");

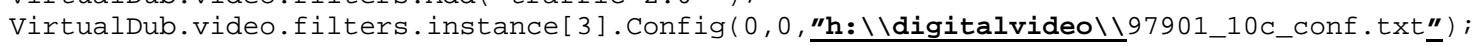

VirtualDub.audio.filters.clear ();

1* VirtualDub. subset.Clear ();

2* VirtualDub. subset. AddRange $(\odot, 112084)$;

//VirtualDub.SaveAVI("h: \\igitalVideo\\2x.avi");

Virtualdub.Preview();

VirtualDub.Close( );

// \$endjob

$/ /$

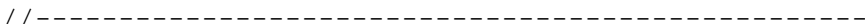

$/ /$ \$done 


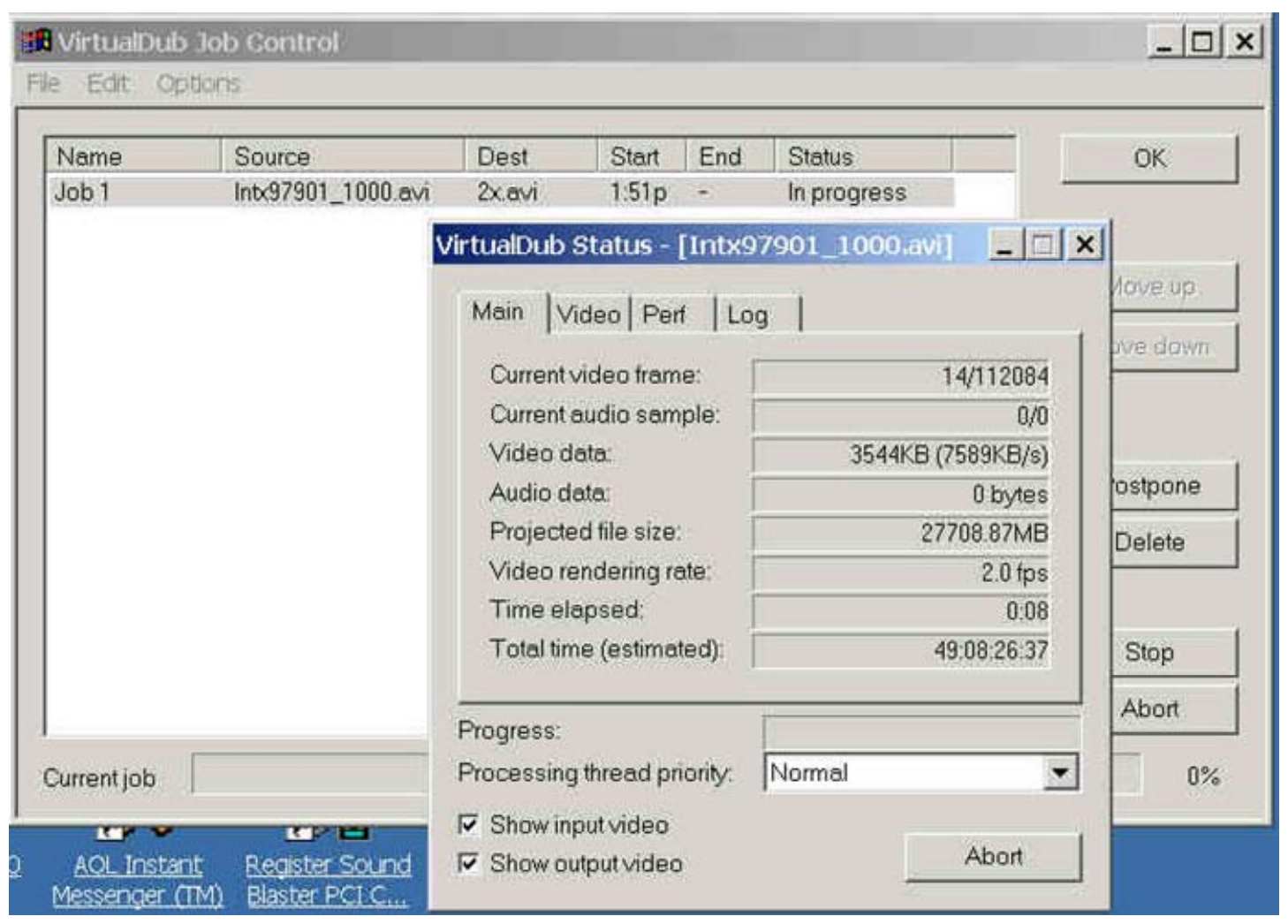

Figure H-21: Executing Jobs in “batch” Mode

\section{S1.H.8 Package ver. 2.0 contents}

The package contains the following files:

Software:

traffic20.vdf: VirtualDub Filter ver. 2.0

Traffic11.exe: program which generates the final REPORT on detected conflicts (ver. 1.1)

traffic2.zip: the two above mentioned files zipped

VirtualDub-1.5.10.zip: VirtualDub program (ver. 1.5.10)

dvdemocodecv2.1.zip: demo (version 2.1) of the DV codec

doc res:

0supp1.doc: the main part of Supplement 1

app_s1_ad.doc: Section H.10

vibra97901c.jobs: the example of the "batch" file

GapData_res5.xls: results of processing compared to the ground data, summary of recognition rates 


\section{H.11 BIBLIOGRAPHY}

Barron, J. L., D. J. Fleet, and S. S. Beauchemin (1994). "Performance of Optical Flow Techniques." Int. Journal of Computer Vision, Vol. 12, No. 1, pp. 43-77.

Beauchemin, S. S. and J. L. Barron (1995). "The Computation of Optical Flow." $A C M$ Computing Surveys, Vol. 27, No. 3, pp. 433-467.

Galvin, B., B. McCane, K. Novins, D. Mason, and S. Mills (1998). "Recovering Motion Fields: An Evaluation of Eight Optical Flow Algorithms." Proc. of the British Machine Vision Conference (BMVC), September.

Horn, B. K. P. and B. G. Schunck (1981). "Determining Optical Flow." Artificial Intelligence, Vol. 17, pp. 185-204.

Horn, B. K. P. and B. G. Schunck (1993). "Determining Optical Flow: A Retrospective." Artificial Intelligence, Vol. 59, pp. 81-87.

Lee, A. (2003). VirtualDub Version 1.5.1. http://virtualdub.org.

Liu, H., T. Hong, M. Herman, T. Camus, and R. Chellappa (1998). "Accuracy vs. Efficiency Trade-offs in Optical Flow Algorithms." Computer Vision and Image Understanding (CVIU), Vol. 72, No. 3, pp. 271-286.

Lucas, B. D. and T. Kanade (1981). "An Iterative Image Registration Technique with an Application to Stereo Vision." Proc. of 7th Intl. Joint Conf. on Artificial Intelligence (IJACAI), August 24-28, Vancouver, pp. 674-679.

Mikrut, Z., and K. Pałczyński (2003). "Segmentation of the Videodetector Images basing on Optical Flow Computation.” AGH UST Automatics, Kraków (in Polish).

Pratt, W. K. (1988). Digital Image Processing. John Wiley \& Sons, New York. 